\title{
Electronic communication in heterometallated porphyrin oligomers
}

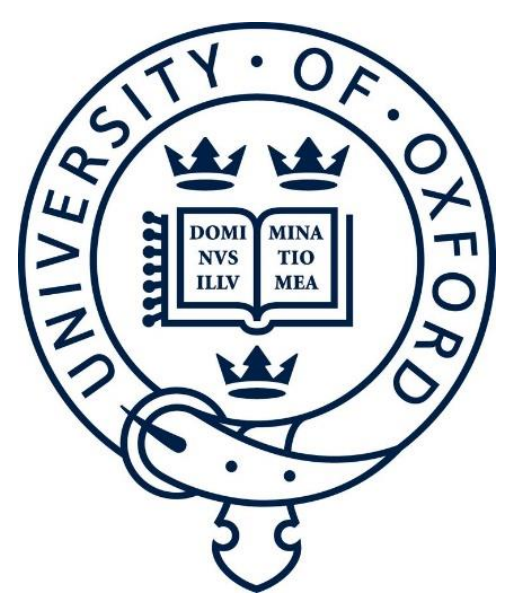

Jonathan Cremers

Keble College

University of Oxford

A thesis submitted for the degree of

Doctor of Philosophy

Michaelmas 2017 



\section{Acknowledgements}

I have been incredibly fortunate to have had the opportunity to work with Prof. Harry Anderson over the past few years. His interest, enthusiasm and dedication as a chemist and supervisor have been genuinely motivating and have been an essential factor in the successful outcome of my DPhil.

Special thanks also go to my co-supervisor Prof. Andrew Briggs. Our termly discussions have been stimulating, encouraging and extremely useful. I truly admire his devotion in the pursuit of good science and I can only hope that some of his passion has rubbed off on me.

A particular thank you to all the members of the Anderson group with whom I have had the pleasure of working. It's a strange thing to realise that not a single member of the group who was here when I started is here when I finish, yet the ambience of the group remains as good today as it was the day I started. It has been genuinely enjoyable to work with every one of you. While every member has contributed to the amazing atmosphere of the group, particular thanks to: Dmitry, Patrick, Arjen, Julien, Ludovic, Levon, Ibrahim, Cecile, Martin, Anjul, Isa, Dan, Renee, James, Will, Thomas, Pernille, Bart, Przemyslaw, Michel, Steffen, Sabine and Yaoyao for their various contributions to an unforgettable few years.

Parts of the results discussed in this thesis have been the work of several excellent collaborators in Oxford: Dr Jennifer le Roy (magnetic studies) and her supervisor Prof. Lapo Bogani, Dr Sabine Richert (EPR) and her supervisor Prof. Chris Timmel, Dr Juliane Gong (photophysics) and her supervisor Prof. Laura Herz. In addition, thanks go to Prof. Tim Claridge, Dr Jan Mol and Dr Pascal Gehring for helpful discussions. I would also like to show my gratitude to Giulia and Isabel for allowing me work with them and teach them the limited amount of chemistry knowledge I've build up over the years.

Most of this work was generously funded by the European Research Council and the John Templeton Foundation. Additional funding came from Keble College and the Department of Chemistry.

Finally I would like to acknowledge a group of people who might not have directly contributed to the science but who have been an important factor for my happiness over the last few years. I am therefore very thankful for the support of my friends and family both in and out of Oxford. 



\section{Abstract}

This thesis presents the synthesis and characterisation of a range of heterometallated porphyrin oligomers and other novel 3D $\pi$-conjugated porphyrin nanostructures. Subsequently, their physical organic properties were evaluated which revealed some fascinating electronic properties.

Chapter 1 summarises some of the work done in the Anderson group on porphyrin nanostructures and reviews the literature regarding heterometallated porphyrin oligomers. In addition it introduces the main concepts and techniques used in the remainder of the thesis.

In Chapter 2 the stabilities of a family of four linear porphyrin pentamer complexes are determined by UV-vis-NIR titrations and analysed using chemical double-mutant cycles which reveal that the binding energy of the copper centre to an axial pyridine ligand is $-6.2 \mathrm{~kJ} \mathrm{~mol}^{-1}$. Subsequently, the $\mathrm{Zn}-\mathrm{Zn}-\mathrm{Cu}-\mathrm{Zn}-\mathrm{Zn}$ pentamer is used in the synthesis of a heterometallated 10-porphyrin nanoring.

Chapter 3 will describe the investigation of quantum interference phenomena in a bis-copper six-porphyrin nanoring by using EPR spectroscopy. We show that the exchange coupling between two spin centres is increased by a factor 4.5 in the ring structure with two parallel coupling pathways as compared to an otherwise identical system with just one coupling path.

In Chapter 4 the syntheses of three isomers of the bis-copper 6-porphyrin nanoring are described. DFT calculations have indicated potential destructive interference phenomena in one of the isomers which would allow for the formation of a molecular system with behaviour resembling that of a hypothetical molecular interferometer.

Chapter 5 reports on the template-directed synthesis of a $\pi$-conjugated 14-porphyrin nanoball. This bicyclic structure consists of two intersecting nanorings of 6 and 10 porphyrin units. Fluorescence up-conversion spectroscopy experiments demonstrate that electronic excitation delocalises over the whole $3 D \pi$-system within $0.3 \mathrm{ps}$ if the nanoball is bound to its templates or within $5 \mathrm{ps}$ if the nanoball is empty.

In Chapter 6 the synthesis and characterisation of a $D_{4 h}$ symmetric analogue of the porphyrin nanoball is described. The structure consists of ten porphyrin units arranged as two perpendicular 6-porphyrin nanorings intersecting at two porphyrins. In the synthesis, a combination of magnesium and zinc porphyrins are used which allows for the introduction of a selective demetallation method crucial for accessing this novel structure. 



\section{Supplement to Statement of Authorship}

In Chapter 2, the EPR distance measurements and DFT calculations summarised in the text have been recorded and processed by $\mathrm{Dr}$ Sabine Richert under the supervision of Prof. C. R. Timmel.

In Chapter 3, the quantification of the interspin exchange coupling between the two copper centres via EPR measurements summarised in the text have been recorded and processed by Dr Sabine Richert under the supervision of Prof. C. R. Timmel.

In Chapter 4, DFT calculations on the three isomers of the bis-copper 6-ring have been performed by Dr Martin Peeks.

In Chapter 5, the fluorescence up-conversion spectroscopy experiments have been performed by Juliane Q. Gong under the supervision of Prof. Laura M. Herz.

The DFT calculations described in Chapters 5 and 6 have been performed by Renee Haver. 

List of Publications xiii

$\begin{array}{lll}\text { List of Abbreviations } & \text { XV }\end{array}$

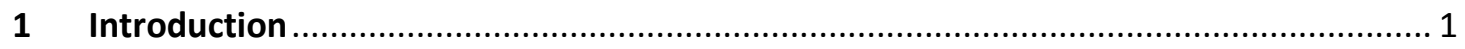

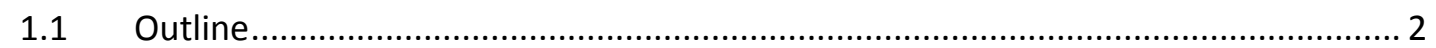

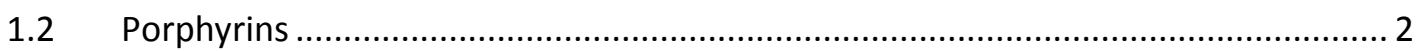

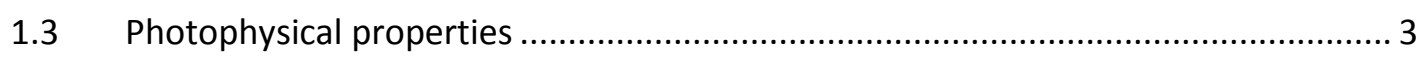

1.4 Axial coordination of metalloporphyrins ................................................... 5

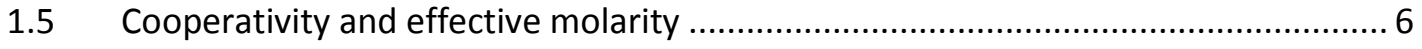

1.6 Template directed synthesis of porphyrin structures ................................... 9

1.7 Heterometallated porphyrin oligomers ...................................................... 14

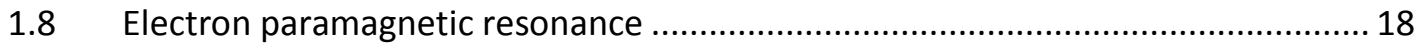

1.9 Quantum interference .............................................................. 21

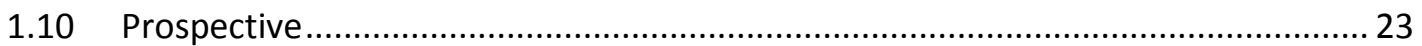

2 10-Porphyrin Nanorings with Copper(II) and Zinc(II) Centres................................. 25

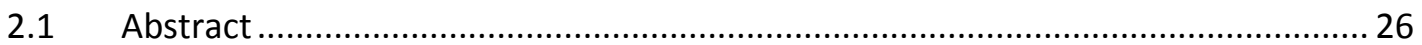

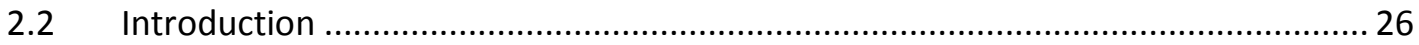

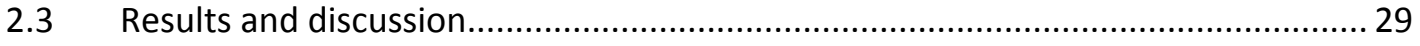

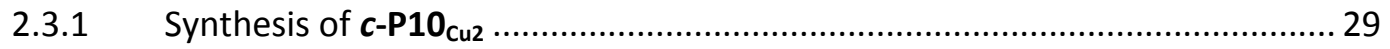

2.3.2 Quantification of the Cu-porphyrin pyridine interaction ............................. 33

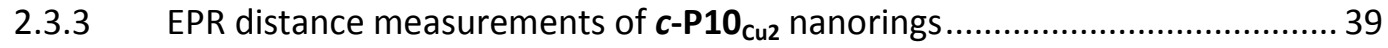

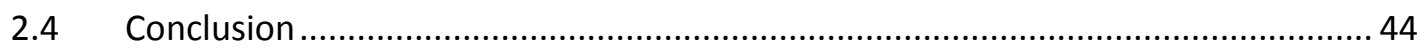

2.5 Experimental procedures and technical details .............................................. 45

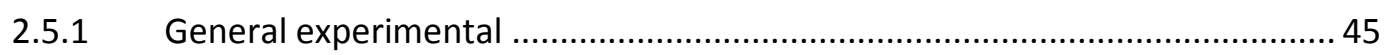

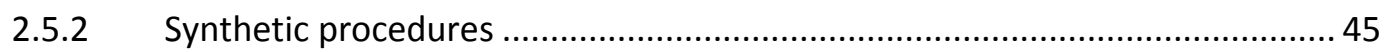

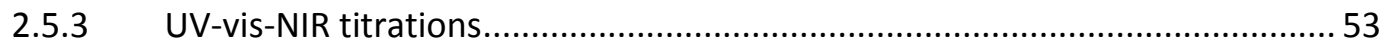

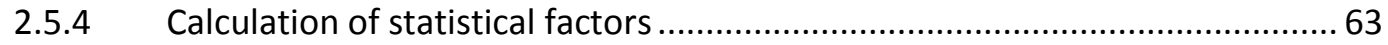

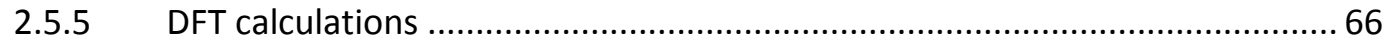

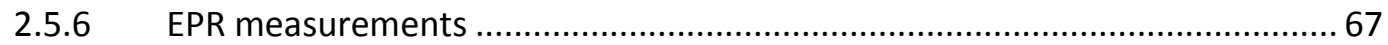

3 Constructive Quantum Interference in a Heterometallated Porphyrin Nanoring ....... 69

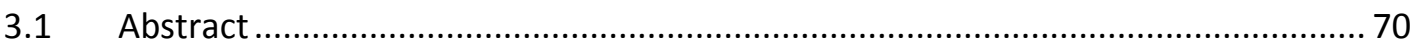




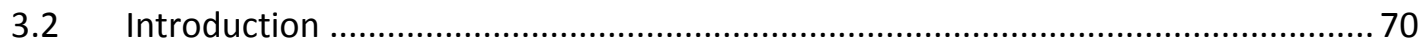

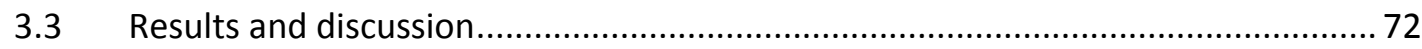

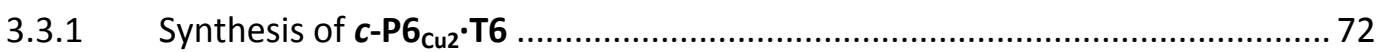

3.3.2 NMR spectroscopy of copper-containing oligomers........................................ 75

3.3.3 Quantification of the constructive interference ............................................. 78

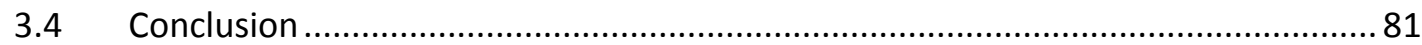

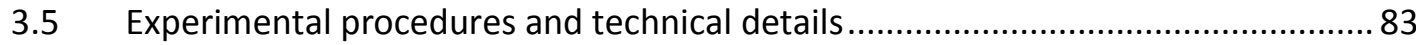

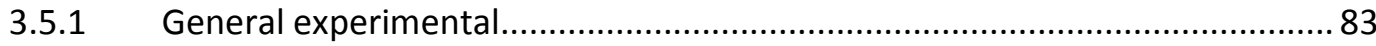

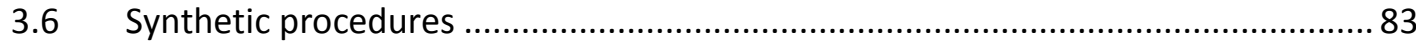

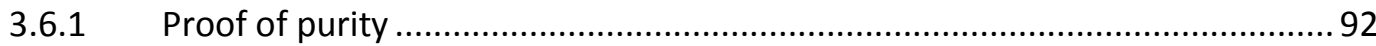

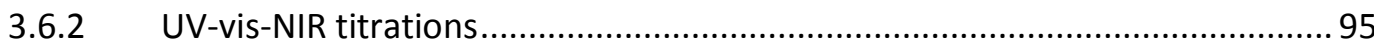

3.6.3 $T_{1}$ and $T_{2}$ relaxation time constant measurements..................................... 100

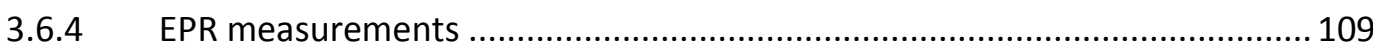

4 Synthesis of Ortho, Meta and Para Bis-Copper Porphyrin Nanorings......................... 111

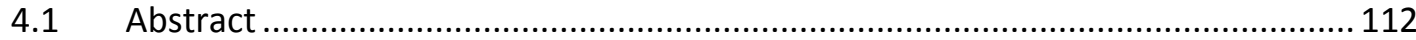

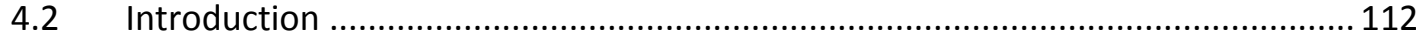

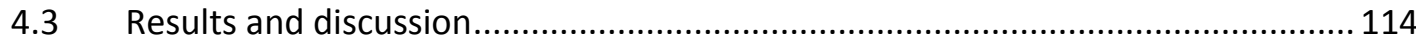

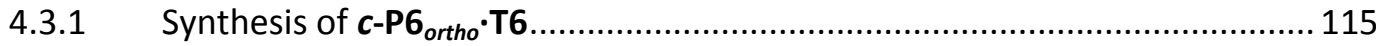

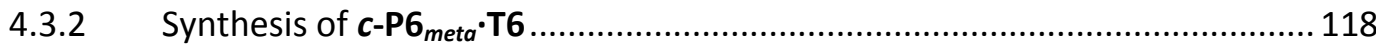

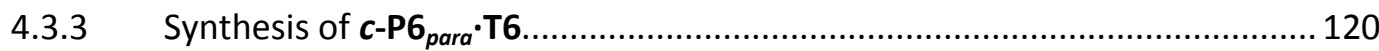

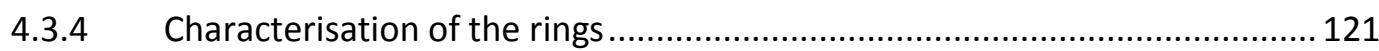

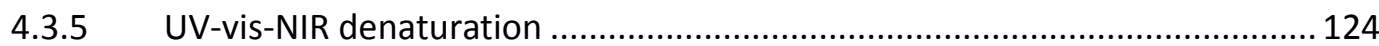

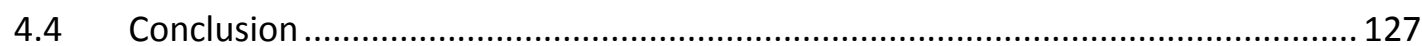

4.5 Experimental procedures and technical details ................................................ 128

4.5.1 General experimental........................................................................... 128

4.5.2 Synthetic procedures ............................................................................ 129

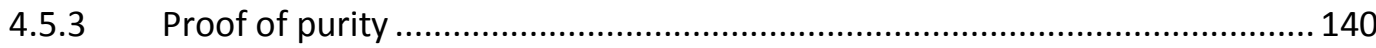

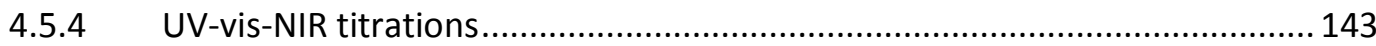

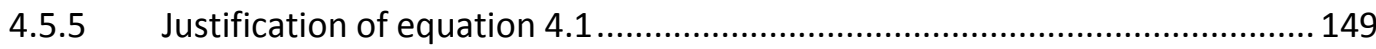

5 The Template-Directed Synthesis of a Fully Conjugated 14-Porphyrin Nanoball ........ 151

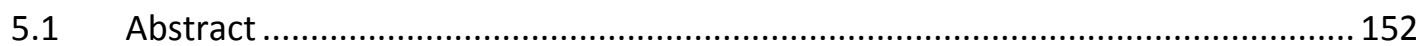

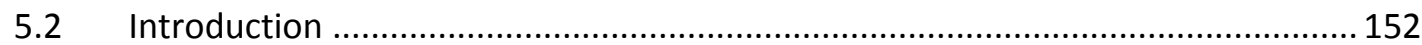

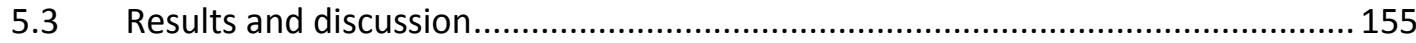




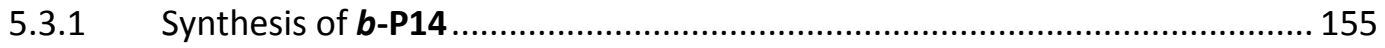

5.3.2 ${ }^{1}$ H NMR assignment of $\boldsymbol{b}$-P14-T6·(T4) $)_{2}, \boldsymbol{b}$-P14·T6 and $\boldsymbol{b}$-P14 ....................... 164

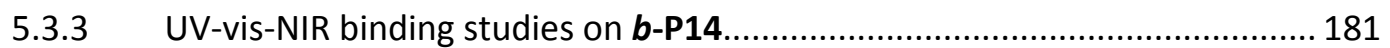

5.3.4 Photophysical properties of $\boldsymbol{b}$-P14 .......................................................... 183

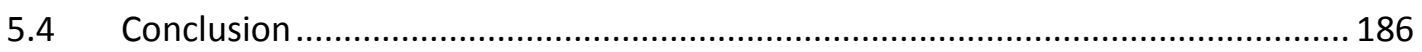

5.5 Experimental procedures and technical details ................................................... 186

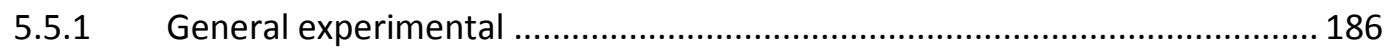

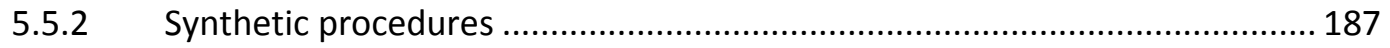

5.5.3 Spectra confirming identity of new compounds ............................................ 202

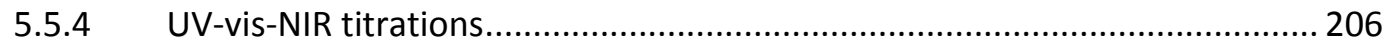

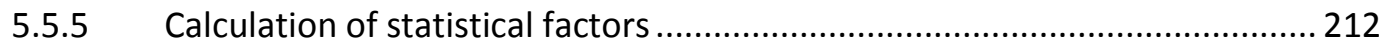

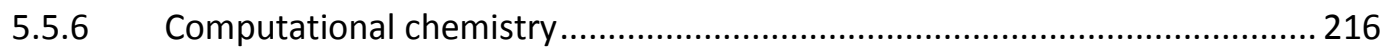

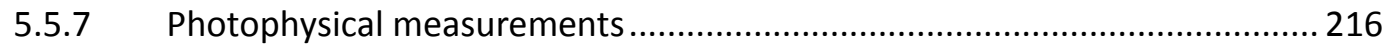

6 The Synthesis of a $D_{4 h}$ Symmetric Porphyrin Nanoball using Selective Demetallation 221

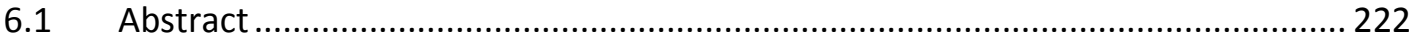

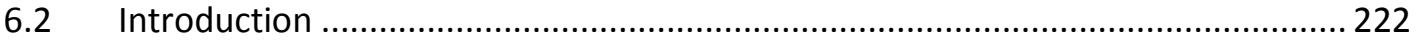

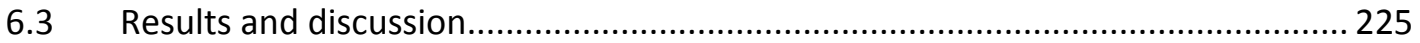

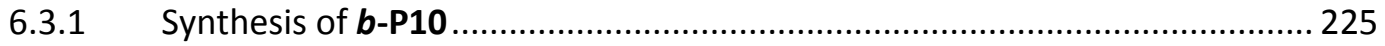

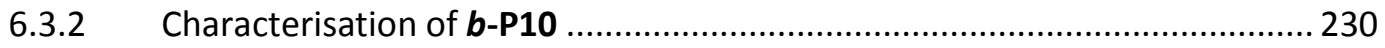

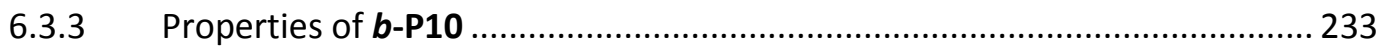

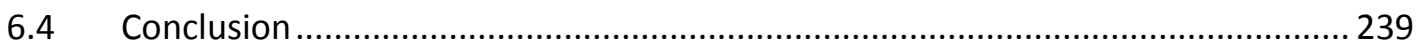

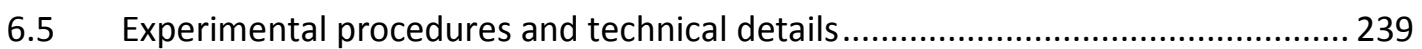

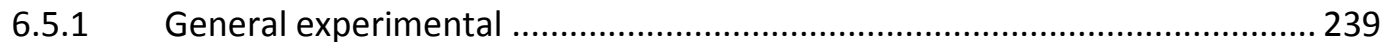

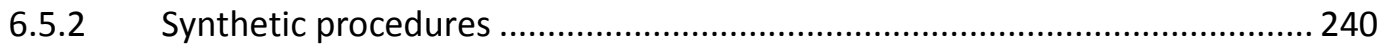

$\begin{array}{lr}\text { Reference } & 254\end{array}$ 



\section{List of Publications}

1. J. Cremers, R. Haver, M. Rickhaus, J. Q. Gong, L. Favereau, M. D. Peeks, T. D. W. Claridge, L. M. Herz, H. L. Anderson, 'Template-Directed Synthesis of a Conjugated Zinc Porphyrin Nanoball', submitted to: J. Am. Chem. Soc. 2018.

2. J. J. Le Roy, J. Cremers, I. A. Thomlinson, M. Slota, W. M. Myers, P. H. Horton, S. J. Coles, H. L. Anderson, L. Bogani, 'Tailored Homo- and Hetero- Lanthanide Porphyrin Dimers: a Synthetic Strategy for Integrating Multiple Spintronic Functionalities into a Single Molecule', submitted to: Chem. Sci. 2017.

3. S. Richert, I. Kuprov, M. D. Peeks, E. A. Suturina, J. Cremers, H. L. Anderson, C. R. Timmel, 'Quantifying the Exchange Coupling in Linear Copper Porphyrin Oligomers', Phys. Chem. Chem. Phys. 2017, 24, 16057-16061.

4. P. Gehring, J. K. Sowa, J. Cremers, Q. Wu, H. Sadeghi, Y. Sheng, J. H. Warner, C. J. Lambert, G. A. D. Briggs, J. A. Mol, 'Distinguishing Lead and Molecule States in Graphene-Based Single-Electron Transistors', ACS Nano 2017, 11, 5325-5331.

5. S. Richert, J. Cremers, I. Kuprov, M. D. Peeks, H. L. Anderson, C. R. Timmel, 'Constructive Quantum Interference in a Bis-Copper Six-Porphyrin Nanoring', Nat. Commun. 2017, 8, 14842-14847.

6. J. Cremers, S. Richert, D. V. Kondratuk, T. D. W. Claridge, C. R. Timmel, H. L. Anderson, 'Nanorings with Copper(II) and Zinc(II) Centers: Forcing Copper Porphyrins to Bind Axial Ligands in Heterometallated Oligomers', Chem. Sci. 2016, 7, 6961-6968.

7. S. Richert, J. Cremers, H. L. Anderson, C. R. Timmel, 'Exploring TemplateBound Dinuclear Copper Porphyrin Nanorings by EPR Spectroscopy', Chem. Sci. 2016, 7, 6952-6960.

8. L. Favereau, A. Cnossen, J. B. Kelber, J. Q. Gong, R. M. Oetterli, J. Cremers, L. M. Herz, H. L. Anderson, 'Six-Coordinate Zinc Porphyrins for TemplateDirected Synthesis of Spiro-Fused Nanorings', J. Am. Chem. Soc. 2015, 137, 14256-14259.

9. S. Liu, D. V. Kondratuk, S. A. L. Rousseaux, G. Gil-Ramirez, M. C. O'Sullivan, J. Cremers, T. D. W. Claridge, H. L. Anderson, 'Caterpillar Track Complexes in Template-Directed Synthesis and Correlated Molecular Motion', Angew. Chem. Int. Ed. 2015, 54, 5355-5359. 



\section{List of Abbreviations}

$\begin{array}{ll}\text { Ar } & \text { Aryl } \\ \text { B3LYP } & \text { Becke, 3-parameter, Lee-Yang-Parr } \\ \text { COSY } & \text { Correlation spectroscopy } \\ \text { CPDIPS } & \text { Cyanopropyldiisopropylsilane } \\ \text { CPDMS } & \text { Cyanopropyldimethylsilane } \\ \text { CPMG } & \text { Carr-Purcell-Meiboom-Gill } \\ \text { CSD } & \text { Cambridge Structural Database } \\ \text { d } & \text { doublet } \\ \text { DABCO } & \text { 1,4-diazabicyclo[2.2.2]octane } \\ \text { dba } & \text { dibenzylideneacetone } \\ \text { DDQ } & \text { 2,3-Dichloro-5,6-dicyano-1,4-benzoquinone } \\ \text { DEER } & \text { Double Electron-Electron Resonance } \\ \text { DFT } & \text { Density Functional Theory } \\ \text { DMC } & \text { Double Mutant Cycle } \\ \text { DNA } & \text { Deoxyribonucleic acid } \\ \text { EM } & \text { Effective Molarity } \\ \text { EPR } & \text { Electron Paramagnetic Resonance } \\ \text { THF } & \text { Tetrahydrofuran } \\ \text { GPC } & \text { Gel Permeation Chromatography } \\ \text { HOMO } & \text { Highest Occupied Molecular Orbital } \\ \text { LUMO } & \text { Lowest Unoccupied Molecular Orbital } \\ \text { m } & \text { multiplet } \\ \text { MALDI } & \text { Matrix-Assisted Laser Desorption/lonisation } \\ \text { NBS } & \text { N-Bromosuccinimide } \\ \text { NMR } & \text { Nuclear Magnetic Resonance } \\ \text { NOESY } & \text { Nuclear Overhauser Effect Spectroscopy } \\ \text { PL } & \text { Photoluminescence } \\ \text { RI } & \text { Resolution of the Identity } \\ \text { S } & \text { singlet } \\ \text { SCF } & \text { Self-Consistent Field } \\ \text { SEC } & \text { Size Exclusion Chromatography } \\ \text { SQUID } & \text { Superconducting Quantum Interference Device } \\ \text { STM } & \text { Scanning Tunneling Microscope } \\ \text { t } & \text { triplet } \\ \text { TBAF } & \text { Tetra-n-butylammonium fluoride } \\ \text { TFA } & \text { trifluoroacetic acid } \\ \text { THS } & \text { Trihexylsilane } \\ \text { TIPS } & \text { Triisopropylsilane } \\ \text { TLC } & \text { Thin Layer Chromatography } \\ \text { TMS } & \text { Trimethylsilane } \\ \text { UV-vis-NIR } & \text { Ultra-violet, visible and near infra-red } \\ & \end{array}$





\section{Introduction}

\section{Contents}

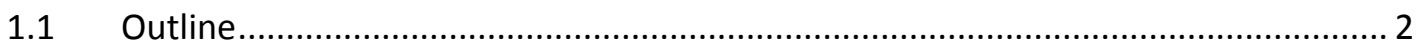

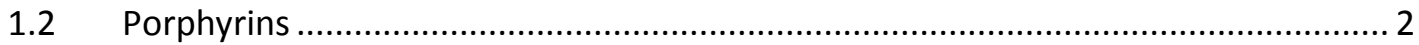

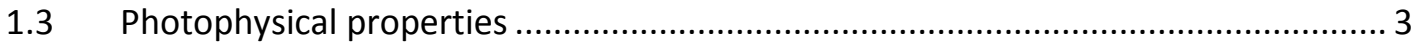

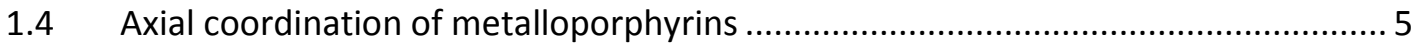

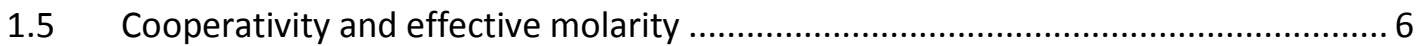

1.6 Template directed synthesis of porphyrin structures........................................... 9

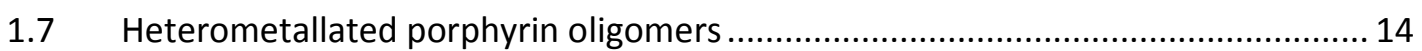

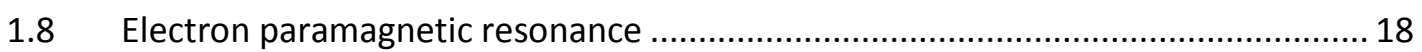

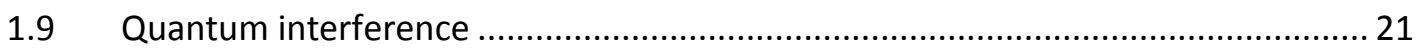

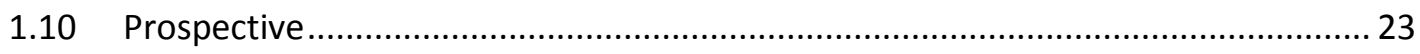




\subsection{Outline}

This thesis explores the design and synthesis of heterometallated oligo-porphyrin nanostructures and presents an investigation into the photophysical and electronic properties of this class of compounds. This introductory chapter presents a literature review of heterometallic porphyrin arrays, followed by an introduction to some of the techniques used to investigate the structures presented throughout this thesis.

\subsection{Porphyrins}

For decades, chemists have taken inspiration from nature in an attempt to mimic biological systems with artificial analogues. There has been a particular emphasis on porphyrins, which have been studied extensively due to their abundance in biological systems and fundamental importance to life on earth. ${ }^{1-5}$

Porphyrins are a family of compounds consisting of four pyrrole moieties connected by methine bridges. Porphine is the simplest example of a porphyrin in which all positions on the periphery of the macrocycle are substituted with hydrogen atoms. ${ }^{6}$ The systematic IUPAC numbering of the atomic positions of porphine is illustrated in Figure 1.1a. In this thesis the different positions on the periphery of the ring will be referred to by their common nomenclature, i.e. the methines will be labelled as meso, the quaternary carbons as $\alpha$ and the pyrrole carbons will be referred to as $\beta$.

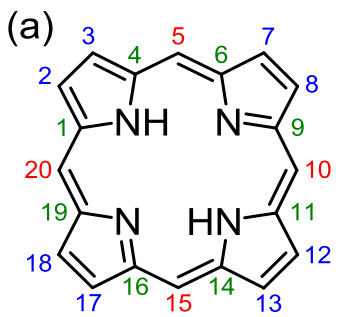

(b)

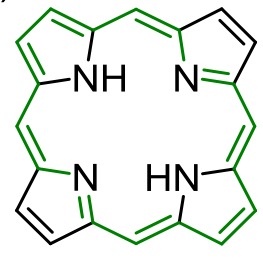

(c)

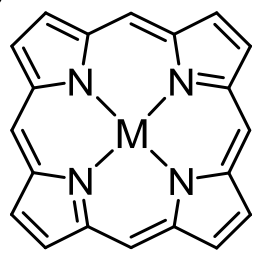

Figure 1.1: (a) Structure of free-base porphine with systematic IUPAC numbering. The common nomenclature for the positions are meso (red), $\alpha$ (green) and $\beta$ (blue). (b) The green marks indicate one of the conjugated $18 \pi$-electron pathways responsible for aromaticity in porphyrins. (c) General structure of a metalloporphyrin.

Porphyrins follow Hückel's rule and are aromatic. The molecular backbone contains 26 delocalised $\pi$-electrons of which 18 form a conjugated pathway (Figure 1.1b). Clear evidence of the aromatic ring current can be found in ${ }^{1} \mathrm{H}$ NMR spectroscopy. The internal $\mathrm{N}-\mathrm{H}$ protons are shielded $\left(\delta_{\mathrm{H}}=-2\right.$ to $\left.-4 \mathrm{ppm}\right)$ and while the external protons are deshielded ( $\delta_{\mathrm{H}}=11$ to $\left.8 \mathrm{ppm}\right)$. 
The centre of a porphyrin contains four nitrogen atoms which render them excellent ligands to accommodate for a wide range of metal centres (Figure 1.1c). Metal centres can be used in order to introduce functionality to the porphyrin. ${ }^{7,8}$ They can also form a convenient handle for the synthesis of supramolecular structures through the axial coordination of additional ligands as will be discussed later in this thesis. ${ }^{9}$

\subsection{Photophysical properties}

The electronic absorption spectrum of a typical porphyrin monomer consists of a strong transition to the second excited state $\left(S_{0} \rightarrow S_{2}\right)$ at approximately $400 \mathrm{~nm}$ (the Soret or B-band) and a weaker transition to the first excited state $\left(S_{0} \rightarrow S_{1}\right)$ at approximately $550 \mathrm{~nm}$ (the Q-band). ${ }^{10}$ There is rapid internal conversion from $\mathrm{S}_{2}$ to $S_{1}$ and therefore fluorescence is only detected from the $S_{1}$ state. The absorption spectrum of a porphyrin can be explained by considering the four frontier orbitals; two $\pi$ orbitals $\left(\mathrm{a}_{1 u}\right.$ and $\left.\mathrm{a}_{2 u}\right)$ and a degenerate pair of $\pi^{*}$ orbitals $\left(\mathrm{e}_{\mathrm{gx}}\right.$ and $\left.\mathrm{e}_{\mathrm{gy}}\right)$. While both the B- and the Q-band arise from $\pi-\pi^{*}$ transitions, the bands have distinctly different intensities. The two highest occupied $\pi$ orbitals, $a_{1 u}$ and $a_{2 u}$, have almost identical energy and one would therefore expect that both transitions from $\mathrm{a}_{1 \mathrm{u}}$ to $\mathrm{e}_{\mathrm{g}}$ and $a_{2 u}$ to $e_{g}$ would have coincident absorption bands. However, internal conversion takes place which causes the two transitions to mix together which results in bands with different intensities and wavelengths. Constructive interference leads to an intense short-wavelength B-band, while the weak long-wavelength Q-band is a consequence of destructive interference (Figure 1.2). ${ }^{10}$ 


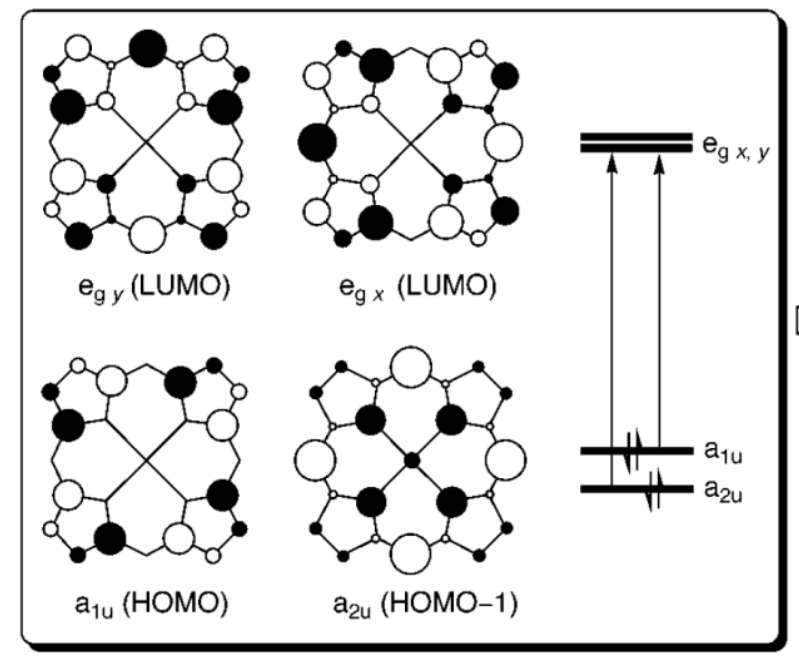

Molecular Orbitals

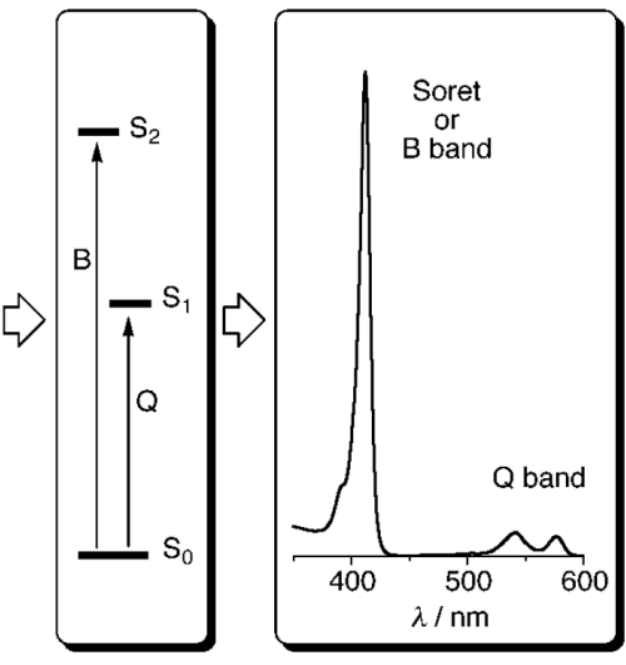

States

Absorption Spectrum

Figure 1.2: The four Gouterman orbitals which can be used to explain the absorption spectra of simple porphyrins. Adapted with permission from ref. 10, Copyright 2017 Royal Society of Chemistry.

Porphyrins can be readily modified at the meso- and $\beta$-positions which can influence their optoelectronic properties. Due to the higher frontier orbital coefficients on the meso-positions in comparison to the $\beta$-positions, alterations at the meso-positions generally result in a more significant change in the optoelectronic properties. Over the years, much effort has been devoted to forming oligo-porphyrins through meso-linkages. Often these molecular wires were not conjugated due to the large porphyrin-linker dihedral angles which results in poor orbital overlap between the individual porphyrins. Various linkages have been introduced to form oligo-porphyrin wires, e.g. direct meso-meso bonded, ${ }^{11}$ phenylene-linked, ${ }^{12,13}$ and diphenylethyne-linked ${ }^{14}$ wires, but none resulted in conjugated porphyrin oligomers due to the meso-aryl twist. The lack of long range conjugation renders their absorption spectra nearly identical to those of the corresponding monomers. ${ }^{15}$ The first example of a conjugated porphyrin oligomer was reported by Arnold in 1978, and has since been the subject of extensive study by his group, and the Anderson group and many others. ${ }^{16-19}$

Conjugation was achieved by using butadiyne-linked porphyrins, which allowed for strong inter-porphyrin electronic coupling and a progressively intensifying red-shifted Q-band in the absorption spectra upon increasing the number of porphyrins in the oligomers. ${ }^{20}$ In addition, the HOMO-LUMO gap decreased as the length of the oligomers increased. ${ }^{21}$ The individual porphyrin units in a butadiyne-linked system 
tend to adopt the lowest energy conformer which is planar. When the length of the oligomer is increased, the diversity of configurations of torsional angles between the porphyrin units increases leading to a broadening of the Q-band in the absorption spectra (Figure 1.3).
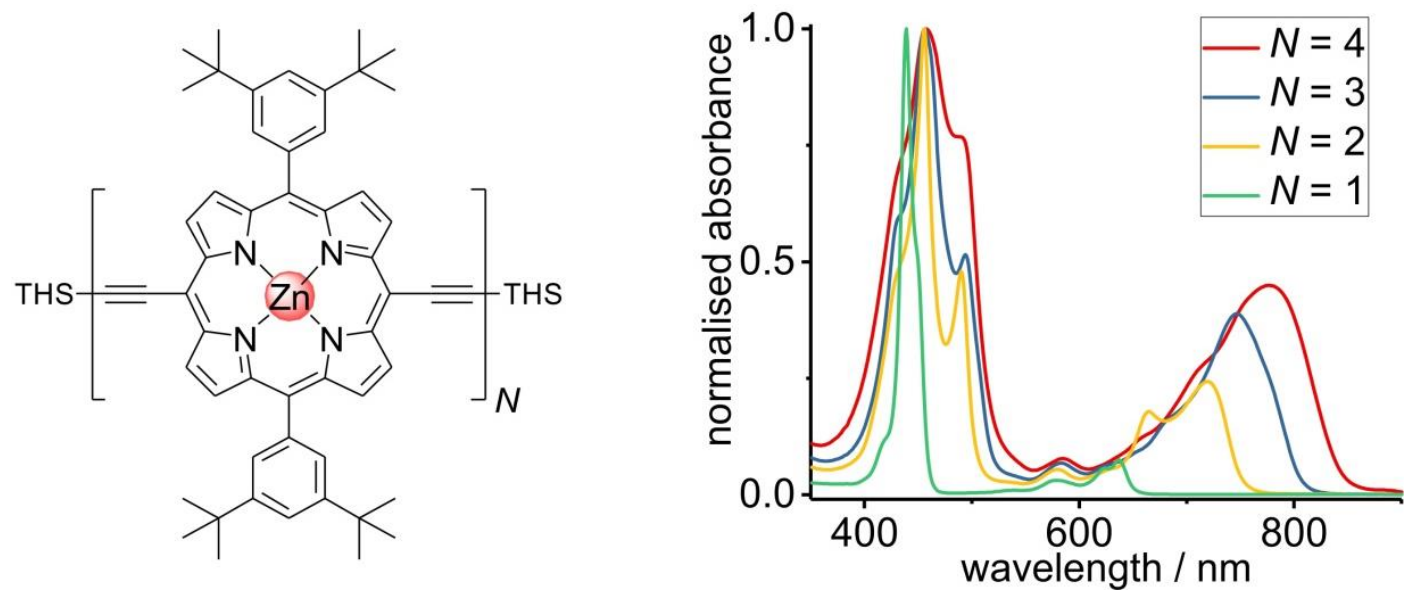

Figure 1.3: Chemical structure of a butadiyne linked $\mathrm{N}$-porphyrin oligomer and the related normalised absorption spectra in $\mathrm{CHCl}_{3}$ at $298 \mathrm{~K}$.

The $\pi$-conjugation between porphyrin units can be enhanced by locking the oligomers in coplanar arrangements using supramolecular templates. The Anderson group has made major efforts in exploring this field by forming ladder compounds with bidentate ligands such as DABCO and 4,4'-bipyridine, ${ }^{22}$ and by complex formation with oligo-pyridine templates to promote a planar conformation. ${ }^{20,23}$

\subsection{Axial coordination of metalloporphyrins}

The use of templates to control the formation of covalent bonds, via non-covalent interactions, has provided access to many fascinating molecular structures. While there are various types of reversible interactions that can be used in template-directed synthetic routes, we will focus mainly on the axial coordination of metalloporphyrins to amine templates, which is arguably the most versatile approach.

The binding of zinc(II) porphyrins to amines has been studied extensively and the association constant $K$ is known to range from 10 to $10^{9} \mathrm{M}^{-1}$ for monovalent amines in organic solvents (Figure 1.4). The strength of this interaction is correlated to the electron density on the nitrogen atoms and is therefore dependent on the basicity of the ligand. In addition, the steric crowding of the ligands influences the binding. 
When the steric hindrance of the ligands is increased, the binding affinity of the ligand decreases. For example, the binding of $n$-propylamine to a zinc porphyrin monomer is approximately an order of magnitude stronger than the binding of the more sterically congested $n$-dipropylamine under identical conditions. Since the stability constant is dominated by the electron density on the nitrogen atom it can be related to the basicity of the ligand. For example, imidazole is more basic than pyridine, leading to an approximate ten-fold increase in its binding strength to a zinc porphyrin monomer. ${ }^{24}$

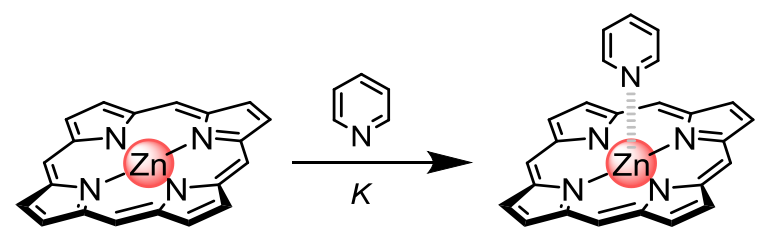

Figure 1.4: Example of a zinc porphyrin axially binding to pyridine. The binding strength is expressed by the association constant $K$.

Solvent can also influence the binding. Generally an increase in binding is observed upon a decrease in the polarity of the solvent. This is explained by the availability of the lone-pair of the nitrogen atom on the ligand to bind to the metal centre. Polar solvents are better able to solvate the ligands and as a result the binding affinity of the ligand to the metalloporphyrin is weaker. Additionally, highly polar solvents are known to bind axially to metalloporphyrins, making the metal centre less available for binding and therefore weaker binding to other ligands is observed.

\subsection{Cooperativity and effective molarity}

The concept of cooperativity is fundamental for understanding supramolecular self-assembly and arises when there is an interplay of two or more interactions in which the system as a whole behaves differently than expected based on the properties of the individual interactions acting in isolation. The coupling of two interactions can have a positive or a negative effect on the binding strength. The concept of cooperativity is widely found in biological systems ${ }^{25,26}$ and is a key feature in systems chemistry that leads to collective properties that are not present in the individual molecular components. ${ }^{27}$

When investigating the binding of a two component system, the binding observed in the isolated state may be different from the binding observed when the components 
are coupled to each other. A simple example is depicted in Figure 1.5. Situation (a) displays the interaction between components $\mathbf{A}$ and $\mathbf{B}$ in isolation. In the chelate system shown in (b) the open form (o-AA·BB) is formed first which can influence the second binding process in which the closed form c-AA·BB is produced. The stability of the closed form can either be higher or lower than the two interactions acting in isolation as a result of positive or negative cooperativity.

(a)

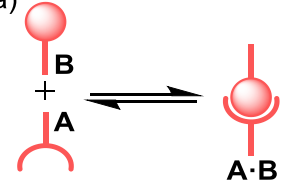

(b)

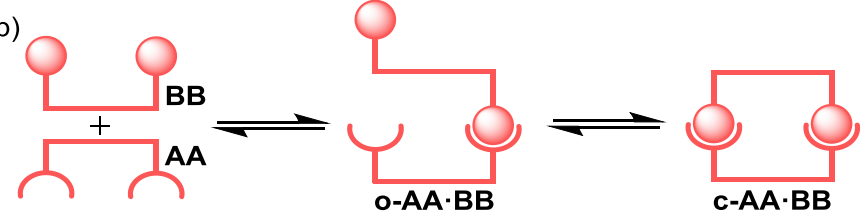

Figure 1.5: Complexation equilibria involving molecules with one or two binding sites. (a) The interaction of a monovalent ligand with a simple receptor. (b) A two-site receptor that interacts with a divalent ligand. The binding of the first site may influence the binding of the second site. ${ }^{27}$

Cooperativity originates from entropy changes. The first interaction may influence subsequent interactions by confining the next binding process to a specific conformation. A key parameter in evaluating the level of cooperativity of chelated systems at the microscopic level is the effective molarity (EM). The $E M$ is a concept which originated in physical chemistry to explain the increased reaction rate caused by proximity and orientation effects in intramolecular reactions versus intermolecular reactions. Figure 1.6 shows an example of kinetic $E M$. When the reaction rate of an intermolecular reaction is compared to the rate of the intramolecular reaction, in the intramolecular reaction the reactive components are in close proximity to each other which results in an increased reaction rate. ${ }^{28}$ This increased rate is reflected by the kinetic $E M$ which can be calculated by the ratio between the intermolecular and intramolecular reaction rate, $E M=k_{\text {intra }} / k_{\text {inter }}$.

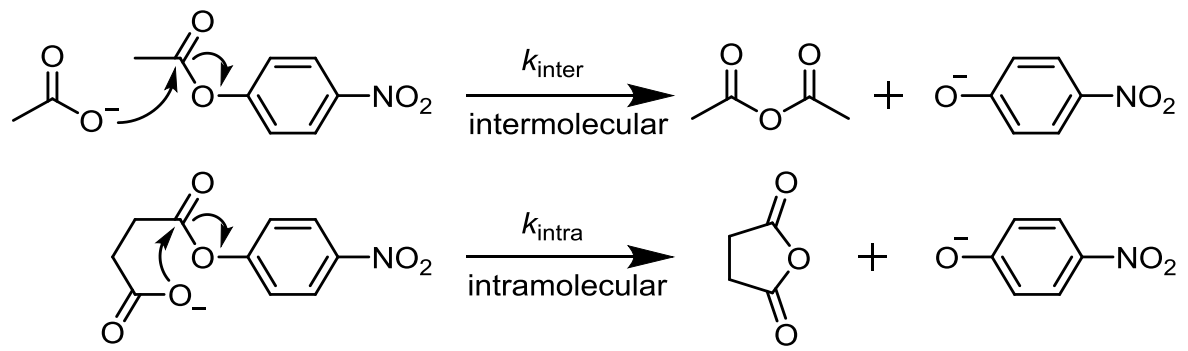

Figure 1.6: An example of a reaction in which the reaction rate is influenced by the proximity of the reactants as a result of inter- or intramolecular interactions. $k_{\text {inter }}=4 \times 10^{-6} \mathrm{~s}^{-1} \mathrm{M}^{-1} ; k_{\text {intra }}=0.8 \mathrm{~s}^{-1} ; \mathrm{EM}=2 \times 10^{5} \mathrm{M}$. 
The affinity between a host and a guest binding through multiple interactions in a supramolecular system is related to the thermodynamic EM. When a single site binding interaction occurs between a host and a guest, as depicted in Figure 1.7a, the strength of the interaction is described by the association constant. In the case of multivalent ligands and hosts with multiple binding sites, the overall stability of the system, $K_{\text {chel, }}$ may differ in stability to the sum of the individual interactions in isolation (Figure 1.7b). The thermodynamic stability of the entire system is influenced by the pre-organisation and potential proximity effects generated by the first binding event. This difference in stability is expressed by the thermodynamic EM.

(a)
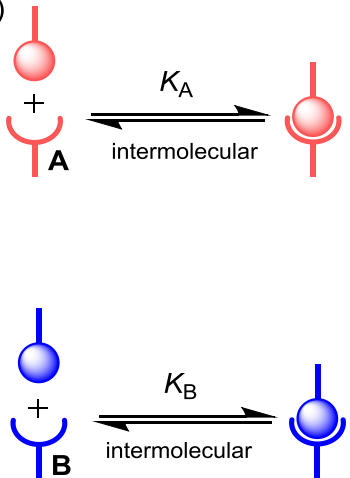

(b)
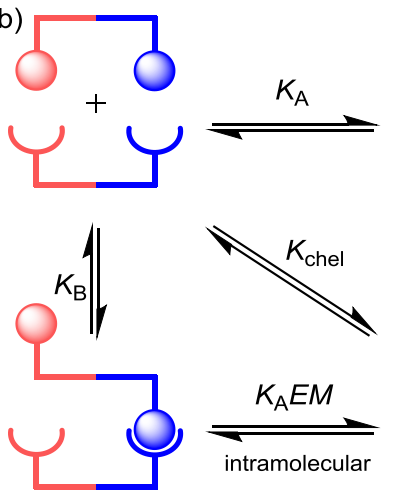
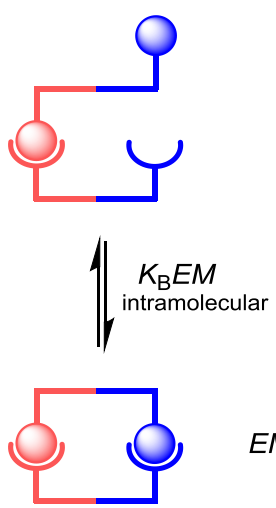

$E M=\frac{K_{\text {chel }}}{K_{\mathrm{A}} K_{\mathrm{B}}}$

Figure 1.7: Thermodynamic relationships between (a) two individual monovalent interactions in isolation and (b) a comparison of these interactions when influenced by cooperativity. ${ }^{27}$

When the system becomes more complex through an increasing number of multivalent interactions between the guest and the host, each additional chelating ligand binding interaction generates a new EM term, as illustrated in Figure 1.8. Many interactions working together can potentially lead to an extremely high overall association constant.

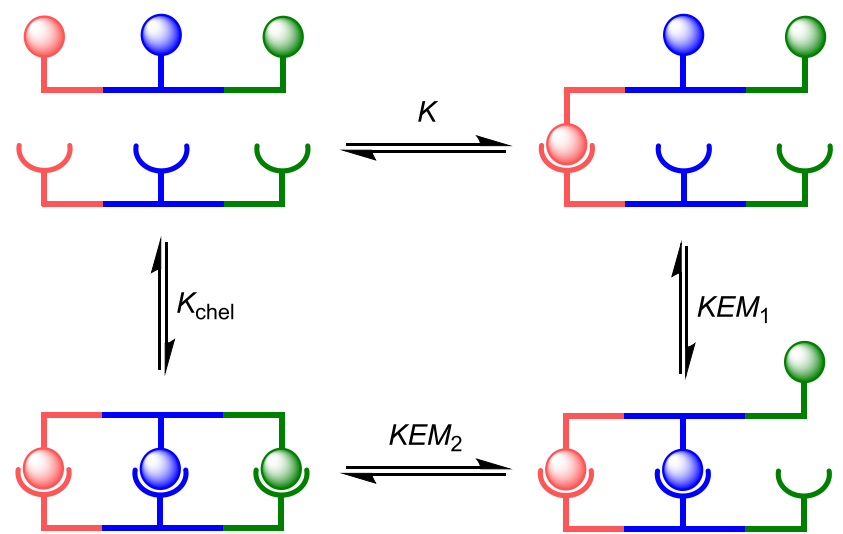

Figure 1.8: When the number of interactions between a host and a guest increases, each binding event is related to its own $E M$ term. $^{27}$ 
Through the years, many groups have investigated cooperativity effects in order to understand and prepare complex molecular systems. After pioneering the field of supramolecular chemistry, the group of Lehn has made many contributions such as the thermodynamic and kinetic understanding of self-sorting helices and other supramolecular systems. ${ }^{29-32}$ More recent contributions have been made by the groups of Hunter, ${ }^{33-35}$ Schalley, ${ }^{36}$ and Anderson. ${ }^{37-39}$

\subsection{Template directed synthesis of porphyrin structures}

The axial coordination between ligands and metalloporphyrins has attracted substantial interest for the preparation of supramolecular porphyrin assemblies. Templates have been used to enable the synthesis of complex covalently-linked oligo-porphyrin compounds which were not accessible through traditional linear synthesis. ${ }^{9,40}$

The first template-directed synthesis of a cyclic oligo-porphyrin system was reported by Anderson and Sanders (Figure 1.9). ${ }^{41}$ In this work, a tridentate pyridyl template was utilised to coordinate three bis-(3-ethynylphenyl) substituted porphyrin monomers, which were subsequently cyclised using Glaser-Hay coupling conditions to give the 3-porphyrin nanoring in a $52 \%$ yield.
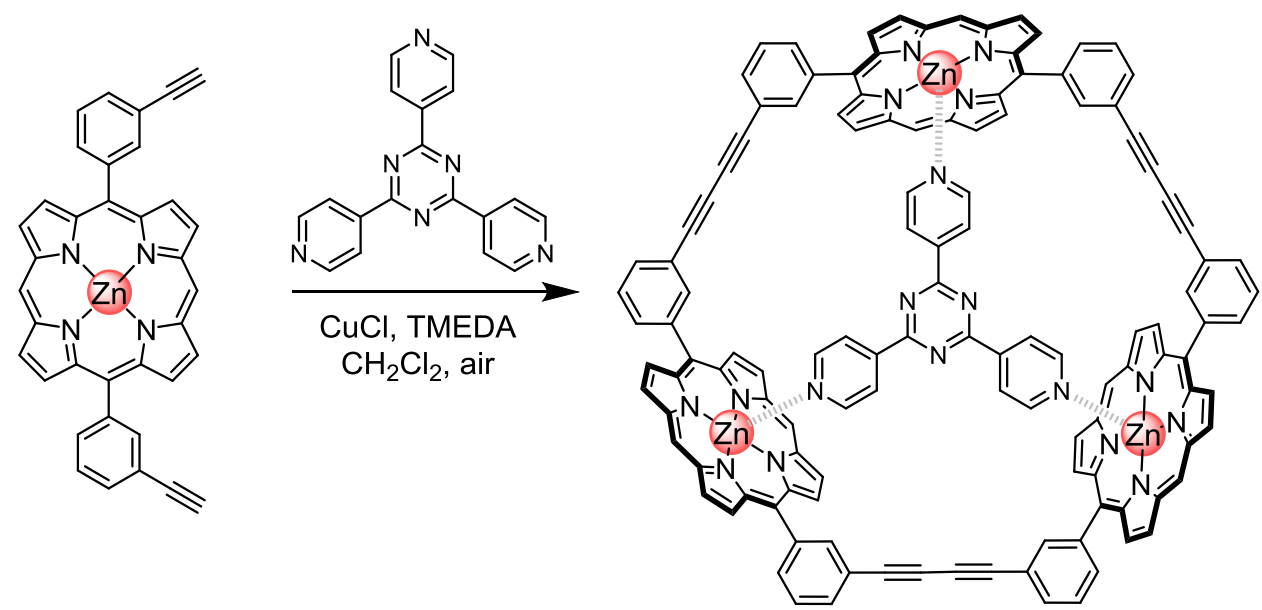

Figure 1.9: The template-directed synthesis of a porphyrin nanoring. Solubilising groups on the $\beta$-positions of the porphyrins have been omitted for clarity. ${ }^{41}$

Other groups have reported on the preparation of a range of related systems. The group of Lindsey extended the line of work by preparing cyclic hexamers comprised of alternating zinc and free-base porphyrins joined by diphenylethyne linkers (Figure 1.10). ${ }^{42-44}$ The cyclic hexamer was prepared using Sonogashira reaction conditions in 
a $14 \%$ yield. In addition, the groups of Gassauer ${ }^{45,46}$ and Aida ${ }^{47-49}$ have reported on the synthesis of rigid and flexible cyclic oligo-porphyrin systems.

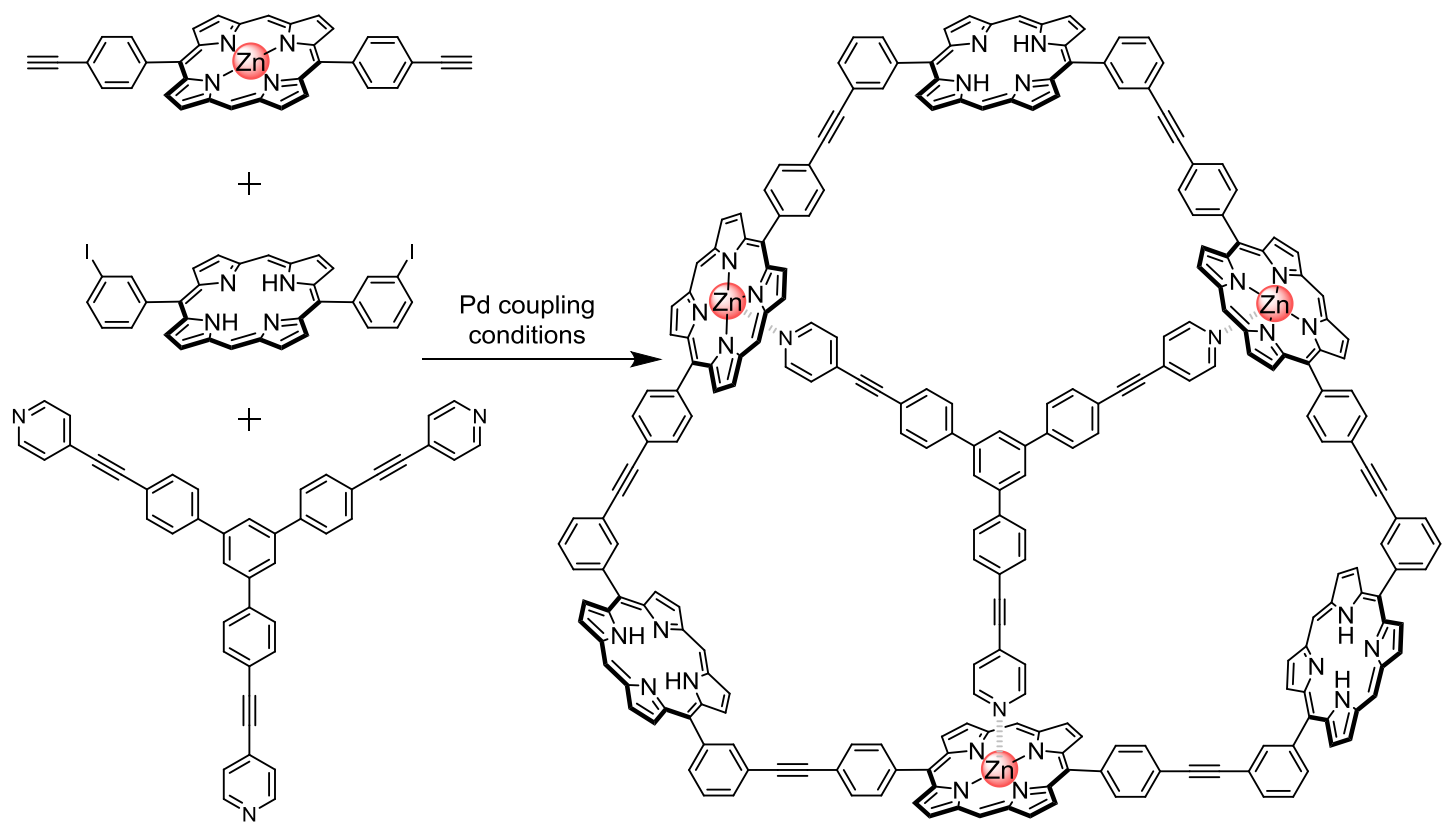

Figure 1.10: The template-directed one-flask synthesis of Lindsey's cyclic hexamer. meso-Aryl solubilising groups have been ommited for clarity. ${ }^{42}$

While the number of porphyrin nanorings increased over the years, it was not until 2008 that Anderson and co-workers reported on the synthesis of the first fully conjugated butadiyne-linked porphyrin nanoring (Figure 1.11). The ring was prepared from a linear octamer after coordination to an octadentate template. Upon coordinating to the template, the conjugated oligomer was pre-organised by bending itself so that the two terminal alkynes came in close proximity to each other. Subjecting the one-to-one complex to oxidative coupling conditions gave the cyclic octamer in a $14 \%$ yield. ${ }^{50}$ Later Anderson and co-workers reported the synthesis of a more strained $\pi$-conjugated 6 -porphyrin nanoring through the template-directed trimerisation of a porphyrin dimer using the hexadentate template T6. The synthesis yielded the $D_{6 h}$ porphyrin nanoring in a $44 \%$ yield. ${ }^{38}$ The highly pre-organised multivalent systems displayed extraordinarily high association constants to the templates. 

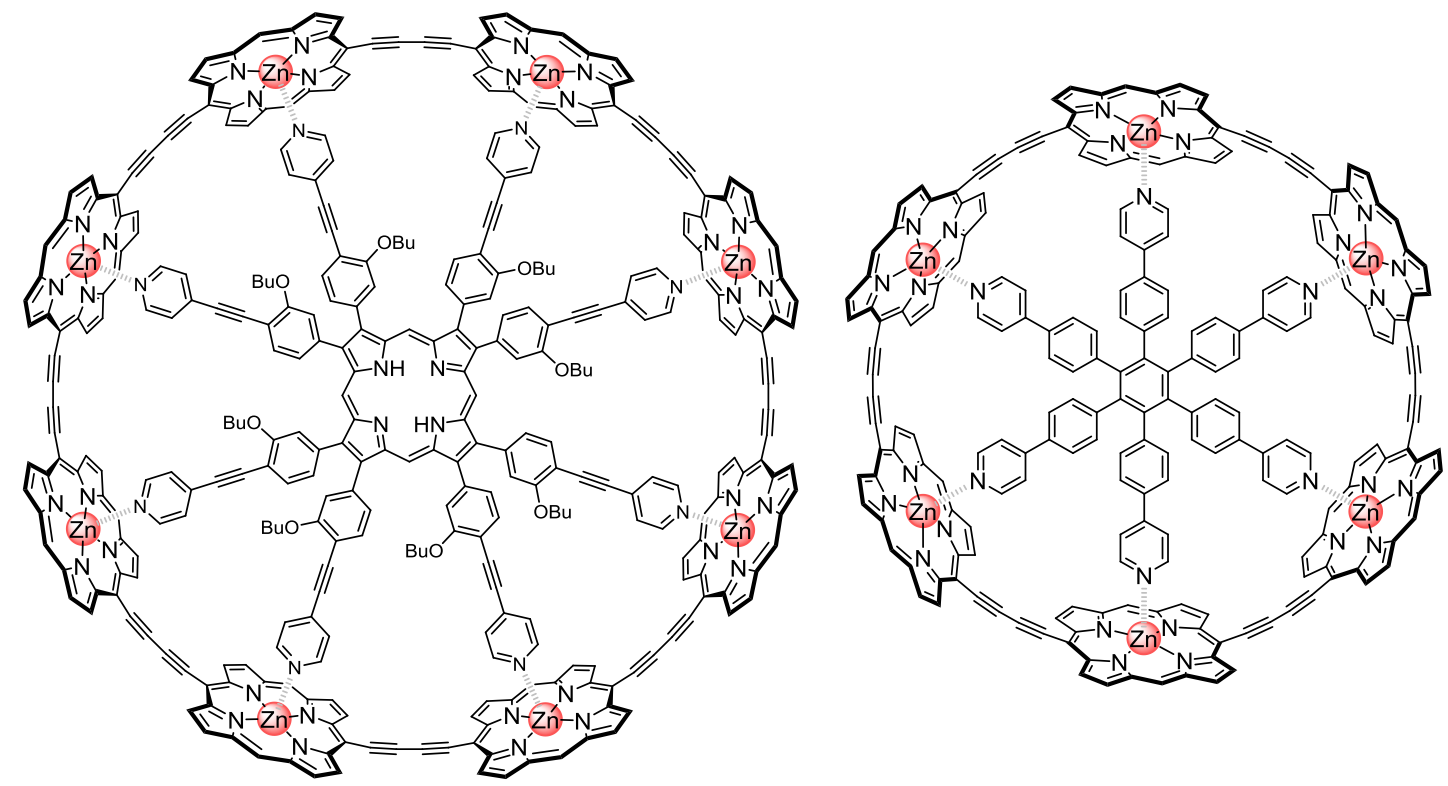

Figure 1.11: The chemical structures of the templated 8- and 6-porphyrin nanorings. meso-Aryl solubilising groups have been ommited for clarity. ${ }^{38,50}$

The template-directed approach demonstrates the power of noncovalent self-assembly as a tool for controlling the formation of porphyrin-based molecular wires. However, the templates used in the synthesis of the 6- and 8-porphyrin nanorings were relatively simple and it is easy to imagine that a larger and more complex target molecule would require a synthetically more demanding template. One way to overcome this problem is by using a Vernier templating strategy. If the number of binding sites on the template, $n_{T}$, is not a multiple of the number of binding sites on the molecular building blocks, $n_{B}$, then small templates can direct the assembly of relatively large Vernier complexes where the number of binding sites in the product, $n_{\mathrm{P}}$, is the lowest common multiple of $n_{\mathrm{B}}$ and $n_{\mathrm{T}}$. Using this approach, the formation of a 12-porphyrin nanoring was achieved from three porphyrin tetramers in combination with two hexadentate templates in a $39 \%$ yield (Figure 1.12). ${ }^{39}$ This approach was later extended to make nanorings containing of up to 50 porphyrin units. $^{51-53}$ 

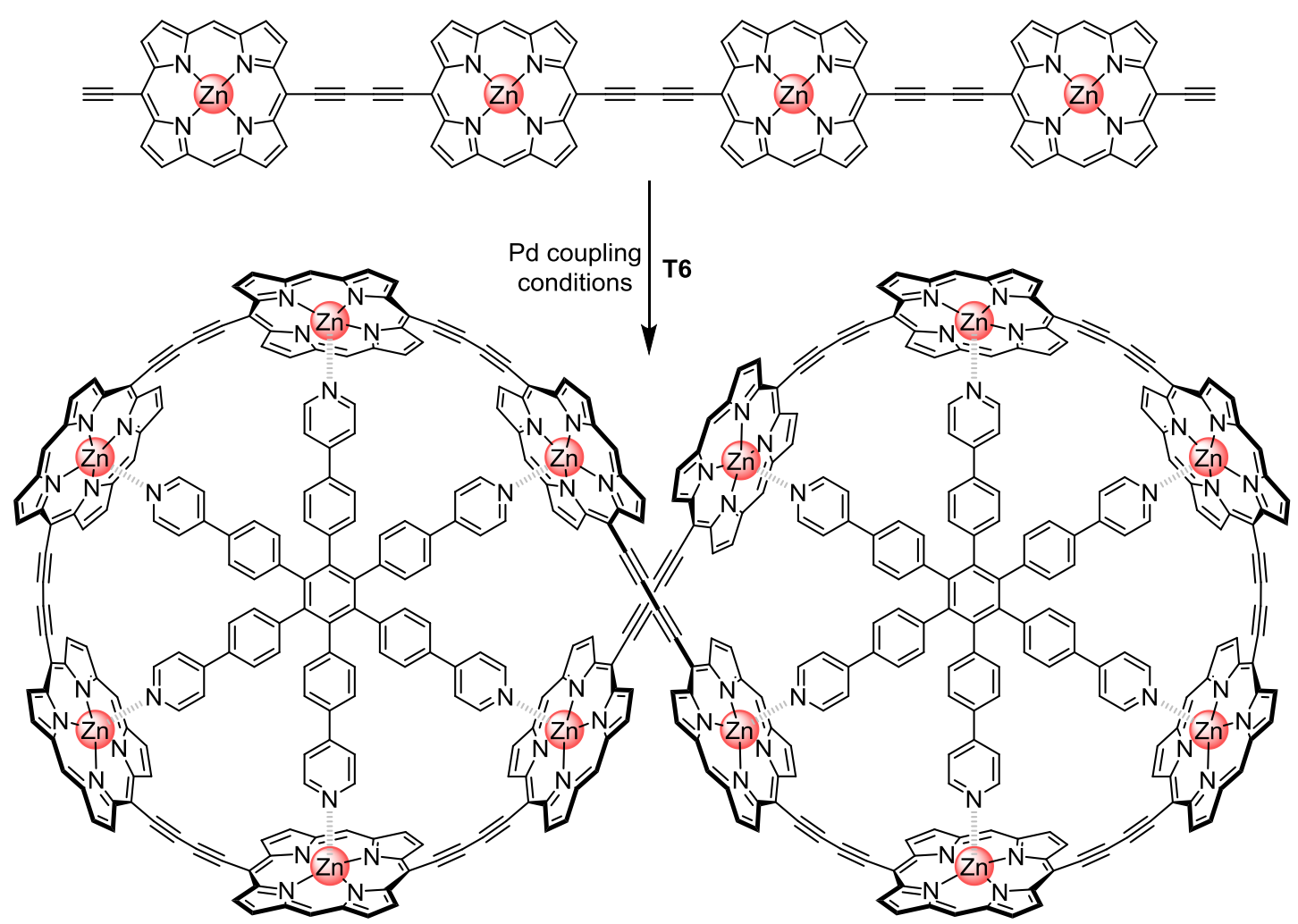

Figure 1.12: The chemical structures of the linear porphyrin tetramer and the templated 12-porphyrin nanoring formed using a Vernier templating strategy. meso-Aryl solubilising groups have been ommited for clarity. ${ }^{39}$

Besides increasing the size of the $\pi$-system by increasing the size of the ring, the structures can also be extended perpendicular to the plane of the ring. The first example of such a 3D conjugated structure by the Anderson group was a molecular tube consisting of twelve porphyrins, each of which was directly $\pi$-conjugated to three of its neighbours (Figure 1.13). The tube can be regarded as a rim-to-rim dimer of the cyclic porphyrin hexamer shown in Figure 1.11. The porphyrin nanotube was prepared using a new type of cooperative templating in which six porphyrin dimers were joined together using oxidative coupling conditions in the presence of the T6 template. The alkyne homo-coupling reaction in the final step of the synthesis involved the formation of twelve new $\mathrm{C}-\mathrm{C}$ bonds giving the tube in a $32 \%$ yield. The absorption and emission maxima of the tube are red-shifted by approximately $300 \mathrm{~cm}^{-1}$ relative to those of a six-porphyrin nanoring which reflects the enhanced conjugation in the extended $\pi$-system. ${ }^{54}$ 


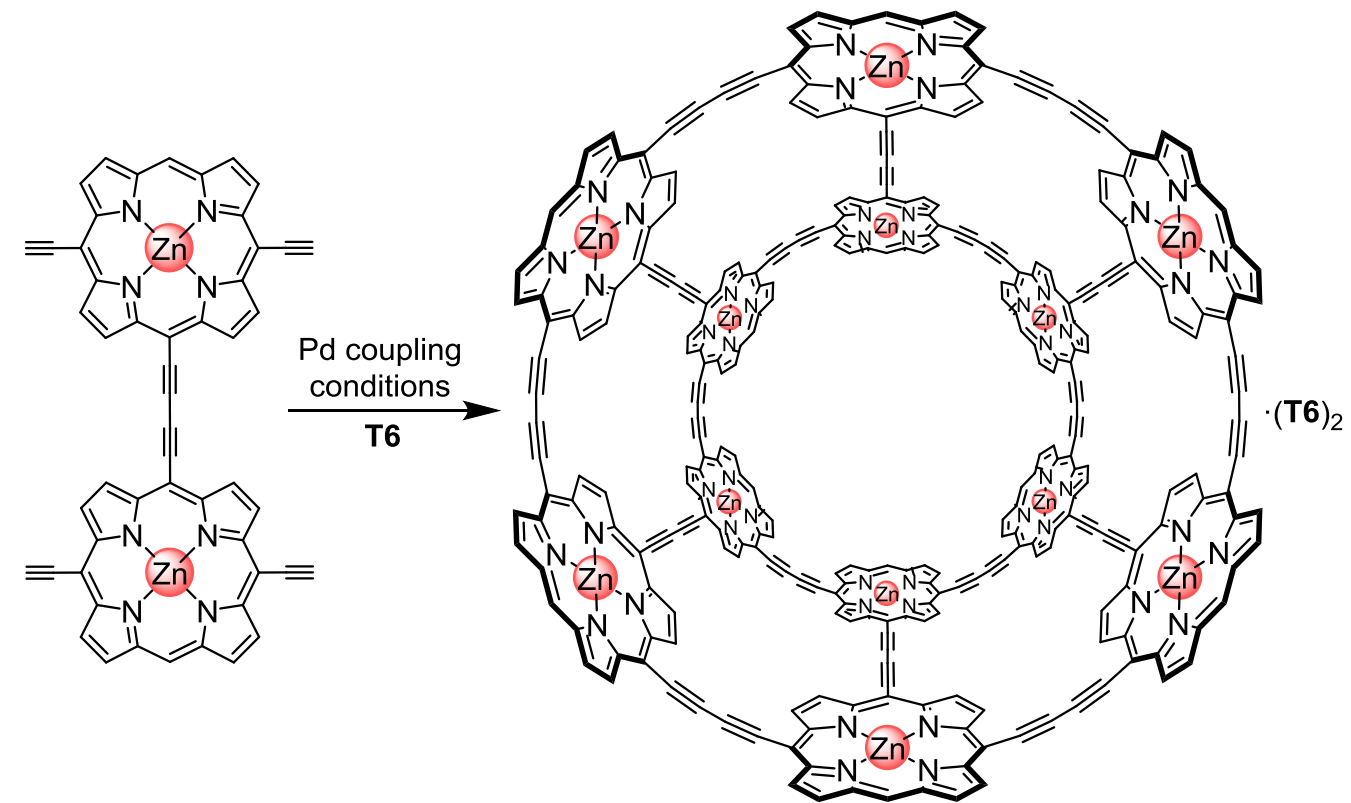

Figure 1.13: The synthetic route for the 12-porphyrin nanotube. The final reaction step involves the formation of twelve C-C bonds. meso-Aryl solubilising groups have been ommited for clarity. ${ }^{54}$

In later work, the Anderson group diverted from the standard five-coordinate binding mode of zinc porphyrins by taking advantage of chelate cooperativity and enforced a pseudo-octahedral six-coordinate zinc porphyrin complex. The formation of the six-coordinate zinc porphyrin complex consequently allowed for the template directed synthesis of a 3D $\pi$-conjugated spiro-fused array of 11 porphyrins (Figure 1.14). The porphyrin units are covalently connected in a nontrivial topology of two porphyrin nanorings sharing one spiro-porphyrin. Similar to the tube, the spiro-system showed an enhancement in the conjugated $\pi$-system through a red-shift in the absorption and emission spectra. Time-resolved fluorescence anisotropy measurements showed that electronic excitation could migrate between the two spiro-fused porphyrin nanorings within $270 \mathrm{fs}^{55}$
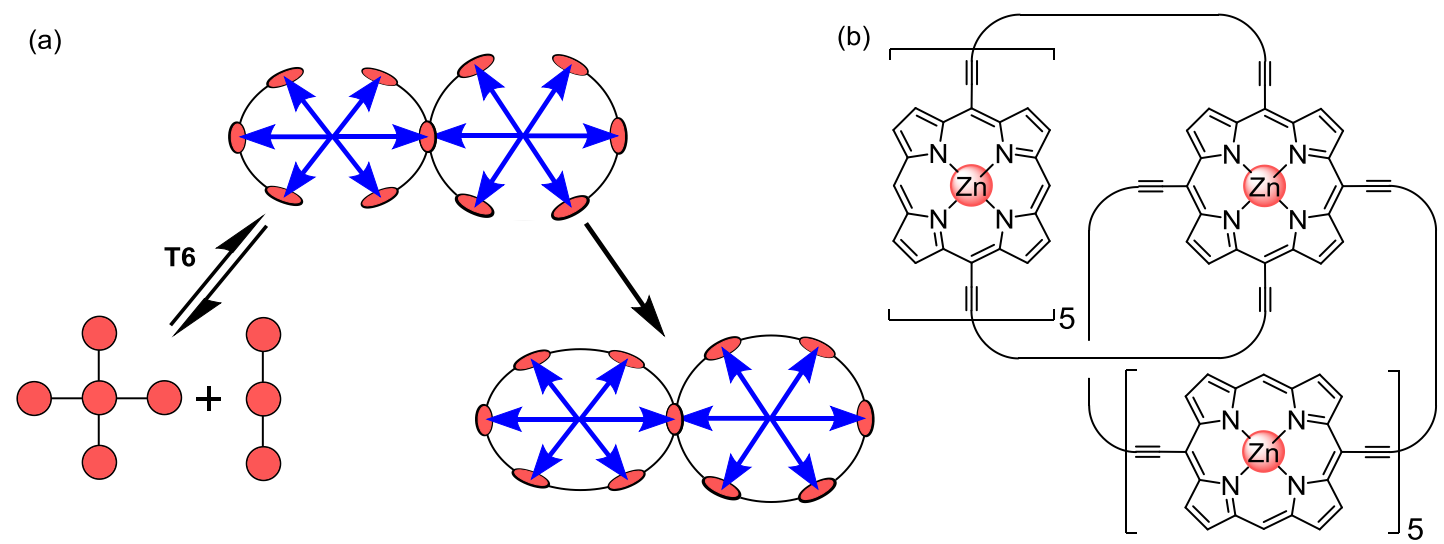

Figure 1.14 (a) Schematic synthetic route to the spiro-fused 11-porphyrin nanostructure. The chelate effect allowed for the formation of a 6-coordinate zinc porphyrin necessary for the formation of the spiro-fused 
system. (b) Chemical structure of the spiro-system. meso-Aryl solubilising groups have been ommited for clarity. ${ }^{55}$

Chemist have sought for rational strategies for the synthesis of 3D $\pi$-conjugated spherical structures ever since the discovery of buckminsterfullerenes. ${ }^{40}$ In Chapter 5 and 6 we will discuss the preparation of 3D $\pi$-conjugated porphyrin nanoballs containing either fourteen or ten porphyrins respectively. The spherical structures form the next generation of porphyrin nanostructures in the Anderson group.

\subsection{Heterometallated porphyrin oligomers}

Over the years, many supramolecular complexes have been prepared using zinc porphyrins. Zinc is often chosen due to its well-defined axial coordination chemistry, high stability and ease of characterisation. Naturally, porphyrin chemistry is not limited to zinc and nearly all transition metals as well as lanthanides have been used in the past to form metalloporphyrins.

The construction of homometallated oligo-porphyrin arrays has resulted in numerous linear, cyclic and polymeric assemblies. ${ }^{56,57}$ The next section will focus on the more scarcely available heterometallated examples. Zinc porphyrins prefer axial nitrogen ligands, but phosphine and oxygen based ligands are frequently used in combination with other metals. An example is depicted in Figure 1.15. This cyclic porphyrin tetramer consists of two bis-phosphine substituted zinc(II) porphyrins and two rhodium(III) porphyrins. Rhodium centres bind phosphine ligands and when the two subcomponents were combined stoichiometrically, a mixture of polymeric species was formed. The addition of half an equivalent 4,4'-bipyridine, coordinating to the zinc centres, resulted in the quantitative formation of the cyclic porphyrin tetramer. ${ }^{58}$ 


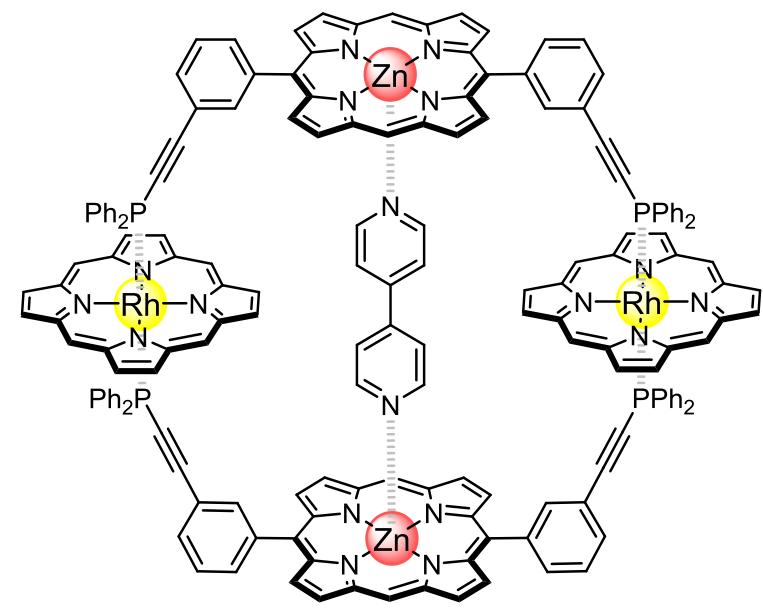

Figure 1.15: Chemical structure of the heterometallated zinc(II) and rhodium(III) cyclic porphyrin assembly. meso-Aryl solubilising groups have been ommited for clarity. ${ }^{58}$

Within the Anderson, group other metal centres besides zinc have been utilised in the preparation of supramolecular structures. Recently it was demonstrated that a ring-in-ring complex, consisting of a 6-porphyrin ring nested inside a 12-porphyrin ring, could be prepared by utilising the coordination chemistry of two different metals in the two rings. The outer 12-ring contained zinc porphyrins that bind amine ligands while the inner 6-ring contained aluminium-porphyrins which are known to bind complementary oxygen-containing ligands. ${ }^{59}$ Aluminium porphyrins can form hexacoordinate complexes upon binding pyridine ligands. As a result, mixing the two rings in combination with the hexapyridyl template T6 and six equivalent of a specifically designed bridging ligand, the nine-component Russian doll complex depicted in Figure 1.16 was formed. ${ }^{60}$ 


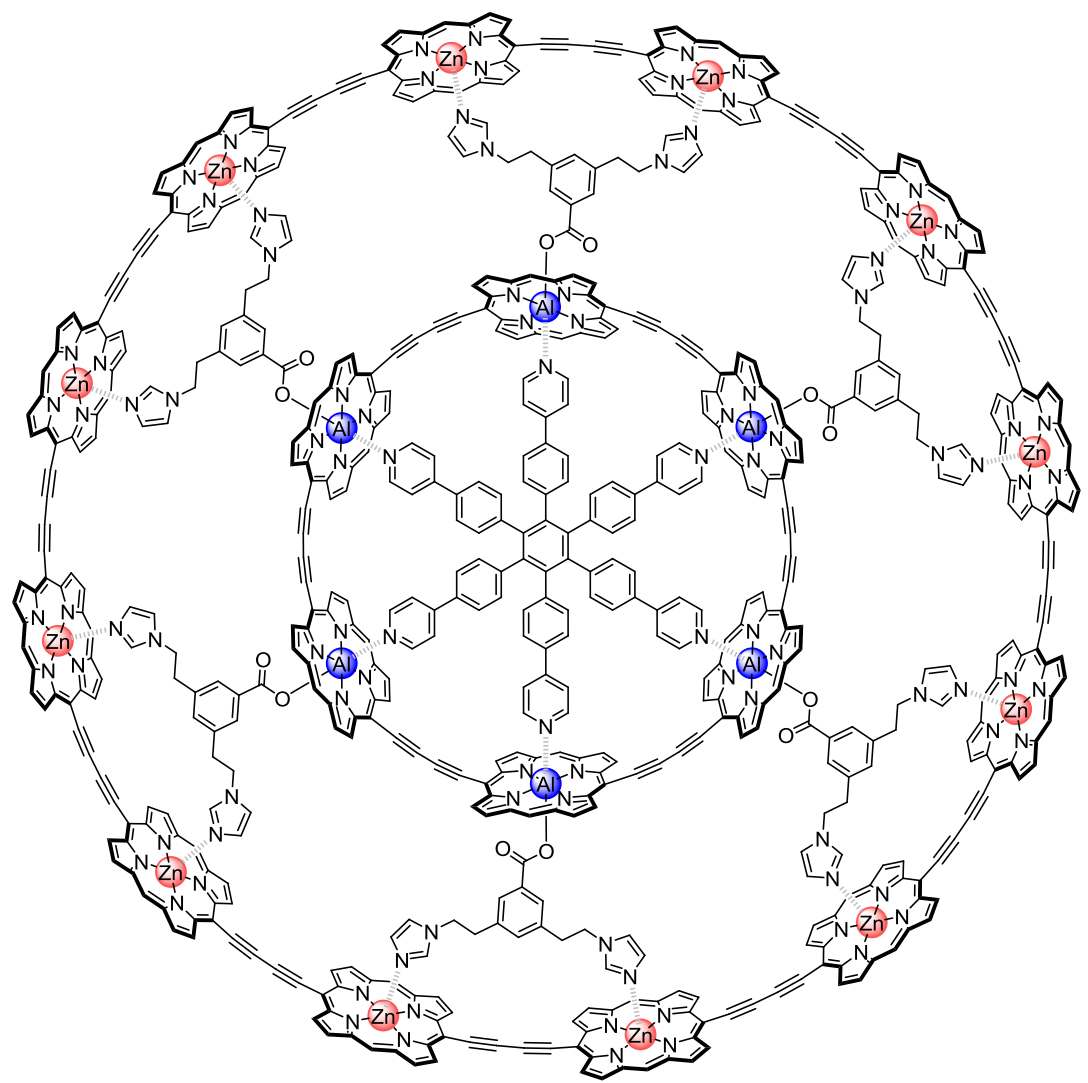

Figure 1.16: Chemical structure of the nine-component Russian doll complex prepared with both zinc(II) and aluminum(III) porphyrins. meso-Aryl solubilising groups have been ommited for clarity. ${ }^{60}$

The examples above illustrate supramolecular heterometallic porphyrin nanostructures. By utilising the different coordination preferences of the metal centres, adequately designed ligands allowed various metal centres to be incorporated into a single structure. Heterometallated porphyrin oligomers connected by covalent bonds are less frequently studied. ${ }^{61,62}$ Most examples in the literature are limited to zinc/gold porphyrin dimers used to study photoinduced electron transfer and charge separation.

One of the earliest examples of a covalently linked heterometallated dimer was reported by Sauvage in $1991 .^{63}$ The structure contained zinc and gold porphyrins connected by a phenanthroline linker (Figure 1.17). Research focussed on the investigation of the electronic coupling between the porphyrins. The system was designed such that the magnitude of electronic coupling between the porphyrin units could be varied through structural modification of the spacer unit. It was found that each porphyrin could be selectively exited and that photoinduced electron transfer occurred from the zinc porphyrin to the more electron deficient gold porphyrin. ${ }^{64,65}$ 
In later studies, the phenanthroline unit was used to form rotaxanes and catenanes to investigate long-range intermolecular electron transfer. ${ }^{66,67}$

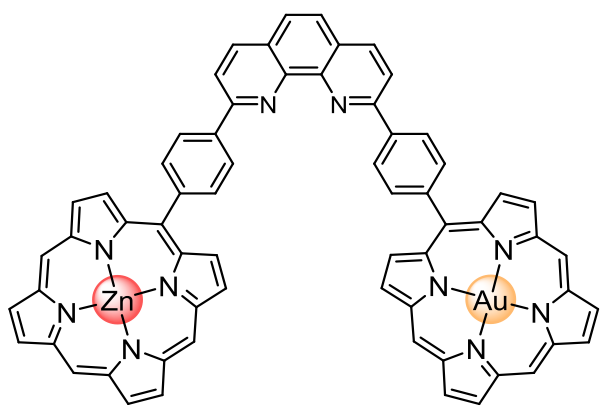

Figure 1.17: Chemical structure of the covalently linked zinc(II)/gold(III) porphyrin dimer prepared by Sauvage. meso-Aryl solubilising groups have been ommited for clarity. ${ }^{63}$

Zinc(II) and gold(III) porphyrins are often chosen as electron donor and acceptor because of their suitable driving force for photoinduced electron transfer. The rate of electron tunnelling in a molecular donor-linker-acceptor system is determined both by the distance between the donor and acceptor, as well as by the energy gap between the donor and the linker moieties. These dependencies were extensively studied by Albinsson et al. on model systems such as those depicted in Figure 1.18. In their work it was shown that the electron tunnelling has an exponential dependence on the distance between the donor and acceptor as well as an inverse dependence on the barrier height invoked by the linker. ${ }^{68,69}$

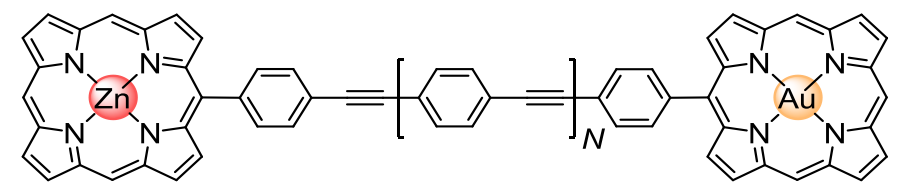

Figure 1.18: Chemical structure of the covalently linked zinc(II)/gold(III) porphyrin dimer prepared by Albinsson. meso-Aryl solubilising groups have been ommited for clarity. ${ }^{68}$

In subsequent work Odobel et al. incorporated ethynyl groups between the porphyrins and the linkers (Figure 1.19). The ethynyl linker increases the distance between the porphyrin and the spacer, and permits extensive $\pi$-conjugation and therefore electronic communication between the porphyrins. The system was found to exhibit strong porphyrin-bridge electronic coupling which resulted in rapid electron and hole transfer processes upon excitation of one of the porphyrin units. The rate constants were found to be two to three orders of magnitude higher than those for similar systems connected via meso-phenyl groups as a result of the stronger conjugation through the linker. ${ }^{70,71}$ 


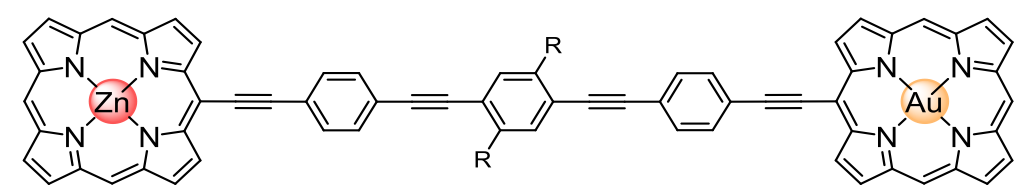

Figure 1.19: Chemical structure of the covalently linked zinc(II)/gold(III) porphyrin dimer prepared by Odobel. meso-Aryl solubilising groups have been ommited for clarity. ${ }^{70,72}$

By changing the bridge with a bis-ethynyl tetrathiophene spacer, highly efficient and long-range photo-induced charge separation between the two porphyrins was observed. The new linker was found to induce fast and quantitative charge separation over a distance of $2.5 \mathrm{~nm}$ which occurred through a superexchange mechanism. In addition, the new linker led to a long-lived charge-separated state of $3.3 \mathrm{~ns}$ in toluene and $100 \mathrm{ps}$ in dichloromethane by prohibiting the decay to the zinc porphyrin's triplet state. ${ }^{71,73}$

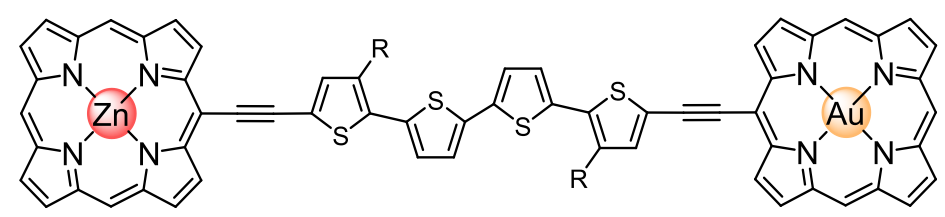

Figure 1.20: Chemical structure of the covalently linked zinc(II)/gold(III) porphyrin dimer prepared by Odobel. meso-Aryl solubilising groups have been ommited for clarity. ${ }^{71,73}$

In Chapter 2, 3, and 4 we will discuss the synthesis and properties of a family of linear and cyclic heterometallated copper and zinc porphyrin oligomers. The copper centres were chosen for their paramagnetic electron configuration which allowed us to measure the through-space dipolar coupling between two paramagnetic centres using electron paramagnetic resonance (EPR) spectroscopy.

\subsection{Electron paramagnetic resonance}

EPR can be used for the study and characterisation of systems containing paramagnetic centres. The fundamental concepts of EPR are similar to those of NMR, except that instead of exciting the spins of atomic nuclei, electrons spins are excited.

All electrons have a spin quantum number $\left(s=\frac{1}{2}\right)$ and a magnetic moment with magnetic components $m_{s}=+\frac{1}{2}$ or $-\frac{1}{2}$. When an external magnetic field with strength $B_{0}$ is applied, the magnetic moment of the electrons aligns either parallel or antiparallel to the field. As a result of the Zeeman effect each alignment has a specific energy which is defined by: 


$$
E=m_{s} g_{e} \mu_{B} B_{0}
$$

where $g_{e}$ is the electron $g$-factor and $\mu_{B}$ is the Bohr magneton. From the equation above we can derive that the energy difference for unpaired electrons between the upper and lower states must equal:

$$
\Delta E=g_{e} \mu_{B} B_{0}
$$

This equation implies that the splitting of the energy levels is directly proportional to the strength of the magnetic field. In order to move an electron between the two energy levels, a photon of energy $h v$ has to be either absorbed or emitted such that the resonance condition $h v=\Delta E$ is obeyed which leads to the fundamental EPR equation:

$$
h v=g_{e} \mu_{B} B_{0}
$$

There are numerous EPR experiments which are based on varying either the microwave photon frequency incident on a sample while maintaining a constant magnetic field or vice versa. Generally the frequency is kept constant while a paramagnetic sample is exposed to microwaves. When the external magnetic field is increased, the gap between the $m_{s}$ energy states widens until it matches the energy of the microwaves allowing the unpaired electrons to move between the two spin states. Most electrons are usually found in the lower energy state as a result of the Maxwell-Boltzmann distribution, this leads to a net absorption of energy which is subsequently converted into a spectrum. EPR experiments can be used to obtain specific information about the interaction of electron spins with the external magnetic fields, interactions between electron spins, or interactions between electron spins and nuclear spins.

To gain information about the electronic structure and molecular environment of spin centres, the recorded magnetic parameters of a system can be related to specific molecular properties. EPR is often used for the study of through-bond and through-space electronic communication between different subunits of complex molecular structures and for the study of structural and conformational dynamics in biological systems. 
Double electron-electron resonance (DEER) is a pulsed EPR technique used to measure distances between paramagnetic centres using dipole-dipole couplings. ${ }^{74,75}$ In the past a series of butadiyne-linked zinc porphyrin oligomers ( $N=1$ to 4 ) have been measured which were spin-labelled at both ends with stable nitroxide TEMPO radicals (Figure 1.21). DEER was used to probe the distribution of intramolecular end-to-end distances to gain insights into the flexibility of these systems. ${ }^{76}$

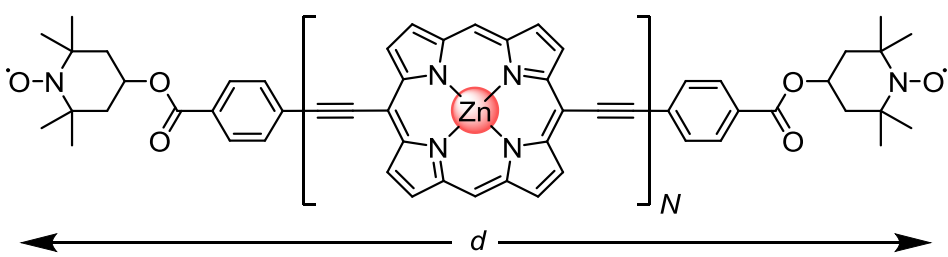

Figure 1.21: Chemical structure of the TEMPO-terminated porphyrin oligomers with $N$ equals 1 to 4 porphyrin units. $d$ represents the distance between the paramagnetic centres. meso-Aryl solubilising groups have been ommited for clarity. ${ }^{76}$

DEER measurements revealed sharp distance distributions around $3.4 \mathrm{~nm}, 4.8 \mathrm{~nm}$, $6.1 \mathrm{~nm}$ and $7.5 \mathrm{~nm}$ for $N=1,2,3,4$ respectively, which agreed with the expected distances calculated by molecular dynamics. The narrow distributions indicate high rigidity in the oligomers. The distance between the spin centres could be influenced by using multidentate amine ligands (Figure 1.22). By bending the oligomer through non-covalent interactions, the distances between the nitroxides was reduced and the new distance could be measured accurately by EPR. These experiments demonstrated that DEER is a valuable technique for gaining structural information on nanoscopic self-assembled supramolecular structures.

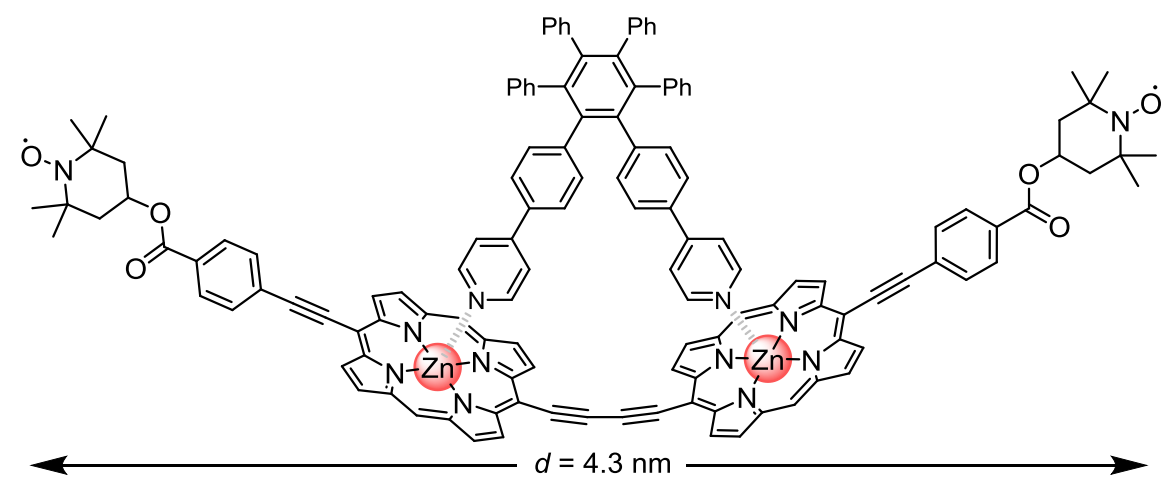

Figure 1.22: The use of multidentate amine ligands allowed for the oligomers to be bend, decreasing the distance between the TEMPO groups. meso-Aryl solubilising groups have been ommited for clarity. ${ }^{76}$

If the molecule under investigation is paramagnetic itself, the laborious need to introduce spin labels into the molecular structure becomes obsolete. As discussed, porphyrin chemistry allows for the introduction of many different metal centres in 
the core of the molecule. When introducing paramagnetic metal centres (e.g. copper(II), manganese(II), cobalt(II) or gadolinium(III)) into the porphyrins, EPR measurements can be conducted on the molecules directly. ${ }^{8}$ In recent work, we have shown the measurement of the exchange interaction between copper(II) centres in a family of linear porphyrin oligomers such as the dimer shown in Figure $1.23 .^{77}$

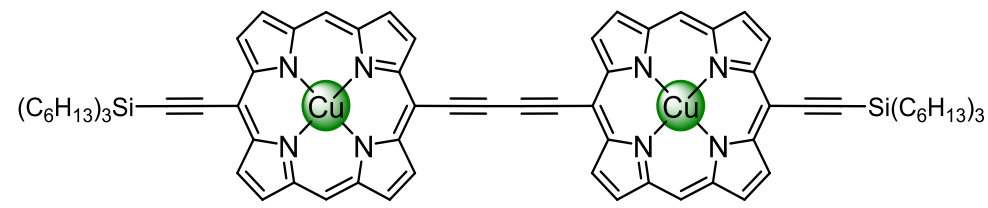

Figure 1.23: Chemical structure of a paramagnetic copper porphyrin dimer which was used to study the coupling between the copper centres via EPR spectroscopy. meso-Aryl solubilising groups have been ommited for clarity. ${ }^{77}$

The EPR measurements on copper containing porphyrin oligomers will be presented in Chapter 2 and 3.

\subsection{Quantum interference}

Kirchhoff's circuit law dictates that the net conductance of two parallel components in an electronic circuit is equal to the sum of the individual conductances. This basic concept predicts that in systems such as those depicted in Figure 1.24, the conductance $G_{A B}$ between points $A$ and $B$ in the two path system is double that of a single path system:

$$
G_{A B}=G_{1}+G_{1}=2 G_{1}
$$

When the dimensions of the circuit are less than the electronic phase coherence length, which means that the electrons can retain their phase information, interference effects may play a role. Quantum interference describes the concept that elementary particles can be in multiple places at any time and that particles can interfere with their own trajectory and direction. As a result, the total conductance in a coherently coupled system equals the sum of the conductances of the individual paths plus an interference term:

$$
G_{A B}=G_{1}+G_{1}+2 \sqrt{G_{1} G_{1}}=4 G_{1}
$$


In the equation above the pathways are assumed to be identical which results in $G_{A B}=4 G_{1}$ instead of the classical result obtained from Kirchhoff's law for two conductors in parallel of $G_{A B}=2 G_{1} \cdot{ }^{78,79}$
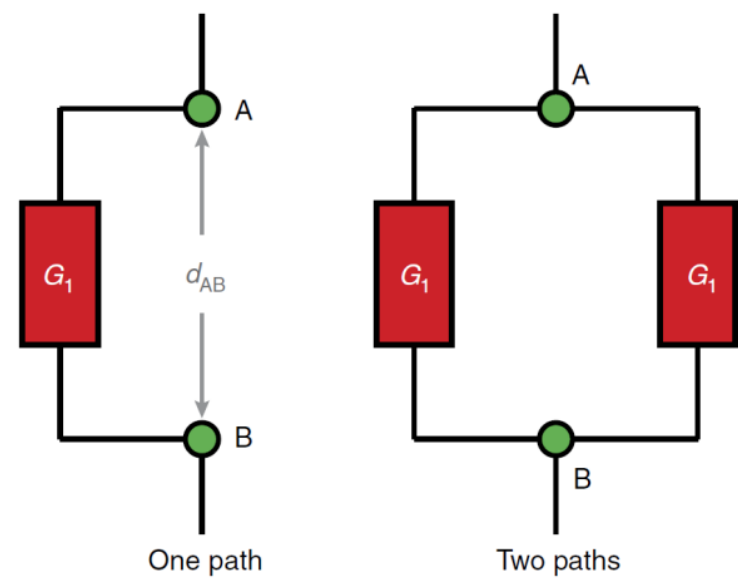

Figure 1.24: Schematic illustration of transmission through one or two paths.

At the molecular scale, interference effects have been shown to dramatically reduce the electron transfer rate through meta-connected benzene rings when compared to para-connected rings (Figure 1.25). Gourdon and co-workers calculated the metal-metal coupling $\left(V_{\mathrm{ab}}\right)$ in the three isomers of diferrocenylbenzene using Hush's equations and found that the values are much higher for the ortho and para isomers than for the meta isomer. The weak interaction in the meta isomer was ultimately traced to quantum interference effects through a cancellation of the contributions of the two electron transfer path. ${ }^{80}$

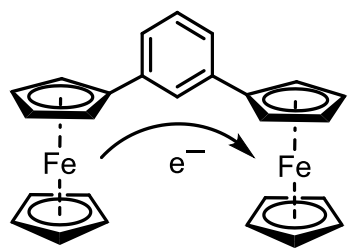

Figure 1.25: Molecular structure of the meta-isomer of the diferrocenylbenzene studied by Gourdon and co-workers. $^{80}$

In 2015, Li and co-workers reported on the calculations and measurements performed on the conductance through a one and two path molecular system (Figure 1.26). By using a scanning tunnelling microscopy (STM) break junction technique and electron transport calculations they were able to show a 40 fold decrease in conductance when comparing the two path system to the single path system. $^{81}$ 


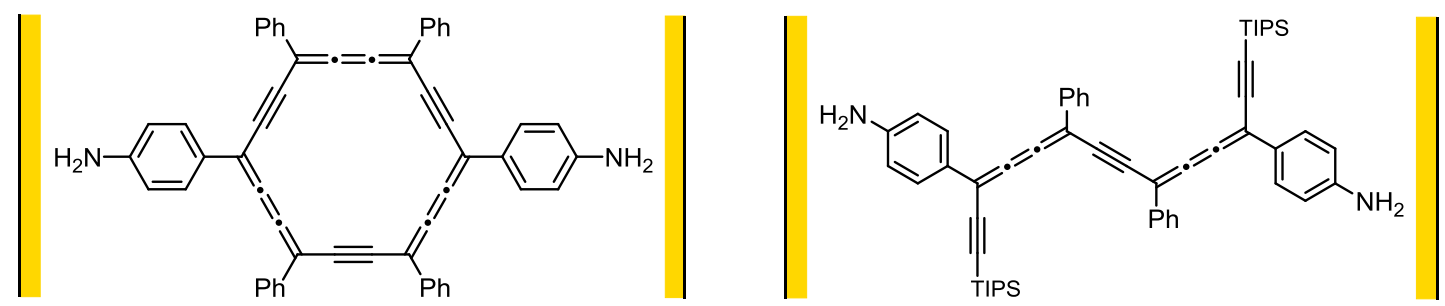

Figure 1.26: Molecular structures of carbo-benzene and carbo-n-butadiene for single-molecular conductance measurements by STM metal junctions. The gold electrodes are depicted by the yellow lines. ${ }^{81}$

However, in this case the higher conductance of the two path systems is unlikely to be purely caused by interference effects and is partly a consequence of the greater conformational rigidity and aromaticity of the system. This example illustrates the difficulty in preparing systems in which the change in conductance can truly be credited to changing the molecule from a one path to a two path system without varying other parameters such as conformational changes. In Chapter 4 we will discuss the synthesis and characterisation of a family of linear and cyclic heterometallated oligomers structurally confined by the T6 template. We measured the through-bond exchange coupling $J$ between two paramagnetic copper centres and demonstrate constructive quantum interference over a remarkably long distance of $3.9 \mathrm{~nm}$.

\subsection{Prospective}

This thesis sets out to develop strategies to prepare heterometallated porphyrin oligomers, which are precursors to mixed-metal nanorings. We start by exploring the possibility of incorporating paramagnetic copper(II) centres into linear oligomers. These structures allows us to investigate the axial binding interaction of copper(II) porphyrins to oligo-pyridine templates through chemical double-mutant cycles.

We continue with the development of template-directed approaches to the synthesis of a family of 10- and 6-porphyrin nanorings with combinations of copper(II) and zinc(II) metals at specific positions around the ring. This work opens up many possibilities for controlling the photophysical behaviour of these supramolecular systems and for probing their conformations by EPR as will be shown in Chapter 2, 3 and 4 .

The thesis concludes with two chapters describing the synthesis and properties of two novel porphyrin nanoballs. In Chapter 5 we discuss the preparation of a 
structure containing 14 porphyrin subunits arranged in a 6-and 10-porphyrin nanoring. The rings are connected at two opposing positions, creating the first example of an atomically precise, 3D $\pi$-conjugated porphyrin nanoball. The synthesis of a smaller 10-porphyrin analogue is described in Chapter 6. The structure consists of two 6-porphyrin nanorings resulting in a $D_{4 \mathrm{~h}}$ symmetric structure. In order to access this structure a selective demetallation strategy was developed using magnesium/zinc mixed-metal porphyrin nanorings. 


\section{0-Porphyrin Nanorings with Copper(II) and Zinc(II) Centres}

\section{Contents}

\begin{tabular}{|c|c|}
\hline 1 & ............... \\
\hline 2 & Introduction . \\
\hline 3 & 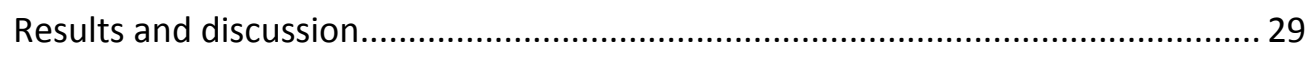 \\
\hline 2.3 .1 & Synthesis of $\boldsymbol{c}-\mathbf{P} 10_{\mathrm{Cu} 2}$. \\
\hline 2.3.2 & Quantification of the Cu-porphyrin pyridine interaction ............. \\
\hline 2.3 .3 & 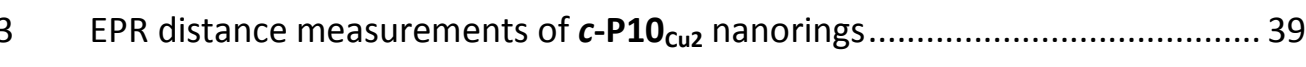 \\
\hline & Conclusion .......... \\
\hline & Experimental procedures and t \\
\hline 2.5 .1 & General experimental \\
\hline 2.5 .2 & Synthetic procedures. \\
\hline 2.5 .3 & UV-vis-NIR titrations.. \\
\hline 2.5 .4 & Calculation of statistical factors .. \\
\hline 2.5 .5 & DFT calcul \\
\hline 2.5 .6 & еплеть \\
\hline
\end{tabular}

\section{Parts of this chapter were published in:}

Nanorings with copper(II) and zinc(II) centers: forcing copper porphyrins to bind axial ligands in heterometallated oligomers

J. Cremers, S. Richert, D. V. Kondratuk, T. D. W. Claridge, C. R. Timmel, H. L. Anderson, Chem. Sci. 2016, 7, 6961-6968.

Exploring template-bound dinuclear copper porphyrin nanorings by EPR spectroscopy

S. Richert, J. Cremers, H. L. Anderson, C. R. Timmel, Chem. Sci. 2016, 7, 6952-6960. 


\subsection{Abstract}

The affinity of copper(II) porphyrins for pyridine ligands is extremely weak, but oligo-pyridine templates can be used to direct the synthesis of $\mathrm{Cu}$-containing cyclic porphyrin oligomers when they also contain zinc porphyrins. Here we describe the synthesis of a heterometallated 10-porphyrin nanoring prepared from a $\mathrm{Zn} / \mathrm{Zn} / \mathrm{Cu} / \mathrm{Zn} / \mathrm{Zn}$ pentamer. The macrocycle has copper porphyrins at two specific positions across the diameter of the ring and zinc porphyrins at the other sites. The $\mathrm{Zn} / \mathrm{Zn} / \mathrm{Cu} / \mathrm{Zn} / \mathrm{Zn}$ linear porphyrin pentamer binds strongly to a penta-pyridyl template, despite the weakness of the $\mathrm{Cu}-\mathrm{N}$ interaction, due to of the chelate cooperativity of the neighbouring $\mathrm{Zn}-\mathrm{N}$ coordinations. The stabilities of a family of four linear porphyrin pentamer complexes were determined by UV-vis-NIR titrations and analysed using a chemical double-mutant cycle. The results show that the binding energy of the copper centre to an axial pyridine ligand is $-6.2 \mathrm{~kJ} \mathrm{~mol}^{-1}$ when the entropy cost of bringing together the two molecules has already been paid by pyridine-zinc interactions. The development of template-directed approaches in the synthesis of nanorings with combinations of different metals around the ring opens up many possibilities for controlling the photophysical behaviour of these supramolecular systems and for probing their conformations by EPR.

\subsection{Introduction}

The use of templates to direct the formation of covalent bonds, via non-covalent interactions, has provided access to many fascinating molecular structures. ${ }^{82-85}$ Various types of reversible interactions can be used in template-directed synthesis. The most versatile of these approaches is arguably the coordination of zinc porphyrins to oligo-amine templates. ${ }^{37,39,42,46,50,54,55,60,86-88}$ Zinc porphyrin nanorings exhibit intriguing photophysical and ligand-binding behaviour, ${ }^{89,90}$ yet their scope would be dramatically expanded if other transition metal centres could be included. Here we present the template directed synthesis of $\mathbf{c}-\mathbf{P} 1 \mathbf{1 0}_{\mathbf{C u 2}}$, a nanoring with copper(II) porphyrins at two specific positions across the diameter of the macrocycle and zinc at the other sites (Figure 2.1). The methodology used should be applicable for the synthesis of many other heterometallated porphyrin nanorings. 


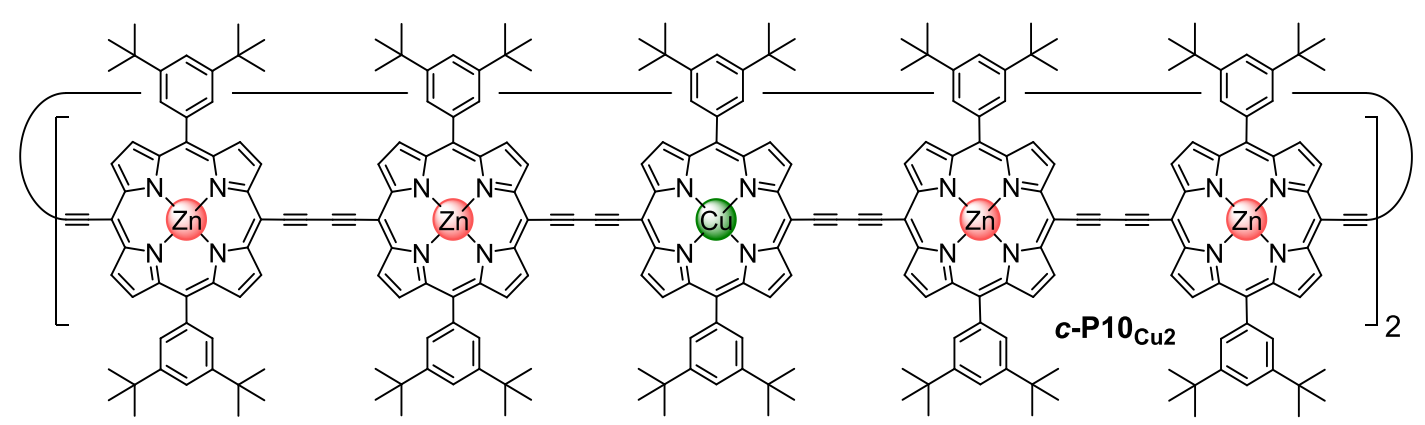

Figure 2.1: Chemical structure of the heterometallated porphyrin nanoring $c-\mathrm{P} 10_{\mathrm{Cu2}}$.

Heterometallated porphyrin oligomers have been widely investigated as model systems for exploring electron transfer, ${ }^{63,70,91}$ energy transfer, ${ }^{92-94}$ nonlinear optical activity $^{72}$ and molecular recognition. ${ }^{95-97}$ The presence of an open-shell copper(II) ion in a porphyrin oligomer provides a local fluorescence-quenching site, due to relaxation via $\mathrm{d}$-d transitions, although phosphorescence from trip-doublet states is sometimes observed at low temperatures. ${ }^{98-101}$ The quenching of fluorescence from a free-base or zinc porphyrin by a remote copper centre provides a test for longrange energy migration. ${ }^{102,103}$ EPR measurements have been used to probe the structure of DNA strands covalently attached to copper(II) porphyrin units. ${ }^{104}$ The through-space dipolar coupling between two paramagnetic copper(II) centres can be measured by EPR using double electron-electron resonance (DEER). ${ }^{105,106}$ This technique provides accurate information on $\mathrm{Cu} \cdots \mathrm{Cu}$ distances in the range 2-5 nm. ${ }^{8,107,108}$ The ability to place two copper(II) centres at precisely defined positions in a nanoring enables the conformation and flexibility of the whole assembly to be probed by EPR. ${ }^{109}$

While the binding of zinc(II) porphyrins to amines has been studied extensively, ${ }^{40,110-}$ 113 little is known about the coordination of axial ligands to copper(II) porphyrins (Figure 2.2). The interaction appears to be extremely weak, as expected from a consideration of the relevant orbitals. In $\mathrm{Cu}(\mathrm{II})\left(\mathrm{d}^{9}\right)$ complexes, there is strong ligand binding in the equatorial plane, due to a single vacancy in the $3 \mathrm{~d}_{x^{2}-y^{2}}$ orbitals. Bonding in the equatorial plane is also enhanced by hybridisation of the $4 \mathrm{~s}$ and $3 \mathrm{~d}_{z^{2}}$ orbitals which leads to a build-up of electron density along the $z$-axis, causing a reduction in axial binding strength. When the d-shell is full, as in $\mathrm{Zn}(\mathrm{II})$, the driving force for binding in the equatorial plane is reduced, as is the $4 \mathrm{~s} / 3 \mathrm{~d}_{z^{2}}$ hybridisation, and axial ligands bind more strongly. During the 1950s, Miller and Dorough reported 
that copper(II) tetraphenyl porphyrins bind pyridine with an association constant of $0.05 \pm 0.02 \mathrm{M}^{-1}$ (in benzene at $303 \mathrm{~K}$ ). ${ }^{110}$ This value was supported by subsequent studies, ${ }^{114}$ but it is not clear whether such a weak interaction can be measured reliably by a simple UV-vis titration, since an association constant of $0.05 \mathrm{M}^{-1}$ requires a pyridine concentration of $20 \mathrm{M}$ for $50 \%$ saturation, which is greater than the concentration of pyridine in neat pyridine $(12 \mathrm{M})$. Here we demonstrate that heterometallated linear porphyrin oligomers can be used to measure the weak interaction of a copper centre with axial nitrogen ligands, without requiring high concentrations of the pyridine ligand, by utilising the cooperative effect of neighbouring zinc centres in a chemical double-mutant cycle. ${ }^{115,116}$

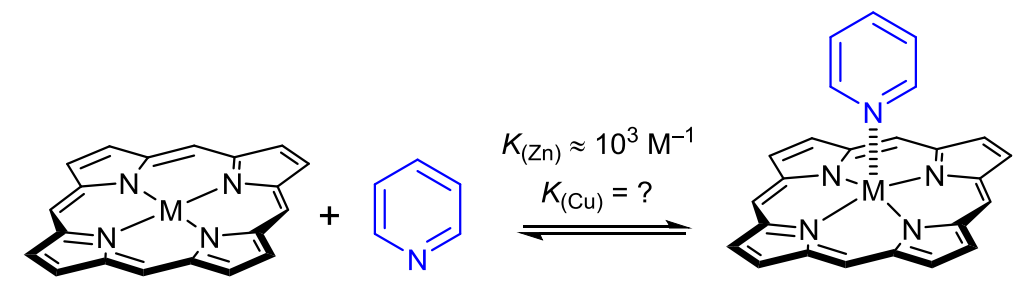

Figure 2.2: Axial coordination of pyridine to a metalloporphyrin ( $\mathrm{M}=\mathrm{Zn}$ or $\mathrm{Cu})$.

Some insights into the interaction of copper and zinc porphyrins with pyridine are provided by DFT calculations. ${ }^{117-121}$ A plot of the self-consistent field (SCF) energy vs. distance $\left(d_{1}\right)$ from constrained DFT geometry optimisations at fixed metal-pyridine distances (Figure 2.3 ) reveals that the binding energy is significantly smaller for copper porphyrins than for zinc porphyrins. As a result, a shorter equilibrium metal-nitrogen bond length $\left(d_{1}\right)$ of $2.18 \AA$ is found for $M=Z n$ compared to $2.35 \AA$ for $\mathrm{M}=\mathrm{Cu}$. The calculations also show that the zinc atom is pulled out of the porphyrin plane by $d_{2}=0.28 \AA$ in the equilibrium geometry, whereas the copper atom is only slightly displaced when it coordinates pyridine, $d_{2}=0.12 \AA$, which reflects stronger equatorial binding (see Section 2.5.5 for more details on the DFT calculations). The calculated distances $d_{1}$ and $d_{2}$ agree well with data from X-ray crystallography. The only known structure of a neutral copper(II) porphyrin with a 5-coordinate metal bound to a nitrogen-ligand is a pyridine complex reported by Lipstman and Goldberg, $^{122-124}$ whereas the Cambridge Structural Database contains 362 crystal structures of zinc porphyrin amine complexes. The mean zinc-pyridine distance is $d_{1}=2.16(3) \AA$ and the out of plane distance is $d_{2}=0.24(6) \AA .{ }^{125}$ The parameters for 
the $\mathrm{Cu}$ (II) complex are $d_{1}=2.47 \AA$ and $d_{2}=0.12 \AA .{ }^{122}$ The scarcity of crystallographic data and the length of this $\mathrm{Cu}-\mathrm{N}$ bond illustrate the weak nature of the interaction.

(a)

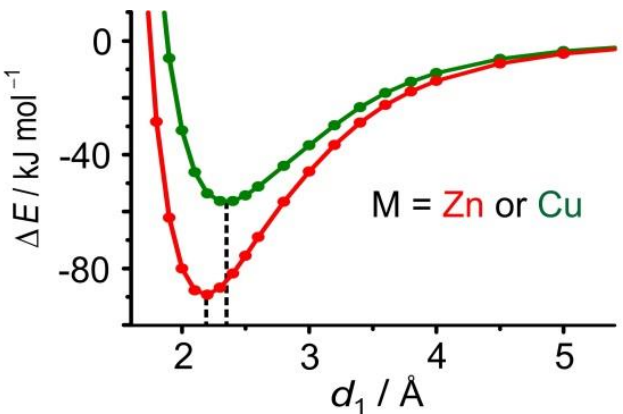

(b)

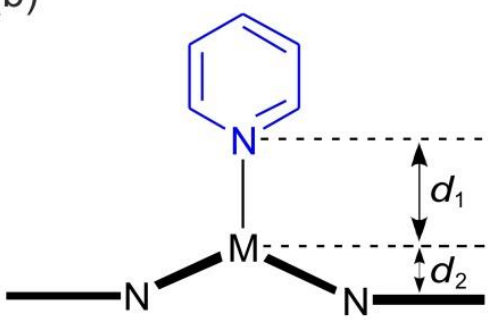

Figure 2.3: (a) Total SCF energy differences as a function of the metal-pyridine separation distance for $\mathrm{M}=\mathrm{Zn}$ (red) and $\mathrm{M}=\mathrm{Cu}$ (green). DFT calculations were carried out with Turbomole V6.1 under $C_{2}$ symmetry, DFT/B3LYP with the TZVP basis set, RI-approximation, empirical dispersion corrected energies. (b) Scheme of a metal centre (M) bound in a porphyrin coordinating an axial pyridine ligand; $d_{1}$ is the distance between the central metal and the axial pyridine, while $d_{2}$ is the displacement of the metal atom from the plane of the porphyrin.

\subsection{Results and discussion}

\subsubsection{Synthesis of $c-\mathrm{P} 10_{\mathrm{Cu} 2}$}

Template-directed synthesis is a powerful strategy for using reversible non-covalent interactions to control the formation of covalent bonds. We have used oligo-pyridine templates to direct the synthesis of zinc porphyrin nanorings, and shown that several small template molecules can cooperate to direct the formation of larger macrocycles, according to a Vernier principle. The ten-porphyrin nanoring with two copper centres was prepared using a 'caterpillar-track' templating strategy by the oxidative homocoupling of two deprotected pentamers in the presence of a pentadentate template.

The synthesis of the 10-ring commenced with the preparation of the zinc porphyrin building block 2.5 (Figure 2.4). Dipyrromethane (2.1) and aryl aldehyde 2.2 were allowed to react in a porphyrin condensation reaction to give a free-base porphyrin intermediate which was immediately metallated with zinc to give porphyrin $\mathbf{2 . 3}$ in a $48 \%$ yield. Bromination of the meso-positions using $N$-bromosuccinimide (NBS) gave 2.4 in a 76\% yield, followed by a Sonogashira coupling with ethynyltrihexylsilane giving 2.5 in a nearly quantitative yield. 


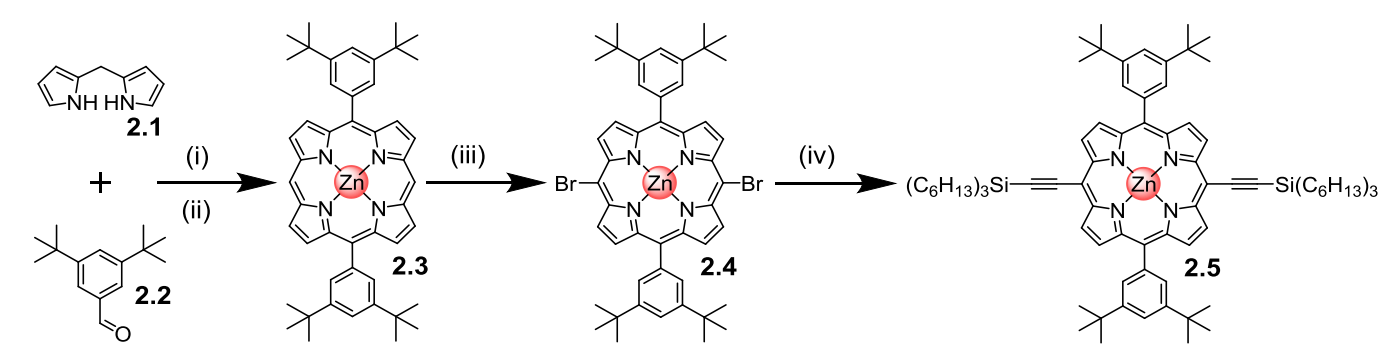

Figure 2.4: Synthetic route to porphyrin 2.5: (i) TFA, DDQ, $\mathrm{CH}_{2} \mathrm{Cl}_{2}$, then (ii) $\mathrm{Zn}(\mathrm{OAc})_{2} \cdot\left(\mathrm{H}_{2} \mathrm{O}\right)_{2}, \mathrm{CHCl}_{3}, 48 \%$; (iii) $\mathrm{NBS}$, $\mathrm{CHCl}_{3}$ /pyridine, 98\%; (iv) $\mathrm{Pd}_{2}$ (dba) ${ }_{3}, \mathrm{Cul}, \mathrm{PPh}_{3}$, ethynyltrihexylsilane, toluene/i- $\mathrm{Pr}_{2} \mathrm{NH}, 99 \%$.

When porphyrin $\mathbf{2 . 5}$ is exposed to a fluoride source, such as tetra- $n$-butylammonium fluoride (TBAF), the trihexylsilyl (THS) protecting groups on the acetylenes can be removed. While the addition of an excess TBAF will deprotect both acetylenes, the addition of small quantities resulted in a statistical mixture of the starting material together with the mono-deprotected and fully-deprotected analogues of 2.5. The mono-deprotected analogue $\mathbf{2 . 6}$ was isolated from the mixture by silica gel column chromatography in a 38\% yield. Oxidative homocoupling of $\mathbf{2 . 6}$ gave the symmetric dimer $\mathbf{2 . 7}$ in a $91 \%$ yield (Figure 2.5 ).

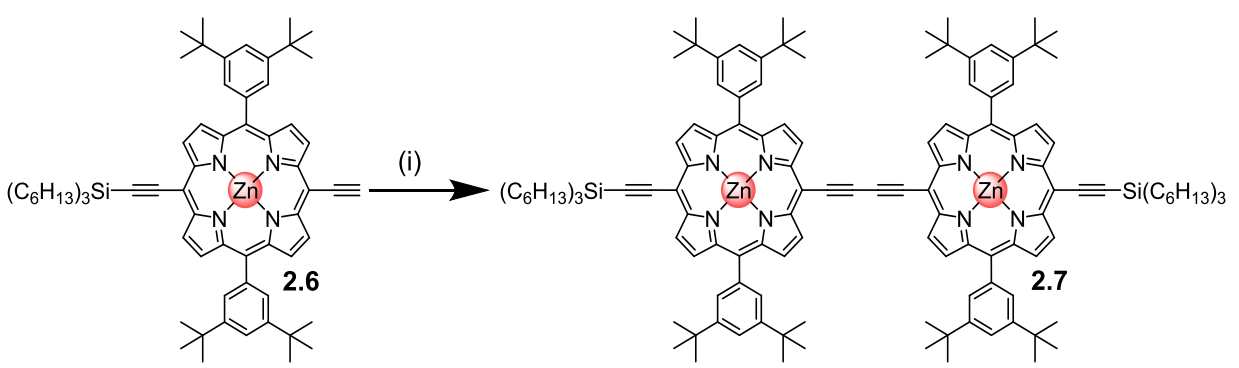

Figure 2.5: Synthetic route to porphyrin dimer 2.7: (i) $\mathrm{Pd}\left(\mathrm{PPh}_{3}\right)_{2} \mathrm{Cl}_{2}$, Cul, 1,4-benzoquinone, toluene/i- $\mathrm{Pr}_{2} \mathrm{NH}$, $91 \%$.

The adaptability of the building block $\mathbf{2 . 5}$ extends to the metal centre in the core of the porphyrin. While zinc porphyrins are commonly used due to their well-established synthetic methods, their stability, and their ability to coordinate nitrogen based ligands, a vast range of other metal centres can be used. As shown in Figure 2.6, the zinc centre of $\mathbf{2 . 5}$ can be removed by the addition of a strong acid such as trifluoroacetic acid (TFA) in a $68 \%$ yield. Subsequently, the THS protecting groups were removed with TBAF to give the free-base, deprotected porphyrin 2.9 in a $76 \%$ yield. 


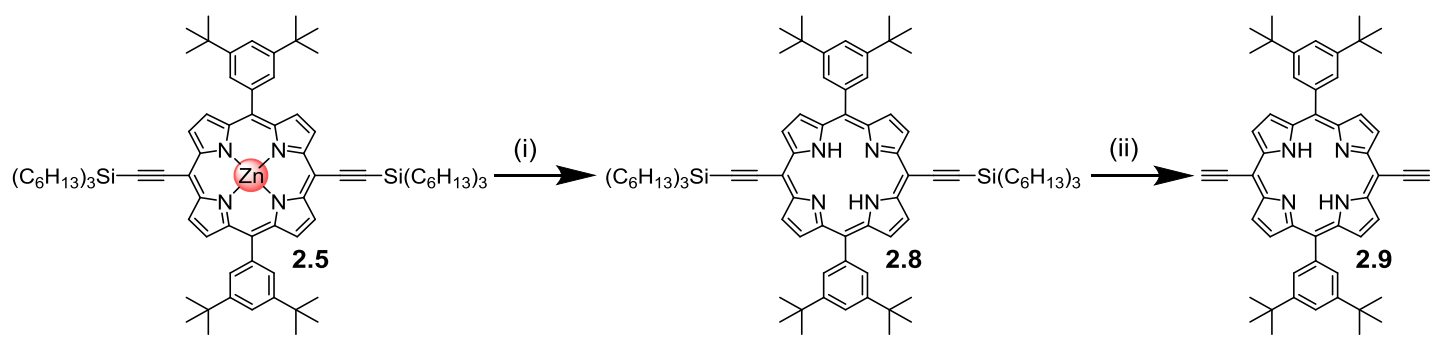

Figure 2.6: Synthetic route to porphyrin 2.9: (i) $\mathrm{TFA}, \mathrm{CHCl}_{3}, 68 \%$; (ii) $\mathrm{TBAF}, \mathrm{CH}_{2} \mathrm{Cl}_{2}, 76 \%$.

The linear pentamer with a free-base porphyrin in the middle of the chain, was prepared in a 33\% yield by coupling porphyrin $\mathbf{2 . 9}$ with a large excess of mono-protected dimer 2.10, as shown in Figure 2.7. Subsequently, copper was inserted into the central free-base porphyrin unit to give the heterometallated oligomer $\mathbf{2 . 1 2}$ in a 73\% yield. The route going through a free-base intermediate was adopted because free-base monomers have better solubility than the corresponding copper porphyrins. In addition, the route can easily be modified to insert other metals at the centres of the oligomers if desired. The use of a free-base intermediate does force us to use copper-free palladium catalysed coupling conditions to avoid premature insertion of copper into the free-base porphyrin units. ${ }^{126}$ However, it was later found that the reaction using classic conditions in the presence of copper proceeds fast enough and no copper insertion is generally observed.

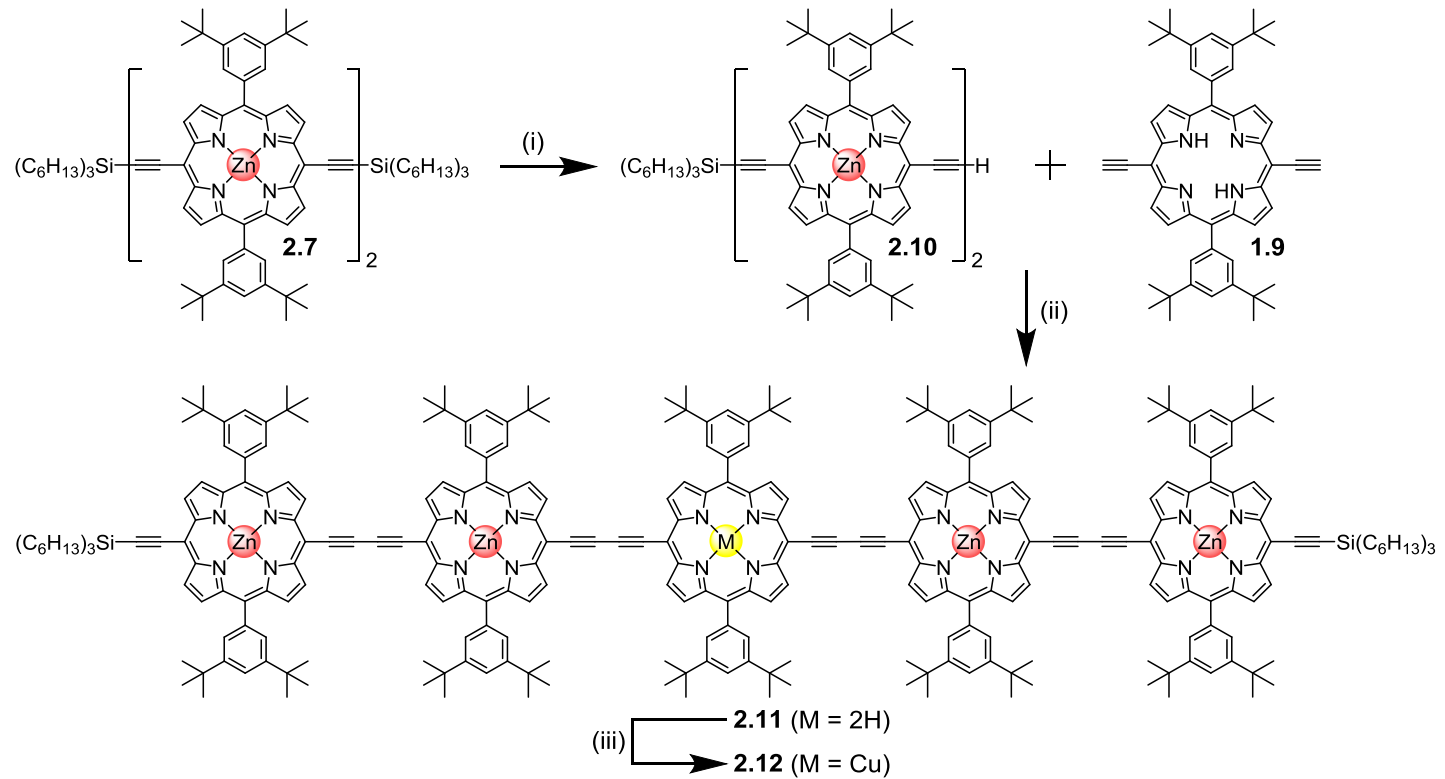

Figure 2.7: Synthetic route to the heterometallated pentamer 2.12: (i) $\mathrm{TBAF}, \mathrm{CH}_{2} \mathrm{Cl}_{2}, 33 \%$; (ii) $\mathrm{Pd}_{2}\left(\mathrm{dba}_{3}\right.$, tri-2-furylphosphine, 1,4-benzoquinone, toluene/ $\mathrm{Et}_{3} \mathrm{~N}, 33 \%$; (iii) $\mathrm{Cu}(\mathrm{OAC})_{2} \cdot \mathrm{H}_{2} \mathrm{O}, \mathrm{CHCl}_{3}, 73 \%$.

The THS protecting groups on the heterometallated pentamer $\mathbf{2 . 1 2}$ were removed in an $86 \%$ yield by the addition of TBAF. The subsequent pentamer 2.13 contains four 
zinc porphyrin units and a central copper porphyrin (Figure 2.8). While the binding of a copper porphyrin to axial pyridyl ligands is nearly non-existing, the presence of the adjacent zinc porphyrin dimers provides a strong cooperative binding interaction with a pentadentate T5 template allowing for the formation of a stable one-to-one complex (Figure 2.8). The ten-porphyrin nanoring with two copper centres, $\mathbf{c}-\mathbf{P} 1 \mathbf{C}_{\mathrm{Cu2}}$, was then prepared using a 'caterpillar-track' templating strategy, ${ }^{88}$ by oxidative homocoupling of the deprotected pentamer, $\mathbf{2 . 1 3}$, in the presence of a pentadentate template T5. The desired heterometallated nanoring was isolated as its template-complex $\boldsymbol{c}-\mathbf{P} 1 \mathbf{C O}_{\mathrm{Cu2}} \cdot(\mathrm{T5})_{\mathbf{2}}$ in a $17 \%$ yield. The $\mathbf{T 5}$ template was removed from this complex in quantitative yield by addition of excess pyridine to yield the target molecule $\boldsymbol{c}-\mathrm{P} 10_{\text {Cu2 }}$. All of the compounds were fully characterised by MALDI-ToF mass spectroscopy, UV-vis-NIR and NMR spectroscopy
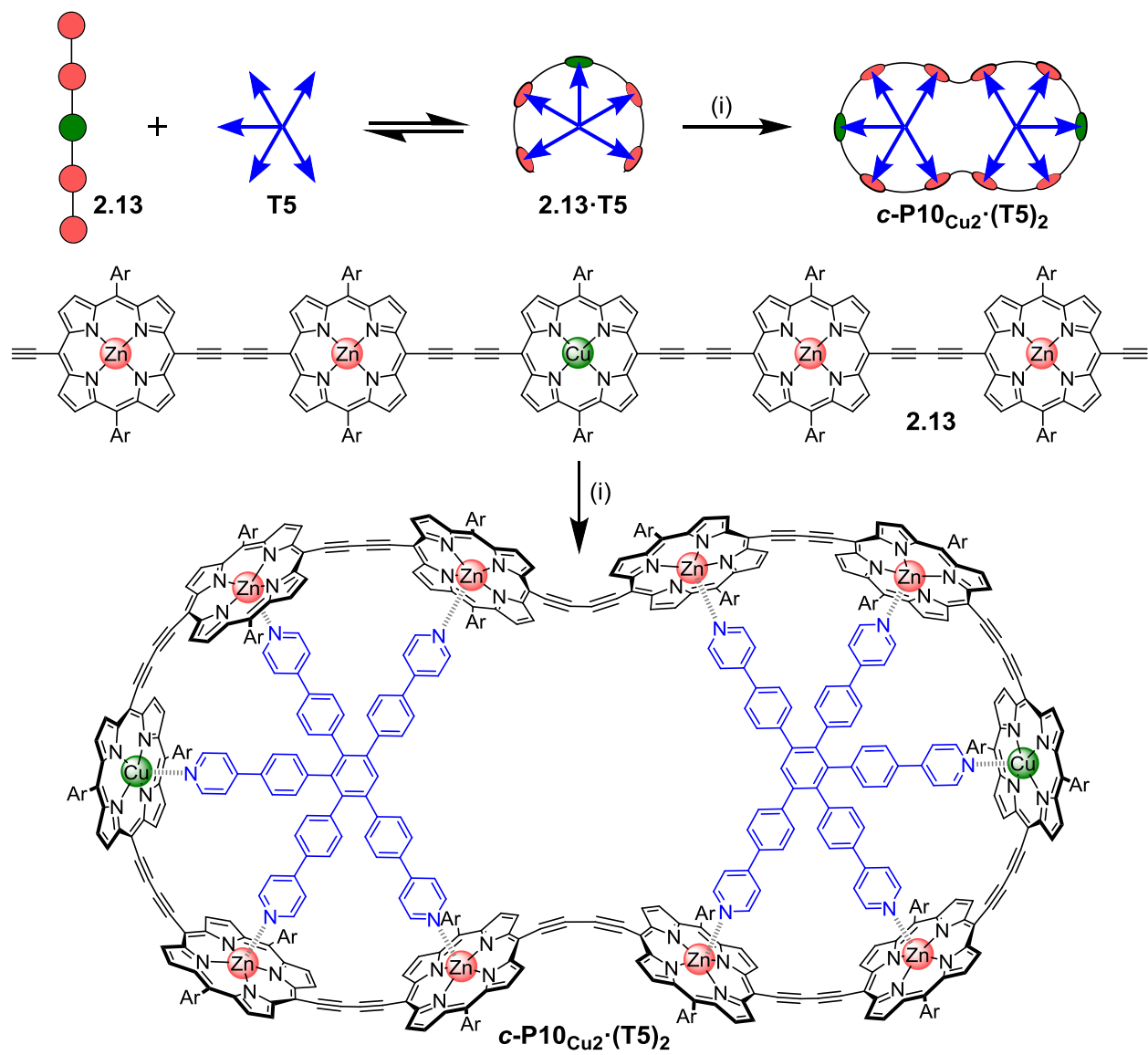

Figure 2.8: Synthesis of $c-\mathrm{P}_{10} \mathrm{Cu2}_{\mathrm{C}} \cdot(\mathrm{T} 5)_{2}$. Two representations of the synthetic route used to prepare c- $-\mathrm{P}_{\mathrm{Cu2}} \cdot(\mathrm{T} 5)_{2}$. Reaction conditions: (i) $\mathrm{PdCl}_{2}\left(\mathrm{PPh}_{3}\right)_{2}$, Cul, 1,4-benzoquinone, $\mathrm{CHCl}_{3} / \mathrm{i}-\mathrm{Pr}_{2} \mathrm{NH}, 17 \%$. Ar = 3,5-bis(tert-butyl)phenyl. 


\subsubsection{Quantification of the Cu-porphyrin pyridine interaction}

Chemical double-mutant cycles (DMCs) are a way to probe weak, non-covalent interactions by utilising the cooperative binding effect of stronger neighbouring interactions. ${ }^{115,116,127,128}$ This approach allows one to disentangle the free energy contribution due to chelate cooperativity associated with the formation of intramolecular non-covalent interactions. We envisioned that we could utilise the cooperative binding effect of the four zinc porphyrins in $\mathbf{2 . 1 2}$ to force the copper centre to interact with the template and determine its contribution to the binding strength. The DMC illustrated in Figure 2.9 quantifies and eliminates all secondary and allosteric effects associated with single mutations and provides a measure of the free energy benefit associated with the $\mathrm{Cu} \cdots \mathrm{N}$ interaction by comparing the affinities of T4 and T5 for 2.11 and 2.12.

Simply comparing the stabilities of 2.12.T5 and 2.11.T5 (complexes A and B respectively) would give an estimate of the energy of the $\mathrm{Cu} \cdots \mathrm{N}$ interaction, but this approach could lead to false conclusions since a mutation in one part of the molecule $(\mathbf{2 . 1 2} \rightarrow \mathbf{2 . 1 1})$ may influence other interactions; for example, a free-base porphyrin might be more flexible than a copper porphyrin, which would add to the stability of 2.11.T5, or there might be a contact between the pyridine ligand and the free-base porphyrin which could destabilise 2.11-T5. Similar issues apply to a simple comparison of complexes $A$ and $C$, where a single mutation is made in the ligand $(\mathrm{T} 5 \rightarrow \mathrm{T} 4)$. The DMC approach overcomes these problems by cancelling the secondary free energy effects of the mutations in a pairwise fashion in the thermodynamic cycle. ${ }^{115,116}$ The cycle is completed by probing the stability of complex D (2.11.T4), in which both the copper centre and the central binding leg are removed. 

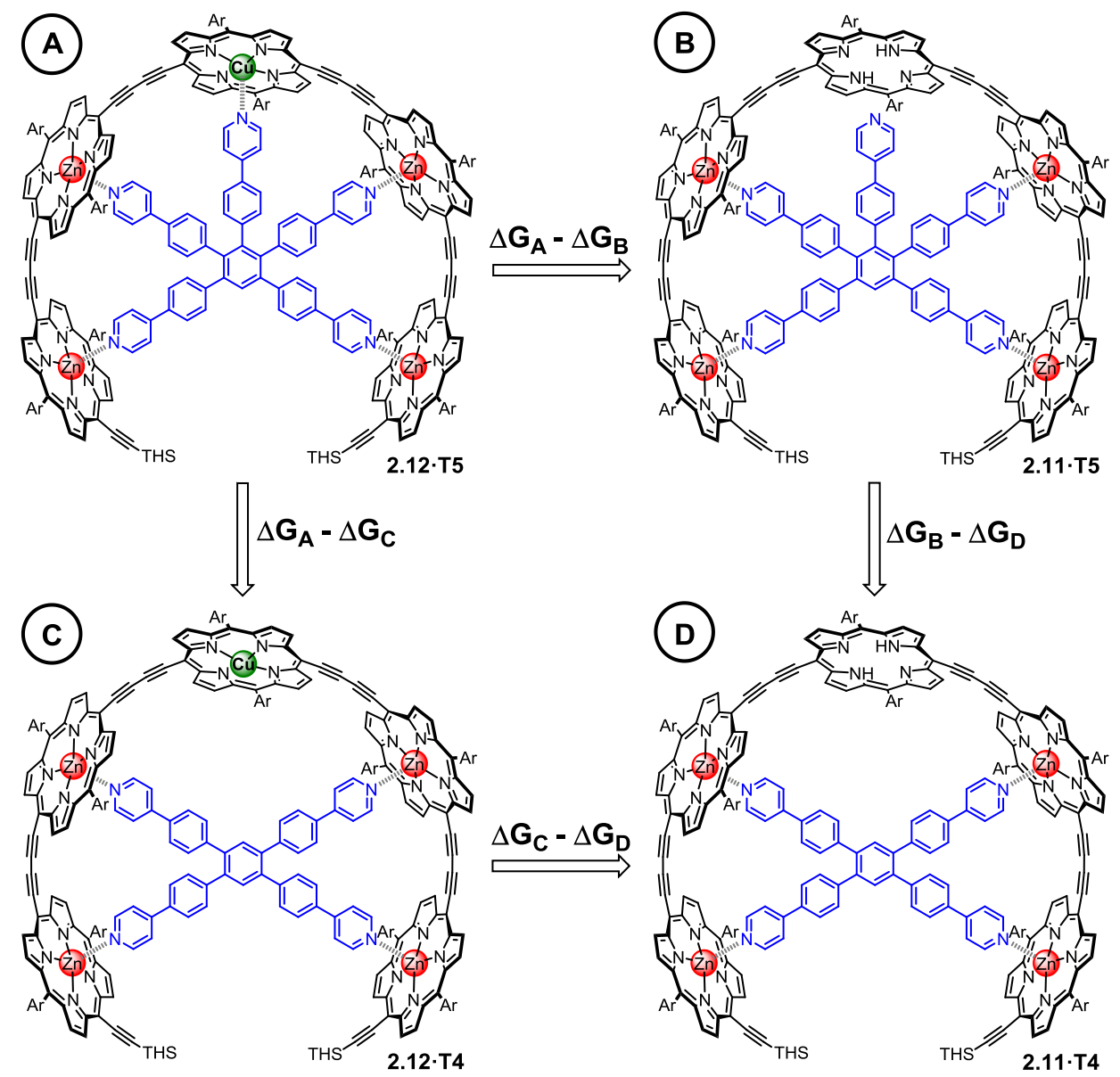

Figure 2.9: The chemical DMC used to investigate the interaction between the copper centre in the central porphyrin and the pyridine leg of the template T5. Ar = 3.5-bis(tert-butyl)phenyl, THS = trihexylsilyl.

The one-to-one complexes shown in Figure 2.9 were generated by titrating solutions of the corresponding porphyrin pentamers dissolved in chloroform with the ligands T5 or T4. These UV-vis-NIR titrations gave sharp end points, and the complexes are too stable for their formation constants to be determined directly from their formation curves. Therefore, denaturation titrations were performed to determine the formation constant $K_{\mathrm{f}}$ via the denaturation constant $K_{\mathrm{dn}} \cdot{ }^{23,55,60} \mathrm{~A}$ large excess of

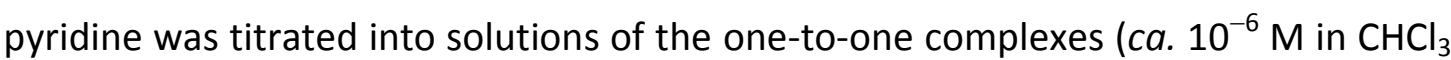
at $298 \mathrm{~K})$ to displace the multidentate ligands. Our analysis assumes that the denaturation processes are essentially all-or-nothing two state equilibria (i.e. that intermediate partially denatured species do not build up to significant concentration). This assumption is supported by the isosbestic nature of the UV-vis-NIR titrations and by the good fits of the curves to the calculated binding isotherm for a two-state equilibrium (Figure 2.10-Figure 2.13). Denaturation constants were used to calculate the formation constants $K_{\mathrm{f}}$ using equation 2.1: 


$$
K_{\mathrm{f}}=\frac{K_{\mathrm{Py}}^{4}}{K_{\mathrm{dn}}}
$$

where $K_{\mathrm{Py}}$ is a reference binding constant which was approximated to the binding of pyridine to porphyrin monomer 2.5 (see section 2.5.3; $K_{\mathrm{Py}}=3.2 \times 10^{3} \mathrm{M}^{-1}$ in $\mathrm{CHCl}_{3}$ at $298 \mathrm{~K})$. The analysis of the titration of 2.12.T5 in terms of the equation 2.1 assumes that coordination of pyridine to the copper porphyrin centre in 2.12.T5 does not significantly contribute to its denaturation, which is valid since copper porphyrins show negligible binding to pyridine at a concentration of $6 \mathrm{mM}$ in $\mathrm{CHCl}_{3}$ at $298 \mathrm{~K}$.
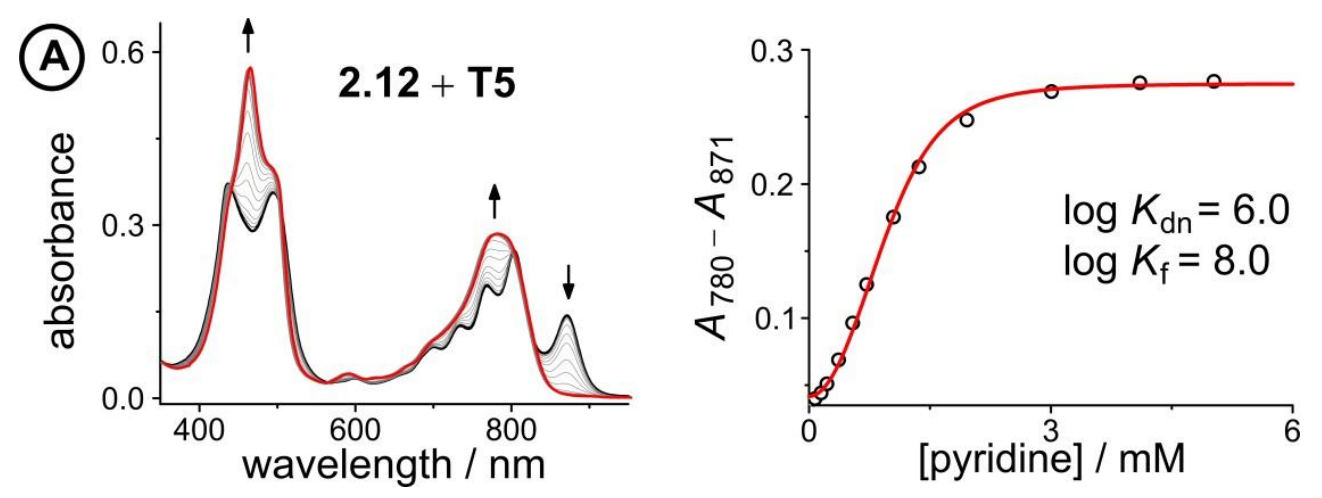

Figure 2.10: UV-vis-NIR denaturation titration of 2.12. $\mathrm{T5}\left(\mathrm{CHCl}_{3}\right.$ at $\left.298 \mathrm{~K}\right)$. (left) The spectra of the one-to-one complex (black) and the end spectra of the pyridine-saturated oligomer (red). (right) The experimental (black circles) and calculated binding isotherms (red line).
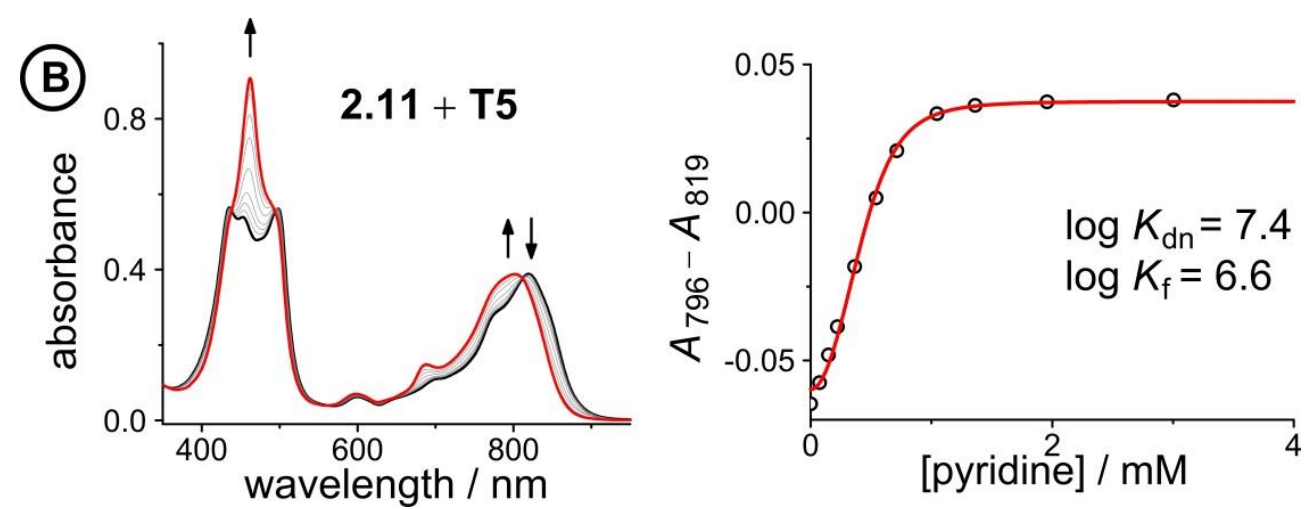

Figure 2.11: UV-vis-NIR denaturation titration of 2.11.T5 $\left(\mathrm{CHCl}_{3}\right.$ at $\left.298 \mathrm{~K}\right)$. (left) The spectra of the one-to-one complex (black) and the end spectra of the pyridine-saturated oligomer (red). (right) The experimental (black circles) and calculated binding isotherms (red line). 

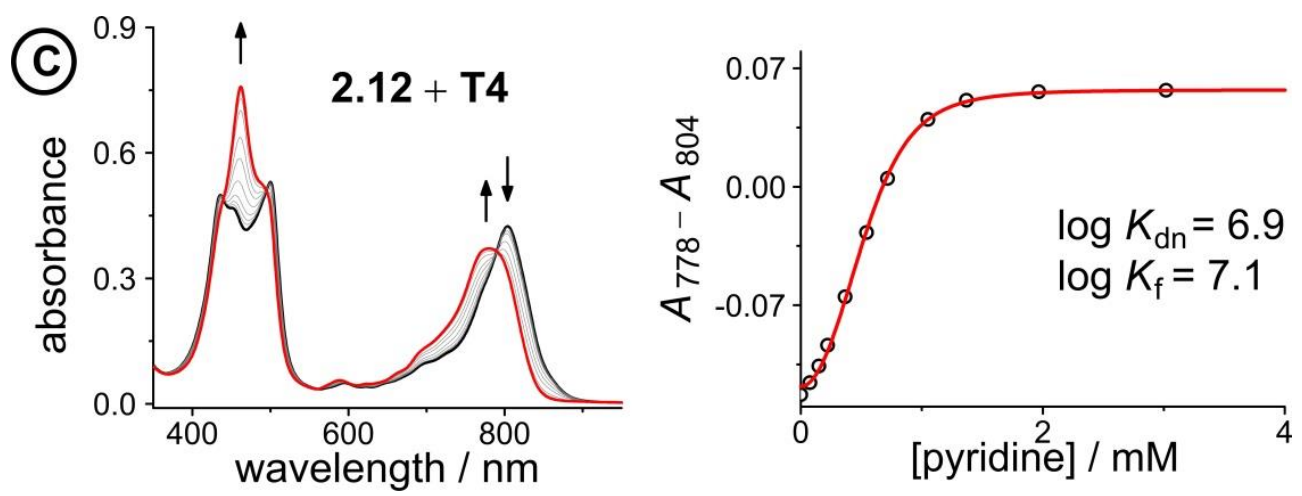

Figure 2.12: UV-vis-NIR denaturation titration of 2.12-T4 ( $\mathrm{CHCl}_{3}$ at $\left.298 \mathrm{~K}\right)$. (left) The spectra of the one-to-one complex (black) and the end spectra of the pyridine-saturated oligomer (red). (right) The experimental (black circles) and calculated binding isotherms (red line).
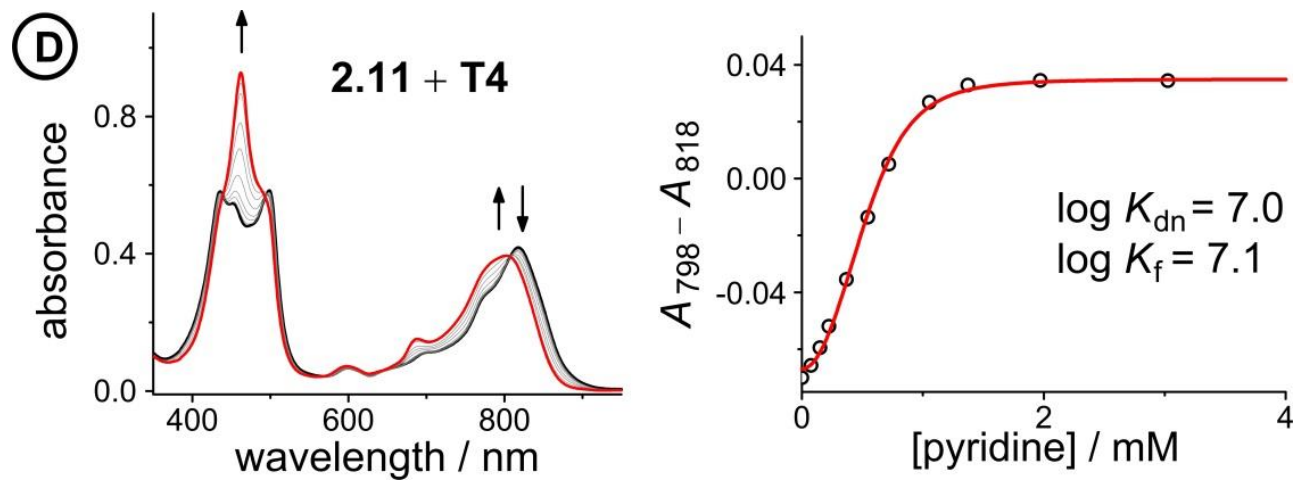

Figure 2.13: UV-vis-NIR denaturation titration of 2.11·T4 $\left(\mathrm{CHCl}_{3}\right.$ at $\left.298 \mathrm{~K}\right)$. (left) The spectra of the one-to-one complex (black) and the end spectra of the pyridine-saturated oligomer (red). (right) The experimental (black circles) and calculated binding isotherms (red line).

The denaturation titrations of the complexes with pyridine are illustrated in Figure 2.10-Figure 2.13 and the results are summarised in Table 2.1. The absorption spectra of the complexes with multidentate ligands are more red-shifted (black lines) than the final spectra (red lines) in which the complex is completely denaturated. The red-shift is caused by the more rigid structures and reduced porphyrin-porphyrin dihedral angles in the template complexes, compared to the free oligomers. The absorption spectrum of the template complex 2.12.T5 (A) clearly reveals more structure in the Q-bands compared to 2.11·T5 (B), 2.12·T4 (C), and 2.11·T4 (D), which is further evidence for the interaction of the copper centre with the template. Binding to T5 locks every porphyrin unit in $\mathbf{2 . 1 2}$ into position and the rotational freedom of the central copper porphyrin is lost, giving rise to the observed characteristic fine structure in the absorption spectrum. In the complexes 2.11.T5 (B), 2.12.T4 (C), and 2.11.T4 (D), motion of the central porphyrin is less restricted resulting in a broader absorption band. The spectral changes observed in the 
denaturation of complexes B-D are similar because in all cases there is no interaction between the central porphyrin and the template.

Table 2.1: Equilibrium constants and free energy changes from the titrations in Figure 2.10-Figure 2.13.

\begin{tabular}{|c|c|c|c|}
\hline 2.12·T5 (A) & $9.1 \pm 2.0 \times 10^{5}$ & $8.0 \pm 0.1$ & $-35.6 \pm 0.3$ \\
\hline 2.11·T5 (B) & $2.4 \pm 0.3 \times 10^{7}$ & $6.6 \pm 0.1$ & $-29.2 \pm 0.2$ \\
\hline 2.12·T4 (C) & $8.1 \pm 0.3 \times 10^{6}$ & $7.1 \pm 0.1$ & $-30.2 \pm 0.2$ \\
\hline 2.11·T4 (D) & $9.0 \pm 0.3 \times 10^{6}$ & $7.0 \pm 0.1$ & $-29.9 \pm 0.2$ \\
\hline
\end{tabular}

The stability constants of complex 2.12-T4 (C) and 2.11·T4 (D) are nearly identical, indicating that there is no inductive effect on the binding interaction due to the presence of copper and that the flexibility of copper and free-base porphyrins are comparable. A slightly lower stability constant is found for complex 2.11.T5 (B), which might reflect steric repulsion of the central template leg with the central free-base unit of the oligomer. The formation constant of the complex 2.12.T5 (A) is roughly an order of magnitude higher than the others, due to interaction of the copper centre with the template.

The energy of the copper-pyridine interaction $\left(\Delta \Delta G_{\mathrm{Cu}}\right)$ was calculated using equation 2.2:

$$
\Delta \Delta G_{\mathrm{Cu}}=\Delta G_{\mathrm{A}}-\Delta G_{\mathrm{B}}-\Delta G_{\mathrm{C}}+\Delta G_{\mathrm{D}}
$$

where $\Delta G_{\mathrm{X}}$ is the statistically corrected energy of formation of complex $\mathrm{X}$ calculated according to equation $2.3 ; K_{\mathrm{chem}(\mathrm{X})}$ is the statistically corrected formation constant and $K_{\sigma(\mathrm{X})}$ is the statistical factor of complex X (see section 2.5.4).

$$
\Delta G_{\mathrm{X}}=-R T \ln K_{\operatorname{chem}(\mathrm{X})}=-R T \ln \left(\frac{K_{\mathrm{f}(\mathrm{X})}}{K_{\sigma(\mathrm{X})}}\right)
$$

Analysis of these data reveal that the energy of interaction between the copper centre and the template is $\Delta \Delta G_{\mathrm{Cu}}=-6.2 \pm 0.4 \mathrm{~kJ} \mathrm{~mol}^{-1}$. This interaction energy is substantially less than the energy of the copper porphyrin pyridine interaction from DFT calculation (56.6 kJ mol${ }^{-1}$, Figure 2.3), which is not surprising as the DFT calculations do not take solvation into account. The free energy changes associated 
with single mutations $\Delta G_{\mathrm{A}}-\Delta G_{\mathrm{B}}=-6.4 \pm 0.3 \mathrm{~kJ} \mathrm{~mol}^{-1}$ and $\Delta G_{\mathrm{A}}-\Delta G_{\mathrm{C}}=-5.4 \pm 0.3$ $\mathrm{kJ} \mathrm{mol}^{-1}$ differ significantly from the value from the double-mutant cycle, showing the benefit of the DMC approach. Its use in this system provided clearer insights into the weak binding interaction than would have been achieved from single mutations.

The free energy term $\Delta \Delta G_{\mathrm{Cu}}=-6.2 \pm 0.4 \mathrm{~kJ} \mathrm{~mol}^{-1}$ is an estimate of the enthalpy of the copper-pyridine interaction, because it is measured in a situation where the loss of translational entropy of bringing together two molecules has already been paid, and the interaction probed by the DMC is effectively intramolecular. In general, the entropy cost of bringing two molecules together to form a non-covalent complex in solution at $298 \mathrm{~K}$ contributes approximately $-T \Delta S \approx+6 \mathrm{~kJ} \mathrm{~mol}^{-1}$ to $\Delta G,{ }^{34,129}$ which would correspond to a free energy for a bimolecular copper porphyrin pyridine interaction of $\Delta G_{\mathrm{Cu}} \approx 0$ (i.e. $K \approx 1 \mathrm{M}^{-1}$ ). However there are reports that the coordination of pyridine to a metalloporphyrin is more entropically unfavourable which would explain the very weak association constant. ${ }^{130-132}$

When two molecules bind together through more than one point of interaction, the increased stability resulting from chelate cooperativity can be quantified by the effective molarity (EM). ${ }^{27,133}$ Comparison of the stability constants of 2.14.T5 $\left(K_{\text {chem }}=1.1 \pm 0.2 \times 10^{12} \mathrm{M}^{-1}\right)$ with those of 2.11.T5 $\left(K_{\text {chem }}=1.3 \pm 0.2 \times 10^{5} \mathrm{M}^{-1}\right)$ and 2.5 with 4-phenylpyridine $\left(K_{\text {chem }}=2.1 \pm 0.1 \times 10^{3} \mathrm{M}^{-1}\right)$ indicates that the effective molarity of the central $\mathrm{Zn}-\mathrm{N}$ interaction in 2.14.T5 is EM $=4 \pm 1 \times 10^{3} \mathrm{M}$ (see Figure 2.14 and section 2.5.3.3). If we assume that the effective molarity is the same for the central Cu- $\mathrm{N}$ interaction in $\mathbf{2 . 1 2} \cdot \mathrm{T5}$, then the single-site microscopic binding constant for copper porphyrins to pyridyl ligands, $K_{\mathrm{Cu}}$, can be estimated by dividing the observed equilibrium constant $\left(\Delta \Delta G_{\mathrm{Cu}}=-6.2 \mathrm{~kJ} \mathrm{~mol}^{-1} \Rightarrow K_{\mathrm{Cu}} \mathrm{EM}=12\right)$ by the effective molarity, giving $K_{\mathrm{Cu}}=3.1 \times 10^{-3} \mathrm{M}^{-1}$. This value illustrates how a high effective molarity enables very weak interactions to be measured.

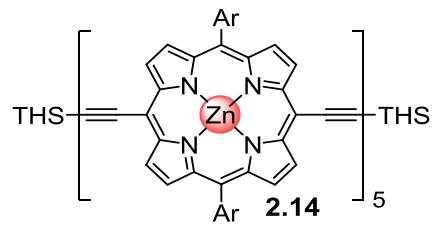

Figure 2.14: Chemical structure of the zinc porphyrin pentamer 2.14. 


\subsubsection{EPR distance measurements of $c-\mathrm{P} 10_{\mathrm{Cu} 2}$ nanorings}

The presence of the copper centres in $\mathbf{c}-\mathbf{P} 10_{\text {Cu2 }}$ complicates the ${ }^{1} \mathrm{H}$ NMR characterisation since the unpaired electrons cause rapid relaxation, resulting in broad signals. EPR is a more suitable technique to study large systems containing paramagnetic centres. Local changes in the electronic or nuclear environment of the paramagnetic centre, which occur upon interaction with any ligand, can be probed sensitively.

The heterometallated ten-membered ring, $\boldsymbol{c}-\mathbf{P} 1 \mathbf{1 0}_{\mathrm{Cu} 2}$, forms an ideal model compound for EPR experiments. In collaboration with Dr Sabine Richert and Prof. Christiane R. Timmel, the structure was investigated in detail by a variety of EPR techniques. EPR is suitable for carrying out distance measurements between paramagnetic centres in the nanometer range and can provide insights into the molecular structure. In addition, information about the flexibility of any investigated structures can be obtained in the form of distance distributions.

We decided to investigate the $\mathrm{Cu} \cdots \mathrm{Cu}$ distance in $\mathbf{c}-\mathrm{P} 10_{\mathrm{Cu} 2}$ by using four-pulse double electron-electron resonance (DEER) measurements at Q-band frequencies to obtain information about the geometry of the ring and the location of the Cu-centres. In addition, the measurements were used to provide information on the flexibility of the ring. We hypothesised that we could control the geometry of the ring and thus the location of the copper centres by using different pyridine based ligands (Figure 2.15).
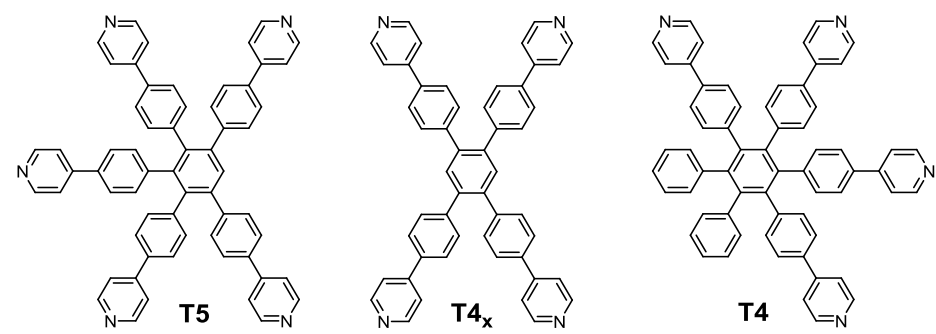

Figure 2.15: Chemical structure of the templates $\mathrm{T5}, \mathrm{T}_{\mathrm{x}}$ and $\mathrm{T} 4$.

As shown before, the axial interaction of the zinc porphyrins with the templates is far stronger than the interaction of copper porphyrins to the templates. As a result, the templates will prefer to bind the zinc porphyrins and potentially force the ring in a specific geometry as illustrated in Figure 2.16. 


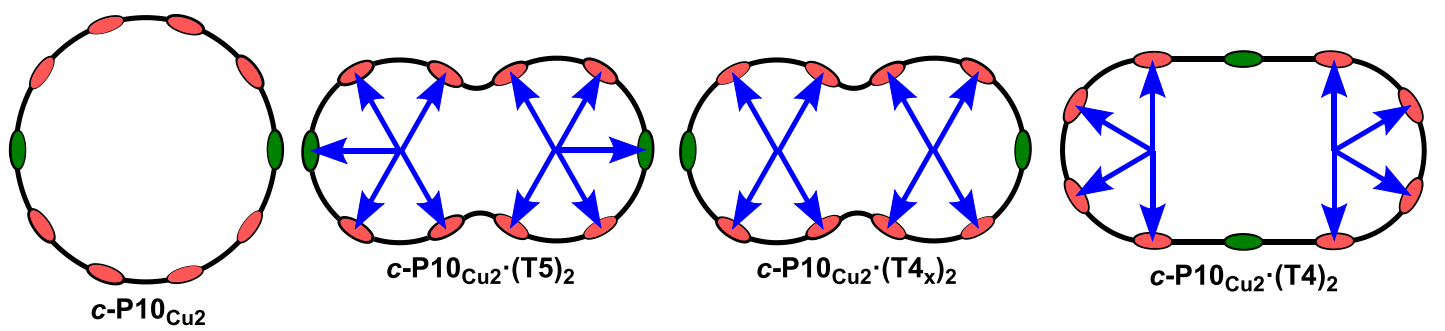

Figure 2.16: Schematic representation of the structures of $c-\mathrm{P} 10_{\mathrm{Cu} 2}, c-\mathrm{P} 10_{\mathrm{Cu} 2} \cdot(\mathrm{T} 5)_{2}, c-\mathrm{P} 10_{\mathrm{Cu} 2} \cdot\left(\mathrm{T} 4_{\mathrm{x}}\right)_{2}$ and $c-\mathrm{P} 10_{\mathrm{Cu2}} \cdot(\mathrm{T} 4)_{2}$. The structure $c-\mathrm{P} 10_{\mathrm{Cu2}} \cdot(\mathrm{T} 5)_{2}$ has been drawn with the two copper centres at a maximum distance from each other. As will be explained later there is no strong preference for the complex to adopt this geometry necessarily.

To confirm the formation of the two-to-one complexes, UV-vis-NIR titrations were carried out between c-P10 $\mathbf{C u 2}_{\text {}}$ and the templates (Figure 2.17Figure 2.19). Upon the addition of small aliquots of templates, a strong red-shift in the Q-bands was observed as a result of the formation of the desired two-to-one complex. A linear, isosbestic change in the absorption spectrum is observed upon the addition of two equivalents of template, indicating the strong formation of the complex (black to red).
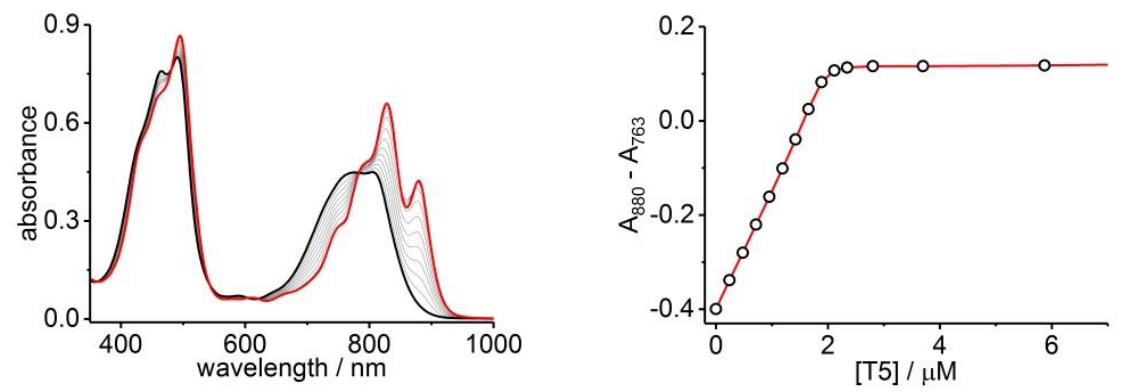

Figure 2.17: UV-vis-NIR formation titration of $c$-P10 $10_{\text {Cu2 }}$ and the T5 template (chloroform, $298 \mathrm{~K}$ ) indicating the formation of a two-to-one complex (black to red).
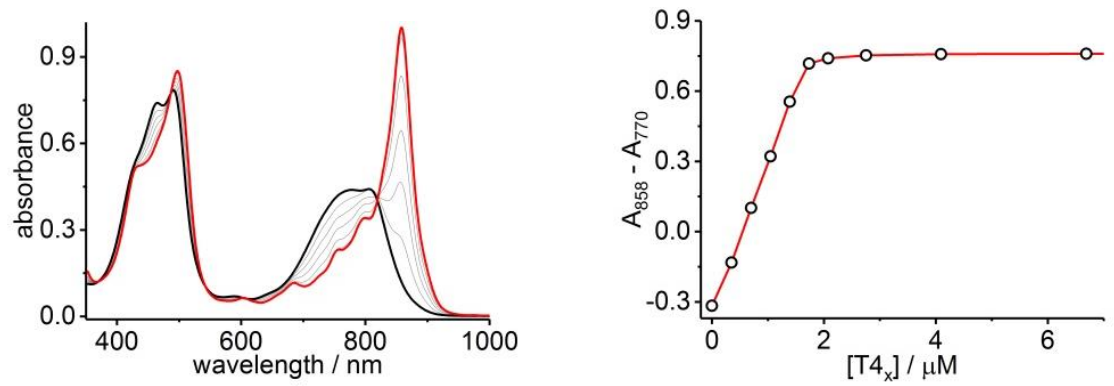

Figure 2.18: UV-vis-NIR formation titration of $c-\mathrm{P} 10_{\mathrm{Cu} 2}$ and the $\mathrm{T} 4_{\mathrm{x}}$ template (chloroform, $298 \mathrm{~K}$ ) indicating the formation of a two-to-one complex (black to red). 

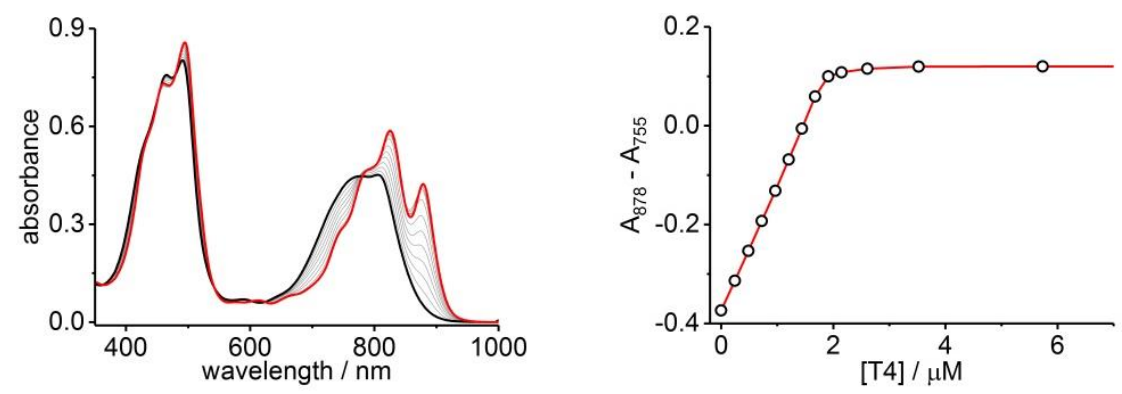

Figure 2.19: UV-vis-NIR formation titration of $c$ - $\mathrm{P} 10_{\mathrm{Cu} 2}$ and the $\mathrm{T} 4$ template (chloroform, $298 \mathrm{~K}$ ) indicating the formation of a two-to-one complex (black to red).

The traces in Figure 2.20 show the background-corrected experimental DEER data for $c-P 10_{\text {Cu2 }}$ and its templated complexes recorded under identical experimental conditions. Clear dipolar modulations can be observed in all traces, pointing towards $\mathrm{Cu} \cdots \mathrm{Cu}$ separation of $c a .2 .5 \mathrm{~nm}$ for the complexes with T4 and T5, $4.3 \mathrm{~nm}$ in the template free ring, and $5.0 \mathrm{~nm}$ in the complex with the $\mathbf{T} \mathbf{4}_{\mathbf{x}}$ template. Since the modulations are well defined in all cases, it can be concluded that the distance distributions are rather narrow and there is little flexibility in the rings.

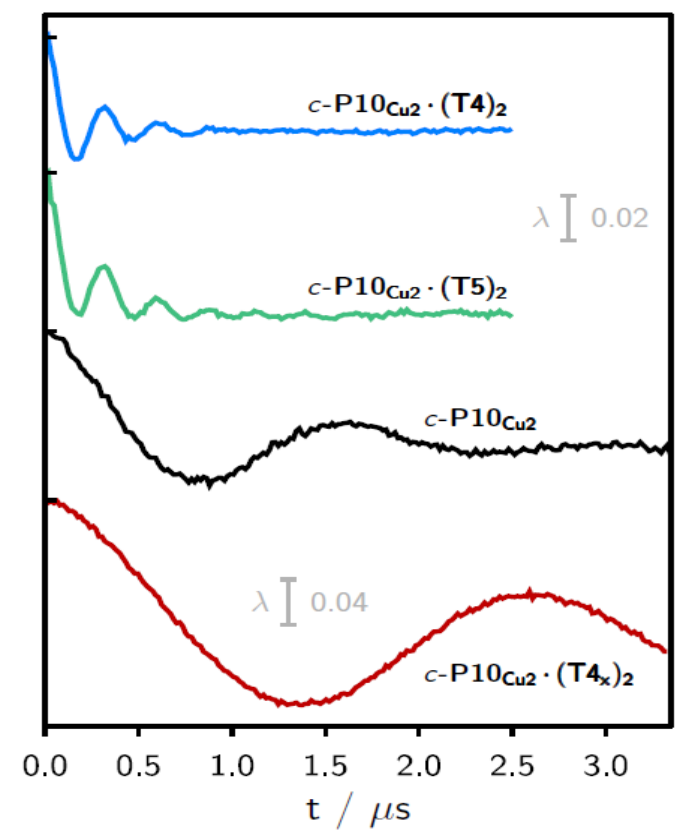

Figure 2.20: Experimental background-corrected DEER traces for $c-\mathrm{P} 10_{\mathrm{Cu2}} \cdot(\mathrm{T} 4)_{2}, c-\mathrm{P} 10_{\mathrm{Cu} 2 \cdot} \cdot(\mathrm{T} 5)_{2}, c-\mathrm{P} 10_{\mathrm{Cu} 2}$ and $c-\mathrm{P} 10_{\mathrm{Cu} 2} \cdot\left(\mathrm{T4}_{\mathrm{x}}\right)_{2}$ recorded at $15 \mathrm{~K}$ in toluene- $d_{8}$. The modulation depth scale $\lambda$ of $2 \%$ refers to the top three traces, whereas the scale of $4 \%$ applies to the bottom trace.

Inter-spin distances can be obtained from the experimental data using Tikhonov regularisation as implemented in DeerAnalysis. The distance distributions obtained from this analysis of the experimental data are shown for all samples in Figure 2.21. 


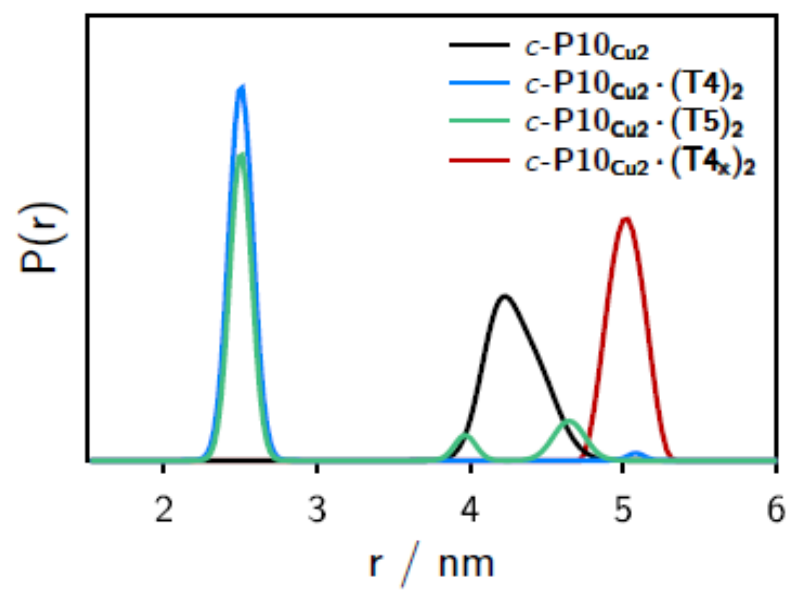

Figure 2.21: Distance distributions for the investigated samples obtained using Tikhonov regularisation as implemented in DeerAnalysis after correction for $g$-factor differences. $P(r)$ is the probability density of spin-spin distance $r$.

The difference in the traces between $c-P 10_{\text {Cu2 }} \cdot(\mathbf{T 4})_{2}$ and $c-P 10_{\text {Cu2 }} \cdot(T 5)_{2}$ likely arises from a larger contribution of potentially longer $\mathrm{Cu} \cdots \mathrm{Cu}$ distances in the T5 sample (Figure 2.22). Since the fifth template leg is forced to interact with the copper porphyrin, other conformations might be populated more than would be the case with T4. Nonetheless, the shortest $\mathrm{Cu} \cdots \mathrm{Cu}$ distance of approximately $2.5 \mathrm{~nm}$ dominates the appearance of the dipolar evolution time trace in both cases

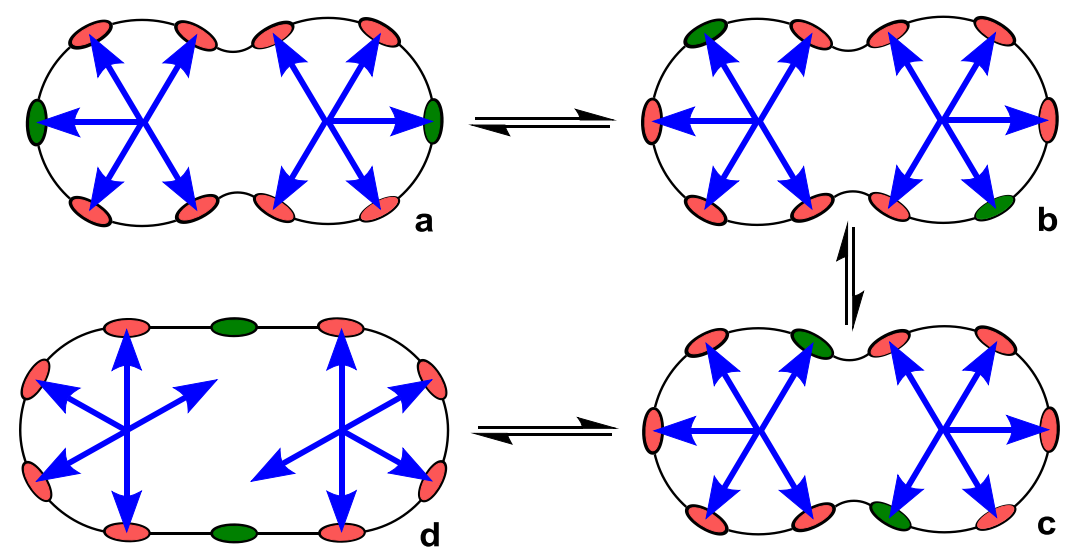

Figure 2.22: Possible conformations of $c-\mathrm{P} 10_{\mathrm{Cu} 2} \cdot(\mathrm{T5})_{2}$ leading to different $\mathrm{Cu} \cdots \mathrm{Cu}$ distances. Expected $\mathrm{Cu} \cdots \mathrm{Cu}$ distances for conformation: $a=5.0 \mathrm{~nm} ; \mathrm{b}=4.3 \mathrm{~nm} ; \mathrm{c}=2.5 \mathrm{~nm} ; \mathrm{d}=2.4 \mathrm{~nm}$.

For $\boldsymbol{c}-\mathrm{P} 10_{\mathrm{Cu} 2}$ a distribution centred at $4.28 \mathrm{~nm}$ is obtained (Figure 2.21, black). Available crystal structures and DFT calculations performed for a porphyrin dimer, ${ }^{134}$ suggest a $\mathrm{Zn} \cdots \mathrm{Zn}$ distance of $1.35 \mathrm{~nm}$ resulting in a diameter of $4.3 \mathrm{~nm}$ for a perfectly circular 10-membered porphyrin ring. The experimentally obtained distance is in excellent agreement with the predicted diameter, which confirms that in solution the ring without templates is fairly shape-persistent. The distance distribution of $\boldsymbol{c}$-P10 $\mathbf{C u 2}$ 
is broad $\left(\sigma_{\mathrm{r}} \sim 0.2 \mathrm{~nm}\right)$ compared to the templated structures which indicates a slightly larger flexibility in the template-free ring.

Distance distributions for the structures with T4 and T5 both show a major contribution of a well-defined short distance of $2.50 \mathrm{~nm}$ with a width of $\sigma_{\mathrm{r}}=0.08 \mathrm{~nm}$ (Figure 2.21, blue and green). The distance corresponds to conformer $\mathbf{c}$ and $\mathbf{d}$ in Figure $2.22\left(\boldsymbol{c}-\mathbf{P} 10_{\mathrm{Cu2}} \cdot(\mathbf{T} 4)_{\mathbf{2}}\right.$ is expected to adopt geometry $\mathbf{d}$ exclusively). X-ray crystallography of a template-bound six-porphyrin nanoring indicates a distance of $1.22 \mathrm{~nm}$ between neighbouring zinc centres. ${ }^{125}$ Imposing this geometric constraint on the template-bound structures proposed in Figure 2.16, results in a predicted $\mathrm{Cu} \cdots \mathrm{Cu}$ distance of ca. $2.5 \mathrm{~nm}$, which is in excellent agreement with the experimentally determined value.

For $\mathbf{c}-\mathbf{P} 10_{\mathrm{Cu2}^{-}} \cdot(\mathbf{T 4})_{2}$, the distance of $2.5 \mathrm{~nm}$ appears to be the major contribution with a relative importance of $>98 \%$. On the contrary, in the sample $\boldsymbol{c}-\mathbf{P} 1 \mathbf{C O}_{\mathrm{Cu2}} \cdot(\mathbf{T 5})_{\mathbf{2}}$, longer distances also appear to contribute. The contribution of the shortest distance is reduced to roughly $80 \%$, and two additional peaks at 4.0 and $4.6 \mathrm{~nm}$ with relative contributions of $5 \%$ and $15 \%$ are observed. It is important to note that only the shortest distance in template-bound structures and its distribution can be determined with high precision. The errors on any additional longer distances and their distributions are expected to be large, due to their small relative contribution to the time evolution and the limited length of the DEER trace. Furthermore, the presented analysis is dependent on the background correction used. In this respect it is not possible to reliably analyse the observed differences between $\mathbf{c}-\mathbf{P} 10_{\text {Cu2 }} \cdot(\mathbf{T 4})_{2}$ and $\mathbf{c}-\mathbf{P} 10_{\mathrm{Cu2}^{2}} \cdot(\mathrm{T} 5)_{2}$ in more detail. However, It can be stated safely that a larger percentage of longer distances contributes to the DEER signal in the case of $c-\mathrm{P} 10_{\mathrm{Cu2}} \cdot(\mathrm{T} 5)_{2}$, as compared to $c-\mathrm{P} 10_{\mathrm{Cu2}} \cdot(\mathrm{T} 4)_{2}$.

Since only the shortest possible $\mathrm{Cu} \cdots \mathrm{Cu}$ distance is observed experimentally for $c-P 10_{\text {Cu2 }} \cdot(\mathbf{T 4})_{2}$, we can conclude that the binding affinity of the pyridine ligands of the template leg to the porphyrin units is considerably smaller for copper than for zinc. This result indicates that we can direct the $\mathrm{Cu} \cdots \mathrm{Cu}$ separation distance using specific templates that impose a certain geometry on the ring. In the $\mathbf{c}-\mathbf{P} 1 \mathbf{O}_{\mathbf{C u 2}} \cdot\left(\mathbf{T} \mathbf{4}_{\mathbf{x}}\right)_{\mathbf{2}}$ complex, 
the geometry of the templates is expected to promote a $\mathrm{Cu} \cdots \mathrm{Cu}$ separation distance of $5.0 \mathrm{~nm}$. The DEER trace and the corresponding distance distribution for c-P10 ${ }_{\text {Cu2 }} \cdot\left(\mathbf{T 4} \mathbf{x}_{\mathbf{x}}\right)_{2}$ obtained are shown in Figure 2.20 and Figure 2.21 , respectively. A well-defined modulation of the DEER trace is observed for this sample corresponding to a distance of $5.0 \mathrm{~nm}$, confirming that the Cu sites are now separated by the largest possible $\mathrm{Cu} \cdots \mathrm{Cu}$ distance. No contributions of shorter distances are observed. The results clearly show that the preferred location of the copper porphyrin units is indeed governed by the differences in binding strength between zinc and copper to axial nitrogen ligands. This opens up the possibility to control the geometry, and thus the specific Cu...Cu distances, in supramolecular complexes at a molecular level.

\subsection{Conclusion}

We have demonstrated that linear porphyrin oligomers can be prepared containing a central free-base porphyrin unit and zinc porphyrins at the other sites. The free-base porphyrin can be metallated with copper(II), without transmetallation at the zinc centres, to prepare heterometallated pentamers, which are precursors to mixed-metal nanorings.

The axial binding interaction between a copper porphyrin and pyridine can be quantified with the help of a chemical double-mutant cycle, revealing that the energy of the $\mathrm{Cu} \cdots \mathrm{N}$ interaction is $\Delta \Delta G_{\mathrm{Cu}}=-6.2 \pm 0.4 \mathrm{~kJ} \mathrm{~mol}^{-1}$. It is tempting to compare this energy with that for coordination of pyridine to a zinc porphyrin monomer $\left(\Delta G_{\mathrm{Zn}}=-20.0 \pm 0.2 \mathrm{~kJ} \mathrm{~mol}^{-1}\right.$ for $2.5 \mathrm{in} \mathrm{CHCl}_{3}$ at $\left.298 \mathrm{~K}\right)$. However such a comparison is misleading because $\Delta G_{\mathrm{Zn}}$ includes the loss of translational entropy associated with bringing two molecules together, whereas $\Delta \Delta G_{\mathrm{Cu}}$ is measured in a situation where the interaction is effectively intramolecular with a high effective molarity.

The heterometallated porphyrin nanoring complexes $c-\mathrm{P} 10_{\mathrm{Cu2}} \cdot(\mathrm{T5})_{2}$ has been prepared by template-directed synthesis. The molecular geometry and metal ligand interactions in the nanoring $\mathbf{c}-\mathbf{P} \mathbf{1 0} \mathbf{C u}_{\mathbf{c} 2}$ have been investigated using EPR. DEER measurements on the nanoring revealed that the $\mathrm{Cu} \cdots \mathrm{Cu}$ distance could be precisely probed. Interestingly, the $\mathrm{Cu} \cdots \mathrm{Cu}$ separation distance could be controlled by the 
addition of multidentate ligands which would force the ring in a specific geometry by strong cooperative binding to the zinc porphyrins.

\subsection{Experimental procedures and technical details}

\subsubsection{General experimental}

Dry toluene and THF were obtained by passing the solvents through columns of alumina, under nitrogen. Diisopropylamine $\left(i-\mathrm{Pr}_{2} \mathrm{NH}\right)$ was distilled from $\mathrm{CaH}_{2}$ and kept over activated molecular sieves (3 A, 8-12 mesh). Unless specified otherwise, all other solvents were used as commercially supplied. Flash chromatography was carried out on silica gel 60 under positive pressure. Analytical thin-layer chromatography was carried out on aluminum-backed silica gel 60 F254 plates. Visualisation was achieved using UV light when necessary.

All UV-vis-NIR spectra were recorded in solution using a Perkin-Lambda 20 spectrometer ( $1 \mathrm{~cm}$ path length quartz cell). Chloroform (containing $\mathrm{ca} .0 .5 \%$ ethanol as stabiliser) or toluene was used for all titrations without any further purification.

Unless stated otherwise, ${ }^{1} \mathrm{H} /{ }^{13} \mathrm{C}$ NMR spectra were recorded at $298 \mathrm{~K}$ using a Bruker AV400 (400/100 MHz) or Bruker AV700 (700/175 MHz) instrument. ${ }^{1} \mathrm{H}$, and ${ }^{13} \mathrm{C}$ NMR spectra are reported in ppm; coupling constants are given in Hertz, to the nearest $0.1 \mathrm{~Hz}$. The solvent used was $\mathrm{CDCl}_{3}$.

MALDI-ToF mass spectra were measured at the EPSRC National Mass Spectrometry service (Swansea) using the Applied Biosystems Voyager DE-STR or at the University of Oxford using Waters MALDI Micro MX spectrometer utilising dithranol as a matrix.

\subsubsection{Synthetic procedures}

\section{2,2'-Dipyrromethane (2.1) $)^{135}$}

Formaldehyde ( $33 \% \mathrm{w} / \mathrm{w}$ solution in water, $5.4 \mathrm{~mL}, 60 \mathrm{mmol}$ ) was added to freshly distilled pyrrole $(100 \mathrm{~mL}, 1.44 \mathrm{~mol})$ and the solution was

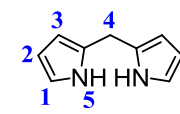
degassed by repeated evacuation and stirring under nitrogen. Trifluoroacetic acid $(0.54 \mathrm{~mL}, 3.10 \mathrm{mmol})$ was added by syringe while stirring vigorously. The reaction was allowed to proceed for $5 \mathrm{~min}$ before $\mathrm{CH}_{2} \mathrm{Cl}_{2}(100 \mathrm{~mL})$ was added, followed immediately by $\mathrm{Na}_{2} \mathrm{CO}_{3}$ (aq.) (sat., $100 \mathrm{~mL}$ ). The organic layer was washed with $\mathrm{Na}_{2} \mathrm{CO}_{3}$ (aq.) (sat., $2 \times 100 \mathrm{~mL}$ ) and water $(100 \mathrm{~mL})$, then dried over $\mathrm{Na}_{2} \mathrm{SO}_{4}$. The 
solvent and then excess pyrrole were removed on the rotovapor. Distillation of the oily residue in a Kugelrohr apparatus $\left(194{ }^{\circ} \mathrm{C}, 2.0 \mathrm{mbar}\right)$ yielded the product as a white crystalline solid (4.61 g, 53\%).

${ }^{1} \mathbf{H}$ NMR $\left(400 \mathrm{MHz}^{\mathrm{C}} \mathrm{CDCl}_{3}, 298 \mathrm{~K}\right): \delta_{\mathrm{H}}(\mathrm{ppm}) 7.98(2 \mathrm{H}, \mathrm{s}, \mathrm{H} 5), 6.63(2 \mathrm{H}, \mathrm{m}, \mathrm{H} 1), 6.61$ $(2 \mathrm{H}, \mathrm{m}, \mathrm{H} 2), 6.04(2 \mathrm{H}, \mathrm{m}, \mathrm{H} 3), 3.95(2 \mathrm{H}, \mathrm{s}, \mathrm{H} 4)$.

\section{Zinc 5,15-bis(3,5-bis(tert-butyl)phenyl porphyrin (2.3)}

3,5-Bis(tert-butyl)benzaldehyde 2.2 (2.81 g, $12.9 \mathrm{mmol})$ and dipyrromethane 2.1 (1.88 g, $12.9 \mathrm{mmol})$ were placed in a dry flask under $\mathrm{N}_{2}$ and dissolved in fresh Winchester bottle of $\mathrm{CH}_{2} \mathrm{Cl}_{2}(2.5 \mathrm{~L})$. The solution was degassed three times by repeated evacuation and stirring under nitrogen. Trifluoroacetic acid $(0.62 \mathrm{~mL}, 8.0 \mathrm{mmol})$ was added and the reaction mixture stirred in the dark for $3 \mathrm{~h}$. DDQ (3.72 g, $16.4 \mathrm{mmol}$ ) was added and stirring continued for $0.5 \mathrm{~h}$. After

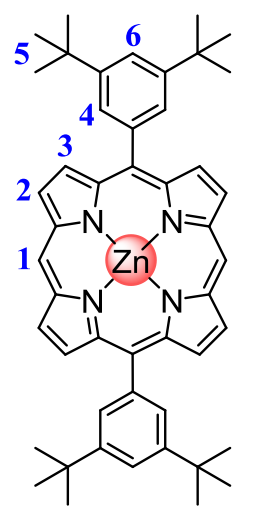
quenching of the acid by addition of triethylamine $(12.5 \mathrm{~mL})$ the mixture was concentrated to a volume of $300 \mathrm{~mL}$ on the rotary evaporator, passed over a silica plug $\left(\mathrm{CH}_{2} \mathrm{Cl}_{2}\right)$, dried and redissolved in $\mathrm{CHCl}_{3}(300 \mathrm{~mL}) \cdot \mathrm{Zn}(\mathrm{OAc})_{2} \cdot 2 \mathrm{H}_{2} \mathrm{O}(3.17 \mathrm{~g}$, $14.46 \mathrm{mmol}$ ) was dissolved in methanol ( $30 \mathrm{~mL}$ ) and added to a solution of free-base porphyrin. The reaction mixture was stirred at room temperature for $1 \mathrm{~h}$. The mixture was passed through a short plug of silica gel $\left(\mathrm{CH}_{2} \mathrm{Cl}_{2}\right)$. The crude product was recrystallised by layer addition $\left(\mathrm{CH}_{2} \mathrm{Cl}_{2} /\right.$ methanol) to give $\mathbf{2 . 3}$ as a purple powder (2.3 g, $48 \%)$.

${ }^{1}$ H NMR $\left(400 \mathrm{MHz}, \mathrm{CDCl}_{3}, 298 \mathrm{~K}\right): \delta_{\mathrm{H}}(\mathrm{ppm}) 10.36(2 \mathrm{H}, \mathrm{s}, \mathrm{H} 1), 9.45(4 \mathrm{H}, \mathrm{d}, J=4.5 \mathrm{~Hz}$, H2), $9.23(4 \mathrm{H}, \mathrm{d}, J=4.5 \mathrm{~Hz}, \mathrm{H} 3), 8.17(4 \mathrm{H}, \mathrm{d}, J=2.0 \mathrm{~Hz}, \mathrm{H} 4), 7.86(2 \mathrm{H}, \mathrm{t}, J=2.0 \mathrm{~Hz}, \mathrm{H} 6)$, $1.59(36 \mathrm{H}, \mathrm{s}, \mathrm{H} 5)$. 


\section{Zinc 5,15-(bis(3,5-bis(tert-butyl)phenyl)-10,20-dibromoporphyrin (2.4) ${ }^{\mathbf{2 2}}$}

To a solution of $2.3(1.5 \mathrm{~g}, 2.0 \mathrm{mmol})$ in $\mathrm{CH}_{2} \mathrm{Cl}_{2}(240 \mathrm{~mL})$ and pyridine $(4 \mathrm{~mL})$, cooled to $-10{ }^{\circ} \mathrm{C}$, was added freshly recrystallised $\mathrm{N}$-bromosuccinimide $(0.72 \mathrm{~g}, 4.0 \mathrm{mmol})$ in one portion. After stirring in the dark for $10 \mathrm{~min}$, the reaction mixture was allowed to warm to room temperature and stirred for $30 \mathrm{~min}$. The reaction mixture was passed through a short silica gel column $\left(\mathrm{CH}_{2} \mathrm{Cl}_{2}\right)$. Recrystallisation by layer addition

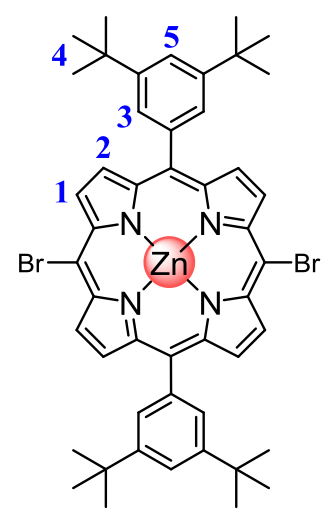
$\left(\mathrm{CH}_{2} \mathrm{Cl}_{2} /\right.$ methanol) gave $\mathbf{2 . 4}$ as a green powder (1.77 g, 98\%).

${ }^{1}$ H NMR $\left(400 \mathrm{MHz} \mathrm{CDCl}_{3}, 298 \mathrm{~K}\right): \delta_{\mathrm{H}}(\mathrm{ppm}) 9.66(4 \mathrm{H}, \mathrm{d}, J=4.5 \mathrm{~Hz}, \mathrm{H} 1), 8.91(4 \mathrm{H}, \mathrm{d}$, $J=4.5 \mathrm{~Hz}, \mathrm{H} 2), 7.99(4 \mathrm{H}, \mathrm{d}, J=2.0 \mathrm{~Hz}, \mathrm{H} 3), 7.80(2 \mathrm{H}, \mathrm{t}, J=2.0 \mathrm{~Hz}, \mathrm{H} 5), 1.50(36 \mathrm{H}, \mathrm{s}$, H4).

\section{Zinc 5,15-(bis(3,5-bis(tert-butyl)phenyl)-10,20-bis(trihexylsilylethynyl)porphyrin} $(2.5)^{22}$

$\mathrm{Pd}_{2}(\mathrm{dba})_{3}(64 \mathrm{mg}, 70 \mu \mathrm{mol})$, Cul $(28 \mathrm{mg}$, $0.14 \mathrm{mmol}), \mathrm{PPh}_{3}(36 \mathrm{mg}, 0.14 \mathrm{mmol})$ and 2.4 (0.64 g, $0.7 \mathrm{mmol}$ ) were placed in a dried Schlenk tube under argon. Toluene (45 $\mathrm{mL}), \quad i-\mathrm{Pr}_{2} \mathrm{NH}(24 \mathrm{~mL})$ and pyridine $(1.27 \mathrm{~mL})$ were added and the reaction mixture deoxygenated [vacuum

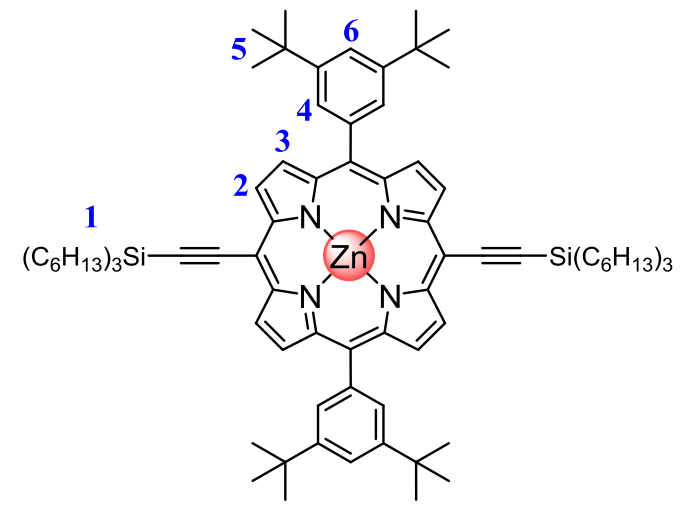
freeze/thaw 3 times]. Trihexylsilyl acetylene $(0.81 \mathrm{~mL}, 2.1 \mathrm{mmol})$ was added by syringe. The reaction mixture was stirred at $50{ }^{\circ} \mathrm{C}$ for $3 \mathrm{~h}$, and monitored by TLC. Solvents removed and the residue passed through a short silica gel column $\left(\mathrm{CH}_{2} \mathrm{Cl}_{2}\right)$. Recrystallisation by layer addition $\left(\mathrm{CH}_{2} \mathrm{Cl}_{2} /\right.$ methanol) gave $\mathbf{2 . 5}$ as a green solid (0.96 g, 99\%).

${ }^{1}$ H NMR (400 MHz, CDCl $\left.3,298 \mathrm{~K}\right): \delta_{\mathrm{H}}(\mathrm{ppm}) 9.74(4 \mathrm{H}, \mathrm{d}, J=4.5 \mathrm{~Hz}, \mathrm{H} 2), 8.98(4 \mathrm{H}, \mathrm{d}$, $J=4.5 \mathrm{~Hz}, \mathrm{H} 3), 8.06(4 \mathrm{H}, \mathrm{d}, J=2.0 \mathrm{~Hz}, \mathrm{H} 4), 7.83(2 \mathrm{H}, \mathrm{t}, J=2.0 \mathrm{~Hz}, \mathrm{H6}), 1.77-0.91(78 \mathrm{H}$, $m, H 1), 1.57(36 \mathrm{H}, \mathrm{s}, \mathrm{H} 5)$. 
Zinc 5,15-(bis(3,5-bis(tert-butyl)phenyl)-10-ethynyl-20-

(trihexylsilylethynyl)porphyrin (2.6) ${ }^{22}$

Porphyrin 2.5 (1.35 g, $1.00 \mathrm{mmol}$ ) was dissolved in $\mathrm{CHCl}_{3}(246 \mathrm{~mL}), \mathrm{CH}_{2} \mathrm{Cl}_{2}(246 \mathrm{~mL})$ and pyridine $(5 \mathrm{~mL})$. Tetra- $n$-butylammonium fluoride $(1.5 \mathrm{~mL}$, 1.0 $\mathrm{M}$ solution in THF, $1.5 \mathrm{mmol}$ ) was added dropwise to the stirred solution. The progress of the reaction was monitored by TLC until an optimal product mixture was reached (30 $\mathrm{min}$ ).

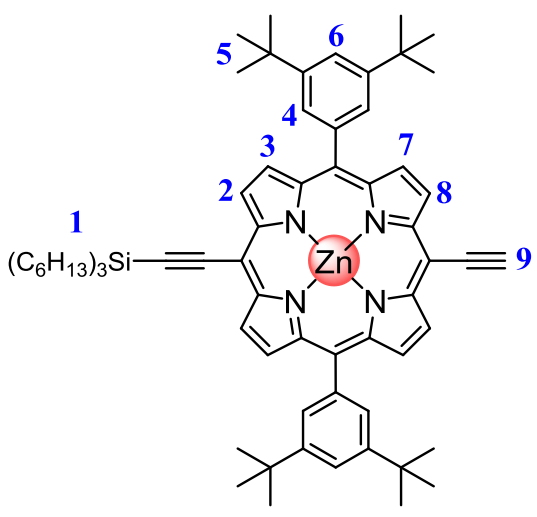

The mixture was immediately passed through a short plug of silica gel (1\% pyridine in $\left.\mathrm{CHCl}_{3}\right)$. Column chromatography $(100: 1: 1=$ petroleum ether : ethyl acetate : pyridine) gave 2.6 as a green powder $(0.41 \mathrm{~g}, 38 \%)$.

${ }^{1} \mathrm{H}$ NMR (400 MHz, CDCl $\left.3,298 \mathrm{~K}\right): \delta_{\mathrm{H}}(\mathrm{ppm}) 9.68(4 \mathrm{H}, \mathrm{m}, \mathrm{H} 2$ and $\mathrm{H} 8), 8.92(4 \mathrm{H}, \mathrm{m}, \mathrm{H} 3$ and H7), $8.03(4 \mathrm{H}, \mathrm{d}, J=2.0 \mathrm{~Hz}, \mathrm{H} 4), 7.81(2 \mathrm{H}, \mathrm{t}, J=2.0 \mathrm{~Hz}, \mathrm{H6}), 4.15(1 \mathrm{H}, \mathrm{s}, \mathrm{H} 9)$, $1.81-0.91(39 H, m, H 1), 1.57(36 \mathrm{H}, \mathrm{s}, \mathrm{H} 5)$.

Zinc porphyrin dimer $(2.7)^{22}$

$\mathrm{Pd}\left(\mathrm{PPh}_{3}\right)_{2} \mathrm{Cl}_{2} \quad(82.9 \quad \mathrm{mg}$,

$0.12 \mathrm{mmol})$, Cul

(221.1 mg, $\quad 1.16 \mathrm{mmol})$

and 1,4-benzoquinone

(165.8 mg, $\quad 1.53 \mathrm{mmol})$

were dissolved in toluene

$(70 \mathrm{~mL})$ and $i-\mathrm{Pr}_{2} \mathrm{NH}$

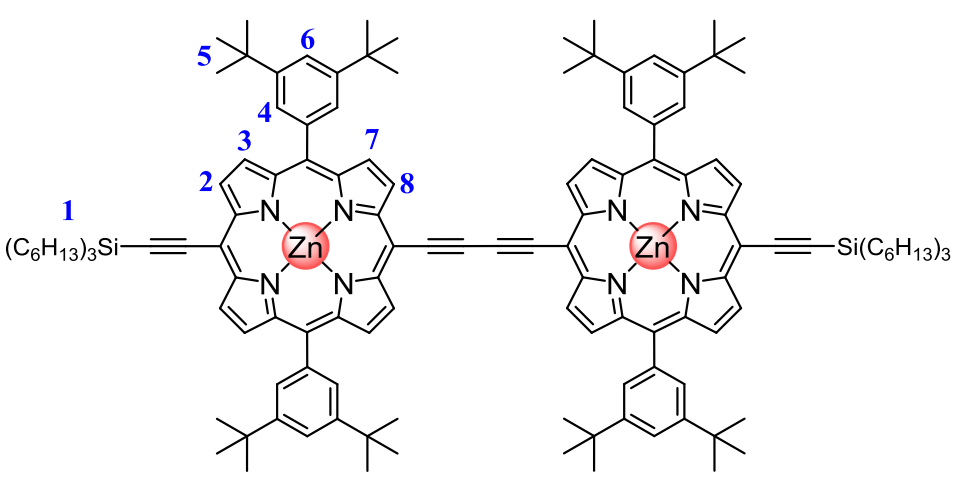

(11 mL). Porphyrin $2.6(1.24 \mathrm{~g}, 1.15 \mathrm{mmol})$ was dissolved in toluene $(42 \mathrm{~mL})$ and the catalyst mixture was added. The reaction mixture was stirred at room temperature for $30 \mathrm{~min}$ and passed through a short silica gel column (1\% pyridine in $\mathrm{CH}_{2} \mathrm{Cl}_{2}$ ). Recrystallisation by layer addition $\left(\mathrm{CH}_{2} \mathrm{Cl}_{2} /\right.$ methanol) gave 2.7 as a brown solid (1.12 g, 91\%).

${ }^{1} \mathrm{H}$ NMR $\left(400 \mathrm{MHz}, \mathrm{CDCl}_{3}, 298 \mathrm{~K}\right): \delta_{\mathrm{H}}(\mathrm{ppm}) 9.87(4 \mathrm{H}, \mathrm{d}, J=4.6 \mathrm{~Hz}, \mathrm{H} 8), 9.65(4 \mathrm{H}, \mathrm{d}$, $J=4.6 \mathrm{~Hz}, \mathrm{H} 2), 8.97(4 \mathrm{H}, \mathrm{d}, J=4.6 \mathrm{~Hz}, \mathrm{H} 7), 8.87(4 \mathrm{H}, \mathrm{d}, J=4.6 \mathrm{~Hz}, \mathrm{H} 3), 8.03(8 \mathrm{H}, \mathrm{d}$, $J=1.6 \mathrm{~Hz}, \mathrm{H} 4), 7.80(4 \mathrm{H}, \mathrm{m}, \mathrm{H} 6), 1.76-0.89(78 \mathrm{H}, \mathrm{m}, \mathrm{H} 1), 1.55(72 \mathrm{H}, \mathrm{s}, \mathrm{H} 5)$. 
Free-base 5,15-(bis(3,5-bis(tert-butyl)phenyl)-10,20-

bis(trihexylsilylethynyl)porphyrin (2.8) ${ }^{136}$

Porphyrin 2.5 (955 mg, $0.7 \mathrm{mmol}$ ) was dissolved in $\mathrm{CHCl}_{3}(250 \mathrm{~mL})$. Trifluoroacetic acid $(2.7 \mathrm{~mL})$ was mixed with $\mathrm{CHCl}_{3}(24 \mathrm{~mL})$ to give a $10 \%$ solution. Both solutions were degassed. The TFA solution was added dropwise to the porphyrin solution and the reaction mixture was stirred at room

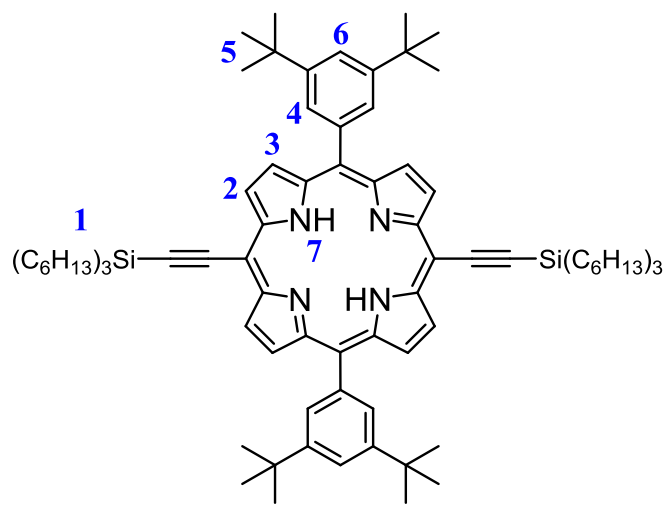
temperature under nitrogen for $15 \mathrm{~min}$. UV and TLC indicated the completion of the reaction and the mixture was passed immediately through a short plug of silica gel (1\% pyridine in $\mathrm{CHCl}_{3}$ ). Column chromatography (50:1:1, petroleum ether : ethyl acetate : pyridine) gave $\mathbf{2 . 8}$ as a purple solid (622 $\mathrm{mg}, 68 \%$ ).

${ }^{1} \mathrm{H}$ NMR $\left(400 \mathrm{MHz}, \mathrm{CDCl}_{3}, 298 \mathrm{~K}\right): \delta_{\mathrm{H}}(\mathrm{ppm}) 9.62(4 \mathrm{H}, \mathrm{d}, J=4.7 \mathrm{~Hz}, \mathrm{H} 2), 8.85(4 \mathrm{H}, \mathrm{d}$, $J=4.7 \mathrm{~Hz}, \mathrm{H} 3), 8.04(4 \mathrm{H}, \mathrm{d}, J=1.5 \mathrm{~Hz}, \mathrm{H} 4), 7.82(2 \mathrm{H}, \mathrm{m}, \mathrm{H} 6), 1.55(36 \mathrm{H}, \mathrm{s}, \mathrm{H} 5)$, 1.75-0.61 (78H, m, H1), $-2.12(2 \mathrm{H}, \mathrm{s}, \mathrm{H} 7)$.

${ }^{13} \mathrm{C}$ NMR $\left(125 \mathrm{MHz}_{\mathrm{CDCl}}, 298 \mathrm{~K}\right): \delta_{\mathrm{C}}$ (ppm) 149.0, 140.3, 129.7, 123.1, 121.3, 108.1, $101.0,100.8,35.0,33.3,31.7,24.4,22.7,14.2,13.8$.

MALDI-TOF: $m / z=1,299\left(\mathrm{C}_{88} \mathrm{H}_{130} \mathrm{~N}_{4} \mathrm{Si}_{2}, \mathrm{M}^{+}\right.$requires 1,300$)$.

Free-base 5,15-(bis(3,5-bis(tert-butyl)phenyl)-10,20-di-ethynyl-porphyrin (2.9) ${ }^{136}$ Porphyrin 2.8 (100 mg, $0.077 \mathrm{mmol}$ ) was dissolved in $\mathrm{CH}_{2} \mathrm{Cl}_{2}$ $(25 \mathrm{~mL})$ under $\mathrm{N}_{2}$. Tetra- $n$-butylammonium fluoride solution (1.0 $\mathrm{M}$ in THF) (1.31 mL, $1.31 \mathrm{mmol})$ was added dropwise to the reaction mixture and was stirred at room temperature for $15 \mathrm{~min}$. The crude reaction mixture was immediately passed through a short plug of silica gel $\left(\mathrm{CHCl}_{3}\right)$. The product was recrystallised by layer addition

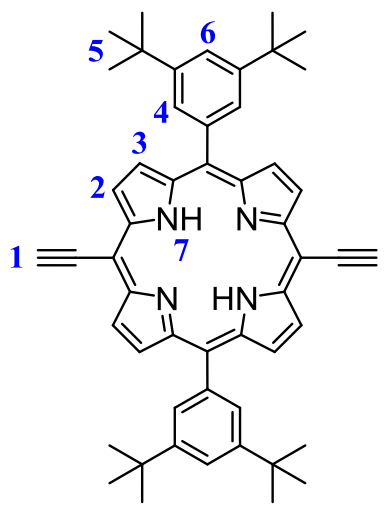
$\left(\mathrm{CH}_{2} \mathrm{Cl}_{2} /\right.$ methanol) to give the title compound as a purple powder (42.4 $\left.\mathrm{mg}, 76 \%\right)$.

${ }^{1} \mathrm{H}$ NMR $\left(400 \mathrm{MHz}^{\mathrm{C} C D C l} 3,298 \mathrm{~K}\right): \delta_{\mathrm{H}}(\mathrm{ppm}) 9.66(4 \mathrm{H}, \mathrm{d}, J=4.7 \mathrm{~Hz}, \mathrm{H} 2), 8.91(4 \mathrm{H}, \mathrm{d}$, $J=4.7 \mathrm{~Hz}, \mathrm{H} 3), 8.05(4 \mathrm{H}, \mathrm{bd}, J=1.6 \mathrm{~Hz}, \mathrm{H} 4), 7.78(2 \mathrm{H}, \mathrm{bt}, \mathrm{H} 6), 4.20(2 \mathrm{H}, \mathrm{s}, \mathrm{H} 1), 1.56$ $(36 \mathrm{H}, \mathrm{s}, \mathrm{H} 5),-2.26(2 \mathrm{H}, \mathrm{s}, \mathrm{H} 7)$. 
${ }^{13} \mathrm{C}$ NMR $(100 \mathrm{MHz}, \mathrm{CDCl} 3,298 \mathrm{~K}): \delta_{\mathrm{C}}(\mathrm{ppm})$ 149.2, 140.4, 130.0, 123.3, 121.5, 99.4, $85.7,84.5,35.2,31.9,29.9$.

MALDI-TOF: $m / z=734\left(\mathrm{C}_{52} \mathrm{H}_{54} \mathrm{~N}_{4}, \mathrm{M}^{+}\right.$requires 735).

Mono-deprotected dimer (2.10) 22

Dimer 2.7 (0.54 g, $0.25 \mathrm{mmol})$

was dissolved in $\mathrm{CH}_{2} \mathrm{Cl}_{2}$

$(60 \mathrm{~mL}), \mathrm{CHCl}_{3}(60 \mathrm{~mL})$ and

pyridine

$\mathrm{mL})$.

Tetra- $n$-butylammonium

fluoride $(0.38 \mathrm{~mL}, 1.0 \mathrm{M}$

solution in THF, $0.38 \mathrm{mmol}$ )

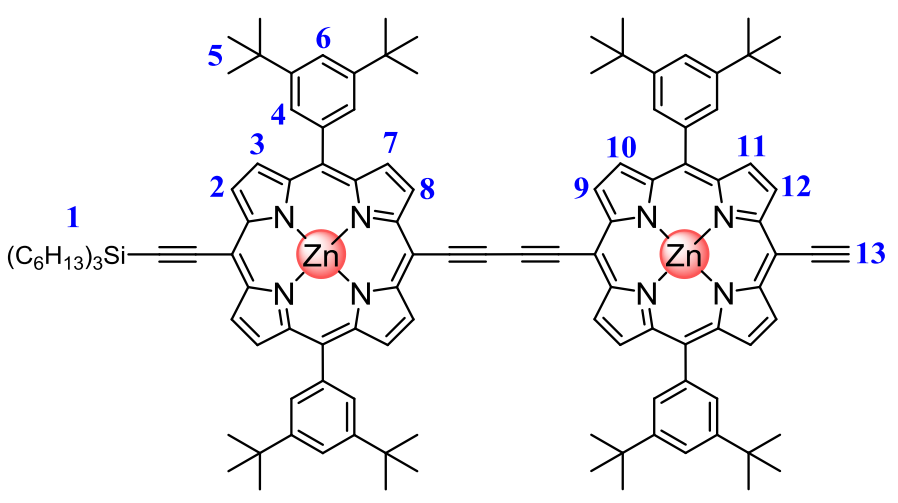

was added to the stirred solution dropwise. The progress of the reaction was monitored by TLC until an optimal product mixture was reached (30 min). The mixture was immediately passed through a short plug of silica gel (1\% pyridine in $\left.\mathrm{CHCl}_{3}\right)$. Column chromatography (30:1:1, petroleum ether : ethyl acetate : pyridine) gave $\mathbf{2 . 1 0}$ as a brown powder $(0.18 \mathrm{~g}, 33 \%)$.

${ }^{1} \mathbf{H}$ NMR $\left(400 \mathrm{MHz}, \mathrm{CDCl}_{3}+1 \%\right.$ pyridine- $\left.d_{5}, 298 \mathrm{~K}\right): \delta_{\mathrm{H}}(\mathrm{ppm}) 9.93(2 \mathrm{H}, \mathrm{d}, J=4.6 \mathrm{~Hz}$, H8 or H9), $9.91(2 \mathrm{H}, \mathrm{d}, J=4.6 \mathrm{~Hz}, \mathrm{H} 8$ or H9), $9.69(2 \mathrm{H}, \mathrm{m}, \mathrm{H} 2$ or H12), $9.68(2 \mathrm{H}, \mathrm{m}, \mathrm{H} 2$ or H12), $9.02(2 \mathrm{H}, \mathrm{m}, \mathrm{H} 7$ or $\mathrm{H} 10), 9.01(2 \mathrm{H}, \mathrm{m}, \mathrm{H} 7$ or $\mathrm{H} 10), 8.94(2 \mathrm{H}, \mathrm{d}, J=4.6 \mathrm{~Hz}, \mathrm{H} 3$ or H11), $8.91(2 \mathrm{H}, \mathrm{d}, J=4.5 \mathrm{~Hz}, \mathrm{H} 3$ or H11), $8.07(8 \mathrm{H}, \mathrm{m}, \mathrm{H} 4), 7.83(4 \mathrm{H}, \mathrm{m}, \mathrm{H} 6), 4.20$ (1H, s, H13), 1.79-0.92 (39H, m, H1), 1.58 (72H, s, H5). 


\section{Pentamer (2.11)}

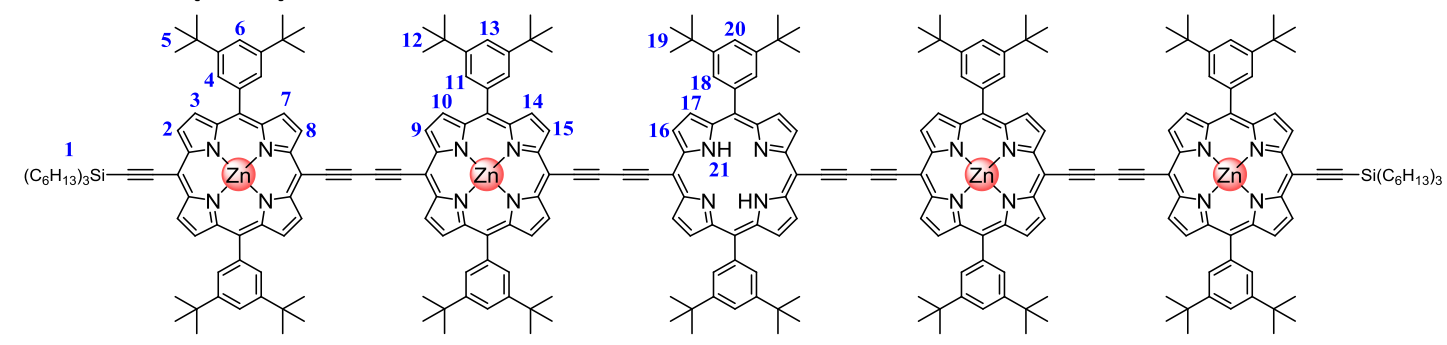

Porphyrin 2.9 (20.0 mg, $0.027 \mathrm{mmol})$, dimer 2.10 (254.1 mg, $0.14 \mathrm{mmol}), \mathrm{Pd}_{2}\left(\mathrm{dba}_{3}\right.$ (12.3 $\mathrm{mg}, 0.014 \mathrm{mmol})$, tri-2-furylphosphine $(25.2 \mathrm{mg}, 0.11 \mathrm{mmol})$ and 1,4-benzoquinone $(37.7 \mathrm{mg}, 0.35 \mathrm{mmol})$ were dissolved in toluene $(30 \mathrm{~mL})$ and triethylamine $(6 \mathrm{~mL})$. The reaction mixture was stirred at $60{ }^{\circ} \mathrm{C}$ overnight. The solvents were removed and the mixture was purified over a plug of silica gel (1\% pyridine in $\mathrm{CHCl}_{3}$ ). A small SEC column (1\% pyridine in $\mathrm{CHCl}_{3}$ ) was used to remove the 1,4-benzoquinone. Recycling GPC (1\% pyridine in toluene) was used for oligomer separation (2.11: $49.3 \mathrm{mg}$, 41\%, linear zinc tetramer: $124.8 \mathrm{mg}, 33 \%$ ).

${ }^{1} \mathrm{H}$ NMR $\left(500 \mathrm{MHz}, \mathrm{CDCl}_{3}, 298 \mathrm{~K}\right): \delta_{\mathrm{H}}(\mathrm{ppm}) 9.92(16 \mathrm{H}, \mathrm{m}, \mathrm{H} 8$ and $\mathrm{H} 9$ and $\mathrm{H} 15$ and $\mathrm{H} 16), 9.68(4 \mathrm{H}, \mathrm{d}, J=4.5 \mathrm{~Hz}, \mathrm{H} 2), 9.02(16 \mathrm{H}, \mathrm{m}, \mathrm{H} 7$ and H10 and H14 and H17), 8.91 $(4 \mathrm{H}, \mathrm{d}, J=4.5 \mathrm{~Hz}, \mathrm{H} 3), 8.16(4 \mathrm{H}, \mathrm{d}, J=1.8 \mathrm{~Hz}, \mathrm{H} 18), 8.12(8 \mathrm{H}, \mathrm{d}, J=1.8 \mathrm{~Hz}, \mathrm{H} 11), 8.07$ $(8 \mathrm{H}, \mathrm{d}, J=1.8 \mathrm{~Hz}, \mathrm{H} 4), 7.89(2 \mathrm{H}, \mathrm{t}, J=1.8 \mathrm{~Hz}, \mathrm{H} 20), 7.86(4 \mathrm{H}, \mathrm{t}, J=1.8 \mathrm{~Hz}, \mathrm{H} 13), 7.83$ $(4 \mathrm{H}, \mathrm{t}, J=1.8 \mathrm{~Hz}, \mathrm{H} 6), 1.77-0.91(78 \mathrm{H}, \mathrm{m}, \mathrm{H} 1), 1.62(36 \mathrm{H}, \mathrm{s}, \mathrm{H} 19), 1.60(72 \mathrm{H}, \mathrm{s}, \mathrm{H} 5$ or 12), $1.59(72 \mathrm{H}, \mathrm{s}, \mathrm{H} 5$ or H12), $-1.48(2 \mathrm{H}, \mathrm{s}, \mathrm{H} 21)$.

MALDI-TOF: $m / z=4,485\left(\mathrm{C}_{296} \mathrm{H}_{330} \mathrm{~N}_{20} \mathrm{Si}_{2} \mathrm{Zn}_{4}, \mathrm{M}^{+}\right.$requires 4,486$)$.

$\lambda_{\text {max }}\left(\mathrm{CHCl}_{3}\right) / \mathrm{nm} \log (\varepsilon): 783$ (5.43), 682 (5.09), 489 (5.64), 458 (5.79).

\section{Pentamer (2.12)}

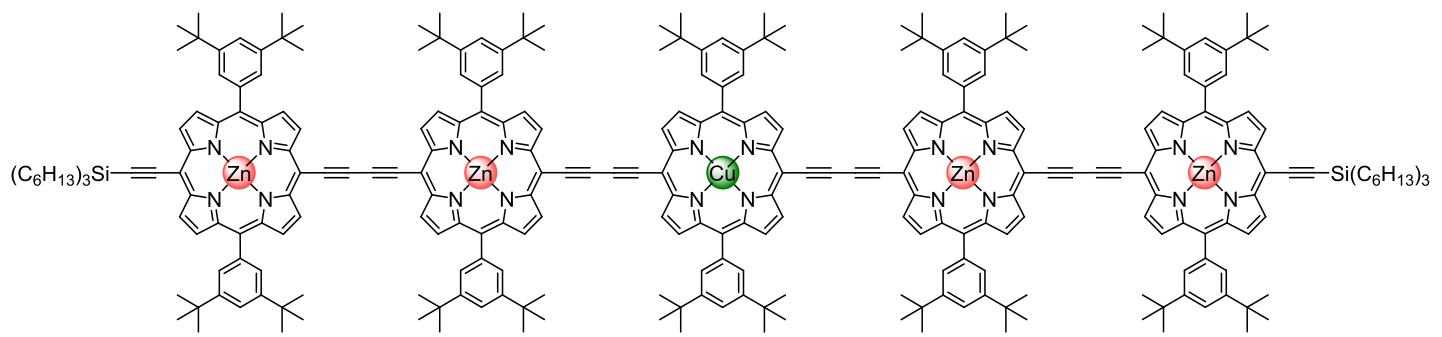

2.11 (40 mg, $10.2 \mu \mathrm{mol}$ ) was dissolved in $\mathrm{CHCl}_{3}(10 \mathrm{~mL})$. $\mathrm{Cu}(\mathrm{OAc})_{2} \cdot \mathrm{H}_{2} \mathrm{O}(12.7 \mathrm{mg}$, $0.383 \mathrm{mmol}$ ) was added as a solid and the reaction was stirred at $60^{\circ} \mathrm{C}$ for $1 \mathrm{~h}$. The reaction mixture was allowed to cool down to room temperature after which methanol was added to the reaction mixture to crash out the product. The 
precipitate was filtered and washed with methanol to give 2.12 as a red/brown solid (29.5 mg, 73\%).

${ }^{1}$ H NMR (400 MHz, CDCl $\left.3,298 \mathrm{~K}\right): \delta_{\mathrm{H}}(\mathrm{ppm}) 9.90\left(\mathrm{~m},-\mathrm{ArH}_{\beta}\right), 9.67\left(\mathrm{~d}, J=4.4 \mathrm{~Hz},-\mathrm{ArH}_{\beta}\right)$, $9.00\left(\mathrm{~m},-\mathrm{ArH}_{\beta}\right), 8.90\left(\mathrm{~d}, J=4.6 \mathrm{~Hz},-\mathrm{ArH}_{\beta}\right), 7.82\left(\mathrm{~m},-\mathrm{ArH}_{\text {ortho }}\right), 8.08\left(\mathrm{~m},-\mathrm{ArH}_{\text {para }}\right), 1.57$ (m, -tBuH), $1.77\left(m,-\mathrm{C}_{6} \mathrm{H}_{13}\right), 1.39\left(\mathrm{~m},-\mathrm{C}_{6} \mathrm{H}_{13}\right), 1.02\left(\mathrm{~m},-\mathrm{C}_{6} \mathrm{H}_{13}\right), 0.90\left(\mathrm{~m},-\mathrm{C}_{6} \mathrm{H}_{13}\right)$.

MALDI-TOF: $m / z=4,545\left(\mathrm{C}_{296} \mathrm{H}_{328} \mathrm{~N}_{20} \mathrm{CuSi}_{2} \mathrm{Zn}_{4}, \mathrm{M}^{+}\right.$requires 4,547).

$\lambda_{\text {max }}\left(\mathrm{CHCl}_{3}\right) / \mathrm{nm} \log (\varepsilon): 764$ (5.45), 693 (5.07), 492 (5.63), 457 (5.75).

\section{Pentamer (2.13)}

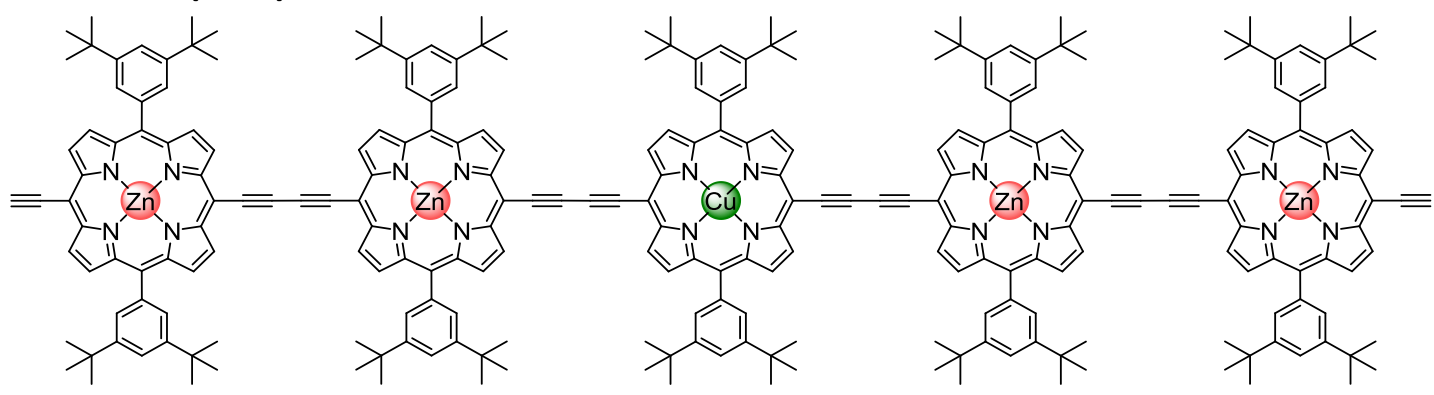

$2.12(28 \mathrm{mg}, 6.16 \mu \mathrm{mol})$ was dissolved in $\mathrm{CH}_{2} \mathrm{Cl}_{2}(9 \mathrm{~mL})$ and pyridine $(9 \mu \mathrm{L})$. Tetra- $n$-butylammonium fluoride $(0.12 \mathrm{~mL}, 1.0 \mathrm{M}$ solution in THF, $0.12 \mathrm{mmol})$ was added dropwise to the reaction mixture and was stirred at room temperature for $15 \mathrm{~min}$. After the completion of the reaction, methanol was added to the reaction mixture to crash out the product. The precipitate was filtered and washed with methanol to give $\mathbf{2 . 1 3}$ as a red/brown solid ( $21.1 \mathrm{mg}, 86 \%)$.

MALDI-TOF: $m / z=3,976\left(\mathrm{C}_{260} \mathrm{H}_{252} \mathrm{CuN}_{20} \mathrm{Zn}_{4}, \mathrm{M}^{+}\right.$requires 3,982). 


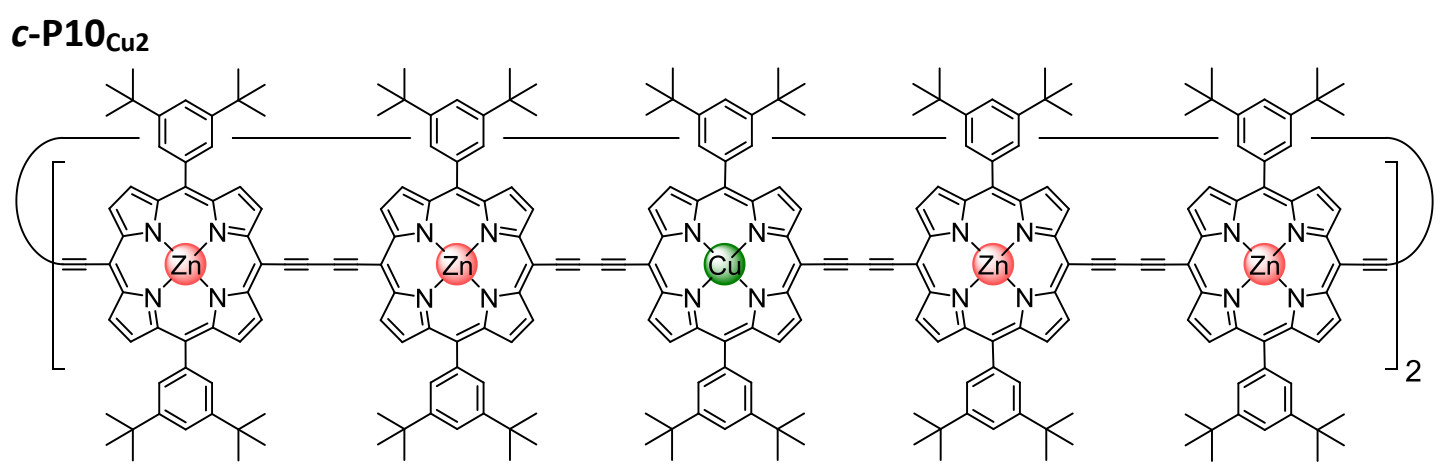

Pentadentate template T5 (5.10 mg, $6.04 \mu \mathrm{mol})$ and 2.13 (8.02 mg, $2.01 \mu \mathrm{mol})$ were dissolved in $\mathrm{CHCl}_{3}(8 \mathrm{~mL})$. A solution of $\mathrm{PdCl}_{2}\left(\mathrm{PPh}_{3}\right)_{2}(0.71 \mathrm{mg}, 1.01 \mu \mathrm{mol})$, Cul (0.71 mg, $3.73 \mu \mathrm{mol}), 1,4$-benzoquinone $(2.18 \mathrm{mg}, 20.1 \mu \mathrm{mol})$ in $\mathrm{CHCl}_{3}(0.7 \mathrm{~mL}$ ) and $i-\operatorname{Pr}_{2} \mathrm{NH}(35 \mu \mathrm{L})$ was added to the porphyrin solution and stirred at room temperature overnight. The reaction mixture was passed through a plug of alumina $\left(\mathrm{CHCl}_{3}\right)$. The solvent was evaporated and redissolved in $20 \%$ pyridine in chloroform $(1 \mathrm{~mL})$ and passed over a SEC column (20\% pyridine in chloroform) to remove the template. Lastly, the ring was purified by recycling GPC (1\% pyridine in toluene) to give $\boldsymbol{c}-\mathbf{P} 1 \mathbf{0}_{\text {cu2 }}$ (1.33 mg, 17\%).

${ }^{1} \mathrm{H}$ NMR $\left(500 \mathrm{MHz}, \mathrm{CDCl}_{3}, 298 \mathrm{~K}\right): \delta_{\mathrm{H}}(\mathrm{ppm})$ 9.87-9.77 (m, $\left.-\mathrm{ArH}_{\beta}\right)$, 9.02-8.85 (m, $\left.-\mathrm{ArH}_{\beta}\right)$, 8.08-7.98 (m, - $\left.\mathrm{ArH}_{\text {ortho }}\right)$, 7.84-7.77 (m, -ArH $\left.\mathrm{para}\right), 1.55\left(\mathrm{~s},-{ }^{t} \mathrm{BuH}_{(\mathrm{Cu})}\right), 1.54\left(\mathrm{~s},-{ }^{t} \mathrm{BuH}_{(\mathrm{Zn})}\right)$.

MALDI-TOF: $m / z=7,960\left(\mathrm{C}_{520} \mathrm{H}_{500} \mathrm{Cu}_{2} \mathrm{~N}_{40} \mathrm{Zn}_{8}, \mathrm{M}^{+}\right.$requires 7,960).

$\lambda_{\text {max }}\left(\mathrm{CHCl}_{3}\right) / \mathrm{nm} \log (\varepsilon)$ : 806 (5.67), 771 (5.67), 495 (5.91).

\subsubsection{UV-vis-NIR titrations}

\subsubsection{Titrations of porphyrin monomer 2.5 and monodentate ligands}

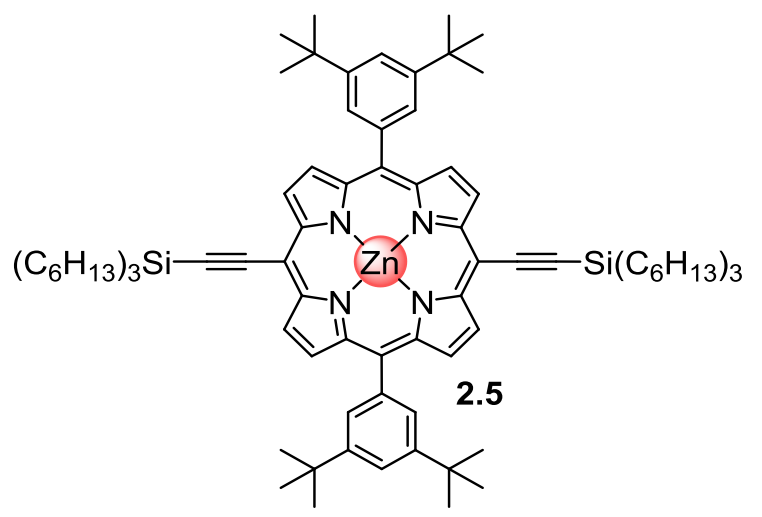

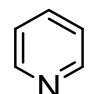

Pyridine<smiles>c1ccc(-c2ccncc2)cc1</smiles>

4-Phenylpyridine

Figure 2.23: Structures of porphyrin monomer 2.5 and monodentate ligands.

Titrations with porphyrin monomer 2.5 and pyridine or 4-phenylpyridine were performed in order to determine reference binding constants ( $K_{\mathrm{Py}}$ and $K_{\mathrm{Ph}}$ ). 
All titrations were performed in chloroform (containing ca. $0.5 \%$ ethanol as stabiliser) at $298 \mathrm{~K}$. Care was taking to keep the porphyrin concentration constant throughout the entire titration by adding porphyrin to the ligand solution before titrations were started. The binding curves were fitted using a 1:1 binding isotherm using the equation:

$$
\frac{A-A_{\text {initial }}}{A_{\infty}-A_{\text {initial }}}=\left(\frac{\left(K_{a}\left([L]+[P]_{0}\right)+1\right)-\sqrt{\left(K_{a}\left([L]+[P]_{0}\right)+1\right)^{2}-4 K_{a}^{2}[P]_{0}[L]}}{2 K_{a}[P]_{0}}\right)
$$

where $A$ is the observed absorption at a specific wavelength or the difference of absorbance between two wavelengths; $A_{\text {initial }}$ is the starting absorption at this wavelength; $A_{\infty}$ is the asymptotic final absorption at this wavelength; $K_{a}$ is the association constant between ligand and porphyrin host; $[\mathrm{L}]$ is the concentration of ligand; $[\mathrm{P}]_{0}$ is the concentration of porphyrin host. The free variables which were adjusted to optimise the fit to the experimental data during the fitting procedure are $A_{\text {initial }}, A_{\infty}$, and $K_{a}$. Fitting analysis was carried out using the Origin software

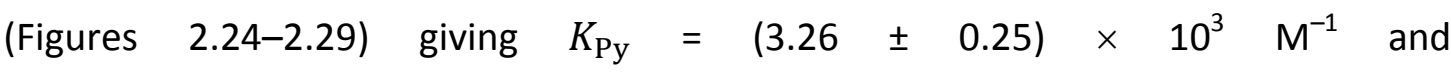
$K_{\mathrm{Ph}}=(4.25 \pm 0.12) \times 10^{3} \mathrm{M}^{-1}$.
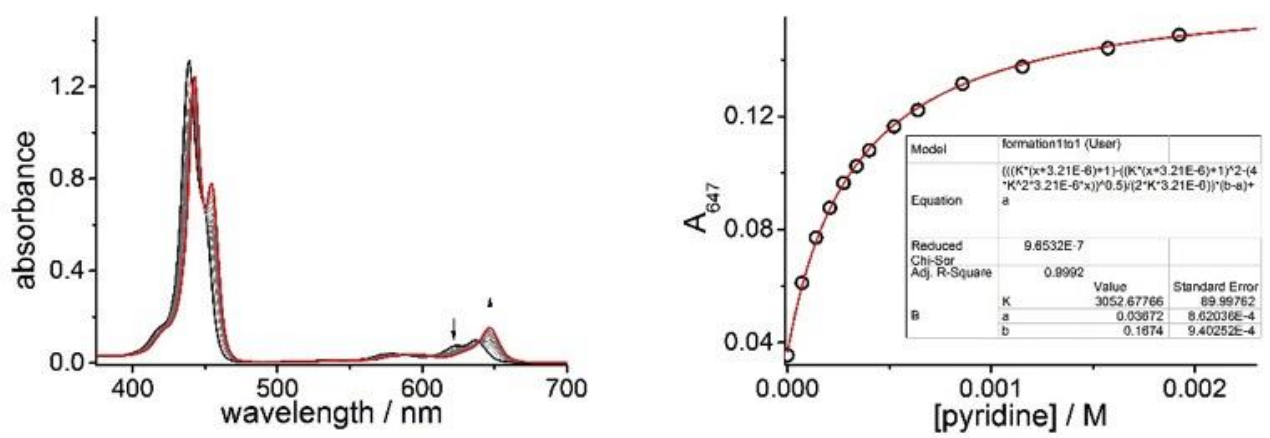

Figure 2.24: UV-vis titration of 2.5 and pyridine, $R^{2}=0.9992$. (Run $1, \mathrm{CHCl}_{3}, 298 \mathrm{~K},[2.5]=3.21 \mu \mathrm{M}$, $\left.K=3.05 \times 10^{3} \mathrm{M}^{-1}\right)$. 

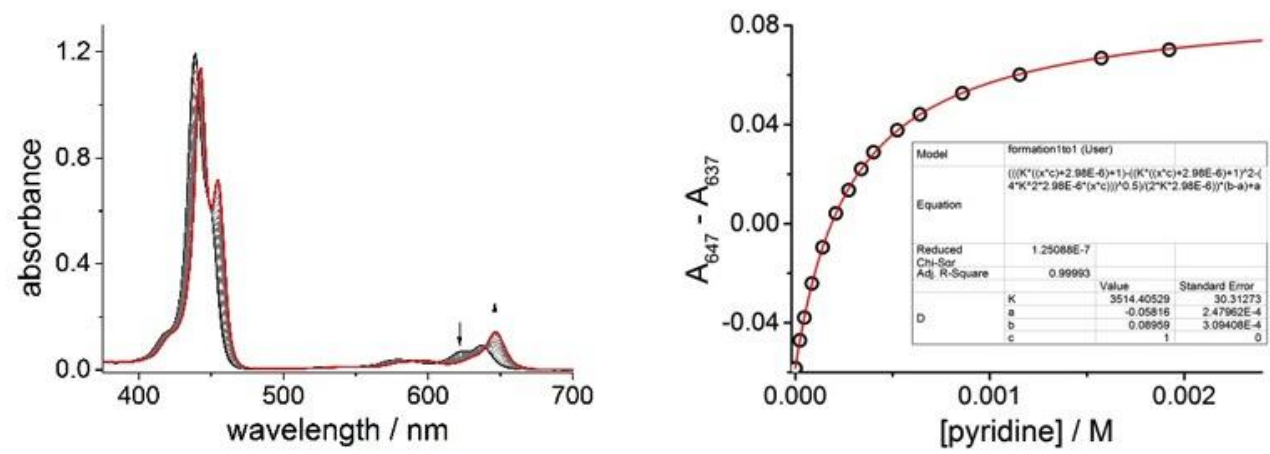

Figure 2.25: UV-vis titration of 2.5 and pyridine, $R^{2}=0.9992$. (Run 2, $\mathrm{CHCl}_{3}, 298 \mathrm{~K},[2.5]=2.98 \mu \mathrm{M}$, $\left.K=3.51 \times 10^{3} \mathrm{M}^{-1}\right)$.
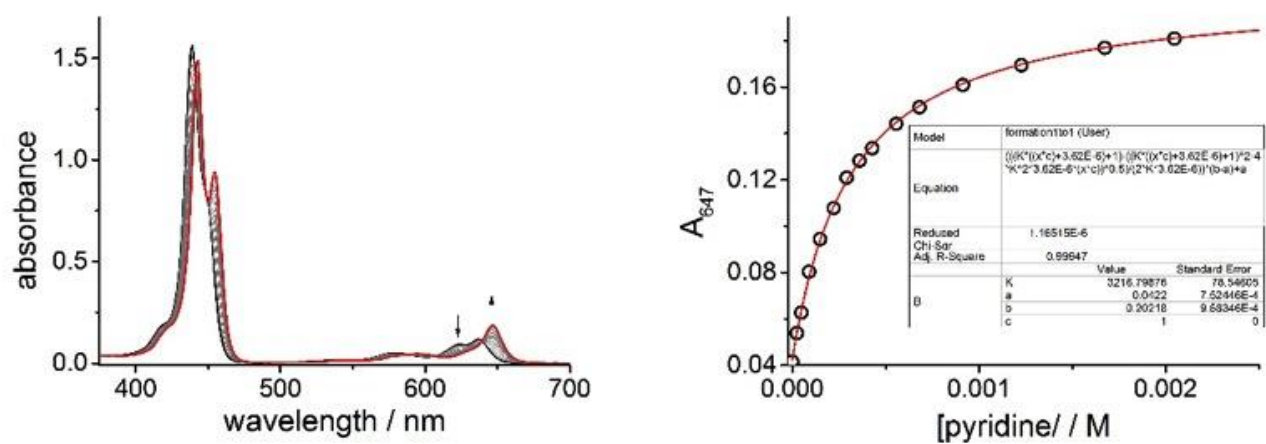

Figure 2.26: UV-vis titration of 2.5 and pyridine, $R^{2}=0.9995$. (Run 3, $\mathrm{CHCl}_{3}, 298 \mathrm{~K},[2.5]=3.62 \mu \mathrm{M}$, $\left.K=3.22 \times 10^{3} \mathrm{M}^{-1}\right)$.
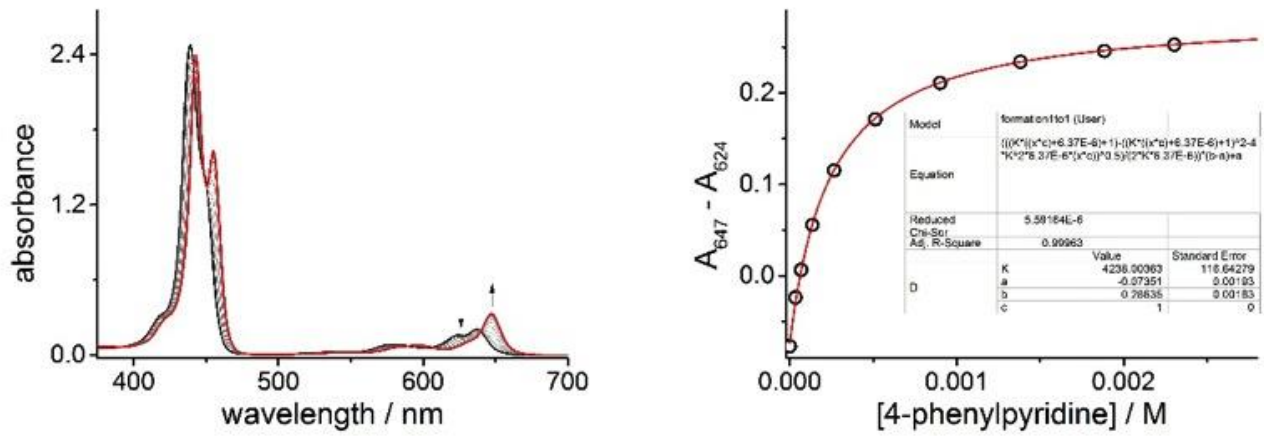

Figure 2.27: UV-vis titration of 2.5 and 4-phenylpyridine, $R^{2}=0.9996$. (Run $1, \mathrm{CHCl}_{3}, 298 \mathrm{~K},[2.5]=6.37 \mu \mathrm{M}$, $\left.K=4.24 \times 10^{3} \mathrm{M}^{-1}\right)$.
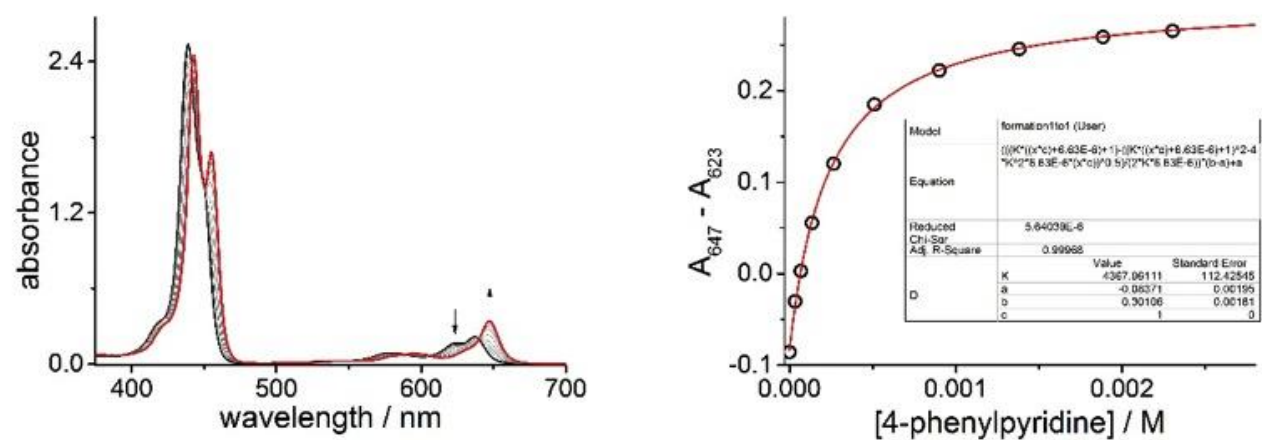

Figure 2.28: UV-vis titration of 2.5 and 4-phenylpyridine, $R^{2}=0.9997$. (Run 2, $\mathrm{CHCl}_{3}, 298 \mathrm{~K},[2.5]=6.63 \mu \mathrm{M}$, $K=4.37 \times 10^{3} \mathrm{M}^{-1}$ ). 

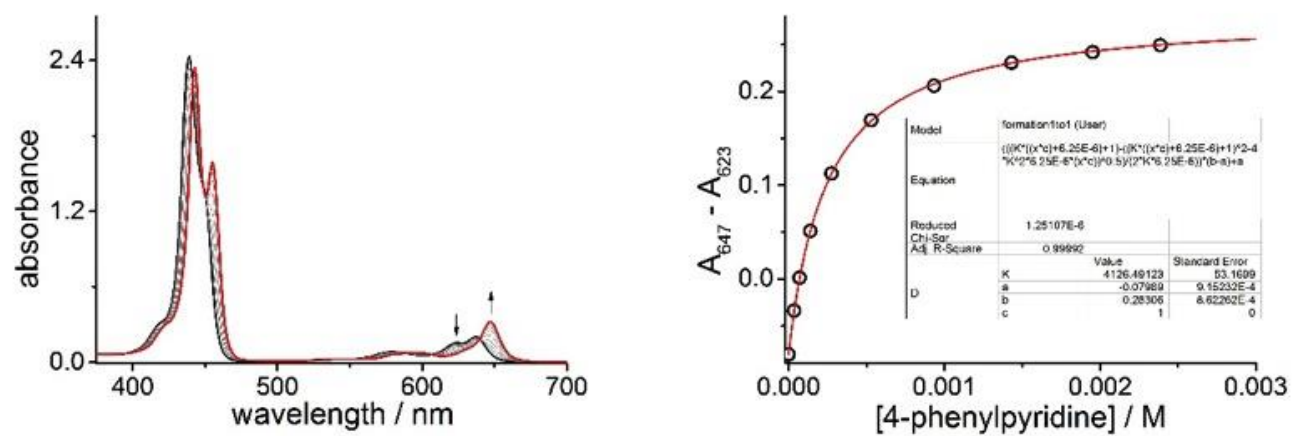

Figure 2.29: UV-vis titration of 2.5 and 4-phenylpyridine, $R^{2}=0.9996$. (Run 3, $\mathrm{CHCl}_{3}, 298 \mathrm{~K},[2.5]=6.25 \mu \mathrm{M}$, $K=4.13 \times 10^{3} \mathrm{M}^{-1}$ ).

\subsubsection{Denaturation titrations with pyridine on heterometallated oligomers}

When binding strength increases, the binding curves become increasingly square, leading to greater uncertainty in the fit. In order to derive a trustworthy binding constant, denaturation titrations (break-up titration) need to be performed with a competing ligand such as pyridine. Using the data from these break-up titrations $\left(K_{\mathrm{dn}}=\right.$ denaturation constant $)$ and the formation constant of the single site binding event of the competing ligand with a zinc porphyrin monomer $\left(K_{\mathrm{Py}}=\right.$ association constant for pyridine to 2.5$)$ allows us to derive the formation binding constant $\left(K_{\mathrm{f}}\right)$ between the oligomers ( $N=$ number of zinc porphyrin binding sites) and the template using the following equation:

$$
K_{\mathrm{f}}=\frac{K_{\mathrm{Py}}^{N}}{K_{\mathrm{dn}}}
$$

via the thermodynamic cycle shown in Figure 2.30.

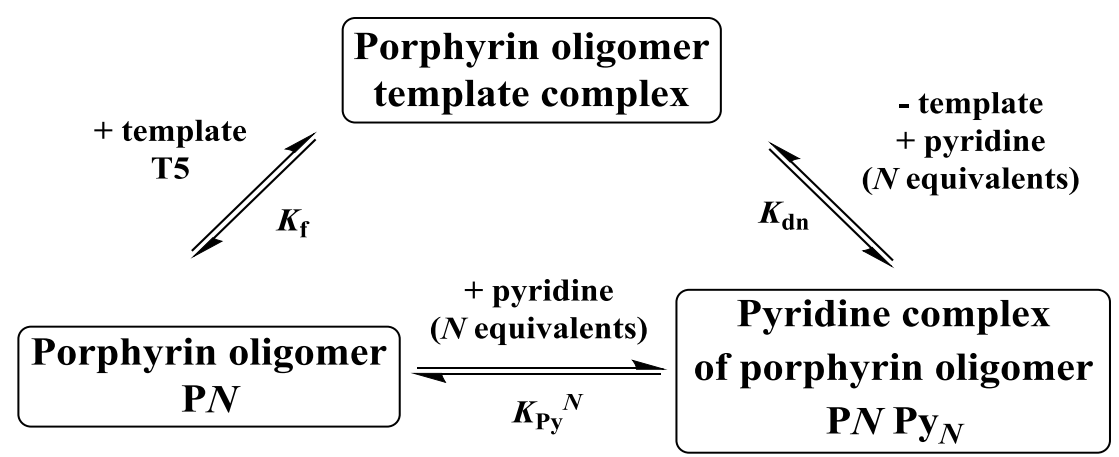

Figure 2.30: Thermodynamic cycle relating the formation constant of the template complex $\left(K_{\mathrm{f}}\right)$ to the denaturation constant $\left(K_{\mathrm{dn}}\right)$ and binding constant of each porphyrin unit for pyridine $\left(K_{\mathrm{Py}}\right)$. 
All the linear pentamers were found to interact strongly with the templates T5 and T4 and therefore denaturation titrations were performed on these complexes in order to determine binding constants.

Denaturation titrations were performed in chloroform at $298 \mathrm{~K}$. The 1:1 complexes between the porphyrin oligomers and templates were prepared in the cuvette prior to the denaturation titration. All formation titrations were carried out at constant porphyrin concentrations by adding porphyrin to the ligand (T5 or T4) stock solution and titrating until a 1:1 complex was formed according to UV-vis-NIR. All denaturation titrations were carried out at constant porphyrin-template complex concentration by adding both porphyrin and template to the ligand (pyridine) stock solution before titrations started.

Data were fitted to the $\mathrm{N}$-dentate denaturation binding isotherm described in the following equation:

$$
\frac{A-A_{\text {initial }}}{A_{\infty}-A_{\text {initial }}}=\left(\frac{-K_{d n}[L]^{N}+\sqrt{K_{d n}^{2}[L]^{2 N}+4 K_{d n}[L]^{N}[P]_{0}}}{2[P]_{0}}\right)
$$

where $A$ is the observed absorption at a specific wavelength or difference of absorption between two wavelengths; $A_{\text {initial }}$ is the starting absorption at a specific wavelength or difference between absorption in two wavelengths; $A_{\infty}$ is the terminal absorption at a specific wavelength or difference of absorption in two wavelengths; $K_{\mathrm{dn}}$ is the dissociation constant between ligand and porphyrin oligomer complex; [L] is the concentration of ligand; $[\mathrm{P}]_{0}$ is the concentration of porphyrin oligomer complex; $N$ is the number of binding sites in the complex (e.g. $N=4$ in 2.12.T4). The titration curves and fittings are shown below.

\begin{tabular}{|l|c|c|c|c|c|}
\hline & \multicolumn{2}{|c|}{ Table 2.2: Results from UV-vis-NIR titrations in Figures 2.31-2.38. } \\
\hline $\mathbf{2 . 1 2} \cdot \mathrm{T5}$ & $9.07 \pm 1.99 \times 10^{5}$ & $1.10 \pm 0.29 \times 10^{8}$ & 64 & $1.72 \pm 0.45 \times 10^{5}$ \\
\hline $\mathbf{2 . 1 1} \cdot \mathrm{T5}$ & $2.44 \pm 0.26 \times 10^{7}$ & $4.10 \pm 0.71 \times 10^{6}$ & 32 & $1.28 \pm 0.22 \times 10^{5}$ \\
\hline $\mathbf{2 . 1 2} \cdot \mathrm{T4}$ & $8.08 \pm 0.27 \times 10^{6}$ & $1.24 \pm 0.17 \times 10^{7}$ & 64 & $1.93 \pm 0.27 \times 10^{5}$ \\
\hline $\mathbf{2 . 1 1} \cdot \mathrm{T4}$ & $8.95 \pm 0.26 \times 10^{6}$ & $1.12 \pm 0.16 \times 10^{7}$ & 64 & $1.74 \pm 0.25 \times 10^{5}$ \\
\hline
\end{tabular}



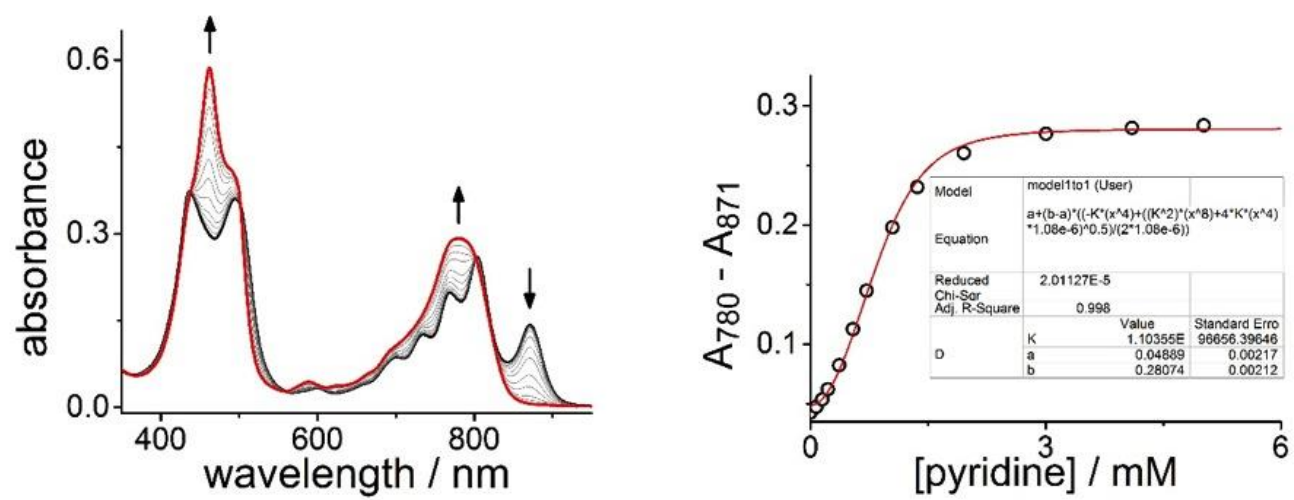

Figure 2.31: UV-vis-NIR titration of pyridine and 2.12·T5. $R^{2}=0.998$. (Run $1, \mathrm{CHCl}_{3}, 298 \mathrm{~K},[2.12 \cdot \mathrm{T5}$ ] $=1.08 \mu \mathrm{M}$, $K=1.10 \times 10^{6} \mathrm{M}^{-3}$ ).
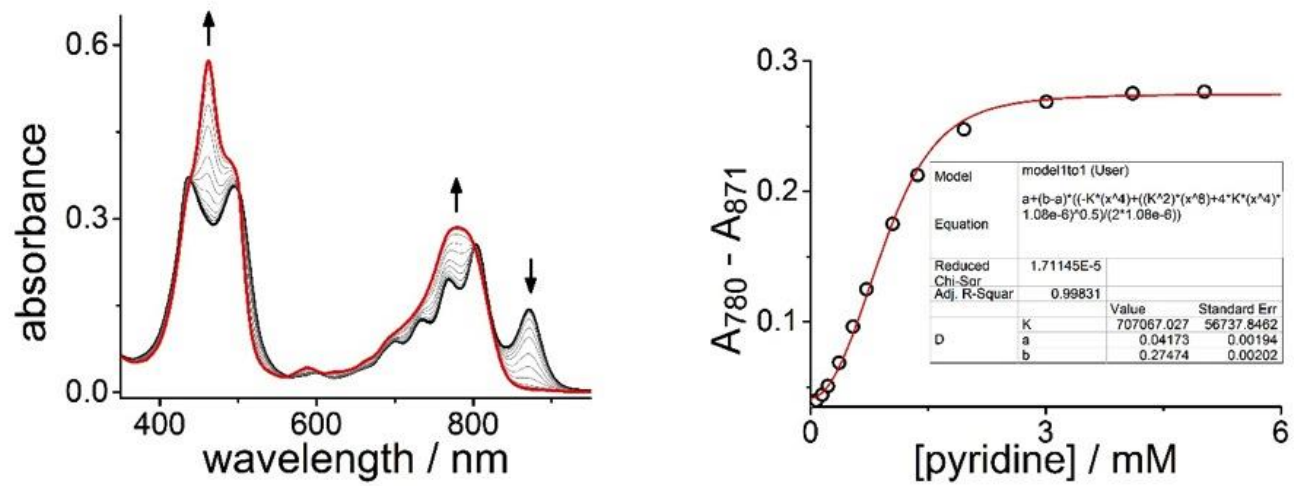

Figure 2.32: UV-vis-NIR titration of pyridine and 2.12·T5. $R^{2}=0.998$. (Run $2, \mathrm{CHCl}_{3}, 298 \mathrm{~K}$, [2.12.T5] = 1.08 $\mu \mathrm{M}$, $K=7.07 \times 10^{5} \mathrm{M}^{-3}$ ).
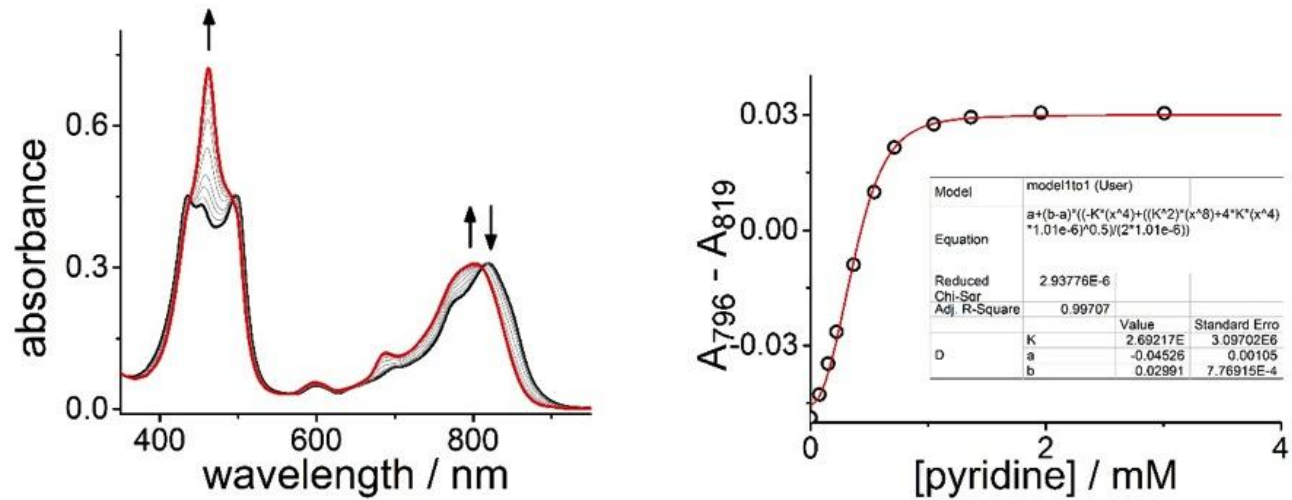

Figure 2.33: UV-vis-NIR titration of pyridine and 2.11·T5. $R^{2}=0.997$. (Run $1, \mathrm{CHCl}_{3}, 298 \mathrm{~K}$, [2.11.T5] $=1.01 \mu \mathrm{M}$, $\left.K=2.69 \times 10^{7} \mathrm{M}^{-3}\right)$. 

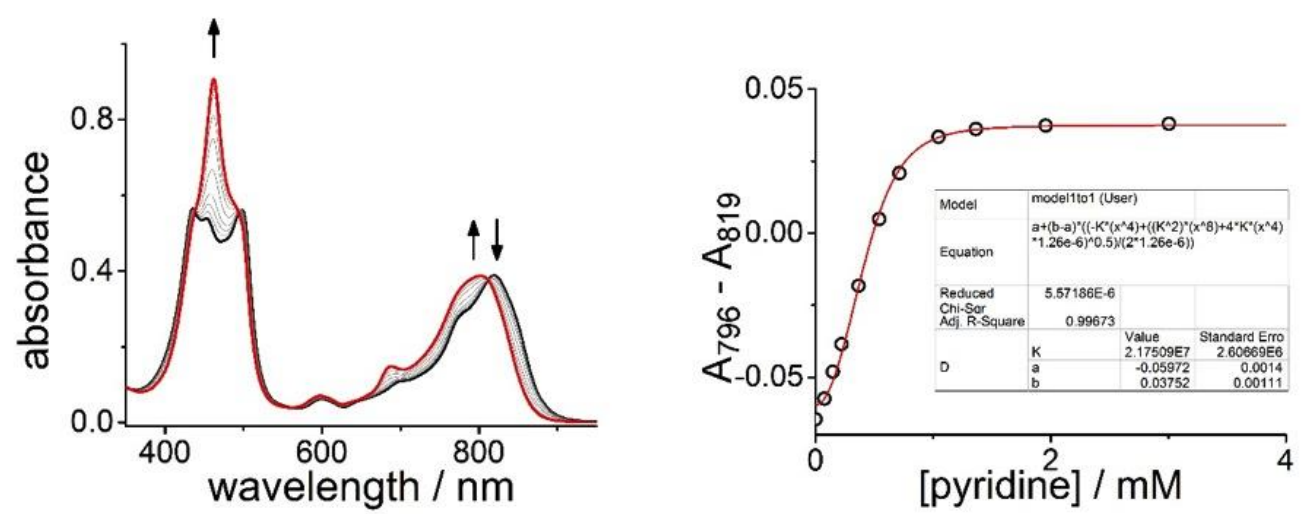

Figure 2.34: UV-vis-NIR titration of pyridine and 2.11.T5. $R^{2}=0.997$. (Run 2, $\mathrm{CHCl}_{3}, 298 \mathrm{~K},[2.11 \cdot \mathrm{T} 5]=1.26 \mu \mathrm{M}$, $\left.K=2.18 \times 10^{7} \mathrm{M}^{-3}\right)$.
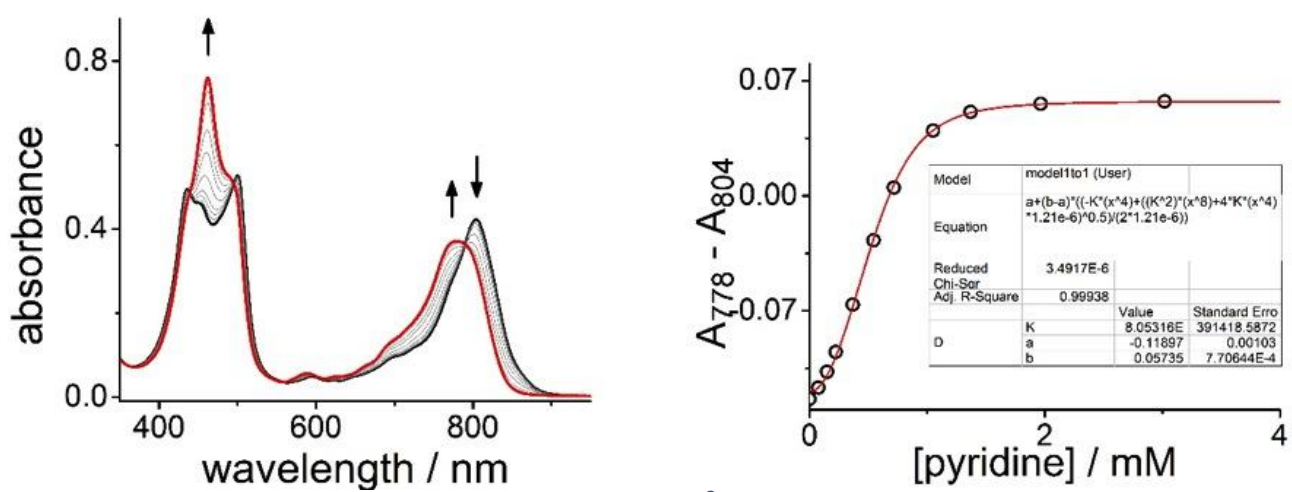

Figure 2.35: UV-vis-NIR titration of pyridine and 2.12·T4. $R^{2}=0.999$. (Run $1, \mathrm{CHCl}_{3}, 298 \mathrm{~K},[2.12 \cdot \mathrm{T} 4]=1.21 \mu \mathrm{M}$, $K=8.05 \times 10^{6} \mathrm{M}^{-3}$ ).
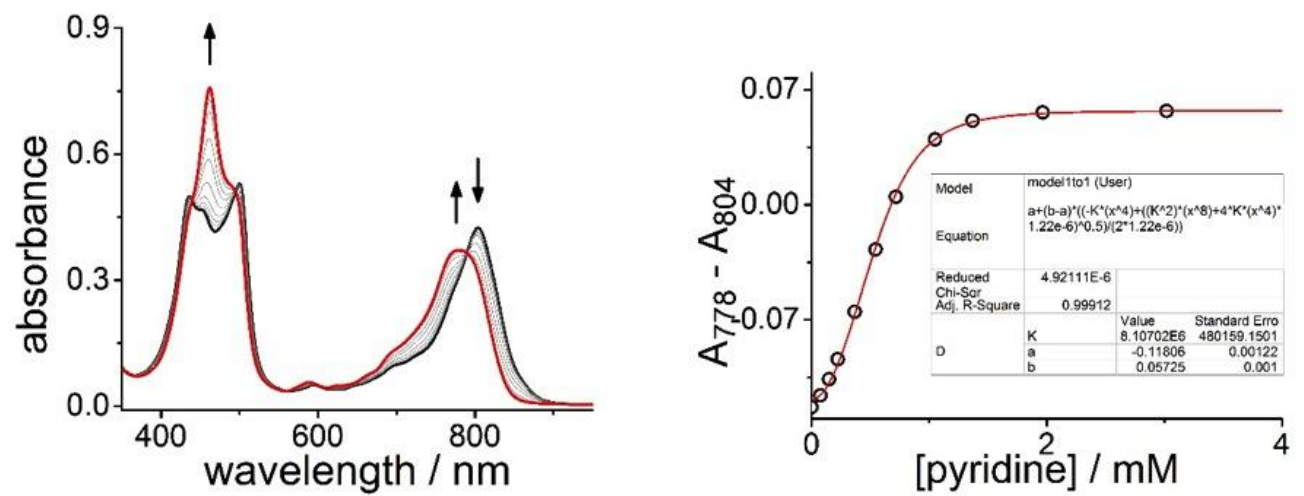

Figure 2.36: UV-vis-NIR titration of pyridine and 2.12·T4. $R^{2}=0.999$. (Run $2, \mathrm{CHCl}_{3}, 298 \mathrm{~K},[2.12 \cdot \mathrm{T} 4]=1.22 \mu \mathrm{M}$, $K=8.11 \times 10^{6} \mathrm{M}^{-3}$ ). 

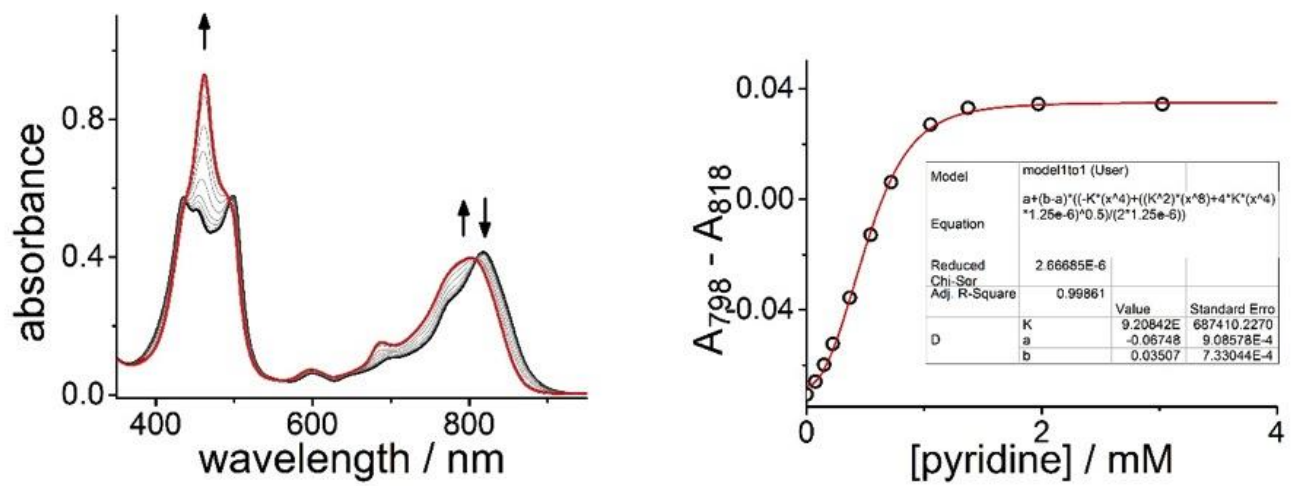

Figure 2.37: UV-vis-NIR titration of pyridine and 2.11·T4. $R^{2}=0.999$. (Run $1, \mathrm{CHCl}_{3}, 298 \mathrm{~K},[2.11 \cdot \mathrm{T} 4]=1.25 \mu \mathrm{M}$, $\left.K=9.21 \times 10^{6} \mathrm{M}^{-3}\right)$.
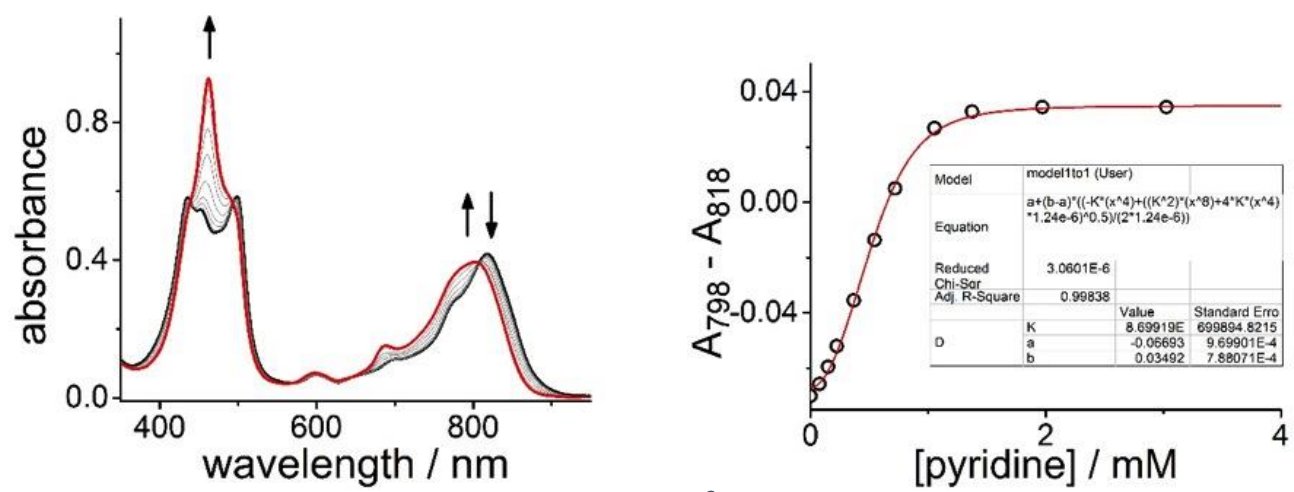

Figure 2.38: UV-vis-NIR titration of pyridine and 2.11·T4. $R^{2}=0.998$. (Run $2, \mathrm{CHCl}_{3}, 298 \mathrm{~K},[2.11 \cdot \mathrm{T} 4]=1.24 \mu \mathrm{M}$, $\left.K=8.70 \times 10^{6} \mathrm{M}^{-3}\right)$.

\subsubsection{Denaturation of 2.14-T5 with pyridine}

Denaturation titrations were performed on the complex 2.14.T5 with pyridine under the same conditions as described in Section 2.5.3.2.
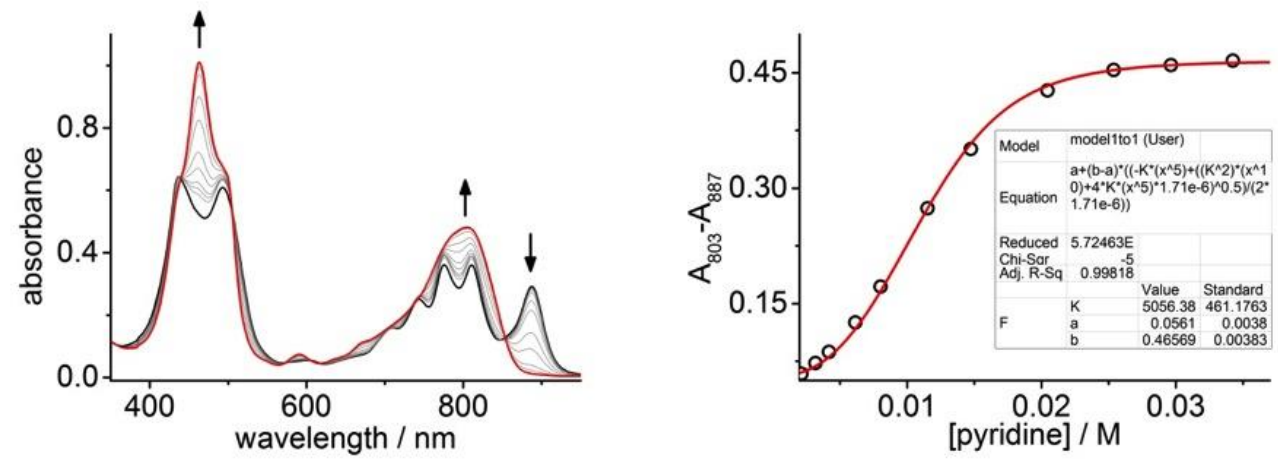

Figure 2.39: UV-vis-NIR titration of pyridine and 2.14·T5. $R^{2}=0.998$. (Run $1, \mathrm{CHCl}_{3}, 298 \mathrm{~K},[2.14 \cdot \mathrm{T} 5]=1.71 \mu \mathrm{M}$, $\left.K=5.06 \times 10^{3} \mathrm{M}^{-4}\right)$. 

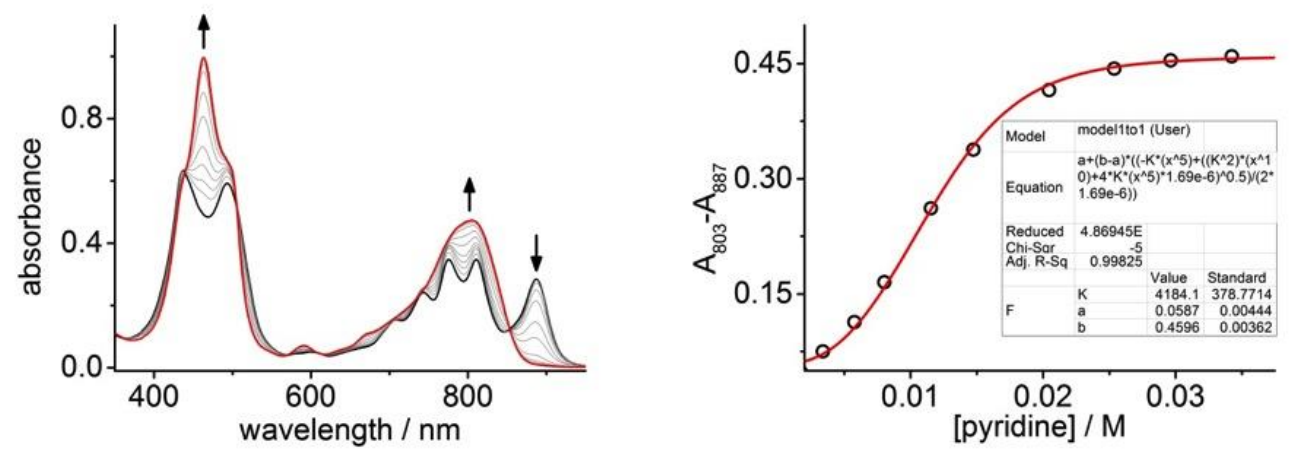

Figure 2.40: UV-vis-NIR titration of pyridine and 2.14·T5. $R^{2}=0.998$. (Run 2, $\mathrm{CHCl}_{3}, 298 \mathrm{~K},[2.14 \mathrm{n} \cdot \mathrm{T5}]=1.69 \mu \mathrm{M}$, $\left.K=4.18 \times 10^{3} \mathrm{M}^{-4}\right)$.
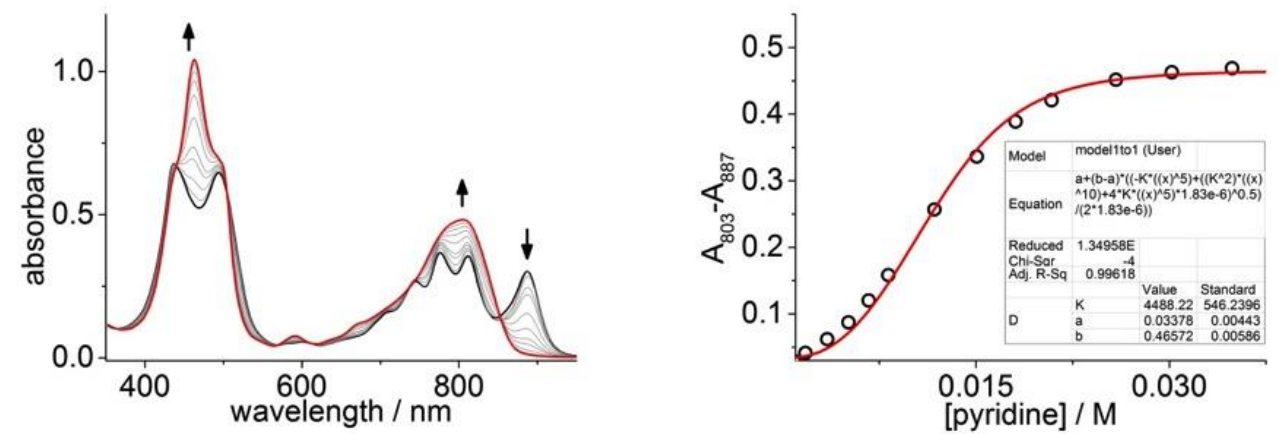

Figure 2.41: UV-vis-NIR titration of pyridine and 2.14·T5. $R^{2}=0.996$. (Run $3, \mathrm{CHCl}_{3}, 298 \mathrm{~K},[2.14 \cdot \mathrm{T} 5]=1.83 \mu \mathrm{M}$, $\left.K=4.49 \times 10^{3} \mathrm{M}^{-4}\right)$.

The denaturation constant, $4.58 \pm 0.48 \times 10^{3} \mathrm{M}^{-4}$, was translated into a statistically corrected association constant of $1.1 \pm 0.4 \times 10^{12} \mathrm{M}^{-1}$. To elucidate the effective molarity of the central porphyrin in the chain, we look at a single mutation in which we compare the stability of 2.14.T5 $\left(1.1 \pm 0.4 \times 10^{12} \mathrm{M}^{-1}\right)$ to the stability of 2.11.T5 $\left(1.3 \pm 0.2 \times 10^{5} \mathrm{M}^{-1}\right)$ according to the thermodynamic cycle below. 


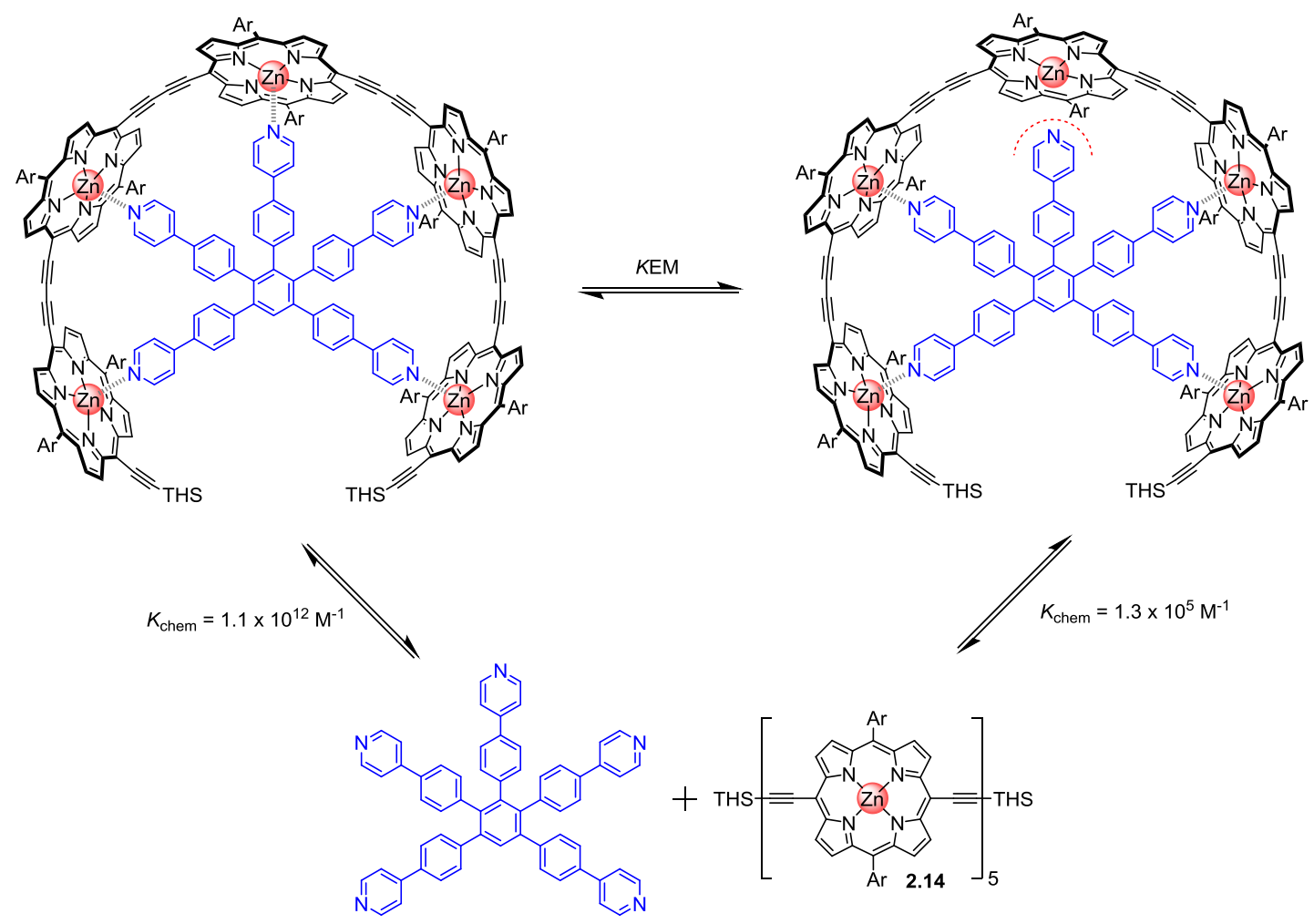

Figure 2.42: Complexes used to determine the interaction between the zinc centre in the central porphyrin and the pyridine leg of the template $\mathrm{T5}$ in $\mathrm{CHCl}_{3}$ at $298 \mathrm{~K}$ in order to determine the effective molarity of the central porphyrin. (Ar = 3,5-bis(tert-butyl)phenyl)

The thermodynamic cycle shows the relationship between the individual species and allows us to determine the EM:

$$
\begin{gathered}
K_{\mathrm{Ph}} E M=\frac{K_{\mathrm{f}}(\mathbf{2 . 1 4} \cdot \mathbf{T} 5)}{K_{\mathrm{f}}(\mathbf{2 . 1 1} \cdot \mathbf{T} 5)} \\
E M=\frac{\left(1.1 \times 10^{12}\right)}{\left(2.1 \times 10^{3}\right) \times\left(1.3 \times 10^{5}\right)}=4.0 \times 10^{3} \mathrm{M}
\end{gathered}
$$

where $K_{\mathrm{Ph}}$ is the equilibrium constant of the porphyrin monomer 2.5 binding to 4-phenylpyridine (see Section 2.5.3.1). We determined the effective molarity of the central porphyrin unit as $4 \pm 1 \times 10^{3} \mathrm{M}$. 


\subsubsection{Calculation of statistical factors}

To understand the stability constants of different complexes, it is useful to factor out statistical contributions. Thus, a measured equilibrium constant $K_{\text {eq }}$ can be factorised into its statistical component $K_{\sigma}$ and its statistically corrected value $K_{\text {chem }}$ according to the following equation:

$$
\begin{gathered}
w \mathrm{~A}+x \mathrm{~B} \rightleftharpoons y \mathrm{C}+z \mathrm{D} \\
K_{\text {eq }}=\frac{Q_{\mathrm{C}}^{y} Q_{\mathrm{D}}^{z}}{Q_{\mathrm{A}}^{w} Q_{\mathrm{C}}^{x}}=\frac{Q_{\mathrm{A}}^{w} Q_{\mathrm{B}}^{x}}{Q_{\mathrm{C}}^{y} Q_{\mathrm{D}}^{z}} \frac{Q_{\mathrm{C}}^{y} Q_{\mathrm{D}}^{\prime z}}{Q_{\mathrm{A}}^{\prime w} Q_{\mathrm{C}}^{\prime x}}=K_{\sigma} K_{\text {chem }}
\end{gathered}
$$

where for each species $i, Q_{i}$ is the partition coefficient, $Q^{\prime}{ }_{i}$ is the statistically corrected partition coefficient, and $\sigma_{i}$ is the symmetry number. ${ }^{23,27}$

Values of $K_{\sigma}$ were calculated using Benson's symmetry number method. ${ }^{137,138}$ The symmetry number $(\sigma)$ of each species is the product of its external symmetry number, $\sigma_{\text {ext, }}$ (calculated from the point group of the molecule) and its internal symmetry number, $\sigma_{\text {int }}$ (calculated from the number of degenerate internal rotors). The values of $\sigma$ for all the complexes involved are shown in Table 2.3. The external symmetry number is defined as the number of different but indistinguishable atomic arrangements that can be obtained by rotating a given molecule as a whole as a rigid object. The internal symmetry number is defined as the number of different but indistinguishable atomic arrangements that can be obtained by internal rotations around single bonds. 
Table 2.3: Internal, external and total symmetry numbers for each component.*

\begin{tabular}{|c|c|c|c|c|}
\hline component & point group & $\boldsymbol{\sigma}_{\text {int }}$ & $\boldsymbol{\sigma}_{\boldsymbol{e x t}}$ & $\boldsymbol{\sigma}$ \\
\hline pyridine & $\mathrm{C}_{2 \mathrm{v}}$ & 1 & 2 & 2 \\
\hline 4-phenylpyridine & $\mathrm{C}_{2 \mathrm{v}}$ & 1 & 2 & 2 \\
\hline T5 & $\mathrm{C}_{2 \mathrm{v}}$ & 1 & 2 & 2 \\
\hline T4 & $\mathrm{D}_{2 \mathrm{~h}}$ & 1 & 4 & 4 \\
\hline $\mathbf{2 . 5}$ & $\mathrm{D}_{2 \mathrm{~h}}$ & 1 & 4 & 4 \\
\hline $\mathbf{2 . 1 1}$ & $\mathrm{D}_{2 \mathrm{~h}}$ & $2^{4}=16$ & 4 & 64 \\
\hline $\mathbf{2 . 1 2}$ & $\mathrm{D}_{2 \mathrm{~h}}$ & $2^{4}=16$ & 4 & 64 \\
\hline $\mathbf{2 . 1 4}$ & $\mathrm{D}_{2 \mathrm{~h}}$ & $2^{4}=16$ & 4 & 64 \\
\hline $\mathbf{2 . 1 1 \cdot T 5}$ & $\mathrm{C}_{2 \mathrm{v}}$ & 2 & 2 & 4 \\
\hline $\mathbf{2 . 1 2} \cdot \mathrm{T5}$ & $\mathrm{C}_{2 \mathrm{v}}$ & 1 & 2 & 2 \\
\hline $\mathbf{2 . 1 4} \cdot \mathrm{T5}$ & $\mathrm{C}_{2 \mathrm{v}}$ & 1 & 2 & 2 \\
\hline $\mathbf{2 . 1 1} \cdot \mathrm{T4}$ & $\mathrm{C}_{2 \mathrm{v}}$ & 2 & 2 & 4 \\
\hline $\mathbf{2 . 1 2}$ T4 & $\mathrm{C}_{2 \mathrm{v}}$ & 2 & 2 & 4 \\
\hline
\end{tabular}

[* Note that when counting internal rotations and calculating $\sigma_{i n t}$, we do not include rotors which are unaffected by the binding process, such as the para-phenylene links in T5 because if a rotor is unaffected by the binding process it has no influence on $K_{\sigma}$.]

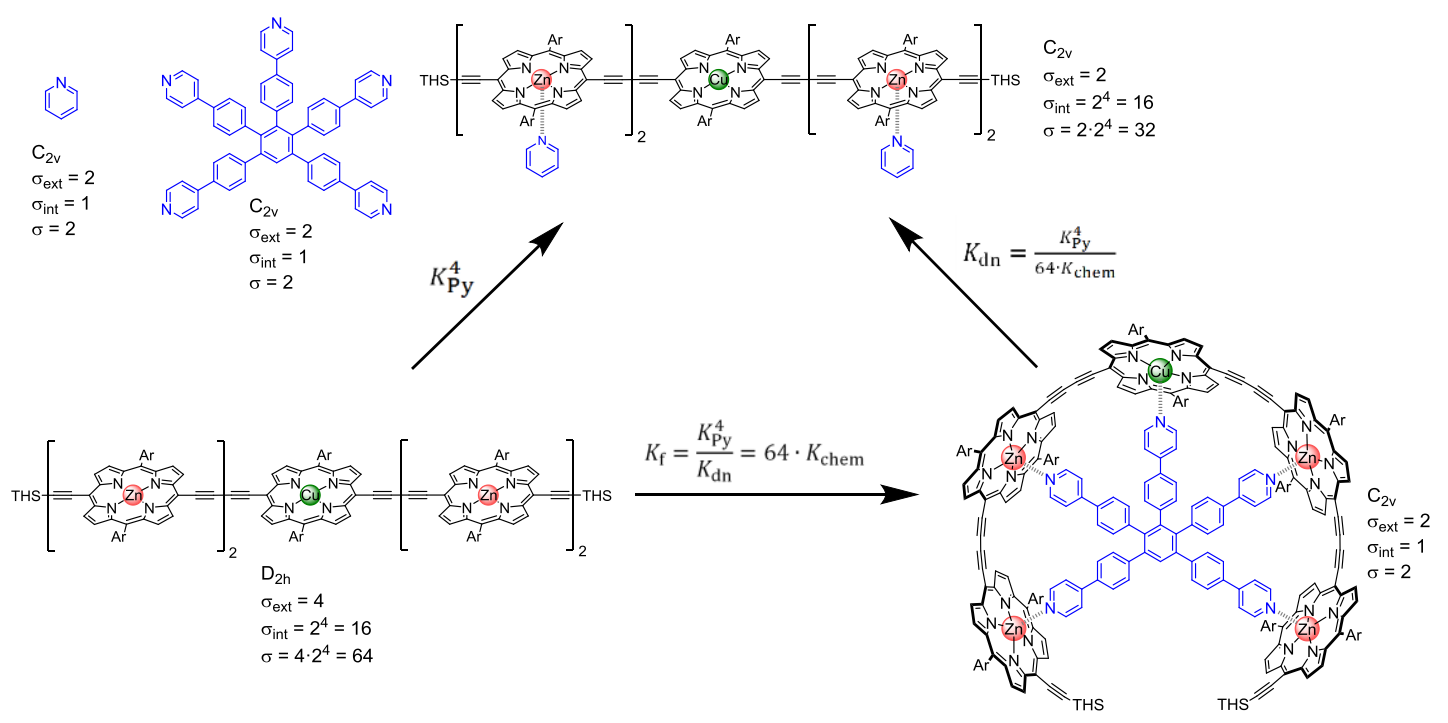

Figure 2.43: Statistical factors involved in the formation and denaturation (pyridine) of the 2.12.T5 complex. $K_{\sigma}=64$. 


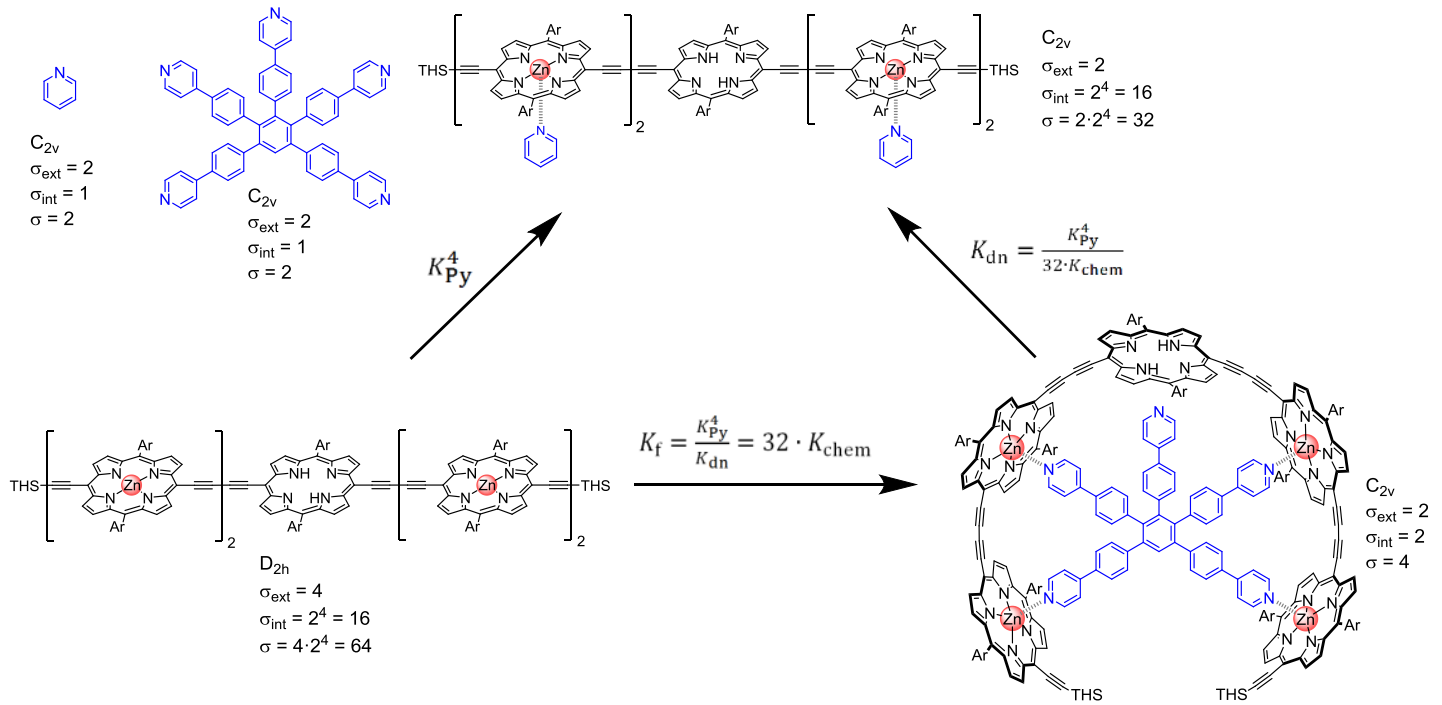

Figure 2.44: Statistical factors involved in the formation and denaturation (pyridine) of the 2.11.T5 complex. $K_{\sigma}=32$.

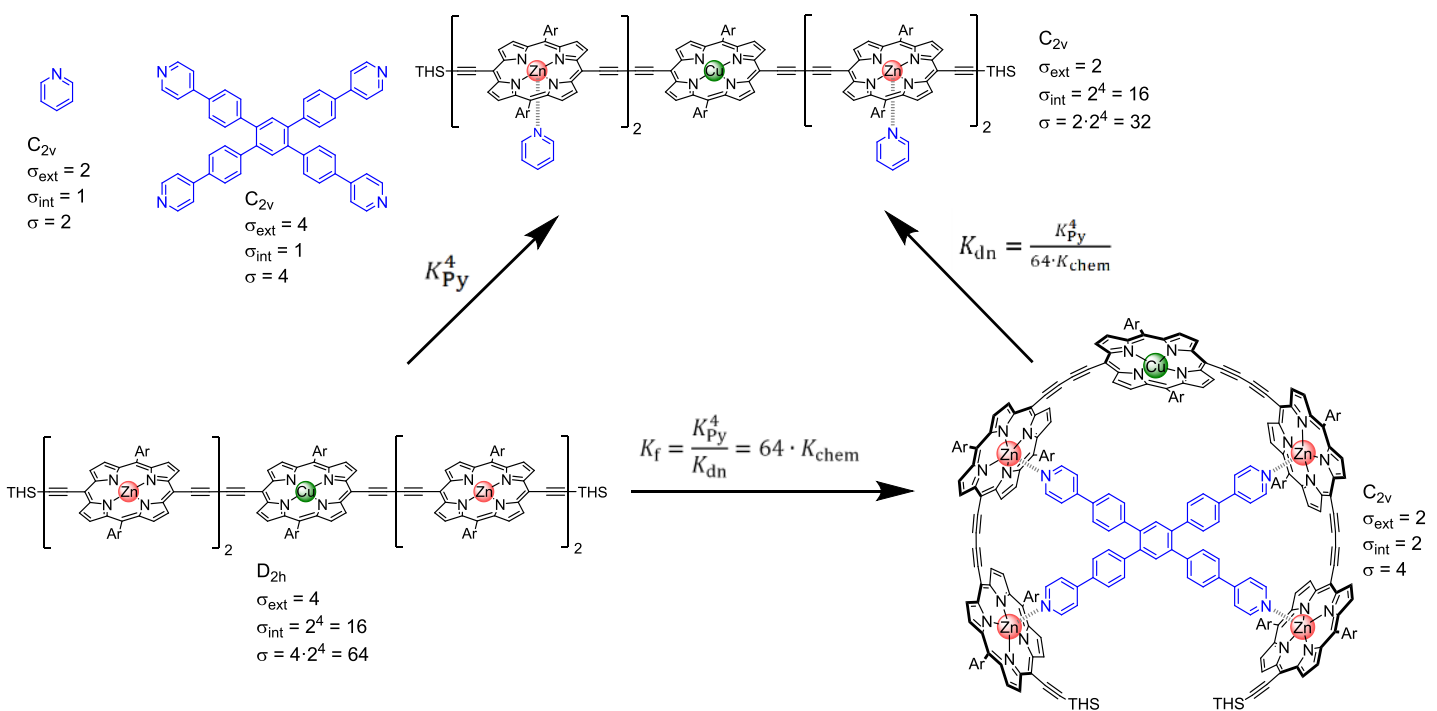

Figure 2.45: Statistical factors involved in the formation and denaturation (pyridine) of the 2.12.T4 complex. $\kappa_{\sigma}=64$. 


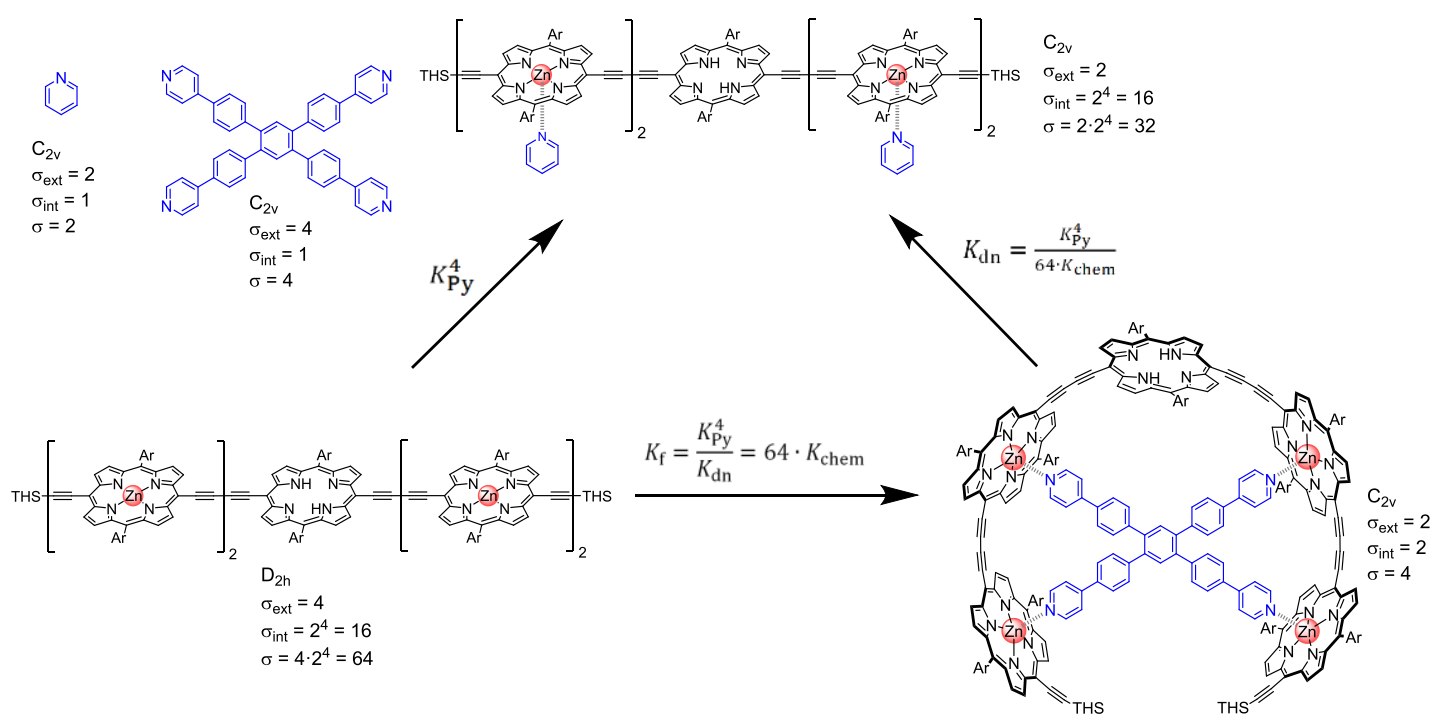

Figure 2.46: Statistical factors involved in the formation and denaturation (pyridine) of the 2.11.T4 complex. $K_{\sigma}=64$.

\subsubsection{DFT calculations}

Constrained DFT geometry optimisations were carried out by Sabine Richert for several fixed separation distances between pyridine and the central metal of the porphyrin unit to explore the binding strength of pyridine to 2.15 in comparison to its copper analogue $\mathbf{2 . 1 5} \mathrm{cu}$. The optimisations were carried out in Turbomole V6.1 $1^{117}$ under $C_{2}$ symmetry using DFT/B3LYP in combination with the TZVP basis set, ${ }^{118}$ $\mathrm{Rl}$-approximation ${ }^{119}$ and an empirical dispersion correction to the energies. ${ }^{120,121}$

The SCF energy differences with respect to the minimum structure were then plotted as a function of the metal-pyridine separation distance and are shown in Figure 2.47.

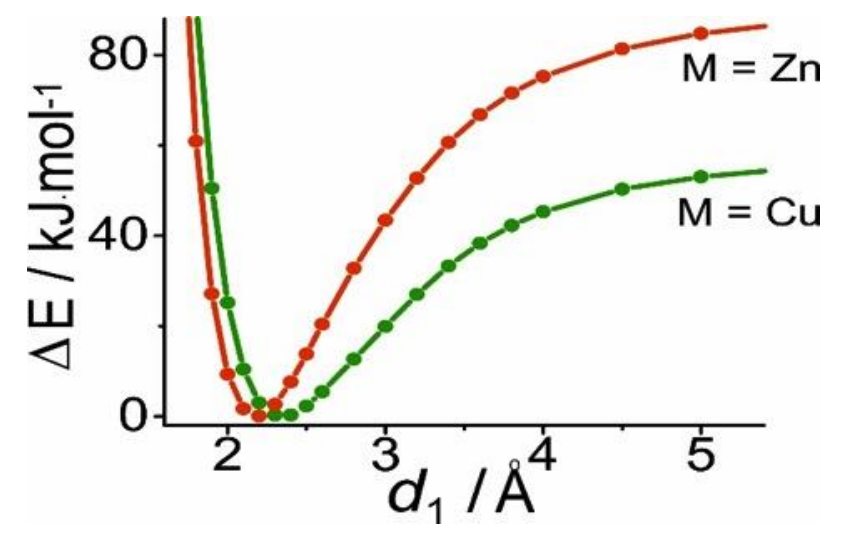

Figure 2.47: Total SCF energy differences with respect to the minimum structure as a function of the metal...pyridine separation distance for $2.15_{\mathrm{cu}}$ (green line) and 2.15 (red line) obtained from a constrained geometry optimization in Turbomole using DFT/B3LYP in combination with the TZVP basis set and including dispersion correction for the energies.

Regarding the geometry of $\mathbf{2 . 1 5} \cdots P y$, experimental data are available from X-ray crystallography in the CSD database. A statistical analysis of all available 
experimental data was found to yield a value of $2.16 \pm 0.03 \AA$ as the mean zinc-pyridine separation distance, ${ }^{125}$ which is in very good agreement with the presented DFT results. A similar analysis based on X-ray data for the distance between the zinc atom and the porphyrin plane results in a mean value of $0.24 \pm 0.06 \AA$. This result compares favourably with the distance of $0.28 \AA$ obtained for $\mathbf{2 . 1 5}$ from the DFT calculations in this work. In the equilibrium geometry, the zinc atom is thus markedly pulled out of the porphyrin plane as is also shown in graphical form in Figure 2.48. For $2.15_{\mathrm{Cu}} \ldots$ Py a distance of $0.12 \AA$ is found by DFT, indicating that the copper atom roughly remains in the centre of the porphyrin plane even when a pyridine ligand is bound.
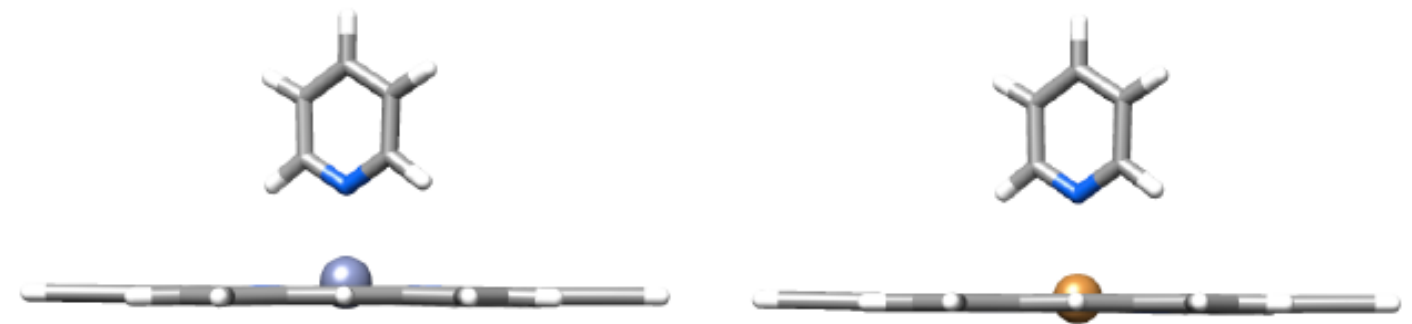

Figure 2.48: Side-view of the optimised minimum geometries of 2.15 ..Py (left) and $2.15_{\mathrm{Cu}} \ldots$ Py (right). The zinc centre is pulled further out of the plane of the porphyrin upon binding pyridine and a smaller separation distance is found.

Figure 2.48 shows a comparison of the optimised structures for $2.15 \cdots P y$ and

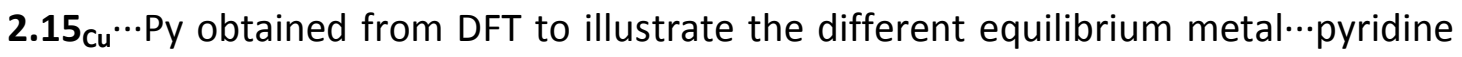
separation distances and the different locations of the metal atom with respect to the porphyrin plane. The calculated equilibrium metal...pyridine separation distance is increased in $2.15_{C u}$..Py $(2.35 \AA)$ as compared to 2.15 ..Py $(2.18 \AA)$. Upon binding of a pyridine ligand, the zinc atom is markedly pulled out of the porphyrin plane, whereas the position of the copper atom with respect to the porphyrin plane is much less affected.

\subsubsection{EPR measurements}

All EPR measurements were carried out by Dr Sabine Richert and the experimentals are described in detail in the main text and supplementary information of the following article:

Exploring template-bound dinuclear copper porphyrin nanorings by EPR spectroscopy S. Richert, J. Cremers, H. L. Anderson, C. R. Timmel, Chem. Sci. 2016, 7, 6952-6960. 


\section{Constructive Quantum Interference in a Heterometallated Porphyrin Nanoring}

\section{Contents}

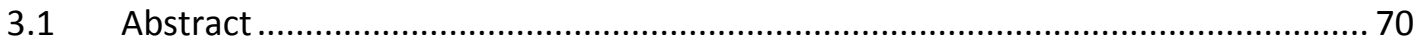

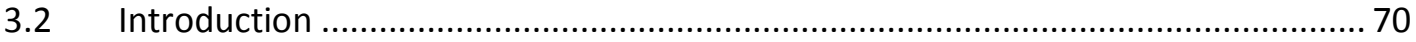

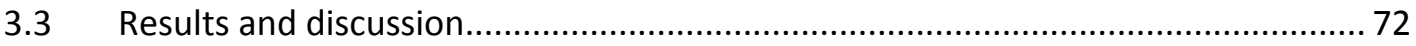

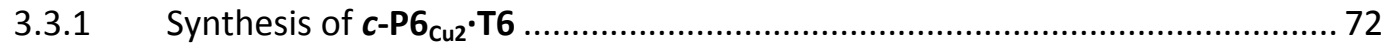

3.3.2 NMR spectroscopy of copper-containing oligomers..................................... 75

3.3.3 Quantification of the constructive interference ............................................. 78

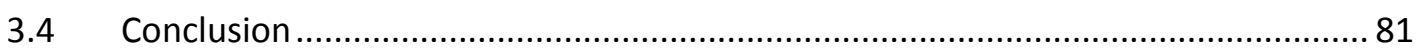

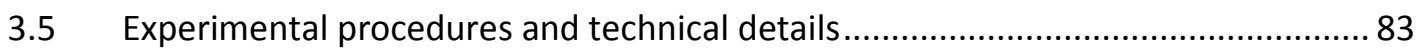

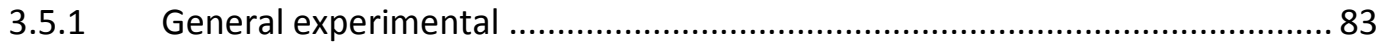

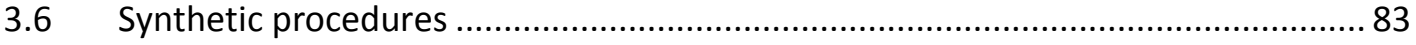

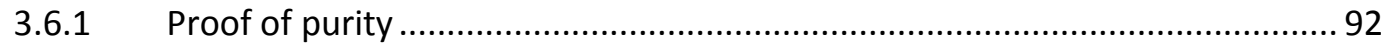

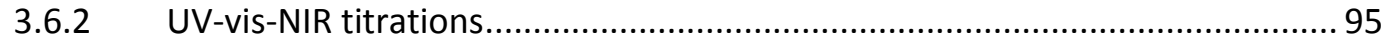

3.6.3 $T_{1}$ and $T_{2}$ relaxation time constant measurements....................................... 100

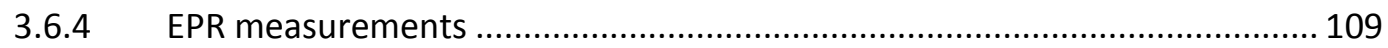

Parts of this chapter were published in:

Constructive quantum interference in a bis-copper six-porphyrin nanoring

S. Richert, J. Cremers, I. Kuprov, M. D. Peeks, H. L. Anderson, C. R. Timmel, Nat. Commun. 2017, 8, 14842-14847.

Nanorings with copper(II) and zinc(II) centers: forcing copper porphyrins to bind axial ligands in heterometallated oligomers

J. Cremers, S. Richert, D. V. Kondratuk, T. D. W. Claridge, C. R. Timmel, H. L. Anderson, Chem. Sci. 2016, 7, 6961-6968.

Quantifying the exchange coupling in linear copper porphyrin oligomers

S. Richert, I. Kuprov, M. D. Peeks, E. A. Suturina, J. Cremers, H. L. Anderson, C. R. Timmel, Phys. Chem. Chem. Phys. 2017, 24, 16057-16061. 


\subsection{Abstract}

The exchange interaction $(J)$ between two spin centres is a convenient measure of through bond electronic communication. This chapter will describe the investigation of quantum interference phenomena in a bis-copper six-porphyrin nanoring $\left(\boldsymbol{c}-\mathbf{P 6}_{\mathrm{Cu2}} \cdot \mathbf{T 6}\right)$ by using electron paramagnetic resonance spectroscopy via measurement of the exchange coupling between the copper centres. Using an analytical expression accounting for both dipolar and exchange coupling to simulate the time traces obtained in a double electron-electron resonance experiment, we demonstrate that $J$ can be quantified to high precision even in the presence of significant through-space coupling $(D)$. We show that the exchange coupling between two spin centres is increased by a factor of 4.5 in the ring structure with two parallel coupling pathways as compared to an otherwise identical system with just one coupling path. This observation is a signature of constructive quantum interference.

\subsection{Introduction}

When electrons tunnel through a molecule via more than one pathway, the wavefunctions corresponding to different routes may be in-phase or out-of-phase with each other, leading to constructive or destructive interference. Quantum interference effects of this type are thought to control electron transfer through proteins. ${ }^{139-141}$ Quantum interference also provides many opportunities for enhancing performance in single-molecule electronic devices, by creating sharp transport resonances. ${ }^{78,142}$ When the distance of the transmission exceeds a certain threshold, interactions with vibrational degrees of freedom result in a change of mechanism from phase-coherent single-step tunnelling to incoherent multistep hopping, erasing interference effects. ${ }^{143,144}$ It is difficult to measure coherence lengths in molecular structures and the size limit for quantum mechanical behaviour is not well established. The simplest test for quantum coherence is to compare transmission between two points, A and B, through two identical channels in parallel, each of conductance $G_{1}$, with that through just one isolated channel as shown in Figure 3.1. 

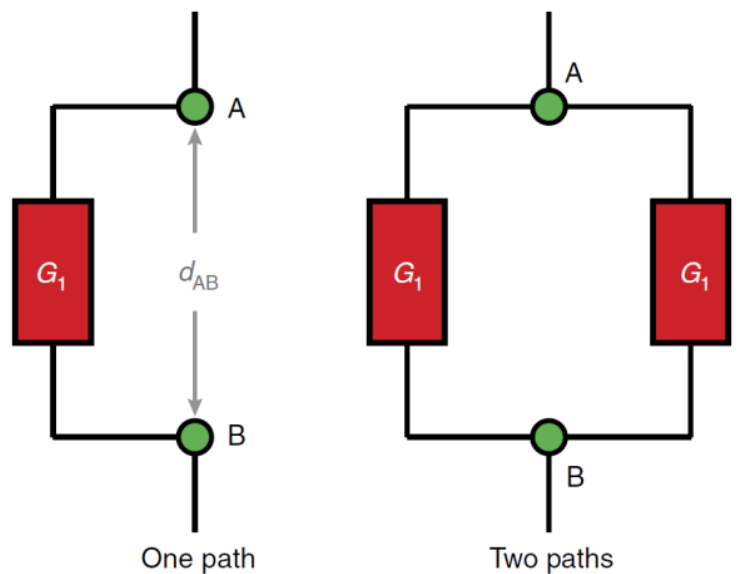

Figure 3.1: Schematic illustration of transmission through one or two paths. Quantum interference can be tested by comparison of the transmission properties. Assuming two identical parallel paths connecting $A$ and $B$ with the total conductance referred to as $G_{A B}$, coherent transmission is expected to yield $G_{A B}=4 G_{1}$, whereas non-coherent transport should result in $G_{A B}=2 G_{1}$. The transmission $G$ is closely related to the exchange coupling $J$.

A classical description predicts that the total conductance for the two channels will be given by Kirchhoff's circuit law as $G_{A B}=2 G_{1}$; this result is expected when the distance $d_{A B}$ is greater than the coherence length. On the other hand, if the system behaves coherently, constructive quantum interference is expected to give a total conductance of $G_{A B}=4 G_{1}{ }^{78,79}$

Recently, this scenario has been tested experimentally for charge transport through single molecules. STM break-junction measurements on a thioether-linked cyclophane $\left(d_{\mathrm{AB}}=0.7 \mathrm{~nm}\right)$ gave the result $G_{\mathrm{AB}}=2.8 G_{1}$, providing evidence for quantum interference. ${ }^{145}$ Similar experiments compared the conductance of a carbobenzene macrocycle $\left(d_{A B}=0.8 \mathrm{~nm}\right)$ with a single-path reference molecule to give $G_{A B}=40 G_{1}$, although in this case the higher conductance of the two-path system is partly a consequence of its greater conformational rigidity. ${ }^{81}$ These results highlight the challenges involved in using single-molecule charge transport measurements to probe quantum coherence.

Despite many advances in methodology, ${ }^{146-148}$ it is difficult to measure molecular conductances accurately enough to detect the predicted fourfold increase in $G_{A B}$ bestowed by constructive interference. This is particularly hard in large molecules, which are expected to be on the threshold between coherent and incoherent transport, and have very low conductances. In addition, it is difficult to synthesise 
pairs of molecules that have identical conformations and only differ in the number of available tunnelling channels.

In this chapter we present solutions to both of these problems: we implement the scheme shown in Figure 3.1 by testing the through-bond exchange coupling $(J)$ between two paramagnetic centres in $\mathbf{c}-\mathbf{P} \mathbf{6}_{\mathrm{Cu2}} \cdot \mathbf{T 6}$ (Figure 3.2 ), which can be measured accurately by electron paramagnetic resonance (EPR), and we lock the molecular wire into a well-defined conformation by complexing the nanoring around the hexadentate T6 template. Comparison of the average value of the exchange coupling between copper(II) centres in compounds $\mathbf{c}-\mathbf{P} \mathbf{6}_{\mathrm{Cu2}} \cdot \mathbf{T 6}$, with two paths, and I-P6 $\mathbf{C u 2}_{\mathrm{Cu}} \cdot \mathbf{T 6}$, with one path gives $J_{A B}=4.5 J_{1}$ for a through-bond tunnelling distance of $d_{A B}=3.9 \mathrm{~nm}$, demonstrating constructive quantum interference over a remarkably long distance.

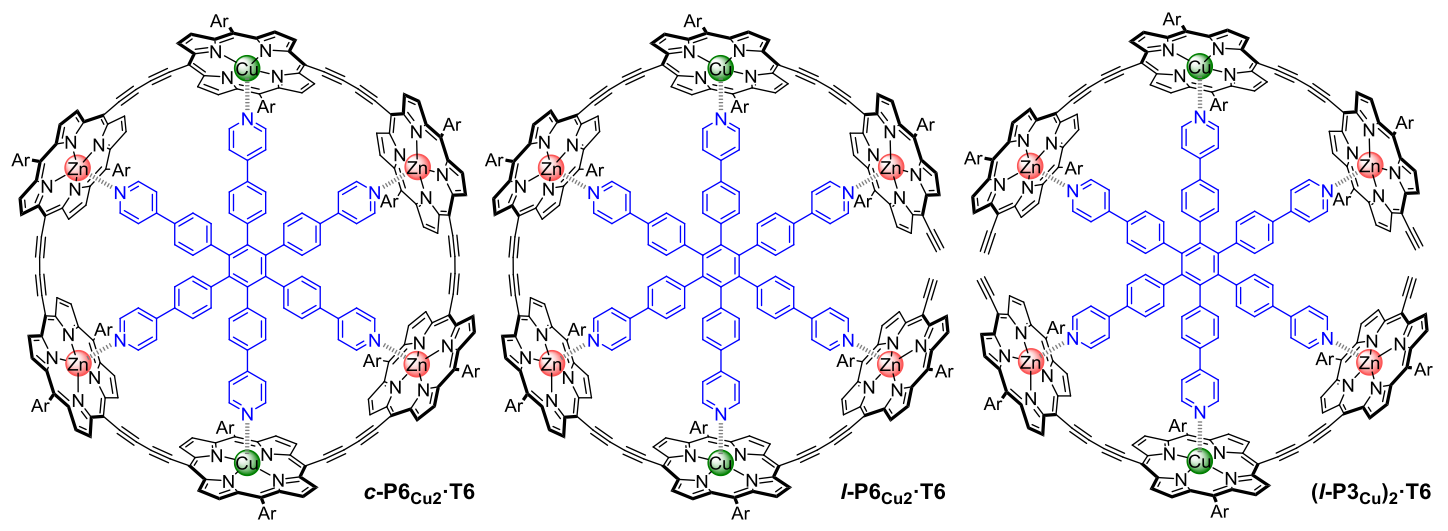

Figure 3.2: Chemical structures of the compounds $c-\mathrm{P} 6_{\mathrm{Cu} 2} \cdot \mathrm{T} 6, I-\mathrm{P} 6_{\mathrm{Cu} 2} \cdot \mathrm{T} 6$ and $\left(I-\mathrm{P} 3_{\mathrm{Cu}}\right)_{2} \cdot \mathrm{T} 6$. The structures differ in the number of pathways between the two copper centres: $c$ - $\mathrm{P} 6_{\mathrm{Cu} 2} \cdot \mathrm{T} 6$ contains two pathways; I-P6 ${ }_{\mathrm{Cu}} \cdot{ }^{-\mathrm{T}} 6$ one pathway; (I-P3 $\left.3_{\mathrm{Cu}}\right)_{2} \cdot \mathrm{T} 6$ has no pathways. The Ar on $c-\mathrm{P} 6_{\mathrm{Cu2}} \cdot \mathrm{T} 6$ represents 3,5-bis(tert-butyl)phenyl; on the other two compounds $\mathrm{Ar}=3,5-b i s($ trihexylsilyl)phenyl.

\subsection{Results and discussion}

\subsubsection{Synthesis of $c-\mathrm{P}_{\mathrm{Cu}} \cdot \mathrm{T} 6$}

The linear porphyrin trimer with a copper porphyrin in the middle of the chain was prepared in two steps from compounds described in Chapter 2. Initially a free-base centred trimer was formed in a $39 \%$ yield by coupling the deprotected free-base porphyrin monomer $\mathbf{2 . 9}$ with a large excess of mono-protected monomer $\mathbf{2 . 6}$ (Figure 3.3). Subsequently, copper was inserted into the central free-base porphyrin unit to give the heterometallated trimer $\mathbf{3 . 2}$ in a nearly quantitative yield. 


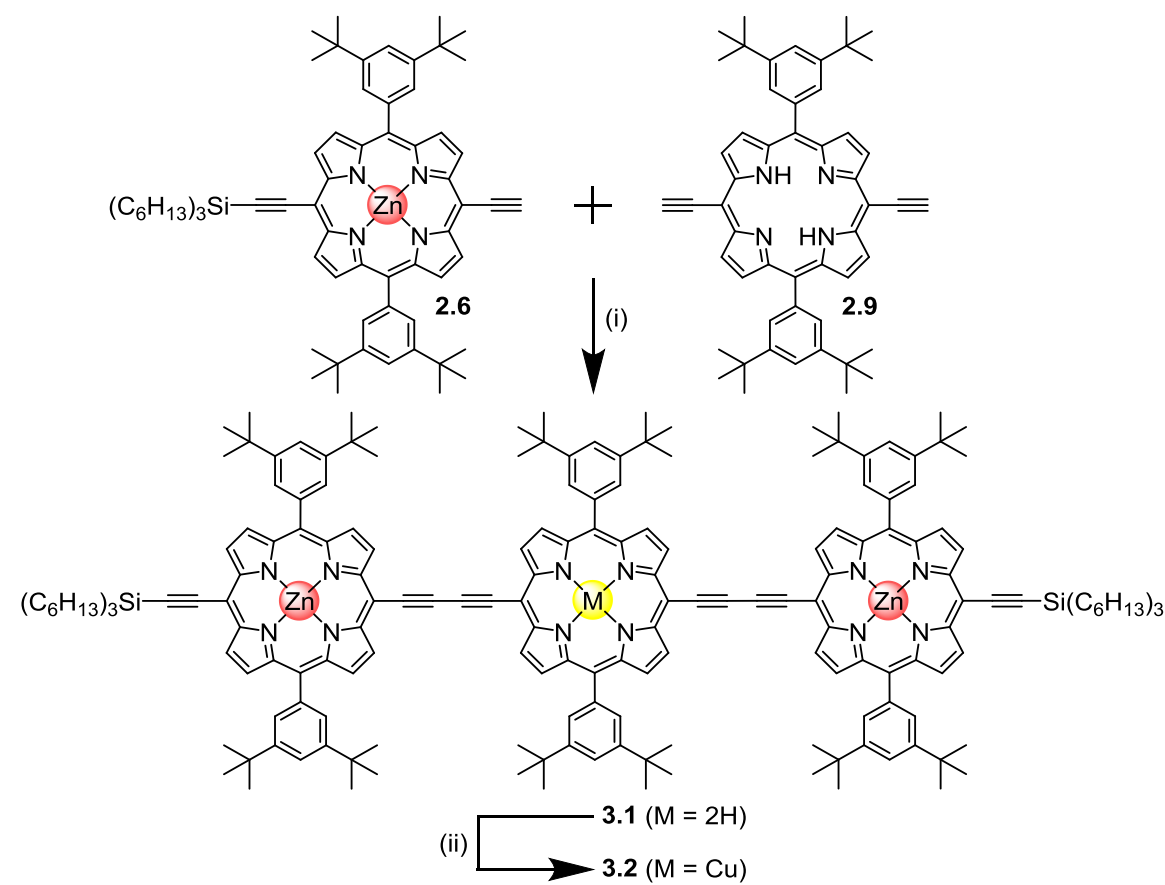

Figure 3.3: Synthetic route of the copper centred trimer 3.2. (i) $\mathrm{Pd}_{2}(\mathrm{dba})_{3}$, tri-2-furylphosphine, 1,4-benzoquinone, toluene/ $\mathrm{Et}_{3} \mathrm{~N}, 39 \%$; (ii) $\mathrm{Cu}(\mathrm{OAC})_{2} \cdot \mathrm{H}_{2} \mathrm{O}, \mathrm{CHCl}_{3}, 95 \%$.

The THS protecting groups on the heterometallated porphyrin oligomer $\mathbf{3 . 2}$ were removed in an $94 \%$ yield by the addition of TBAF giving trimer 3.3. In order to prepare the target nanoring, two trimers have to be preorganised around the hexadentate template $\mathbf{T 6}$ before the acetylenes can be coupled. As discussed in Chapter 2, the axial binding affinity of a copper monomer to a pyridyl ligand is weak. In the preparation of $\boldsymbol{c}-\mathbf{P} 10_{\mathrm{Cu} 2}$ we could utilise the strong cooperative binding of four zinc porphyrins on each oligomer to stabilise the templated intermediate before forming the ring. In the case of $\mathbf{3 . 3}$ there are only two zinc-nitrogen interactions per oligomer to the template and the binding is considerably weaker. UV-vis-NIR titrations were performed with $\mathbf{3 . 3}$ and T6 to investigate the binding (Figure 3.4).
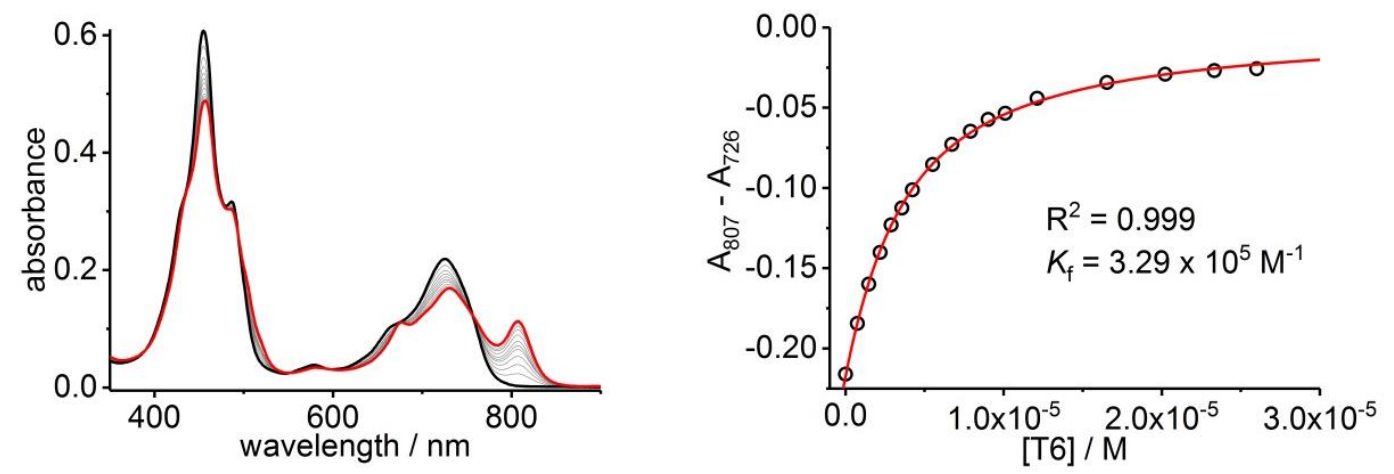

Figure 3.4: UV-vis-NIR titrations of 3.3 and T6, $\mathrm{R}^{2}=0.999 .\left(\mathrm{CHCl}_{3}, 298 \mathrm{~K},[3.3]=1.35 \mu \mathrm{M}, K=3.29 \times 10^{5} \mathrm{M}^{-1}\right)$. 
Upon the addition of T6, complex formation is indicated by a characteristic red-shift in the Q-band. The rise of the band around $800 \mathrm{~nm}$ is typically observed as a result of planarisation of oligomers and restriction of the rotation of individual porphyrins. While there is clear complex formation, the binding strength is relatively weak. As a comparison, a linear trimer containing solely zinc porphyrins, has been reported to bind approximately five orders of magnitude stronger to T6 than the heterometallated trimer, demonstrating the importance of the central binding site in the overall cooperative binding event. ${ }^{23}$

In spite of the weak binding affinity of the trimer to the template, we proceeded in the synthesis of the nanoring. The six-porphyrin nanoring with two copper centres was prepared by the oxidative homocoupling of the deprotected trimers $\mathbf{3 . 3}$ in the presence of the hexadentate template T6 (Figure 3.5). Polymerisation dominated in this reaction, and the desired product, $\mathbf{c}-\mathbf{P 6} \mathbf{C}_{\mathrm{Cu2}} \cdot \mathbf{T 6}$, was isolated in only $2 \%$ yield, reflecting the weak binding of the trimer to the template. ${ }^{55}$
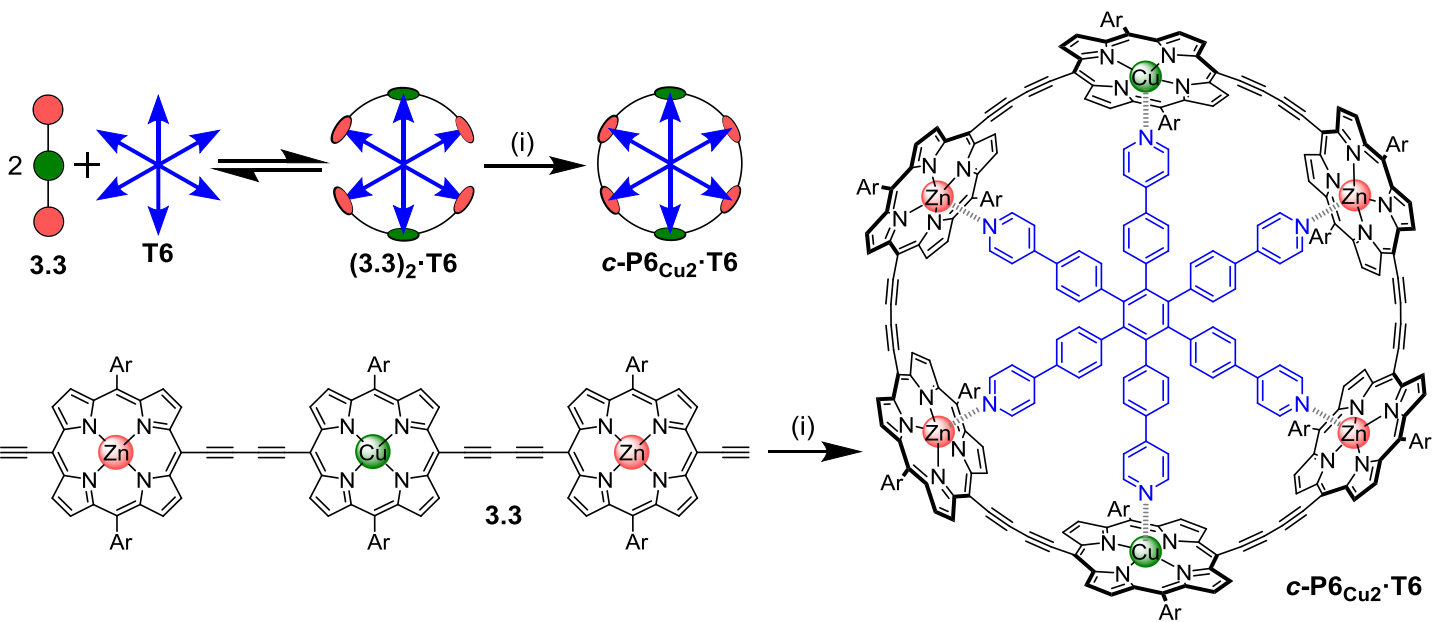

Figure 3.5: Synthesis of $c-\mathrm{P} 6_{\mathrm{Cu} 2} \cdot \mathrm{T} 6$. Two representations of the synthetic route used to prepare the nanoring. (i) T6, $\mathrm{PdCl}_{2}\left(\mathrm{PPh}_{3}\right)_{2}$, Cul, 1,4-benzoquinone, i- $\mathrm{Pr}_{2} \mathrm{NH}, \mathrm{CHCl}_{3}, 2 \%$. $\mathrm{Ar}=3,5-$ bis(tert-butyl)phenyl.

During the course of the synthesis, it was found at multiple stages that the solubility of the deprotected porphyrin oligomers with 3,5-bis(tert-butyl)phenyl solubilising groups was low. It was therefore decided to prepare the reference compounds I-P6 Cu2 $\cdot \mathbf{T 6}$ and $\left(I-P 3_{\mathrm{Cu}}\right)_{2} \cdot \mathbf{T 6}$ with 3,5-bis(trihexylsilyl)phenyl aryls to improve solubility. The heterometallated trimer $\mathbf{3 . 1 1}$ with THS-aryls was prepared via a comparable synthetic strategy as was used to prepare the tert-butyl analogue 3.2. 
Subjecting 3.11 to an excess of TBAF removed the protecting groups on the acetylenes to give $\mathbf{I - P 3 _ { C u }}$ in a $96 \%$ yield (Figure 3.6). When trimer $\mathbf{3 . 1 1}$ was subjected to small quantities of TBAF, a statistical deprotection of the acetylenes was achieved. Unfortunately it proved to be impossible to purify the reaction mixture to yield pure 3.12. The difference in polarity between the starting material and the mono- and fully-deprotected products is too small to separate the products via column chromatography. Additionally, the difference in size between the different products is negligible and size exclusion chromatography does not provide a suitable way for purification. It was decided to subject the mixture of products to oxidative coupling conditions without further purification. The reaction gave a mixture of oligomers which vary in size by multiples of three porphyrins (potential products: 3-mer, 6-mer, 9-mer, etc.). The difference in size between the resulting oligomers is large which allows for isolation of $\mathbf{3 . 1 3}$ from the mixture of oligomers by recycling GPC. Deprotection of the acetylenes of $\mathbf{3 . 1 3}$ by the addition of TBAF gave the desired hexamer $\mathbf{I - P 6 _ { \text { Cu2 } }}$ in a $76 \%$ yield.

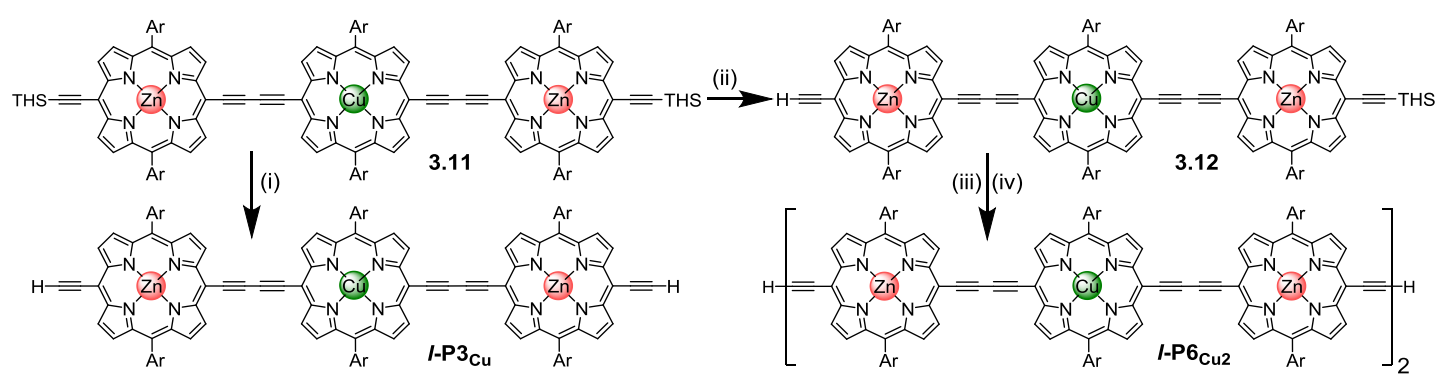

Figure 3.6: Synthesis of I-P3 $3_{\mathrm{Cu}}$ and I-P6 $6_{\mathrm{Cu} 2}$. (i) TBAF, $\mathrm{CH}_{2} \mathrm{Cl}_{2}, 96 \%$; (ii) TBAF, $\mathrm{CH}_{2} \mathrm{Cl}_{2} / \mathrm{CHCl}_{3}$, then; (iii) $\mathrm{PdCl}_{2}\left(\mathrm{PPh}_{3}\right)_{2}$, Cul, 1,4-benzoquinone, toluene/i- $\mathrm{Pr}_{2} \mathrm{NH}$, 43\%; (iv) TBAF, $\mathrm{CH}_{2} \mathrm{Cl}_{2}, 76 \%$. Ar = 3,5-bis(trihexylsilyl)phenyl.

\subsubsection{NMR spectroscopy of copper-containing oligomers}

Many copper porphyrins have been synthesised, but their NMR spectra are rarely reported, because they tend to give broad unresolved resonances. ${ }^{149,150}$ NMR measurements on paramagnetic compounds are often uninformative since the unpaired electron causes rapid relaxation, resulting in broad signals. ${ }^{151}$ However, the heterometallated porphyrin oligomers used in this study are large enough to give informative ${ }^{1} \mathrm{H}$ NMR spectra, and the effect of the paramagnetic centre provides extra information. Protons close to the copper centre give extremely broad peaks, whereas those further away from the paramagnetic centre are well resolved. 
The aromatic regions of the ${ }^{1} \mathrm{H}$ NMR spectra of the linear trimers containing either a free-base, 3.1, or a copper porphyrin, 3.2, are compared in Figure 3.7. The NMR spectrum of the diamagnetic compound 3.1 shows sharp signals for both the $\beta$-protons (at 8.9-10.0 ppm) and the aryl-protons (at 7.8-8.2 ppm). In the spectrum of the copper-containing trimer, 3.2, the signals for the $\beta$-protons nearest to the copper (g and $\mathrm{h}$ ) are completely unobservable, whereas the signals further removed from the copper $(a-d)$ are well resolved.
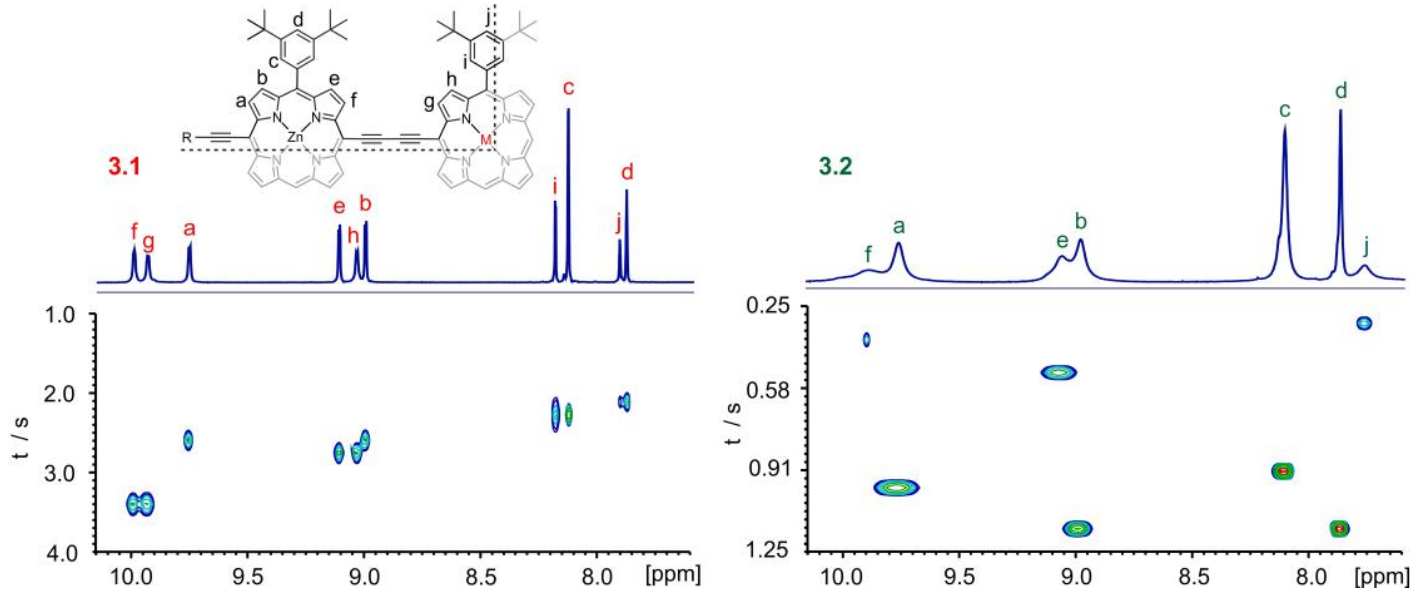

Figure 3.7: ${ }^{1} \mathrm{H}$ NMR spectra $\left(\mathrm{CDCl}_{3}, 700 \mathrm{MHz}, 298 \mathrm{~K}\right)$ of 3.1 (left) and 3.2 (right) and the general assignment $(\mathrm{M}=2 \mathrm{H}$ or $\mathrm{Cu})$. Only the aromatic regions of the spectra are shown. The graphs below the spectra correlate the chemical shift and the corresponding $T_{1}$ relaxation time of each proton signal.

The deterioration observed in the ${ }^{1} \mathrm{H}$ NMR spectrum of $\mathbf{3 . 2}$ is a direct result of the presence of the paramagnetic copper centre and can be attributed to shortening of the $T_{1}$ and $T_{2}$ relaxation time constants. Values of $T_{1}$ and $T_{2}$ were determined for $\mathbf{3 . 2}$ and 3.1 using the inversion-recovery and Carr-Purcell-Meiboom-Gill (CPMG) sequences, respectively. ${ }^{152}$ As expected, analysis of the data for the signals a-j in $\mathbf{3 . 1}$ shows little variation; the $T_{1}$ times are in the range $2-3$ seconds (Figure 3.7, left) and the $T_{2}$ times are $0.05-0.78$ seconds (see Section 3.6.3). These values fall in the range expected for molecules of this size and reflect the local environment of the protons within the molecule. As a result of the paramagnetic copper centre, $\mathbf{3 . 2}$ has considerably shorter relaxation times; $T_{1}=0.10-1.12$ seconds (Figure 3.7 , right) and $T_{2}=0.005-0.083$ seconds for signals $a-j$ (see Section 3.6.3). Although many factors contribute to relaxation rates, a clear trend can be observed when comparing oligomers with and without copper. The relaxation rates are considerably faster for 3.2 than for $\mathbf{3 . 1}$ indicating that all protons are affected by the copper centre. 
Additionally, the relaxation rates are noticeably greater for the signals in closer spatial proximity to the copper centre in $\mathbf{3 . 2}$, allowing us to estimate the relative distances between the protons and the copper centre based on their relaxation times.

The difference in relaxation rate $R_{1}$ between protons in $\mathbf{3 . 2}$ and $\mathbf{3 . 1}$ can be defined as $\Delta R_{1}$ according to equation 3.1 .

$$
\Delta R_{1}=\frac{1}{T_{1}(3.2)}-\frac{1}{T_{1}(3.1)}
$$

Dipolar relaxation rates are expected to be proportional to the inverse $6^{\text {th }}$ power of the distance between two magnetic dipoles as shown in equation 3.2. ${ }^{153-155}$

$$
\Delta R_{1} \propto \frac{1}{r^{6}}
$$

Figure 3.8 shows a plot of the experimental values of $\Delta R_{1}$ against the distance between the copper centre and the corresponding protons $(r)$, estimated from crystal structures of similar oligomers. ${ }^{134}$ The good fit to equation (3.2) confirms the expected distance dependence and shows that changes in $T_{1}$ relaxation rate can be used to gain structural information.

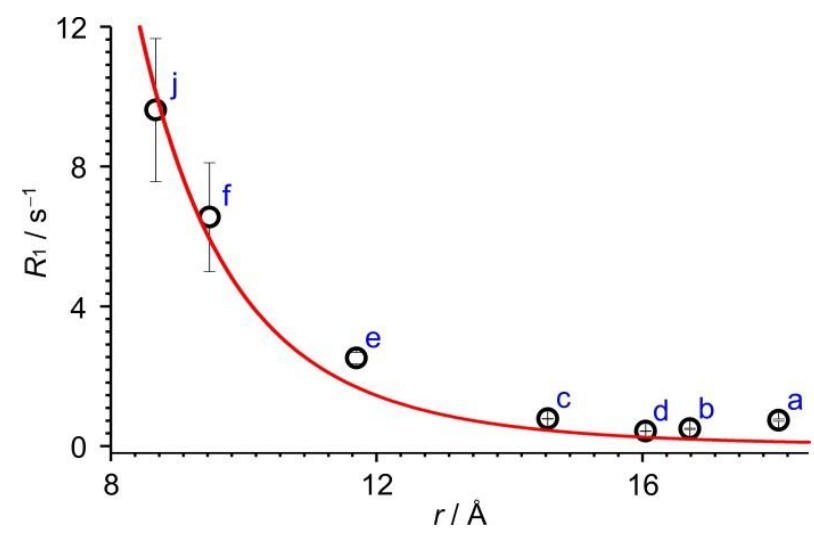

Figure 3.8: Graph depicting the change in $R_{1}$ relaxation rates $\left(R_{1}=1 / T_{1}\right)$ between 3.2 and 3.1 with respect to the distance between the two nuclei, with a fit for $\Delta R_{1} \propto r^{-6}$. The experiment error in $\Delta R_{1}$ is only evident when $T_{1}$ is short (signals $j$ and $f$ ).

The NMR spectra of the porphyrin pentamer 2.12 reported in Chapter 2 are more complicated than those of $\mathbf{3 . 2}$, because there are twice as many zinc porphyrin environments. Protons further from the copper centre give rise to sharp signals, but many of the signals overlap. 


\subsubsection{Quantification of the constructive interference}

Solutions of the complexes depicted in Figure 3.2 were prepared in toluene and the formation of the complexes was confirmed by UV-vis-NIR titrations. The ring c-P6 $\mathbf{C u 2}_{\mathbf{2}} \cdot \mathbf{T 6}$ was isolated with the T6 template complexed in the cavity. Porphyrin 6-rings have been reported to form extremely stable complexes with T6 $\left(K_{\mathrm{f}}=7 \times 10^{38} \mathrm{M}^{-1}\right)$ and we therefore expect that a one-to-one complex with T6 will be formed despite the presence of copper. ${ }^{23,38}$ The formation of I-P6 $\mathbf{C u 2}_{\mathbf{C u 2}} \cdot \mathbf{T 6}$ was confirmed by UV-vis-NIR titrations and revealed the formation of a highly stable complex with a formation constant exceeding $10^{8} \mathrm{M}^{-1}$ (Figure 3.9). In principle, denaturation titrations can be performed to reveal the exact formation constant of the complex. Since we are solely interested in knowing if a complex has formed, which can be concluded from the formation curve, we did not investigate this any further.
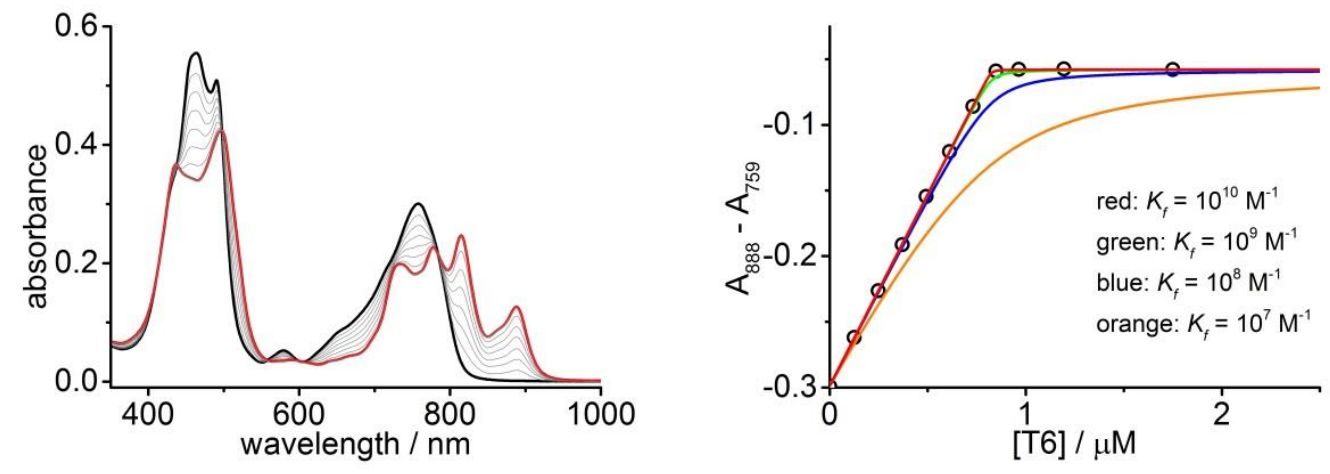

Figure 3.9: UV-vis-NIR titration of $I-P 6_{\mathrm{Cu} 2}$ and T6. (Toluene, $\left.298 \mathrm{~K},\left[I-\mathrm{P} 6_{\mathrm{Cu} 2}\right]=0.78 \mu \mathrm{M}\right)$. On the right an evaluation of the magnitude of the binding constant is shown. Fits to the experimental data are shown for four (fixed) values of the binding constant $K_{\mathrm{f}}$. The other parameters entering the simulation were allowed to vary.

The formation of $\left(I-P 3_{\mathrm{Cu}}\right)_{2} \cdot \mathrm{T} 6$ was confirmed by UV-Vis-NIR titrations (Figure 3.10$)$. This titration is comparable to the measurements shown in Figure 3.4 except that the aryls of the porphyrins are different and that the solvent is changed to toluene which results in a slightly stronger binding. While the formation constant is still small, EPR measurements are carried out at a considerably higher concentration (ca. $200 \mu \mathrm{M})$ than the UV-vis-NIR titrations (ca. $5 \mu \mathrm{M})$. The formation constant of $4.7 \times 10^{5} \mathrm{M}^{-1}$ implies that at EPR concentrations of $0.2 \mathrm{mM}$ the mole fraction of the two-to-one complex will be approximately 0.8 in toluene at $298 \mathrm{~K}$. 

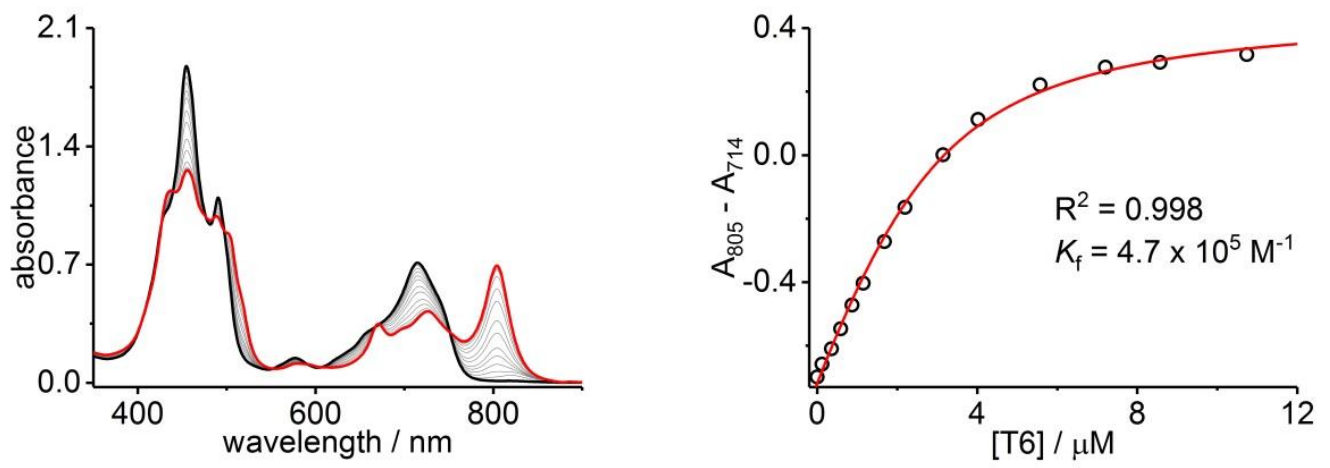

Figure 3.10: UV-vis-NIR titrations of $I-P 3_{C u}$ and $T 6, R^{2}=0.998$. (Toluene, $298 \mathrm{~K},\left[/-\mathrm{P} 3_{\mathrm{Cu}}\right]=5.28 \mu \mathrm{M}$, $K=4.7 \times 10^{5} \mathrm{M}^{-1}$ ).

We now focus on the quantification of the interspin exchange coupling $(J)$ between the two copper centres which reports on wavefunction overlap and interference phenomena. Large exchange couplings $\left(\gg>1 \mathrm{~cm}^{-1}\right.$ ) are commonly measured using SQUID, ${ }^{156,157}$ whereas EPR can be used to determine $J$ values in the sub-cm ${ }^{-1}$ regime. In addition to the through-bond coupling $J$, the unpaired electrons can also couple through space via dipolar interactions $(D)$. Since the distance between the copper centres is the same in all three structures shown in Figure $3.2, D$ is identical for all three structures.

With this information in hand, we continued to employ the technique of double electron-electron resonance (DEER) to quantify and compare the exchange interaction in the structures $\mathbf{c}-\mathbf{P} \mathbf{6}_{\mathrm{Cu2}} \cdot \mathbf{T} \mathbf{6}$ and $\mathbf{I}-\mathbf{P} \mathbf{6}_{\mathrm{Cu2}} \cdot \mathbf{T 6}$. DEER is usually employed to elucidate molecular structures and their flexibility via the determination of interspin distances and their distance distributions. The latter are extracted from modulations in the DEER traces presumed to be solely due to the dipolar interaction, which, for the typically addressed interspin distances $(1.5-8 \mathrm{~nm})$, much exceed the magnitude of the exchange interaction. ${ }^{106,158,159}$ However, in highly $\pi$-conjugated systems such as ours, the two couplings might be of comparable magnitude and their respective contributions to the DEER time trace need to be disentangled. To determine $J$ from the experimental DEER data, we needed to disentangle dependence of the dipolar evolution (DEER) time trace which contains contributions from both $D$ and $J$. It was found that to a first approximation the experimental signal oscillates with a frequency approximately corresponding to $D+J .{ }^{160}$ 
Measurements were commenced on $\left(I-P 3_{\mathrm{Cu}}\right)_{2} \cdot \mathbf{T 6}$. This system contains no exchange coupling pathways between the copper centres since there is no conjugated through-bond pathway between the copper centres, and allows for the quantification of the dipolar coupling $(D)$. Figure 3.11 (left) shows the experimental background-corrected DEER time trace recorded for $\left(I-\mathbf{P} \mathbf{C}_{\mathrm{Cu}}\right)_{2} \cdot \mathbf{T 6}$. The frequency of the pronounced dipolar modulation corresponds to a distance of roughly $2.5 \mathrm{~nm}$ as determined by Fourier transformation of the time trace. This result is in good agreement with published X-ray crystallography and density functional theory (DFT) data for a similar template-bound six-membered porphyrin ring for which a ring diameter of $2.5 \mathrm{~nm}$ was determined. ${ }^{125,136}$
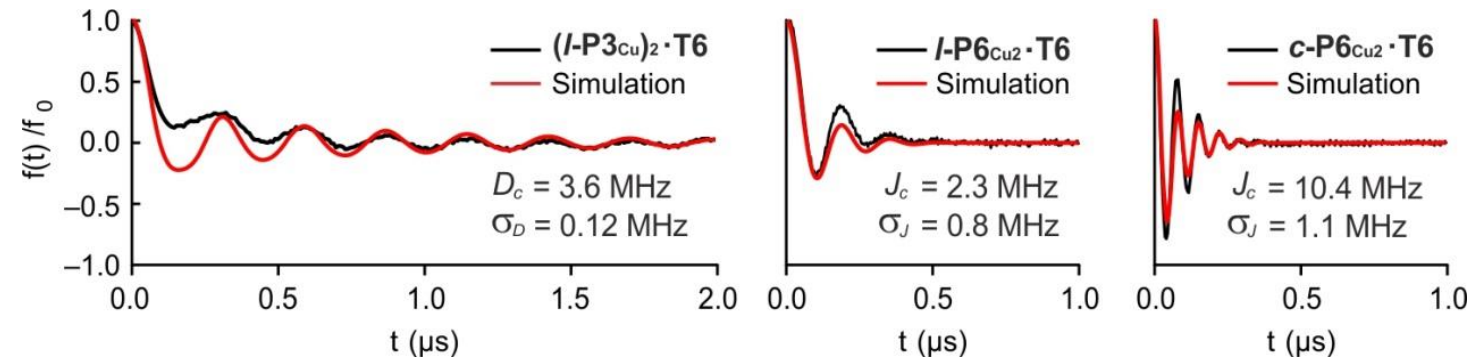

Figure 3.11: Experimental and simulated DEER data. (black) Experimental background-corrected DEER traces of $\left(I-P 3_{\mathrm{Cu}}\right)_{2} \cdot \mathrm{T6}, \quad I-\mathrm{P} 6_{\mathrm{Cu} 2} \cdot \mathrm{T} 6$ and $c-\mathrm{P} 6_{\mathrm{Cu} 2} \cdot \mathrm{T} 6$. (red) Simulations of the individual experimental traces with the simulation parameters indicated in the graphs.

A first inspection of the DEER trace for the two exchange coupled systems $c$-P6 $\mathbf{C u 2}_{\mathrm{Cu}} \cdot \mathbf{T 6}$ and $\mathbf{I}-\mathbf{P} \mathbf{G}_{\mathrm{Cu2}} \cdot \mathbf{T 6}$ in Figure 3.11 (centre and right, respectively) reveals that the introduction of through-bond coupling pathways leads to a significant increase in the modulation frequency. Fourier transformation of the traces further establishes the dominant frequency components $I-\mathbf{P} 6_{\mathrm{Cu2}} \cdot \mathbf{T 6}$ as $5.2 \mathrm{MHz}$ and for $\mathbf{c}-\mathbf{P} \mathbf{6}_{\mathrm{Cu} 2} \cdot \mathbf{T} 6$ as $13 \mathrm{MHz}$, providing first proof that the exchange interaction has pronounced but distinct effects in the two coupled systems. To quantify the magnitude of $J$, the background-corrected experimental data were simulated and compared (Figure 3.11). Although the relative modulation amplitudes cannot be reproduced exactly, partially due to uncertainties in the DEER background correction, the simulations can be considered satisfactory since the modulation frequencies and the dampening of the oscillations, which contain all the relevant information for our purposes, can be reproduced accurately and determined with high precision. 
The centres and widths of the frequency distributions in $J$ and $D$, obtained from the simulations, are indicated in the corresponding graphs in Figure 3.11. First, the distribution in $D$ was determined using the $\left(I-\mathbf{P 3}_{\mathbf{C u}_{2}}\right)_{2} \cdot \mathbf{T 6}$ sample with two breaks in the conjugation. The resulting distribution then served as an input into the simulations of the $I-\mathbf{P} \mathbf{6}_{\mathrm{Cu} 2} \cdot \mathbf{T 6}$ and $\left(I-\mathbf{P} \mathbf{3}_{\mathrm{Cu}}\right)_{2} \cdot \mathrm{T} 6$ DEER traces in which, consequently, only the centre and width of the distribution in $J$ were adapted. The distribution in $D$ was found to be relatively narrow corresponding to a width of $\sigma=0.12 \mathrm{MHz}$, whereas the distributions in $J$ are generally found to be much wider. The centre frequency of 3.6 $\mathrm{MHz}$ determined for $D$ corresponds to a $\mathrm{Cu}$... Cu distance of $2.47 \mathrm{~nm}$ which is in excellent agreement with expectations from available experimental data and DFT calculations. $^{125,136}$

For $\boldsymbol{c}-\mathbf{P} \mathbf{6}_{\mathrm{Cu} 2} \cdot \mathbf{T 6}$, an exchange coupling of $10.4 \mathrm{MHz}\left(\sigma_{J}=1.1 \mathrm{MHz}\right)$ could be determined from the simulation. The magnitude of the exchange interaction between the two copper centres is remarkable given the large through-bond interspin distance of $3.9 \mathrm{~nm}$, which is a consequence of the high degree of conjugation between the paramagnetic centres. ${ }^{161}$ Interestingly, the interruption of one coupling path in I-P6 cu2 $\cdot \mathbf{T 6}$ leads to attenuation of $J$ to $2.3 \mathrm{MHz}$.

We observe an approximately fourfold increase in the size of the exchange coupling in the two-path structure as compared to the one-path case. This result is in excellent agreement with the prediction of a fourfold increase in the transmission of a closed system with two parallel branches as compared to a single linear chain under conditions of constructive quantum interference. ${ }^{78}$ Although quantum interference has been observed previously in two-path systems by conductance measurements, ${ }^{81,145}$ this is the first time that it has been detected via exchange coupling.

\subsection{Conclusion}

In this chapter we have described the synthesis of the first heterometallated 6-porphyrin nanoring $\mathbf{c - P 6} \mathbf{C u 2}_{\mathbf{C}} \cdot \mathbf{T} 6$ using the hexadentate template $\mathbf{T 6}$ and mixed metal linear oligomers. The binding interaction between the starting materials was found to be relatively weak as a result of the central copper centre. As a result, the final 
nanoring structure could only be isolated in $2 \%$ yield and polymerisation dominated the last reaction step.

${ }^{1} \mathrm{H}$ NMR spectroscopy can be used to gain structural information on porphyrin oligomers containing paramagnetic copper(II) centres. While the signals corresponding to protons in close proximity to the copper are broadened to the extent that they can no longer be observed, protons further from the copper are well resolved. The changes in proton relaxation rate constants $\left(R_{1}=1 / T_{1}\right)$ due to the presence of the copper centre depend on the inverse $6^{\text {th }}$ power to the distance, providing information on the molecular geometry.

The nanoring $\boldsymbol{c}-\mathbf{P} \mathbf{6}_{\mathbf{C u 2} \cdot} \cdot \mathbf{T 6}$ was used in DEER measurements designed to probe the through-bond $(J)$ interaction between the copper centres. A remarkably high exchange coupling of $10.4 \mathrm{MHz}$ was observed. When one of the coupling paths between the copper centres was broken, the exchange coupling was found to drop to $2.3 \mathrm{MHz}$. This result is in excellent agreement with the prediction of a fourfold increase in the transmission of a closed system with two parallel branches as compared to a single linear chain under conditions of constructive quantum interference.

The major advantage of employing DEER is that the data allow the direct quantification of the exchange coupling between the spins, alleviating the need for any connecting electrodes. Our study does not report on conductance phenomena directly but rather addresses quantum interference via the exchange interaction between the two paramagnetic centres. However, it has been shown previously that conductance and antiferromagnetic coupling exhibit strongly correlated trends ${ }^{162,163}$ so that the fourfold increase in the exchange coupling by introduction of a second, parallel path is consistent with the prediction based on transmission considerations.

These results provide an unambiguous demonstration of constructive quantum interference in a closed model system with two identical parallel paths. They illustrate the applicability of DEER for measuring long-range exchange couplings in molecular wires and indicate that efficient long-range electronic communication is favoured by a highly rigid molecular framework. 


\subsection{Experimental procedures and technical details}

\subsubsection{General experimental}

Dry toluene and THF were obtained by passing the solvents through columns of alumina, under nitrogen. Diisopropylamine $\left(i-\mathrm{Pr}_{2} \mathrm{NH}\right)$ was distilled from $\mathrm{CaH}_{2}$ and kept over activated molecular sieves ( $3 \AA, 8-12$ mesh). Unless specified otherwise, all other solvents were used as commercially supplied. Flash chromatography was carried out on silica gel 60 under positive pressure. Analytical thin-layer chromatography was carried out on aluminum-backed silica gel 60 F254 plates. Visualisation was achieved using UV light when necessary.

All UV-vis-NIR spectra were recorded in solution using a Perkin-Lambda 20 spectrometer ( $1 \mathrm{~cm}$ path length quartz cell). Chloroform (containing $c a .0 .5 \%$ ethanol as stabiliser) or toluene was used for all titrations without any further purification.

Unless stated otherwise, ${ }^{1} \mathrm{H} /{ }^{13} \mathrm{C}$ NMR spectra were recorded at $298 \mathrm{~K}$ using a Bruker AV400 (400/100 MHz) or Bruker AV700 (700/175 MHz) instrument. ${ }^{1} \mathrm{H}$, and ${ }^{13} \mathrm{C} N M R$ spectra are reported in ppm; coupling constants are given in Hertz, to the nearest $0.1 \mathrm{~Hz}$. The solvent used was $\mathrm{CDCl}_{3}$.

MALDI-ToF spectra were measured at the EPSRC National Mass Spectrometry service (Swansea) using the Applied Biosystems Voyager DE-STR or at the University of Oxford using Waters MALDI Micro MX spectrometer utilising dithranol as a matrix.

\subsection{Synthetic procedures}

Trimer (3.1)

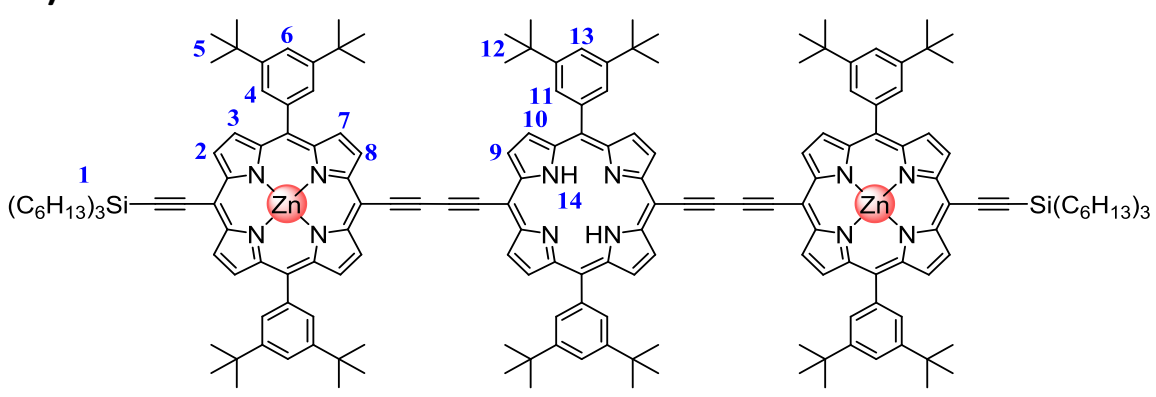

Porphyrin 2.9 (10.0 mg, $0.013 \mathrm{mmol})$, porphyrin $2.6(73.5 \mathrm{mg}, 0.068 \mathrm{mmol}), \mathrm{Pd}_{2}\left(\mathrm{dba}_{3}\right.$ (4.36 mg, $0.0047 \mathrm{mmol})$, tri-2-furylphosphine $(8.84 \mathrm{mg}, 0.038 \mathrm{mmol}$ ) and 1,4-benzoquinone (13.2 $\mathrm{mg}, 0.122 \mathrm{mmol})$ were dissolved in a mixture of toluene : $\mathrm{Et}_{3} \mathrm{~N}(5: 1)(16 \mathrm{~mL})$. The reaction mixture was stirred at $60^{\circ} \mathrm{C}$ overnight. The 
solvents were removed and the mixture was purified over a plug of silica gel (1\% pyridine in $\mathrm{CHCl}_{3}$ ). A small SEC column (1\% pyridine in $\mathrm{CHCl}_{3}$ ) was used to remove the 1,4-benzoquinone. A large SEC column (1\% pyridine in toluene) was used for separation of the trimer species (15.4 $\mathrm{mg}, 39 \%)$.

${ }^{1} \mathrm{H}$ NMR $\left(700 \mathrm{MHz}, \mathrm{CDCl}_{3}, 298 \mathrm{~K}\right): \delta_{\mathrm{H}}(\mathrm{ppm}) 9.89(4 \mathrm{H}, \mathrm{d}, J=4.5 \mathrm{~Hz}, \mathrm{H} 8), 9.88(4 \mathrm{H}, \mathrm{d}$, $J=4.5 \mathrm{~Hz}, \mathrm{H9}), 9.66(4 \mathrm{H}, \mathrm{d}, J=4.5 \mathrm{~Hz}, \mathrm{H} 2), 9.00(4 \mathrm{H}, \mathrm{d}, J=4.6 \mathrm{~Hz}, \mathrm{H} 7), 8.98(4 \mathrm{H}, \mathrm{d}$, $J=4.7 \mathrm{~Hz}, \mathrm{H} 10), 8.89(4 \mathrm{H}, \mathrm{d}, J=4.5 \mathrm{~Hz}, \mathrm{H} 3), 8.14(4 \mathrm{H}, \mathrm{d}, J=1.8 \mathrm{~Hz}, \mathrm{H} 11), 8.05(8 \mathrm{H}, \mathrm{d}$ $J=1.8 \mathrm{~Hz}, \mathrm{H} 4), 7.87(2 \mathrm{H}, \mathrm{t}, J=1.8 \mathrm{~Hz}, \mathrm{H} 13), 7.81(4 \mathrm{H}, \mathrm{t}, J=1.8 \mathrm{~Hz}, \mathrm{H6}), 1.77-0.90(78 \mathrm{H}$, $m, H 1), 1.59(36 \mathrm{H}, \mathrm{s}, \mathrm{H} 12), 1.56(72 \mathrm{H}, \mathrm{s}, \mathrm{H} 5),-1.51$ (2H, s, H14).

${ }^{13} \mathrm{C}$ NMR $\left(100 \mathrm{MHz} \mathrm{CDCl}_{3}, 298 \mathrm{~K}\right): \delta_{\mathrm{C}}$ (ppm) 153.1, 152.4, 151.0, 150.5, 149.3, 149.1, $141.2,133.7,133.2$, 131.5, 131.0, 130.2, 129.9, 124.8, 124.4, 121.7, 121.3, 35.3, 35.3, $33.5,32.0,31.9,24.5,22.9,14.3,14.0$.

MALDI-TOF: $m / z=2,893\left(\mathrm{C}_{192} \mathrm{H}_{230} \mathrm{~N}_{12} \mathrm{Si}_{2} \mathrm{Zn}_{2}, \mathrm{M}^{+}\right.$requires 2,894).

$\lambda_{\text {max }}\left(\mathrm{CHCl}_{3}\right) / \mathrm{nm} \log (\varepsilon): 752$ (5.15), 661 (4.96), 485 (5.29), 455 (5.70).

Trimer 3.2

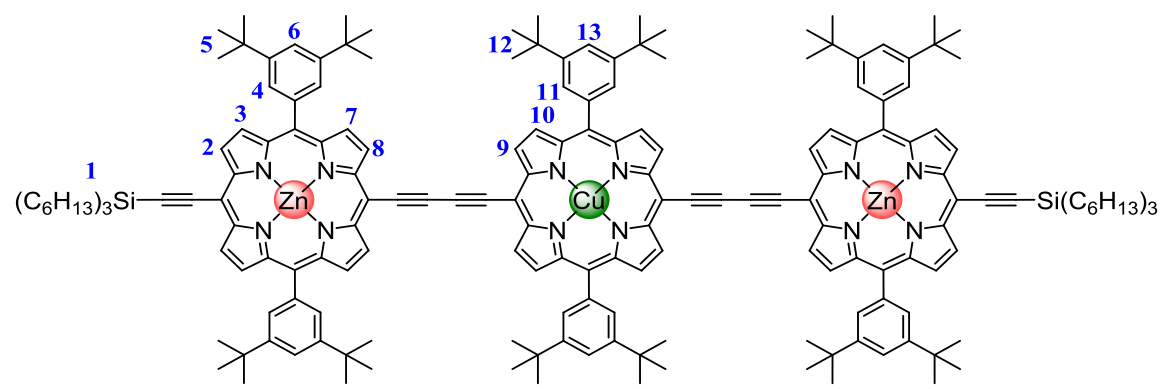

Trimer 3.1 (4.5 mg, $1.6 \mu \mathrm{mol})$ was dissolved in $\mathrm{CHCl}_{3}(4 \mathrm{~mL}) . \mathrm{Cu}(\mathrm{OAc})_{2} \cdot \mathrm{H}_{2} \mathrm{O}(12.2 \mathrm{mg}$, $67 \mathrm{mmol}$ ) was added as a solid and the reaction was stirred at $60^{\circ} \mathrm{C}$ for $1 \mathrm{~h}$. The reaction mixture was allowed to cool down to room temperature after which methanol was added to the reaction mixture to crash out the product. The precipitate was filtered and washed with methanol to give a red/brown solid $(4.8 \mathrm{mg}$, $100 \%)$.

${ }^{1} \mathrm{H}$ NMR (700 MHz, $\left.\mathrm{CDCl}_{3}, 298 \mathrm{~K}\right): \delta_{\mathrm{H}}$ (ppm) $9.87(4 \mathrm{H}, \mathrm{m}, \mathrm{H} 8), 9.74(4 \mathrm{H}, \mathrm{m}, \mathrm{H} 2), 9.05$ (4H, m, H7), $8.96(4 \mathrm{H}, \mathrm{m}, \mathrm{H} 3), 8.09(8 \mathrm{H}, \mathrm{m}, \mathrm{H} 4), 7.86(4 \mathrm{H}, \mathrm{m}, \mathrm{H} 6), 7.75(2 \mathrm{H}, \mathrm{m}, \mathrm{H} 13)$, $1.59(108 \mathrm{H}, \mathrm{m}, \mathrm{H} 5$ and $\mathrm{H} 12), 1.89-0.80(78 \mathrm{H}, \mathrm{m}, \mathrm{H} 1)$.

MALDI-TOF: $m / z=2,953\left(\mathrm{C}_{192} \mathrm{H}_{228} \mathrm{CuN}_{12} \mathrm{Si}_{2} \mathrm{Zn}_{2}, \mathrm{M}^{+}\right.$requires 2,954$)$. 
$\lambda_{\text {max }}\left(\mathrm{CHCl}_{3}\right) / \mathrm{nm} \log (\varepsilon): 726$ (5.20), 666 (4.89), 486 (5.37), 454 (5.65).

Trimer 3.3

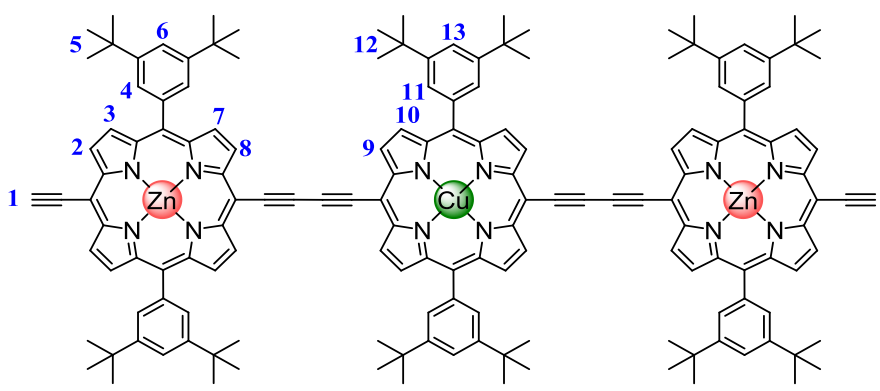

Trimer $3.2(25 \mathrm{mg}, 8.5 \mu \mathrm{mol})$ was dissolved in $\mathrm{CH}_{2} \mathrm{Cl}_{2}(10 \mathrm{~mL})$ and pyridine $(10 \mu \mathrm{L})$. Tetra-n-butylammonium fluoride $(170 \mu \mathrm{L}, 1.0 \mathrm{M}$ solution in THF, $170 \mu \mathrm{mol})$ was added and the reaction mixture was stirred at room temperature for $15 \mathrm{~min}$. The reaction mixture was immediately passed through a short plug of silica gel (1\% pyridine in $\mathrm{CHCl}_{3}$ ) to give the title compound as a brown powder (19 $\mathrm{mg}, 94 \%$ ) which was immediately used in the next reaction.

c-P6 Cu2 $\cdot \mathrm{T6}$

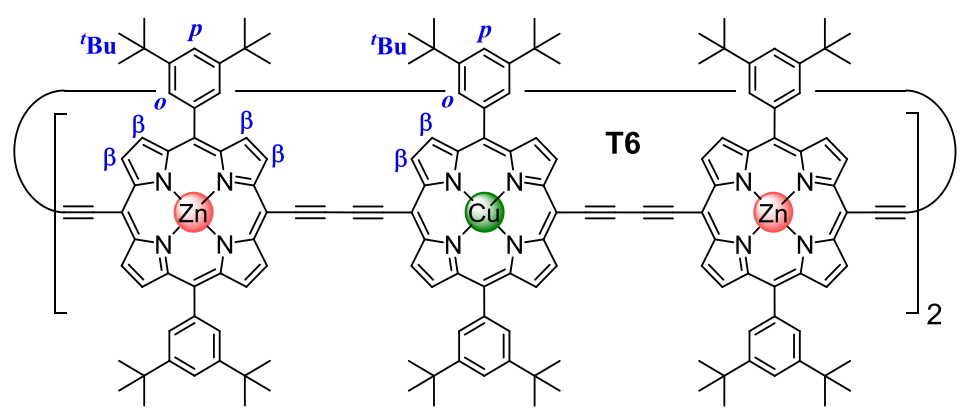

Hexadentate template T6 (81 mg, $81.6 \mu \mathrm{mol})$ and $3.3(65 \mathrm{mg}, 27.2 \mu \mathrm{mol}$ ) were dissolved in $150 \mathrm{~mL} \mathrm{CHCl}$. A solution of $\mathrm{PdCl}_{2}\left(\mathrm{PPh}_{3}\right)_{2}(9.5 \mathrm{mg}, 13.6 \mu \mathrm{mol})$, Cul (9.5 mg, $50.3 \mu \mathrm{mol})$, 1,4-benzoquinone $(29.4 \mathrm{mg}, 272 \mu \mathrm{mol})$ in $\mathrm{CHCl}_{3}(10 \mathrm{~mL})$ and $i-\mathrm{Pr}_{2} \mathrm{NH}(0.5 \mathrm{~mL})$ was added to the porphyrin solution and stirred at room temperature overnight. The reaction mixture was passed through a plug of alumina using $\mathrm{CHCl}_{3}$ as eluent. The solvent was evaporated and redissolved in a minimum amount of $1 \%$ pyridine in $\mathrm{CHCl}_{3}$ and passed over a SEC column (1\% pyridine in $\mathrm{CHCl}_{3}$ ). Lastly, the ring was purified by recycling GPC (1\% pyridine in toluene) to give the title compound (1.8 mg, 2\%). 
${ }^{1} \mathrm{H}$ NMR $\left(600 \mathrm{MHz}, \mathrm{CDCl}_{3}, 298 \mathrm{~K}\right): \delta_{\mathrm{H}}(\mathrm{ppm})$ 9.71-9.31 (m, $\left.\beta\right), 8.93-8.68(\mathrm{~m}, \beta), 8.05(\mathrm{~s}$, ortho), 7.83 (s, para), 7.78 (s, para), 5.77 (m, T6), 5.68 (m, T6), 5.14 (m, T6), 2.34 (m, T6), 2.02 (m, T6), 1.59 (s, $\left.{ }^{t} \mathrm{Bu}\right)$.

MALDI-TOF: $m / z=4,787\left(\mathrm{C}_{320} \mathrm{H}_{300} \mathrm{Cu}_{2} \mathrm{~N}_{24} \mathrm{Zn}_{4}, \mathrm{M}^{+}\right.$requires 4,774). $\lambda_{\text {max }}\left(\mathrm{CHCl}_{3}\right) / \mathrm{nm} \log (\varepsilon): 480$ (5.67), 761 (5.47), 797 (5.58), 838 (5.55).

\section{Zinc 5,15-bis(3,5-bis(trihexylsilyl)phenyl)porphyrin (3.4) ${ }^{164}$}

3,5-Bis(trihexylsilyl)benzaldehyde $(9.20 \mathrm{~g}, 13.7 \mathrm{mmol})$ and 2,2'-dipyrromethane 2.1 ( $2.00 \mathrm{~g}, 13.7 \mathrm{mmol})$ were dissolved in $\mathrm{CH}_{2} \mathrm{Cl}_{2}(2,500 \mathrm{~mL})$. The solution was saturated with nitrogen by bubbling nitrogen through the solution for $30 \mathrm{~min}$, then TFA (2.1 $\mathrm{mL}, 27.4 \mathrm{mmol}$ ) was added and the mixture was stirred for

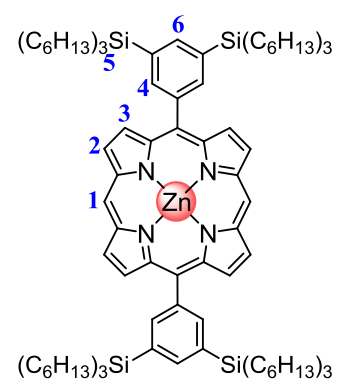
$3 \mathrm{~h}$ in the dark. DDQ (4.66 g, $20.5 \mathrm{mmol})$ was added and the mixture was stirred for a further $20 \mathrm{~min}$. Finally, triethylamine $(13.6 \mathrm{~mL})$ was added to quench the acid. The solution was concentrated and passed through a short silica plug eluting with a mixture of petroleum ether $40-60$ and $\mathrm{CH}_{2} \mathrm{Cl}_{2}(80: 20)$. The solvent was removed under reduced pressure to give the free base porphyrin as a dark pink oil. The oil was dissolved in $\mathrm{CHCl}_{3}(500 \mathrm{~mL})$ and a solution of $\mathrm{Zn}(\mathrm{OAc})_{2} \cdot 2 \mathrm{H}_{2} \mathrm{O}(6.80 \mathrm{~g}, 30.8 \mathrm{mmol})$ in $\mathrm{MeOH}(75 \mathrm{~mL})$ was added. The mixture was stirred at $40{ }^{\circ} \mathrm{C}$ for $90 \mathrm{~min}$ and monitored by TLC. The solution was evaporated and passed through a short silica plug, eluting with a mixture of petroleum ether $40-60, \mathrm{CH}_{2} \mathrm{Cl}_{2}$ and pyridine $(80: 20: 1 \mathrm{v} / \mathrm{v} / \mathrm{v})$. The solvent was removed to give the title compound as a dark pink oil (9.27 g, 91\%).

${ }^{1} \mathrm{H}$ NMR $\left(400 \mathrm{MHz}, \mathrm{CDCl}_{3}\right) \delta_{\mathrm{H}}=10.21(2 \mathrm{H}, \mathrm{s}, \mathrm{H} 1), 9.36(4 \mathrm{H}, \mathrm{d}, J=4.4 \mathrm{~Hz}, \mathrm{H} 2), 9.07(4 \mathrm{H}$, d, $J=4.4 \mathrm{~Hz}, \mathrm{H} 3), 8.32(4 \mathrm{H}, \mathrm{bd}, J=1.04 \mathrm{~Hz}, \mathrm{H} 4), 7.98(2 \mathrm{H}, \mathrm{bt}, \mathrm{H} 7), 1.63-0.77(156 \mathrm{H}, \mathrm{m}$, $\mathrm{H} 5)$. 


\section{Zinc 5,15-bis(3,5-bis(trihexylsilyl)phenyl)-10,20-dibromoporphyrin (3.5) ${ }^{164}$}

To a stirred solution of porphyrin $3.4(2.95 \mathrm{~g}, 1.78 \mathrm{mmol})$ in $\mathrm{CHCl}_{3}(275 \mathrm{~mL})$ and pyridine $(1.25 \mathrm{~mL})$ was added $\mathrm{N}$-bromosuccinimide $(0.63 \mathrm{~g}, 3.56 \mathrm{mmol})$. The reaction mixture was stirred for $15 \mathrm{~min}$ after which acetone $(2 \mathrm{~mL})$ was added to quench any excess $N$-bromosuccinimide. The solution was

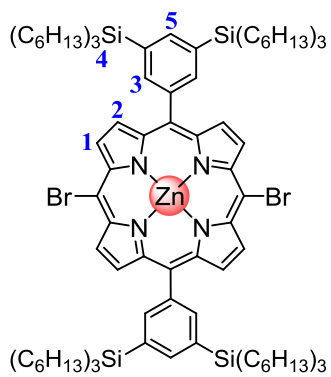
passed through a short silica plug eluting with a mixture of petroleum ether 40-60, $\mathrm{CH}_{2} \mathrm{Cl}_{2}$ and pyridine $(80: 20: 1 \mathrm{v} / \mathrm{v} / \mathrm{v})$. The solvent was removed to give the title compound as a green/purple oil $(2.79 \mathrm{~g}, 86.5 \%)$.

${ }^{1} \mathrm{H}$ NMR $\left(400 \mathrm{MHz}, \mathrm{CDCl}_{3}\right) \delta_{\mathrm{H}}=9.36(4 \mathrm{H}, \mathrm{d}, J=4.7 \mathrm{~Hz}, \mathrm{H} 1), 8.82(4 \mathrm{H}, \mathrm{d}, J=4.7 \mathrm{~Hz}, \mathrm{H} 2)$, $8.19(4 \mathrm{H}, \mathrm{bd}, \mathrm{H} 3), 7.97(2 \mathrm{H}, \mathrm{bt}, \mathrm{H} 5), 1.55-0.78(156 \mathrm{H}, \mathrm{m}, \mathrm{H} 4)$.

Zinc 5,15-bis(3,5-bis(trihexylsilyl)phenyl)-10,20-bis-trihexylsilanylethynylporphyrin $(3.6)^{164}$

Porphyrin $3.5(0.30 \mathrm{~g}, 0.17 \mathrm{mmol}), \mathrm{Pd}_{2}(\mathrm{dba})_{3}$ (15.1 mg, $0.017 \mathrm{mmol})$, Cul (6.3 mg, $0.033 \mathrm{mmol})$ and $\mathrm{PPh}_{3}(8.7 \mathrm{mg}, 0.033 \mathrm{mmol})$ were dissolved in a mixture of toluene $(7.5 \mathrm{~mL})$ and $i-\operatorname{Pr}_{2} \mathrm{NH}(7.5 \mathrm{~mL})$ and the solution was degassed by three freeze-pump-thaw cycles. Trihexylsilylacetylene $(0.2 \mathrm{~mL}, 0.51 \mathrm{mmol})$ was added by syringe and the mixture was stirred at $50{ }^{\circ} \mathrm{C}$ for $2 \mathrm{~h}$. TLC indicated full conversion. The reaction mixture was filtered over a plug of silica eluting with a mixture of petroleum ether 40-60, $\mathrm{CH}_{2} \mathrm{Cl}_{2}$ and pyridine $(80: 20: 1 \mathrm{v} / \mathrm{v} / \mathrm{v})$. Purification by column chromatography on silica gel eluting with a gradient of petroleum ether 40-60, $\mathrm{CH}_{2} \mathrm{Cl}_{2}$, and pyridine (100:0:1 to 94:6:1 $\mathrm{v} / \mathrm{v} / \mathrm{v}$ ) gave the title compound as a green oil (284 mg, 76\%).

${ }^{1} \mathbf{H}$ NMR $\left(400 \mathrm{MHz}, \mathrm{CDCl}_{3}\right) \delta_{\mathrm{H}}=9.74(4 \mathrm{H}, \mathrm{d}, J=4.7 \mathrm{~Hz}, \mathrm{H} 2), 8.09(4 \mathrm{H}, \mathrm{d}, J=4.7 \mathrm{~Hz}, \mathrm{H} 3)$, $8.25(4 \mathrm{H}, \mathrm{m}, \mathrm{H} 4), 8.00$ (2H, m, H6), 1.82-0.83 (234H, m, H1 and H5). 
Zinc 5,15-bis(3,5-bis(trihexylsilyl)phenyl)-10-trihexylsilanylethynyl-20ethynylporphyrin (3.7)

Porphyrin 3.6 (863 mg, $0.38 \mathrm{mmol}$ ) was dissolved in $\mathrm{CH}_{2} \mathrm{Cl}_{2}(60 \mathrm{~mL}), \mathrm{CHCl}_{3}(30 \mathrm{~mL})$ and pyridine $(7 \mathrm{~mL})$. Tetra- $n$-butylammonium fluoride $(0.57 \mathrm{~mL}, 1.0 \mathrm{M}$ solution in THF, $0.57 \mathrm{mmol}$ ) was added to the stirred solution. The progress of the reaction was monitored by

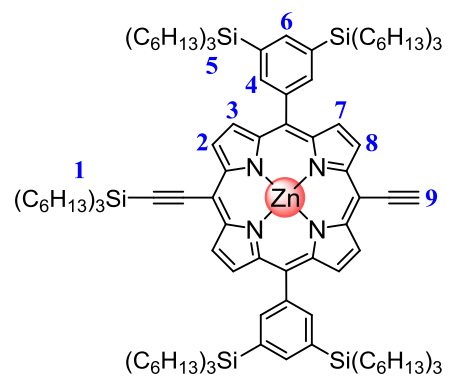
TLC until an optimal product mixture was reached ( $30 \mathrm{~min})$. The mixture immediately was passed through a short plug of silica gel $\left(\mathrm{CHCl}_{3}\right)$. Purification by column chromatography on silica gel eluting with a gradient of petroleum ether 40-60, $\mathrm{CH}_{2} \mathrm{Cl}_{2}$, and pyridine $(10: 1: 0.1 \mathrm{v} / \mathrm{v} / \mathrm{v})$ gave the title compound as a green oil $(0.37 \mathrm{~g}$, $48 \%)$.

${ }^{1} \mathrm{H}$ NMR $\left(400 \mathrm{MHz}, \mathrm{CDCl}_{3}\right) \delta_{\mathrm{H}}=9.73(4 \mathrm{H}, \mathrm{m}, \mathrm{H} 2$ and $\mathrm{H} 8), 8.93(4 \mathrm{H}, \mathrm{m}, \mathrm{H} 3$ and $\mathrm{H} 7), 8.26$ (4H, m, H4), 8.00 (2H, m, H6), 4.18 (1H, s, H9), 1.76-0.84 (195H, m, H1 and H5).

MALDI-TOF: $m / z=1,703\left(\mathrm{C}_{108} \mathrm{H}_{172} \mathrm{~N}_{4} \mathrm{Si}_{4} \mathrm{Zn}, \mathrm{M}^{+}\right.$requires 1,701$)$.

\section{Free-base 5,15-bis(3,5-bis(trihexylsilyl)phenyl)-10,20-bis-} trihexylsilanylethynylporphyrin (3.8)

Porphyrin 3.6 ( $280 \mathrm{mg}, 0.12 \mathrm{mmol}$ ) was dissolved in $\mathrm{CHCl}_{3}(40 \mathrm{~mL})$. Trifluoroacetic acid $(0.47 \mathrm{~mL})$ was mixed with $\mathrm{CHCl}_{3}(5 \mathrm{~mL})$ to give a $10 \%$ solution. The TFA solution was added dropwise to the porphyrin solution and the reaction mixture

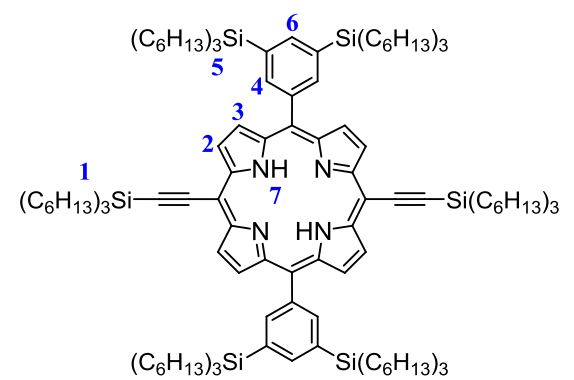
was stirred at room temperature for $15 \mathrm{~min}$. The reaction mixture was passed through a short plug of silica gel $\left(\mathrm{CHCl}_{3}\right)$ to give the title compound as a dark purple oil (260 mg, 96\%).

${ }^{1} \mathrm{H}$ NMR $\left(400 \mathrm{MHz}, \mathrm{CDCl}_{3}, 298 \mathrm{~K}\right): \delta_{\mathrm{H}}(\mathrm{ppm}) 9.61(4 \mathrm{H}, \mathrm{d}, J=4.7 \mathrm{~Hz}, \mathrm{H} 2), 8.78(4 \mathrm{H}, \mathrm{d}$, $J=4.7 \mathrm{~Hz}, \mathrm{H} 3), 8.24(4 \mathrm{H}, \mathrm{m}, \mathrm{H} 4), 8.00(2 \mathrm{H}, \mathrm{m}, \mathrm{H} 6), 1.80-0.82(234 \mathrm{H}, \mathrm{m}, \mathrm{H} 1$ or $H 5),-2.11(2 \mathrm{H}, \mathrm{s}, \mathrm{H} 7)$.

MALDI-TOF: $m / z=2,207\left(\mathrm{C}_{144} \mathrm{H}_{250} \mathrm{~N}_{4} \mathrm{Si}_{6}, \mathrm{M}^{+}\right.$requires 2,206). 
Copper 5,15-bis(3,5-bis(trihexylsilyl)phenyl)-10,20-bis-

trihexylsilanylethynylporphyrin (3.9)

Porphyrin 3.8 (150 mg, $0.068 \mathrm{mmol})$ was

dissolved in $\mathrm{CHCl}_{3}(30 \mathrm{~mL})$. $\mathrm{Cu}(\mathrm{OAc})_{2} \cdot \mathrm{H}_{2} \mathrm{O}$ (494 mg, $2.72 \mathrm{mmol}$ ) was added and the reaction mixture was stirred at $60{ }^{\circ} \mathrm{C}$ for $15 \mathrm{~min}$. The mixture was allowed to cool to room

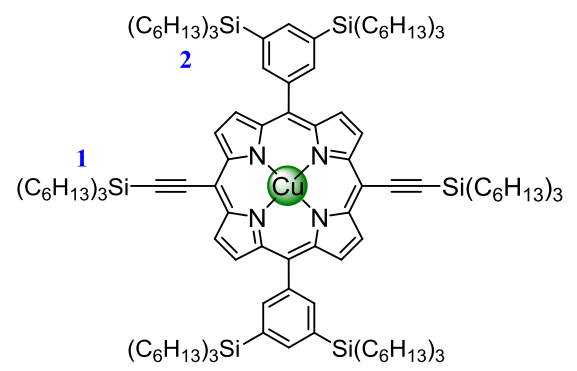
temperature after which it was passed through a short plug of silica gel $\left(\mathrm{CHCl}_{3}\right)$ to give the title compound as a dark green oil (110 mg, 71\%).

${ }^{1} \mathrm{H}$ NMR (400 MHz, $\left.\mathrm{CDCl}_{3}, 298 \mathrm{~K}\right): \delta_{\mathrm{H}}$ (ppm) broad, 1.80-0.61 (234H, m, H1 and H2).

MALDI-TOF: $m / z=2,267\left(\mathrm{C}_{144} \mathrm{H}_{248} \mathrm{CuN}_{4} \mathrm{Si}_{6}, \mathrm{M}^{+}\right.$requires 2,266).

$\lambda_{\text {max }}\left(\mathrm{CHCl}_{3}\right) / \mathrm{nm} \log (\varepsilon): 434$ (5.58), 567 (4.20), 610 (4.53).

\section{Copper 5,15-bis(3,5-bis(trihexylsilyl)phenyl)-10,20-bis-ethynylporphyrin (3.10)}

Porphyrin 3.9 (110 mg, $0.049 \mathrm{mmol}$ ) was dissolved in $\mathrm{CH}_{2} \mathrm{Cl}_{2}$ $(15 \mathrm{~mL})$. Tetra- $n$-butylammonium fluoride $(0.97 \mathrm{~mL}, 1.0 \mathrm{M}$ solution in THF, $0.97 \mathrm{mmol}$ ) was added dropwise and the reaction mixture was stirred at room temperature for $15 \mathrm{~min}$. The reaction mixture was immediately passed through a short

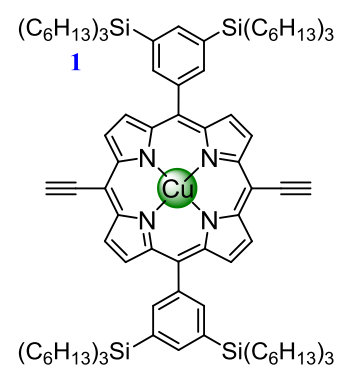
plug of silica gel $\left(\mathrm{CHCl}_{3}\right)$ to give the title compound as a purple oil (80 mg, 97\%).

${ }^{1} \mathrm{H}$ NMR $\left(400 \mathrm{MHz}, \mathrm{CDCl}_{3}, 298 \mathrm{~K}\right): \delta_{\mathrm{H}}(\mathrm{ppm})$ broad, 1.72-0.70 (156H, m, H1).

MALDI-TOF: $m / z=1,702\left(\mathrm{C}_{108} \mathrm{H}_{172} \mathrm{CuN}_{4} \mathrm{Si}_{4}, \mathrm{M}^{+}\right.$requires 1,702$)$.

Trimer (3.11)

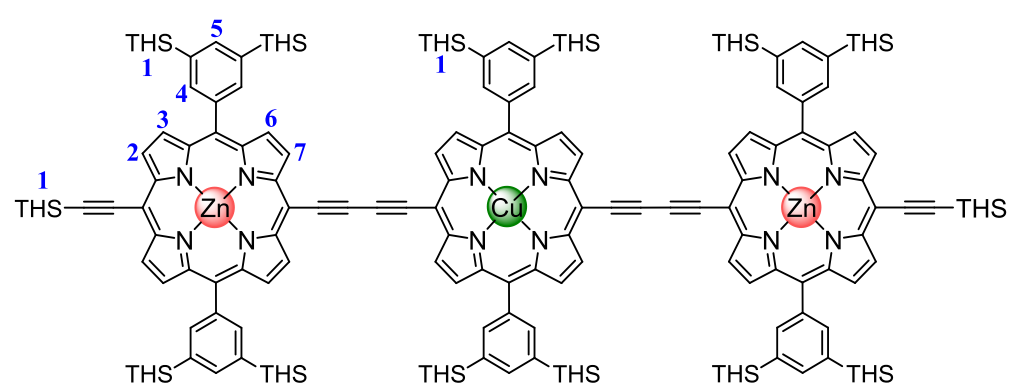

Zinc porphyrin $3.7(410 \mathrm{mg}, 0.21 \mathrm{mmol})$ and copper porphyrin 3.10 (70 mg, $0.04 \mathrm{mmol})$ were dissolved in $\mathrm{CHCl}_{3}(25 \mathrm{~mL})$. A catalyst solution was prepared by dissolving $\mathrm{PdCl}_{2}\left(\mathrm{PPh}_{3}\right)_{2}(21.7 \mathrm{mg}, 0.03 \mathrm{mmol})$, Cul $(21.6 \mathrm{mg}, 0.11 \mathrm{mmol})$ and 
1,4-benzoquinone $(63.8 \mathrm{mg}, 0.59 \mathrm{mmol})$ in $\mathrm{CHCl}_{3}(14 \mathrm{~mL})$ and $i-\mathrm{Pr}_{2} \mathrm{NH}(0.7 \mathrm{~mL})$. The catalyst solution was added to the porphyrin solution and the reaction mixture was stirred at room temperature for $1 \mathrm{~h}$. The crude reaction mixture was passed through a short plug of silica gel ( $1 \%$ pyridine in $\mathrm{CHCl}_{3}$ ), passed over a SEC column (1\% pyridine in $\mathrm{CHCl}_{3}$ ), and finally the oligomers were separated by recycling GPC (1\% pyridine in toluene) to give $\mathbf{3 . 1 1}$ as a dark oil (77.5 $\mathrm{mg}, 34 \%$ ).

${ }^{1} \mathrm{H}$ NMR $\left(400 \mathrm{MHz}, \mathrm{CDCl}_{3}, 298 \mathrm{~K}\right): \delta_{\mathrm{H}}(\mathrm{ppm}) 9.77(4 \mathrm{H}, \mathrm{m}, \mathrm{H} 7), 9.67(4 \mathrm{H}, \mathrm{d}, J=4.3 \mathrm{~Hz}$, $\mathrm{H} 2), 8.91(4 \mathrm{H}, \mathrm{m}, \mathrm{H} 6), 8.84(4 \mathrm{H}, \mathrm{d}, J=4.3 \mathrm{~Hz}, \mathrm{H} 3), 8.25(8 \mathrm{H}, \mathrm{m}, \mathrm{H} 4), 8.00(4 \mathrm{H}, \mathrm{m}, \mathrm{H} 5)$, 1.81-0.77 (546H, m, H1).

MALDI-TOF: $m / z=5,672\left(\mathrm{C}_{360} \mathrm{H}_{588} \mathrm{CuN}_{12} \mathrm{Si}_{14} \mathrm{Zn}_{2}, \mathrm{M}^{+}\right.$requires 5,672).

$\lambda_{\text {max }}\left(\mathrm{CHCl}_{3}\right) / \mathrm{nm} \log (\varepsilon): 457$ (5.69), 488 (3.79), 579 (4.16), 669 (2.80), 726 (1.93).

\section{Trimer (3.12)}

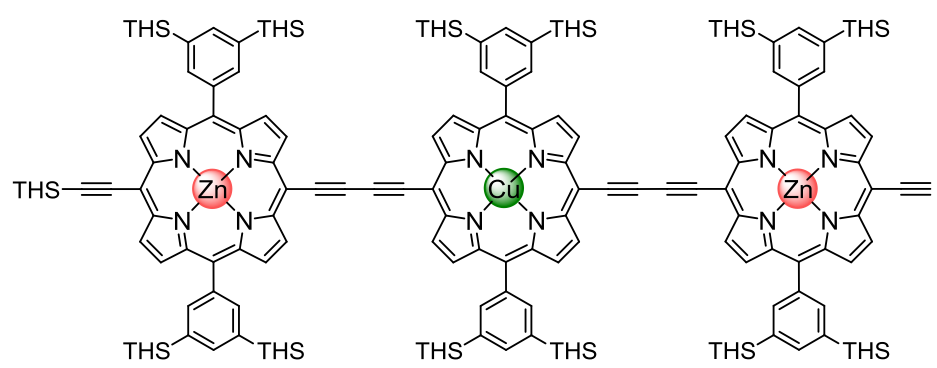

Trimer 3.11 (77.5 mg, $13.7 \mu \mathrm{mol})$ was dissolved in $\mathrm{CH}_{2} \mathrm{Cl}_{2}(4.5 \mathrm{~mL})$ and $\mathrm{CH}_{3} \mathrm{Cl}(4.5 \mathrm{~mL})$. Tetra-n-butylammonium fluoride $(20.5 \mu \mathrm{L}, 1.0 \mathrm{M}$ solution in THF, $20.5 \mu \mathrm{mol})$ was added to the stirred solution. The progress of the reaction was monitored by TLC (hexane, ethyl acetate, pyridine $=90: 5: 0.5 \mathrm{v} / \mathrm{v} / \mathrm{v}$ ) until an optimal mixture was reached $(20 \mathrm{~min})$. The mixture was immediately passed through a short plug of silica gel (1\% pyridine in $\mathrm{CHCl}_{3}$ ). We were unable purify the reaction mixture further and the fraction containing the desired product were concentrated to dryness and used in the next step to form the hexamer $\mathbf{3 . 1 3}$ without further purification assuming that half of the starting material was converted to the title compound. 


\section{Hexamer (3.13)}

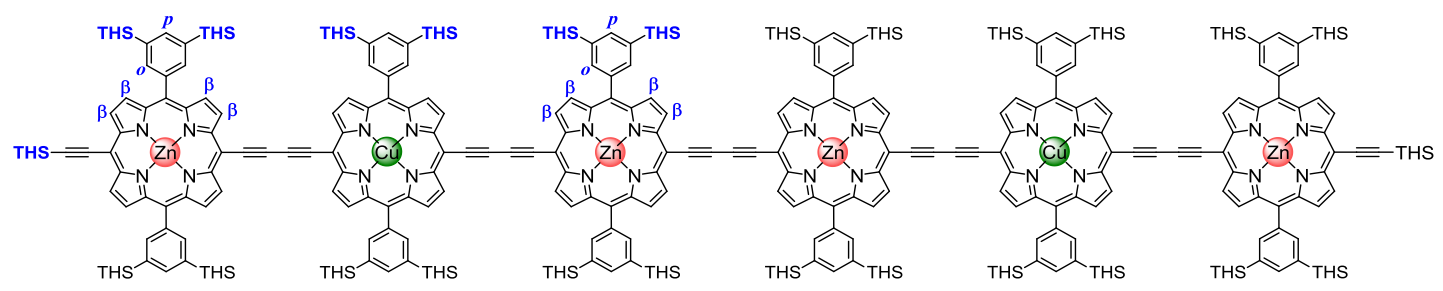

Trimer 3.12 (35 mg, $6.5 \mu \mathrm{mol})$ was dissolved in toluene $(1 \mathrm{~mL})$. $\mathrm{PdCl}_{2}\left(\mathrm{PPh}_{3}\right)_{2}(0.23 \mathrm{mg}$, $0.32 \mu \mathrm{mol})$, Cul $(0.62 \mathrm{mg}, 0.32 \mu \mathrm{mol})$ and 1,4-benzoquinone $(1.40 \mathrm{mg}, 13.0 \mu \mathrm{mol})$ were dissolved in toluene $(0.3 \mathrm{~mL})$ and $i-\mathrm{Pr}_{2} \mathrm{NH}(0.3 \mathrm{~mL})$. The catalyst solution was immediately added and the mixture was stirred at room temperature for $1 \mathrm{~h}$. The crude reaction mixture was passed through a short plug of silica gel (1\% pyridine in $\mathrm{CHCl}_{3}$ ), passed over a SEC column (1\% pyridine in $\mathrm{CHCl}_{3}$ ), and finally the oligomers were separated by recycling GPC (1\% pyridine in toluene) to give $\mathbf{3 . 1 3}$ as a dark oil (15.1 mg, 43\%).

${ }^{1} \mathrm{H}$ NMR $\left(400 \mathrm{MHz}, \mathrm{CDCl}_{3}, 298 \mathrm{~K}\right): \delta_{\mathrm{H}}(\mathrm{ppm}) 9.90(8 \mathrm{H}, \mathrm{m}, \beta), 9.67(8 \mathrm{H}, \mathrm{m}, \beta), 8.96(8 \mathrm{H}$, $m, \beta), 8.85(8 \mathrm{H}, \mathrm{m}, \beta), 8.31(8 \mathrm{H}, \mathrm{m}$, ortho $), 8.25(8 \mathrm{H}, \mathrm{m}$, ortho $), 8.02(4 \mathrm{H}, \mathrm{m}$, para $)$, $8.00(4 \mathrm{H}, \mathrm{m}$, para $), 1.82-0.79(1014 \mathrm{H}, \mathrm{m}, \mathrm{THS})$.

MALDI-TOF: $m / z=10,779\left(\mathrm{C}_{584} \mathrm{H}_{1098} \mathrm{Cu}_{2} \mathrm{~N}_{24} \mathrm{Si}_{26} \mathrm{Zn}_{4}, \mathrm{M}^{+}\right.$requires 10,777$)$.

$\lambda_{\text {max }}\left(\mathrm{CHCl}_{3}\right) / \mathrm{nm} \log (\varepsilon): 458$ (5.88), 488 (5.78), 581 (4,76), 663 (5.07), 766 (5.54). I-P3 $\mathrm{Cu}$

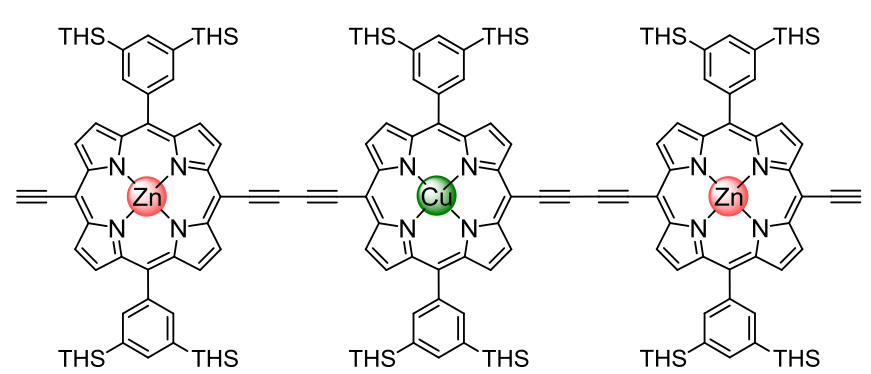

Trimer 3.11 (6 mg, $1.2 \mu \mathrm{mol})$ was dissolved in $\mathrm{CH}_{2} \mathrm{Cl}_{2}(1.5 \mathrm{~mL})$. Tetra- $n$-butylammonium fluoride ( $24 \mu \mathrm{L}, 1.0 \mathrm{M}$ solution in THF, $24 \mu \mathrm{mol}$ ) was added and the reaction mixture was stirred at room temperature for $15 \mathrm{~min}$. The reaction mixture was immediately passed through a short plug of silica gel $\left(\mathrm{CHCl}_{3}\right)$ to give the title compound as a dark oil (5.2 $\mathrm{mg}, 96 \%)$.

MALDI-TOF: $m / z=5,106\left(\mathrm{C}_{324} \mathrm{H}_{512} \mathrm{CuN}_{12} \mathrm{Si}_{12} \mathrm{Zn}_{2}, \mathrm{M}^{+}\right.$requires 5,105).

$\lambda_{\text {max }}\left(\mathrm{CHCl}_{3}\right) / \mathrm{nm} \log (\varepsilon): 454$ (5.55), 487 (5.21), 577 (4.38), 720 (5.08). 


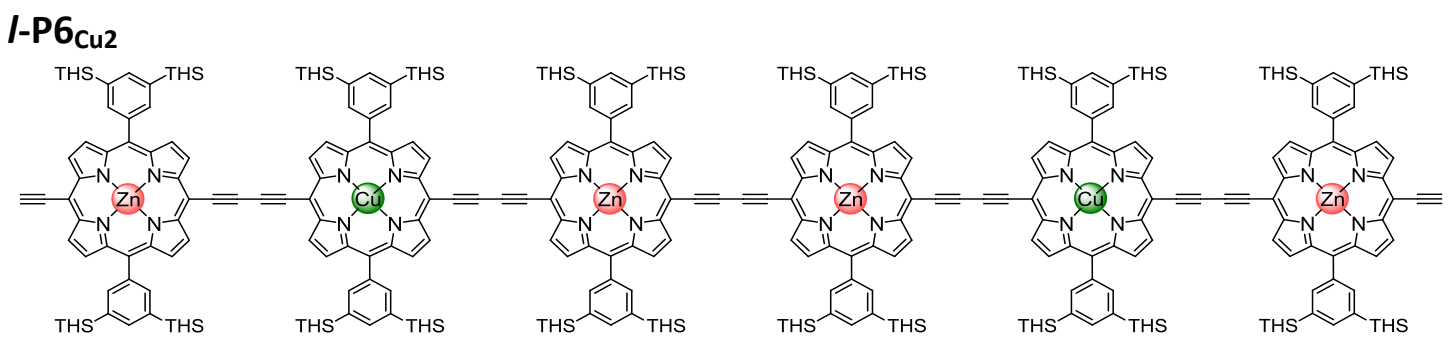

Hexamer $3.13(9.0 \mathrm{mg}, 0.88 \mu \mathrm{mol})$ was dissolved in $\mathrm{CH}_{2} \mathrm{Cl}_{2} \quad(1 \mathrm{~mL})$.

Tetra- $n$-butylammonium fluoride ( $18 \mu \mathrm{L}, 1.0 \mathrm{M}$ solution in THF, $18 \mu \mathrm{mol})$ was added and the reaction mixture was stirred at room temperature for $15 \mathrm{~min}$. The crude mixture was immediately passed through a short plug of silica gel $\left(\mathrm{CHCl}_{3}\right)$ to give the title compound as a dark oil (6.5 mg, 76\%).

MALDI-TOF: $m / z=10,207\left(\mathrm{C}_{648} \mathrm{H}_{1022} \mathrm{Cu}_{2} \mathrm{~N}_{24} \mathrm{Si}_{24} \mathrm{Zn}_{4}, \mathrm{M}^{+}\right.$requires 10,212).

$\lambda_{\text {max }}\left(\mathrm{CHCl}_{3}\right) / \mathrm{nm} \log (\varepsilon): 455$ (6.09), 488 (6.01), 579 (5.00), 659 (5.26), 766 (5.76).

\subsubsection{Proof of purity}

To prove the purity of the relevant compounds, clean MALDI-MS spectra are provided for $\mathbf{I}-\mathbf{P} \mathbf{3}_{\mathrm{Cu}}, \mathbf{I}-\mathbf{P} \mathbf{6}_{\mathrm{Cu} 2}$ and $\mathbf{c}-\mathbf{P} \mathbf{6}_{\mathrm{Cu} 2} \cdot \mathbf{T} \mathbf{6}$. Additionally, clean analytical GPC traces

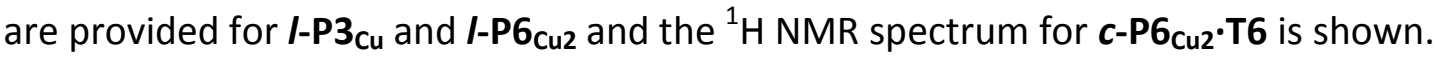
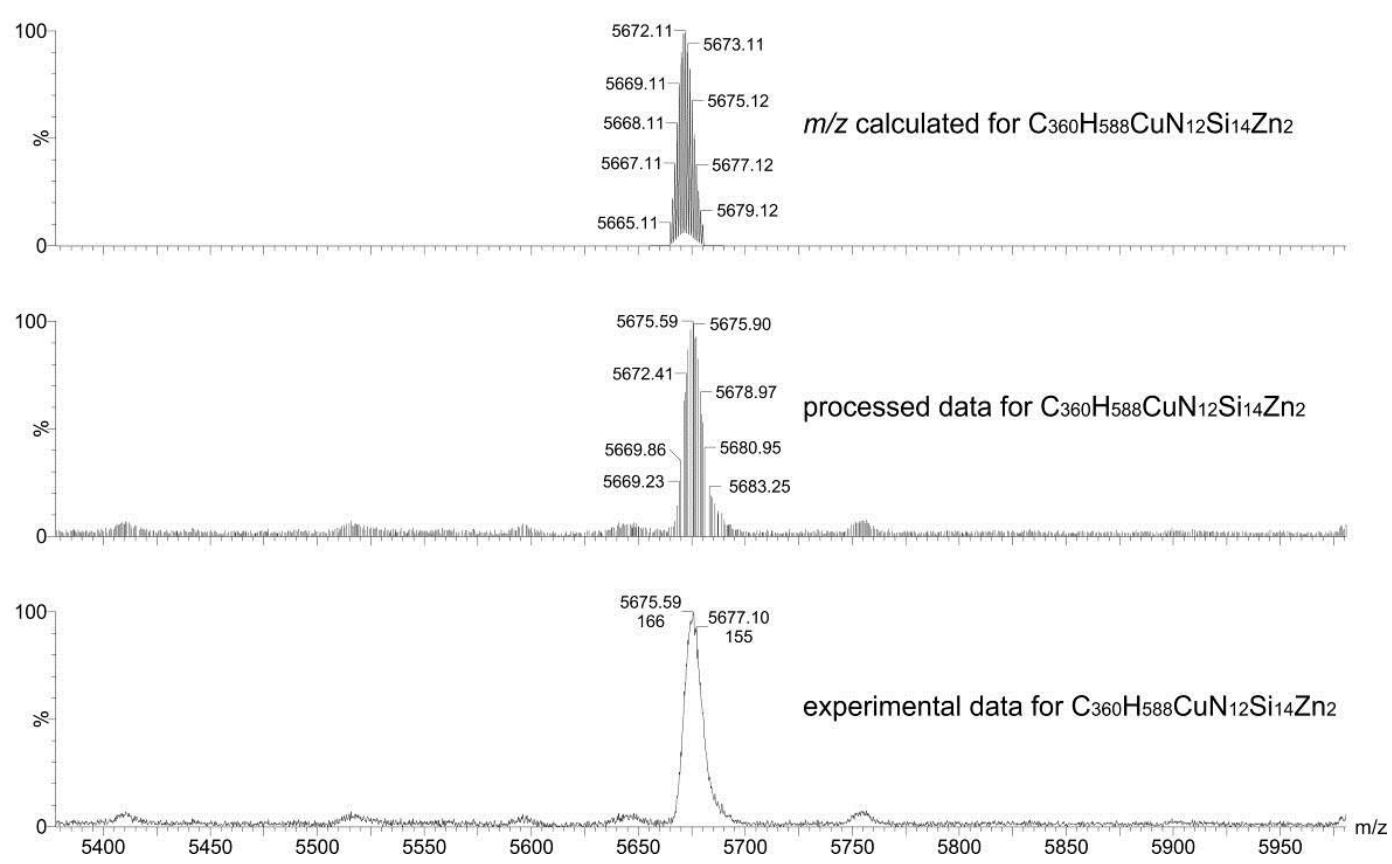

Figure 3.12: The MALDI-MS spectrum of $I-\mathrm{P}_{\mathrm{Cu}}\left(\mathrm{m} / \mathrm{z}=5,676\left(\mathrm{C}_{360} \mathrm{H}_{588} \mathrm{CuN}_{12} \mathrm{Si}_{14} \mathrm{Zn}_{2}, \mathrm{M}^{+}\right.\right.$requires 5,672), matrix: dithranol). 

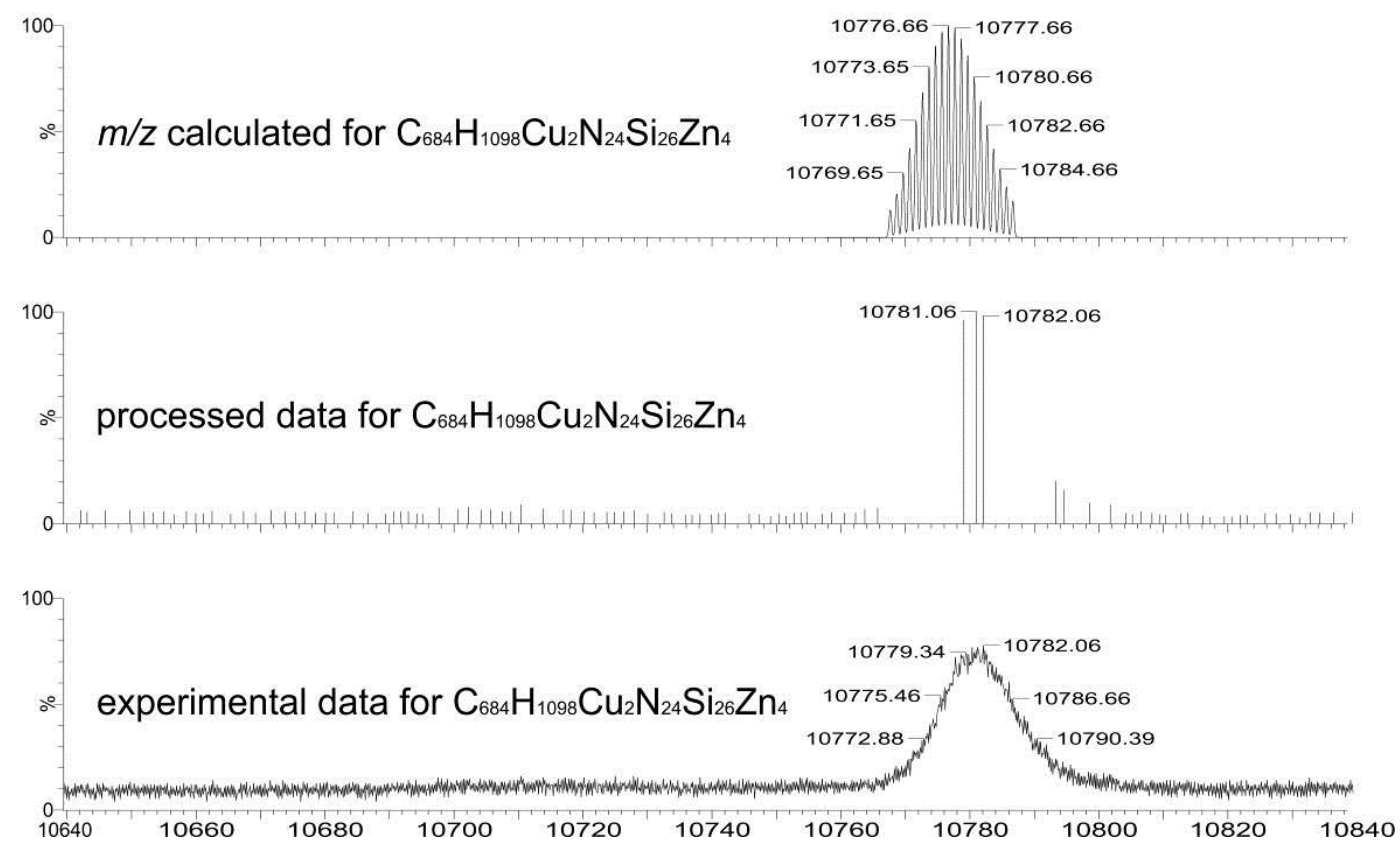

Figure 3.13: The MALDI-MS spectrum of I-P6 ${ }_{\text {Cu2 }}\left(m / z=10,779\left(C_{684} \mathrm{H}_{1098} \mathrm{Cu}_{2} \mathrm{~N}_{24} \mathrm{Si}_{26} \mathrm{Zn}_{4}, \mathrm{M}^{+}\right.\right.$requires 10,777), matrix: dithranol).
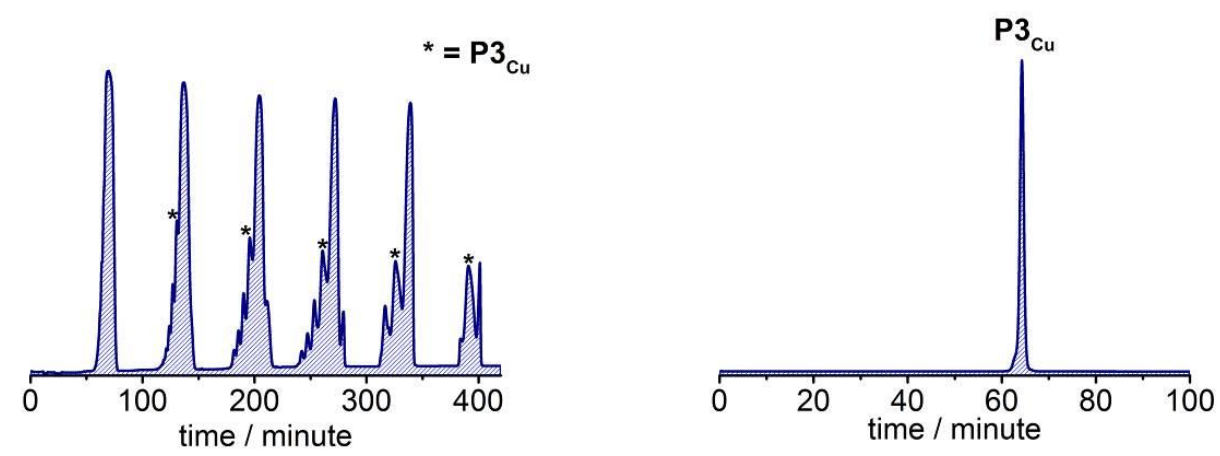

Figure 3.14: Analytical recycling GPC trace of the crude reaction mixture and the purified trace of $I-\mathrm{P} 3_{\mathrm{Cu}}$ (eluent: $1 \%$ pyridine in toluene, recorded at $800 \mathrm{~nm}$ ). 

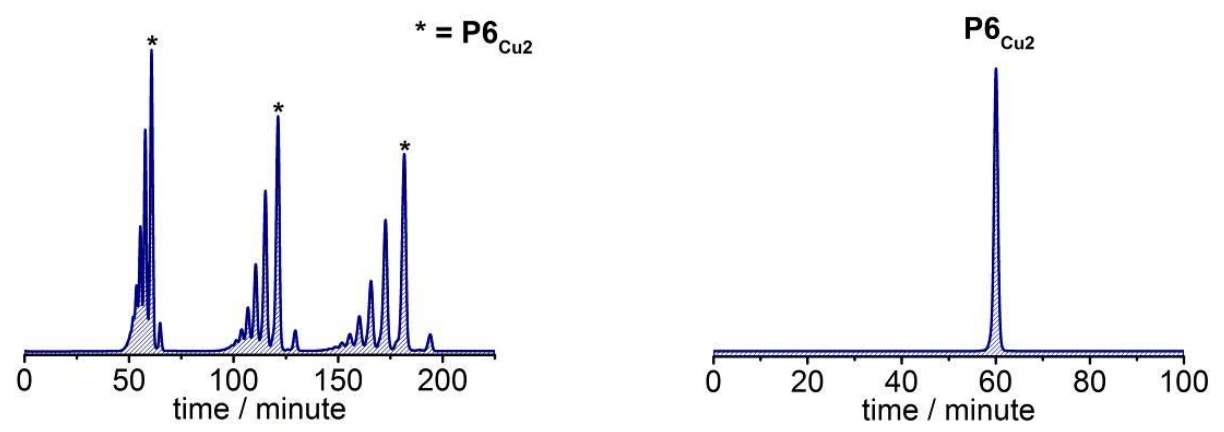

Figure 3.15: Analytical recycling GPC trace of the crude reaction mixture and the purified trace of $I-\mathrm{P}_{\mathrm{Cu}}$ (eluent: $1 \%$ pyridine in toluene, recorded at $800 \mathrm{~nm}$ ).

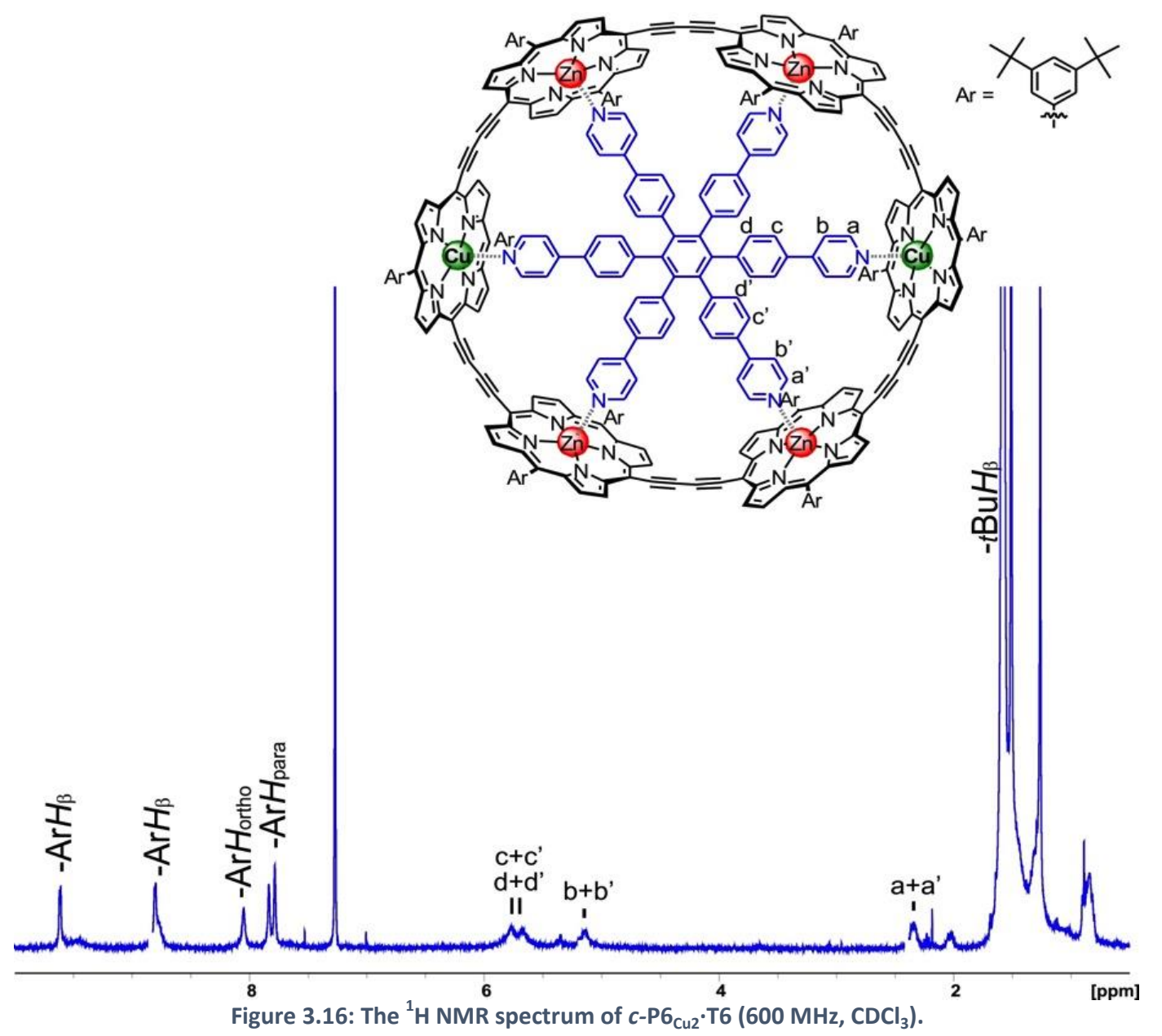




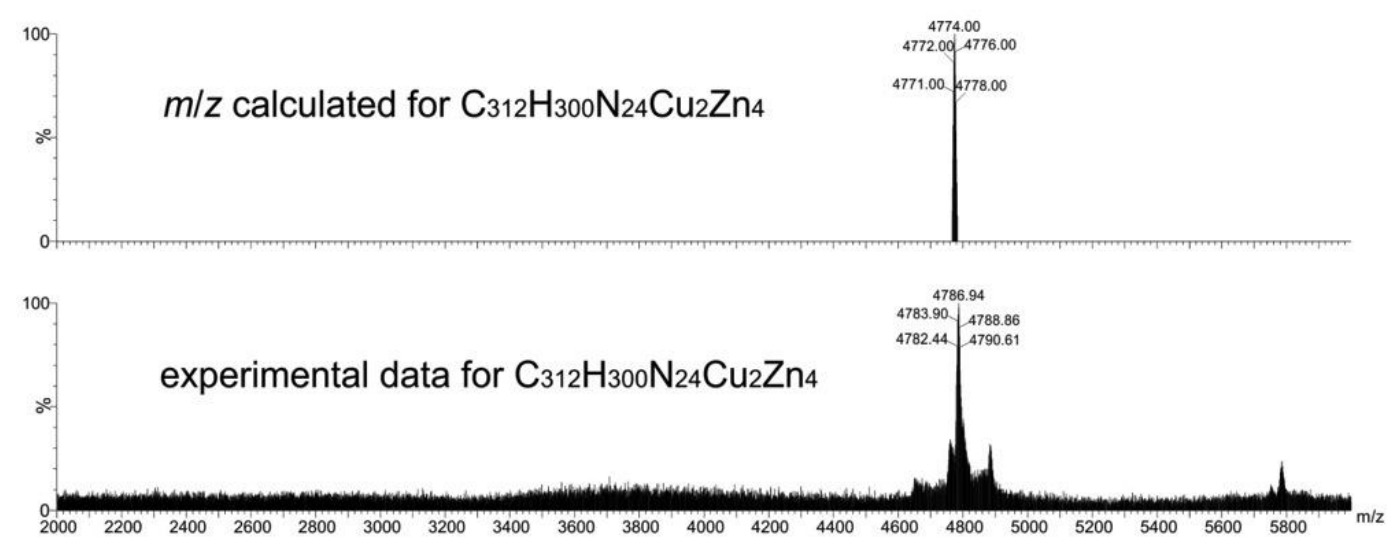

Figure 3.17: The MALDI-MS spectrum of $c-\mathrm{P}_{\mathrm{Cu2}}\left(\mathrm{m} / \mathrm{z}=4,787\left(\mathrm{C}_{312} \mathrm{H}_{300} \mathrm{Cu}_{2} \mathrm{~N}_{24} \mathrm{Zn}_{4}, \mathrm{M}^{+}\right.\right.$requires 4,774). matrix: DCTB).

\subsubsection{UV-vis-NIR titrations}

\subsubsection{Titrations of porphyrin monomer 3.6 and DABCO}

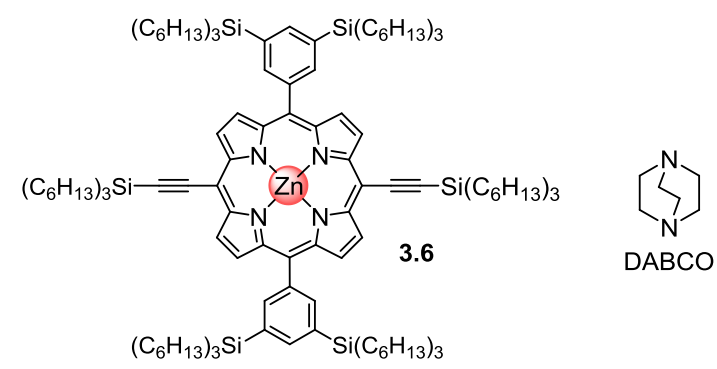

Figure 3.18: Structures of reference porphyrin monomer 3.6 and DABCO.

Titrations with porphyrin monomer 3.6 and DABCO were performed in order to determine the reference binding constant $K_{\mathrm{DABCO}}$.

All titrations were performed in toluene at $298 \mathrm{~K}$. Care was taking to keep the porphyrin concentration constant throughout the entire titration by adding porphyrin to the ligand solution before titrations were started. The binding curves were fitted using a 1:1 binding isotherm using the equation:

$$
\frac{A-A_{\text {initial }}}{A_{\infty}-A_{\text {initial }}}=\left(\frac{\left(K_{a}\left([L]+[P]_{0}\right)+1\right)-\sqrt{\left(K_{a}\left([L]+[P]_{0}\right)+1\right)^{2}-4 K_{a}^{2}[P]_{0}[L]}}{2 K_{a}[P]_{0}}\right)
$$

where $A$ is the observed absorption at a specific wavelength or the difference of absorbance between two wavelengths; $A_{\text {initial }}$ is the starting absorption at this wavelength; $A_{\infty}$ is the asymptotic final absorption at this wavelength; $K_{a}$ is the association constant between ligand and porphyrin host; $[L]$ is the concentration of ligand; $[\mathrm{P}]_{0}$ is the concentration of porphyrin host. The free variables which were 
adjusted to optimise the fit to the experimental data during the fitting procedure are $A_{\text {initial }}, A_{\infty}$, and $K_{a}$. Fitting analysis was carried out using the Origin software (Figure 3.19-Figure 3.21) giving $K_{\mathrm{DABCO}}=(1.45 \pm 0.20) \times 10^{6} \mathrm{M}^{-1}$.
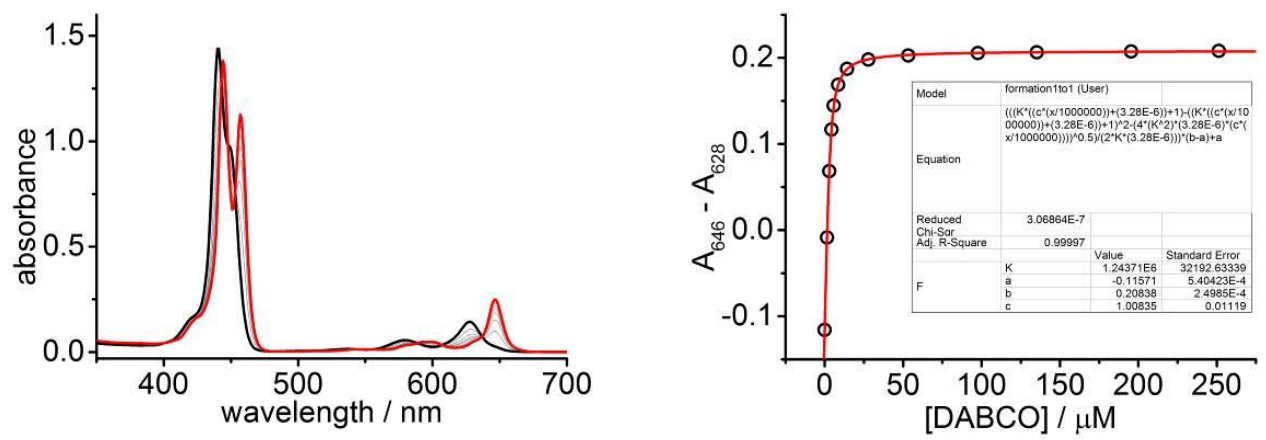

Figure 3.19: UV-vis titration of 3.6 and $\mathrm{DABCO}, R^{2}=1.000$. (Run 1 , toluene, $298 \mathrm{~K},[3.6]=3.28 \mu \mathrm{M}$, $K=1.24 \times 10^{6} \mathrm{M}^{-1}$ ).
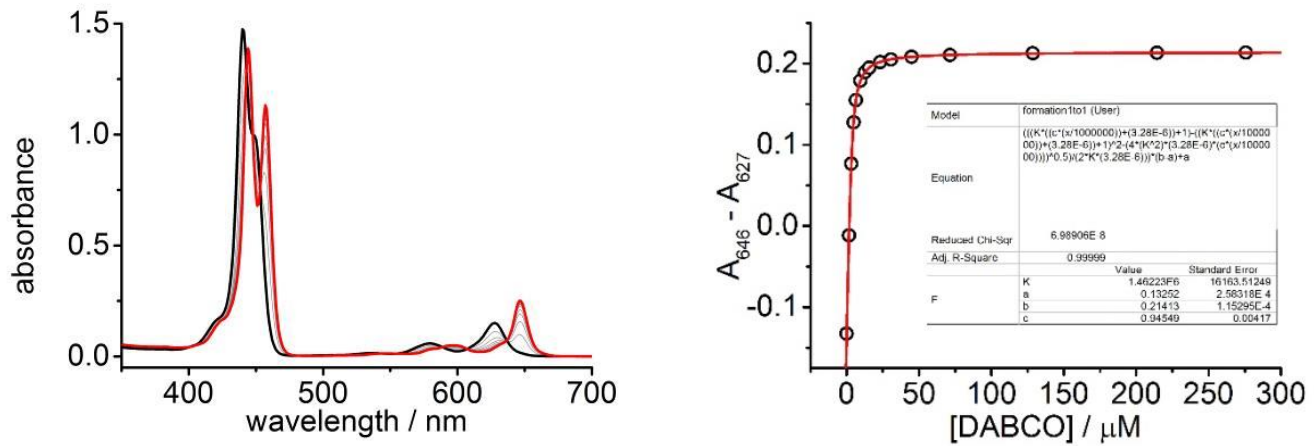

Figure 3.20: UV-vis titration of 3.6 and DABCO, $R^{2}=1.000$. (Run 2, toluene, $298 \mathrm{~K},[3.6]=3.28 \mu \mathrm{M}$, $K=1.46 \times 10^{6} \mathrm{M}^{-1}$ ).
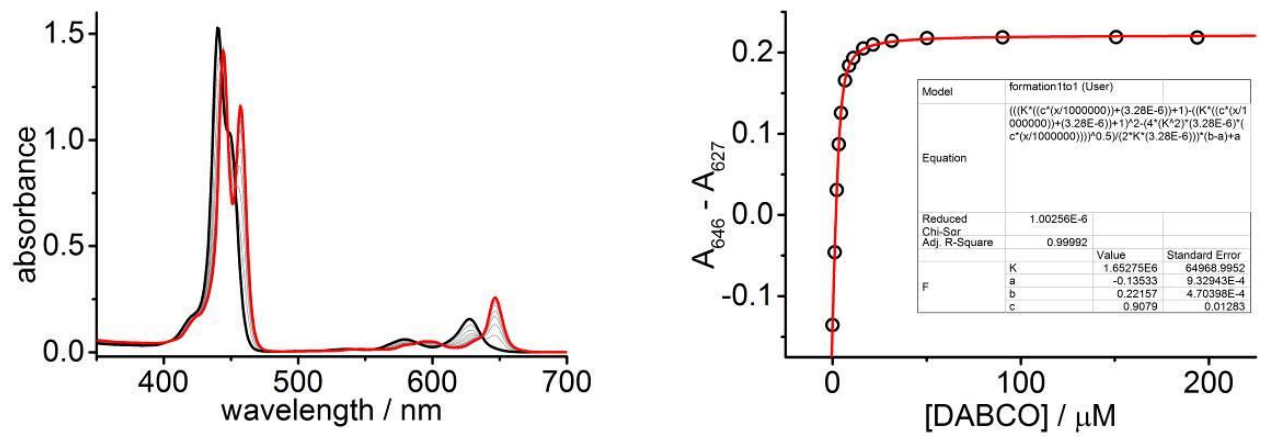

Figure 3.21: UV-vis titration of 3.6 and DABCO, $R^{2}=1.000$. (Run 3, toluene, $298 \mathrm{~K},[3.6]=3.28 \mu \mathrm{M}$, $K=1.65 \times 10^{6} \mathrm{M}^{-1}$ ).

\subsubsection{Formation titrations of $I-P 3_{\mathrm{Cu}}$ and $I-P 6_{\mathrm{Cu} 2}$ with T6}

UV-vis-NIR titrations were performed with the linear oligomers I-P3 $\mathbf{C u}$ and I-P6 $\mathbf{C u 2}_{\mathbf{C}}$ with the template T6 to verify the formation of the desired two-to-one and one-to-one complexes respectively. The titrations of I-P3 $\mathbf{C u}_{\mathrm{u}}$ are shown in Figure 3.22 
and Figure 3.23 which reveal a binding isotherm weak enough to fit to the $1: 1$ binding isotherm described in Section 3.6.2.1. Figure 3.24 shows the titrations carried out with I-P6 $6_{\text {Cu2 }}$ which reveals a binding constant of $8.81 \times 10^{9} \mathrm{M}^{-1}$ which is too strong to be fit reliably. We therefore show in Figure 3.25 a plot in which the binding constant is fixed at different values while allowing the other variables to fit. This allows us to say that the binding constant is greater than $10^{8} \mathrm{M}^{-1}$ guaranteeing full complex formation.
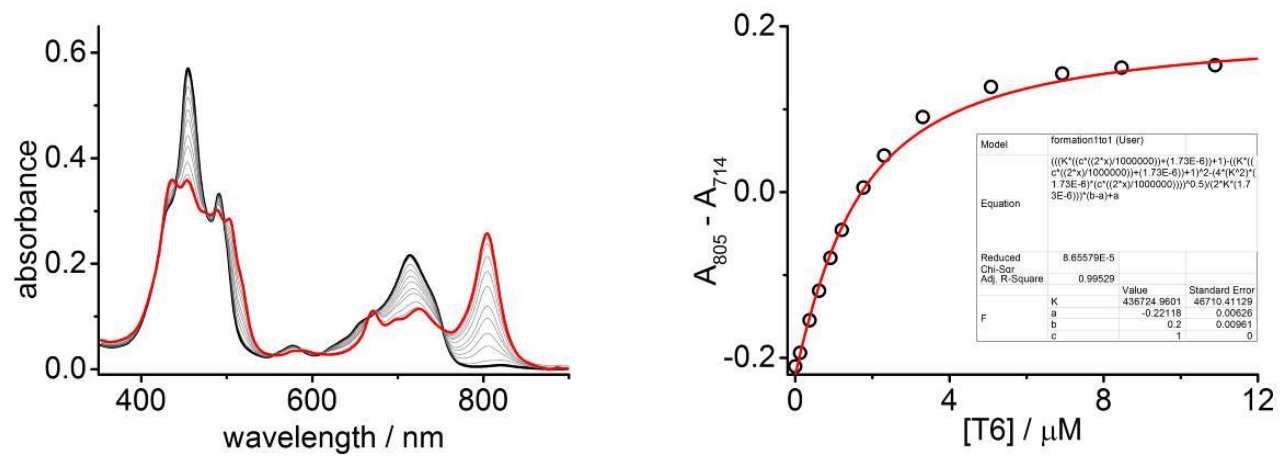

Figure 3.22: UV-vis titration of $I-\mathrm{P}_{\mathrm{Cu}}$ and $\mathrm{T} 6, R^{2}=0.995$. (Run 1 , toluene, $298 \mathrm{~K},\left[I-\mathrm{P} 3_{\mathrm{Cu}}\right]=1.73 \mu \mathrm{M}$, $K=4.37 \times 10^{5} \mathrm{M}^{-1}$ ).
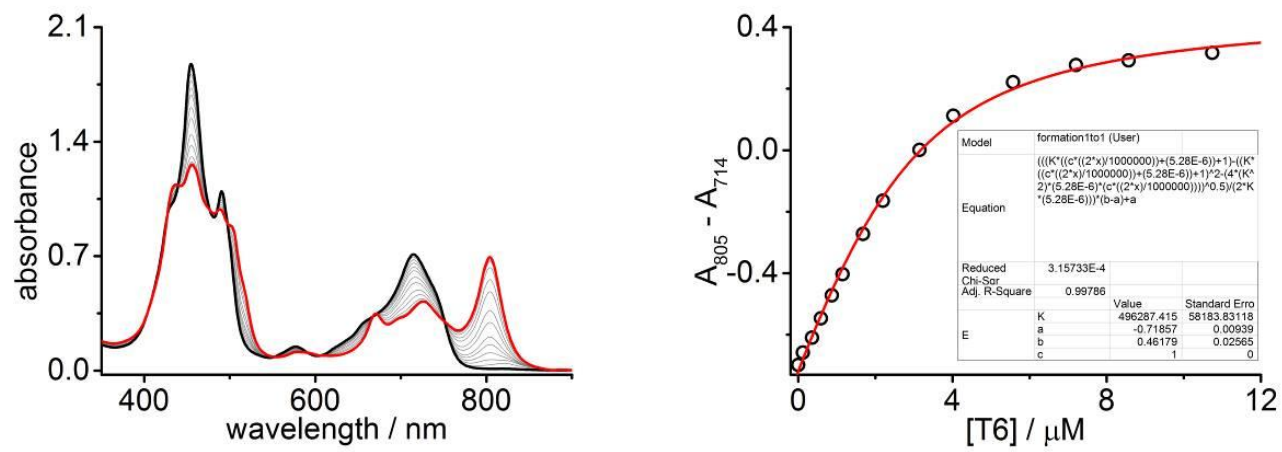

Figure 3.23: UV-vis titration of $I-P 3_{C u}$ and $T 6, R^{2}=0.998$. (Run 2, toluene, $298 \mathrm{~K},\left[I-P 3_{C u}\right]=5.28 \mu \mathrm{M}$, $K=4.96 \times 10^{5} \mathrm{M}^{-1}$ ).
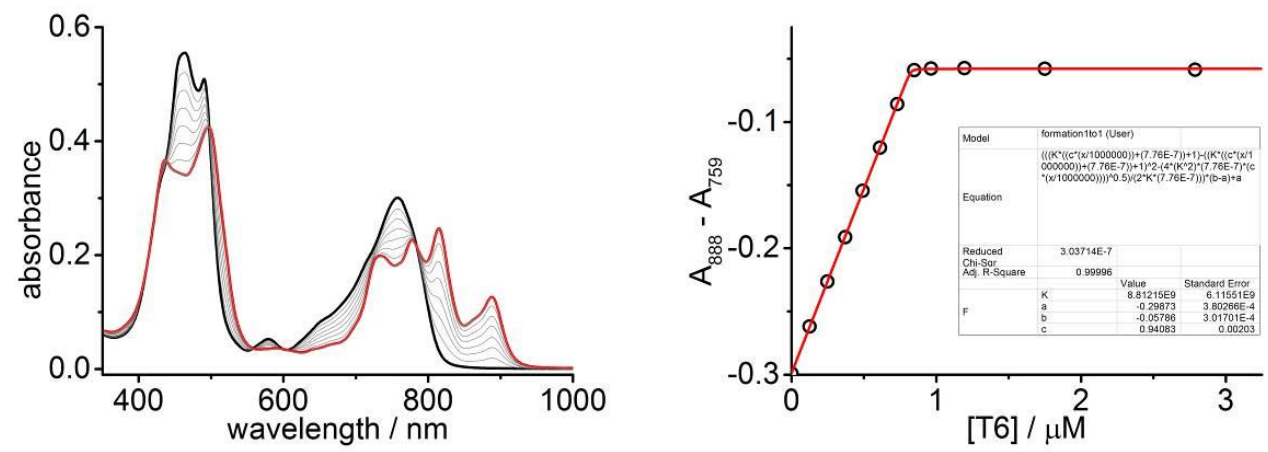

Figure 3.24: UV-vis titration of $I-P 6_{\mathrm{Cu} 2}$ and $\mathrm{T} 6, R^{2}=1.000$. (Run 1 , toluene, $298 \mathrm{~K},\left[I-\mathrm{P} 6_{\mathrm{Cu}}\right]=0.78 \mu \mathrm{M}$, $K=8.81 \times 10^{9} \mathrm{M}^{-1}$ ). 


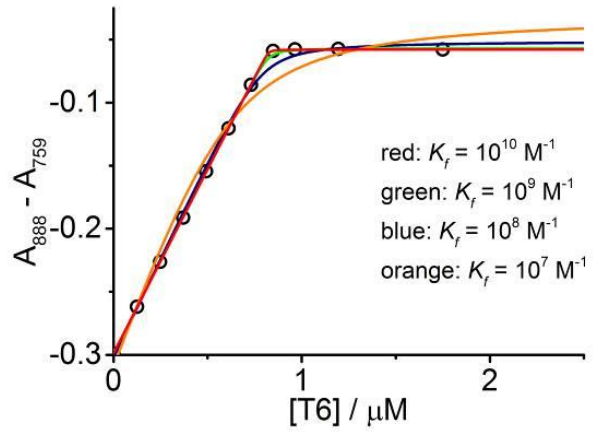

Figure 3.25: UV-vis titration of $I-\mathrm{P}_{\mathrm{Cu}}$ and T6. Binding constant is fixed while the other variables are allowed to fit.

\subsubsection{Denaturation titration with DABCO on $c-\mathrm{P}_{\mathrm{Cu}} \mathrm{C}_{2} \cdot \mathrm{T} 6$}

When binding strength increases, the binding curves become increasingly square, leading to greater uncertainty in the fit. In order to derive a trustworthy binding constant, denaturation titrations (break-up titration) need to be performed with a competing ligand such as DABCO. Using the data from these break-up titrations $\left(K_{\mathrm{dn}}=\right.$ denaturation constant $)$ and the formation constant of the single site binding event of the competing ligand with a zinc porphyrin monomer $\left(K_{\mathrm{DABCO}}=\right.$ association constant for DABCO to 3.6) allows us to derive the formation binding constant $\left(K_{\mathrm{f}}\right)$ between the ring ( $N=$ number of zinc porphyrin binding sites) and the template using the following equation:

$$
K_{\mathrm{f}}=\frac{K_{\mathrm{DABCO}}^{N}}{K_{\mathrm{dn}}}
$$

via the thermodynamic cycle shown in Figure 3.26.

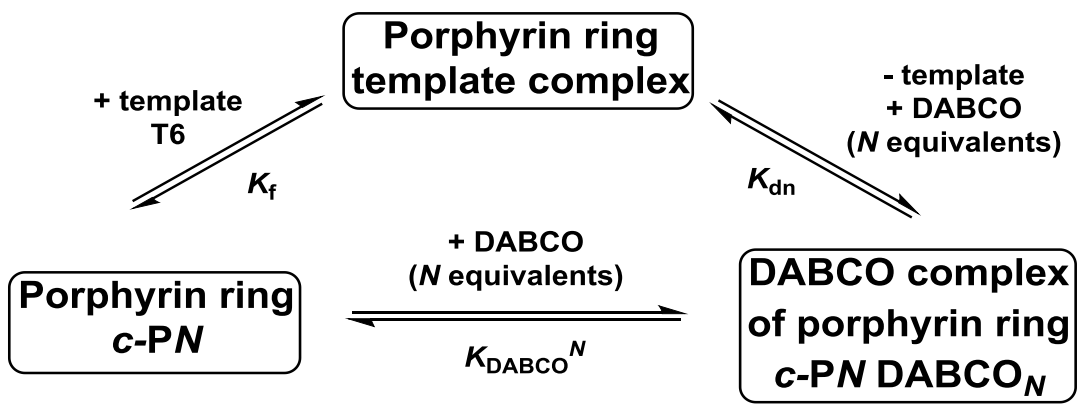

Figure 3.26: Thermodynamic cycle relating the formation constant of the template complex $\left(K_{\mathrm{f}}\right)$ to the denaturation constant $\left(K_{\mathrm{dn}}\right)$ and binding constant of each porphyrin unit for DABCO $\left(K_{\mathrm{DABCO}}\right)$.

Denaturation titrations were performed in toluene at $298 \mathrm{~K}$. The 1:1 complexes between the ring and template were formed in the cuvette prior to the denaturation titration. All denaturation titrations were carried out at constant complex 
concentration by adding the complex to the ligand (DABCO) stock solution before titrations started.

Data were fitted to the $\mathrm{N}$-dentate denaturation binding isotherm described in the following equation:

$$
\frac{A-A_{\text {initial }}}{A_{\infty}-A_{\text {initial }}}=\left(\frac{-K_{d n}[L]^{N}+\sqrt{K_{d n}^{2}[L]^{2 N}+4 K_{d n}[L]^{N}[P]_{0}}}{2[P]_{0}}\right)
$$

where $A$ is the observed absorption at a specific wavelength or difference of absorption between two wavelengths; $A_{\text {initial }}$ is the starting absorption at a specific wavelength or difference between absorption in two wavelengths; $A_{\infty}$ is the terminal absorption at a specific wavelength or difference of absorption in two wavelengths; $K_{\mathrm{dn}}$ is the dissociation constant between ligand and porphyrin oligomer complex; [L] is the concentration of ligand; $[\mathrm{P}]_{0}$ is the concentration of porphyrin complex; $N$ is the number of binding sites in the complex (e.g. $N=4$ in $\mathbf{c}-\mathbf{P} \mathbf{6}_{\mathrm{Cu2}} \cdot \mathbf{T 6}$ ). The titration curves and fittings are shown below.
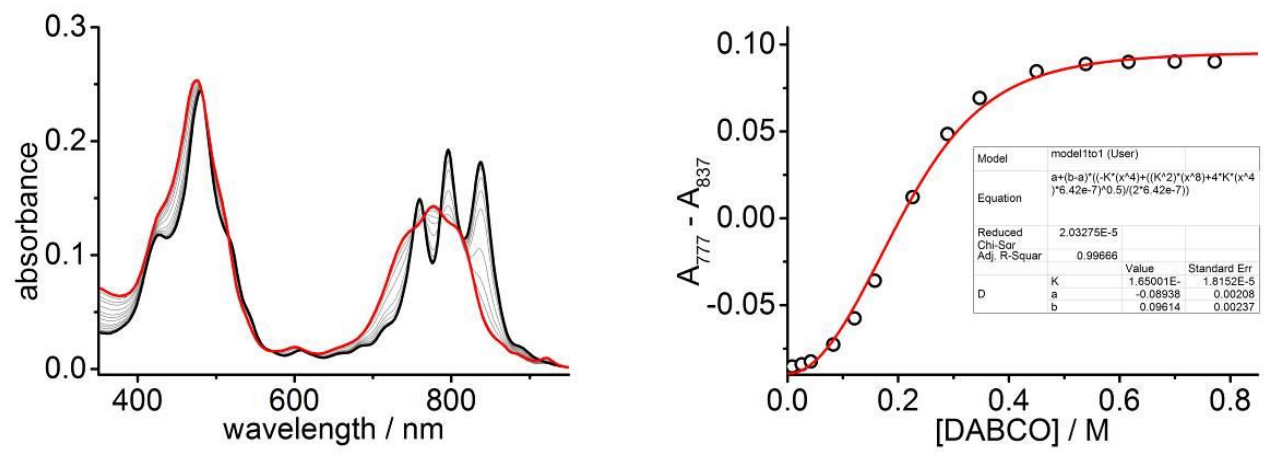

Figure 3.27: UV-vis titration of $c-\mathrm{P}_{\mathrm{Cu}} \cdot{ } \cdot \mathrm{T} 6$ and $\mathrm{DABCO}, R^{2}=0.997$. (Run 1 , toluene, $298 \mathrm{~K},\left[c-\mathrm{P}_{\mathrm{Cu}} \cdot{ }^{\circ} \mathrm{T} 6\right]=0.64 \mu \mathrm{M}$, $\left.K_{\mathrm{dn}}=1.65 \times 10^{-4} \mathrm{M}^{-3}\right)$.
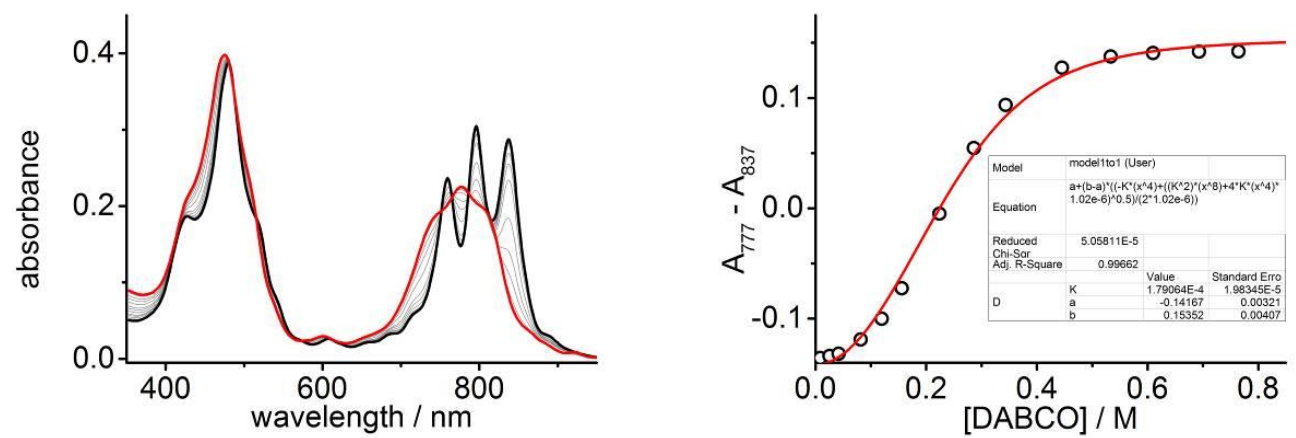

Figure 3.28: UV-vis titration of $c-\mathrm{P}_{\mathrm{Cu2}} \cdot \mathrm{T} 6$ and DABCO, $R^{2}=0.997$. (Run 2, toluene, $298 \mathrm{~K},\left[c-\mathrm{P}_{\mathrm{Cu}} \cdot{ }^{\circ} 6\right]=1.02 \mu \mathrm{M}$, $\left.K_{\mathrm{dn}}=1.79 \times 10^{-4} \mathrm{M}^{-3}\right)$. 
The data shows $K_{\mathrm{dn}}=(1.72 \pm 0.07) \times 10^{-4} \mathrm{M}^{-3}$ which is equivalent to $K_{\mathrm{f}}=2.57 \times 10^{28} \mathrm{M}^{-1}$ clearly indicating that under the conditions of the EPR experiments a 1:1 complex will be formed.

\subsection{3 $T_{1}$ and $T_{2}$ relaxation time constant measurements}

Relaxation time constant measurements were carried out by Prof. Tim Clardige.

\subsubsection{1 $T_{1}$ measurements on free-base trimer 3.1}

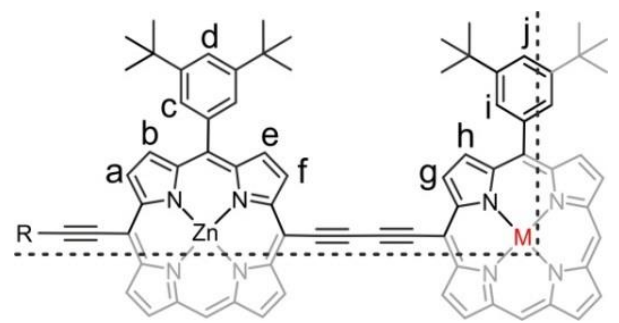

Figure 3.29: The general assignment of the labels for the ${ }^{1} \mathrm{H}$ NMR spectra below $(\mathrm{M}=2 \mathrm{H}$ or $\mathrm{Cu}$ ).

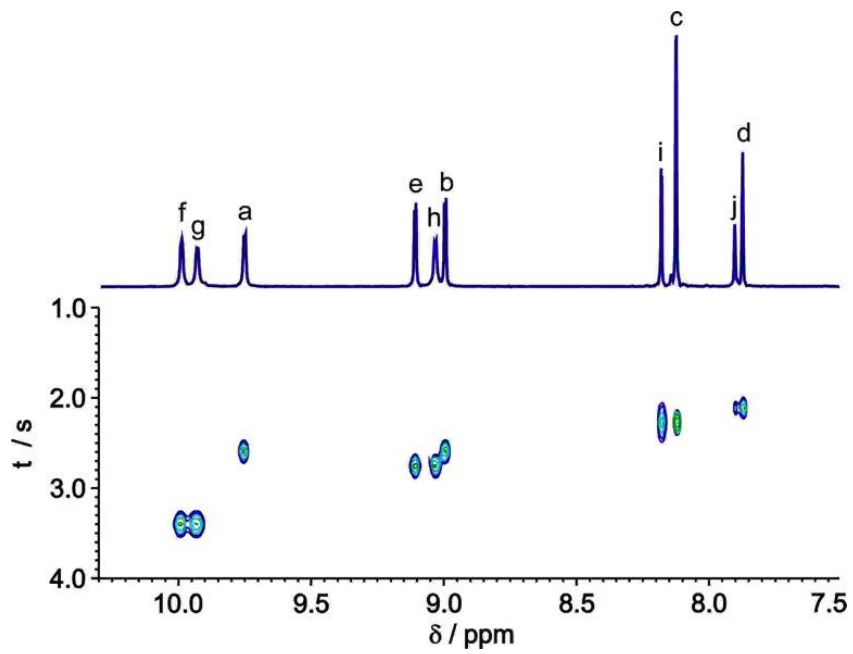

Figure 3.30: The ${ }^{1} \mathrm{H}$ NMR spectrum of $3.1\left(\mathrm{CDCl}_{3}, 700 \mathrm{MHz}, 298 \mathrm{~K}\right)$. Only the aromatic part of the spectrum is shown. The graph below shows the cross signals between chemical shift of the proton signals with respect to the corresponding $T_{1}$ relaxation time constants.

Table 3.1: Summary of the data describing the chemical shift of the aromatic proton signal of 3.1 with the accompanying $T_{1}$ relaxation time constants.

\begin{tabular}{|cccc|}
\hline Peak name & $\delta(\mathbf{p p m})$ & $\boldsymbol{T}_{\mathbf{1}}(\mathbf{s})$ & error (s) \\
\hline $\mathbf{f}$ & 9.987 & 3.33 & 0.098 \\
\hline $\mathbf{g}$ & 9.928 & 3.32 & 0.10 \\
\hline $\mathbf{a}$ & 9.749 & 2.53 & 0.048 \\
\hline $\mathbf{e}$ & 9.106 & 2.68 & 0.024 \\
\hline $\mathbf{h}$ & 9.031 & 2.78 & 0.041 \\
\hline $\mathbf{b}$ & 8.992 & 2.51 & 0.026 \\
\hline $\mathbf{i}$ & 8.178 & 2.24 & 0.16 \\
\hline $\mathbf{c}$ & 8.121 & 2.22 & 0.015 \\
\hline j & 7.901 & 2.15 & 0.020 \\
\hline $\mathbf{d}$ & 7.872 & 2.08 & 0.020 \\
\hline
\end{tabular}



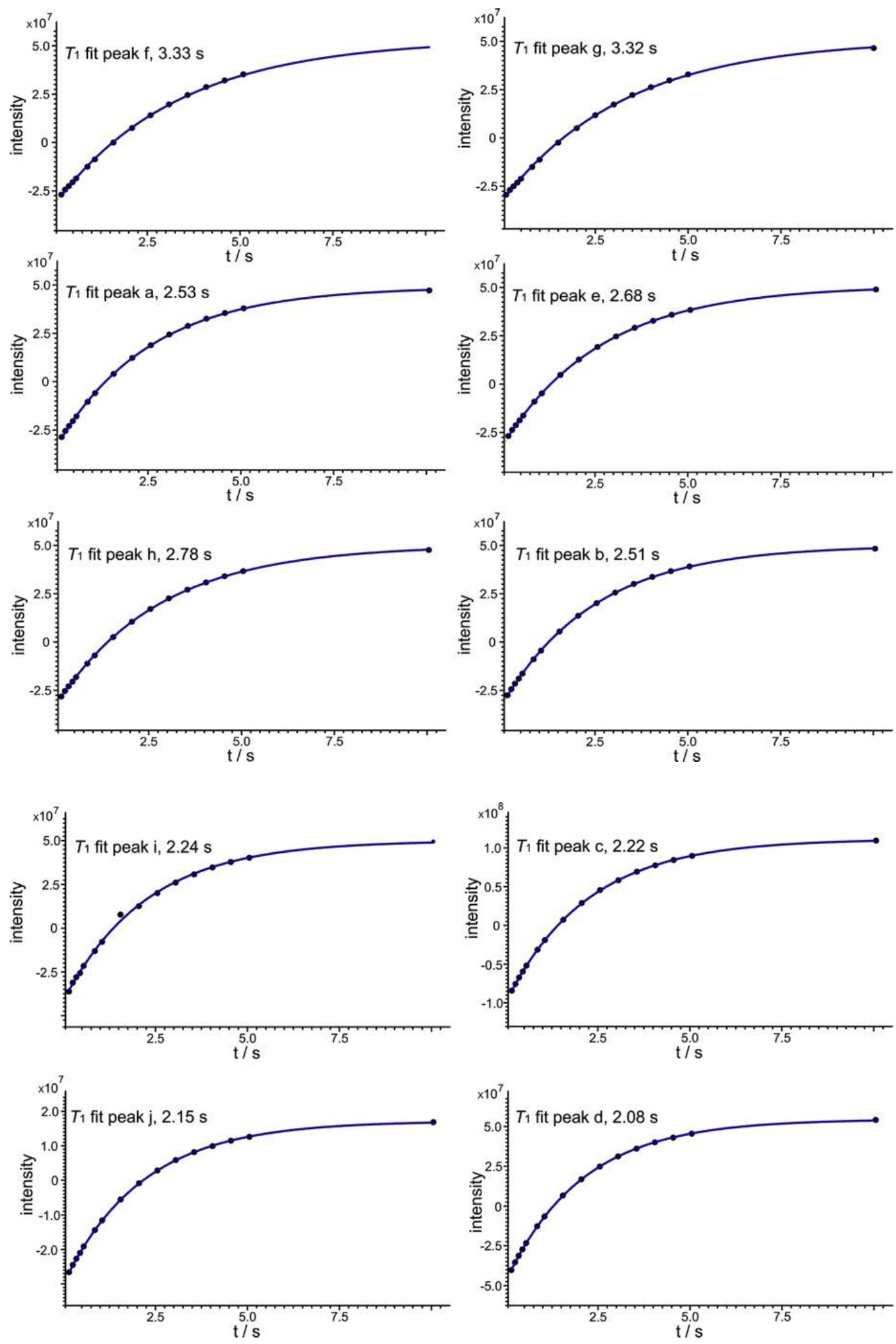

Figure 3.31: Inversion recovery curves and exponential fits for the $T_{1}$ 's of 3.1 peaks a-j. 


\subsubsection{2 $T_{2}$ measurements on free-base trimer 3.1}

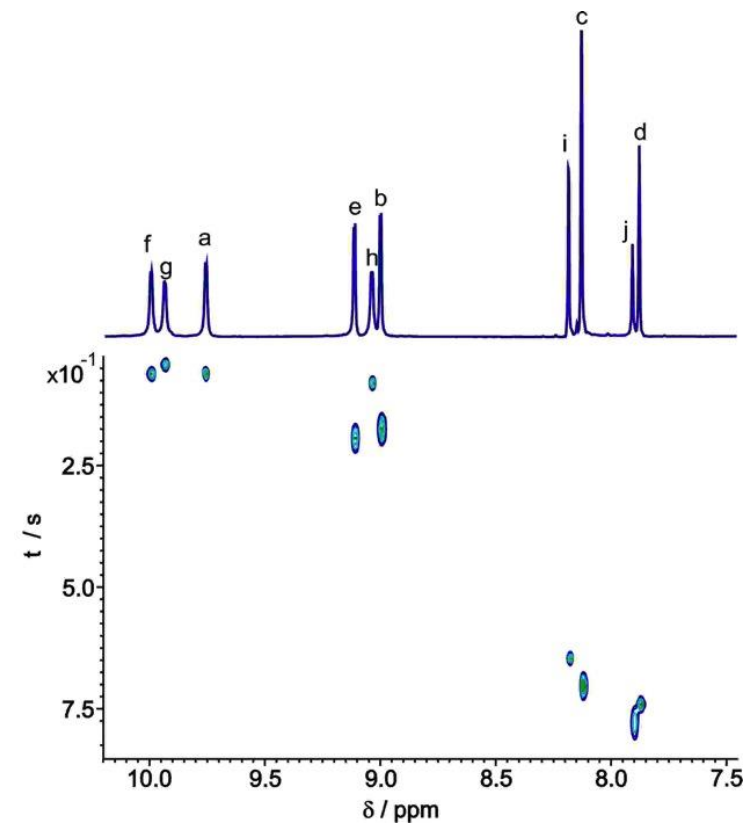

Figure 3.32: The ${ }^{1} \mathrm{H}$ NMR spectrum of $3.1\left(\mathrm{CDCl}_{3}, 700 \mathrm{MHz}, 298 \mathrm{~K}\right)$. Only the aromatic part of the spectrum is shown. The graph below shows the cross signals between chemical shifts of the proton signals with respect to the corresponding $T_{2}$ relaxation time constants.

Table 3.2: Summary of the data describing the chemical shift of the aromatic proton signal of 3.1 with the accompanying $T_{2}$ relaxation time constants.

\begin{tabular}{|cccc|}
\hline Peak name & $\delta(\mathrm{ppm})$ & $T_{2}(\mathrm{~s})$ & error $(\mathrm{s})$ \\
\hline $\mathbf{f}$ & 9.987 & 0.0564 & 0.0019 \\
\hline $\mathbf{g}$ & 9.927 & 0.0520 & 0.0017 \\
\hline $\mathbf{a}$ & 9.750 & 0.0599 & 0.0058 \\
\hline $\mathbf{e}$ & 9.106 & 0.190 & 0.0209 \\
\hline $\mathbf{h}$ & 9.031 & 0.0729 & 0.0036 \\
\hline b & 8.991 & 0.177 & 0.0281 \\
\hline $\mathbf{i}$ & 8.178 & 0.639 & 0.0092 \\
\hline $\mathbf{c}$ & 8.122 & 0.698 & 0.0165 \\
\hline j & 7.901 & 0.775 & 0.0321 \\
\hline d & 7.872 & 0.748 & 0.0135 \\
\hline
\end{tabular}



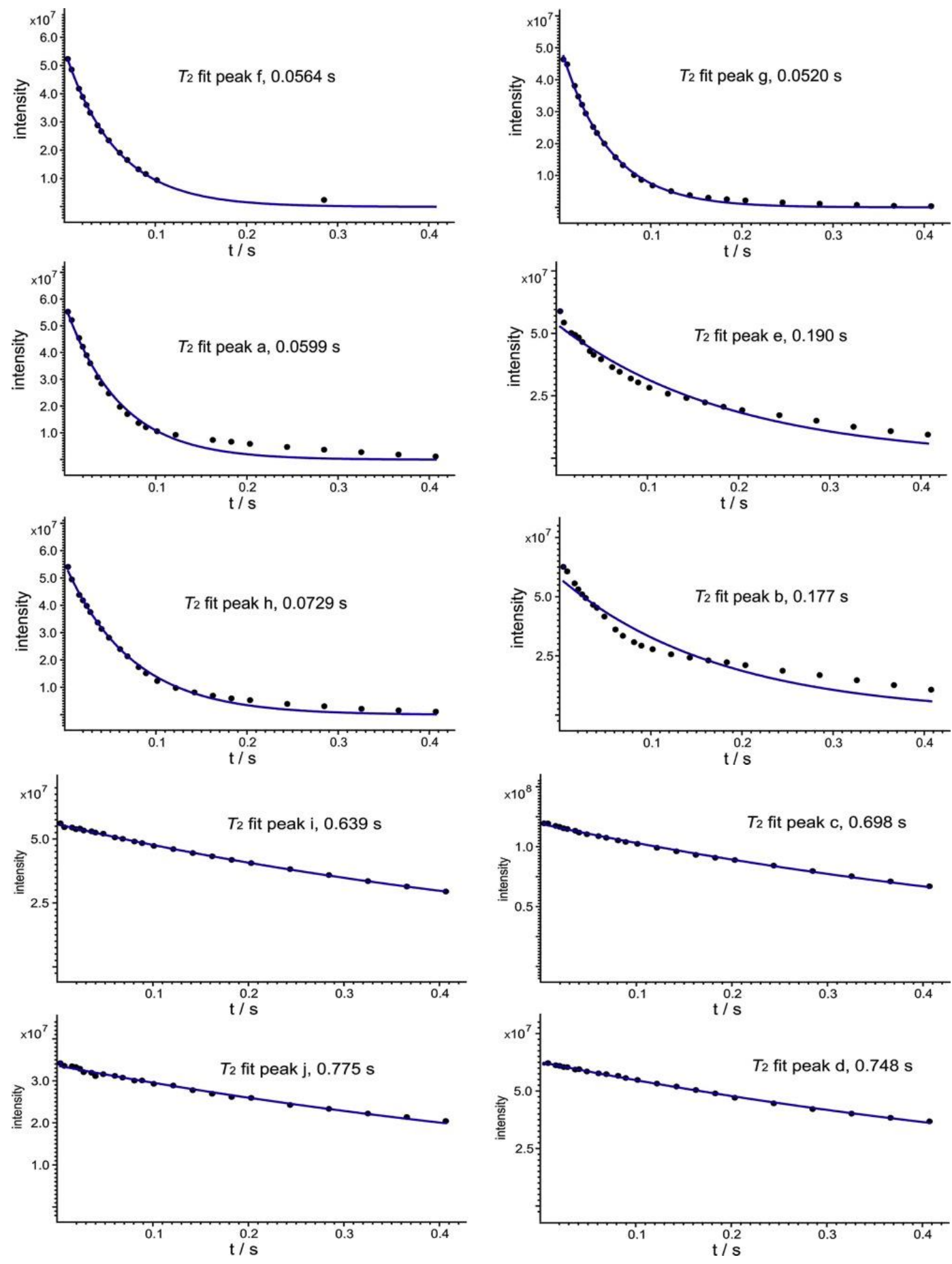

Figure 3.33: CPMG relaxation curves and exponential fits for the $T_{2}$ 's of 3.1 peaks a-j. 


\subsubsection{3 $T_{1}$ measurements on copper trimer 3.2}

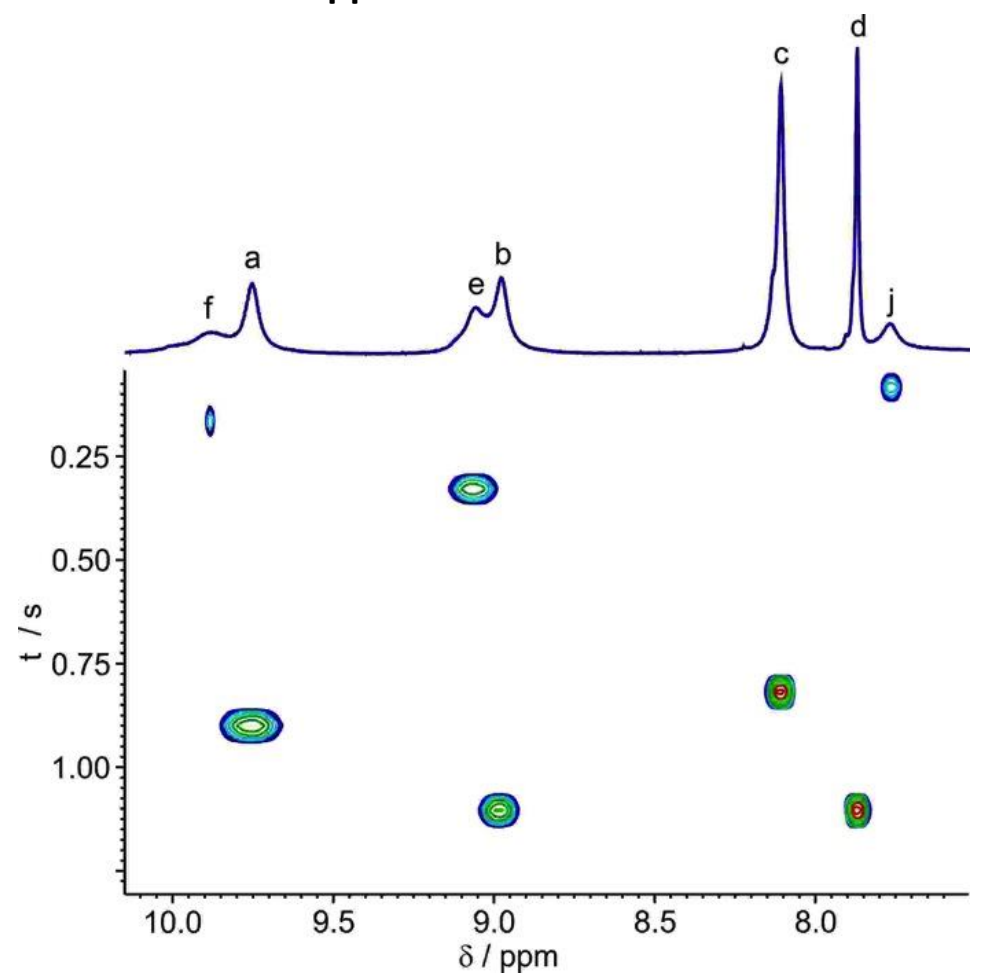

Figure 3.34: The ${ }^{1} \mathrm{H}$ NMR spectrum of $3.2\left(\mathrm{CDCl}_{3}, 700 \mathrm{MHz}, 298 \mathrm{~K}\right)$. Only the aromatic part of the spectrum is shown. The graph below shows the cross signals between chemical shift of the proton signals with respect to the corresponding $T_{1}$ relaxation time constants.

Table 3.3: Summary of the data describing the chemical shift of the aromatic proton signal of 3.2 with the accompanying $T_{1}$ relaxation time constant.

\begin{tabular}{|cccc|}
\hline Peak name & $\boldsymbol{\delta}(\mathbf{p p m})$ & $\boldsymbol{T}_{\mathbf{1}}(\mathbf{s})$ & error $(\mathbf{s})$ \\
\hline $\mathbf{f}$ & 9.878 & 0.146 & 0.033 \\
\hline $\mathbf{a}$ & 9.753 & 0.880 & 0.024 \\
\hline e & 9.063 & 0.346 & 0.019 \\
\hline b & 8.983 & 1.12 & 0.022 \\
\hline c & 8.110 & 0.813 & 0.004 \\
\hline d & 7.871 & 1.10 & 0.003 \\
\hline j & 7.767 & 0.099 & 0.020 \\
\hline
\end{tabular}



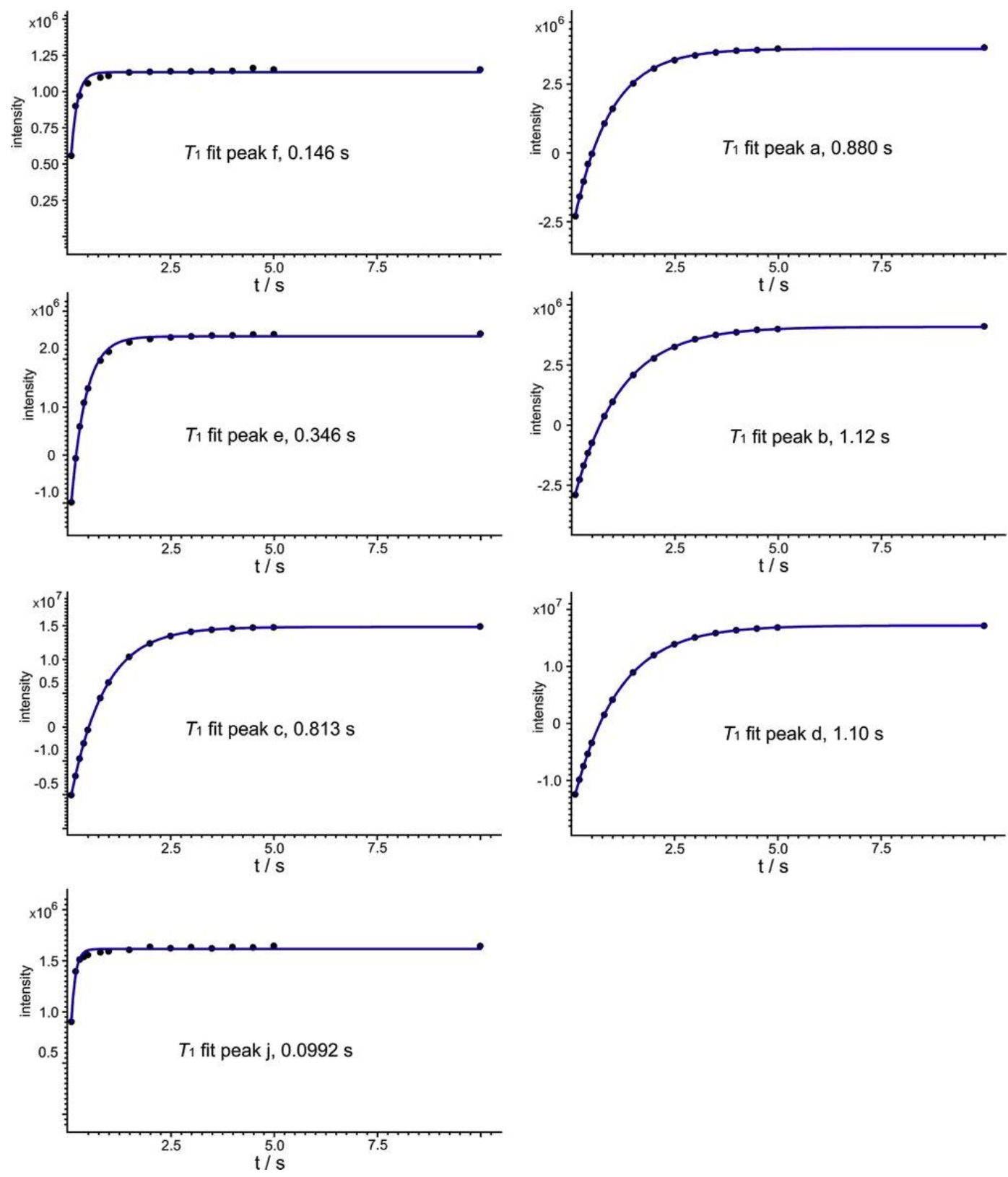

Figure 3.35: Inversion recovery curves and exponential fits for the $T_{1}$ 's of 3.2 peaks a-j. 


\subsubsection{4 $T_{2}$ measurements on copper trimer 3.2}

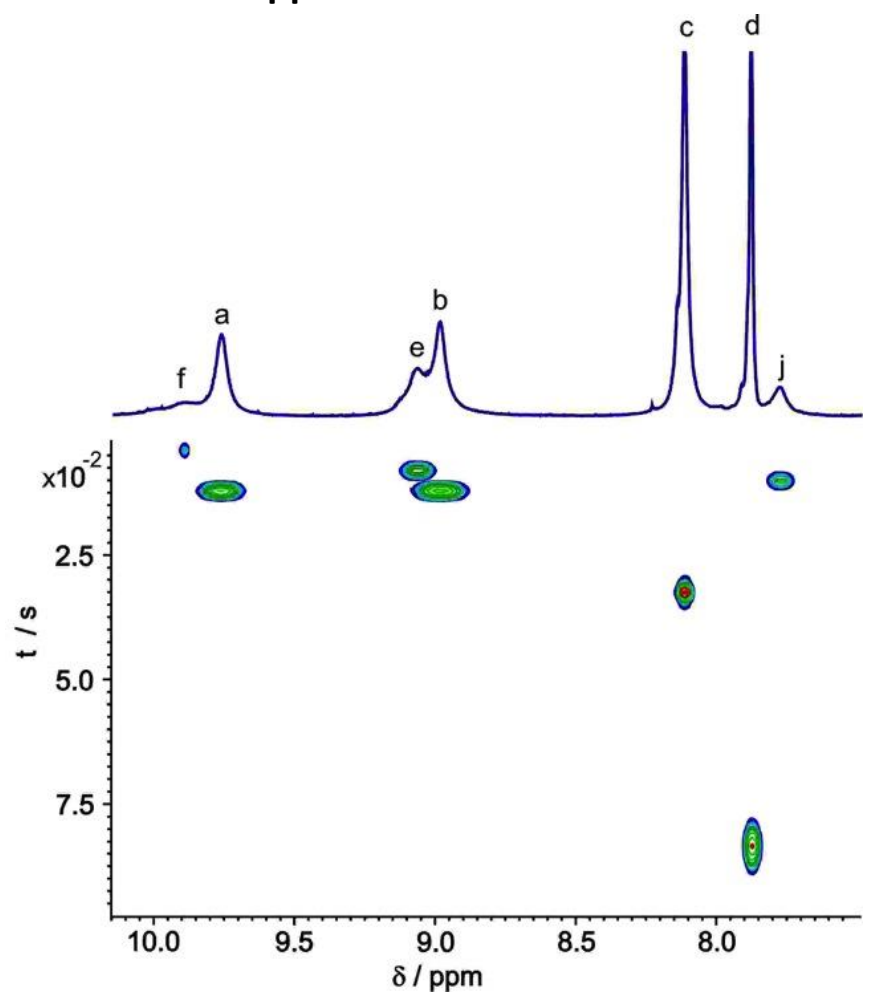

Figure 3.36: The ${ }^{1} \mathrm{H}$ NMR spectrum of $3.2\left(\mathrm{CDCl}_{3}, 700 \mathrm{MHz}, 298 \mathrm{~K}\right)$. Only the aromatic part of the spectrum is shown. The graph below shows the cross signals between chemical shift of the proton signals with respect to the corresponding $T_{2}$ relaxation time constants.

Table 3.4: Summary of the data describing the chemical shift of the aromatic proton signal of 3.2 with the accompanying $T_{2}$ relaxation time constants.

\begin{tabular}{|cccc|}
\hline Peak name & $\delta(\mathrm{ppm})$ & $T_{2}(\mathbf{s})$ & error (s) \\
\hline $\mathbf{f}$ & 9.887 & 0.00474 & 0.00018 \\
\hline a & 9.756 & 0.0116 & 0.0003 \\
\hline e & 9.060 & 0.00867 & 0.00040 \\
\hline b & 8.980 & 0.0128 & 0.0004 \\
\hline c & 8.114 & 0.0318 & 0.0012 \\
\hline d & 7.875 & 0.0831 & 0.0028 \\
\hline j & 7.774 & 0.0106 & 0.0009 \\
\hline
\end{tabular}



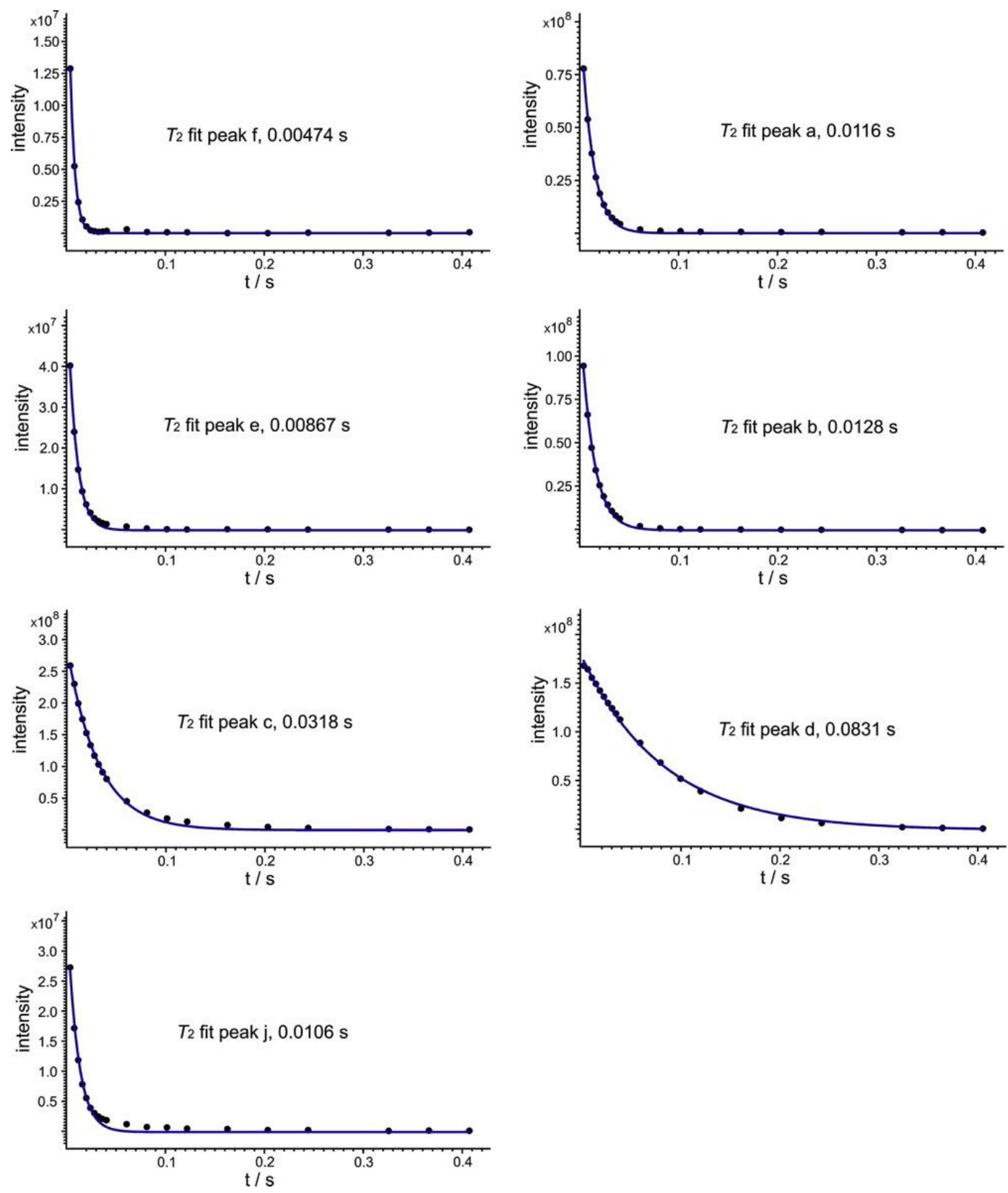

Figure 3.37: CPMG relaxation curves and exponential fits for the $T_{2}$ 's of 3.2 peaks a-j. 


\subsubsection{5 $R_{1}$ and $R_{2}$ decay rate versus distance plots}

The dipolar relaxation rate is expected to depend on the inverse of the $6^{\text {th }}$ power of the distance between a nucleus and an electron. We have taken the experimental $T_{1}$ and $T_{2}$ relaxation time constants and plotted the change in the corresponding rates $\left(R_{1}=1 / T_{1}\right.$ and $\left.R_{2}=1 / T_{2}\right)$ between 3.2 and 3.1 to the distance between the copper centre and the corresponding proton. The distance is estimated from crystal structures of similar oligomers. The experimental data were fitted to equation:

$$
y=\frac{A}{r^{6}}
$$

where $y$ is the change in relaxation rate $\left(\frac{1}{T_{1}(3.2)}-\frac{1}{T_{1}(3.1)}\right.$ or $\left.\frac{1}{T_{2}(3.2)}-\frac{1}{T_{2}(3.1)}\right), r$ is the distance between the proton and metal centre and $A$ is a free fitting parameter. The $T_{1}$ data fit with relatively good accuracy, confirming the expected distance dependence. The trend can also be observed for the $T_{2}$ data but the variation in the points in much larger and only an overall trend can be observed relating dipolar relaxation and distance between the two nuclei.
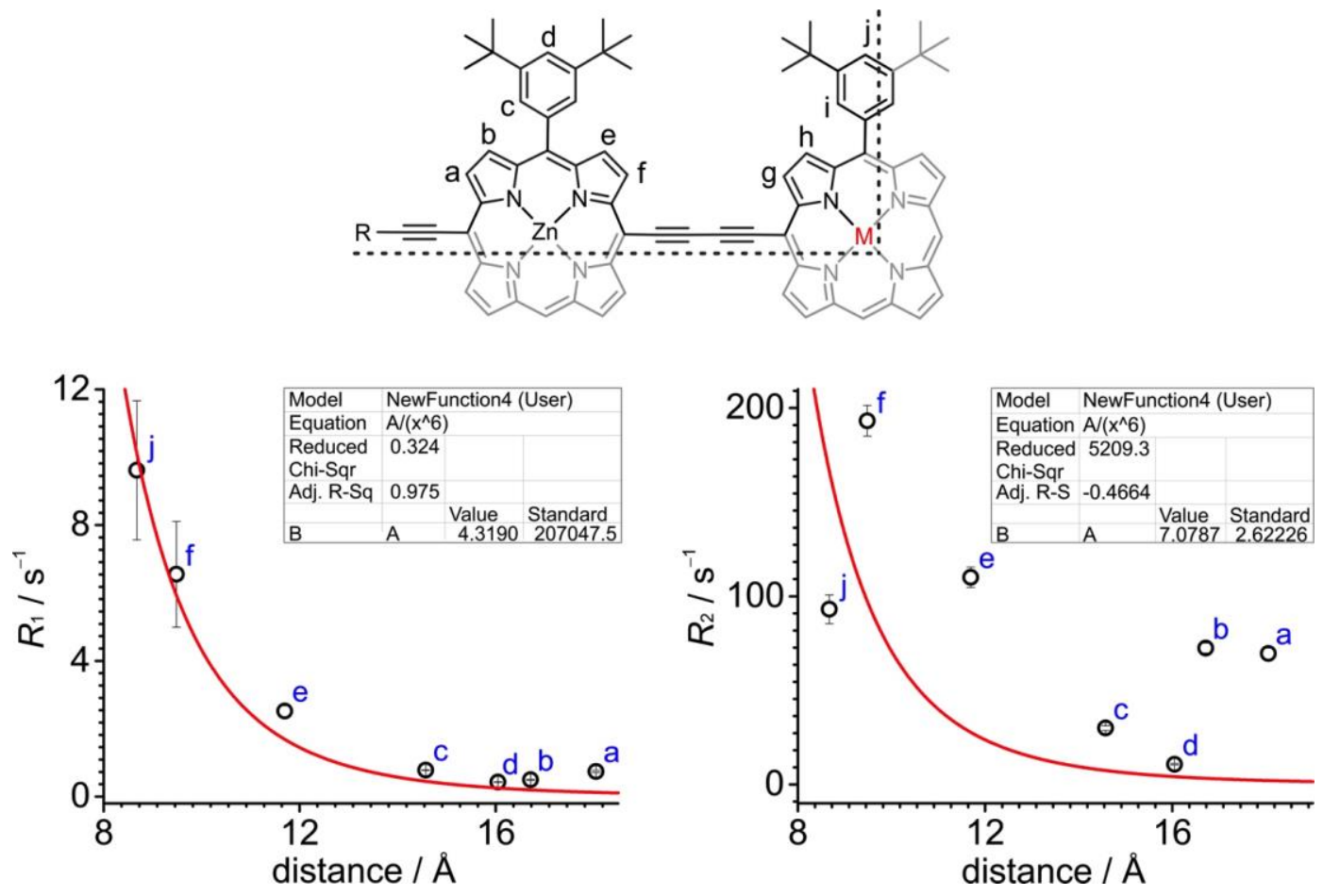

Figure 3.38: Changes in relaxation rates from comparing $\mathbf{R}_{1}$ and $\mathbf{R}_{2}$ values in $\mathbf{3 . 2}$ and 3.1 for peaks a-j. 


\subsubsection{EPR measurements}

All work relating to EPR and calculation has been performed by Dr Sabine Richert, Dr Ilya Kuprov and Dr Martin Peeks. For all experimental procedures related to these experiments, please refer main text and supplementary information reported in:

Constructive quantum interference in a bis-copper six-porphyrin nanoring

S. Richert, J. Cremers, I. Kuprov, M. D. Peeks, H. L. Anderson, C. R. Timmel, Nat. Commun. 2017, 8, 14842-14847. 


\section{Synthesis of Ortho, Meta and Para Bis-Copper Porphyrin Nanorings}

\section{Contents}

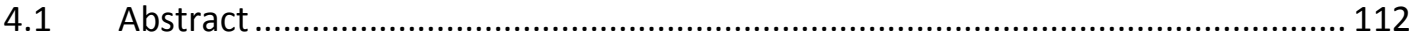

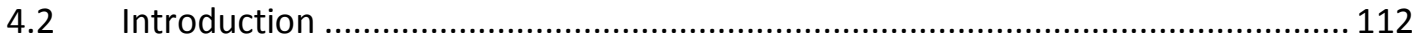

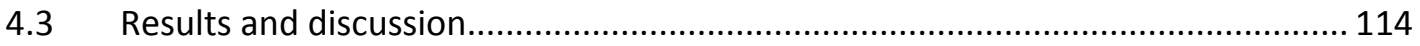

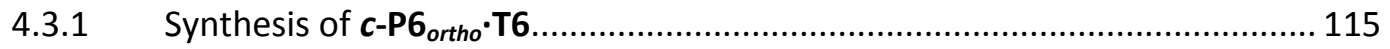

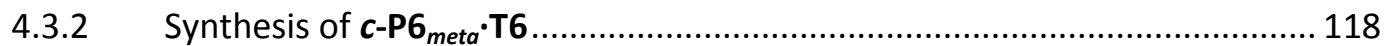

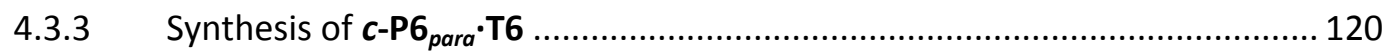

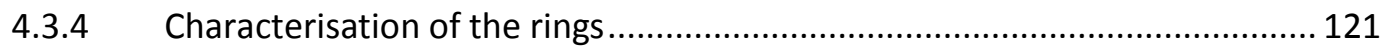

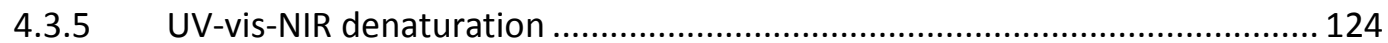

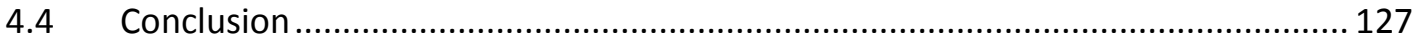

4.5 Experimental procedures and technical details ................................................... 128

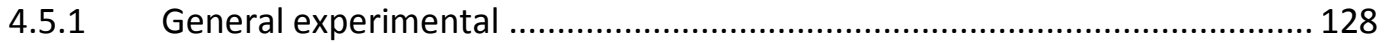

4.5.2 Synthetic procedures ................................................................................. 129

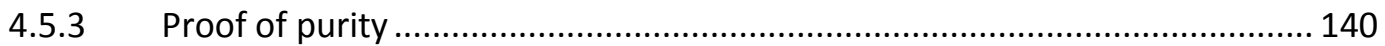

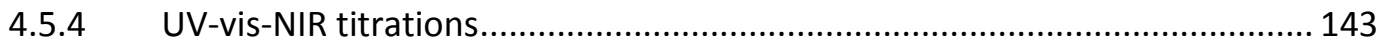

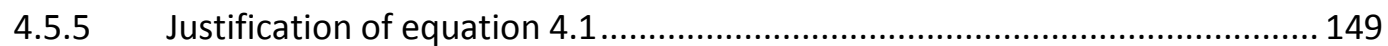




\subsection{Abstract}

The synthesis of three isomers of bis-copper 6-porphyrin nanorings is described. The structures differ only in the relative positions of the copper porphyrins within the ring. Generally $J$ coupling is expected to decay exponentially as a function of through-bond distance. However, DFT calculations performed by Dr Martin Peeks suggest that the coupling between the two copper centres in a 6-porphyrin nanoring does not solely depend on the distance between the ions, indicating potential interference phenomena. Experimental evidence to support these calculations would provide a relatively simple molecular system with behaviour resembling that of a hypothetical molecular interferometer. This chapter only describes the synthesis and characterisation of the rings. EPR measurements are currently underway.

\subsection{Introduction}

In Chapter 3 we demonstrated how the exchange interaction $(J)$ between two copper centres in a 6-porphyrin nanoring can be used as a measure for through-bond electronic communication. It was shown that the exchange coupling between the two spin centres was increased by a factor of 4.5 in a ring structure with two parallel coupling pathways as compared to an otherwise identical system with just one coupling path. ${ }^{160}$ This observation provides a signature of constructive quantum interference. This chapter considers the DFT calculated exchange coupling in three analogues of a bis-copper 6-porphyrin nanoring. The three structures differ only in the relative arrangement of the copper centres (Figure 4.1). The structures have been named by analogy to the benzene substitution pattern and will be referred to as the ortho, meta and para isomers of the bis-copper 6-ring.
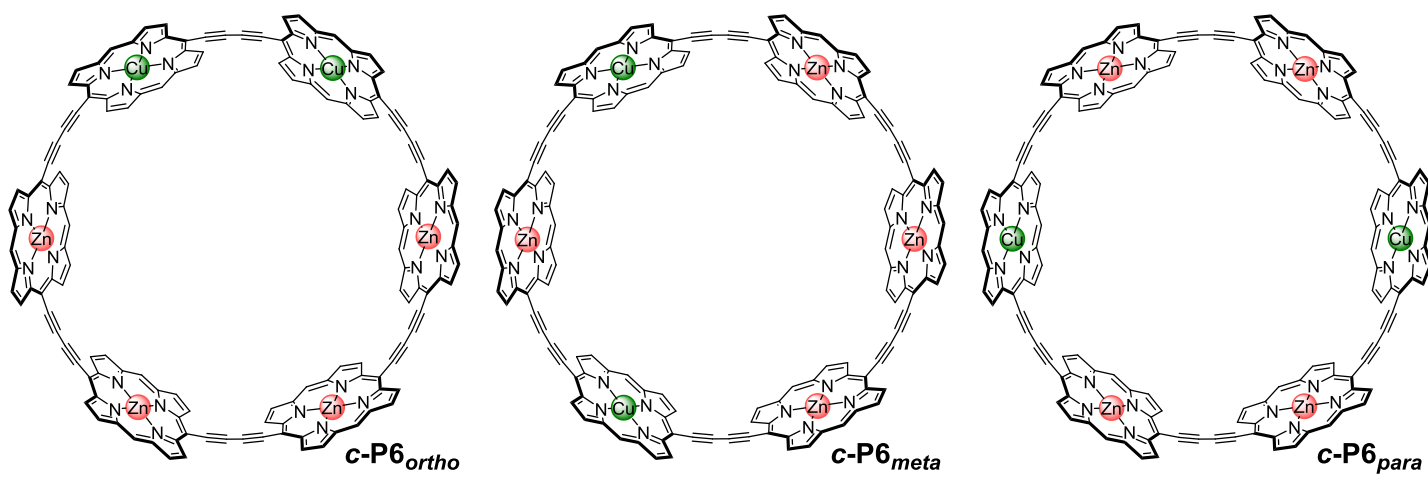

Figure 4.1: Chemical structures used in the DFT calculations. All calculations were conducted on the structures as shown, i.e. in the absence of template and with all porphyrin substituents truncated to hydrogens. 
Generally, one would expect the exchange coupling $J$ to decay rapidly as a function of through-bond distance between the copper centres (i.e. the largest coupling is expected in the ortho isomer and the lowest coupling in the para system). DFT calculations indicate the expected decrease in coupling when comparing the ortho to the meta isomer (Table 4.1). However, when comparing the meta to the para isomer, the coupling appears to increase despite the increase in the distance between the copper centres. This calculation potentially points towards interference effects. The sign of $J$ should not be misinterpreted and should be regarded as an absolute value. This means that the coupling in the para system of $-27 \mathrm{MHz}$ is larger than the coupling of $-2 \mathrm{MHz}$ in the meta isomer.

Table 4.1: Calculated (B3LYP/6-31G*) exchange coupling for the three isomers of the ring.

\begin{tabular}{|c|c|}
\hline Isomer & $J_{\text {DFT }}(\mathrm{MHz})$ \\
\hline c-P6 ortho & 160 \\
\hline$c-P 6_{m e t a}$ & -2 \\
\hline c-P6 para & -27 \\
\hline
\end{tabular}

Calculated spin density isosurfaces of the rings reveal that the spin is primarily localised on the copper centres when using high isovalues $\left(10^{-3}\right)$ (Figure 4.2).
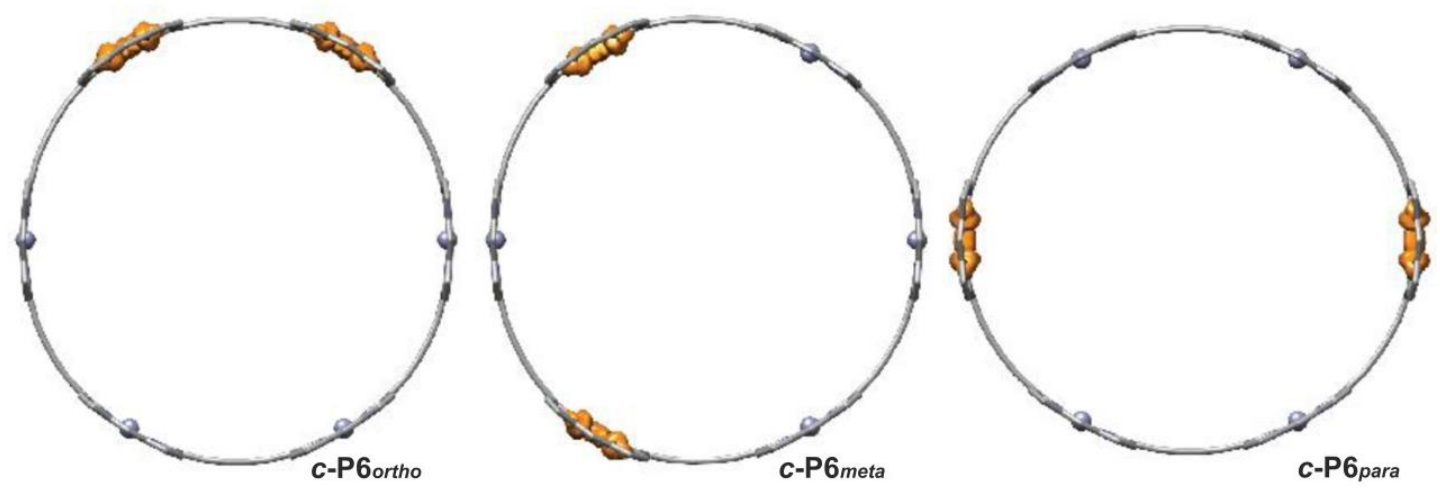

Figure 4.2: Spin density isosurfaces for the ortho, meta and para isomers of the ring at an isovalue of $10^{-3}$. The plots indicate that the triplet spin is primarily localised on the copper centres.

However, when the isovalues are decreased $\left(10^{-7}\right)$ the spin appears to spread further across the molecule (Figure 4.3). The plots show that spin was located on every porphyrin monomer in both $\mathbf{c}-\mathbf{P} \mathbf{6}_{\text {ortho }}$ and $\boldsymbol{c}-\mathbf{P} \mathbf{6}_{\text {para }}$. In contrast, in the $\boldsymbol{c}-\mathbf{P} \mathbf{6}_{\text {meta }}$ isomer the zinc porphyrin located meta to both copper centres appears to have no spin density. 


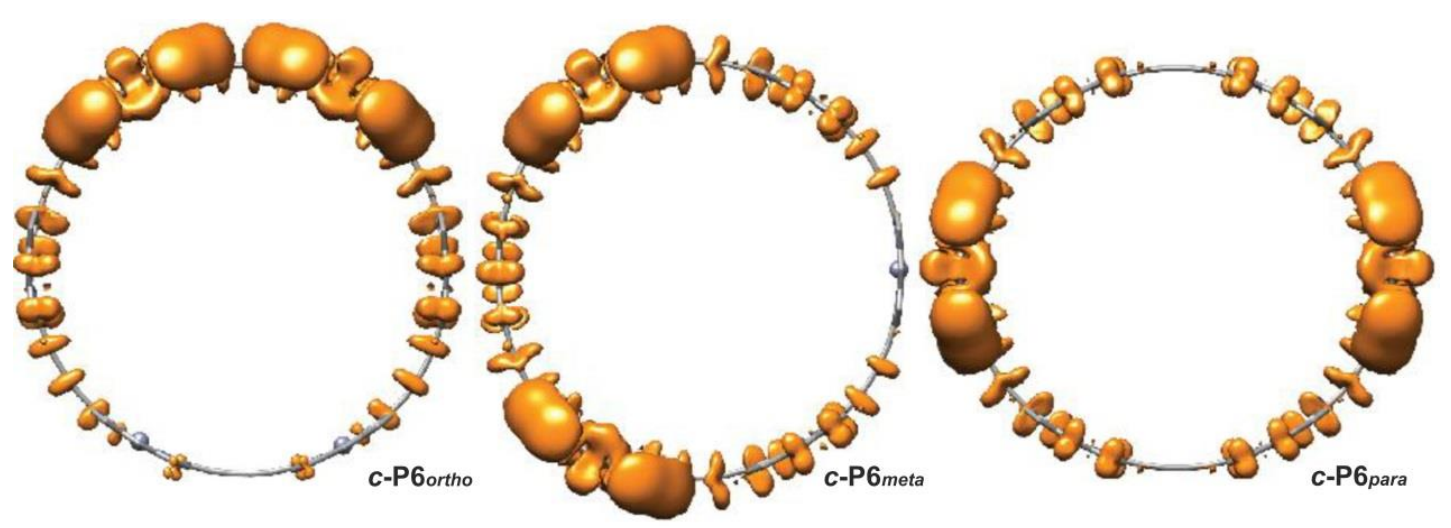

Figure 4.3: Spin density isosurfaces for the ortho, meta and para isomers of the ring at an isovalue of $10^{-7}$. The plots indicate that the triplet spin is spread over multiple porphyrins. Interestingly, in the meta system, one of the porphyrins shows no spin density.

The low-isovalue spin density plots suggest that the extent of spin delocalisation is not purely a function of distance. While in the ortho and para isomers, the spin appears to extend over two zinc porphyrin centres in both directions, in the meta isomer delocalisation only seems to extend over a single porphyrin unit. This observation could be an indication of destructive quantum interference. The increase in coupling between the copper centres in $\mathbf{c}-\mathbf{P} \mathbf{6}_{\text {para }}$ vs. $\mathbf{c}-\mathbf{P} \mathbf{6}_{\text {meta }}$ despite the increased separation distance is reminiscent of the behaviour of a hypothetical molecular interferometer. Experimental evidence to support the calculations would be extremely exciting. This chapter will describe the synthesis and characterisation of the three isomers of the bis-copper 6-ring. EPR measurements are currently underway.

\subsection{Results and discussion}

In the design of the synthetic routes for the three isomers of the ring it is important to incorporate the functionality desired in the final product in the linear starting materials. Since the binding of copper to the pyridyl templates is weak, it is necessary to integrate neighbouring zinc porphyrins to each copper centre to ensure a strong interaction with the template. This is essential in order to pre-organise the system such that a ring can be formed. As discussed in Chapter 3, the para substituted bis-copper ring was prepared from two linear trimers containing a central copper porphyrin with two adjacent zinc porphyrins. This reaction relied on the binding of the zinc porphyrins to the template which pre-organised the system sufficiently in order to couple the trimers and form the ring (Figure 4.4, left). 

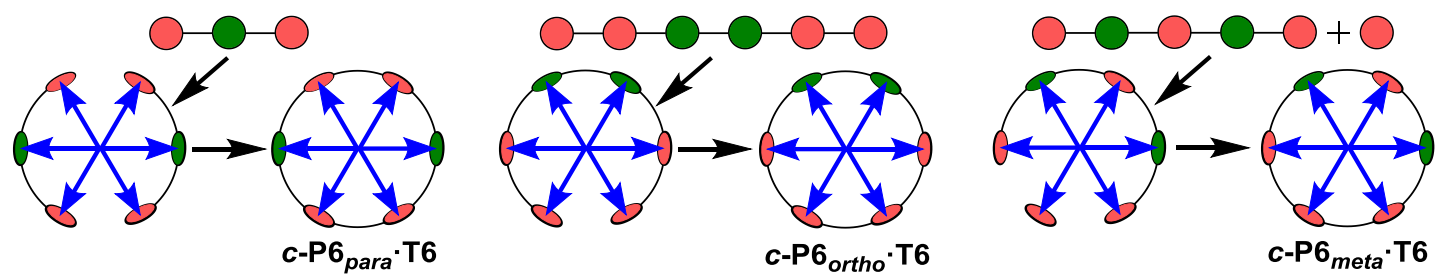

Figure 4.4: Schematic representation of synthetic routes to prepare the three isomers of the ring starting with linear oligomers. Zinc porphyrins are represented by red spheres and copper porphyrins are green.

The synthesis of the ortho ring was envisioned by using a linear hexamer containing a central copper dimer with two adjacent zinc dimers. The hexamer is expected to bind strongly to the $\mathbf{T 6}$ template and should allow for the formation of $\mathbf{c - P \mathbf { 6 } _ { \text { ortho } }}{ }^{\circ} \mathbf{T 6}$ (Figure 4.4, middle). The meta isomer does not contain a symmetric 6-porphyrin precursor that would exclusively lead to the formation of the desired product. However, it was anticipated that a linear pentamer containing alternating zinc and copper porphyrins would bind to the T6 template. The subsequent addition of a zinc monomer to the one-to-one complex was expected to bind to the remaining leg of the T6 template as shown on the right of Figure 4.4. Subjecting this three component complex to oxidative coupling conditions should lead to the formation of $\boldsymbol{c}-\mathbf{P} \mathbf{6}_{\text {meta }} \cdot \mathbf{T 6}$.

Throughout this project, THS-aryl solubilising groups were used due to its increased solubility with respect to the tert-butyl-aryls. In Chapter 2, the synthesis of the copper/zinc heterometallated oligomers was described going through a free-base intermediate due to the low solubility of the deprotected copper porphyrin monomers. When THS-aryls are utilised, the solubility of the copper monomers is sufficiently high that they can be used directly in the synthesis of heterometallated oligomers.

\subsubsection{Synthesis of $c-\mathrm{PG}_{\text {ortho }} \cdot \mathrm{T} 6$}

The synthesis of the ortho isomer of the ring was commenced with the preparation of the central copper dimer. Copper monomer $\mathbf{3 . 9}$ was statistically deprotected by the addition of TBAF to give 4.1 in a $42 \%$ yield. Homocoupling of 4.1 yielded the symmetrical dimer $\mathbf{4 . 2}$ in an $81 \%$ yield. Lastly, a deprotection of the acetylenes gave 4.3 in a quantitative yield (Figure 4.5). 


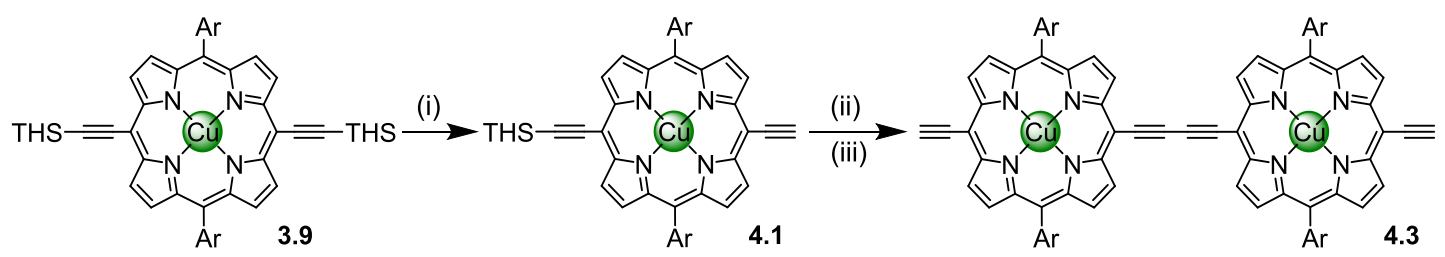

Figure 4.5: Synthesis of 4.3. (i) $\mathrm{TBAF}, \mathrm{CH}_{2} \mathrm{Cl}_{2} / \mathrm{CHCl}_{3}, 42 \%$; (ii) $\mathrm{PdCl}_{2}\left(\mathrm{PPh}_{3}\right)_{2}$, Cul, 1,4-benzoquinone, toluene/i- $\mathrm{Pr}_{2} \mathrm{NH}, 81 \%$; (iii) TBAF, $\mathrm{CH}_{2} \mathrm{Cl}_{2}, 100 \%$. Ar = 3,5-bis(trihexylsilyl)phenyl.

Next, a zinc porphyrin dimer was prepared (Figure 4.6). A zinc porphyrin analogue of 3.9 was prepared with cyanopropyldiisopropylsilyl (CPDIPS) protected acetylenes. A statistical deprotection on a porphyrin monomer with THS-aryl and THS protected acetylenes allows for the purification of the statistical mixture of porphyrins. However, on a THS-aryl dimer the polarity difference between starting material and mono/fully deprotected products is too small and does allow for the isolation of the desired mono-deprotected product. Introducing the cyano-moiety in the protecting group increases the polarity difference between the products and allows for an easy separation using silica gel column chromatography. Dimer 4.7 was prepared in three steps starting from monomer $\mathbf{4 . 4}$.

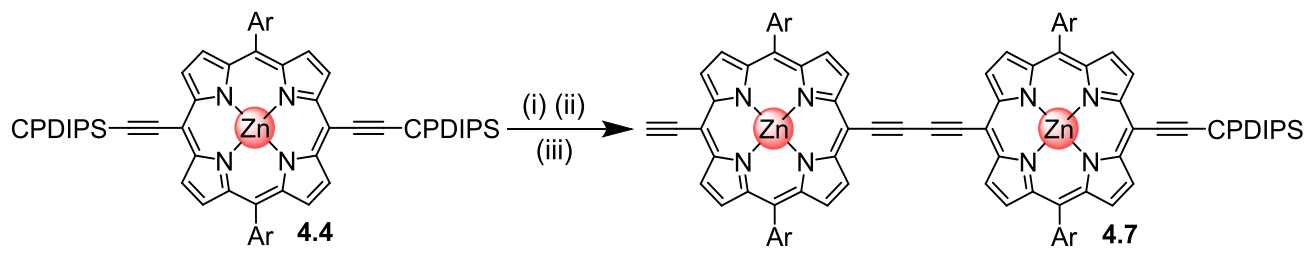

Figure 4.6: Synthesis of 4.7. (i) $\mathrm{TBAF}, \mathrm{CH}_{2} \mathrm{Cl}_{2} / \mathrm{CHCl}_{3}, 27 \%$; (ii) $\mathrm{PdCl}_{2}\left(\mathrm{PPh}_{3}\right)_{2}$, Cul, 1,4-benzoquinone, toluene/i- $\mathrm{Pr}_{2} \mathrm{NH}, 64 \%$; (iii) TBAF, $\mathrm{CH}_{2} \mathrm{Cl}_{2} / \mathrm{CHCl}_{3}, 25 \%$. $\mathrm{Ar}=3,5-$ bis(trihexylsilyl)phenyl.

When a five-to-one mixture of $\mathbf{4 . 7}$ and $\mathbf{4 . 3}$ was subjected to oxidative coupling conditions, a statistical mixture of oligomers was formed from which the hexamer 4.8 was isolated in a $42 \%$ yield after recycling GPC (Figure 4.7). Subsequent deprotection with TBAF afforded the hexamer 4.9 in a quantitative yield. 


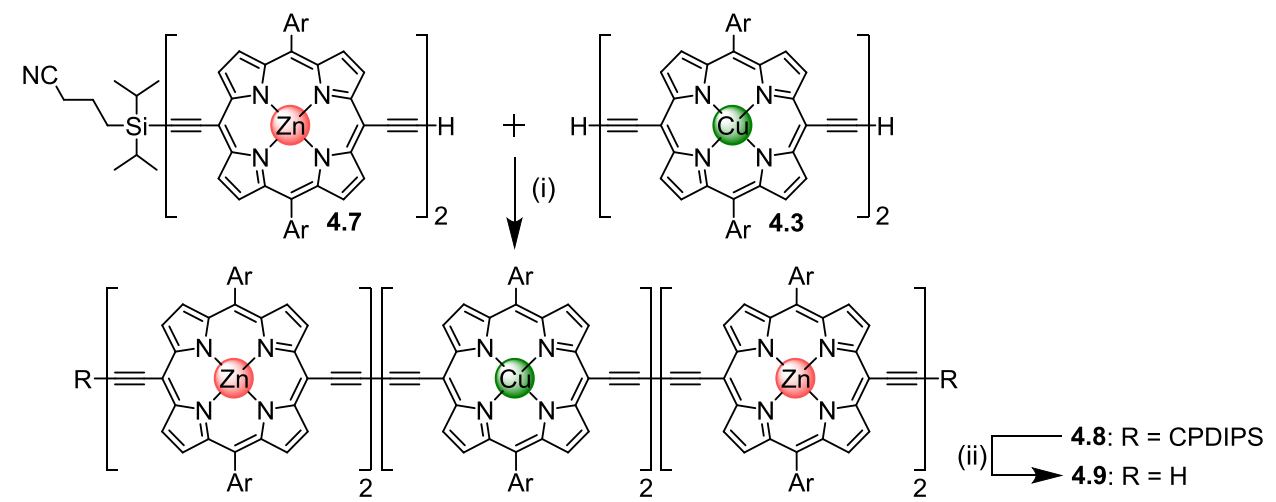

Figure 4.7: Synthesis of 4.9. (i) $\mathrm{PdCl}_{2}\left(\mathrm{PPh}_{3}\right)_{2}$, Cul, 1,4-benzoquinone, $\mathrm{CHCl}_{3} / i-\mathrm{Pr}_{2} \mathrm{NH}, 42 \%$; (ii) $\mathrm{TBAF}, \mathrm{CH}_{2} \mathrm{Cl}_{2}, 100 \%$. Ar = 3,5-bis(trihexylsilyl)phenyl.

The last step of the synthetic route depends on the formation of a stable one-to-one complex of the hexamer to the T6 template. The formation of the complex was probed by UV-vis-NIR titrations which showed a linear change in the absorbance upon the addition of T6 to a solution of $\mathbf{4 . 9}$ until one equivalent was reached indicating the formation of the complex. While there was a clear red shift in the Q-band, no characteristic splitting in the band was observed.
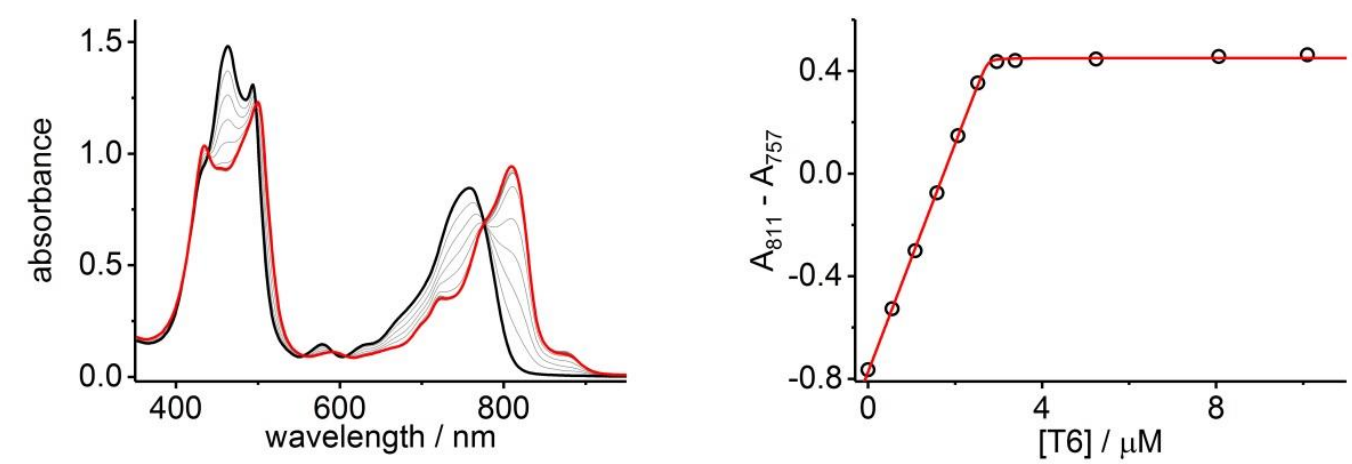

Figure 4.8: UV-vis-NIR titration of 4.9 and T6 in toluene at $298 \mathrm{~K}$. The formation of a stable one-to-one complex is indicated by the linear change up to the addition of one equivalent T6 after which no further change is observed in the spectra.

Subjecting the one-to-one complex to oxidative coupling conditions resulted in the formation $\mathbf{c}-\mathbf{P 6} \mathbf{o r t h o}_{\text {or }} \cdot \mathbf{T 6}$ in a $6 \%$ yield after purification by recycling GPC (Figure 4.9). Despite the apparent strong binding between the 4.9 and T6, the yield is low considering the high pre-organisation of the precursor and the fact that only a single carbon-carbon bond was formed in the course of this reaction. Nevertheless, $2.5 \mathrm{mg}$ of the product $\mathbf{c}-\mathbf{P 6 _ { o r t h o }}$.T6 was isolated which was sufficient for a full characterisation (see Section 4.3.4) of the structure and the planned EPR measurements. 

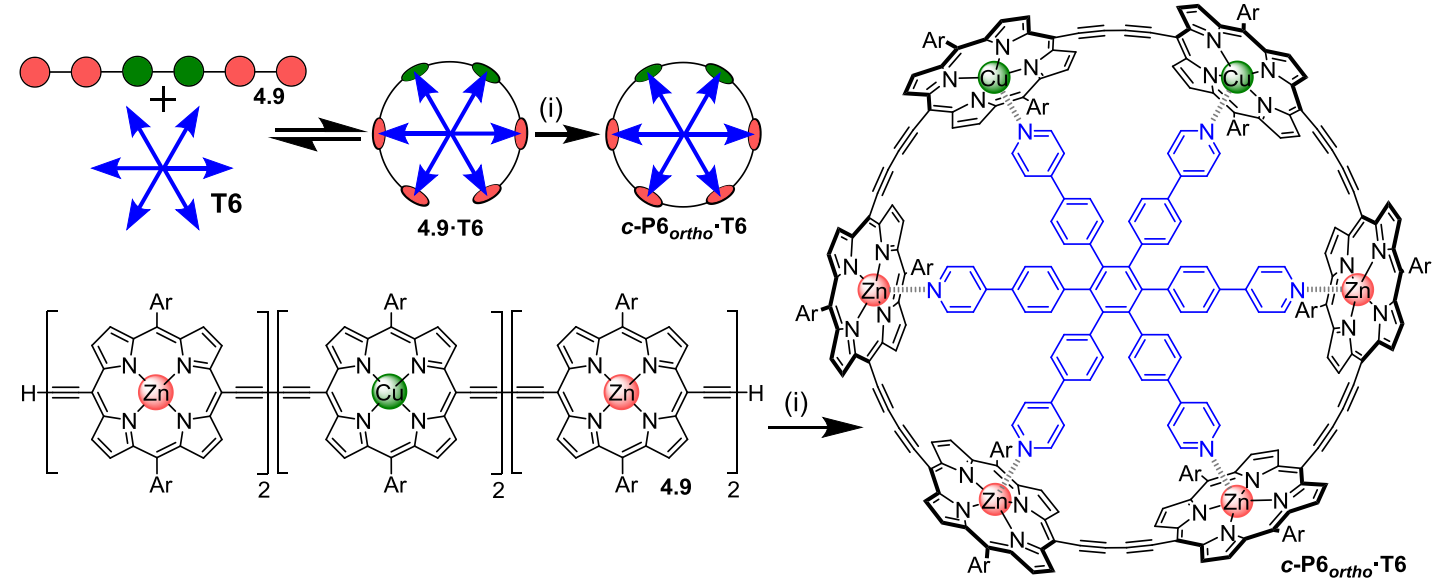

Figure 4.9: Synthesis of $c-\mathrm{PG}_{\text {ortho }} \cdot \mathrm{T6}$. Two representations of the synthetic route used to prepare the nanoring. (i) T6, $\mathrm{PdCl}_{2}\left(\mathrm{PPh}_{3}\right)_{2}, \mathrm{Cul}$, 1,4-benzoquinone, toluene/i- $\mathrm{Pr}_{2} \mathrm{NH}, 6 \%$. Ar = 3,5-bis(trihexylsilyl)phenyl.

\subsubsection{Synthesis of $c-\mathrm{P} 6_{m e t a} \cdot \mathrm{T} 6$}

The precursor to the meta isomer was prepared in a succession of statistical deprotection and coupling reaction steps. The first step was the oxidative coupling of 4.10 in the presence of an excess 4.1 which resulted in the formation of trimer $\mathbf{4 . 1 1}$ in a $28 \%$ yield. Subsequent deprotection of the trimer with TBAF gave 4.12 in a quantitative yield (Figure 4.10).
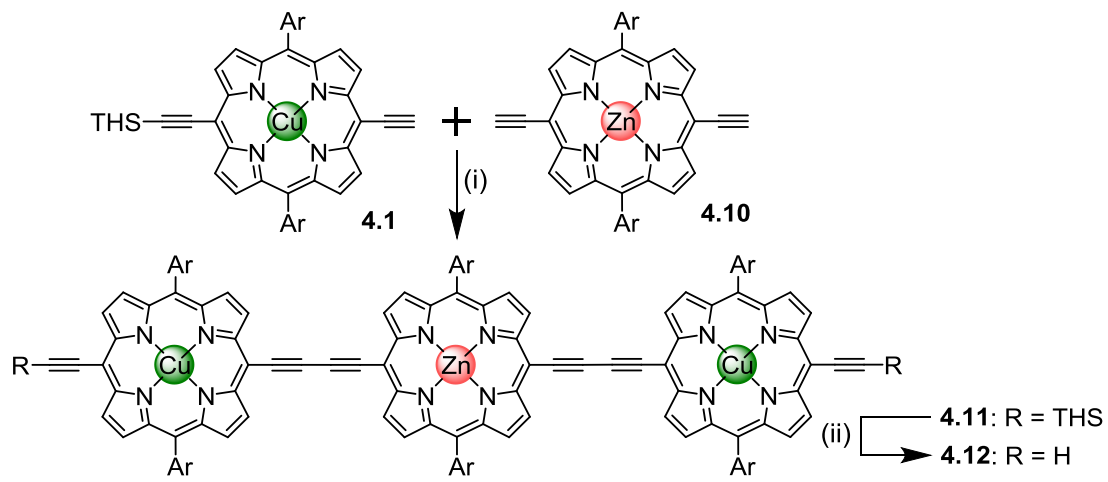

Figure 4.10: Synthesis of 4.12. (i) $\mathrm{PdCl}_{2}\left(\mathrm{PPh}_{3}\right)_{2}$, Cul, 1,4-benzoquinone, $\mathrm{CHCl}_{3} / i-\mathrm{Pr}_{2} \mathrm{NH}, 28 \%$; (ii) $\mathrm{TBAF} \mathrm{CH}_{2} \mathrm{Cl}_{2}$, $100 \%$. Ar = 3,5-bis(trihexylsilyl)phenyl.

The fully deprotected trimer $\mathbf{4 . 1 2}$ was subjected to oxidative coupling conditions in the presence of a large excess of the mono-deprotected zinc porphyrin monomer $\mathbf{4 . 5}$ to yield the pentamer $\mathbf{4 . 1 3}$ in a 55\% yield. Lastly, the pentamer was deprotected by the addition of TBAF in a $96 \%$ yield (Figure 4.11). 


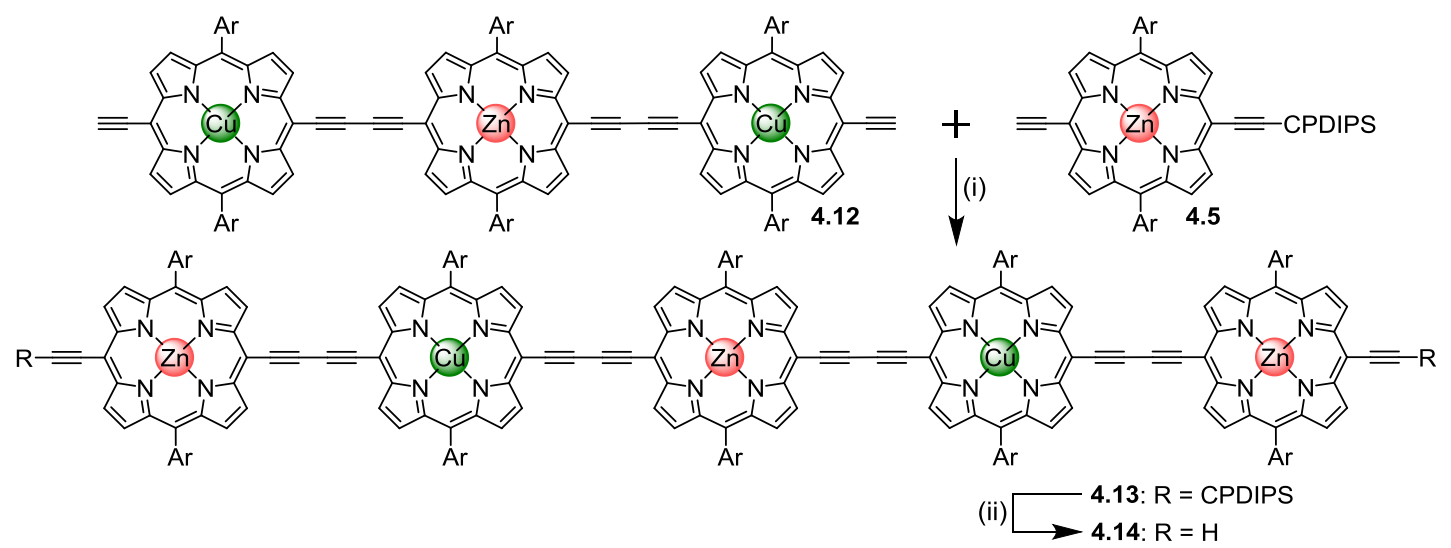

Figure 4.11: Synthesis of 4.14. (i) $\mathrm{PdCl}_{2}\left(\mathrm{PPh}_{3}\right)_{2}$, Cul, 1,4-benzoquinone, $\mathrm{CHCl}_{3} / i-\mathrm{Pr}_{2} \mathrm{NH}, 55 \%$; (ii) $\mathrm{TBAF}, \mathrm{CH}_{2} \mathrm{Cl}_{2}$, $96 \%$. Ar = 3,5-bis(trihexylsilyl)phenyl.

The binding of the pentamer $\mathbf{4 . 1 4}$ to T6 was investigated by UV-vis-NIR titrations (Figure 4.12). The formation of the desired one-to-one complex was observed with an association constant of $\mathrm{ca} \cdot 10^{7} \mathrm{M}^{-1}$.
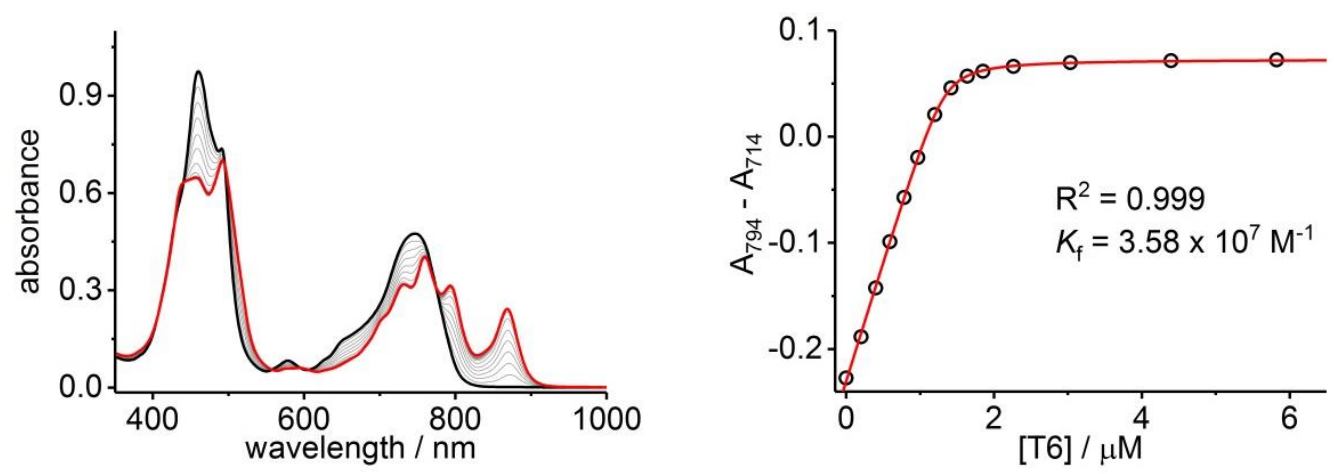

Figure 4.12: UV-vis-NIR titration of 4.14 and T6 in toluene at $298 \mathrm{~K}$. The formation of a one-to-one complex was observed. The titration shows that a slight excess of T6 will ensure full complex formation of the pentamer in solution.

Ten equivalents of the zinc porphyrin monomer $\mathbf{4 . 1 0}$ were added to the one-to-one mixture of 4.14 and T6. The large excess of monomer is necessary since the binding of a monomer to the remaining leg of the template is too low to form a stable three component complex. However, if a monomer couples to the pentamer complex in solution, the resulting hexamer will bind strongly to the template and pre-organise the system and allow for the formation of the target ring. When the mixture was subjected to oxidative coupling conditions, the ring was formed in a $5 \%$ yield after purification by recycling GPC (Figure 4.13). For characterisation of the ring refer to Section 4.3.4. 

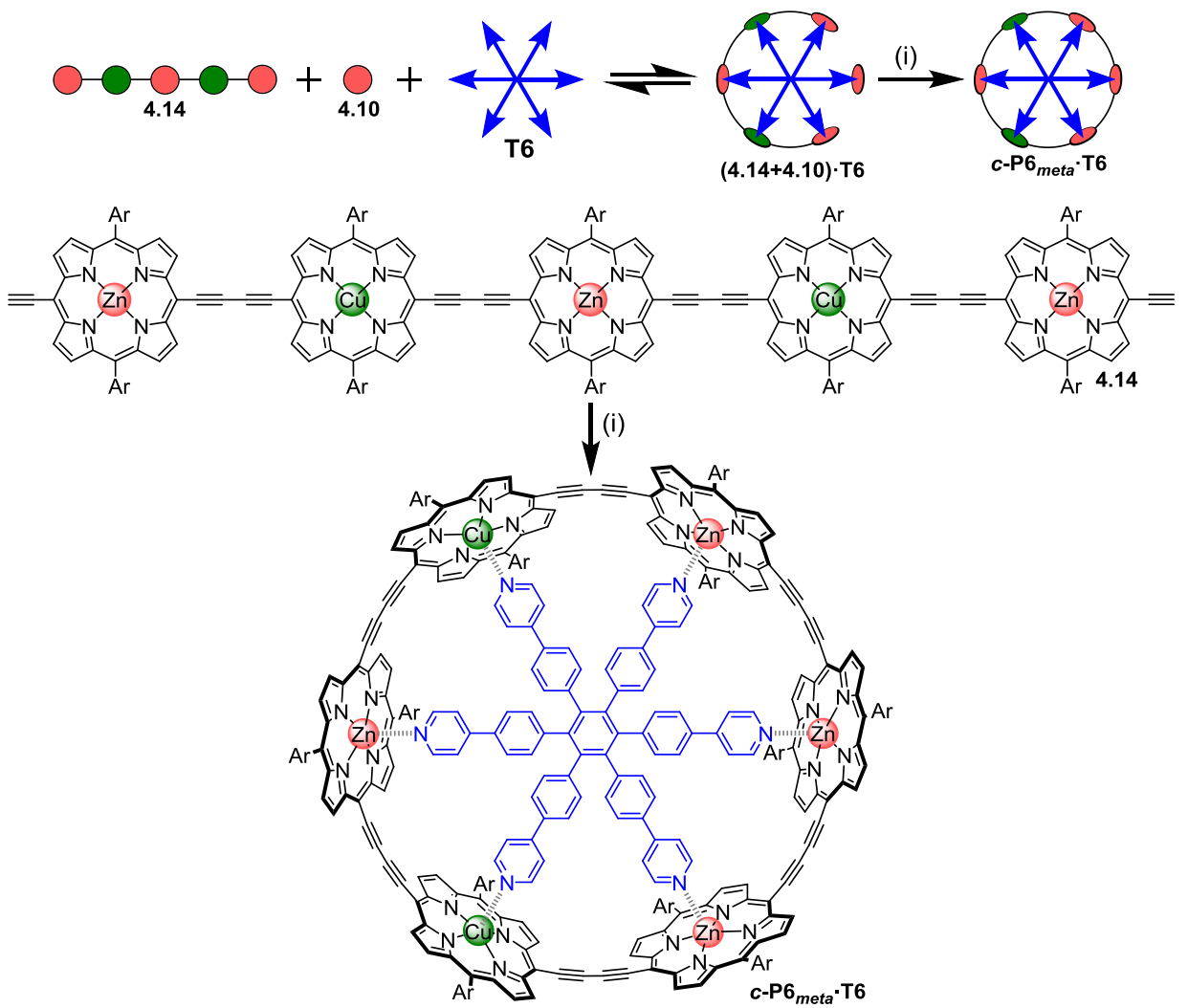

Figure 4.13: Synthesis of $c-\mathrm{P} 6_{\text {meta }} \cdot \mathrm{T} 6$. Two representations of the synthetic route used to prepare the nanoring. (i) T6, $\mathrm{PdCl}_{2}\left(\mathrm{PPh}_{3}\right)_{2}, \mathrm{Cul}$, 1,4-benzoquinone, toluene/i- $\mathrm{Pr}_{2} \mathrm{NH}, 5 \%$. Ar = 3,5-bis(trihexylsilyl)phenyl.

\subsubsection{Synthesis of $c-\mathrm{PG}_{\text {para }} \cdot \mathrm{T} 6$}

For homogeneity in the solubilising groups in all rings, a THS-aryl analogue of the para isomer was prepared. The synthesis was adapted from the synthetic route described in Chapter 3 with two variations. Due to the increased solubility of the THS-aryls, a heterometallated trimer was formed directly from zinc and copper porphyrin precursors without going through a free-base trimer intermediate. In addition, the solvent in the ring-forming step was changed from $\mathrm{CHCl}_{3}$ to toluene which increased the yield from $2 \%$ to $14 \%$, potentially due to the increased binding affinity between the template and the oligomer (Figure 4.14). The characterisation of the ring will be described in Section 4.3.4. 


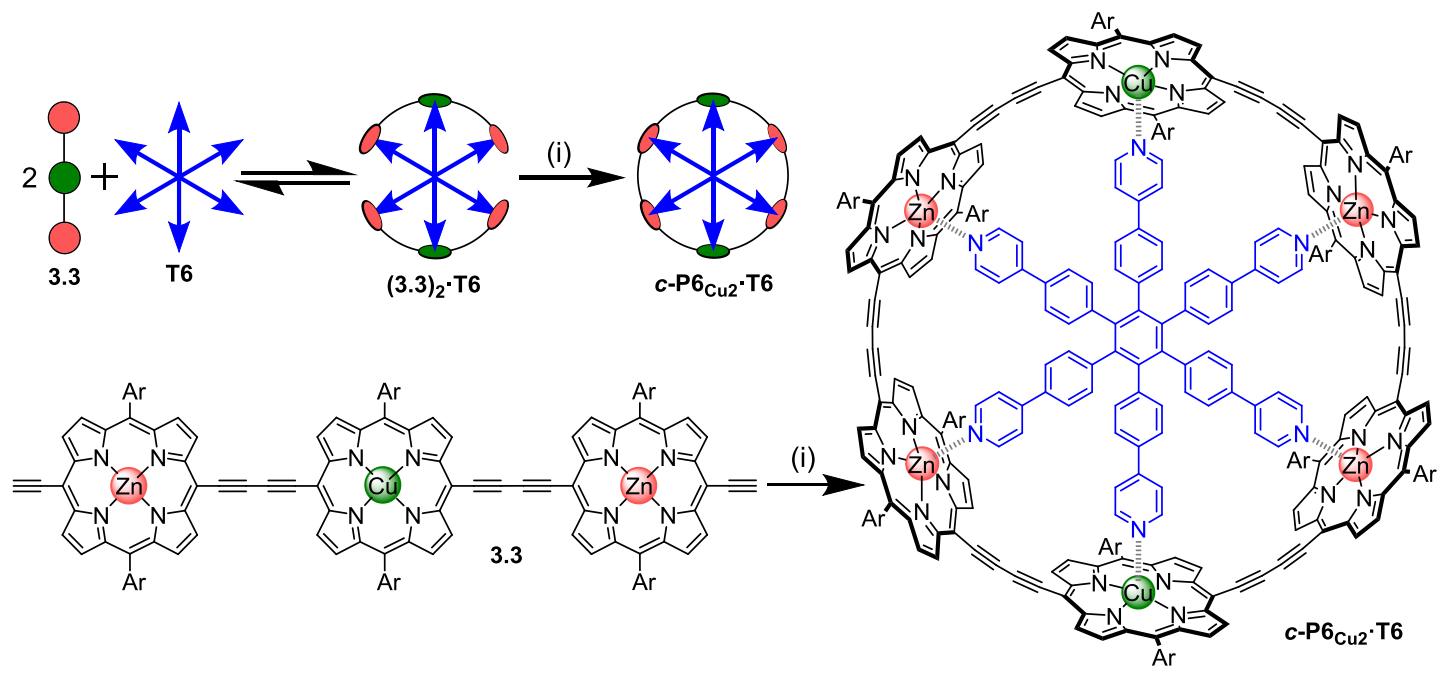

Figure 4.14: Synthesis of $c-\mathrm{P} 6_{\text {para }} \cdot \mathrm{T} 6$. Two representations of the synthetic route used to prepare the nanoring. (i) T6, $\mathrm{PdCl}_{2}\left(\mathrm{PPh}_{3}\right)_{2}$, Cul, 1,4-benzoquinone, toluene/i- $\mathrm{Pr}_{2} \mathrm{NH}, 14 \%$. Ar = 3,5-bis(trihexylsilyl)phenyl.

\subsubsection{Characterisation of the rings}

The presence of the paramagnetic centres complicates the characterisation of the rings by ${ }^{1} \mathrm{H}$ NMR spectroscopy. By combining several characterisation techniques it is possible to confirm the structures. The preparative GPC traces of the reaction mixtures and the clean ring samples are shown in Figure 4.15. The rings are all similar in size and therefore have identical retention times of 62.5 min on GPC, comparable to its fully zinc analogue. 

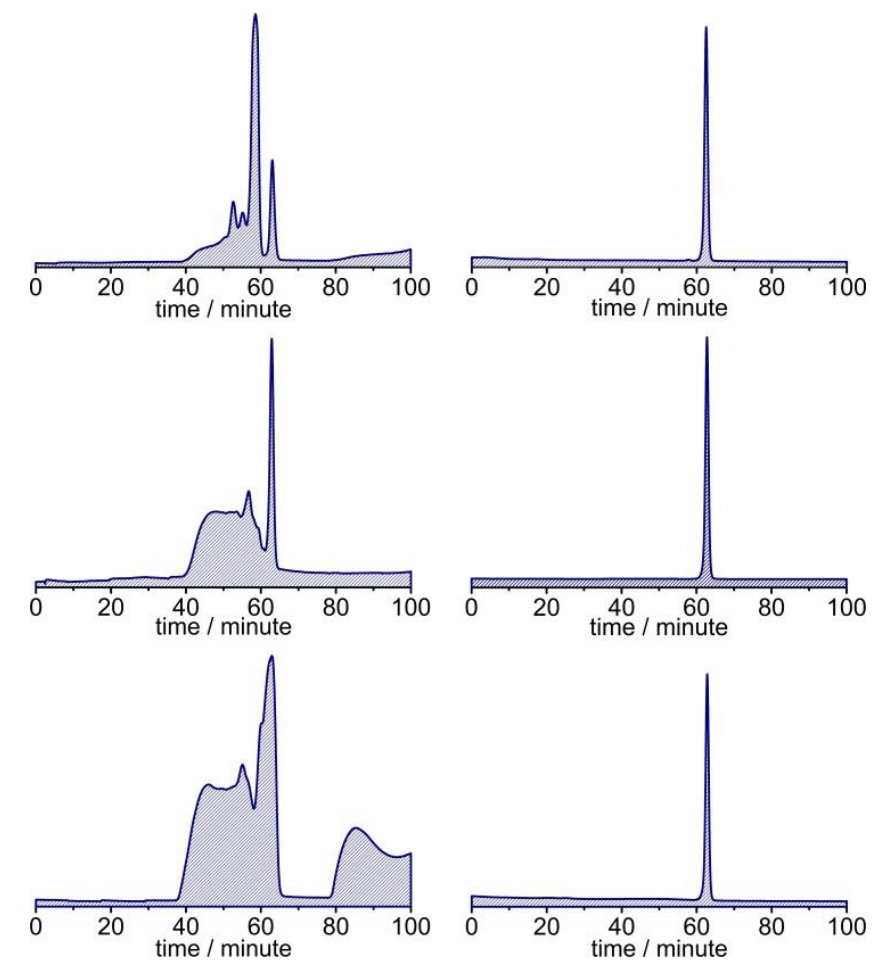

Figure 4.15: Preperative GPC traces of the reaction mixtures of the ring forming reactions (left) and the clean traces of the rings (right). From top to bottom the spectra correspond to the ortho, meta and para isomers of the bis-copper 6-ring. (JAIGEL columns, $1^{\text {st }}$ cycle shown, $1 \%$ pyridine in toluene, detection at $800 \mathrm{~nm}$ )

To obtain high resolution mass spectra, samples of the rings were submitted to the National Mass Spectrometry Facility in Swansea. Figure 4.16 shows the data recorded for the $\boldsymbol{c}-\mathbf{P} \mathbf{6}_{\text {para }} \cdot \mathbf{T 6}$ sample which illustrate the quality of the data that they are able to record for molecules with an $\mathrm{m} / \mathrm{z}$ exceeding 11,200 .

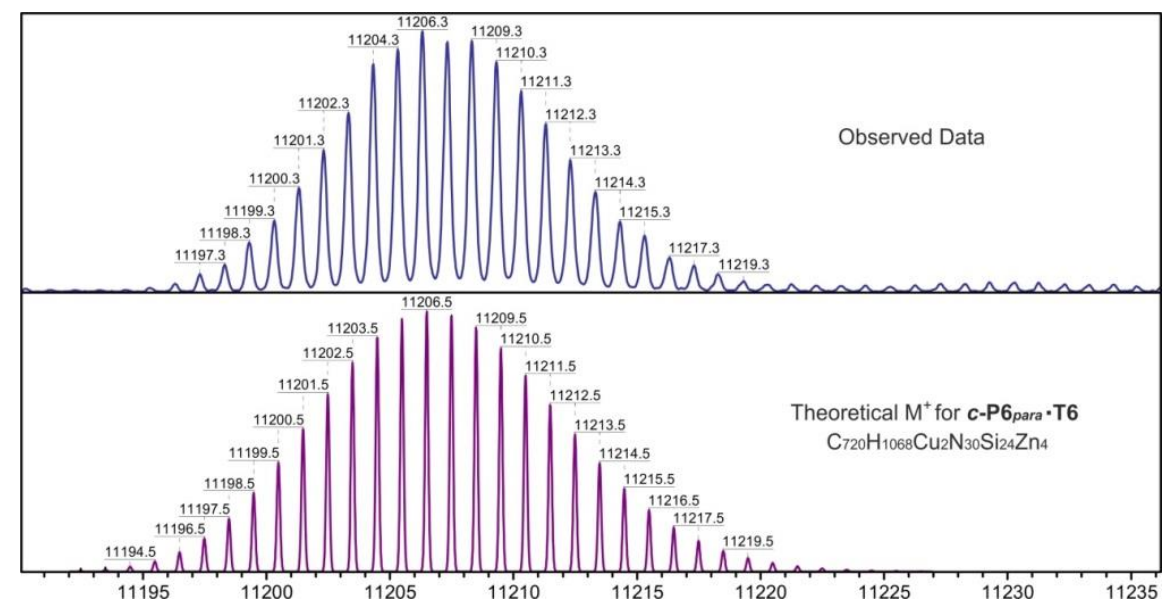

Figure 4.16: The MALDI-MS spectrum of $c$ - $\mathrm{P6}$ para $\cdot \mathrm{T6}\left(\mathrm{m} / \mathrm{z}=11,206\left(\mathrm{C}_{720} \mathrm{H}_{1068} \mathrm{Cu}_{2} \mathrm{~N}_{30} \mathrm{Si}_{24} \mathrm{Zn}_{4}, \mathrm{M}^{+}\right.\right.$requires 11,207$)$, matrix: DCTB).

As demonstrated in Chapter 3 the extend of broadening in the ${ }^{1} \mathrm{H}$ NMR spectra of linear trimers containing copper centres in the central porphyrin provides information on the distance of the protons with respect to the copper centre. Due to 
the large amount of spectral overlap in the ring samples, it was not possible to carry out $T_{1}$ and $T_{2}$ relaxation time measurements on the rings. However, the aromatic region of the ${ }^{1} \mathrm{H}$ NMR spectra provides some structural information (Figure 4.17).

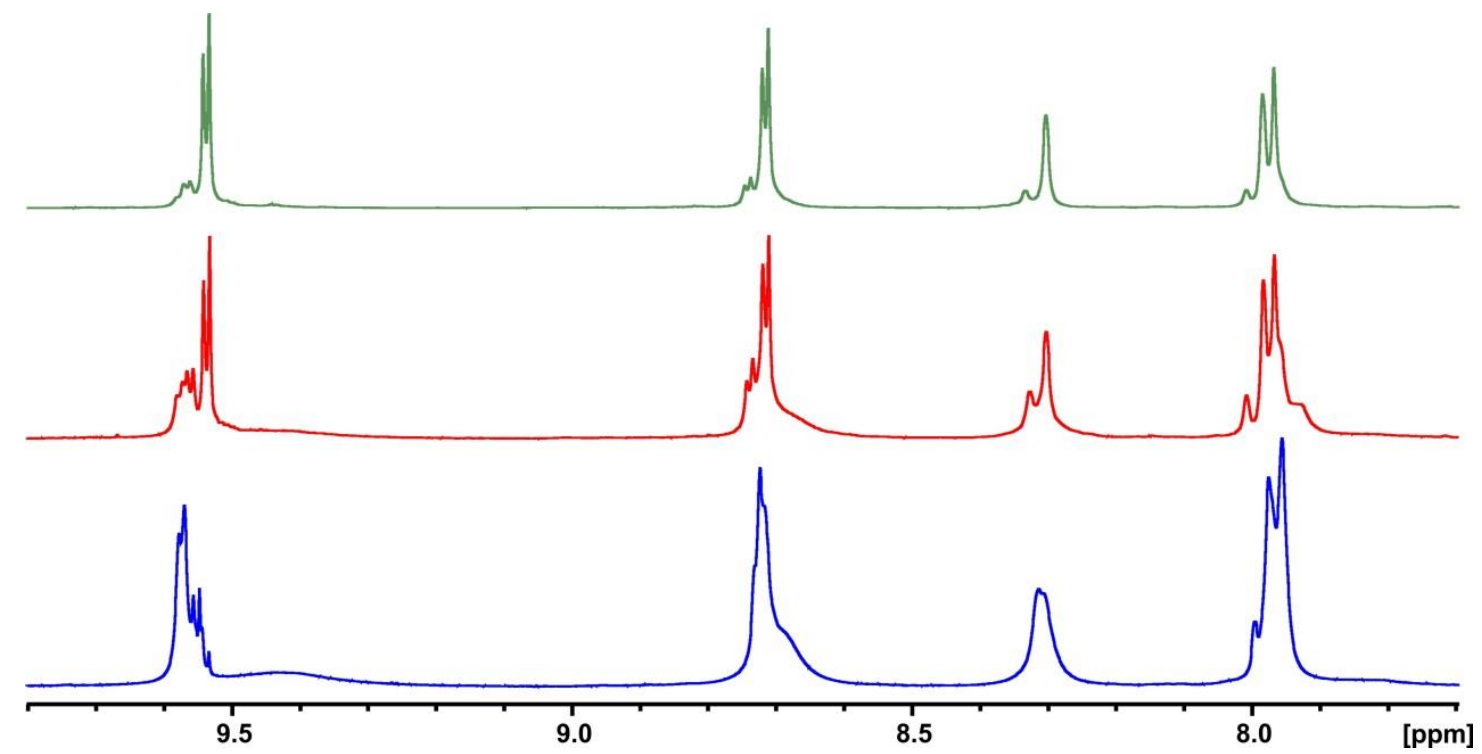

Figure 4.17: ${ }^{1} \mathrm{H}$ NMR spectra of the ortho, meta and para isomers of the bis-copper 6-ring (from top to bottom respectively) recorded in $\mathrm{CDCl}_{3}$ at $500 \mathrm{MHz}$.

A regular 6-porphyrin nanoring containing solely zinc centres gives rise to a highly symmetric ${ }^{1} \mathrm{H}$ NMR spectrum. ${ }^{165}$ In the bis-copper rings the symmetry is lost due to the presence of the heterometallated porphyrins (Figure 4.18). The $\beta$-protons (A) on the copper porphyrin have broadened beyond recognition due to their close proximity to the copper. The signals for $\mathbf{B}$ and $\mathbf{C}$ have preserved some structure and the $\beta$ protons positioned furthest away from the copper centre (D) have remained sharp (9.54 ppm). As a result of the broadening and overlap of the signals, integrating the peaks does not provide accurate information, but it can be seen that the ratio between the sharp and broad multiplets corresponding to the $\beta$-protons (ca. $9.56 \mathrm{ppm}$ and $8.28 \mathrm{ppm}$ ) vary between the rings which is in agreement with the expected ratio shown in Figure 4.18. 

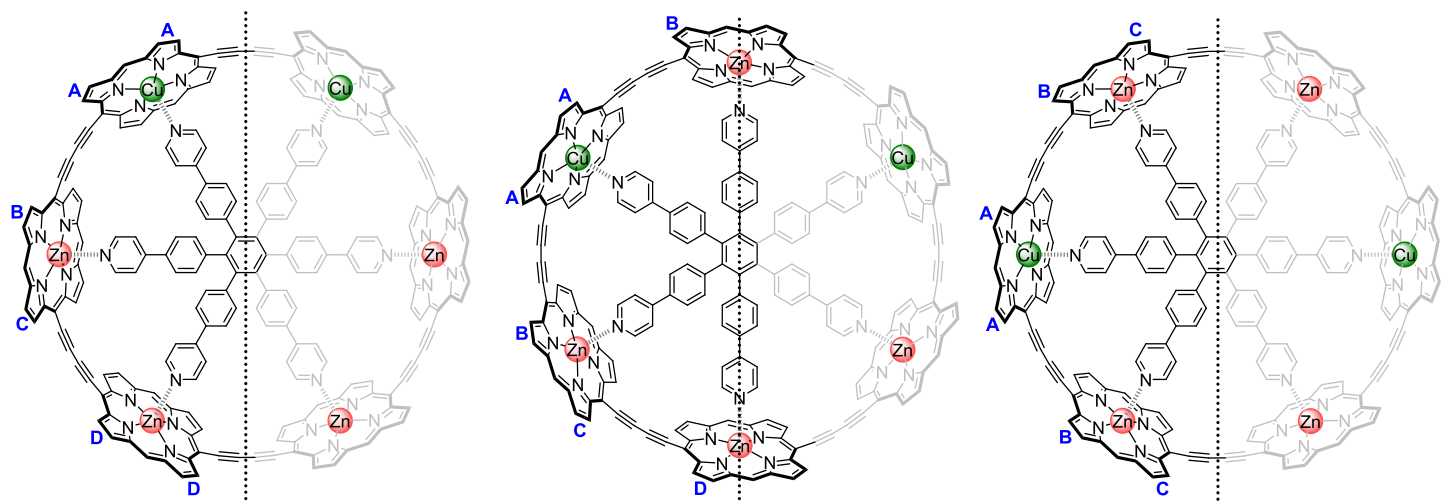

Figure 4.18: Structures of the three isomers of the ring with labels on the $\beta$-protons based on the distance to the copper centres.

\subsubsection{UV-vis-NIR denaturation}

The cooperative binding effect of the hexadentate ligand in combination with the good size match between the rings and T6 results in a high binding affinity. Despite the strength of the interaction, the template can be displaced by the addition of a large excess of a competing ligand such as DABCO. In the past, all measurements on the binding strength of T6 within porphyrin nanorings have been performed on rings containing solely zinc centres. The binding of the template in the ortho, meta and para systems was measured to investigate the effect of the copper interaction on the overall binding to the template.

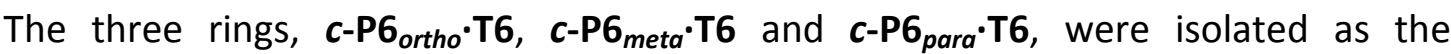
one-to-one complex with the template T6. Denaturation titrations were performed in order to examine the stability of the complexes (Figure 4.19). A large excess of DABCO was titrated into solutions of the one-to-one complexes ( $c a .0 .6 \mathrm{M}$ in toluene at $298 \mathrm{~K}$ ) to displace the T6 template. Additionally, a fully zinc 6-porphyrin nanoring, c-P6·T6, was investigated under identical conditions for comparison reasons. 

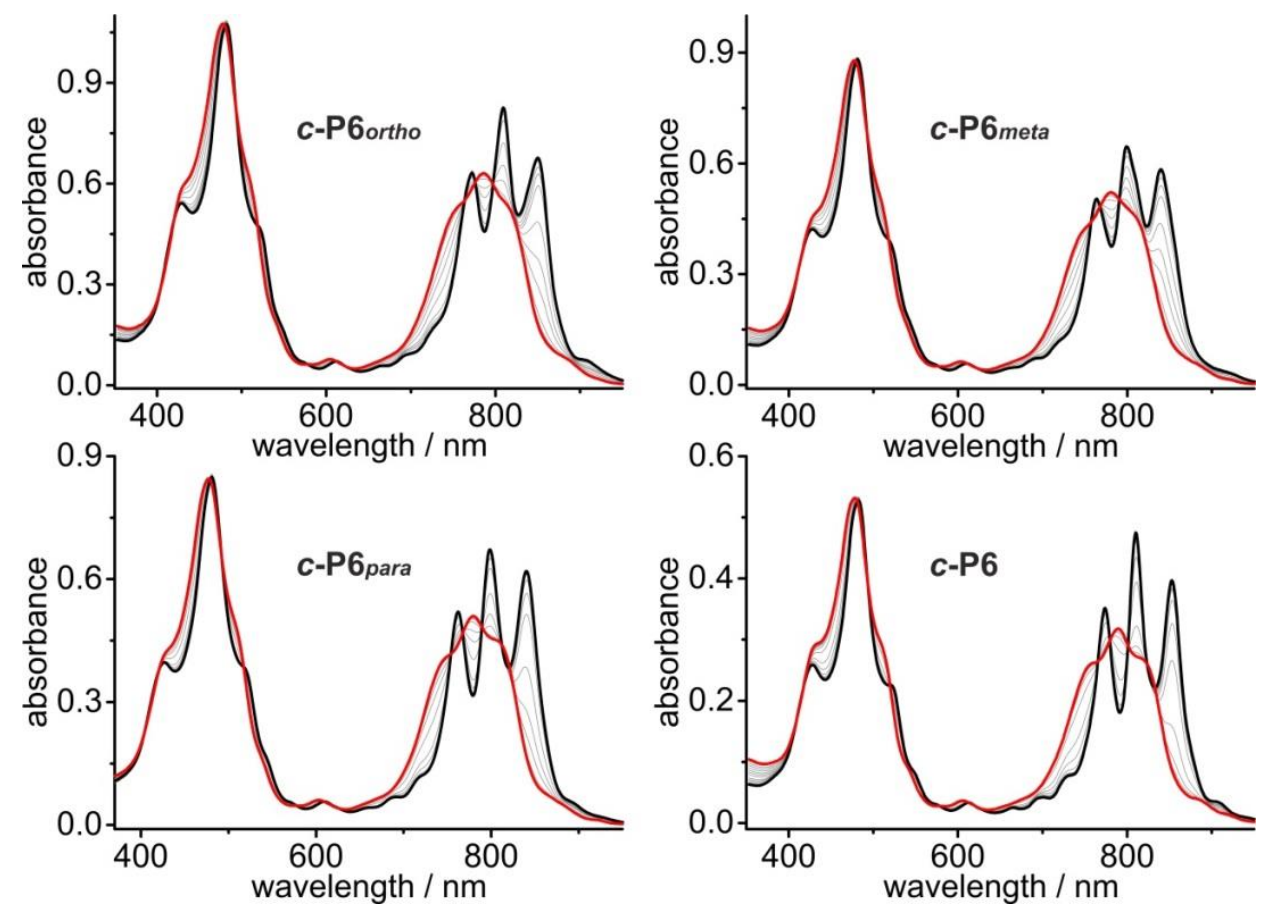

Figure 4.19: UV-vis-NIR denaturation titrations of the 6-ring complexes with DABCO (toluene at $298 \mathrm{~K}$ ). The spectra of the one-to-one complexes are shown in black and the spectra of the DABCO-saturated rings in red.

The absorption spectra of the templated rings are more red-shifted (black lines) than the final spectra (red lines) in which the complex is completely denatured. A characteristic three-finger pattern is observed for all templated structures which is caused by the more rigid structures and reduced freedom of the individual porphyrins to rotate when the template is bound. The sharpest features are observed in c-P6.T6 were every porphyrin is locked into position by the template. Slightly broader Q-band features are observed in the bis-copper rings. The broadening is potentially caused by the increased freedom to vary the porphyrin-porphyrin dihedral angles of the copper porphyrins. The fully denatured complexes gave rise to nearly indistinguishable absorption spectra in all cases.

The experimental and calculated binding isotherms for the denaturation titrations fit a 6-site binding model and suggest comparable denaturation constants for all complexes (Figure 4.20). This observation suggests that the DABCO concentration needed to displace T6 is equal for the fully zinc and the bis-copper systems. This result is counter-intuitive since we expected that the presence of copper porphyrins, which bind weaker to pyridyl ligands than zinc porphyrins, would make it easier to displace the T6 template in the bis-copper rings. This would be reflected by the displacement of T6 at lower DABCO concentrations but this seems not to the case. 

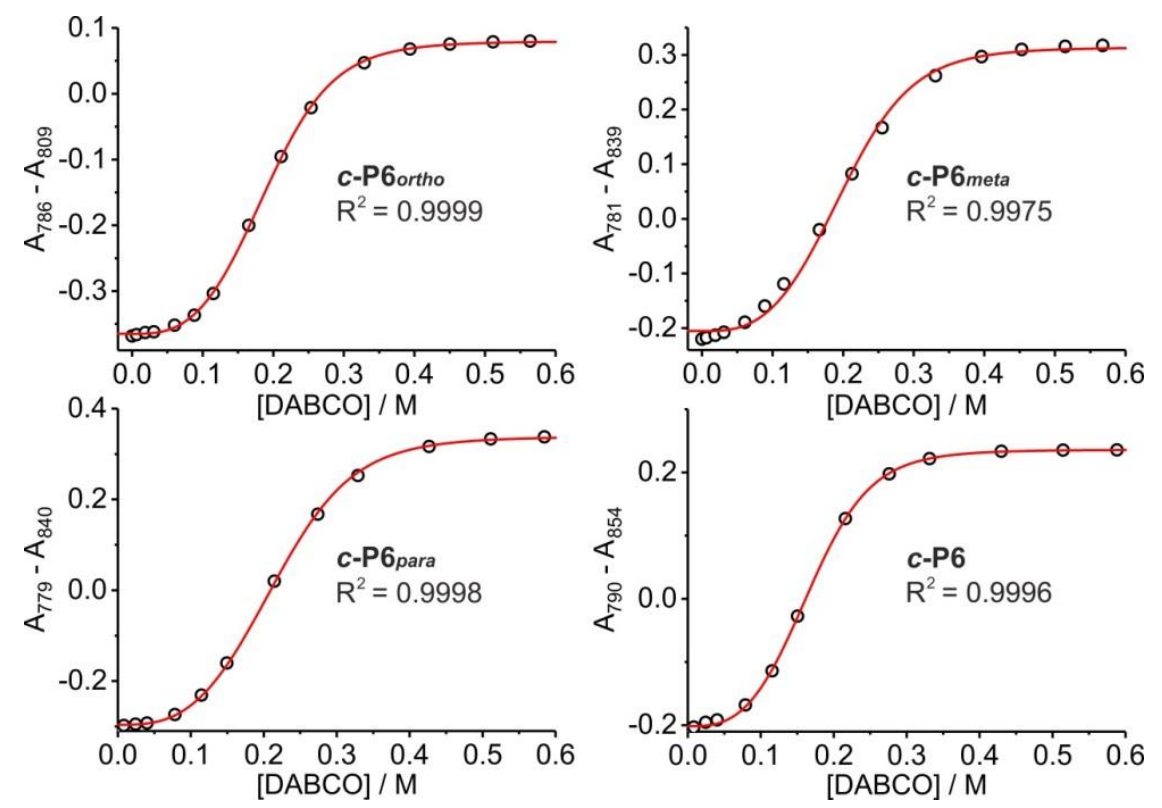

Figure 4.20: The experimental (black circles) and calculated binding isotherms (red lines) of the denaturation titrations of the rings with DABCO.

In order to explain the observation that all binding curves fit to a 6-site binding isotherm and that the template is displaced at comparable DABCO concentrations for all systems, we hypothesise that DABCO binds to both zinc and copper porphyrins in the ligand concentration range of the denaturation titration. If this is true, the data indicates that despite the weaker binding between T6 and the copper porphyrin, the binding of DABCO to an individual copper porphyrin is equally weaker. As a result, the template is only displaced at the moment that the DABCO concentration is equal to the $E M$ of the template which suggests that the $E M$ is constant in all rings despite the presence of the weaker individual binding constants.

In Chapter 2 we have described that the binding of pyridine to a copper porphyrin in $\mathrm{CHCl}_{3}$ is too weak to be measured directly. However, DABCO is a stronger binding ligand than pyridine and by changing the solvent to toluene the binding is also expected to increase. We performed the reference titrations which revealed that under similar conditions to the denaturation titrations the binding of DABCO to a zinc or copper porphyrin is $c a .10^{5} \mathrm{M}^{-1}$ and $10 \mathrm{M}^{-1}$ respectively (Figure 4.21). 


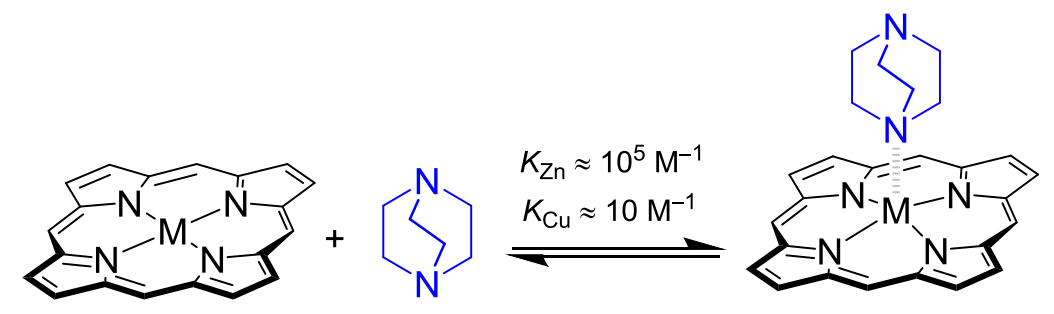

Figure 4.21: Axial coordination of DABCO to a metalloporphyrin ( $\mathrm{M}=\mathrm{Zn}$ or $\mathrm{Cu}$ ) in toluene at $298 \mathrm{~K}$.

While the binding to copper is relatively weak, the fact that binding does occur validates the hypothesis stated above. We can support the hypothesis by looking at the relationship between the denaturation constant $K_{\mathrm{dn}}$ and the average effective molarity $(\overline{E M})$ for each porphyrin unit to the template by using equation 4.1 :

$$
K_{\mathrm{dn}} \propto \frac{1}{\overline{\mathrm{EM}}}
$$

From this equation we can conclude that since the denaturation constants for the fully zinc and the bis-copper systems are similar, $\overline{E M}$ must also be the same in all systems measured. This means that the $E M$ of a zinc porphyrin positioned in the nanoring binding to the template is equal to a much weaker axially coordinating copper porphyrin. In Chapter 2 we investigated the axial binding affinity of copper porphyrins to pyridyl ligands via double mutant cycles. ${ }^{136}$ In our calculations we assumed that the effective molarity of a central copper porphyrin in a heterometallated pentamer was identical to a zinc porphyrin positioned in the centre of the chain. Our observations here support this assumption.

The calculated denaturation constants can be used to determine the formation constants between the T6 template and the rings (see Section 4.5.4). The stability constant for the three bis-copper rings are similar and average around $10^{22} \mathrm{M}^{-1}$. The reference fully zinc system, $\mathbf{c - P 6}$, was found to bind the template approximately 10 orders of magnitude stronger $\left(10^{32} \mathrm{M}^{-1}\right)$, clearly showing the effect of the copper centres on the overall stability of the complex.

\subsection{Conclusion}

Three isomers of the bis-copper 6-porphyrin nanoring were successfully synthesised. Initially heterometallated linear oligomers were prepared which were subsequently 
cyclised to prepare the rings. Purification of the rings was achieved using recycling GPC to yield the ortho, meta and para isomers in a 6, 5 and $14 \%$ yield respectively.

A combination of ${ }^{1} \mathrm{H}$ NMR, mass spectroscopy, UV-vis-NIR and analytical GPC was used to confirm the structures. The presence of the copper centres complicated the characterisation of the complexes through ${ }^{1} \mathrm{H}$ NMR spectroscopy; although a close inspection of the spectra still provided structural information based on broadening effects.

UV-vis-NIR titrations were performed on the rings which revealed similar denaturation constants for all systems indicating that the effective molarity of a copper porphyrin binding the T6 template in a porphyrin nanoring is identical to the effective molarity of a zinc porphyrin in a similar system. This observation supports the assumptions made in Chapter 2 where we determined the axial binding constant of a copper porphyrin to pyridyl ligands in linear pentamers. While the denaturation constants were similar, the association constant of the template in a bis-copper ring to a full zinc ring was ca. 10 orders of magnitude lower than in fully zinc c-P6.

DFT calculation on the three isomers indicated that the decay in $J$ coupling might not solely be a function of through-bond distance between the copper centres. EPR measurements, performed by Dr Alice Bowen, are currently underway in order to provide experimental data to confirm or refute these calculations.

\subsection{Experimental procedures and technical details}

\subsubsection{General experimental}

Dry toluene and THF were obtained by passing the solvents through columns of alumina, under nitrogen. Diisopropylamine $\left(i-\mathrm{Pr}_{2} \mathrm{NH}\right)$ was distilled from $\mathrm{CaH}_{2}$ and kept over activated molecular sieves ( $3 \AA$, 8-12 mesh). Unless specified otherwise, all other solvents were used as commercially supplied. Flash chromatography was carried out on silica gel 60 under positive pressure. Analytical thin-layer chromatography was carried out on aluminum-backed silica gel 60 F254 plates. Visualisation was achieved using UV light when necessary. 
All UV-vis-NIR spectra were recorded in solution using a Perkin-Lambda 20 spectrometer (1 $\mathrm{cm}$ path length quartz cell). Toluene was used for all titrations without any further purification.

Unless stated otherwise, ${ }^{1} \mathrm{H} /{ }^{13} \mathrm{C}$ NMR spectra were recorded at $298 \mathrm{~K}$ using a Bruker AV400 (400/100 MHz) or Bruker AV500 (500/125 MHz) instrument. ${ }^{1} \mathrm{H}$, and ${ }^{13} \mathrm{C}$ NMR spectra are reported in ppm; coupling constants are given in Hertz, to the nearest $0.1 \mathrm{~Hz}$. The solvent used was $\mathrm{CDCl}_{3}$.

MALDI-ToF spectra were measured at the EPSRC National Mass Spectrometry service (Swansea) using the Applied Biosystems Voyager DE-STR or at the University of Oxford using Waters MALDI Micro MX spectrometer utilising dithranol as a matrix.

\subsubsection{Synthetic procedures}

\section{Porphyrin monomer (4.1) ${ }^{77}$}

Porphyrin 3.9 (131 mg, $57 \mu \mathrm{mol}$ ) was dissolved in $\mathrm{CHCl}_{3}$ (3.5 $\mathrm{mL}$ ) and $\mathrm{CH}_{2} \mathrm{Cl}_{2}(13 \mathrm{~mL})$. Tetra- $n$-butylammonium fluoride ( $86 \mu \mathrm{L}, 1.0 \mathrm{M}$ solution in THF, $86 \mu \mathrm{mol}$ ) was added to the stirred solution. The progress of the reaction was monitored by TLC (petrol ether) until an

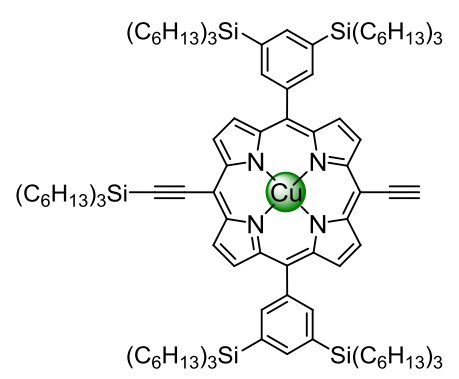
optimal mixture was reached ( $20 \mathrm{~min})$. The mixture was immediately passed through a short plug of silica gel $\left(\mathrm{CHCl}_{3}\right)$ to give the title compound as a purple oil (48 $\mathrm{mg}$, $42 \%)$.

${ }^{1} \mathbf{H}$ NMR (400 MHz, CDCl 3 , 298 K): $\delta_{H}$ (ppm) broad, 1.70-0.68 (m).

MALDI-TOF: $m / z=1,986\left(\mathrm{C}_{126} \mathrm{H}_{210} \mathrm{CuN}_{4} \mathrm{Si}_{5}, \mathrm{M}^{+}\right.$requires 1,985$)$.

\section{Porphyrin dimer $(4.2)^{77}$}

$\mathrm{PdCl}_{2}\left(\mathrm{PPh}_{3}\right)_{2}(0.4 \mathrm{mg}, 0.53 \mu \mathrm{mol})$,

Cul $(4.0 \mathrm{mg}, 21 \mu \mathrm{mol})$ and

1,4-benzoquinone (19.8 $\mathrm{mg}$,

$0.18 \mathrm{mmol}$ ) were dissolved in toluene $(1 \mathrm{~mL})$ and $i-\mathrm{Pr}_{2} \mathrm{NH}(1 \mathrm{~mL})$.

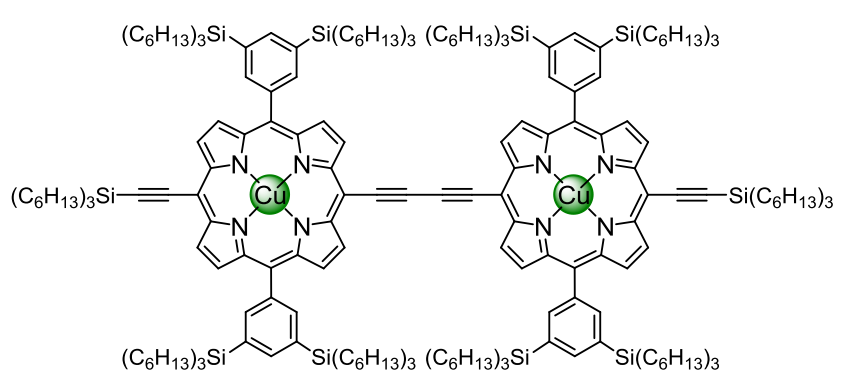

Porphyrin 4.1 (21 mg, $0.64 \mathrm{mmol})$ was dissolved in toluene $(1 \mathrm{~mL})$ and the catalyst mixture was added. The reaction mixture was stirred at room temperature for $2 \mathrm{~h}$ 
and passed through a short silica gel column $\left(\mathrm{CH}_{2} \mathrm{Cl}_{2}\right)$ to give 4.2 as a dark oil (15 mg, $81 \%)$.

${ }^{1}$ H NMR $\left(400 \mathrm{MHz}, \mathrm{CDCl}_{3}, 298 \mathrm{~K}\right): \delta_{\mathrm{H}}(\mathrm{ppm})$ broad, 8.02-7.75 (m), 1.76-0.68 (m).

MALDI-TOF: $m / z=3,967\left(\mathrm{C}_{252} \mathrm{H}_{418} \mathrm{Cu}_{2} \mathrm{~N}_{8} \mathrm{Si}_{10}, \mathrm{M}^{+}\right.$requires 3,967).

$\lambda_{\text {max }}\left(\mathrm{CHCl}_{3}\right) / \mathrm{nm} \log (\varepsilon): 453$ (5.56), 482 (5.30), 568 (4.40), 634 (4.87), 687 (5.00).

\section{Porphyrin dimer (4.3)}

Dimer 4.2 (15 mg, $3.5 \mu \mathrm{mol}$ ) was dissolved in $\mathrm{CH}_{2} \mathrm{Cl}_{2} \quad(1 \mathrm{~mL})$. Tetra- $n$-butylammonium fluoride $(69 \mu \mathrm{L}, 1.0 \mathrm{M}$ solution in THF, $69 \mu \mathrm{mol})$ was added and the reaction mixture was stirred at room temperature for $15 \mathrm{~min}$. The

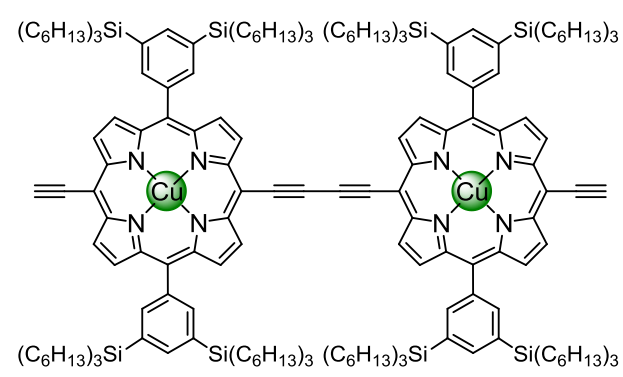
reaction mixture was immediately passed through a short plug of silica gel $\left(\mathrm{CHCl}_{3}\right)$ to give the title compound as a dark oil (12.8 mg, 100\%).

${ }^{1}$ H NMR $\left(400 \mathrm{MHz}, \mathrm{CDCl}_{3}, 298 \mathrm{~K}\right): \delta_{\mathrm{H}}(\mathrm{ppm})$ broad, $7.89(\mathrm{~m})$, 1.69-0.44 (m).

MALDI-TOF: $m / z=3,403\left(\mathrm{C}_{216} \mathrm{H}_{342} \mathrm{Cu}_{2} \mathrm{~N}_{8} \mathrm{Si}_{8}, \mathrm{M}^{+}\right.$requires 3,402$)$.

$\lambda_{\text {max }}\left(\mathrm{CHCl}_{3}\right) / \mathrm{nm} \log (\varepsilon): 448$ (5.47), 479 (5.23), 567 (4.34), 629 (4.73), 677 (4.87).

Porphyrin monomer (4.4)

$\mathrm{Pd}_{2}(\mathrm{dba})_{3}$ (223 $\left.\mathrm{mg}, 0.24 \mathrm{mmol}\right)$, Cul (93 mg, $0.49 \mathrm{mmol}), \mathrm{PPh}_{3}(128 \mathrm{mg}, 0.49 \mathrm{mmol})$ and 3.5 $(4.42 \mathrm{~g}, 2.44 \mathrm{mmol})$ were placed in a dried Schlenk tube under argon. Toluene $(112 \mathrm{~mL})$ and $i-\operatorname{Pr}_{2} \mathrm{NH}(112 \mathrm{~mL})$ were added and the reaction

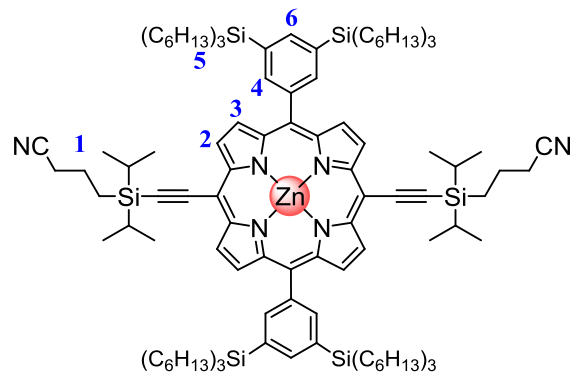
mixture deoxygenated [vacuum freeze/thaw 3 times]. cyanopropyldiisopropylsilyl acetylene $(1.52 \mathrm{~g}, 7.31 \mathrm{mmol})$ was added by syringe. The reaction mixture was stirred at $50{ }^{\circ} \mathrm{C}$ for $3 \mathrm{~h}$. Solvents removed and the residue passed through a short silica gel column $\left(\mathrm{CH}_{2} \mathrm{Cl}_{2}\right)$ to give 4.4 as a green oil $(5.00 \mathrm{~g}, 99 \%)$.

${ }^{1} \mathrm{H}$ NMR $\left(400 \mathrm{MHz}, \mathrm{CDCl}_{3}, 298 \mathrm{~K}\right): \delta_{\mathrm{H}}(\mathrm{ppm}) 9.63(4 \mathrm{H}, \mathrm{d}, J=4.5 \mathrm{~Hz}, \mathrm{H} 2), 8.85(4 \mathrm{H}, \mathrm{d}$, $J=4.5 \mathrm{~Hz}, \mathrm{H} 3), 8.21(4 \mathrm{H}, \mathrm{m}, \mathrm{H} 4), 7.99(2 \mathrm{H}, \mathrm{m}, \mathrm{H} 6), 2.54(4 \mathrm{H}, \mathrm{t}, J=6.8 \mathrm{~Hz}, \mathrm{H} 1), 2.20$ $(4 \mathrm{H}, \mathrm{m}, \mathrm{H} 1), 1.55-0.70(188 \mathrm{H}, \mathrm{m}, \mathrm{H} 1$ and $\mathrm{H} 5)$. 


\section{Porphyrin monomer (4.5)}

Porphyrin 4.4 (256 $\mathrm{mg}, 0.22 \mathrm{mmol})$ was dissolved in $\mathrm{CHCl}_{3}(15 \mathrm{~mL}), \mathrm{CH}_{2} \mathrm{Cl}_{2}(15 \mathrm{~mL})$, and pyridine $(2 \mathrm{~mL})$. Tetra- $n$-butylammonium fluoride $(0.22 \mathrm{~mL}, 1.0 \mathrm{M}$ solution in THF, $0.22 \mathrm{mmol}$ ) was added to the stirred solution. The progress of the reaction was monitored by

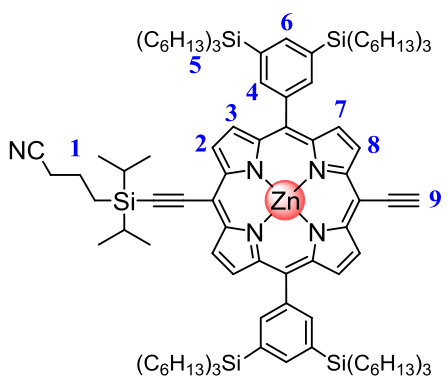
TLC (PE: $\mathrm{CH}_{2} \mathrm{Cl}_{2}=5: 2$ ) until an optimal mixture was reached $(20 \mathrm{~min}$ ). The mixture was immediately passed through a short plug of silica gel $\left(\mathrm{CHCl}_{3}\right)$. The title compound was obtained by silica gel chromatography (PE: $\mathrm{CH}_{2} \mathrm{Cl}_{2}=5: 2$ ) as a dark oil (62 $\mathrm{mg}, 27 \%$ ).

${ }^{1} \mathbf{H}$ NMR $\left(400 \mathrm{MHz}, \mathrm{CDCl}_{3}, 298 \mathrm{~K}\right): \delta_{\mathrm{H}}(\mathrm{ppm}) 9.75(2 \mathrm{H}, \mathrm{d}, J=4.7 \mathrm{~Hz}, \mathrm{H} 2$ or $\mathrm{H} 8), 9.72$ $(2 \mathrm{H}, \mathrm{d}, J=4.7 \mathrm{~Hz}, \mathrm{H} 2$ or H8), $8.96(2 \mathrm{H}, \mathrm{d}, J=4.7 \mathrm{~Hz}, \mathrm{H} 3$ or H7), $8.93(2 \mathrm{H}, \mathrm{d}, J=4.7 \mathrm{~Hz}$, $\mathrm{H} 3$ or H7), $8.25(4 \mathrm{H}, \mathrm{m}, \mathrm{H} 4), 8.01(2 \mathrm{H}, \mathrm{m}, \mathrm{H} 6), 4.20(1 \mathrm{H}, \mathrm{s}, \mathrm{H} 9), 2.56(2 \mathrm{H}, \mathrm{t}, \mathrm{J}=6.8 \mathrm{~Hz}$, $\mathrm{H} 1), 2.21(2 \mathrm{H}, \mathrm{m}, \mathrm{H} 1), 1.55-0.84(172 \mathrm{H}, \mathrm{m}, \mathrm{H} 1$ and $\mathrm{H} 5)$.

MALDI-TOF: $m / z=1,886\left(\mathrm{C}_{118} \mathrm{H}_{191} \mathrm{~N}_{5} \mathrm{Si}_{5} \mathrm{Zn}, \mathrm{M}^{+}\right.$requires 1,886).

$\lambda_{\text {max }}\left(\mathrm{CHCl}_{3}\right) / \mathrm{nm} \log (\varepsilon)$ : 436 (5.57), 448 (5.42), 580 (4.15), 619 (4.30), 632 (4.49).

Porphyrin dimer (4.6)

$\mathrm{PdCl}_{2}\left(\mathrm{PPh}_{3}\right)_{2}(23 \mathrm{mg}, 32 \mu \mathrm{mol})$, Cul (61 mg, $0.32 \mathrm{mmol}$ ) and 1,4-benzoquinone (139 mg, $1.28 \mathrm{mmol}$ ) were dissolved in toluene $(60 \mathrm{~mL})$ and $i-\operatorname{Pr}_{2} \mathrm{NH}$

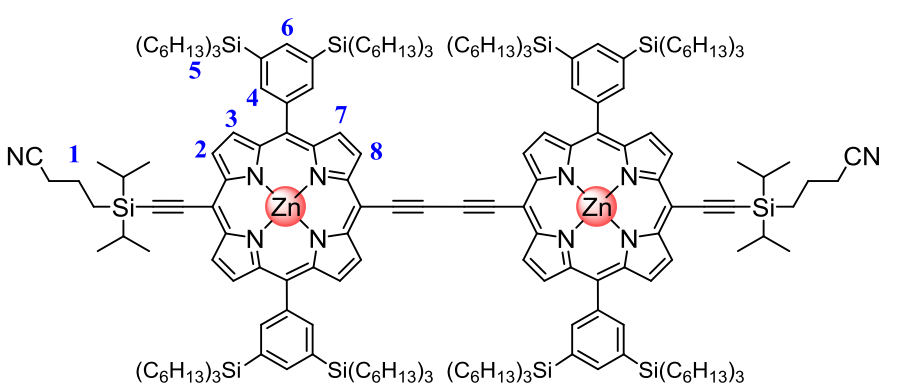
$(30 \mathrm{~mL})$. Porphyrin $3.5(1.21 \mathrm{~g}, 0.64 \mathrm{mmol})$ was dissolved in toluene $(60 \mathrm{~mL})$ and the catalyst mixture was added. The reaction mixture was stirred at room temperature for $2 \mathrm{~h}$ and passed through a short silica gel column $\left(\mathrm{CH}_{2} \mathrm{Cl}_{2}\right)$ to give 4.6 as a dark oil (0.77 g, 64\%).

${ }^{1} \mathbf{H}$ NMR $\left(500 \mathrm{MHz}, \mathrm{CDCl}_{3}, 298 \mathrm{~K}\right): \delta_{\mathrm{H}}(\mathrm{ppm}) 9.91(4 \mathrm{H}, \mathrm{d}, J=4.6 \mathrm{~Hz}, \mathrm{H} 2$ or H8), 9.66 $(4 \mathrm{H}, \mathrm{d}, J=4.6 \mathrm{~Hz}, \mathrm{H} 2$ or H8), $8.96(4 \mathrm{H}, \mathrm{d}, J=4.6 \mathrm{~Hz}, \mathrm{H} 3$ or $\mathrm{H} 7), 8.90(4 \mathrm{H}, \mathrm{d}, J=4.6 \mathrm{~Hz}$, H3 or H7), $8.26(8 \mathrm{H}, \mathrm{m}, \mathrm{H} 4), 7.99(4 \mathrm{H}, \mathrm{m}, \mathrm{H6}), 4.20(1 \mathrm{H}, \mathrm{s}, \mathrm{H} 9), 2.54(4 \mathrm{H}, \mathrm{t}, \mathrm{J}=6.9 \mathrm{~Hz}$, $\mathrm{H} 1), 2.23-2.14(4 \mathrm{H}, \mathrm{m}, \mathrm{H} 1), 1.53-0.83(344 \mathrm{H}, \mathrm{m}, \mathrm{H} 1$ and $\mathrm{H} 5)$.

MALDI-TOF: $m / z=3,765\left(\mathrm{C}_{236} \mathrm{H}_{380} \mathrm{~N}_{10} \mathrm{Si}_{10} \mathrm{Zn}_{2}, \mathrm{M}^{+}\right.$requires 3,769$)$. 


\section{Porphyrin dimer (4.7)}

Dimer 4.6 (215 mg, $57 \mu \mathrm{mol})$ was dissolved in $\mathrm{CHCl}_{3}(15 \mathrm{~mL}), \mathrm{CH}_{2} \mathrm{Cl}_{2}$ $(15 \mathrm{~mL})$, and pyridine $(2 \mathrm{~mL})$. Tetra- $n$-butylammonium fluoride $(86 \mu \mathrm{L}, \quad 1.0 \mathrm{M}$ solution in THF, $86 \mu \mathrm{mol})$ was added to the stirred

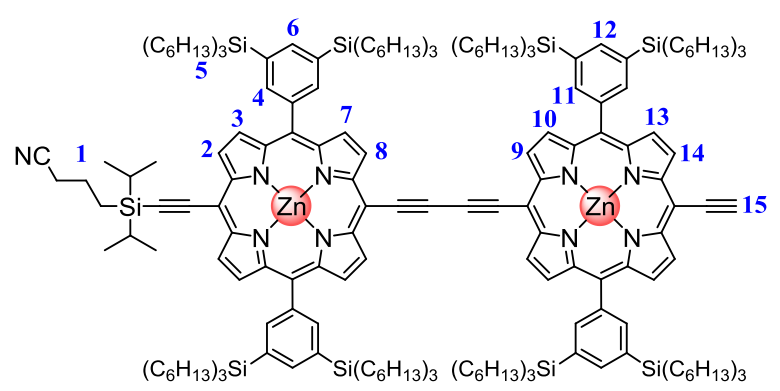
solution. The progress of the reaction was monitored by TLC $\left(P E: \mathrm{CH}_{2} \mathrm{Cl}_{2}=5: 2\right)$ until an optimal mixture was reached ( $20 \mathrm{~min}$ ). The mixture was immediately passed through a short plug of silica gel $\left(\mathrm{CHCl}_{3}\right)$. The title compound was obtained by silica gel chromatography (PE: $\left.\mathrm{CH}_{2} \mathrm{Cl}_{2}=5: 2\right)$ as a dark oil (51.9 mg, 25\%).

${ }^{1} \mathrm{H}$ NMR $\left(400 \mathrm{MHz}, \mathrm{CDCl}_{3}, 298 \mathrm{~K}\right): \delta_{\mathrm{H}}(\mathrm{ppm}) 9.98(2 \mathrm{H}, \mathrm{d}, J=4.7 \mathrm{~Hz}, \mathrm{H} 8$ or H9), 9.97 $(2 \mathrm{H}, \mathrm{d}, J=4.7 \mathrm{~Hz}, \mathrm{H} 8$ or H9), $9.76(2 \mathrm{H}, \mathrm{d}, J=4.7 \mathrm{~Hz}, \mathrm{H} 2$ or H14), $9.72(2 \mathrm{H}, \mathrm{d}, J=4.7 \mathrm{~Hz}$, $\mathrm{H} 2$ or H14), $9.05(2 \mathrm{H}, \mathrm{d}, J=4.7 \mathrm{~Hz}, \mathrm{H} 7$ or $\mathrm{H} 10), 9.01(2 \mathrm{H}, \mathrm{d}, J=4.7 \mathrm{~Hz}, \mathrm{H} 7$ or H10), 8.97 $(2 \mathrm{H}, \mathrm{d}, J=4.7 \mathrm{~Hz}, \mathrm{H} 3$ or $\mathrm{H} 13), 8.86(2 \mathrm{H}, \mathrm{d}, J=4.7 \mathrm{~Hz}, \mathrm{H} 3$ or $\mathrm{H} 13), 8.32(4 \mathrm{H}, \mathrm{m}, \mathrm{H} 4$ or $\mathrm{H} 11), 8.30(4 \mathrm{H}, \mathrm{m}, \mathrm{H} 4$ or $\mathrm{H} 11), 8.04(4 \mathrm{H}, \mathrm{m}, \mathrm{H} 6$ and $\mathrm{H} 12), 4.22(1 \mathrm{H}, \mathrm{s}, \mathrm{H} 15), 2.57(2 \mathrm{H}$, $\mathrm{t}, J=6.80 \mathrm{~Hz}, \mathrm{H} 1), 2.22(2 \mathrm{H}, \mathrm{m}, \mathrm{H} 1), 1.96-0.84(328 \mathrm{H}, \mathrm{m}, \mathrm{H} 1$ and $\mathrm{H} 5)$.

MALDI-TOF: $m / z=3589\left(\mathrm{C}_{226} \mathrm{H}_{361} \mathrm{~N}_{9} \mathrm{Si}_{9} \mathrm{Zn}_{2}, \mathrm{M}^{+}\right.$requires 3589).

$\lambda_{\text {max }}\left(\mathrm{CHCl}_{3}\right) / \mathrm{nm} \log (\varepsilon): 455$ (5.54), 490 (5.23), 581 (4.26), 662 (4.77), 720 (4.88).

\section{Porphyrin hexamer (4.8)}

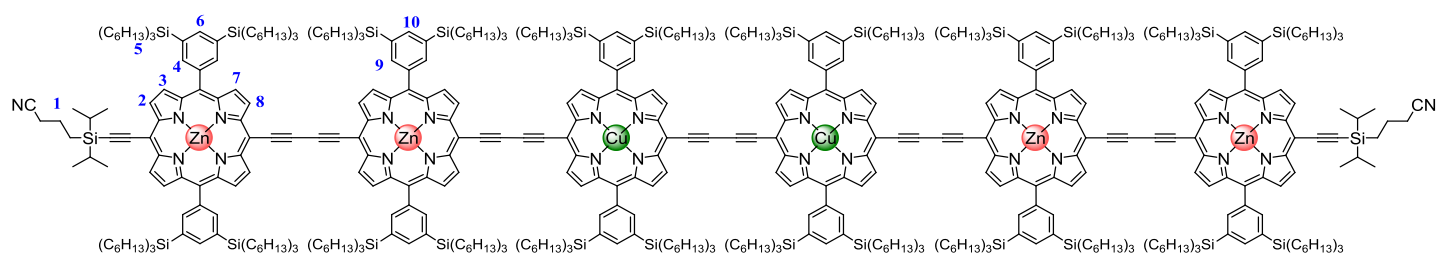

Dimer 4.7 (52 mg, $14.5 \mu \mathrm{mol})$ and dimer 3.3 (10 mg, $2.9 \mu \mathrm{mol})$ were dissolved in $\mathrm{CHCl}_{3}(5 \mathrm{~mL})$. A catalyst solution was prepared by dissolving $\mathrm{PdCl}_{2}\left(\mathrm{PPh}_{3}\right)_{2}(1.5 \mathrm{mg}$, $2.2 \mu \mathrm{mol})$, Cul (1.5 mg, $8.0 \mu \mathrm{mol}$ ) and 1,4-benzoquinone (4.5 mg, $41.4 \mu \mathrm{mol})$ in $\mathrm{CHCl}_{3}$ $(1 \mathrm{~mL})$ and $i-\operatorname{Pr}_{2} \mathrm{NH}(50 \mu \mathrm{L})$. The catalyst solution was added to the porphyrin solution and the reaction mixture was stirred at room temperature for $1 \mathrm{~h}$. The crude reaction mixture was passed through a short plug of silica gel (1\% pyridine in $\mathrm{CHCl}_{3}$ ), passed 
over a SEC column ( $1 \%$ pyridine in $\mathrm{CHCl}_{3}$ ), and finally the oligomers were separated by recycling GPC (1\% pyridine in toluene) to give 4.8 as a dark solid (12.8 $\mathrm{mg}, 42 \%)$.

${ }^{1} \mathrm{H}$ NMR $\left(400 \mathrm{MHz}, \mathrm{CDCl}_{3}, 298 \mathrm{~K}\right): \delta_{\mathrm{H}}(\mathrm{ppm}) 9.91(\mathrm{~d}, J=4.5 \mathrm{~Hz}, \mathrm{H} 8), 9.67(\mathrm{~d}, J=4.5 \mathrm{~Hz}$, H2), 8.96 (d, J = 4.5 Hz, H7), 8.90 (d, J = 4.5 Hz, H3), 8.31 (m, H9), 8.28 (m, H4), 8.03 (m, H10), $8.02(m, H 6), 2.57(t, J=6.74 \mathrm{~Hz}, \mathrm{H} 1), 2.22(\mathrm{~m}, \mathrm{H} 1), 1.59-0.80(\mathrm{~m}, \mathrm{H} 1$ and H5).

MALDI-TOF: $m / z=10,566\left(\mathrm{C}_{668} \mathrm{H}_{1060} \mathrm{Cu}_{2} \mathrm{~N}_{26} \mathrm{Si}_{26} \mathrm{Zn}_{4}, \mathrm{M}^{+}\right.$requires 10,574$)$.

$\lambda_{\text {max }}\left(\mathrm{CHCl}_{3}\right) / \mathrm{nm} \log (\varepsilon): 459$ (5.90), 492 (5.76), 582 (4.80), 759 (5.61).

\section{Porphyrin hexamer (4.9)}

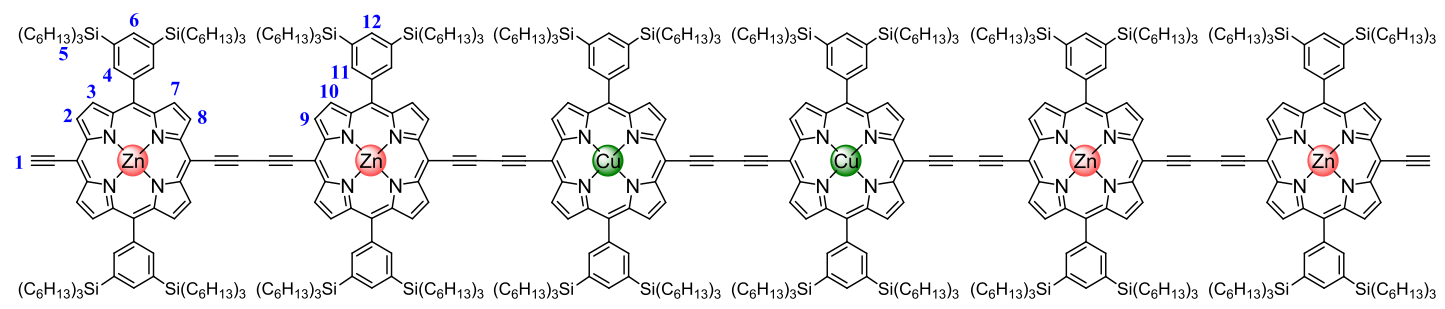

Hexamer $4.8(10.0 \mu \mathrm{mg}, 1.0 \mu \mathrm{mol})$ was dissolved in $\mathrm{CH}_{2} \mathrm{Cl}_{2}(2 \mu \mathrm{mL})$. Tetra- $n$-butylammonium fluoride ( $20 \mu \mathrm{L}, 1.0 \mathrm{M}$ solution in THF, $20 \mu \mathrm{mol}$ ) was added and the reaction mixture was stirred at room temperature for $15 \mathrm{~min}$. The reaction mixture was immediately passed through a short plug of silica gel $\left(\mathrm{CHCl}_{3}\right)$ to give the title compound as a dark oil (10.0 mg, 100\%).

${ }^{1} \mathbf{H}$ NMR (400 MHz, CDCl, $298 \mathrm{~K}$ ): $\delta_{\mathrm{H}}(\mathrm{ppm})$ 9.94-9.87 (m, H8 and H9), 9.70 (d, $J=4.6 \mathrm{~Hz}, \mathrm{H} 2), 9.00-8.93(\mathrm{~m}, \mathrm{H} 7$ and $\mathrm{H} 10), 8.90(\mathrm{~d}, J=4.6 \mathrm{~Hz}, \mathrm{H} 3), 8.32-8.25(\mathrm{~m}, \mathrm{H} 4$ and $\mathrm{H} 11)$, 8.04-7.98 (m, H6 and H12), 4.19 (s, H1), 1.61-0.89 (m, H5).

MALDI-TOF: $m / z=10,206\left(\mathrm{C}_{648} \mathrm{H}_{1022} \mathrm{Cu}_{2} \mathrm{~N}_{24} \mathrm{Si}_{24} \mathrm{Zn}_{4}, \mathrm{M}^{+}\right.$requires 10,212$)$.

$\lambda_{\text {max }}\left(\mathrm{CHCl}_{3}\right) / \mathrm{nm} \log (\varepsilon): 463$ (5.79), 494 (5.74), 579 (4.78), 630 (4.78), 757 (5.55).

\section{Porphyrin monomer $(4.10)^{52}$}

Monomer 3.4 (200 mg, $97 \mu \mathrm{mol})$ was dissolved in $\mathrm{CH}_{2} \mathrm{Cl}_{2}(30 \mathrm{~mL})$. Tetra- $n$-butylammonium fluoride $(0.97 \mathrm{~mL}, 1.0 \mathrm{M}$ solution in THF, $0.97 \mathrm{mmol}$ ) was added and the reaction mixture was stirred at room temperature for $15 \mathrm{~min}$. The reaction mixture was immediately passed through a short plug of silica gel $\left(\mathrm{CHCl}_{3}\right)$ to give the title compound as a dark oil (161 mg, 98\%).

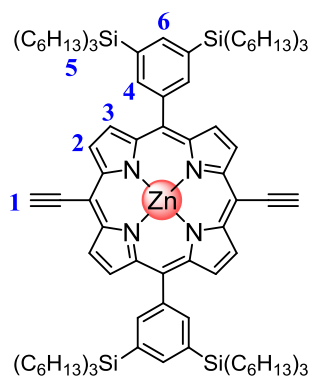


${ }^{1} \mathrm{H}$ NMR $\left(400 \mathrm{MHz}, \mathrm{CDCl}_{3}, 298 \mathrm{~K}\right): \delta_{\mathrm{H}}(\mathrm{ppm}) 9.68(4 \mathrm{H}, \mathrm{d}, J=4.6 \mathrm{~Hz}, \mathrm{H} 2), 8.89(4 \mathrm{H}, \mathrm{d}$, $J=4.6 \mathrm{~Hz}, \mathrm{H} 3), 8.24(4 \mathrm{H}, \mathrm{m}, \mathrm{H} 4), 7.98(2 \mathrm{H}, \mathrm{m}, \mathrm{H} 6), 4.16(2 \mathrm{H}, \mathrm{s}, \mathrm{H} 1), 1.59-0.80(156 \mathrm{H}$, $m, \mathrm{H} 5)$.

\section{Trimer (4.11)}

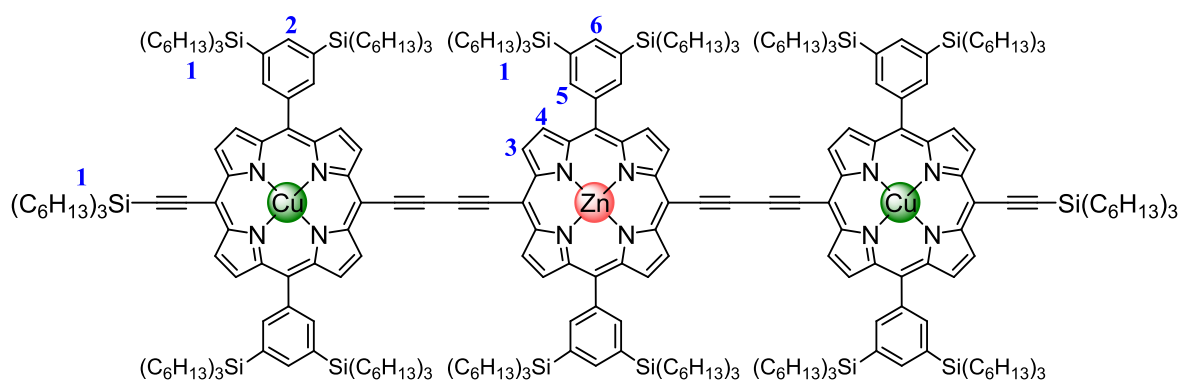

Monomer 4.1 (48 mg, $24 \mu \mathrm{mol}$ ) and monomer 4.10 (8 mg, $8 \mu \mathrm{mol}$ ) were dissolved in $\mathrm{CHCl}_{3}(3 \mathrm{~mL})$. A catalyst solution was prepared by dissolving $\mathrm{PdCl}_{2}\left(\mathrm{PPh}_{3}\right)_{2}(2.6 \mathrm{mg}$, $4 \mu \mathrm{mol})$, Cul $(2.5 \mathrm{mg}, 13 \mu \mathrm{mol})$ and 1,4-benzoquinone $(7.5 \mathrm{mg}, 69 \mu \mathrm{mol})$ in $\mathrm{CHCl}_{3}$ $(1.7 \mathrm{~mL})$ and $i-\operatorname{Pr}_{2} \mathrm{NH}(84 \mu \mathrm{L})$. The catalyst solution was added to the porphyrin solution and the reaction mixture was stirred at room temperature for $1 \mathrm{~h}$. The crude reaction mixture was passed through a short plug of silica gel (1\% pyridine in $\mathrm{CHCl}_{3}$ ), passed over a SEC column (1\% pyridine in toluene), and finally the oligomers were separated by recycling GPC (1\% pyridine in toluene) to give 4.11 as a dark oil ( $8 \mathrm{mg}$, $28 \%)$.

${ }^{1} \mathrm{H}$ NMR $\left(400 \mathrm{MHz}, \mathrm{CDCl}_{3}, 298 \mathrm{~K}\right): \delta_{\mathrm{H}}(\mathrm{ppm}) 9.82(\mathrm{~m}, \mathrm{H} 3), 8.95(\mathrm{~m}, \mathrm{H} 4), 8.29(\mathrm{~m}, \mathrm{H} 5)$, 8.02 (m, H6), $7.89(m, H 2), 1.59-1.18(m, H 1), 1.01-0.73(m, H 1)$.

MALDI-TOF: $m / z=5,670\left(\mathrm{C}_{360} \mathrm{H}_{588} \mathrm{Cu}_{2} \mathrm{~N}_{12} \mathrm{Si}_{14} \mathrm{Zn}, \mathrm{M}^{+}\right.$requires 5,669).

$\lambda_{\max }\left(\mathrm{CHCl}_{3}\right) / \mathrm{nm} \log (\varepsilon): 427$ (5.42), 450 (5.60), 490 (5.42), 572 (4.57), 614 (4.64), 727 (5.20). 


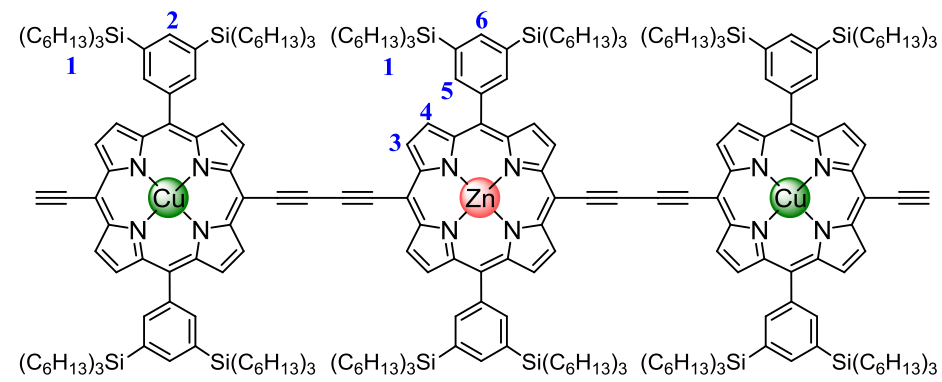

Trimer 4.11 (7.7 $\mathrm{mg}, 1.4 \mu \mathrm{mol})$ was dissolved in $\mathrm{CH}_{2} \mathrm{Cl}_{2}(2 \mu \mathrm{mL})$. Tetra- $n$-butylammonium fluoride ( $27 \mu \mathrm{L}, 1.0 \mathrm{M}$ solution in THF, $27 \mu \mathrm{mol}$ ) was added and the reaction mixture was stirred at room temperature for $15 \mathrm{~min}$. The reaction mixture was immediately passed through a short plug of silica gel $\left(\mathrm{CHCl}_{3}\right)$ to give $\mathbf{4 . 1 2}$ as a dark oil (7.4 mg, 100\%).

${ }^{1} \mathrm{H}$ NMR $\left(400 \mathrm{MHz}, \mathrm{CDCl}_{3}, 298 \mathrm{~K}\right): \delta_{\mathrm{H}}(\mathrm{ppm})$ broad, $9.79(\mathrm{~m}, \mathrm{H} 3), 8.94(\mathrm{~m}, \mathrm{H} 4), 8.28$ (m, H5), 8.01 (m, H6), 7.89 (m, H2), 1.59-1.17 (m, H1), 1.00-0.76 (m, H1).

MALDI-TOF: $m / z=5,104\left(C_{324} \mathrm{H}_{512} \mathrm{Cu}_{2} \mathrm{~N}_{12} \mathrm{Si}_{12} \mathrm{Zn}, \mathrm{M}^{+}\right.$requires 5,105).

$\lambda_{\text {max }}\left(\mathrm{CHCl}_{3}\right) / \mathrm{nm} \log (\varepsilon): 424$ (5.37), 447 (5.52), 489 (5.39), 570 (4.52), 609 (4.57), 723 (5.12).

\section{Pentamer (4.13)}

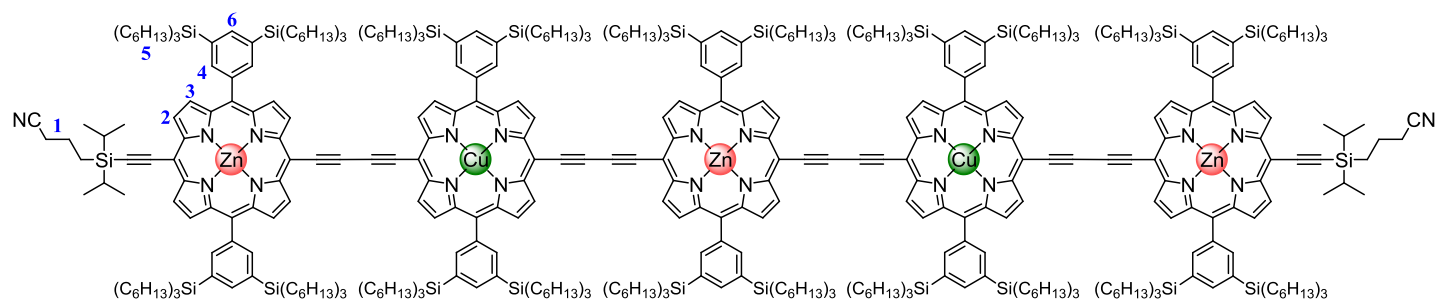

Trimer $4.12(7.5 \mathrm{mg}, 1.5 \mu \mathrm{mol})$ and monomer $3.5(27.7 \mathrm{mg}, 14.7 \mu \mathrm{mol})$ were dissolved in $\mathrm{CHCl}_{3}(3 \mathrm{~mL})$. A catalyst solution was prepared by dissolving $\mathrm{PdCl}_{2}\left(\mathrm{PPh}_{3}\right)_{2}$ (1.3 mg, $1.9 \mu \mathrm{mol})$, Cul (1.3 mg, $6.7 \mu \mathrm{mol}$ ) and 1,4-benzoquinone (3.8 mg, $24.5 \mu \mathrm{mol})$ in $\mathrm{CHCl}_{3}(1 \mathrm{~mL})$ and $i-\mathrm{Pr}_{2} \mathrm{NH}(50 \mu \mathrm{L})$. The catalyst solution was added to the porphyrin solution and the reaction mixture was stirred at room temperature for $1 \mathrm{~h}$. The crude reaction mixture was passed through a short plug of silica gel (1\% pyridine in $\mathrm{CHCl}_{3}$ ), passed over a SEC column (1\% pyridine in $\mathrm{CHCl}_{3}$ ), and finally the oligomers were separated by recycling GPC (1\% pyridine in toluene) to give 4.13 as a dark solid (7.0 mg, 55\%). 
${ }^{1} \mathrm{H}$ NMR $\left(400 \mathrm{MHz}, \mathrm{CDCl}_{3}, 298 \mathrm{~K}\right): \delta_{\mathrm{H}}(\mathrm{ppm}) 9.68(\mathrm{~m}, \mathrm{H} 2), 8.09(\mathrm{~m}, \mathrm{H} 3), 8.26(\mathrm{~m}, \mathrm{H} 4)$, $8.01(m, H 6), 2.56(t, J=6.75 \mathrm{~Hz}, \mathrm{H} 1), 2.21(\mathrm{~m}, \mathrm{H} 1), 1.57-0.81(\mathrm{~m}, \mathrm{H} 1$ and $\mathrm{H} 5)$.

MALDI-TOF: $m / z=8,872\left(\mathrm{C}_{560} \mathrm{H}_{890} \mathrm{Cu}_{2} \mathrm{~N}_{22} \mathrm{Si}_{22} \mathrm{Zn}_{3}, \mathrm{M}^{+}\right.$requires 8,872).

$\lambda_{\text {max }}\left(\mathrm{CHCl}_{3}\right) / \mathrm{nm} \log (\varepsilon): 458$ (5.83), 488 (5.68), 579 (4.71), 661 (5.03), 749 (5.48).

\section{Pentamer (4.14)}

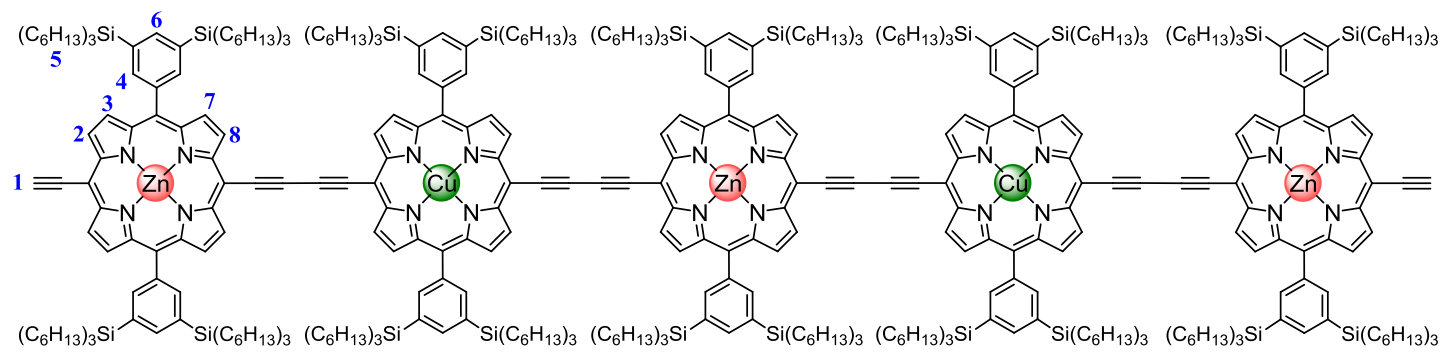

Pentamer $4.13(7.0 \mu \mathrm{mg}, \quad 0.8 \mu \mathrm{mol})$ was dissolved in $\mathrm{CH}_{2} \mathrm{Cl}_{2}(1.5 \mu \mathrm{mL})$. Tetra- $n$-butylammonium fluoride $(15.8 \mu \mathrm{L}, 1.0 \mathrm{M}$ solution in THF, $15.8 \mu \mathrm{mol})$ was added and the reaction mixture was stirred at room temperature for $15 \mathrm{~min}$. The reaction mixture was immediately passed through a short plug of silica gel $\left(\mathrm{CHCl}_{3}\right)$ to give 4.14 as a dark oil (6.5 $\mathrm{mg}, 96 \%)$.

${ }^{1} \mathrm{H}$ NMR $\left(400 \mathrm{MHz}, \mathrm{CDCl}_{3}, 298 \mathrm{~K}\right): \delta_{\mathrm{H}}(\mathrm{ppm}) 9.85(\mathrm{~m}, \mathrm{H} 8), 9.75(\mathrm{~m}, \mathrm{H} 2), 9.00(\mathrm{~m}, \mathrm{H} 7)$, 8.95 (m, H3), 8.29 (m, H4), 8.03 (m, H6), 7.93 (m, H9), 4.22 (s, H1), 1.60-0.77 (m, H5). MALDI-TOF: $m / z=8,505\left(\mathrm{C}_{540} \mathrm{H}_{852} \mathrm{Cu}_{2} \mathrm{~N}_{20} \mathrm{Si}_{20} \mathrm{Zn}_{3}, \mathrm{M}^{+}\right.$requires 8,509).

$\lambda_{\text {max }}\left(\mathrm{CHCl}_{3}\right) / \mathrm{nm} \log (\varepsilon): 455$ (5.79), 487 (5.67), 579 (4.70), 653 (4.95), 747 (5.44). 


\section{Porphyrin nanoring $\left(c-\mathrm{P} 6_{\text {ortho }} \cdot \mathrm{T} 6\right)$}

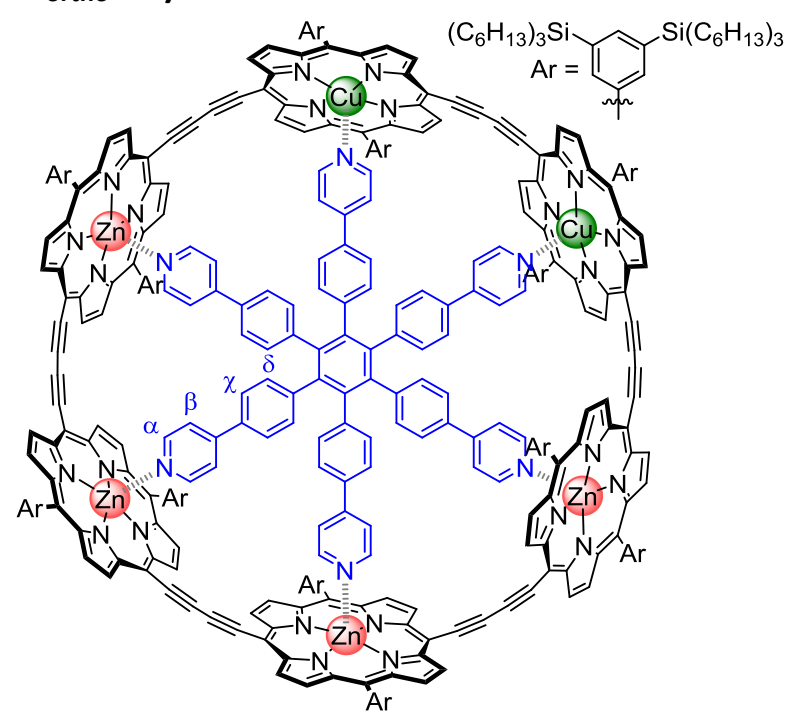

Hexadentate template T6 $(7.8 \mathrm{mg}, 7.8 \mu \mathrm{mol})$ and hexamer 4.9 (39.7 mg, $3.9 \mu \mathrm{mol})$ were dissolved in $\mathrm{CHCl}_{3}(20 \mathrm{~mL})$. The solvent was removed and the complex was dissolved in toluene $(20 \mathrm{~mL})$. A solution of $\mathrm{PdCl}_{2}\left(\mathrm{PPh}_{3}\right)_{2}(1.4 \mathrm{mg}, 2.0 \mu \mathrm{mol})$, Cul (1.4 mg, $7.2 \mu \mathrm{mol}), 1,4$-benzoquinone $(4.2 \mathrm{mg}, 39.0 \mu \mathrm{mol})$ in toluene $(1.4 \mathrm{~mL})$ and $i-\operatorname{Pr}_{2} \mathrm{NH}(72 \mu \mathrm{L})$ was added to the porphyrin solution and stirred at room temperature for $16 \mathrm{~h}$. The reaction mixture was passed through a plug of alumina using $\mathrm{CHCl}_{3}$ as eluent. The solvent was evaporated and dissolved in toluene and passed over a SEC column (toluene). Lastly, the ring was purified by recycling GPC (1\% pyridine in toluene) to give $\boldsymbol{c}-\mathbf{P} 6_{o r t h o} \cdot \mathbf{T 6}$ as a red solid $(2.5 \mathrm{mg}, 6 \%)$.

${ }^{1}$ H NMR $\left(500 \mathrm{MHz}, \mathrm{CDCl}_{3}, 298 \mathrm{~K}\right): \delta_{\mathrm{H}}(\mathrm{ppm})$ 9.62-9.47 (m, $\left.-H_{\beta}\right)$, 8.77-8.64 $\left(\mathrm{m},-H_{\beta}\right)$, 8.37-8.25 (m, -ArH para $)$, 8.04-7.91 ( $m,-$ ArH $\left._{\text {ortho }}\right), 5.82-5.37\left(m, \mathbf{T 6}_{\delta}\right.$ and $\left.\mathbf{T 6}_{\chi}\right), 5.21-4.89$ $\left(m, \mathbf{T 6}_{\beta}\right), 2.49-2.35\left(\mathrm{~m}, \mathrm{T6}_{\alpha}\right), 1.60-0.70(\mathrm{~m},-\mathrm{THS})$.

MALDI-TOF: $m / z=11,209\left(\mathrm{C}_{720} \mathrm{H}_{1068} \mathrm{Cu}_{2} \mathrm{~N}_{30} \mathrm{Si}_{24} \mathrm{Zn}_{4}, \mathrm{M}^{+}\right.$requires 11,207$)$.

$\lambda_{\text {max }}\left(\mathrm{CHCl}_{3}\right) / \mathrm{nm} \log (\varepsilon)$ : 430 (5.53), 481 (5.77), 764 (5.55), 800 (5.62), 840 (5.50). 


\section{Porphyrin nanoring $\left(c-\mathrm{P}_{m e t a} \cdot \mathrm{T} 6\right)$}

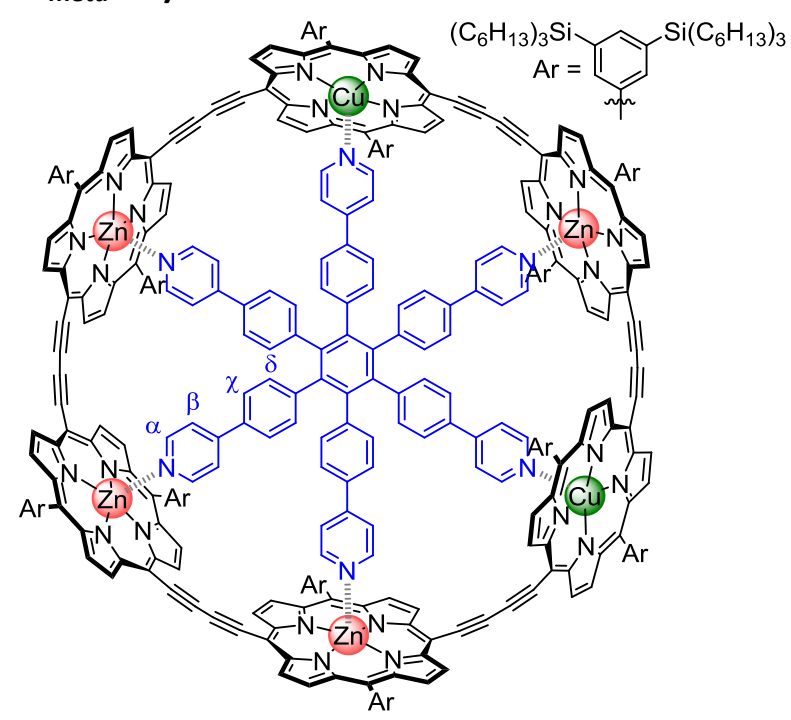

Hexadentate template T6 (3.8 mg, $3.8 \mu \mathrm{mol})$, pentamer 4.14 (32.5 mg, $3.8 \mu \mathrm{mol})$ and monomer 4.10 (13.0 mg, $7.6 \mu \mathrm{mol})$ were dissolved in $\mathrm{CHCl}_{3}(50 \mathrm{~mL})$. The solvent was removed and the complex was dissolved in toluene $(15 \mathrm{~mL})$. A solution of $\mathrm{PdCl}_{2}\left(\mathrm{PPh}_{3}\right)_{2}(1.3 \mathrm{mg}, 1.9 \mu \mathrm{mol})$, Cul (1.3 mg, $\left.7.1 \mu \mathrm{mol}\right)$, 1,4-benzoquinone (4.1 mg, $38.1 \mu \mathrm{mol})$ in toluene $(1.4 \mathrm{~mL})$ and $i-\mathrm{Pr}_{2} \mathrm{NH}(70 \mu \mathrm{L})$ was added to the porphyrin solution and stirred at room temperature for $16 \mathrm{~h}$. The reaction mixture was passed through a plug of alumina using $\mathrm{CHCl}_{3}$ as eluent. The solvent was evaporated and dissolved in toluene and passed over a SEC column (toluene). Lastly, the ring was purified by recycling GPC (1\% pyridine in toluene) to give $\mathbf{c}-\mathbf{P} \mathbf{6}_{\text {meta }} \cdot \mathbf{T 6}$ as a red solid (2.0 mg, 5\%).

${ }^{1}$ H NMR $\left(500 \mathrm{MHz}, \mathrm{CDCl}_{3}, 298 \mathrm{~K}\right): \delta_{\mathrm{H}}(\mathrm{ppm})$ 9.62-9.30 (m, $\left.-H_{\beta}\right)$, 8.77-8.61 (m, $\left.-H_{\beta}\right)$, 8.37-8.24 ( $\left.\mathrm{m},-\mathrm{ArH}_{\text {para }}\right), 8.03-7.89\left(\mathrm{~m},-\mathrm{ArH}_{\text {ortho }}\right), 6.03-5.40\left(\mathrm{~m}, \mathbf{T 6}_{\delta}\right.$ and $\left.\mathbf{T 6}_{\chi}\right)$, 5.18-4.92 $\left(\mathrm{m}, \mathbf{T 6}_{\beta}\right), 2.50-2.38\left(\mathrm{~m}, \mathrm{T6}_{\alpha}\right), 1.60-0.70(\mathrm{~m},-\mathrm{THS})$.

MALDI-TOF: $m / z=11,208\left(\mathrm{C}_{720} \mathrm{H}_{1068} \mathrm{Cu}_{2} \mathrm{~N}_{30} \mathrm{Si}_{24} \mathrm{Zn}_{4}, \mathrm{M}^{+}\right.$requires 11,207).

$\lambda_{\text {max }}\left(\mathrm{CHCl}_{3}\right) / \mathrm{nm} \log (\varepsilon): 429$ (5.37), 481 (5.64), 767 (5.41), 805 (5.51), 843 (5.42). 


\section{Porphyrin nanoring $\left(c-\mathrm{P} 6_{\text {para }} \cdot \mathrm{T} 6\right)$}

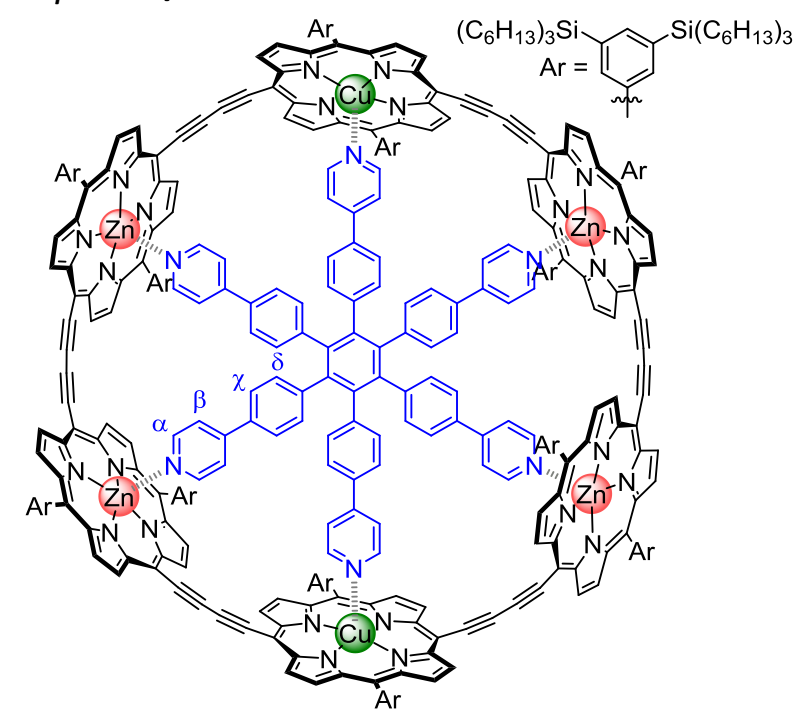

Hexadentate template T6 (61 mg, $61 \mu \mathrm{mol})$ and trimer $/$-P3 $\mathbf{C u}_{\mathrm{Cu}}(155 \mathrm{mg}, 30 \mu \mathrm{mol})$ were dissolved in $\mathrm{CHCl}_{3}(100 \mathrm{~mL})$. The solvent was removed and the complex was dissolved in toluene $(120 \mathrm{~mL})$. A solution of $\mathrm{PdCl}_{2}\left(\mathrm{PPh}_{3}\right)_{2}(10.7 \mathrm{mg}, 15 \mu \mathrm{mol})$, Cul $(11 \mathrm{mg}$, $56 \mu \mathrm{mol}), 1,4$-benzoquinone $(32.8 \mathrm{mg}, 0.30 \mathrm{mmol})$ in toluene $(11 \mathrm{~mL})$ and $i-\operatorname{Pr}_{2} \mathrm{NH}$ $(0.56 \mathrm{~mL})$ was added to the porphyrin solution and stirred at room temperature for $16 \mathrm{~h}$. The reaction mixture was passed through a plug of alumina using $\mathrm{CHCl}_{3}$ as eluent. The solvent was evaporated and dissolved in toluene and passed over a SEC column (toluene). Lastly, the ring was purified by recycling GPC ( $1 \%$ pyridine in toluene) to give $\boldsymbol{c}-\mathbf{P} \mathbf{6}_{\text {para }} \cdot \mathbf{T 6}$ as a red solid (24 mg, $\left.14 \%\right)$.

${ }^{1} \mathbf{H}$ NMR $\left(500 \mathrm{MHz}, \mathrm{CDCl}_{3}, 298 \mathrm{~K}\right): \delta_{\mathrm{H}}(\mathrm{ppm})$ 9.65-9.27 (m, $\left.-H_{\beta}\right), 8.81-8.59\left(\mathrm{~m},-H_{\beta}\right)$, $8.31\left(\mathrm{~m},-\mathrm{Ar} H_{\text {para }}\right), 7.97\left(\mathrm{~m},-\mathrm{ArH}_{\text {ortho }}\right)$, 5.99-5.72 (m, T6 $\delta$ ), 5.72-5.55 (m, T6 $\left.\boldsymbol{6}_{\chi}\right)$, 5.22-5.04 $\left(\mathrm{m}, \mathbf{T 6}_{\beta}\right), 3.03-2.53\left(\mathrm{~m}, \mathbf{T 6}_{\alpha}\right), 1.50-0.77$ (m, -THS).

MALDI-TOF: $m / z=11,206\left(\mathrm{C}_{720} \mathrm{H}_{1068} \mathrm{Cu}_{2} \mathrm{~N}_{30} \mathrm{Si}_{24} \mathrm{Zn}_{4}, \mathrm{M}^{+}\right.$requires 11,207).

$\lambda_{\text {max }}\left(\mathrm{CHCl}_{3}\right) / \mathrm{nm} \log (\varepsilon): 427$ (5.47), 480 (5.80), 762 (5.59), 799 (5.70), 840 (5.66). 


\subsubsection{Proof of purity}

To confirm the identity and the purity of the rings, the MALDI-MS spectra and

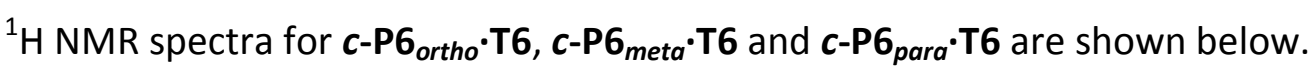

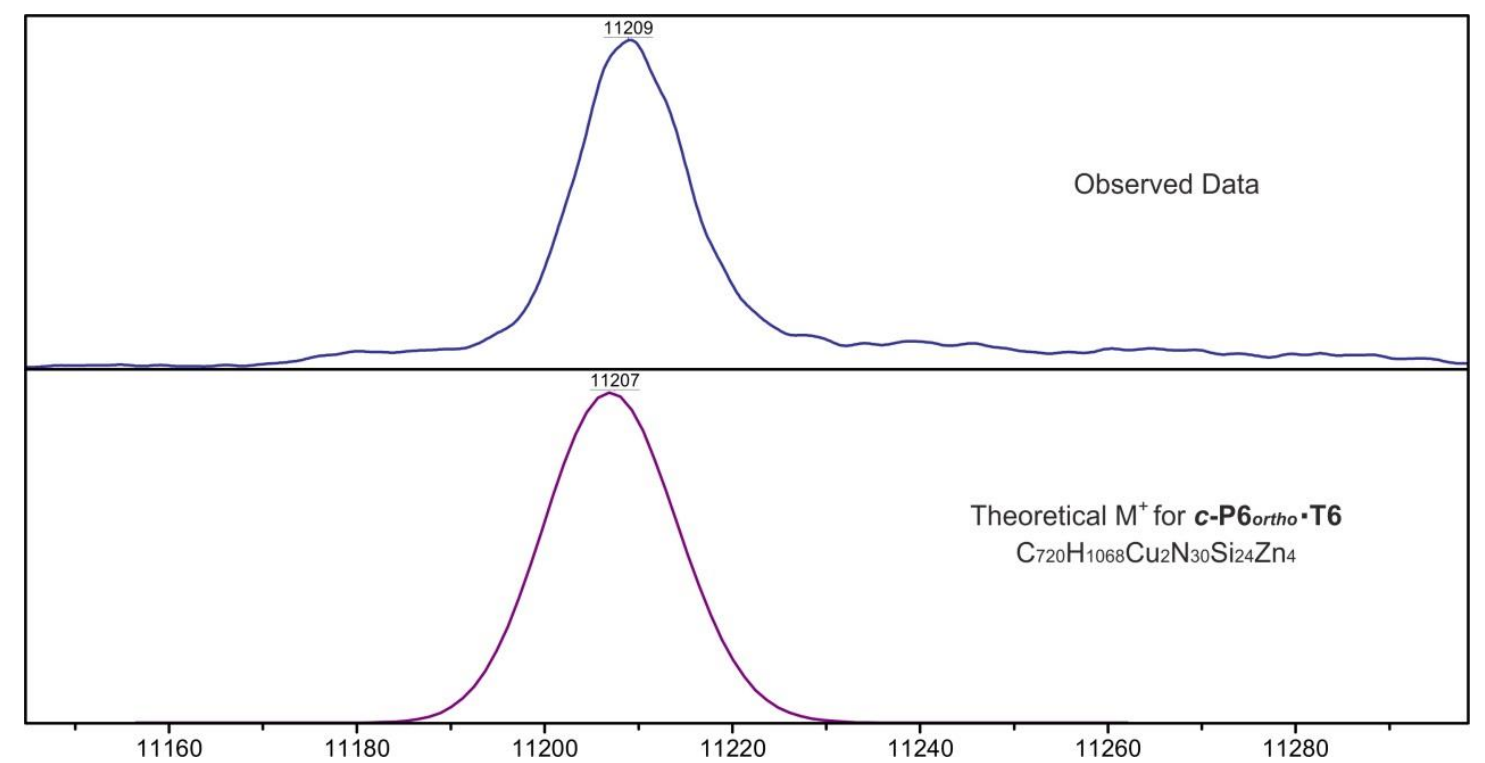

Figure 4.22: The MALDI-MS spectrum of $c$ - $\mathrm{P} 6_{\text {ortho }} \cdot \mathrm{T6}\left(\mathrm{m} / \mathrm{z}=11,209\left(\mathrm{C}_{720} \mathrm{H}_{1068} \mathrm{Cu}_{2} \mathrm{~N}_{30} \mathrm{Si}_{24} \mathrm{Zn}_{4}, \mathrm{M}^{+}\right.\right.$requires 11,207$)$, matrix: DCTB).

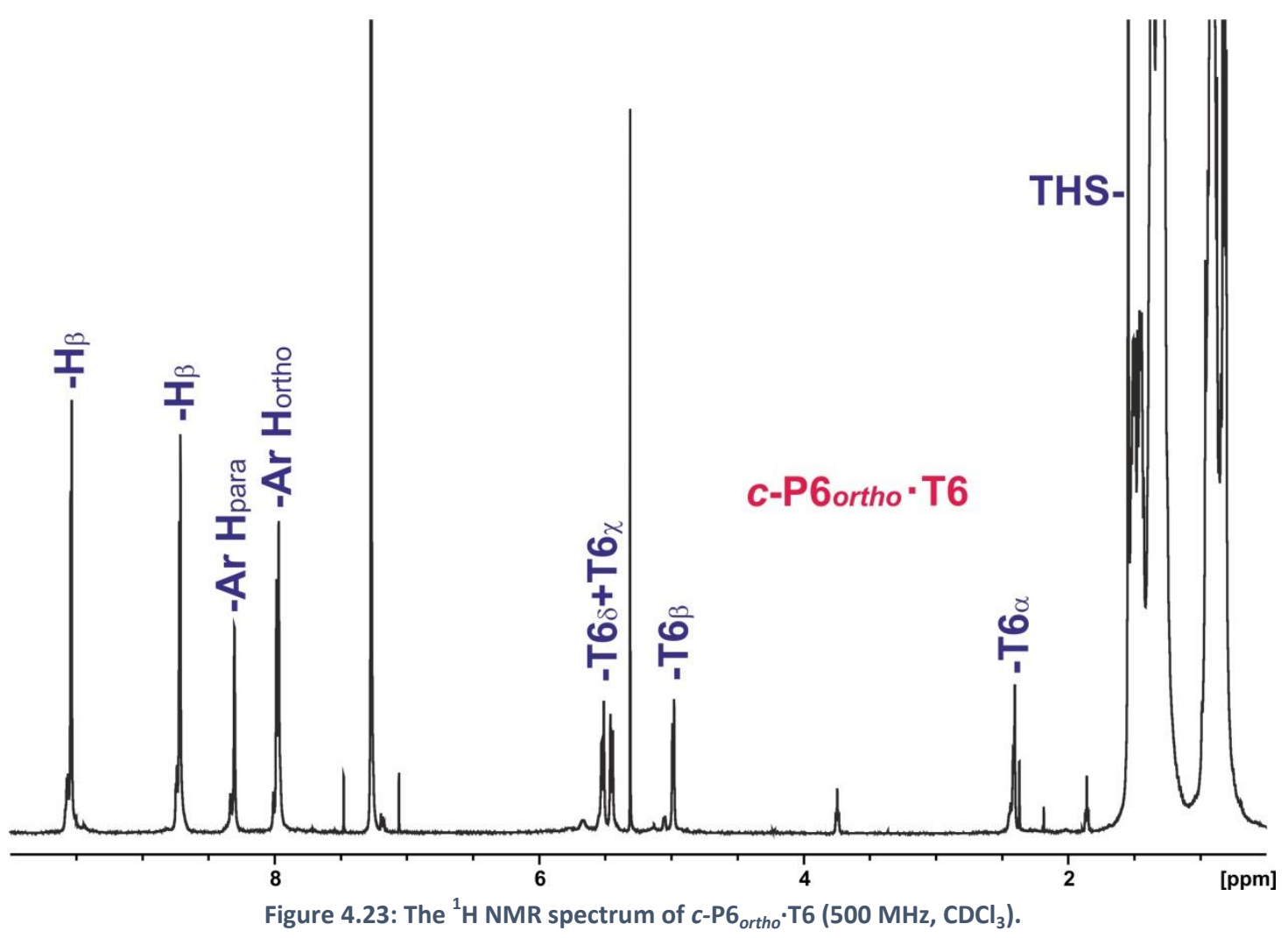




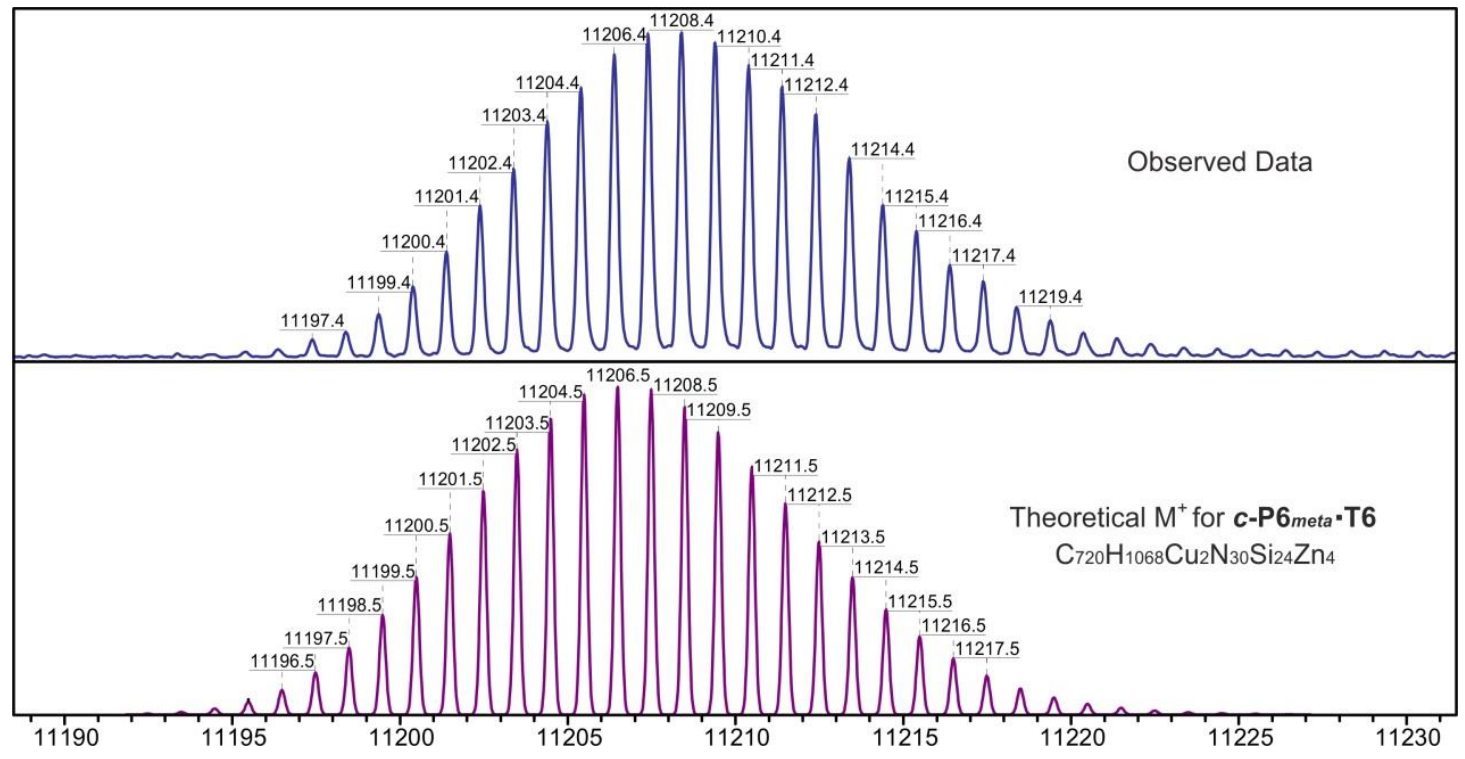

Figure 4.24: The MALDI-MS spectrum of $c$ - $\mathrm{P} 6_{\text {meta }} \cdot \mathrm{T6}\left(\mathrm{m} / \mathrm{z}=11,208\left(\mathrm{C}_{720} \mathrm{H}_{1068} \mathrm{Cu}_{2} \mathrm{~N}_{30} \mathrm{Si}_{24} \mathrm{Zn}_{4}, \mathrm{M}^{+}\right.\right.$requires 11,207), matrix: DCTB).

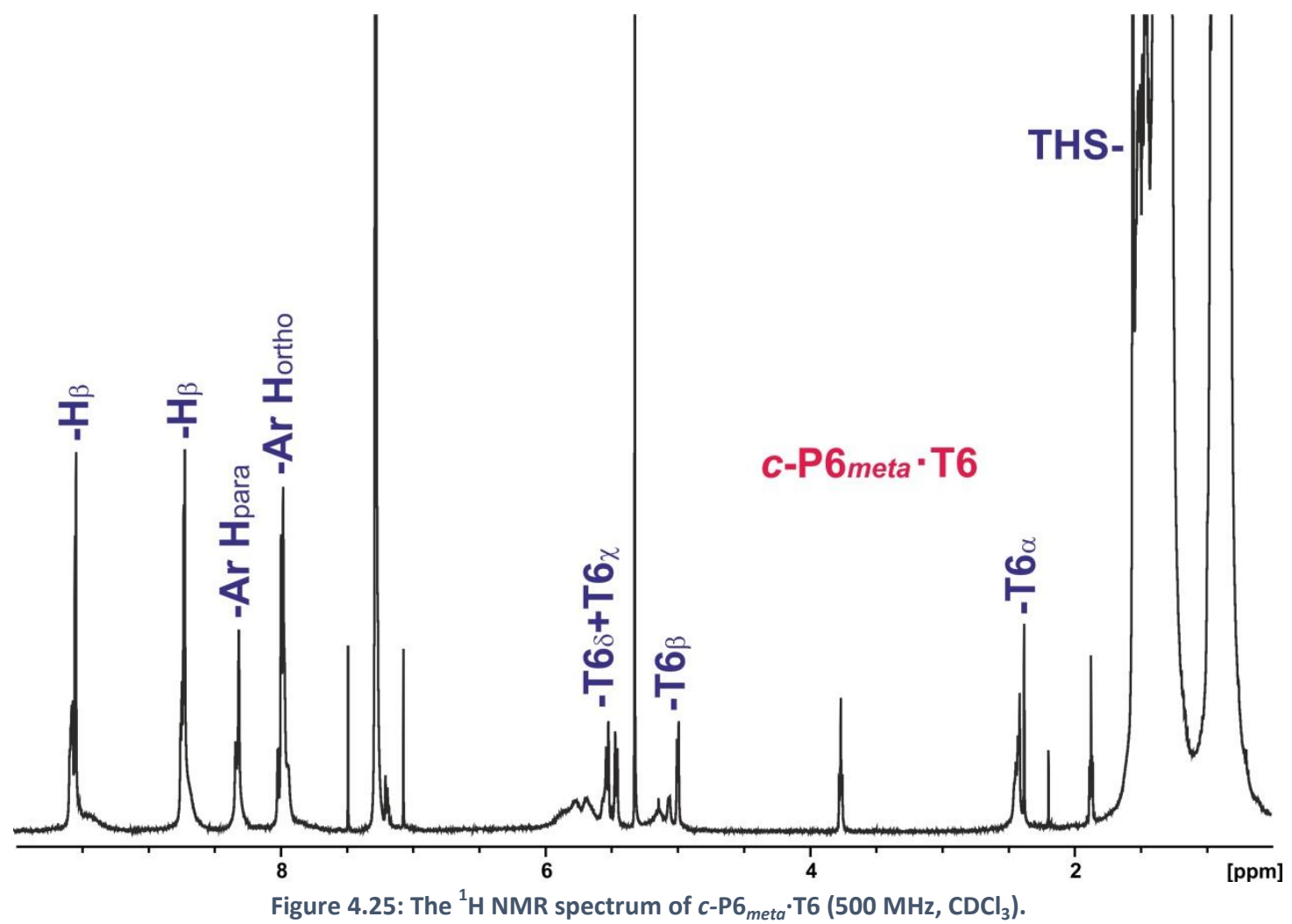




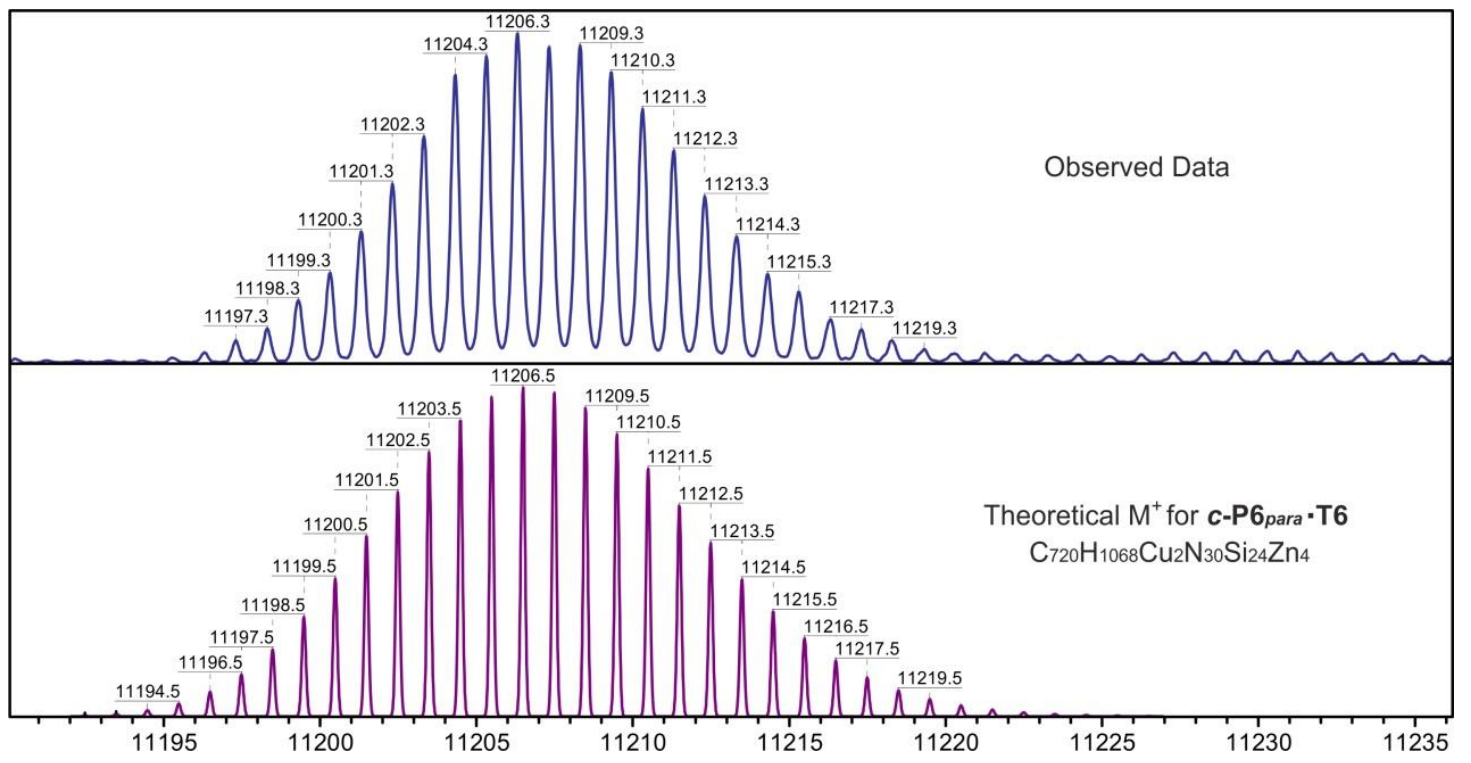

Figure 4.26: The MALDI-MS spectrum of $c$ - $\mathrm{P} 6_{\text {para }} \cdot \mathrm{T} 6\left(\mathrm{~m} / \mathrm{z}=11,206\left(\mathrm{C}_{720} \mathrm{H}_{1068} \mathrm{Cu}_{2} \mathrm{~N}_{30} \mathrm{Si}_{24} \mathrm{Zn}_{4}, \mathrm{M}^{+}\right.\right.$requires 11,207), matrix: DCTB).

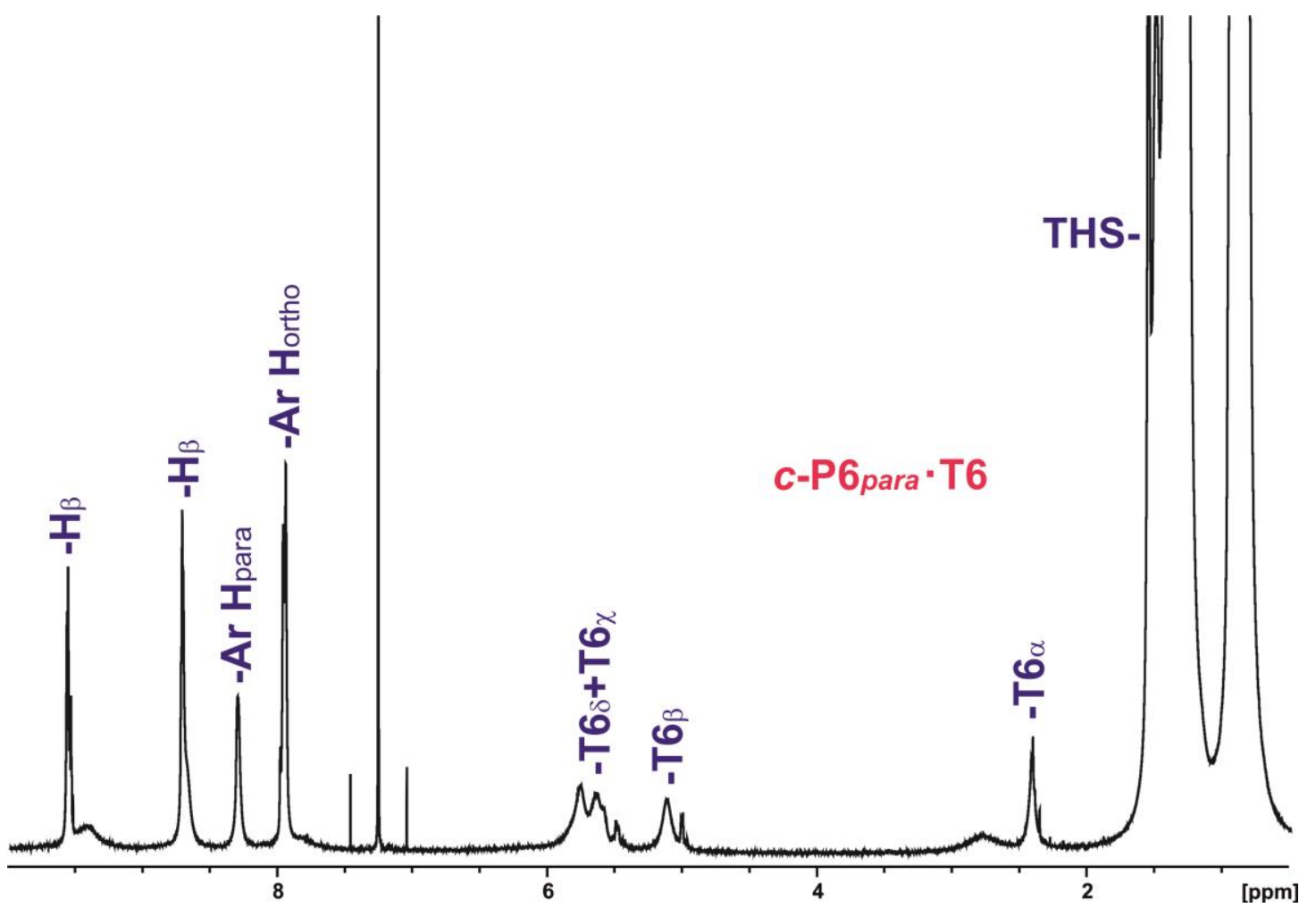

Figure 4.27: The ${ }^{1} \mathrm{H} N M R$ spectrum of $c-\mathrm{P}_{\text {para }} \cdot \mathrm{T6}\left(500 \mathrm{MHz}, \mathrm{CDCl}_{3}\right)$. 


\subsubsection{UV-vis-NIR titrations}

\subsubsection{Titrations of porphyrin monomers and DABCO}
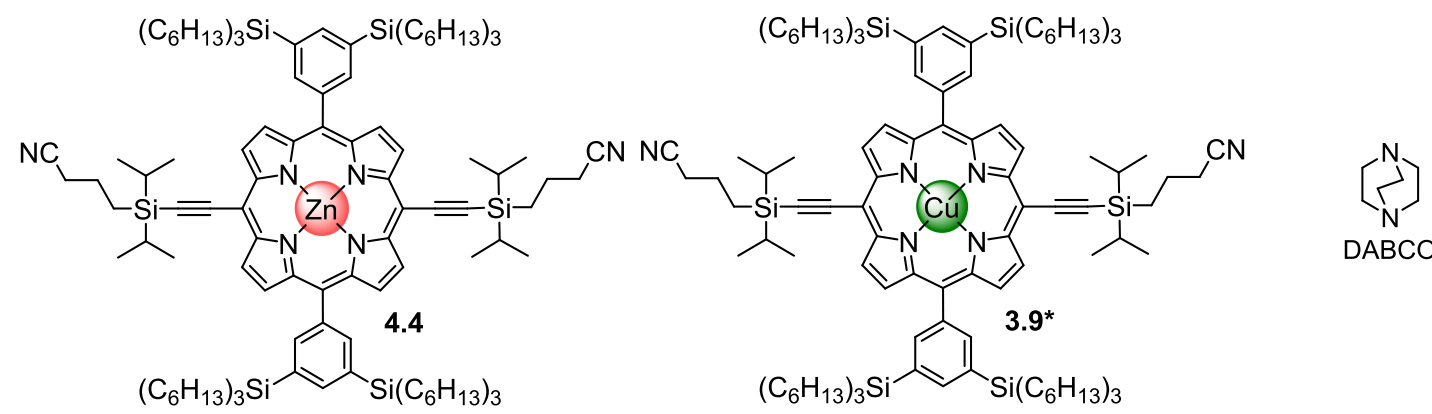

DABCO

Figure 4.28: Structures of reference porphyrin monomers 4.4 and $3.9^{*}$ and the ligand DABCO.

Titrations with porphyrin monomers 4.4 and $3.9 *$ and DABCO were performed in order to determine reference binding constants ( $K_{\mathrm{Zn}}$ and $\left.K_{\mathrm{Cu}}\right)$.

All titrations were performed in toluene at $298 \mathrm{~K}$. Care was taking to keep the porphyrin concentration constant throughout the entire titration by adding porphyrin to the ligand solution before titrations were started. The binding curves were fitted using a 1:1 binding isotherm using the equation:

$$
\frac{A-A_{\text {initial }}}{A_{\infty}-A_{\text {initial }}}=\left(\frac{\left(K_{a}\left([L]+[P]_{0}\right)+1\right)-\sqrt{\left(K_{a}\left([L]+[P]_{0}\right)+1\right)^{2}-4 K_{a}^{2}[P]_{0}[L]}}{2 K_{a}[P]_{0}}\right)
$$

where $A$ is the observed absorption at a specific wavelength or the difference of absorbance between two wavelengths; $A_{\text {initial }}$ is the starting absorption at this wavelength; $A_{\infty}$ is the asymptotic final absorption at this wavelength; $K_{a}$ is the association constant between ligand and porphyrin host; [L] is the concentration of ligand; $[\mathrm{P}]_{0}$ is the concentration of porphyrin host. The free variables which were adjusted to optimise the fit to the experimental data during the fitting procedure are $A_{\text {initial }}, A_{\infty}$, and $K_{a}$. Fitting analysis was carried out using the Origin software (Figures 4.29-4.32) giving $K_{\mathrm{Zn}}=(1.58 \pm 0.03) \times 10^{6} \mathrm{M}^{-1}$ and $K_{\mathrm{Cu}}=(9.11 \pm 0.02) \mathrm{M}^{-1}$. 

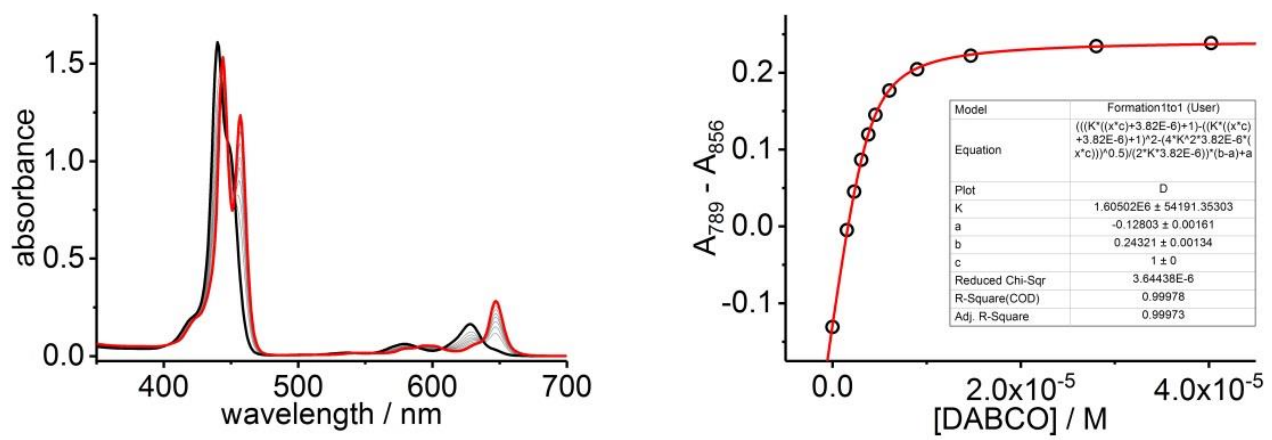

Figure 4.29: UV-vis titration of 4.4 and DABCO, $R^{2}=0.9998$. (Run 1 , toluene, $298 \mathrm{~K},[4.4]=3.82 \mu \mathrm{M}$, $\left.K=1.61 \times 10^{6} \mathrm{M}^{-1}\right)$.
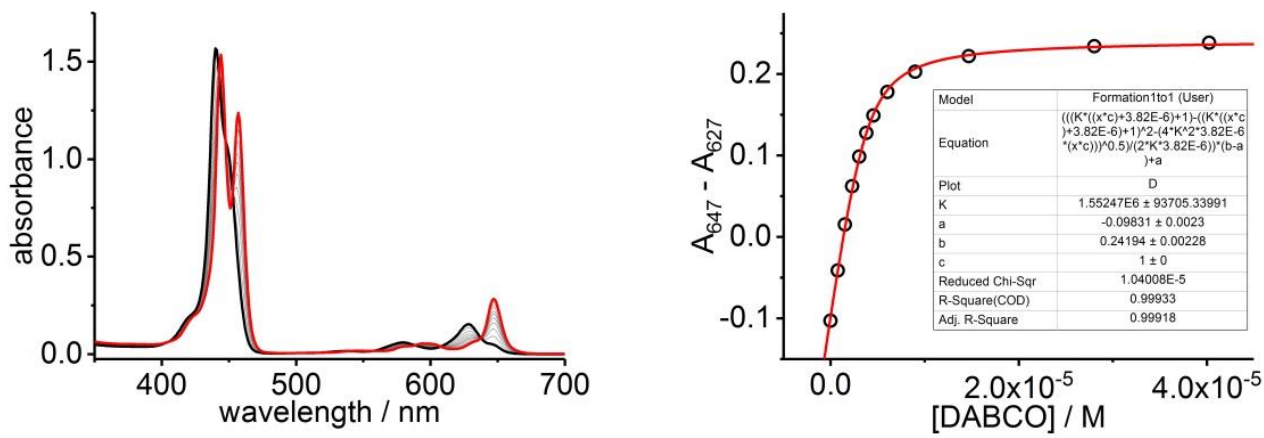

Figure 4.30: UV-vis titration of 4.4 and $\mathrm{DABCO}, R^{2}=0.9993$. (Run 2, toluene, $298 \mathrm{~K},[4.4]=3.82 \mu \mathrm{M}$, $K=1.55 \times 10^{6} \mathrm{M}^{-1}$ ).
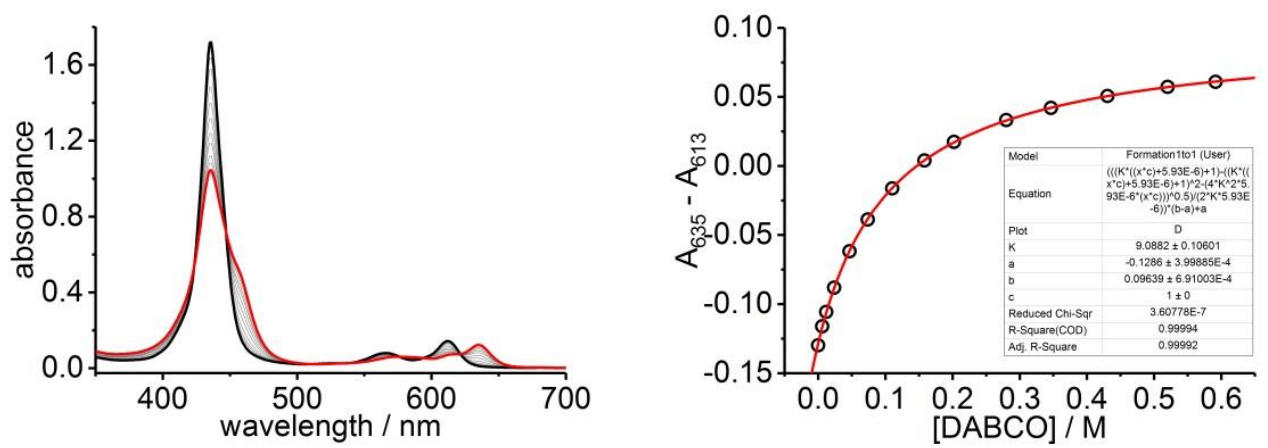

Figure 4.31: UV-vis titration of $3.9^{*}$ and DABCO, $R^{2}=0.9999$. (Run 1 , toluene, $298 \mathrm{~K},[3.9 *]=5.93 \mu \mathrm{M}$, $K=9.09 \mathrm{M}^{-1}$ ).
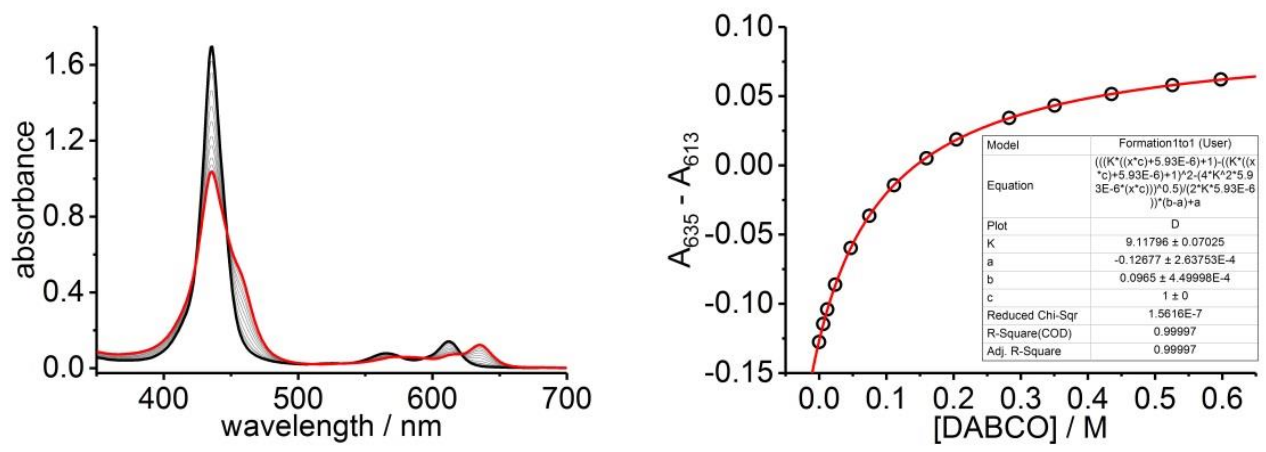

Figure 4.32: UV-vis titration of $3.9^{*}$ and $\mathrm{DABCO}, R^{2}=1.0000$. (Run 2, toluene, $298 \mathrm{~K},\left[3.9^{*}\right]=5.93 \mu \mathrm{M}$, $K=9.12 \mathrm{M}^{-1}$ ). 


\subsubsection{Denaturation titrations of the rings}

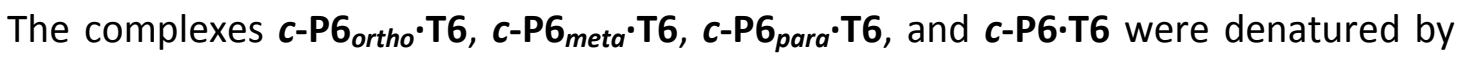
UV-vis-NIR titration by the addition of a large excess of DABCO. As described in Section 2.5.3.2, using the data from these break-up titrations $\left(K_{\mathrm{dn}}=\right.$ denaturation constant) and the formation constant of the single site binding event of DABCO with a zinc or copper porphyrin monomer $\left(K_{\mathrm{zn}}=\right.$ association constant for DABCO to 4.4; $K_{\mathrm{Cu}}=$ association constant for $\mathrm{DABCO}$ to $\mathbf{3 . 9}^{*}$ ) allows us to derive the formation binding constant $\left(K_{\mathrm{f}}\right)$ between the rings and the template.

Table 4.1: Results from UV-vis-NIR titrations in Figures 4.33-4.41.

\begin{tabular}{|c|c|c|c|c|c|}
\hline & $K_{\mathrm{dn}}\left(\mathrm{M}^{-5}\right)$ & $K_{\mathrm{f}}\left(\mathrm{M}^{-1}\right)$ & $K_{\sigma}$ & $K_{\text {chem }}\left(\mathrm{M}^{-1}\right)$ & $\log \left(K_{\text {chem }}\right)$ \\
\hline c-P6 & ortho & & & \\
\hline c-P6 & $(1.85 \pm 0.04) \times 10^{-2}$ & $(6.83 \pm 0.54) \times 10^{24}$ & 768 & $(8.89 \pm 0.70) \times 10^{22}$ & $21.9 \pm 0.1$ \\
\hline c-P6 & $(1.09 \pm 0.01) \times 10^{-2}$ & $(1.16 \pm 0.09) \times 10^{25}$ & 768 & $(1.51 \pm 0.12) \times 10^{22}$ & $22.2 \pm 0.1$ \\
\hline c-P6 & $(0.94 \pm 0.29) \times 10^{-2}$ & $(1.34 \pm 0.43) \times 10^{25}$ & 768 & $(1.75 \pm 0.56) \times 10^{22}$ & $22.2 \pm 0.1$ \\
\hline
\end{tabular}
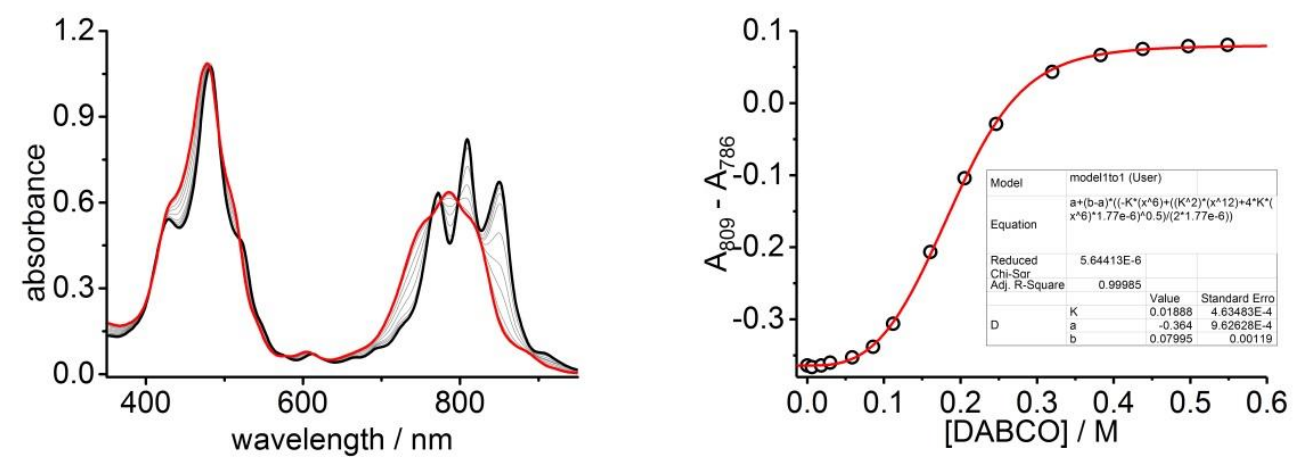

Figure 4.33: UV-vis-NIR titration of $\mathrm{DABCO}$ and $c$-P6 ortho ${ }^{\circ} \mathrm{T6} . R^{2}=1.000$. (Run 1, toluene, $298 \mathrm{~K}$, $\left[c-\mathrm{P} 6_{\text {ortho }} \cdot \mathrm{T6}\right]=1.77 \mu \mathrm{M}, K_{\mathrm{dn}}=1.89 \times 10^{-2} \mathrm{M}^{-5}$ ).
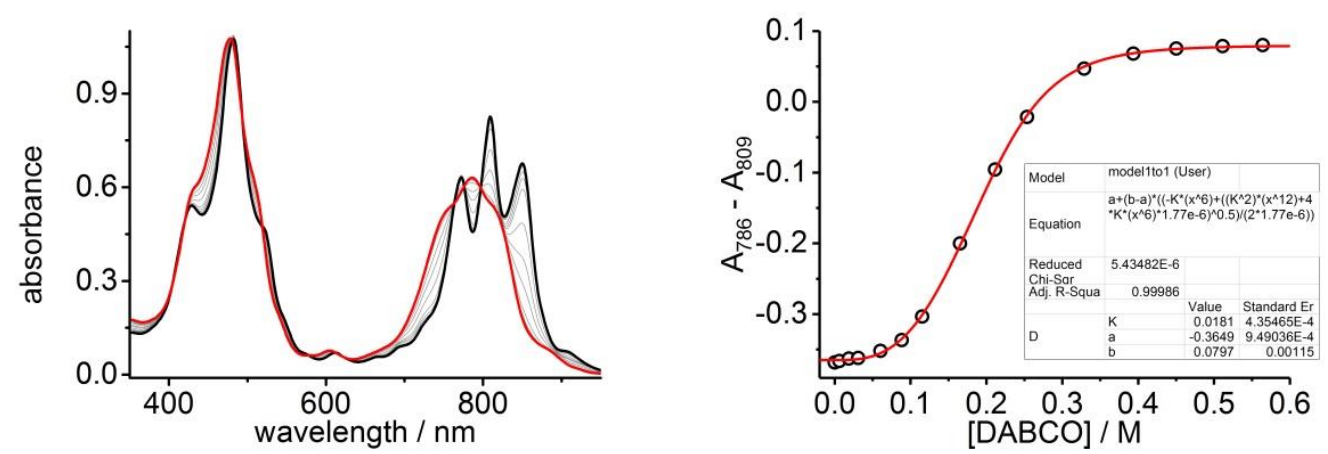

Figure 4.34: UV-vis-NIR titration of $\mathrm{DABCO}$ and $c$-P6 ortho ${ }^{\circ} \mathrm{T6} . R^{2}=1.000$. (Run 2, toluene, $298 \mathrm{~K}$, $\left[c-\mathrm{P}_{\text {ortho }} \cdot \mathrm{T6}\right]=1.77 \mu \mathrm{M}, K_{\mathrm{dn}}=1.81 \times 10^{-2} \mathrm{M}^{-5}$ ). 

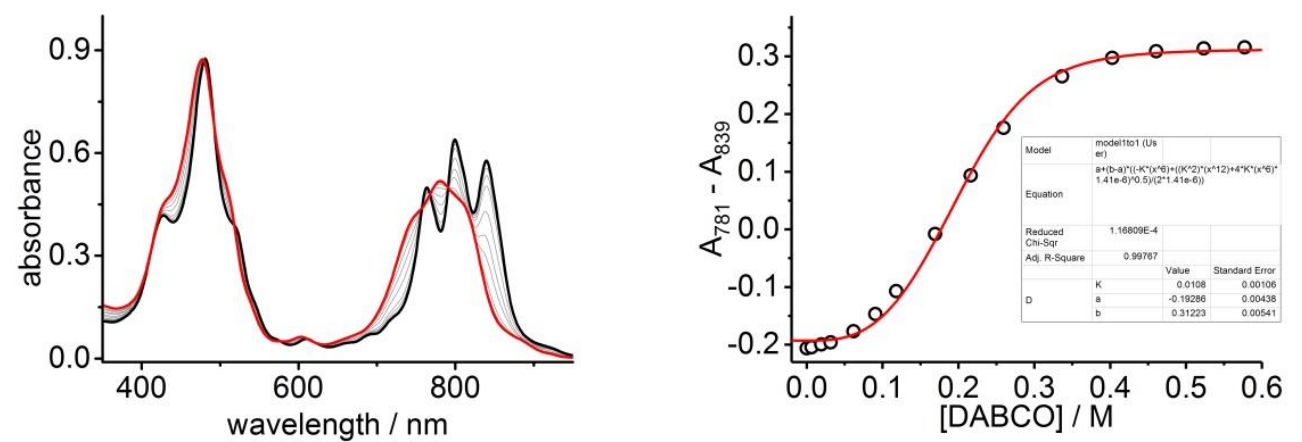

Figure 4.35: UV-vis-NIR titration of $\mathrm{DABCO}$ and $c$-P6 meta $\cdot \mathrm{T6} . R^{2}=0.998$. (Run 1, toluene, $298 \mathrm{~K}$, $\left.\left[c-\mathrm{P}_{\text {meta }} \cdot \mathrm{T6}\right]=1.41 \mu \mathrm{M}, K_{\mathrm{dn}}=1.08 \times 10^{-2} \mathrm{M}^{-5}\right)$.
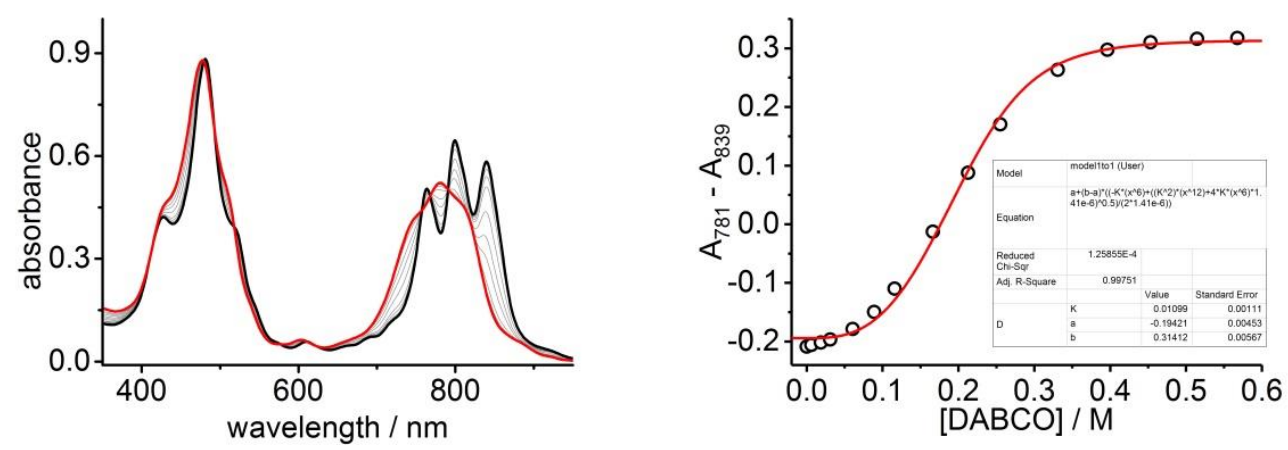

Figure 4.36: UV-vis-NIR titration of DABCO and c-P6 meta $\cdot{ }^{\mathrm{T} 6 .} R^{2}=0.998$. (Run 2, toluene, $298 \mathrm{~K}$, $\left[c-\mathrm{P}_{\text {meta }} \cdot \mathrm{T6}\right]=1.41 \mu \mathrm{M}, K_{\mathrm{dn}}=1.10 \times 10^{-2} \mathrm{M}^{-5}$ ).
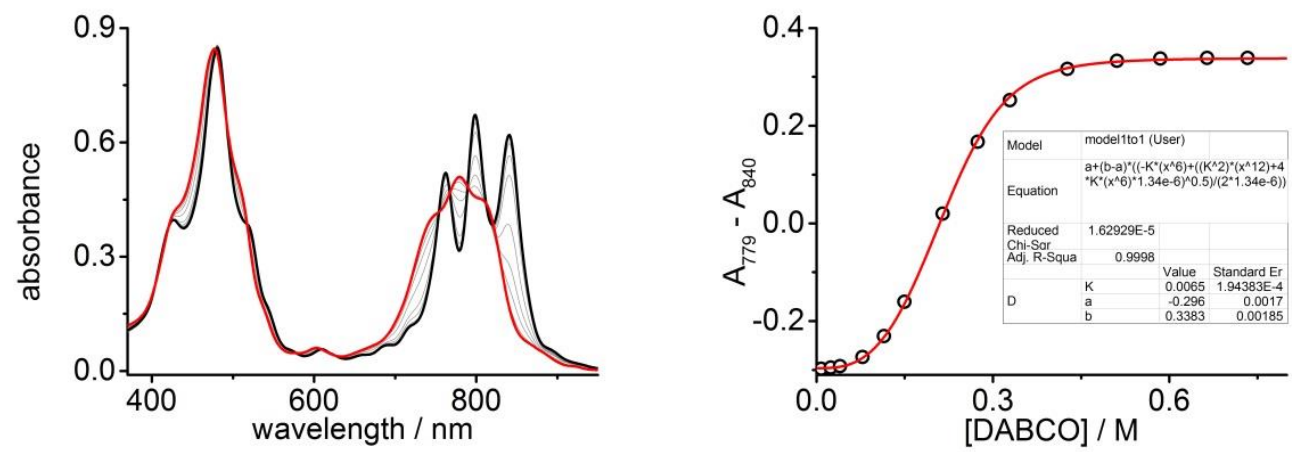

Figure 4.37: UV-vis-NIR titration of DABCO and c-P6 para $\cdot$ T6. $R^{2}=1.000$. (Run 1, toluene, $298 \mathrm{~K}$, $\left.\left[c-P 6_{\text {para }} \cdot \mathrm{T} 6\right]=1.34 \mu \mathrm{M}, K_{\mathrm{dn}}=0.65 \times 10^{-2} \mathrm{M}^{-5}\right)$. 

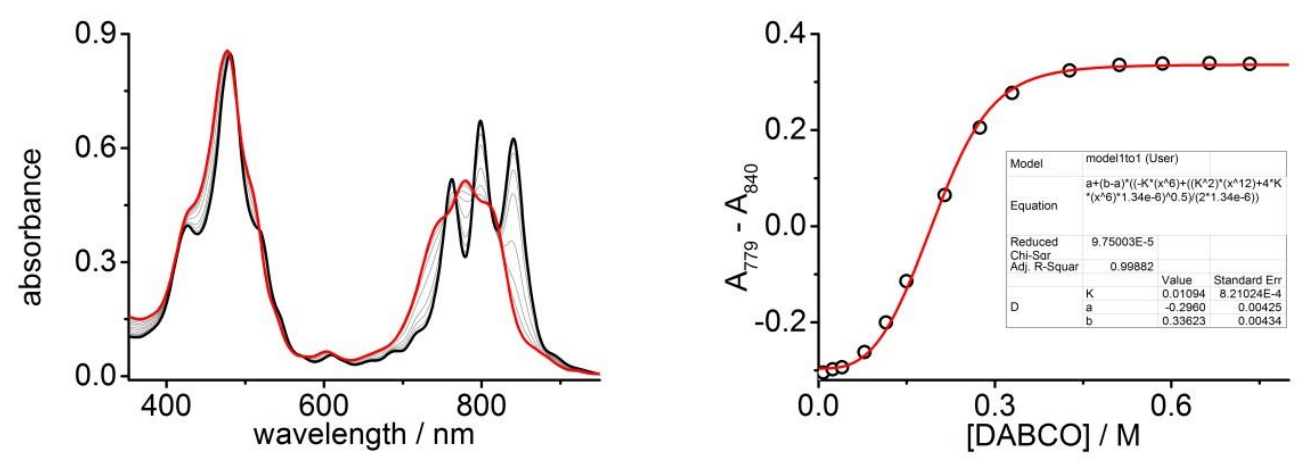

Figure 4.38: UV-vis-NIR titration of $\mathrm{DABCO}$ and $c$-P6 para $\cdot \mathrm{T6} . R^{2}=0.999$. (Run 2, toluene, $298 \mathrm{~K}$, $\left[c-\mathrm{P} 6_{\text {para }} \cdot \mathrm{T6}\right]=1.34 \mu \mathrm{M}, K_{\mathrm{dn}}=1.09 \times 10^{-2} \mathrm{M}^{-5}$ ).
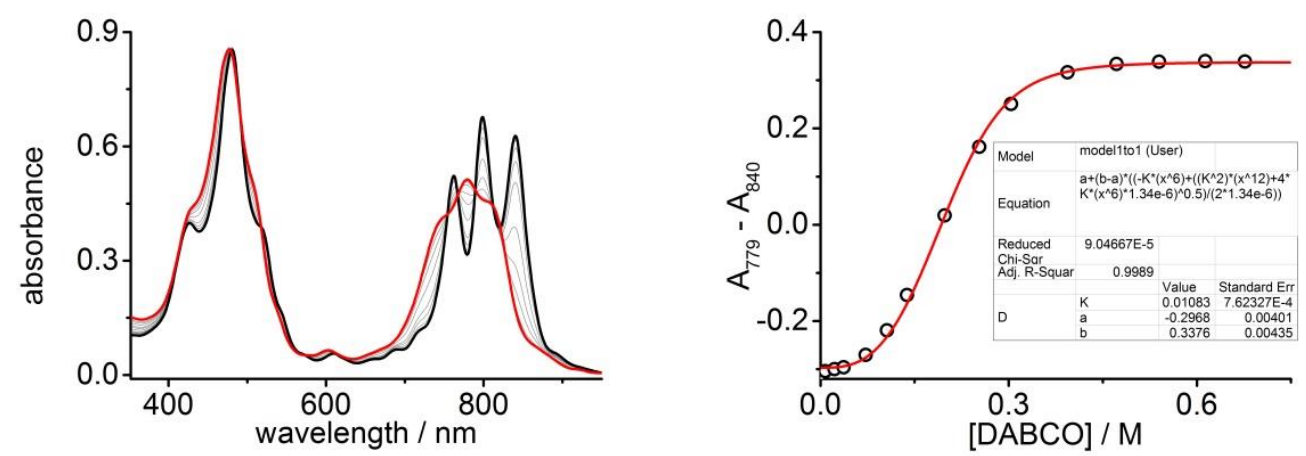

Figure 4.39: UV-vis-NIR titration of DABCO and c-P6 para $\cdot$ T6. $R^{2}=0.999$. (Run 3, toluene, 298 K, $\left[c-\mathrm{P} 6_{\text {para }} \cdot \mathrm{T6}\right]=1.34 \mu \mathrm{M}, K_{\mathrm{dn}}=1.08 \times 10^{-2} \mathrm{M}^{-5}$ ).
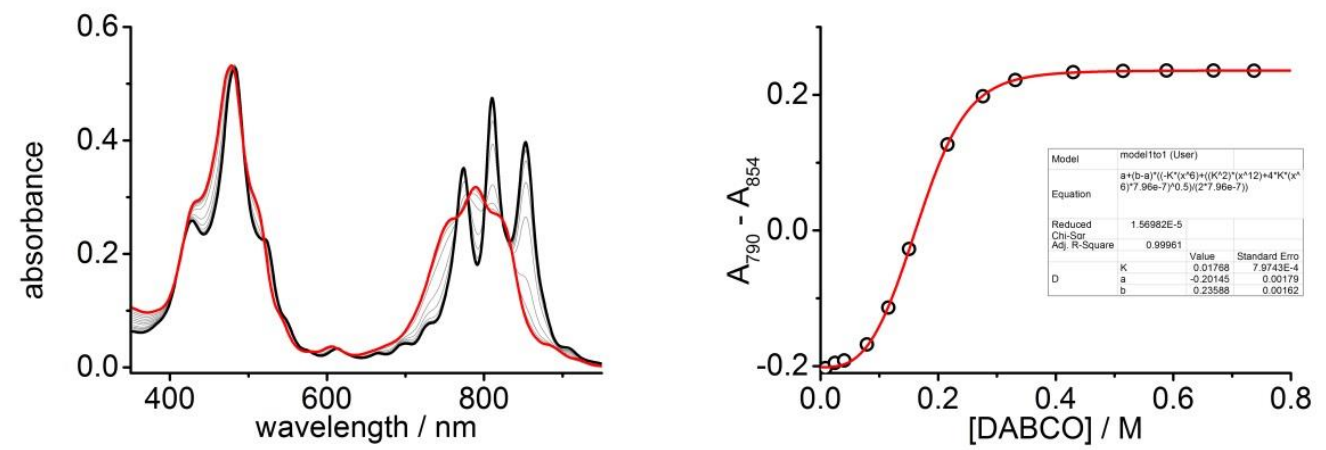

Figure 4.40: UV-vis-NIR titration of DABCO and c-P6·T6. $R^{2}=1.000$. (Run 1, toluene, $298 \mathrm{~K},[c-\mathrm{P} 6 \cdot \mathrm{T} 6]=0.80 \mu \mathrm{M}$, $K_{\mathrm{dn}}=1.77 \times 10^{-2} \mathrm{M}^{-5}$ ). 

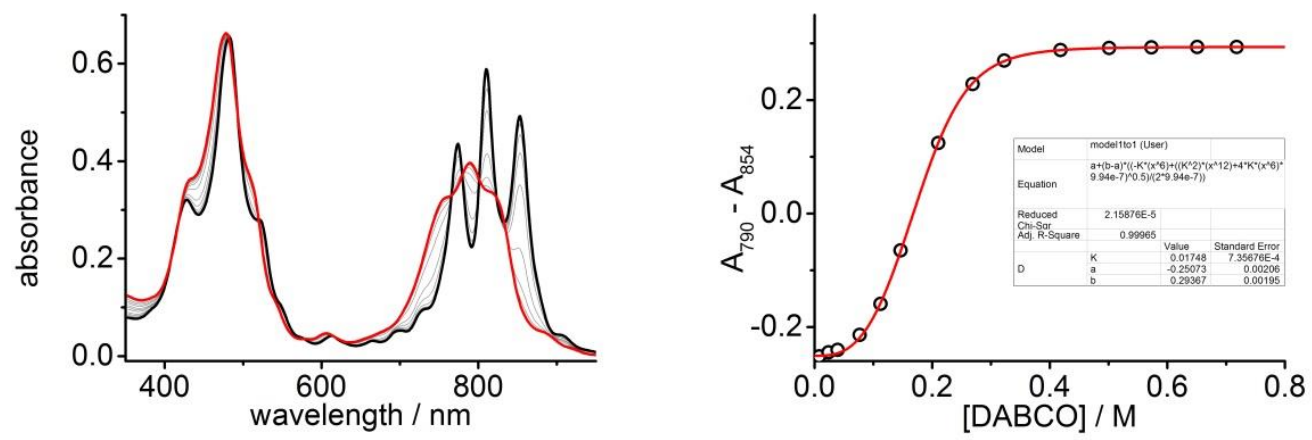

Figure 4.41: UV-vis-NIR titration of DABCO and c-P6·T6. $R^{2}=1.000$. (Run 2, toluene, $298 \mathrm{~K},[c-\mathrm{P} 6 \cdot \mathrm{T} 6]=0.99 \mu \mathrm{M}$, $K_{\mathrm{dn}}=1.75 \times 10^{-2} \mathrm{M}^{-5}$ ).

\subsubsection{Calculation of statistical factors}

To understand the stability constants of different complexes, it is useful to factor out statistical contributions. The values of $K_{\sigma}$ were calculated using Benson's symmetry number method as described in Section 2.5.4.

Table 4.2: Internal, external and total symmetry numbers for each component.*

\begin{tabular}{|c|c|c|c|c|}
\hline component & point group & $\sigma_{\text {int }}$ & $\sigma_{e x t}$ & $\boldsymbol{\sigma}$ \\
\hline DABCO & $\mathrm{C}_{3 \mathrm{v}}$ & 1 & 6 & 6 \\
\hline T6 & $\mathrm{D}_{6 h}$ & 1 & 12 & 12 \\
\hline 4.4 & $\mathrm{D}_{2 \mathrm{~h}}$ & 1 & 4 & 4 \\
\hline $3.9 *$ & $\mathrm{D}_{2 \mathrm{~h}}$ & 1 & 4 & 4 \\
\hline C-P6 ortho & $\mathrm{C}_{2 \mathrm{v}}$ & $2^{6}=64$ & 2 & 128 \\
\hline$c-P 6_{m e t a}$ & $\mathrm{C}_{2 \mathrm{v}}$ & $2^{6}=64$ & 2 & 128 \\
\hline$c-P 6_{p a r a}$ & $\mathrm{D}_{2 \mathrm{~h}}$ & $2^{6}=64$ & 4 & 256 \\
\hline c-P6 & $\mathrm{D}_{6 \mathrm{~h}}$ & $2^{6}=64$ & 12 & 768 \\
\hline c-P6 $6_{\text {ortho }} \cdot \mathrm{T6}$ & $\mathrm{C}_{2 \mathrm{v}}$ & 1 & 2 & 2 \\
\hline$c-\mathrm{P} 6_{m e t a} \cdot \mathrm{T} 6$ & $\mathrm{C}_{2 \mathrm{v}}$ & 1 & 2 & 2 \\
\hline$c-P 6_{p a r a} \cdot T 6$ & $\mathrm{D}_{2 \mathrm{~h}}$ & 1 & 4 & 4 \\
\hline c-P6-T6 & $D_{6 h}$ & 1 & 12 & 12 \\
\hline
\end{tabular}

[* Note that when counting internal rotations and calculating $\sigma_{i n t}$, we do not include rotors which are unaffected by the binding process, such as the para-phenylene links in T5 because if a rotor is unaffected by the binding process it has no influence on $K_{\sigma}$.] 


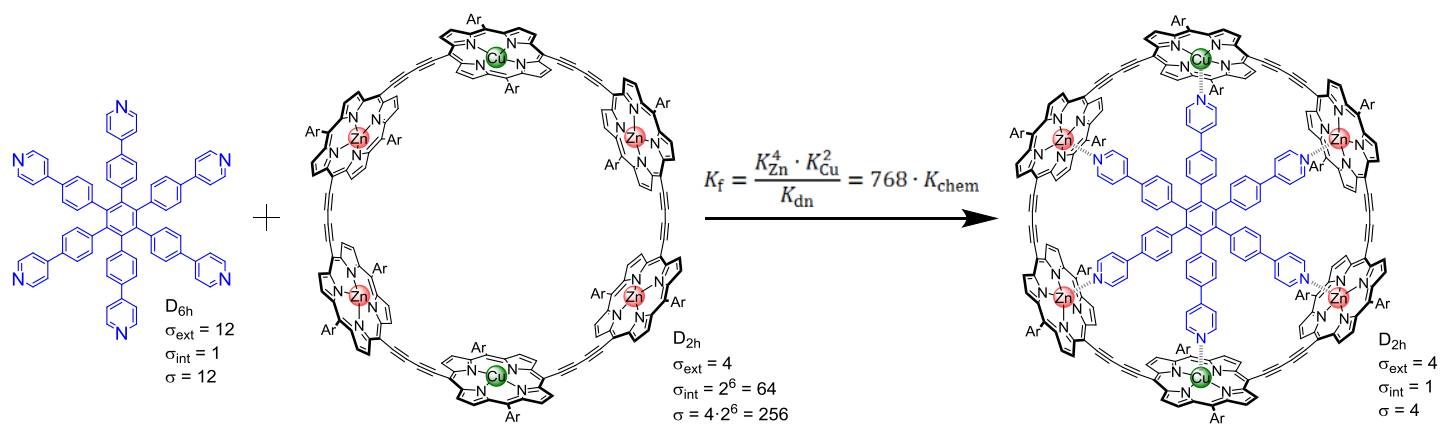

Figure 4.42: Statistical factors involved in the formation of the complex $c-\mathrm{P} 6_{\text {para }} \cdot \mathrm{T} 6 \cdot K_{\sigma}=768$.

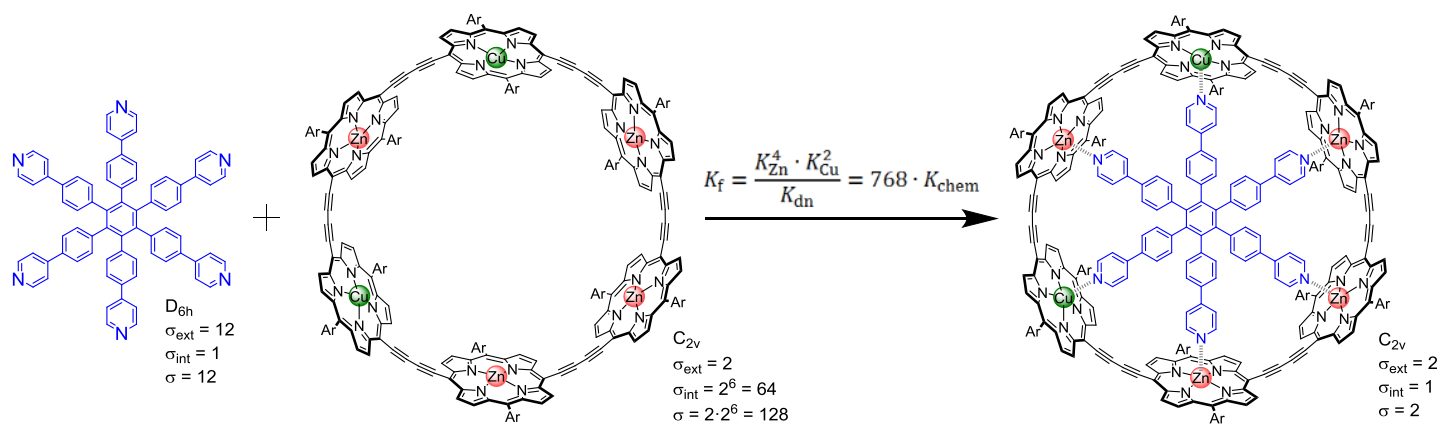

Figure 4.43: Statistical factors involved in the formation of the complex $c-\mathrm{P} 6_{\text {meta }} \cdot \mathrm{T} 6 . K_{\mathrm{\sigma}}=768$.

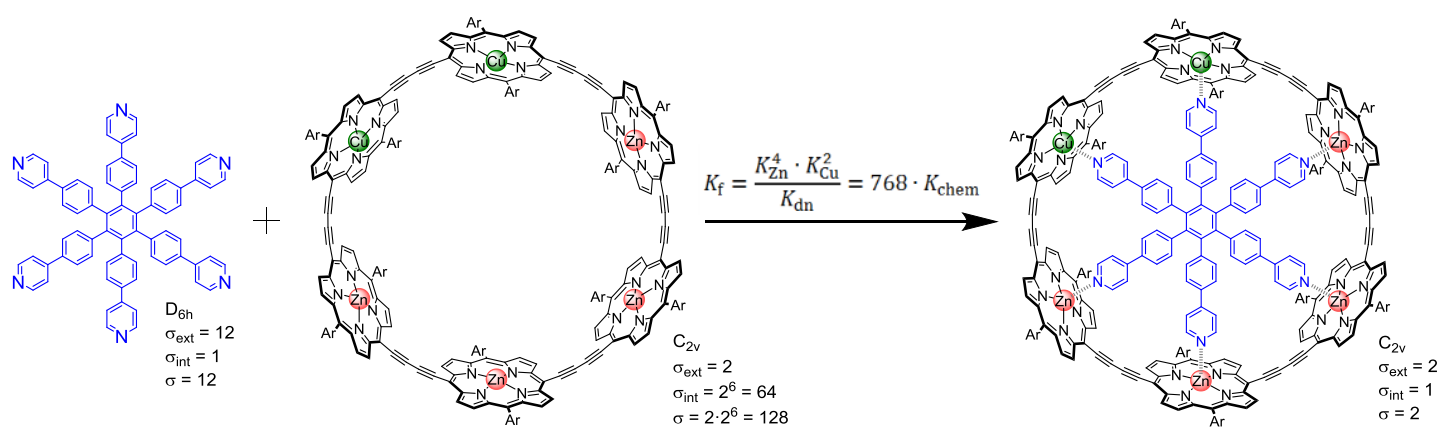

Figure 4.44: Statistical factors involved in the formation of the complex $c-\mathrm{P} 6_{\text {ortho }}{ }^{\cdot T 6} \cdot K_{\sigma}=768$.

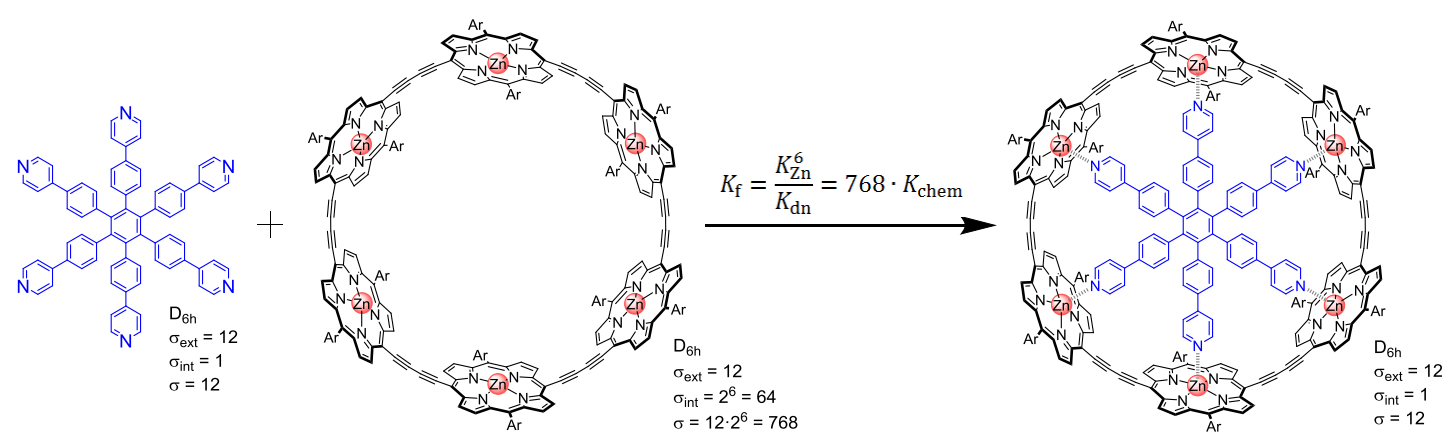

Figure 4.45: Statistical factors involved in the formation of the complex $c$-P6 $\cdot$ T6. $K_{\sigma}=768$.

\subsubsection{Justification of equation 4.1}

In Section 4.3.5, we used an equation relating the average effective molarity $(\overline{E M})$ to the denaturation constant. The justification of this equation is a result of the thermodynamic cycle describing the binding events which are schematically depicted in Figure 4.46. 


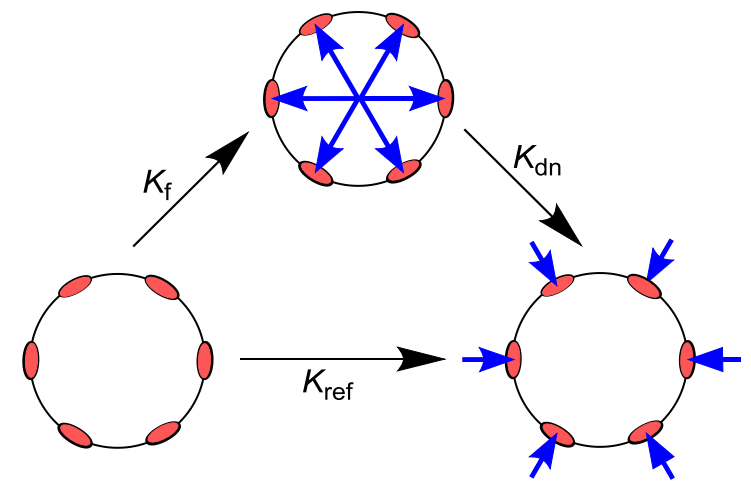

Figure 4.46: Thermodynamic cycle relating the formation, denaturation and reference binding constants.

The stability constants for the three processes are described by:

$$
\begin{gathered}
K_{\mathrm{f}}=\frac{[c \mathbf{P 6} \cdot \mathbf{T 6}]}{[c \mathbf{P 6}] \cdot[\mathbf{T 6}]} \\
K_{\mathrm{dn}}=\frac{\left[c \mathbf{P 6} \cdot \mathbf{L}_{6}\right] \cdot[\mathbf{T 6}]}{[c \mathbf{P 6} \cdot \mathbf{T} 6] \cdot[\mathbf{L}]^{6}} \\
K_{\mathrm{ref}}=\frac{\left[c \mathbf{P 6} \cdot \mathbf{L}_{6}\right]}{[c \mathbf{P 6}] \cdot[\mathbf{L}]^{6}}
\end{gathered}
$$

We also know that the effective molarity is described by the difference between the intramolecular and intermolecular binding events which reveals:

$$
E M=\frac{K_{\text {intra }}}{K_{\text {inter }}}=\frac{K_{\mathrm{f}}}{K_{\text {ref }}}=\frac{\frac{[c \mathbf{P 6} \cdot \mathbf{T 6}]}{[c \mathbf{P 6}] \cdot[\mathbf{T 6}]}}{\frac{\left[c \mathbf{P 6} \cdot \mathbf{L}_{6}\right]}{[c \mathbf{P 6}] \cdot[\mathbf{L}]^{6}}}=\frac{[c \mathbf{P 6} \cdot \mathbf{T 6}] \cdot[\mathbf{L}]^{6}}{\left[c \mathbf{P 6} \cdot \mathbf{L}_{\mathbf{6}}\right] \cdot[\mathbf{T 6}]}=\frac{1}{K_{\mathrm{dn}}}
$$

As can be seen from the equation, the $E M$ and $K_{\mathrm{dn}}$ are directly related to each other but since the isolated interactions of the individual legs of the T6 template and the competing ligand (DABCO) with porphyrin monomers are different, it would be false to claim that they are equal, they are merely proportional as described by the formula used in the main text:

$$
K_{\mathrm{dn}} \propto \frac{1}{\overline{\mathrm{EM}}}
$$




\section{The Template-Directed Synthesis of a Fully Conjugated 14-Porphyrin Nanoball}

\section{Contents}

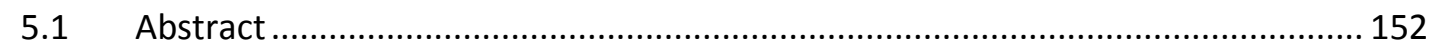

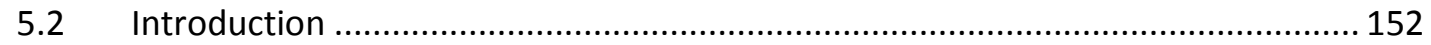

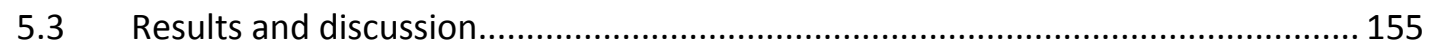

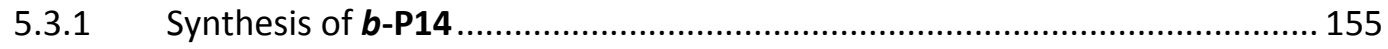

5.3.2 ${ }^{1}$ H NMR assignment of $\boldsymbol{b}$-P14-T6-(T4) $)_{2}, \boldsymbol{b}$-P14·T6 and $\boldsymbol{b}$-P14 ........................ 164

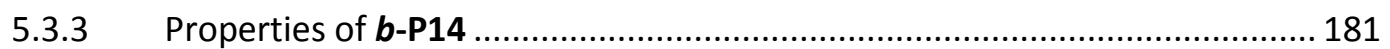

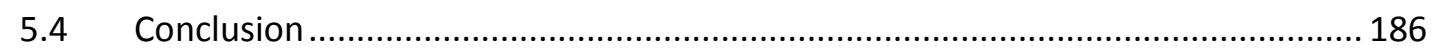

5.5 Experimental procedures and technical details................................................ 186

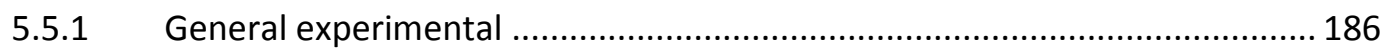

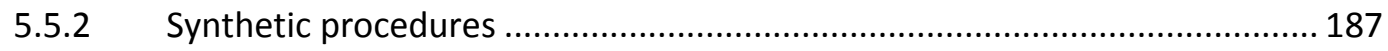

5.5.3 Spectra confirming identity of new compounds ........................................... 202

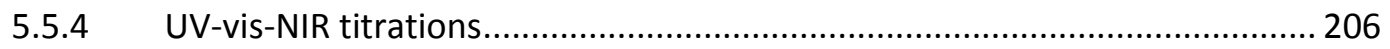

5.5.5 Calculation of statistical factors ............................................................... 212

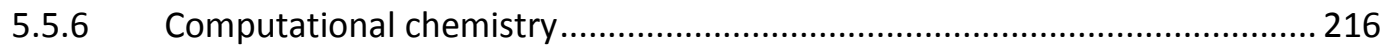

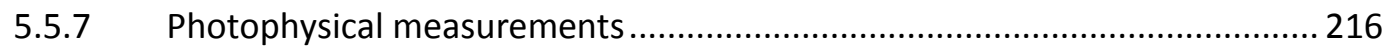

Parts of this chapter have been submitted for publication in:

Template-directed synthesis of a conjugated zinc porphyrin nanoball

J. Cremers, R. Haver, M. Rickhaus, J. Q. Gong, L. Favereau, M. D. Peeks, T. D. W. Claridge, L. M. Herz, H. L. Anderson, submitted to: J. Am. Chem. Soc. 2018. 


\subsection{Abstract}

The preparation of conjugated organic systems with strong $\pi$-orbital overlap between the molecular components is both challenging and laborious. This chapter describes the template-directed synthesis and characterisation of a $\pi$-conjugated 14-porphyrin nanoball. The bicyclic structure consists of a 6- and 10-porphyrin nanoring intersecting at two points. UV-vis-NIR titrations were performed to investigate the binding behaviour of the templates inside the cavity of the ball. In addition, photoluminescence $(\mathrm{PL})$ upconversion spectroscopy revealed strong coupling between the two rings. By removing the templates, the flexibility in the system increases which results in a decrease in the PL anisotropy. This indicates that excitations may migrate between the two ring components of the nanoball.

\subsection{Introduction}

Conjugated organic molecules have been extensively studied to investigate the correlation between structure and electronic properties. By increasing the size and dimensionality of a $\pi$-system, electronic delocalisation can be enhanced, potentially leading to electronic features such as global aromaticity and quantum interference. ${ }^{160,165}$ Porphyrins are versatile building blocks which can be used to produce elaborate $\pi$-conjugated systems. Their chemistry is well-established and allows for the combination of rationally designed linear synthesis and a supramolecular template-directed approach. Within our group, we have utilised this methodology to prepare cyclic porphyrin arrays with up to 50 porphyrins in the perimeter of the ring by using either a direct or Vernier templating method (Figure 5.1). ${ }^{38,39,50,51,53}$ 

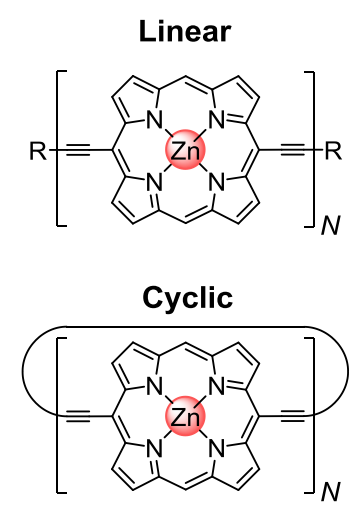
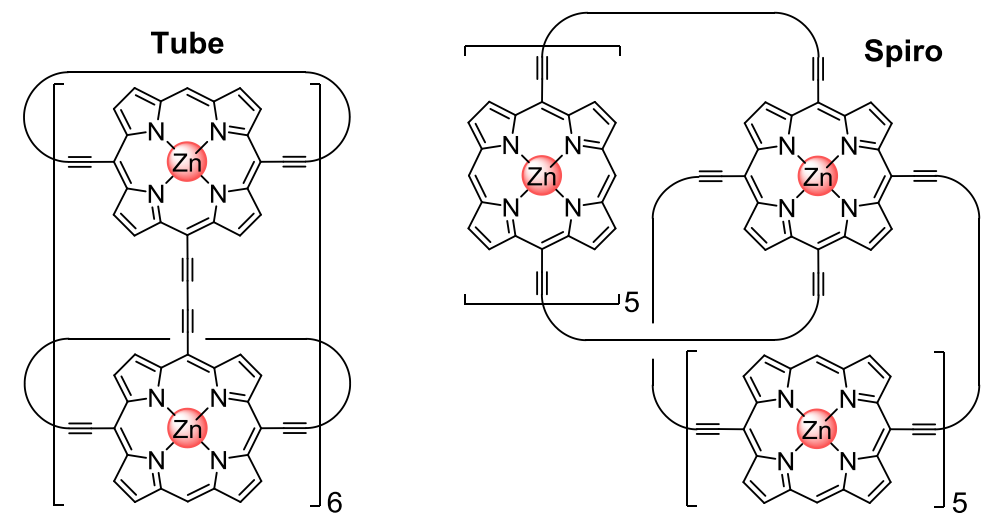

Figure 5.1: Chemical structures of linear porphyrin oligomers, rings, tubes and spiro-systems. The smallest porphyrin nanoring to date consists of $N=5$ porphyrins, the largest system contains $N=50$. Aryl solubilising groups have been omitted for clarity.

Recent effort has been directed at the development of synthetic strategies to expand these cyclic porphyrin systems in the third dimension. By modifying the zinc porphyrin monomers to contain three acetylene substituents in the meso-positions, the formation of a 12-porphyrin tube nanotube was achieved consisting of two stacked 6-porphyrin rings (Figure 5.1, middle). ${ }^{54}$ More recently, a tetra-mesosubstituted porphyrin unit was used in the template-directed synthesis of a spirofused array of 11 porphyrin units (Figure 5.1, right). ${ }^{55}$

Ever since the discovery of buckminsterfullerene, ${ }^{167}$ chemists have sought rational strategies for the synthesis of three-dimensional $\pi$-conjugated geodesic cages. Stepwise chemical synthesis has not yet been used to prepare any fullerenes, except $\mathrm{C}_{60},{ }^{168-170}$ but several fullerene-like ball-shaped $\pi$-conjugated hydrocarbons have been synthesised recently. ${ }^{171-174}$ The high dimensionality of these $\pi$-systems is expected to enhance their electronic delocalization, compared with conventional 1D or 2D molecular semiconductors. ${ }^{175-177}$ Shape-persistent molecular cages are also in demand for their gas adsorption properties, which mimic those of zeolites. ${ }^{178,179}$ The best reactions for preparing molecular cages or capsules are reversible processes that allow error correction, such as metal coordination ${ }^{180-182}$ or boronic ester condensation, ${ }^{183}$ but these reactions do not produce $\pi$-conjugated connections. In principle, reversible reactions such as imine formation, ${ }^{184}$ alkene metathesis ${ }^{87}$ and alkyne metathesis ${ }^{185,186}$ could be used to construct $\pi$-conjugated cages, but so far the cages made by these reactions lack long-range conjugation. Template-directed coupling, under kinetic control, is an alternative strategy for preparing large 
macrocycles and cages. ${ }^{37,39,54,55}$ Here we show how simple molecular templates can be used to synthesise the first fully $\pi$-conjugated porphyrin ball $\boldsymbol{b}$-P14 (Figure 5.2). This 14-porphyrin prolate ellipsoidal cage consists of two perpendicular intersecting conjugation pathways, defined by six and ten porphyrin units respectively. Structures of this type are valuable models for photosynthetic light-harvesting systems. ${ }^{39,54,55,187-189}$ When all 14 porphyrin units in $\boldsymbol{b}$-P14 are bound to templates, to lock the conformation, excited state energy delocalisation occurs over the whole system within $0.3 \mathrm{ps}$, where in the absence of the templates excitation is distributed over the ball with a time constant of about 2 ps.

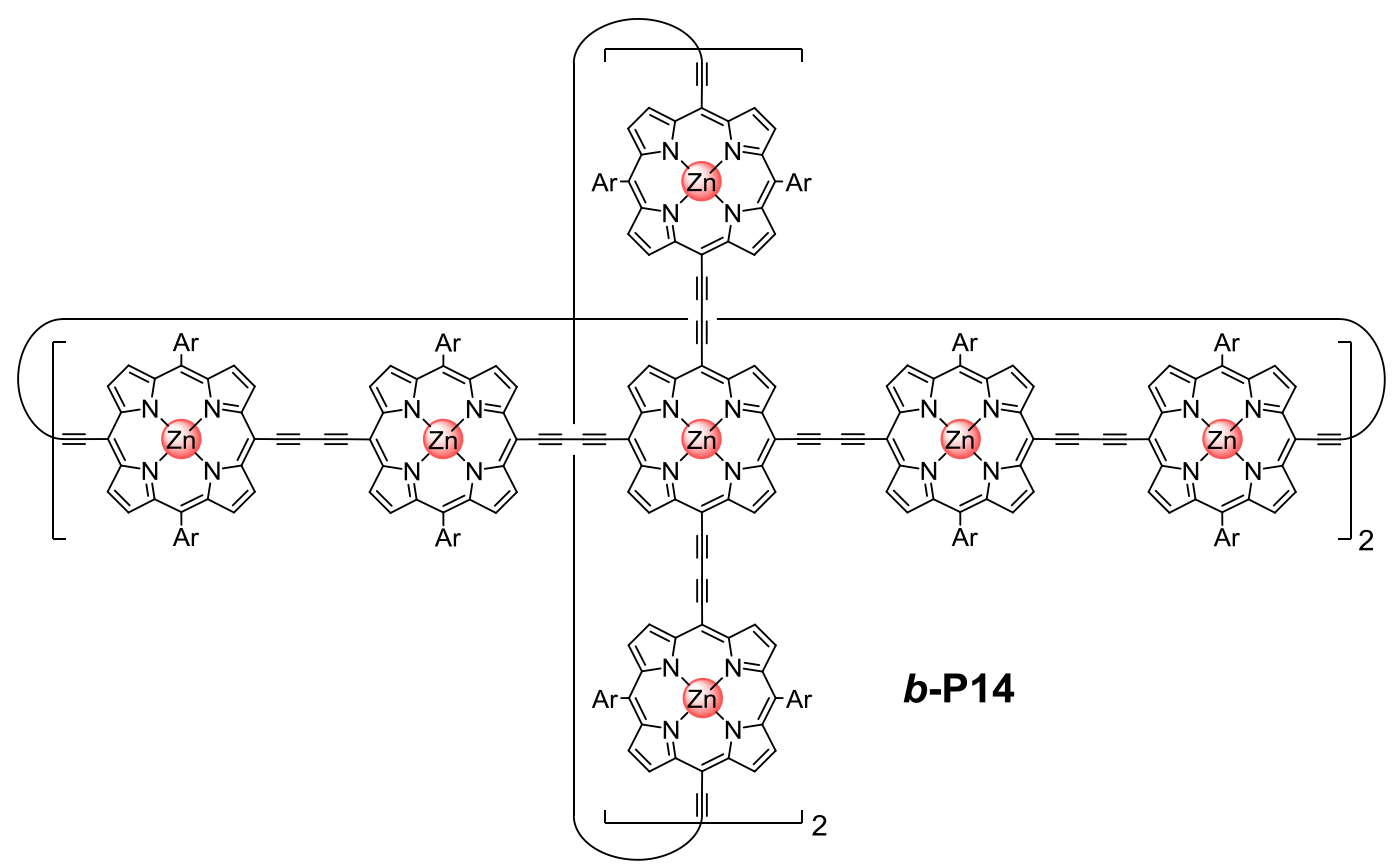

Figure 5.2: Proposed chemical structure of the 14-porphyrin nanoball $b$-P14. Ar will be defined later in the chapter.

The schematic synthetic plan for the synthesis of $\boldsymbol{b}$-P14 is shown in Figure 5.3. Initially a 6-porphyrin nanoring is prepared which contains two porphyrins with acetylene moieties at all four meso-positions in opposing positions of the ring. These acetylenes are coupled to four porphyrin dimers to produce a 14-porphyrin precursor. The tetra-dentate template $\mathbf{T 4}$ is then used to pre-organise the system before oxidative coupling conditions are used to form the last two carbon-carbon bonds. Finally, an excess of a competing ligand is used in order to remove the templates and obtain the template free target molecule, $\boldsymbol{b}$-P14. The 
porphyrin-porphyrin distance along the length of the 10-ring exceeds $5 \mathrm{~nm}$, rendering it one of the largest $\pi$-conjugated molecular structures to date.

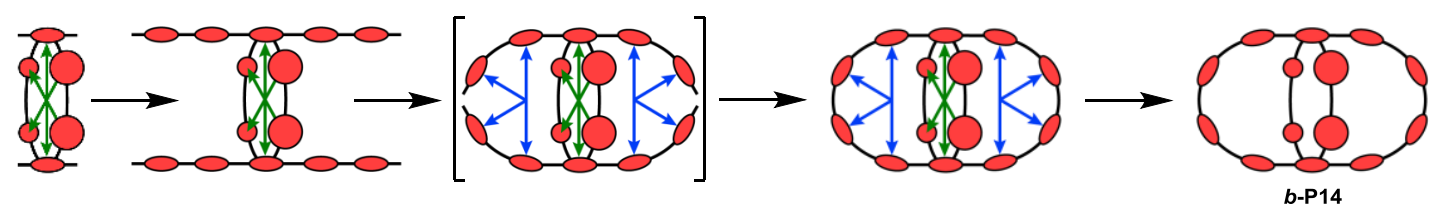

Figure 5.3: Schematic synthetic route for $b-\mathrm{P} 14$.

\subsection{Results and discussion}

\subsubsection{Synthesis of $b$-P14}

The first building block in the synthesis of $\boldsymbol{b}$-P14 is the central-porphyrin functionalised in all four meso-positions with acetylene units. The synthetic route is depicted in Figure 5.4 and is an adaption from a literature procedure reported by Anderson et al. ${ }^{190}$ Initially, dipyrromethane (2.1) and triisopropylpropynal (5.1) are condensed with boron trifluoride, followed by oxidation with DDQ to give porphyrin 5.2 in a $32 \%$ yield. Magnesium was inserted into the centre of the porphyrin by refluxing 5.2 overnight with $\mathrm{Mgl}_{2}$ in a mixture of pyridine and triethylamine. Subsequently, the meso-positions of the porphyrin were selectively brominated in a $66 \%$ yield. While it seems laborious to go via a magnesium porphyrin intermediate, direct bromination of the free-base or zinc porphyrin analogues are reported to result in significant side reactions at the $\beta$-positions and on the alkynes. The electron withdrawing 5,15-dialkynyl substituents reduce the reactivity of $\mathbf{5 . 2}$ and therefore magnesium needs to be inserted to make the porphyrin more electron-rich and ensure a clean reaction at the meso-positions of the porphyrin. ${ }^{190,191}$ The addition of TFA was subsequently used to remove the magnesium after which zinc was inserted to give 5.6. Subjecting $\mathbf{5 . 6}$ to Sonogashira coupling conditions in the presence of ethynyltrimethylsilane afforded the tetra-meso-functionalised porphyrin monomer 5.7 in an $86 \%$ yield. The trimethylsilane (TMS) groups are more labile than the triisopropylsilane (TIPS) groups, which allows for the selective deprotection of the TMS-acetylenes in later stages of the synthesis. 


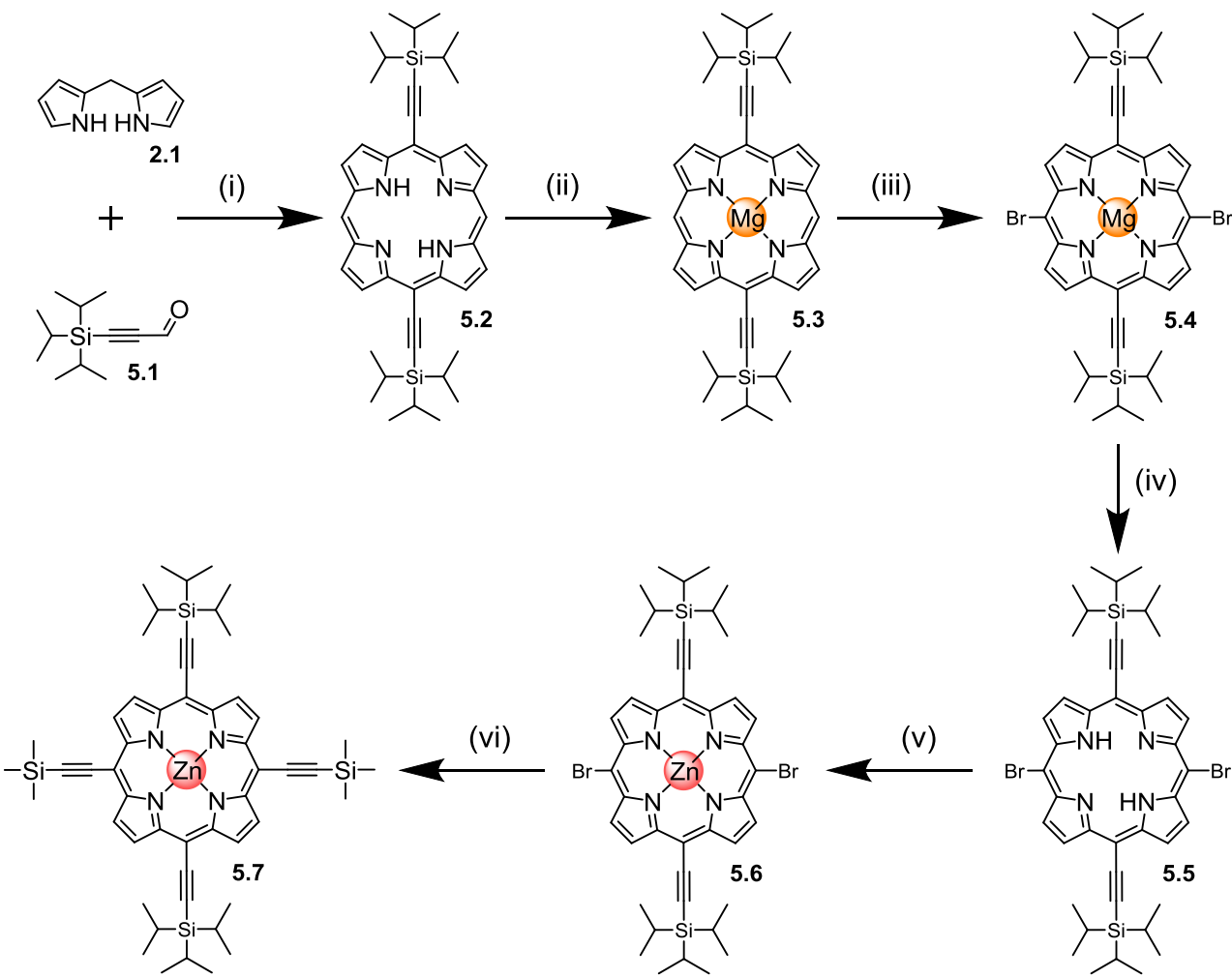

Figure 5.4: Synthetic route to porphyrin 5.7: (i) $\mathrm{BF}_{3} \cdot \mathrm{Et}_{2} \mathrm{O}, \mathrm{DDQ}, \mathrm{CH}_{2} \mathrm{Cl}_{2}, 32 \%$; (ii) $\mathrm{Mgl}_{2}, \mathrm{pyridine} \mathrm{Et}_{3} \mathrm{~N}, 97 \%$; (iii) NBS, $\mathrm{CHCl}_{3} /$ pyridine, 63\%; (iv) TFA, $\mathrm{CHCl}_{3}, 72 \%$; (v) $\mathrm{Zn}(\mathrm{OAC})_{2} \cdot\left(\mathrm{H}_{2} \mathrm{O}\right)_{2}, \mathrm{CHCl}_{3} /$ methanol, $100 \%$; (vi) $\mathrm{Pd}_{2}\left(\mathrm{dba}_{3}\right.$, Cul, $\mathrm{PPh}_{3}$, ethynyltrimethylsilane, toluene/ $i-\mathrm{Pr}_{2} \mathrm{NH}, 86 \%$.

The second building block contains zinc porphyrins accommodating solubilising aryl groups. A variety of solubilising groups have been utilised in the past which range from highly solubilising 3,5-bis-(trihexylsilyl)phenyl groups to more crystalline 3,5-bis(tert-butyl)phenyl groups. The aryl substituents in the meso-position of porphyrins are known to twist perpendicular to the porphyrin plane. As a result, solubilising chains pointing towards the centre of the ball are anticipated to lead to steric congestion, particularly between the aryl groups on the four porphyrins directly adjacent to the central tetra-meso-acetylene porphyrins, hindering the formation of the ball in the final stages of the synthesis. It was decided to use 3-((2-ethylhexyl-alkoxy)phenyl as the aryl group which contains a single chain solubilising chain. It was anticipated that the chain would rotate towards the periphery of the ball, minimising steric congestion towards the centre of the molecule.

Porphyrin $\mathbf{5 . 1 1}$ was prepared in four steps (Figure 5.5). In the first step, dipyrromethane (2.1) and the corresponding aldehyde are used in a porphyrin condensation reaction followed directly by zinc insertion to give $\mathbf{5 . 9}$ in a $44 \%$ overall 
yield. Bromination of the meso-positions followed by Sonogashira coupling with ethynyltrimethylsilane resulted in the porphyrin $\mathbf{5 . 1 1}$ in nearly quantitative yields.

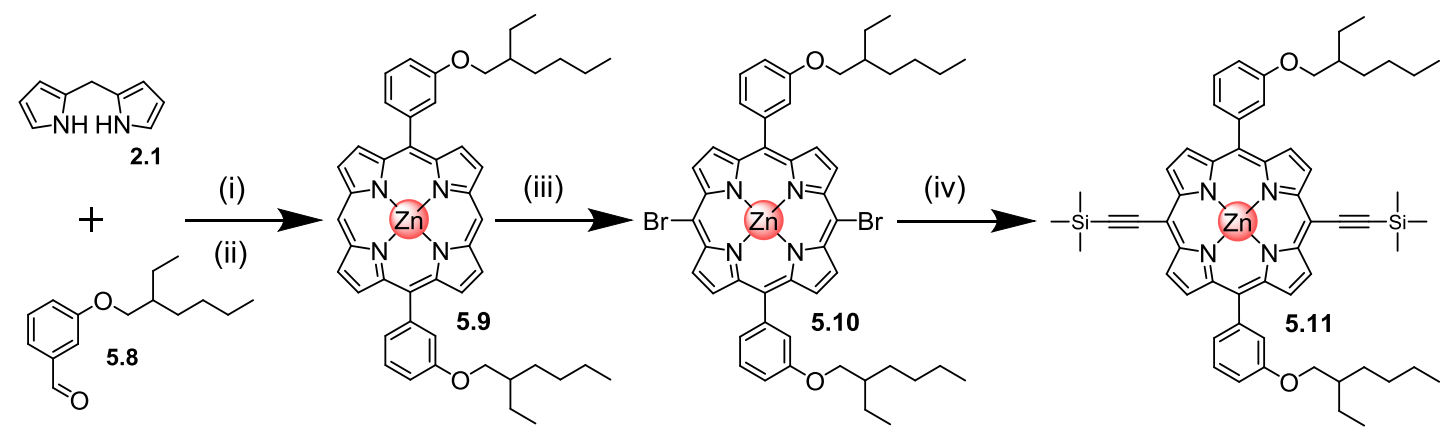

Figure 5.5: Synthetic route to porphyrin 5.11: (i) TFA, DDQ, $\mathrm{CH}_{2} \mathrm{Cl}_{2}$; (ii) $\mathrm{Zn}(\mathrm{OAc})_{2} \cdot\left(\mathrm{H}_{2} \mathrm{O}\right)_{2}, \mathrm{CHCl}_{3}, 44 \%$; (iii) $\mathrm{NBS}$, $\mathrm{CHCl}_{3}$ /pyridine, 92\%; (iv) $\mathrm{Pd}_{2}$ (dba) $)_{3}, \mathrm{Cul}$, $\mathrm{PPh}_{3}$, ethynyltrimethylsilane, toluene/i- $\mathrm{Pr}_{2} \mathrm{NH}, 96 \%$.

The functionalisation of $\mathbf{5 . 7}$ with both TIPS and TMS protecting groups allowed for the selective removal of the more labile TMS group by reacting 5.7 with $\mathrm{K}_{2} \mathrm{CO}_{3}$ in a mixture of THF and methanol giving porphyrin $\mathbf{5 . 1 2}$ in a 94\% yield (Figure 5.6). Careful addition of small quantities of TBAF, while constantly monitoring the reaction progress, allowed for the statistical deprotection of one of the TMS groups on 5.11, giving the mono-deprotected porphyrin $\mathbf{5 . 1 3}$ in a 31\% yield. Porphyrins $\mathbf{5 . 1 2}$ and $\mathbf{5 . 1 3}$ were mixed in a one-to-five ratio after which oxidative coupling conditions were used to form a statistical mixture of oligomers. The desired porphyrin trimer $\mathbf{5 . 1 4}$ was isolated from the mixture by recycling GPC in a $47 \%$ yield. The use of an excess of 5.13 resulted in the formation of large quantities of a symmetric homo-coupled dimer (5.18) which will be utilised later in the synthesis (Figure 5.8). 


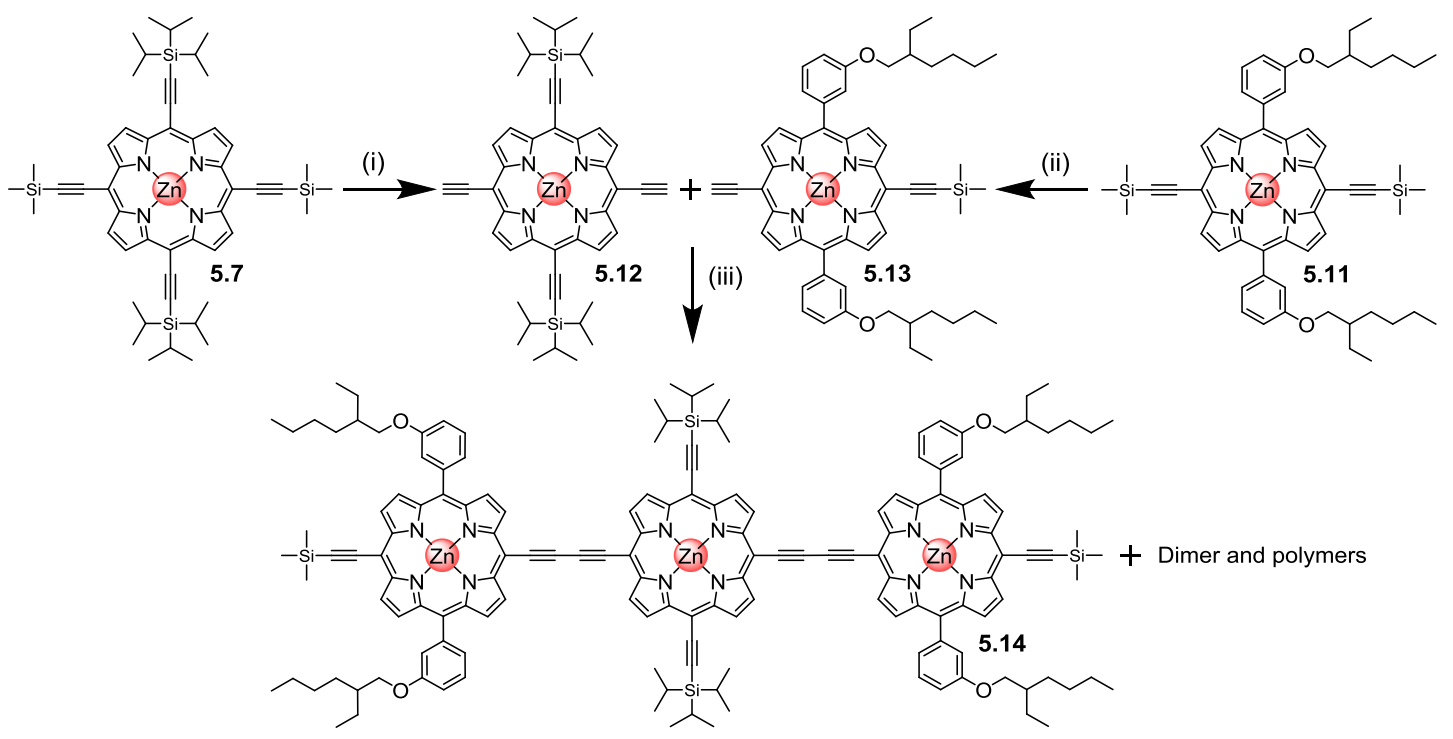

Figure 5.6: Synthetic route to trimer 5.14: (i) $\mathrm{K}_{2} \mathrm{CO}_{3}, \mathrm{THF} /$ methanol, $94 \%$; (ii) $\mathrm{TBAF}, \mathrm{CHCl}_{3} /$ pyridine, $31 \%$; (iii) $\mathrm{PdCl}_{2}\left(\mathrm{PPh}_{3}\right)_{2}, \mathrm{Cul}, 1,4$-benzoquinone, $\mathrm{CHCl}_{3} / i-\mathrm{Pr}_{2} \mathrm{NH}, 47 \%$.

Similar to 5.7, 5.14 contains both TMS and TIPS protecting groups which allows for the selective deprotection of the more labile TMS groups with $\mathrm{K}_{2} \mathrm{CO}_{3}$ to give $\mathbf{5 . 1 5}$ in an $85 \%$ yield (Figure 5.7). Butadiyne linked zinc porphyrin trimers are known to bind strongly to the T6 template. ${ }^{23}$ When trimer $\mathbf{5 . 1 5}$ and T6 were combined in a two-to-one ratio, a highly pre-organised complex was formed. Subsequently subjecting the complex to oxidative coupling conditions resulted in the formation of the 6-porphyrin nanoring $\mathbf{5 . 1 6}$ in a $70 \%$ yield after purification by recycling GPC. Ring 5.16 contains two porphyrins at opposing positions of the ring substituted with TIPS-protected acetylenes suitable for extension in the perpendicular direction of the ring. The addition of TBAF resulted in the removal of the TIPS protecting group, forming nanoring 5.17 in an $87 \%$ yield. 


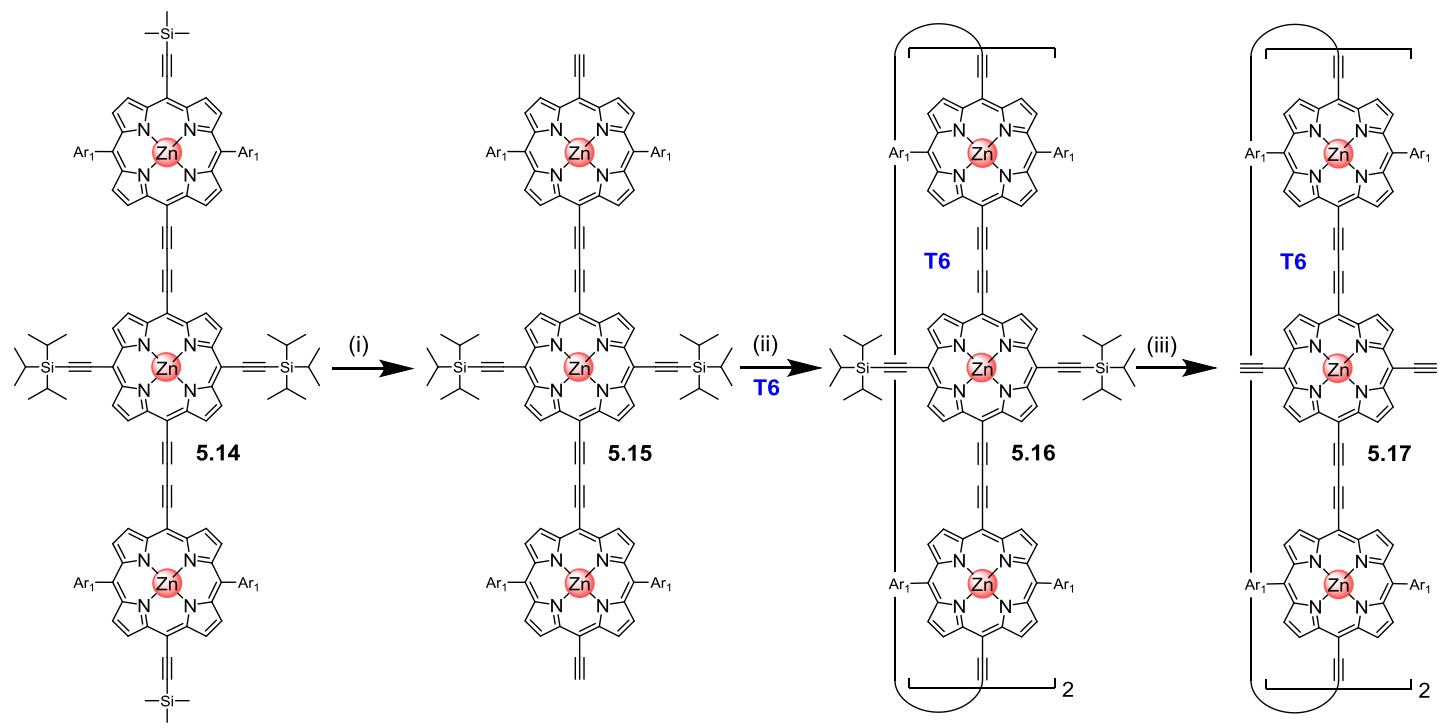

Figure 5.7: Synthetic route of 6-porphyrin nanoring 5.17. (i) $\mathrm{K}_{2} \mathrm{CO}_{3}, \mathrm{THF} /$ methanol, 85\%; (ii) $\mathrm{T6}, \mathrm{Pd}\left(\mathrm{PPh}_{3}\right)_{2} \mathrm{Cl}_{2}$, Cul, 1,4-benzoquinone, $\mathrm{CHCl}_{3} / i-\mathrm{Pr}_{2} \mathrm{NH}, 70 \%$; (iii) TBAF, $\mathrm{CH}_{2} \mathrm{Cl}_{2}, 87 \%$. ( $\mathrm{Ar}_{1}$ = 3-(2-ethylhexyl-alkoxy)phenyl).

The statistical coupling of $\mathbf{5 . 1 2}$ and $\mathbf{5 . 1 3}$ discussed in Figure 5.6 resulted in the formation of a symmetric homo-coupled dimer $\mathbf{5 . 1 8}$ which was used in the extension of the ring perpendicular to the ring plane (Figure 5.8). Careful addition of TBAF allowed for the statistical removal of one of the protecting groups of the dimer in a $32 \%$ yield. To suppress the polymerisation of $\mathbf{5 . 1 7}$ in the subsequent oxidative coupling reaction, a 40-fold excess of the mono-deprotected dimer $\mathbf{5 . 1 9}$ was used with respect to 5.17 resulting in the formation of the extended porphyrin nanoring 5.20.

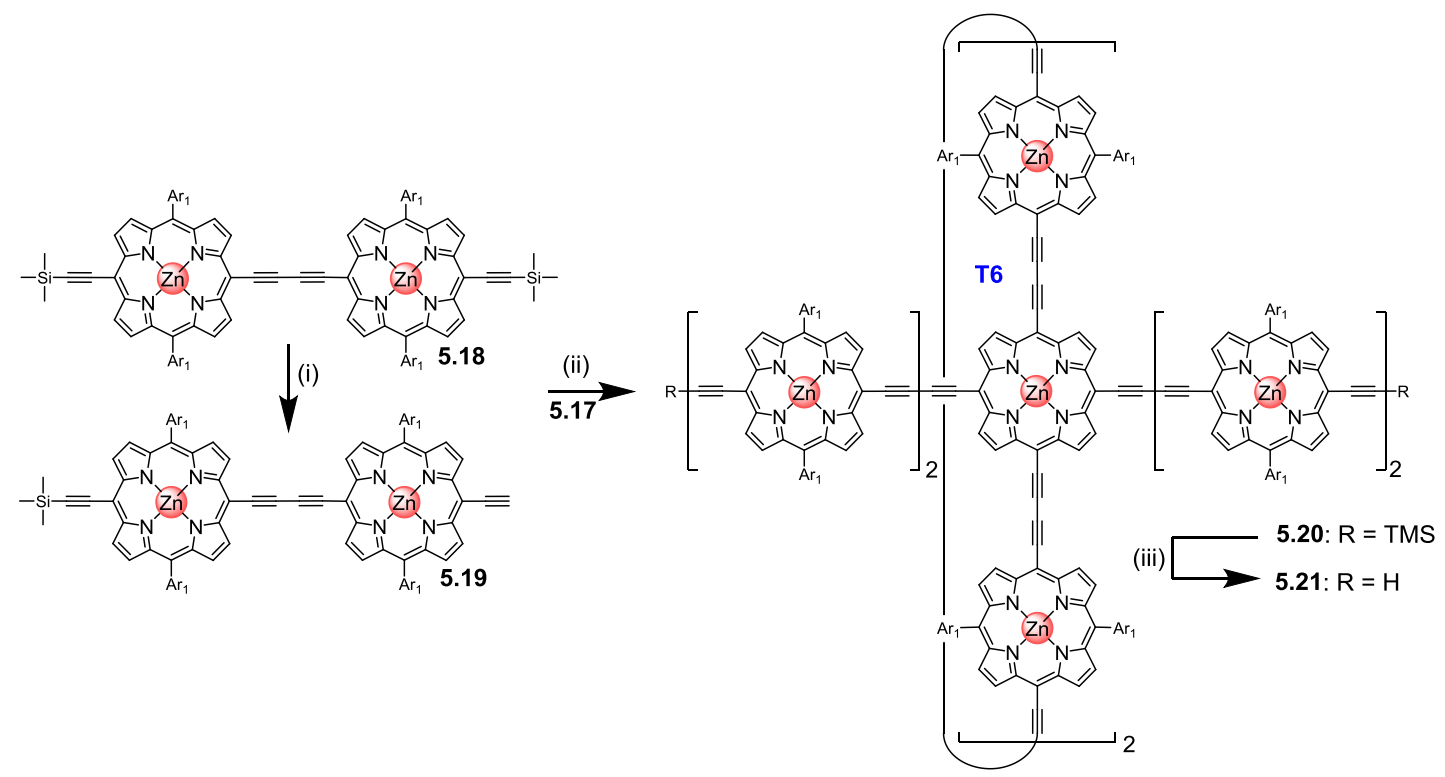

Figure 5.8: Synthetic route of the extended 6-porphyrin nanoring 5.21. (i) $\mathrm{TBAF}, \mathrm{CHCl}_{3}, 32 \%$; (ii) 5.17, $\mathrm{Pd}\left(\mathrm{PPh}_{3}\right)_{2} \mathrm{Cl}_{2}$, Cul, 1,4-benzoquinone, toluene/i- $\mathrm{Pr}_{2} \mathrm{NH}, 52 \%$; (iii) $\quad \mathrm{TBAF}, \mathrm{CH}_{2} \mathrm{Cl}_{2}, 96 \%$. $\left(A r_{1}=3\right.$-(2-ethylhexyl-alkoxy)phenyl). 
Naturally, the use of an excess dimer $\mathbf{5 . 1 9}$ in the reaction resulted in the formation of large quantities of a linear tetramer. The difference in size between $\mathbf{5 . 2 0}$ and the side-products is large which allowed for the purification of the desired product by recycling GPC in a $52 \%$ yield (Figure 5.9 ).

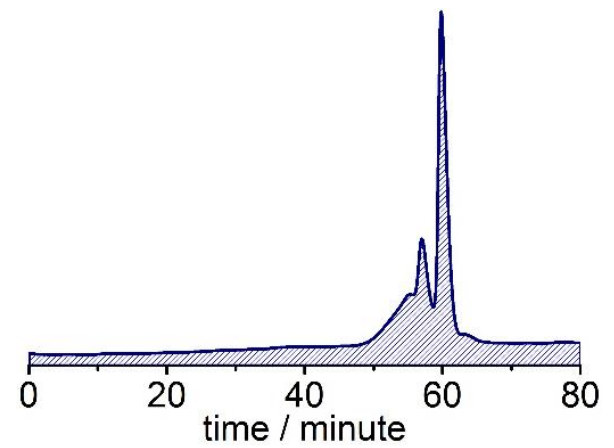

Figure 5.9: The first cycle of the recycling GPC trace (JAIGEL columns, $1^{\text {st }}$ cycle shown, $1 \%$ pyridine in toluene, detection at $590 \mathrm{~nm}$ ) in the formation of 5.20 . The main product is a linear tetramer at ca. 60 minutes preceded by the desired product and longer oligomers at a shorter retention time.

The TMS protecting groups on $\mathbf{5 . 2 0}$ were removed with an excess of TBAF, after which the complexation of $\mathbf{5 . 2 1}$ to the four-legged template T4 was investigated (Figure 5.10). The formation of a two-to-one complex with the T4 template is necessary in order to pre-organise $\mathbf{5 . 2 1}$ before an intramolecular oxidative coupling of the acetylenes is attempted to form the target ball.

(a)

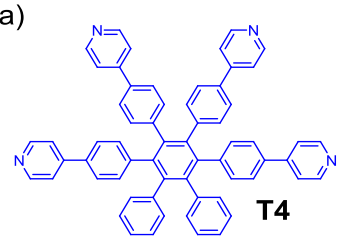

(b)

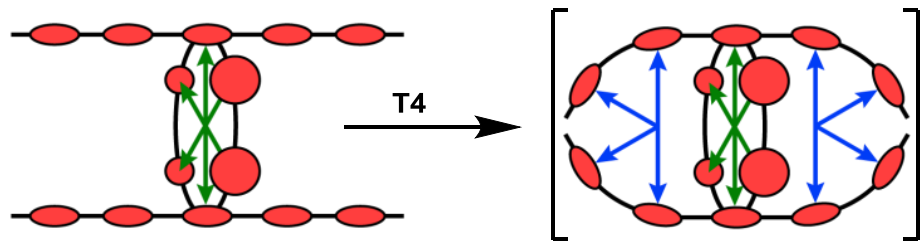

Figure 5.10: (a) Chemical structure of T4. (b) Schematic representation of the formation of the desired two-to-one complex.

UV-vis-NIR titrations were carried out in order to gain insights into the binding process. The UV-vis-NIR spectrum of $\mathbf{5 . 2 1}$ (Figure 5.11, black) shows a three-finger pattern in the Q-band region which is characteristically observed for a templated porphyrin 6-ring. Upon the addition of small aliquots of T4, drastic changes in the spectrum are observed (Figure 5.11, red). The formation of a sharp red-shifted Q-band is formed upon bending linear oligomers and restricting their rotation which indicates complex formation. ${ }^{23,136,192}$ When plotting the change in absorbance as a 
function of the T4 concentration, a sharp end-point is observed after the addition of two eq. T4 indicating the formation of a stable two-to-one complex.
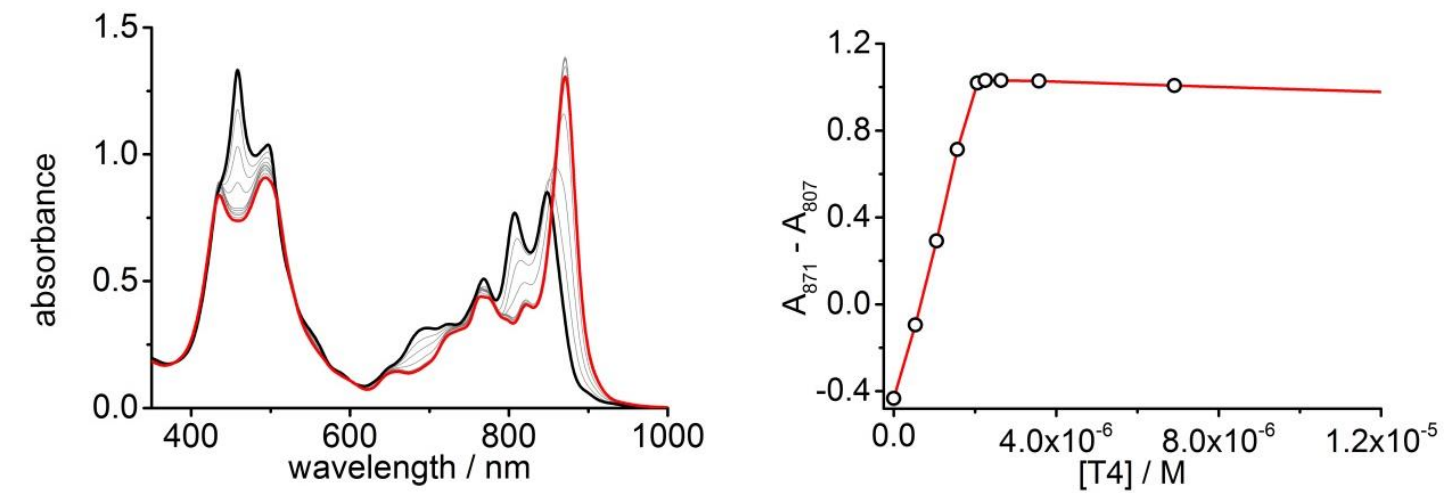

Figure 5.11: UV-vis-NIR formation titration of 5.21 with the template T4 (in toluene at $298 \mathrm{~K}$ ). The spectrum shown on the left are the extended ring 5.21 (black) and the end spectrum of the 2:1 complex with T4 (red). On the right the experimental (black circles) and the line plot (red line) are shown.

While the titration shows the formation of the desired complex, the intensity of the absorption spectra was observed to decrease slowly over time (most clearly observed in the loss of intensity in the large Q-band around $880 \mathrm{~nm}$ in Figure 5.11). This observation indicates the slow precipitation of the complex from solution as a result of a decrease in solubility caused by the increase in rigidity of the complex with respect to its precursor. The low solubility of the two-to-one complex was subsequently observed when the complex was quantitatively prepared prior to the intramolecular ball-forming reaction which did not result in the desired target ball.

In order to increase the solubility, it was decided to use a dimer containing the highly soluble 3,5-bis-(trihexylsilyl)phenyl aryl groups to couple to the 6-porphyrin ring 5.17, which resulted in $\mathbf{5 . 2 3}$ in a $41 \%$ yield after purification by recycling GPC (Figure $5.12 a)$. 

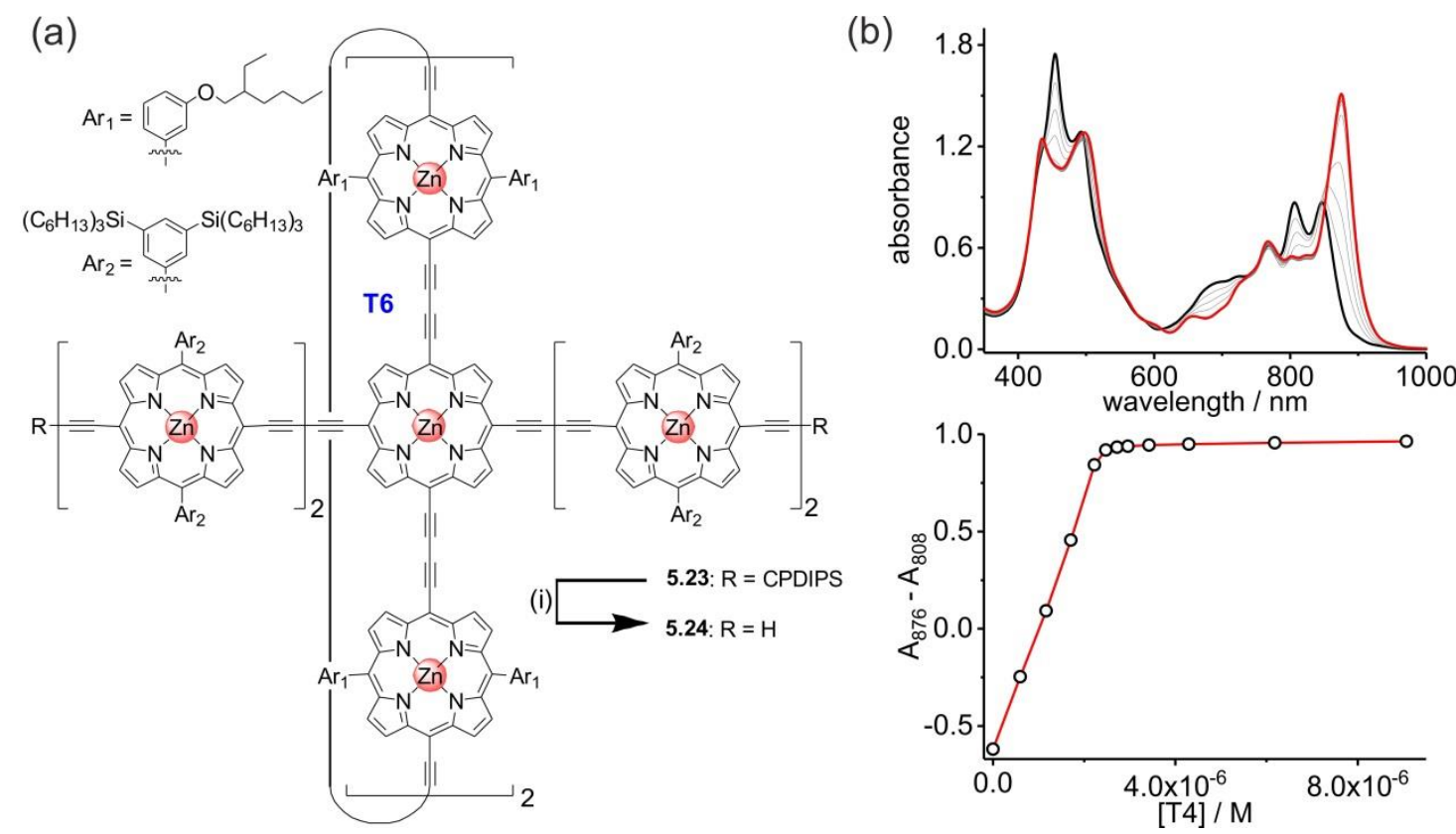

Figure 5.12: (a) Deprotection to give the extended porphyrin ring 5.24. (i) $\mathrm{TBAF}, \mathrm{CH}_{2} \mathrm{Cl}_{2}, 97 \%$. CPDIPS = cyanopropyldiisopropyl silane (b) UV-vis-NIR formation titration of 5.24 with the template T4 (in $\mathrm{CHCl}_{3}$ at $298 \mathrm{~K}$ ). The top spectrum the extended ring 5.24 (black) and the end spectrum of the two-to-one complex with T4 (red). On the bottom the experimental (black circles) and the line plot (red line) are shown.

After the removal of the protecting groups, the binding to the T4 templates was investigated by UV-vis-NIR titrations. The formation of the desired two-to-one complex was confirmed and there was no indication of precipitation (Figure 5.12b). Interestingly, the anticipated steric congestion between the aryl groups, which motivated the original choice of aryl groups, did not appear to hinder the formation of the complex.

With the encouraging titration data in hand, the final step in the synthesis was attempted. The ball precursor $\mathbf{5 . 2 4}$ and T4 were mixed in a one-to-two ratio after which the reaction mixture was subjected to oxidative coupling conditions. The reaction mixture was allowed to react for 18 hours after which UV-vis-NIR spectroscopy of the crude reaction mixture indicated the completion of the reaction. The recorded spectrum resembles that of an overlap of a 6-porphyrin nanoring, with its distinctive three finger pattern, and a large red shifted Q-band characteristic for the formation of a 10-ring (Figure 5.13, red). The crude reaction mixture was purified by silica gel chromatography, SEC and recycling GPC (Figure 5.13, right) to give the nanoball $\boldsymbol{b}$-P14·T6·(T4) $)_{2}$ in a $51 \%$ yield. 

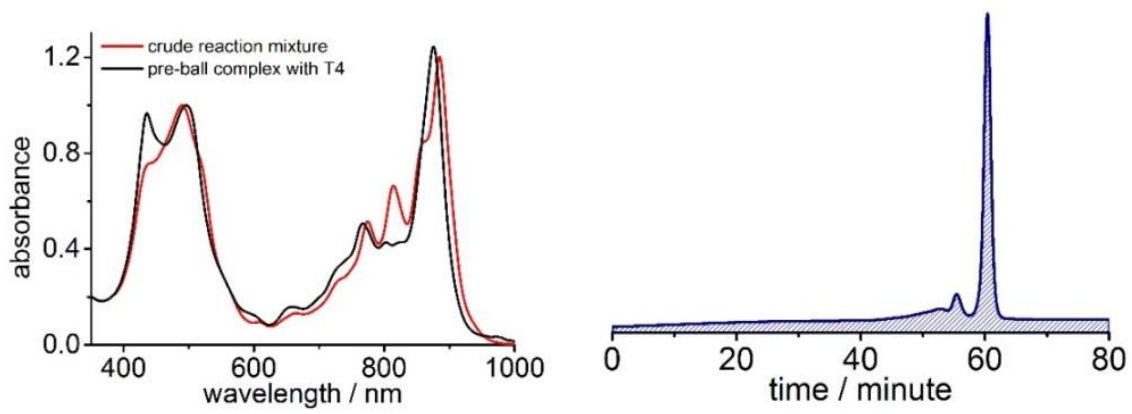

Figure 5.13: (left) UV-vis-NIR spectra of the extended ring 5.24 complexed with two T4 and one T6 templates (black) and the crude reaction mixture after coupling of the acetylenes (black). (right) The recycling GPC trace (JAIGEL columns, $1^{\text {st }}$ cycle shown, $1 \%$ pyridine in toluene, detection at $590 \mathrm{~nm}$ ) of the crude reaction mixture of $b$-P14·T6·(T4) .

The T6 and T4 templates bound in the ball have a different binding affinity as a consequence of the different number of binding sites. The tetra-dentate T4 ligand binds weaker to the host and can be selectively removed from the cavity by the addition of a large excess of pyridine. When using a stronger coordinating competing ligand such as quinuclidine or DABCO, both the T6 and T4 templates are removed to yield the empty ball $\boldsymbol{b}$-P14 after purification by size exclusion chromatography.

The structure of $\boldsymbol{b}$-P14 and the templated analogues $\boldsymbol{b}$-P14-T6 and $\boldsymbol{b}$-P14-T6-(T4) 2 were confirmed by analytical GCP, MALDI, UV-vis-NIR, and ${ }^{1} \mathrm{H}$ NMR spectroscopy. The ${ }^{1} \mathrm{H}$ NMR spectra were fully assigned with the aid of COSY and NOESY spectroscopy. The molecule, with a molecular mass exceeding $20 \mathrm{kDa}$, contains 1,708 protons but gives rise to a remarkably interpretable spectrum due to the $D_{2 h}$ symmetry of the complex (Figure 5.14 and Figure 5.15). With the exception of an unresolved multiplet of 1,368 protons resonating between 2.0 and $0.4 \mathrm{ppm}$, corresponding to the solubilising chains on the aryls, all signals were assigned.

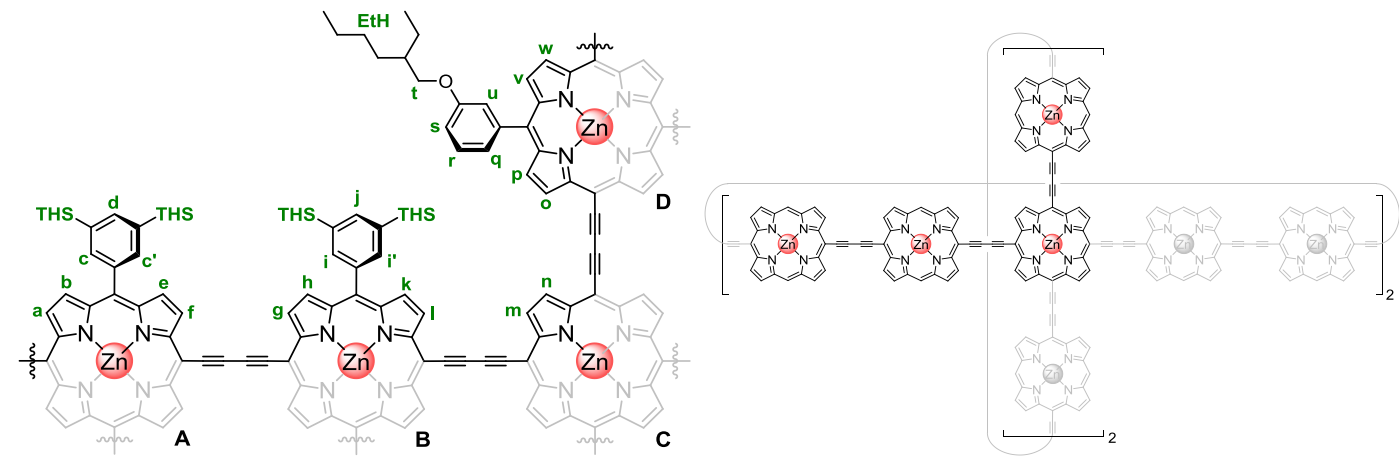

Figure 5.14: Symmetric unit of the porphyrins in $b$-P14-T6 $(\mathrm{T} 4)_{2}$ with proton labelling. Templates are not shown for clarity. Protons $\mathrm{c}$ and $\mathrm{i}$ point towards the centre of the ball whereas $\mathrm{c}$ ' and $\mathrm{i}$ ' point outwards. There are two diastereomers of porphyrin $\mathrm{D}$, as drawn the EtH group is pointing towards the centre of the ball, the signals in which the EtH group is pointing outwards will be denoted with a *. (THS = trihexylsilyl; EtH = ethylhexyl). 


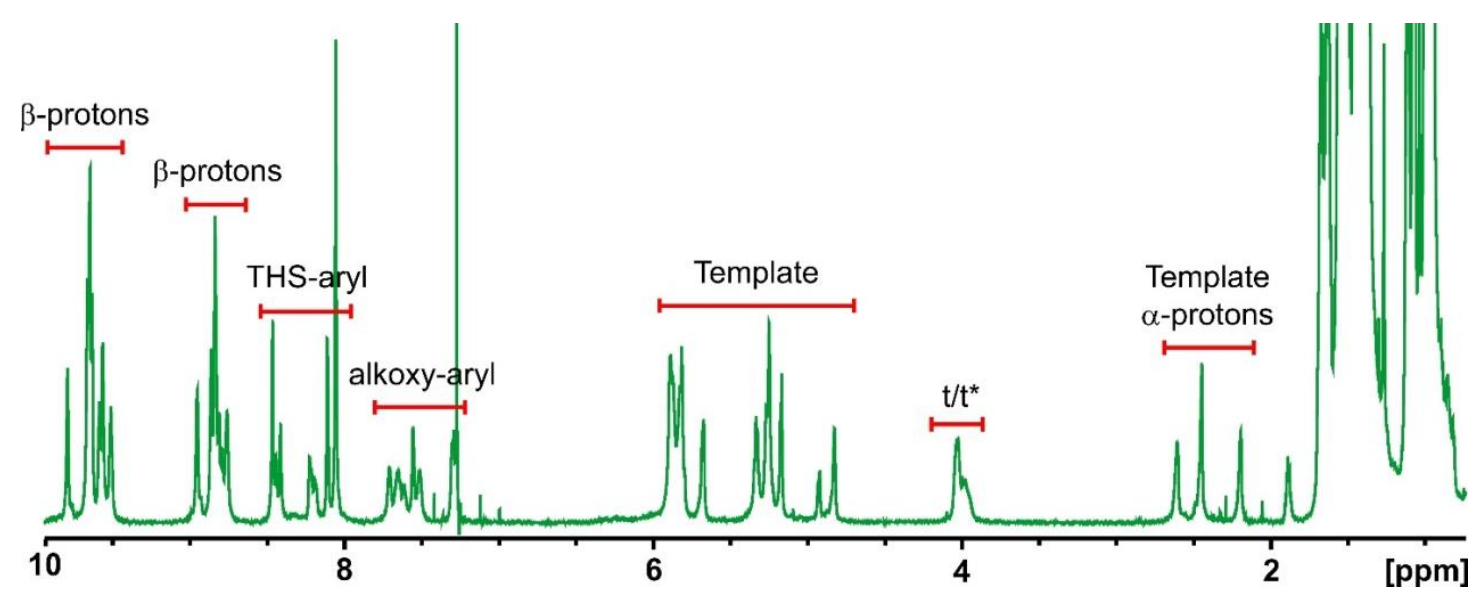

Figure 5.15: ${ }^{1} \mathrm{H}$ NMR spectrum of $b$-P14.T6·(T4) $)_{2}$, the full assignment of the spectrum is described in the following section. (700 MHz, $\left.\mathrm{CDCl}_{3}\right)$.

\subsection{2 ${ }^{1} \mathrm{H}$ NMR assignment of $b$-P14-T6 $(\mathrm{T} 4)_{2}, b-\mathrm{P} 14 \cdot \mathrm{T} 6$ and $b$-P14}

In the following section, the full assignments of the ${ }^{1} \mathrm{H}$ NMR spectra of $\boldsymbol{b}$-P14-T6-(T4) $2, b$-P14-T6 and $\boldsymbol{b}$-P14 are described. The spectra were recorded by Prof. Tim Claridge using a Bruker AV700 (700 MHz) instrument with $\mathrm{CDCl}_{3}$ as the solvent at $298 \mathrm{~K}$. The 2D-NMR techniques COSY and NOESY were used to achieve full assignment of the signals. Throughout this section, COSY correlations are indicated in blue, both in the molecular structures and in the cross-peaks. NOESY correlations are indicated in red. In addition, 1D-NOESY experiments were carried out with increased mixing times to detect weak long-range NOESY correlations between protons across the butadiynes between the porphyrin units. Selective 1D-NOESY experiments were collected using single gradient spin-echoes employing $80 \mathrm{~ms} 180^{\circ}$ selective Gaussian pulses. In situations where closely neighbouring resonances prevented clean selective excitation, 1D-NOESY sequences employing chemical shift selective filters were used. ${ }^{193,194}$ These were performed using $80 \mathrm{~ms} 180^{\circ}$ selective Gaussian pulses with data collected over 16 incremented time steps for suppression of undesired signals and NOE mixing times of 300 or 500 ms.

The assignments of the three complexes will be discussed systematically. By considering symmetry, the molecule has been split in four parts. First the signals corresponding to the central-porphyrin (C) are assigned, followed by the alkoxy-porphyrin (D) and the THS-porphyrins (A and B). Where necessary, the templates were assigned last. The spectra are complex as a result of the presence of rotamers (diastereomers) in which the EtH solubilising groups can either point 
towards the centre or periphery of the ball. Upon removal of the templates the spectra simplify due to free rotation of the porphyrins. The solubilising groups (THS and EtH) on the aryls gave rise to an unresolved envelope of 1,368 protons resonating between 2.0 and 0.4 ppm which has not been assigned in detail. Various peaks in the ${ }^{1} \mathrm{H}$ NMR spectra were found to be too broad to resolve the coupling pattern and are labelled as multiplets $(\mathrm{m})$.

We start with the assignment of $\boldsymbol{b}-\mathbf{P} 14 \cdot \mathbf{T 6} \cdot(\mathbf{T 4})_{2}$. The aromatic region of the spectrum is shown in Figure 5.16 which will be discussed systematically.

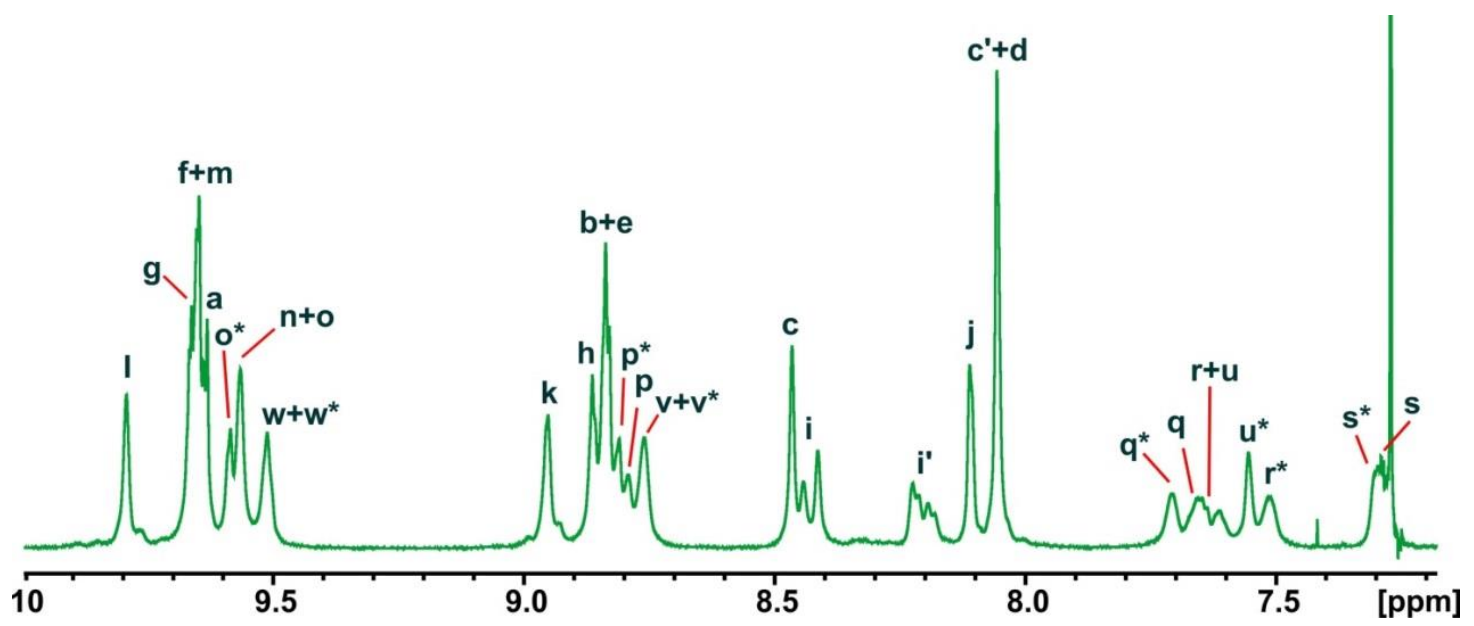

Figure 5.16: The assigned aromatic region of the ${ }^{1} \mathrm{H}$ NMR spectrum of $b-\mathrm{P} 14 \cdot \mathrm{T} 6 \cdot(\mathrm{T} 4)_{2}$. The signals corresponding to o-u are split due to the existence of rotamers with the EtH chains pointing inwards or outwards (the signals for the diastereomers in which the EtH group is pointing outwards is denoted with *). The signal at $7.27 \mathrm{ppm}$ corresponds to residual $\mathrm{CDCl}_{3}$.

The central-porphyrin $\mathbf{C}$ (Figure 5.17) gives rise to two non-identical proton signals, $\mathbf{m}$ and $\mathbf{n}$, in the aromatic region of the spectrum.
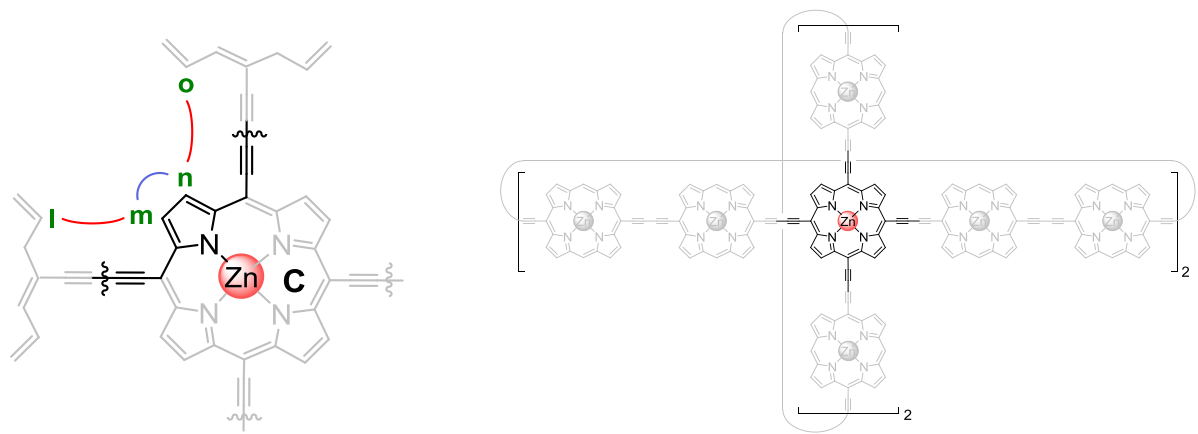

Figure 5.17: Proton labelling of the central-porphyrin and its position within the ball.

Beta-pyrrole proton signals in close proximity to acetylenes were found between 10.0 and $9.4 \mathrm{ppm}$ (Figure 5.16). In this region, only the protons corresponding to $\mathbf{m}$ 
and $\mathbf{n}$ are expected to show a COSY correlation which allowed for the identification of the protons $\mathbf{m}$ and $\mathbf{n}$ as two multiplets at 9.65 and $9.58 \mathrm{ppm}$ (Figure 5.18).

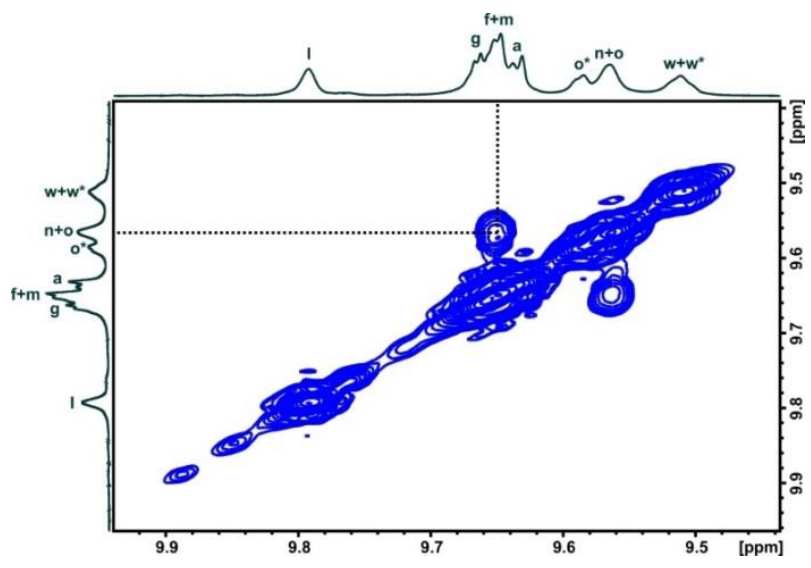

Figure 5.18: COSY correlation between the protons $m$ and $n$.

The signals for $\mathbf{m}$ and $\mathbf{n}$ were distinguished from each other through 1D-NOESY experiments (Figure 5.19). By irradiating at $9.806 \mathrm{ppm}$, the signal corresponding to I was selectively excited and revealed a weak NOESY correlation to $\mathbf{m}$ and no correlation to $\mathbf{n}$ was observed. Due to signal overlap between $\mathbf{f}$ and $\mathbf{m}$ it was not possible to directly irradiate $\mathbf{m}$. Irradiation of the signal corresponding to an overlap of $\mathbf{n}$ and $\mathbf{o}$ at $9.579 \mathrm{ppm}$ showed no NOESY correlation to I, which demonstrates that the signal at $9.579 \mathrm{ppm}$ corresponds to $\mathbf{n}$ rather than $\mathbf{m}$.

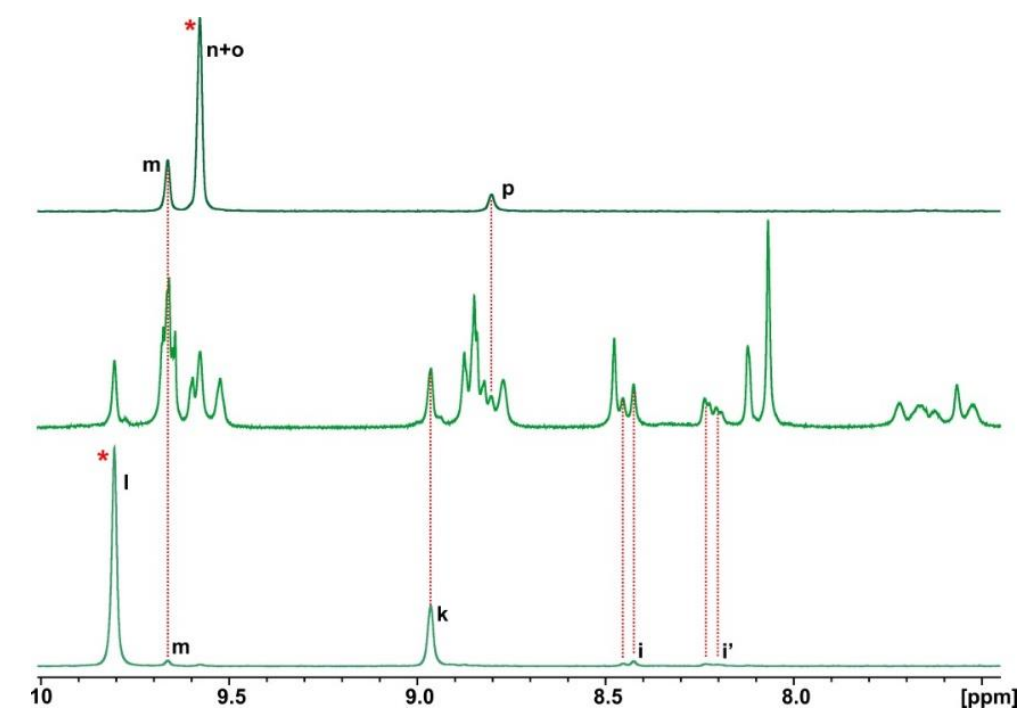

Figure 5.19: 1D-NOESY spectrum irradiating the signals for $n+o$ and I. Irradiated signals are denoted by *.

As will be discussed later, the alpha-protons $(\alpha 4)$ of the template T6 are in close proximity to the $\mathbf{m}$ and $\mathbf{n}$ protons and show an NOE which agrees with the assignment (Figure 5.32). 
The alkoxy-porphyrins (D) are located in the 6-porphyrin nanoring of the ball (Figure 5.20) and give rise to a multitude of signals.
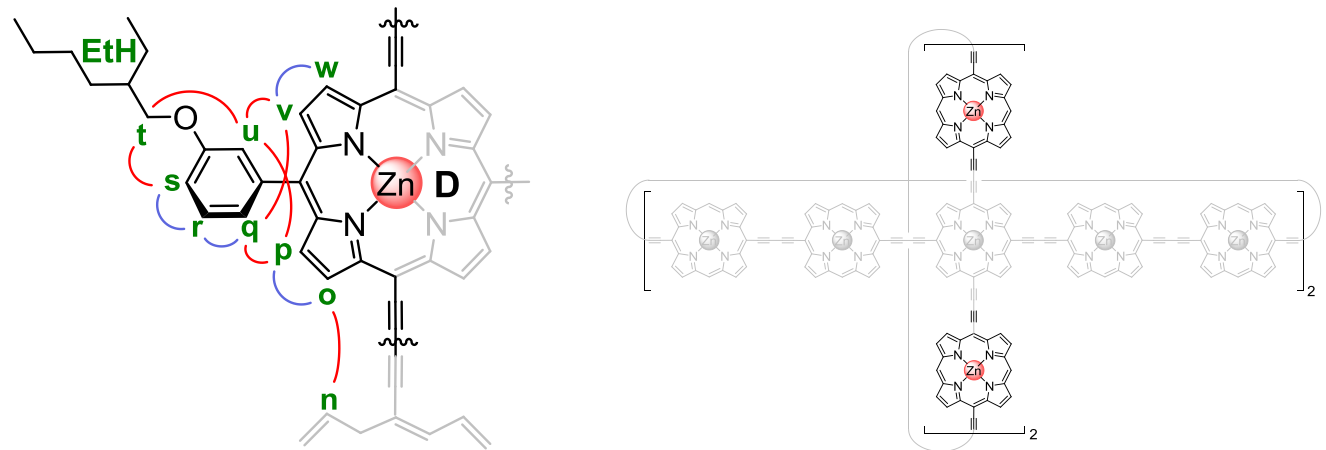

Figure 5.20: Proton labelling of the alkoxy-porphyrin and its position within the ball.

The aromatic protons of the aryl-groups give rise to a range of multiplets between 7.8 and $7.2 \mathrm{ppm}$. Due to signal overlap with the residual solvent signal, diffusion editing experiments were performed to suppress the residual chloroform signal at 7.27 ppm (Figure 5.21).

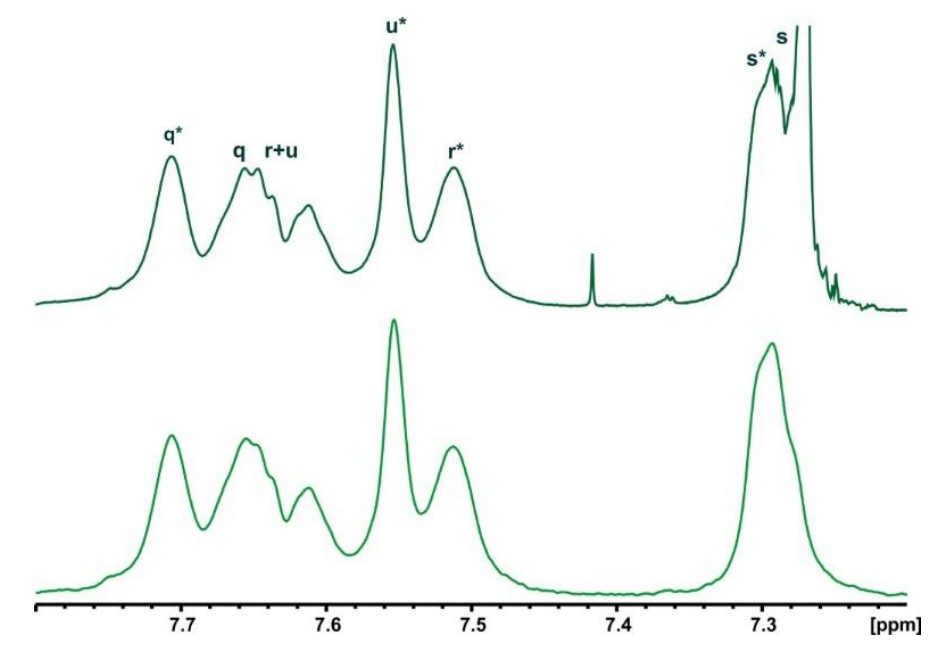

Figure 5.21: Region of the ${ }^{1} \mathrm{H}$ NMR spectrum of $b$-P14-T6·(T4) $)_{2}$, the residual solvent signal overlaps with the signal for $s$ and is suppressed in the bottom spectrum. (The signal in the top spectrum at $7.42 \mathrm{ppm}$ is the ${ }^{13} \mathrm{C}$ satellite of $\mathrm{CDCl}_{3}$ ).

Splitting in the signals is observed which can be rationalised by the presence of rotamers (splitting might also be expected due to the racemic nature of the EtH solubilising group but this was not observed). The rotation of the aryl-groups is expected to be slow on the NMR timescale (but faster than $1 \mathrm{~Hz}$ ) with the solubilising groups either pointing towards the centre or towards the periphery of the ball. The signals corresponding to the diastereomer in which the EtH group is pointing outwards are denoted with *. The protons for $\mathbf{t}$ give rise to two multiplets at 3.97 
and $4.03 \mathrm{ppm}$ (each corresponding to one rotamer) which were used to identify the protons $\mathbf{s} / \mathbf{s}^{*}$ and $\mathbf{u} / \mathbf{u}^{*}$ through NOESY correlations (Figure 5.22).
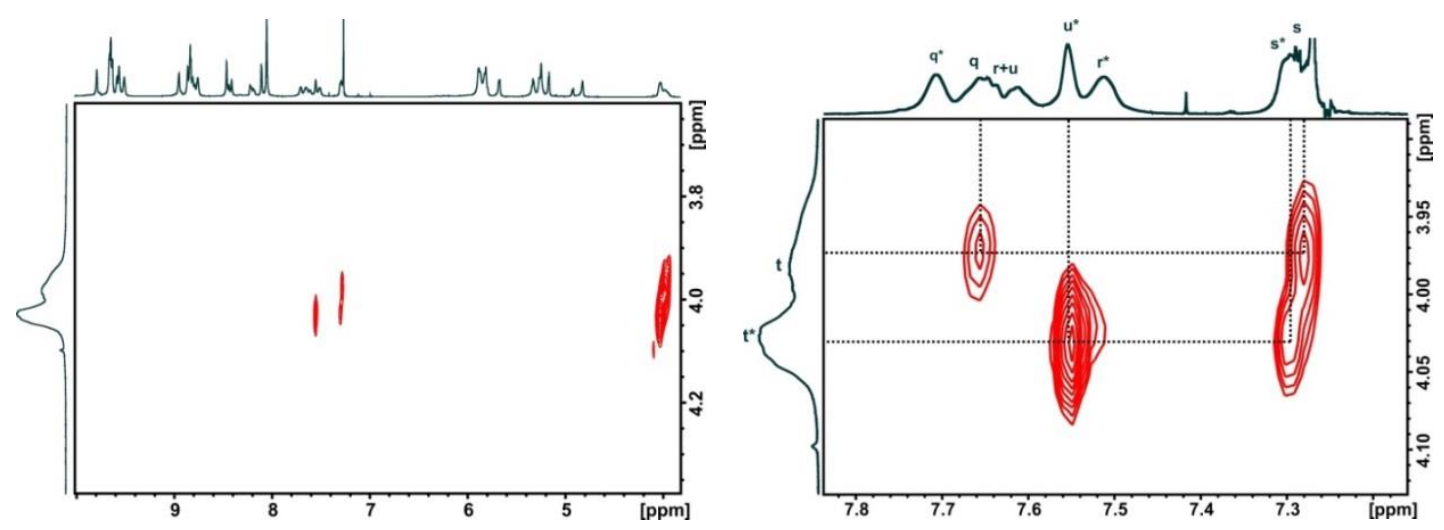

Figure 5.22: (left) NOESY correlations from $t$ to $s$ and $u$. (right) Region of the same spectrum.

The signal for $\mathbf{u}$ is split in two multiplets at 7.65 and $7.55 \mathrm{ppm}$ respectively and as expected they shows no COSY correlations (Figure 5.23, left). The protons pointing towards the centre of the ball show NOEs to the $\alpha 3$ protons of the template T6 (Figure 5.23, right).
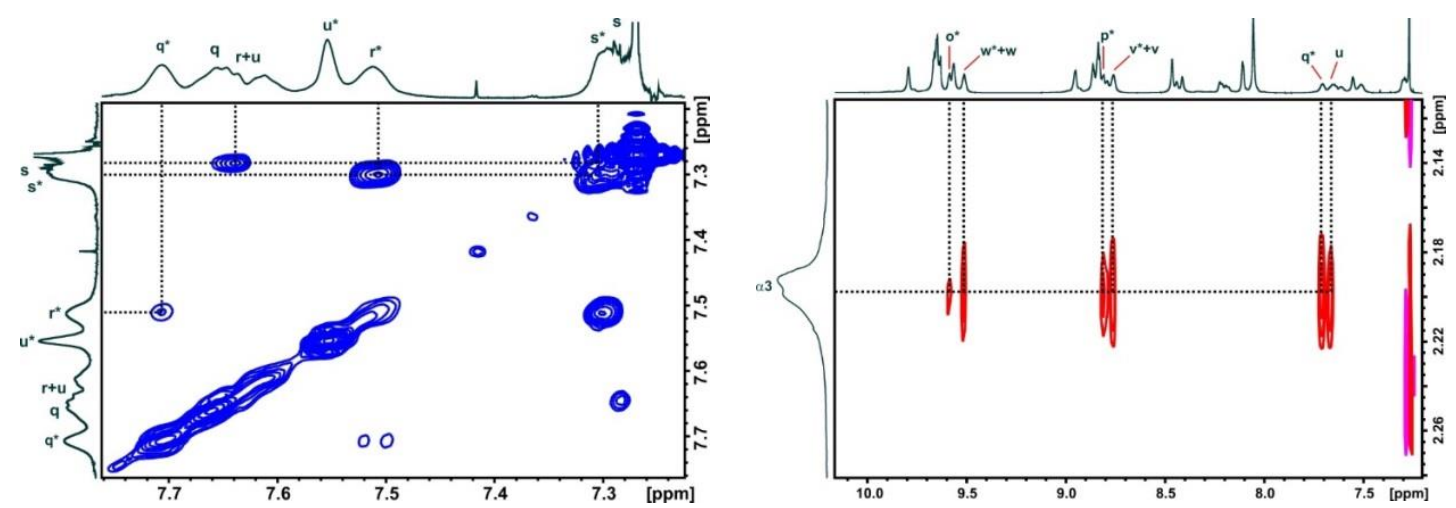

Figure 5.23: (left) COSY correlations from $s$ to $r$ and from $r$ to $q$. (right) NOESY correlations between the template protons $\alpha 3$ and the protons of porphyrin $D$.

No NOE was observed between the signal for $\mathbf{u}^{*}$ at $7.55 \mathrm{ppm}$ and $\alpha 3$, this proton is therefore expected to point towards the periphery of the ball. In agreement with this hypothesis, the signal at $7.65 \mathrm{ppm}(\mathbf{u})$ does show an NOE to the template and is expected to point towards the centre. COSY and NOESY correlations were used to distinguish between $\mathbf{q} / \mathbf{q}^{*}, \mathbf{r} / \mathbf{r}^{*}$ and $\mathbf{s} / \mathbf{s}^{*}$.

The 8-proton multiplet at $7.30 \mathrm{ppm}$ was identified as an overlap of $\mathbf{s}$ and $\mathbf{s}^{*}$, the NOEs to $\mathbf{t}$ and $\mathbf{t}^{*}$ were used to distinguish between the diastereomers (Figure 5.22). The signal for $\mathbf{s}^{*}$ shows a COSY correlation to the multiplet at $7.51 \mathrm{ppm}$ identified as 
$\mathbf{r}^{*}$. An additional COSY correlation is found between $\mathbf{r}^{*}$ and $\mathbf{q}^{*}$ (Figure 5.23, left). The signal for $\mathbf{q}$ is split in two multiplets resonating at 7.71 and $7.65 \mathrm{ppm}$. The NOESY correlation from $\mathbf{u}$ to the $\alpha 3$ protons of the template allow us to the signals corresponding to the diastereomer with the EtH group pointing towards the centre of the ball. The signal corresponding to s shows a COSY to $r$ which overlaps with $\mathbf{u}$ (the COSY correlation between $\mathbf{t}$ and $\mathbf{u}$ discussed above confirms that the signal for $\mathbf{t}$ at $3.98 \mathrm{ppm}$ belongs to this rotamer, i.e. pointing towards the centre of the ball). Integration of the signals corresponding to $\mathbf{t}$ and $\mathbf{t}^{*}$ indicates that approximately $60 \%$ of the EtH solubilising groups are pointing towards the periphery of the ball.

The signals for $\mathbf{p} / \mathbf{p}^{*}$ and $\mathbf{v} / \mathbf{v}^{*}$ can be identified through NOESY correlations to both $\mathbf{u} / \mathbf{u}^{*}$ and $\mathbf{q} / \mathbf{q}^{*}$ (Figure 5.24, left). COSY correlations from $\mathbf{p} / \mathbf{p}^{*}$ and $\mathbf{v} / \mathbf{v}^{*}$ to o/o* and $\mathbf{w} / \mathbf{w}^{*}$ respectively were used to identify the last protons on this porphyrin (Figure 5.24 , right).
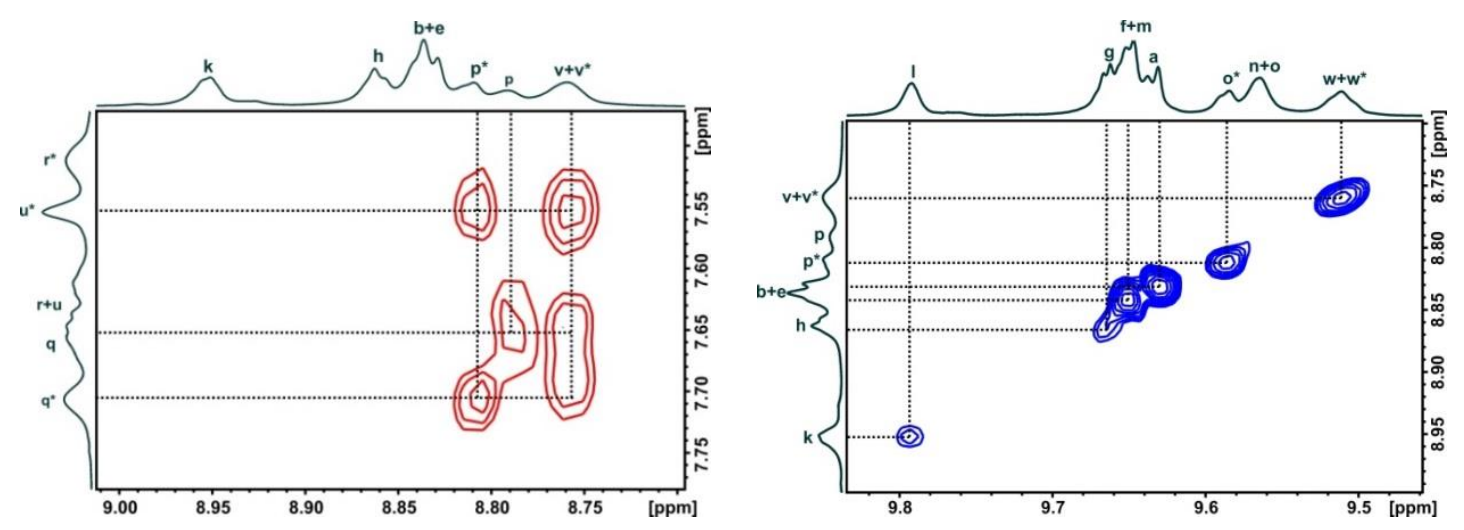

Figure 5.24: (left) NOESY cross-peaks from $p$ and $v$ to $q$ and $u$. (right) COSY correlations between the porphyrin beta-protons.

To distinguish between the signals for $\mathbf{0}, \mathbf{p}, \mathbf{v}$, and $\mathbf{w}$, we performed 1D-NOESY experiments to see the NOEs across the butadiynes. As shown previously in Figure 5.19, a weak correlation was observed between $\mathbf{o}$ and $\mathbf{m}$ in addition to a stronger correlation to $\mathbf{p}$. In Figure 5.25, selective irradiation of the signals corresponding to $\mathbf{p}$ and $\mathbf{v}$ shows the correlations to $\mathbf{o}$ and $\mathbf{w}$. Upon irradiation of $\mathbf{w}$, no correlation to $\mathbf{n}$ was observed, which is in agreement with the assignment. In addition, selectively irradiating $\mathbf{p}^{*}$ shows NOESY correlations to $\mathbf{q}^{*}$ and $\mathbf{u}^{*}$, while selectively irradiating $\mathbf{p}$ shows NOEs to $\mathbf{q}$ and $\mathbf{u}$, confirming the assignments. As expected, when the overlap of $\mathbf{v}$ and $\mathbf{v}^{*}$ is irradiated, $\mathbf{q}, \mathbf{q}^{*}, \mathbf{u}$ and $\mathbf{u}^{*}$ all show NOESY correlations. 


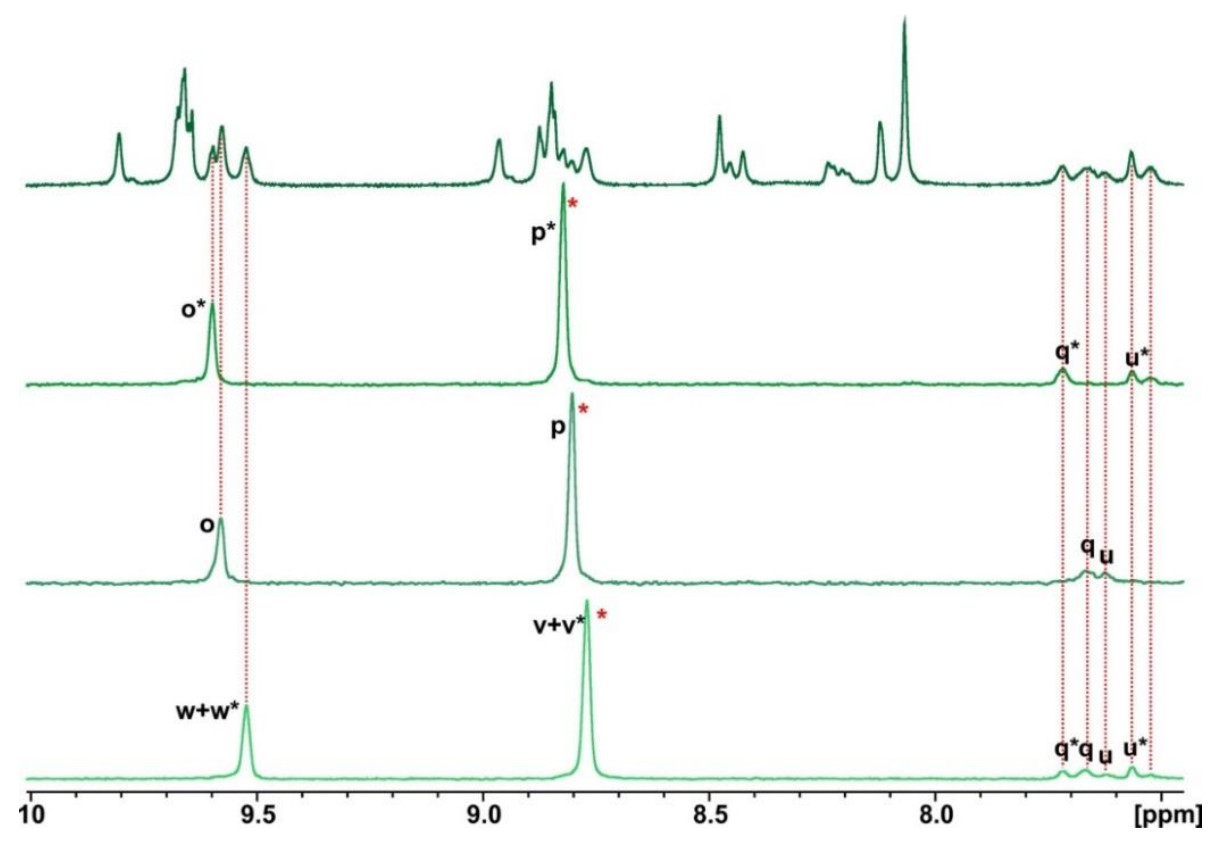

Figure 5.25: 1D-NOESY spectrum irradiating the signals for $p$ and $v$. Irradiated signal is denoted by *.

There are three distinct regions where the aromatic signals of the THS-porphyrins $\mathbf{A}$ and $\mathbf{B}$ resonate (Figure 5.26). Protons $\mathbf{a}, \mathbf{f}, \mathbf{g}$, and $\mathbf{I}$ are in close spatial proximity to the acetylenes and are shifted furthest downfield (between 10.0 and 9.4 ppm). The signals for $\mathbf{b}, \mathbf{e}, \mathbf{h}$, and $\mathbf{k}$ are found between 9.0 and $8.7 \mathrm{ppm}$, the aryl protons $\mathbf{c}, \mathbf{d}, \mathbf{i}$, and $\mathbf{j}$ resonate between 8.5 and $8.0 \mathrm{ppm}$. The ortho-protons of the aryls give rise to four signals ( $\mathbf{c}, \mathbf{c}^{\prime}, \mathbf{i}$, and $\left.\mathbf{i}^{\prime}\right)$. The signals for $\mathbf{i}$ and $\mathbf{i}^{\prime}$ are split further as a consequence of the close proximity to the diastereomeric alkoxy-porphyrin.
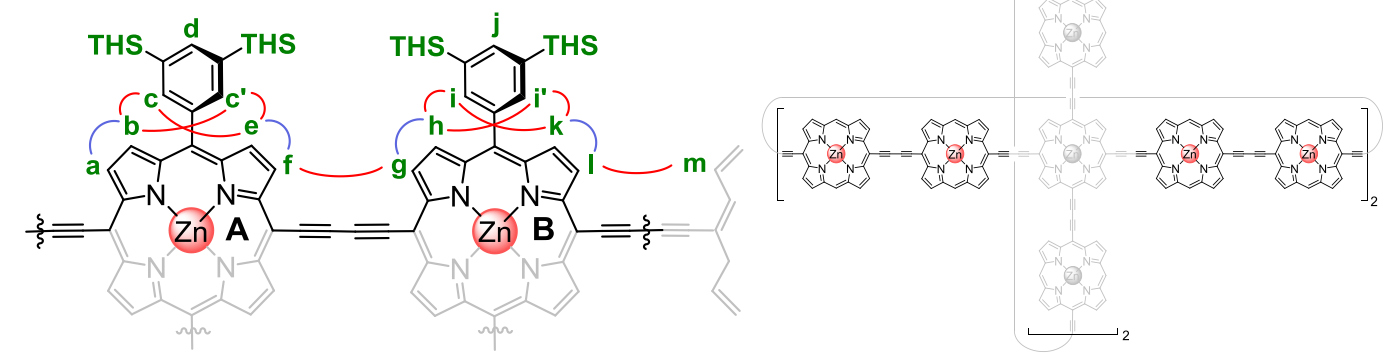

Figure 5.26: Proton labelling of the THS-porphyrins and an illustration of their position within the nanoball. (THS = trihexylsilyl).

The protons $\mathbf{c}$ and $\mathbf{i}$ point towards the centre of the ball causing them to be shifted downfield with respect to $\mathbf{c}^{\prime}$ and $\mathbf{i}^{\prime}$ which are directed towards the periphery of the ball. This assignment is confirmed by the NOEs of $\mathbf{c}$ and $\mathbf{i}$ to the $\alpha$-protons of the T4 template (Figure 5.33). Exchange peaks between $\mathbf{c}$ and $\mathbf{c}^{\prime}$, and between $\mathbf{i}$ and $\mathbf{i}$ ' were observed in agreement with the slow rotation of the aryl groups. The ortho-protons 
of the aryl group show NOEs to the porphyrin beta-protons (Figure 5.27), this information was used in combination with the COSY spectrum shown in Figure 5.24 (right) to identify $\mathbf{a}, \mathbf{f}, \mathbf{b}+\mathbf{e}, \mathbf{g}, \mathbf{h}, \mathbf{k}$, and $\mathbf{I}$. Weak COSY correlation from $\mathbf{c}$ to $\mathbf{d}$ and from $\mathbf{i}$ to $\mathbf{j}$ were observed which were used to confirm the remaining two signals.

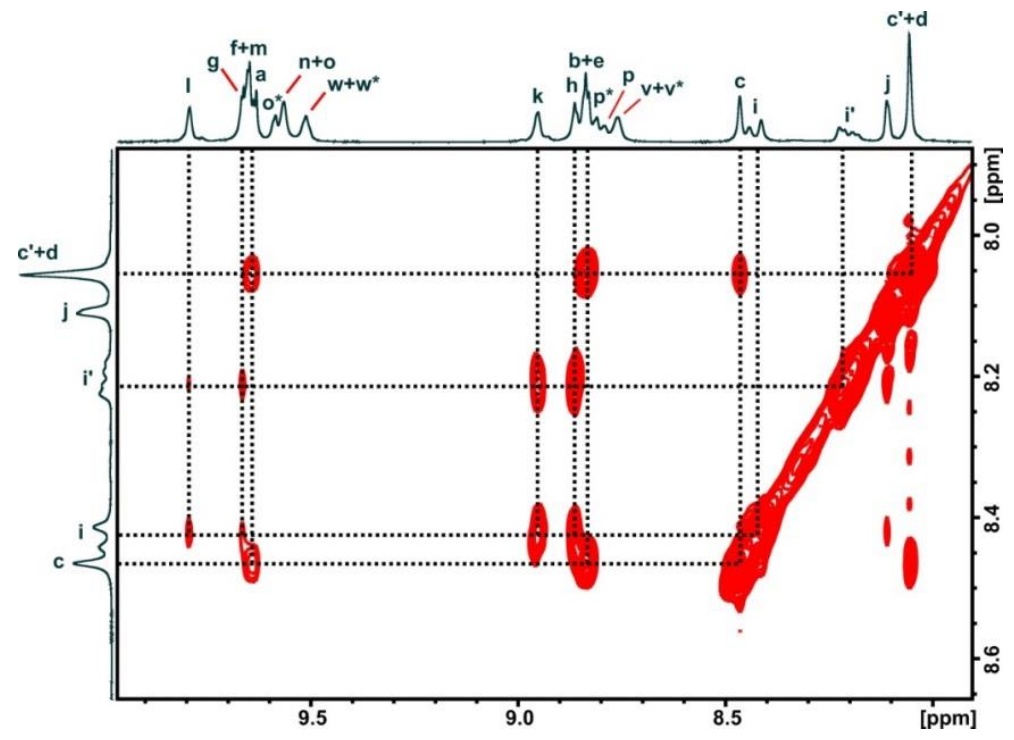

Figure 5.27: NOESY correlations between the THS-aryl protons to the porphyrin beta-protons.

By performing 1D-NOESY experiments we were able to differentiate between the different beta-pyrrole signals (Figure 5.28). Selective irradiation of I revealed the expected correlation with $\mathbf{m}$ which subsequently showed a relayed NOE to $\mathbf{n}$. Upon irradiation of $\mathbf{k}$, a direct correlation was observed to I which subsequently relays to m. In addition, both experiments show NOESY correlations to the aryl group.

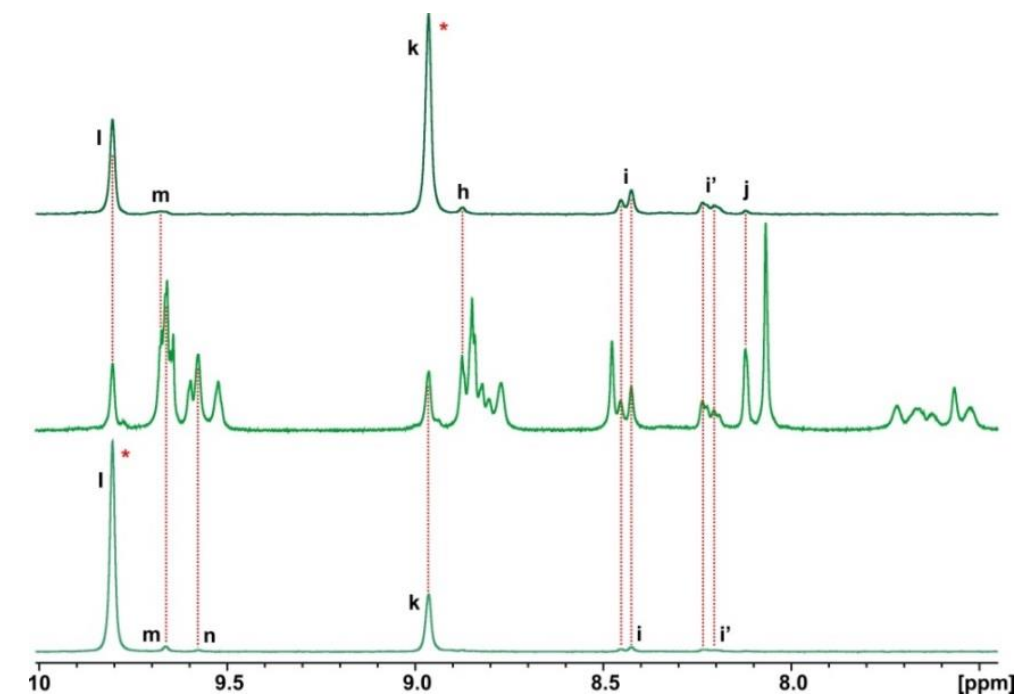

Figure 5.28: 1D-NOESY spectrum irradiating the signals for $\mathrm{k}$ and $\mathrm{I}$. Irradiated signal is denoted by * 
Due to signal overlap, it was not possible to selectively irradiate $\mathbf{g}$ or $\mathbf{f}$ to see its NOE across the butadiyne. However, by irradiating the more isolated signal corresponding to $\mathbf{h}$ and increasing the mixing time, a small shoulder appears at $9.64 \mathrm{ppm}$, through relayed NOE effects from $\mathbf{g}$, which allowed for the assignment of $\mathbf{f}$. In addition, the absence of a relayed signal for a at 9.63 ppm confirms the assignment (Figure 5.29).

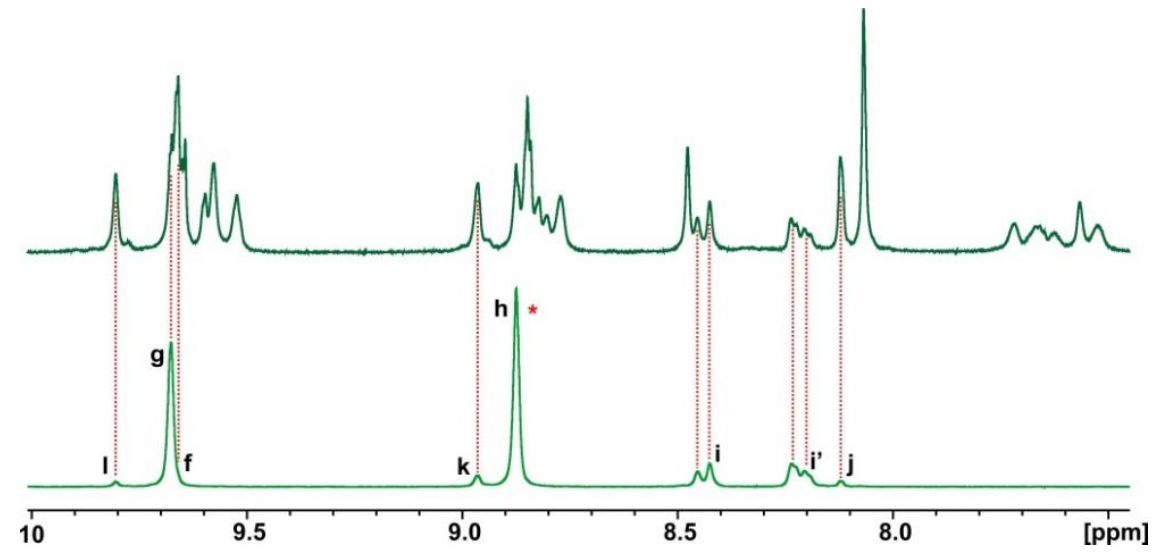

Figure 5.29: 1D-NOESY spectrum irradiating the signal for h. Irradiated signal is denoted by *.

Lastly the T6 and the two T4 templates in the cavity were assigned (Figure 5.30).
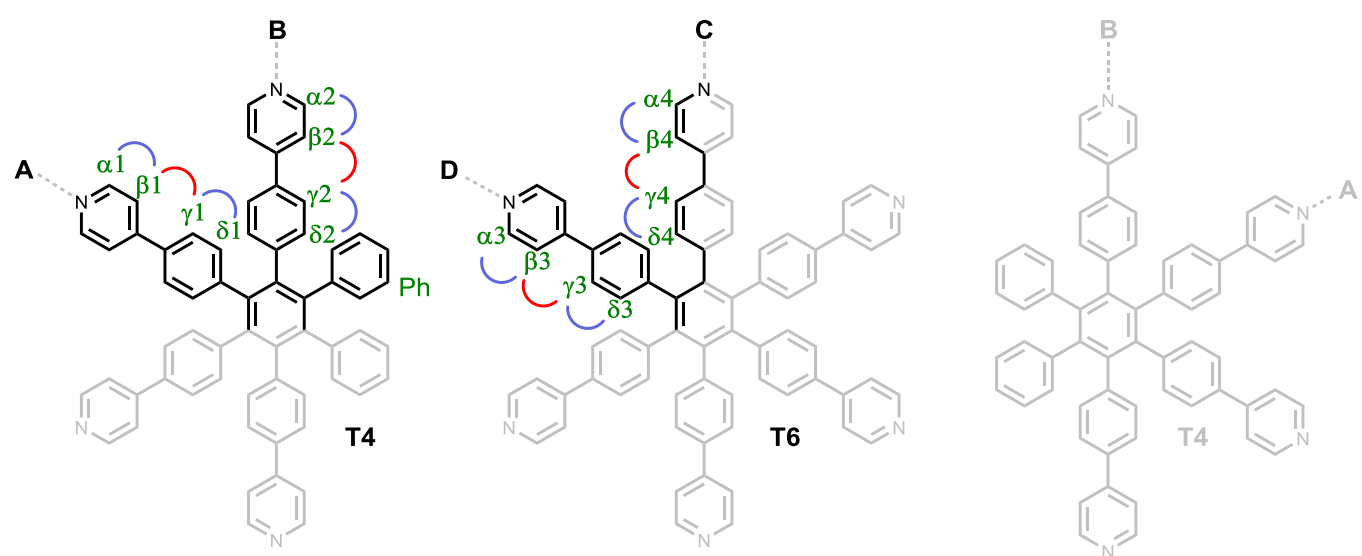

Figure 5.30: Symmetric unit of the templates within $b$-P14·T6·(T4 $)_{2}$ with proton labelling. The dotted lines indicate which porphyrins the legs are pointing towards.

The templates give rise to a number of signals between 6 and 2 ppm (Figure 5.31). Due to strong shielding by the porphyrin $\pi$-system, the alpha-protons are found upfield and give rise to three multiplets. By utilising the NOEs to the porphyrin beta-pyrrole protons, the 8-proton multiplet at $2.20 \mathrm{ppm}$ was identified as $\alpha \mathbf{3}$, the 12-proton multiplet at $2.45 \mathrm{ppm}$ is an overlap of the signals $\boldsymbol{\alpha} \mathbf{1}$ and $\boldsymbol{\alpha} \mathbf{4}$, and the 8-proton multiplet at $2.61 \mathrm{ppm}$ was identified as $\boldsymbol{\alpha 2}$ (Figure 5.33). 


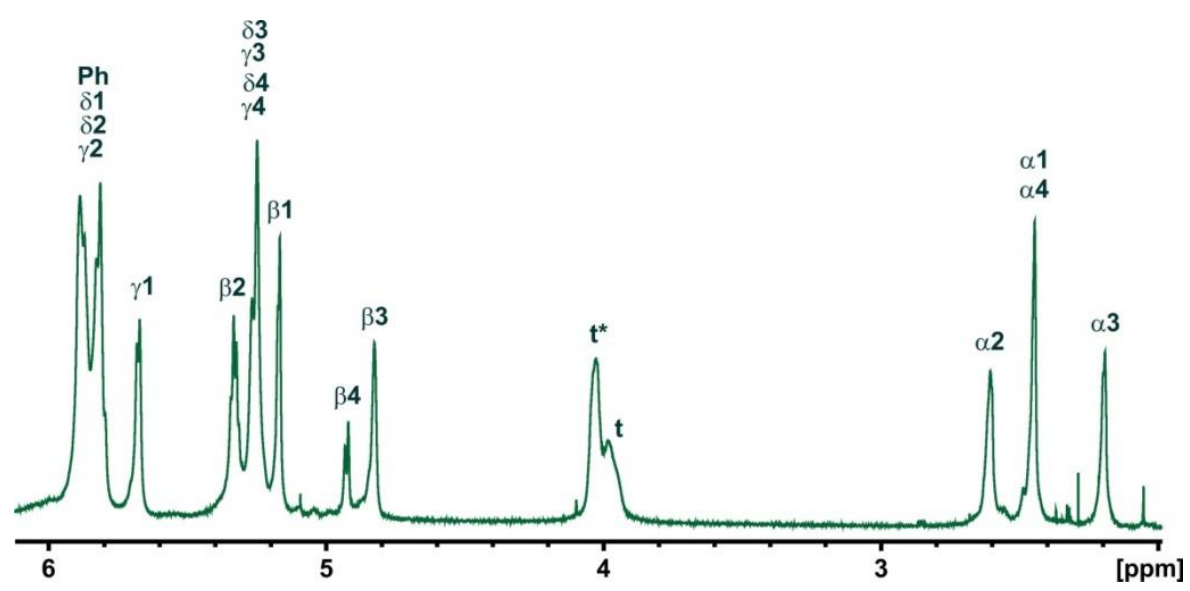

Figure 5.31: The template region of the ${ }^{1} \mathrm{H} N M R$ spectrum of $b$-P14·T6·(T4) ${ }_{2}$.

All four of the protons with the label " $\beta$ " were identified through COSY correlations to the $\alpha$-protons (Figure 5.32, left). Subsequently, NOESY correlations were used to identify the signals for $\boldsymbol{\gamma} \mathbf{1 - 4}$ (Figure 5.32, right).
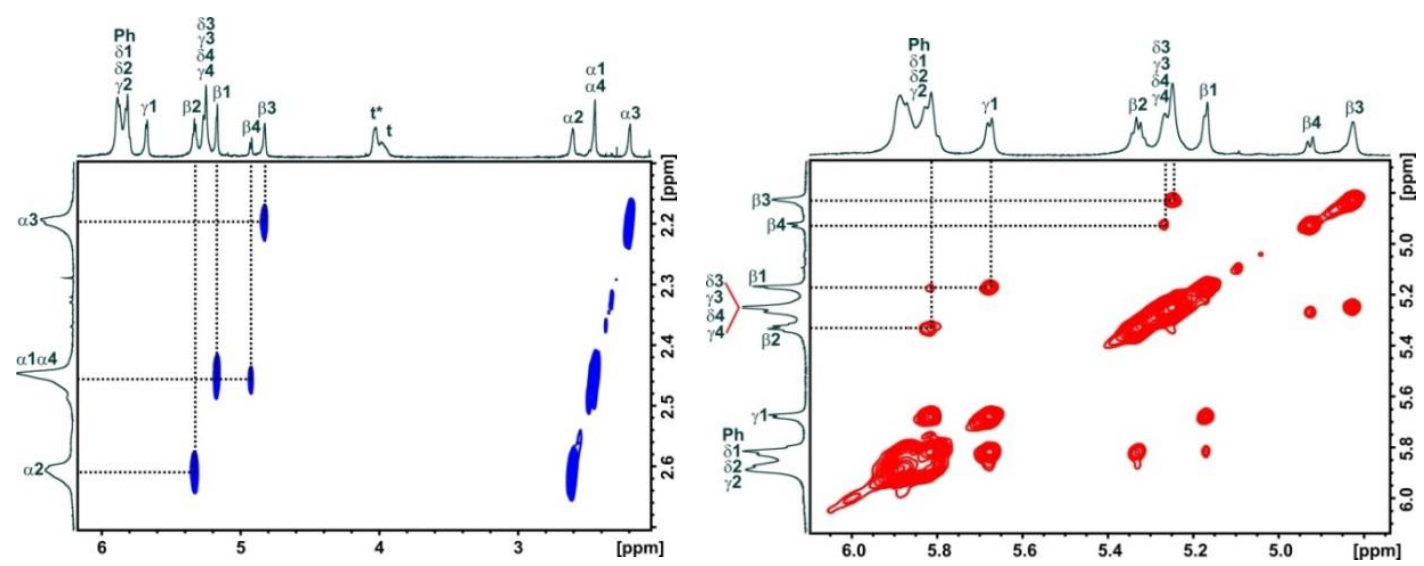

Figure 5.32: (left) COSY correlations between the templates $\alpha$ and $\beta$ signals. (right) NOESY correlations between the templates $\beta$ and $\gamma$ signals.

The signals for $\gamma 3, \gamma 4, \delta 3$, and $\delta 4$ resonate at nearly identical chemical shifts (5.26 ppm) and are not resolved. $\gamma \mathbf{1}$ is a multiplet at $5.68 \mathrm{ppm}$ and shows a COSY correlation with $\delta \mathbf{1}$. The signals for $\delta \mathbf{1}, \boldsymbol{\delta} \mathbf{2}, \boldsymbol{\gamma} \mathbf{2}$, and the ten protons for $\mathrm{Ph}$ give rise to an overlapping multiplet at $5.85 \mathrm{ppm}$.

As mentioned before, the $\alpha$-protons of the templates are in close proximity to the porphyrins and show NOESY correlations (Figure 5.33). The signal for $\boldsymbol{\alpha} \mathbf{2}$ shows five NOESY correlations to the THS-porphyrin (B). Intense cross-peaks were observed to $\mathbf{g}$ and $\mathbf{h}$ and slightly less intense cross-peaks were found relate to $\mathbf{k}$ and $\mathbf{I}$. In addition, a cross-peak was observed to $\mathbf{i}$ confirming that $\mathbf{i}$ is pointing towards the centre of the ball (and consequently is in closer proximity to the template than i'). The signal for 
$\alpha 3$ was confirmed by its NOESY correlations to the alkoxy-porphyrin (D) by showing cross-peaks to $\mathbf{0}, \mathbf{w}, \mathbf{p}$ and $\mathbf{v}$. In addition, weak cross-peaks to $\mathbf{q}$ and $\mathbf{u}$ were observed. The signals for $\alpha \mathbf{1}$ and $\alpha \mathbf{4}$ were found to overlap and show four NOESY correlations. The proton $\alpha \mathbf{4}$ is in close proximity to the central-porphyrin (C) and shows a cross-peak to $\mathbf{m}$ and $\mathbf{n}$. The signals for $\mathbf{a}, \mathbf{f}, \mathbf{b}$, e and $\mathbf{c}$ of the THS-porphyrin (A) show cross-peaks to $\alpha \mathbf{1}$. Again, the correlation between $\alpha \mathbf{1}$ and c confirms that $\mathrm{c}$ is pointing towards the centre of the ball.

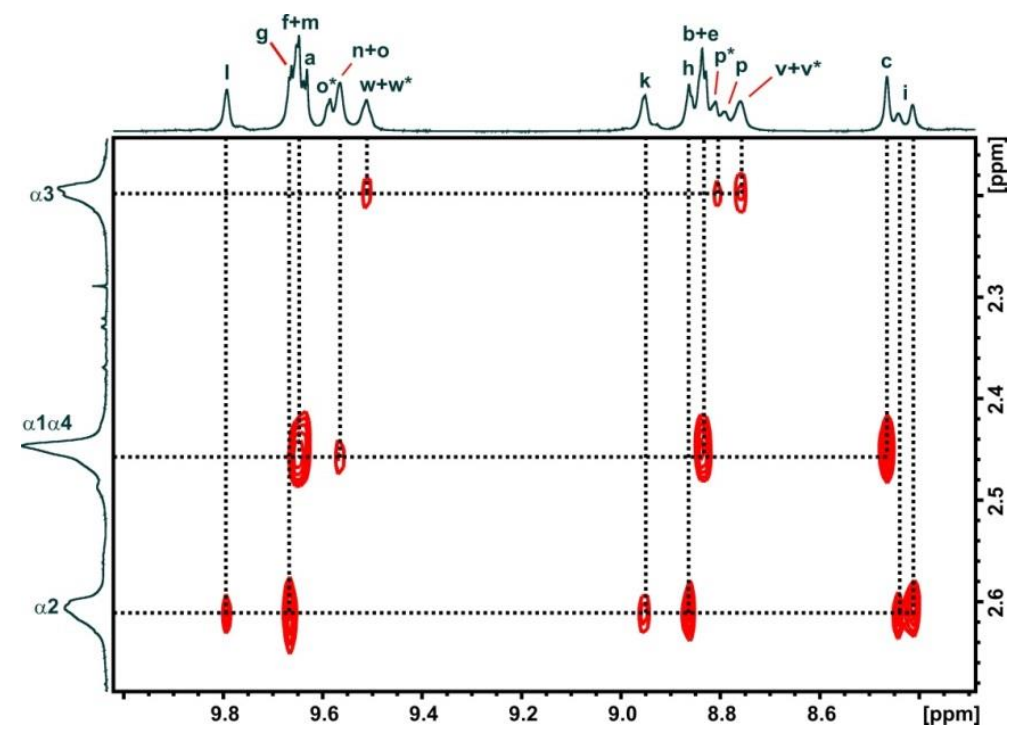

Figure 5.33: NOESY correlations between the template $\alpha$-protons and the protons of the porphyrins.

Next, the ${ }^{1}$ H NMR spectrum of $\boldsymbol{b}$-P14-T6 were interpreted. The displacement of the T4 templates resulted in subtle changes in the aromatic region of the spectrum (Figure 5.34). The most notable changes occured in the THS-aryl region between 8.0 and $8.5 \mathrm{ppm}$. With the T4 templates in place, rotation of the THS-porphyrins around the butadiynes was restricted. After the displacement of the templates, the porphyrins are free to rotate resulting in a simpler spectrum. 


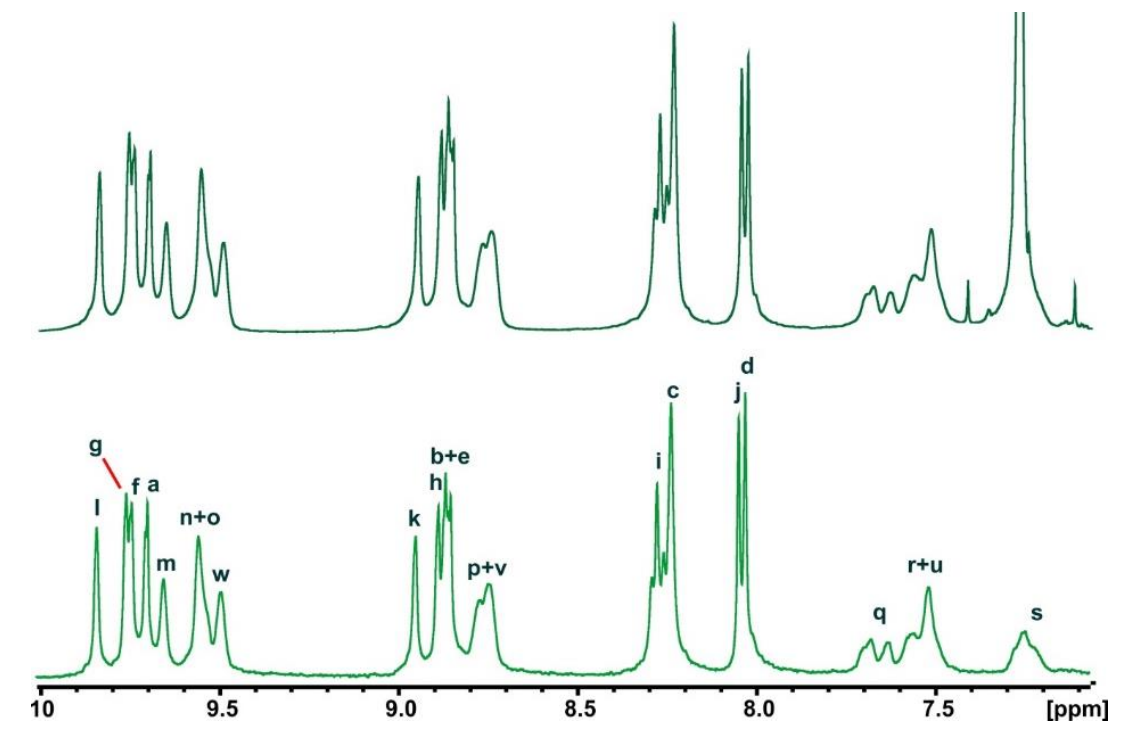

Figure 5.34: (top) The aromatic region of the ${ }^{1} \mathrm{H}$ NMR spectrum of $b$-P14-T6. (bottom) The diffusion edited ${ }^{1} \mathrm{H}$ NMR spectrum.

Similar to the interpretation of $\boldsymbol{b}$-P14.T6.(T4) $\mathbf{2}_{\mathbf{2}}$ the signals corresponding to the central-porphyrin (C) are discussed first. Porphyrin $\mathbf{C}$ gives rise to two 8-proton multiplets at 9.66 and $9.55 \mathrm{ppm}$. The signals were distinguished from the other beta-signals of the porphyrins through a COSY cross-peak (Figure 5.35, left). An NOE was observed between the template $\boldsymbol{\alpha} \mathbf{2}$ protons and $\mathbf{m}$ and $\mathbf{n}$ as a result of their close spatial proximity (Figure 5.35, right).
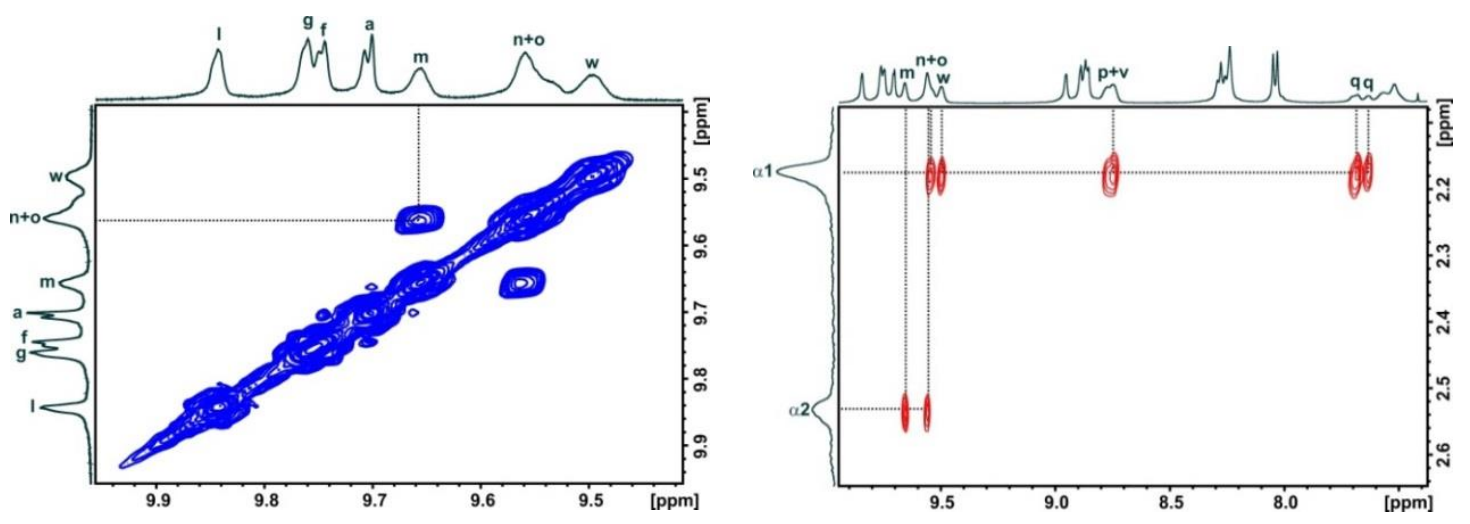

Figure 5.35: (left) COSY correlations between the $m$ and $n$ protons. (right) NOESY correlations between the template $\alpha$-protons and the porphyrin signals.

The aromatic region of the ${ }^{1} \mathrm{H}$ NMR spectrum of $\boldsymbol{b}$-P14-T6, one of the signals on the alkoxy-porphyrin shows spectral overlap with the solvent signal. Diffusion editing experiments were performed to suppress the chloroform signal and revealed the proton signals corresponding to $\mathbf{s}$ (Figure 5.34). Similar to $\boldsymbol{b}$-P14·T6·(T4) $)_{2}$, the presence of rotamers, in which the EtH chain can either point towards the centre or the periphery of the ball, gave rise to splitting and broad signals for $\mathbf{q}-\mathbf{u}$. The ratio 
between the diastereomers could be obtained by integrating the signals for $\mathbf{t}$ which revealed a 50-50 split.

The NOESY correlations from t, illustrated in Figure 5.36 (left), were used to identify the signals for $\mathbf{s}$ and $\mathbf{u}$. The COSY spectrum (Figure 5.36, right) was used to distinguish between $\mathbf{s}$ and $\mathbf{u}$. The protons corresponding to $\mathbf{s}$ show a COSY cross-peak to $\mathbf{r}$ which subsequently couples to q. No COSY correlations were found for $\mathbf{u}$.

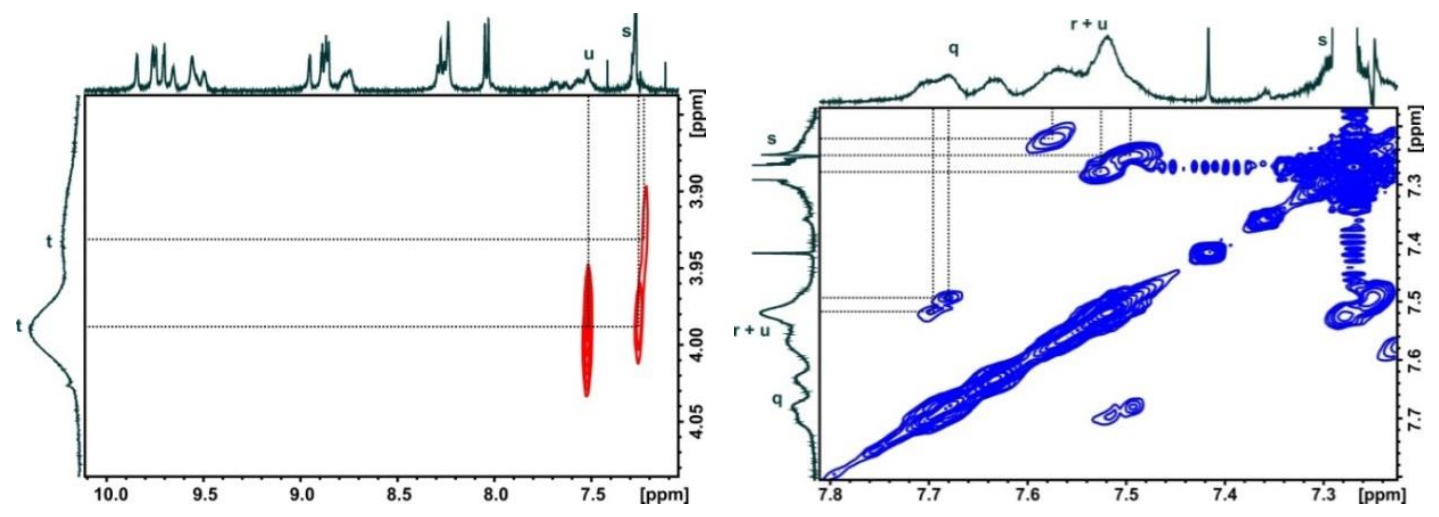

Figure 5.36: (left) NOESY correlations from $t$ to $s$ and $u$. (right) COSY correlations between $s, r$ and $q$.

The aryl protons $\mathbf{q}$ and $\mathbf{u}$ are in close proximity to the porphyrin protons $\mathbf{p}$ and $\mathbf{v}$ and show NOESY correlations (Figure 5.37, left). The signals for $\mathbf{0}$ and $\mathbf{w}$ were identified through their cross-peak in the COSY spectrum with $\mathbf{p}$ and $\mathbf{v}$ (Figure 5.37 , right).
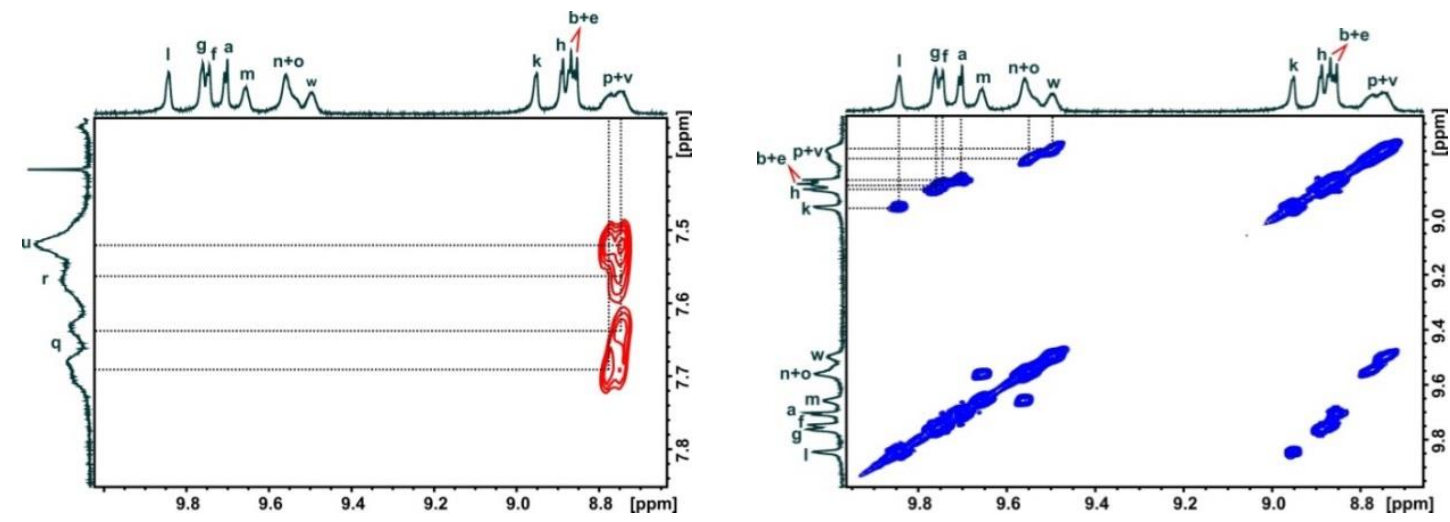

Figure 5.37: (left) NOESY correlations from $p$ and $v$ to $q$ and $u$. (right) COSY cross-peaks between the porphyrin's beta-protons.

The signals for $\mathbf{0}, \mathbf{w}, \mathbf{p}$ and $\mathbf{v}$ were confirmed through a NOESY to the template $\boldsymbol{\alpha} \mathbf{1}$ protons depicted in Figure 5.35 (right).

The THS-porphyrins (A and B) were interpreted next. By analogy to $\boldsymbol{b}$-P14-T6 $(\mathbf{T 4})_{2}$ the beta-protons I was found furthest downfield as the 8-proton multiplet at $9.84 \mathrm{ppm}$. A COSY coupling from I was observed to the 8-proton multiplet at 
$9.76 \mathrm{ppm}$ identified as $\mathbf{k}$ (Figure 5.37, right). The NOESY spectrum showed a correlation from $\mathbf{k}$ to the 16 -proton multiplet at $8.28 \mathrm{ppm}$ characterised as $\mathbf{i}$ (Figure 5.38 , left). In the absence of the T4 templates, the porphyrins $\mathbf{A}$ and $\mathbf{B}$ are free to rotate. As a result the signals for $\mathbf{c}$ and $\mathbf{i}$ are no longer split as was observed in $\boldsymbol{b}$-P14-T6-(T4) $)_{2}$. An additional NOESY coupling from $\mathbf{i}$ was used to identify $h$, which shows a COSY correlation itself to g (Figure 5.37, right).

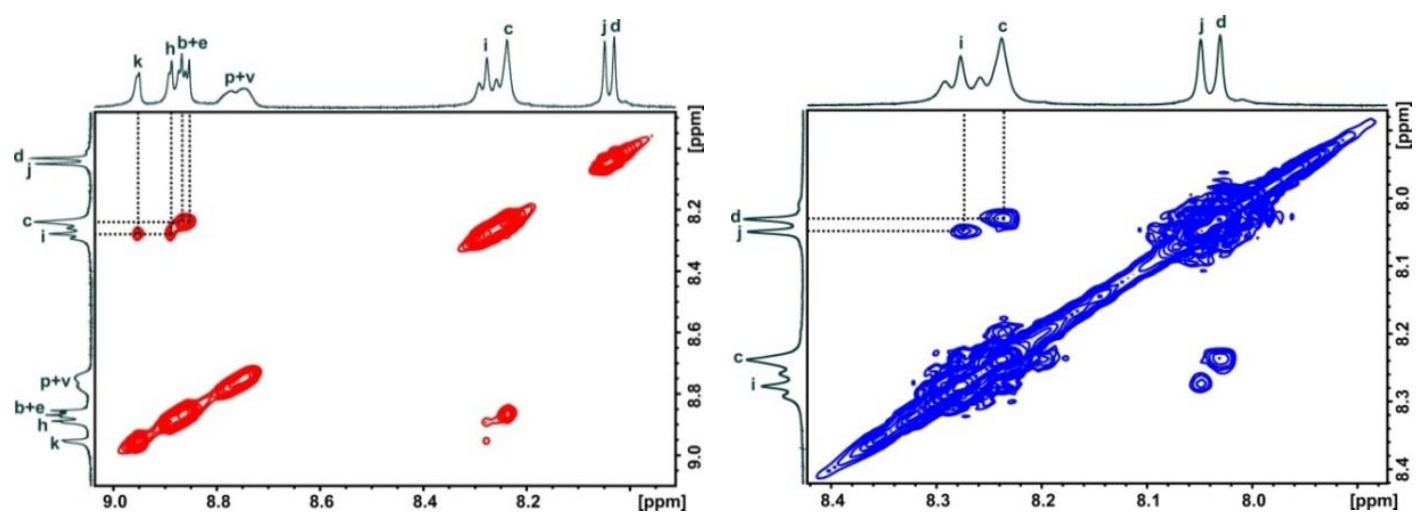

Figure 5.38: (left) NOESY correlations from $c$ and $i$ to $b+e, h$ and $k$. (right) COSY cross-peaks from $c$ and $i$ to $\mathrm{d}$ and $\mathrm{j}$.

A long range COSY coupling from $\mathbf{i}$ was used to identify $\mathbf{j}$ as the 8-proton singlet at $8.05 \mathrm{ppm}$ (Figure 5.38, right). Similarly, $\mathbf{c}$ and $\mathbf{d}$ were identified as the 16-proton multiplet and the 8-proton singlet at $8.24 \mathrm{ppm}$ and $8.03 \mathrm{ppm}$ respectively. An NOE between $\mathbf{c}$ and $\mathbf{b}+\mathbf{e}$ is depicted in Figure 5.38 (left) and confirms this assignment. Lastly, COSY correlations from $\mathbf{a}$ to $\mathbf{b}$ and from $\mathbf{e}$ to $\mathbf{f}$ were found (Figure 5.37, right).

The proton labelling on the T6 template is shown in Figure 5.39 (left) and the template region of the diffusion edited ${ }^{1} \mathrm{H}$ NMR spectrum is shown on the right which is considerably simpler after the removal of the T4 templates. The diffusion edited ${ }^{1} \mathrm{H}$ NMR allowed us to differentiate between signals from the template and small solvent impurities. 

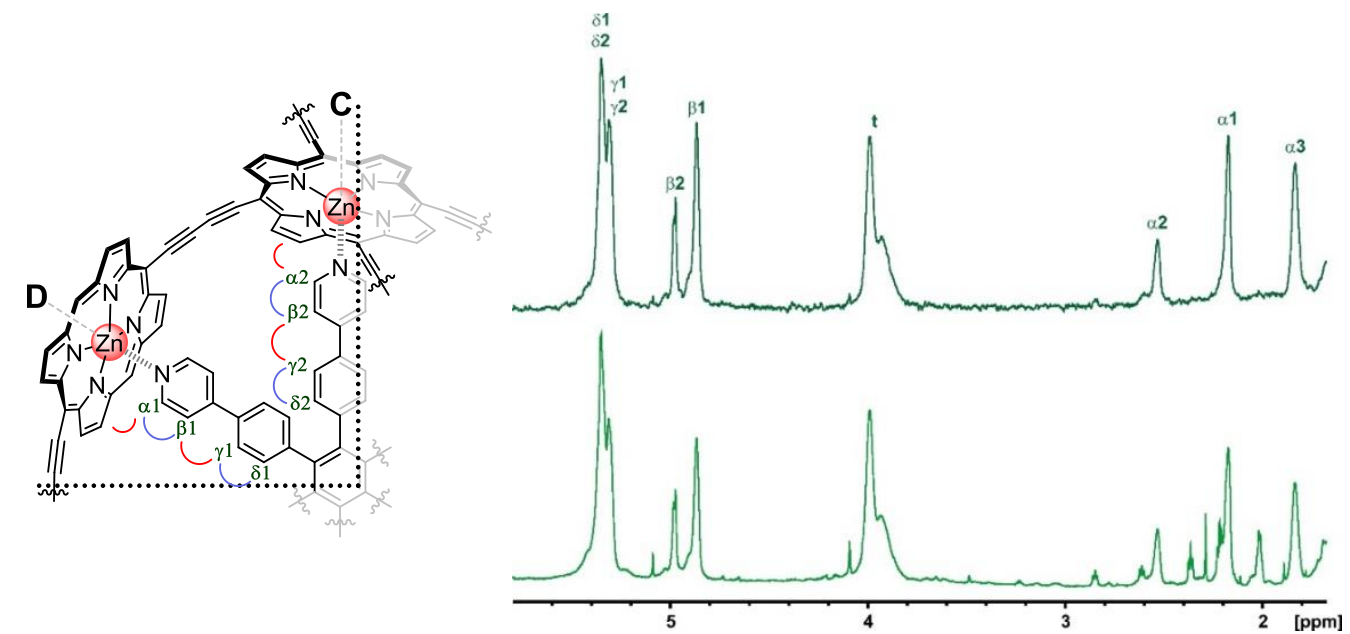

Figure 5.39: (left) Proton labelling for the symmetric unit of the template T6. (right) Template region of the diffusion edited ${ }^{1} \mathrm{H}$ NMR spectrum of $b$-P14.T6.

The 4-proton and 8-proton multiplets at 2.53 and $2.22 \mathrm{ppm}$ were assigned as $\alpha 2$ and $\alpha 1$ respectively. COSY correlation between these signals and the 4-proton and 8-proton multiplets at 4.98 and 4.87 ppm allows these signals to be identified as $\beta 2$ and $\beta 1$ respectively. A subsequent NOESY correlation to the 24-proton multiplet between 5.41 and $5.26 \mathrm{ppm}$ characterised this signal as an overlap for the protons $\gamma \mathbf{1}, \gamma \mathbf{2}, \delta 1$, and $\delta 2$ (Figure 5.40).

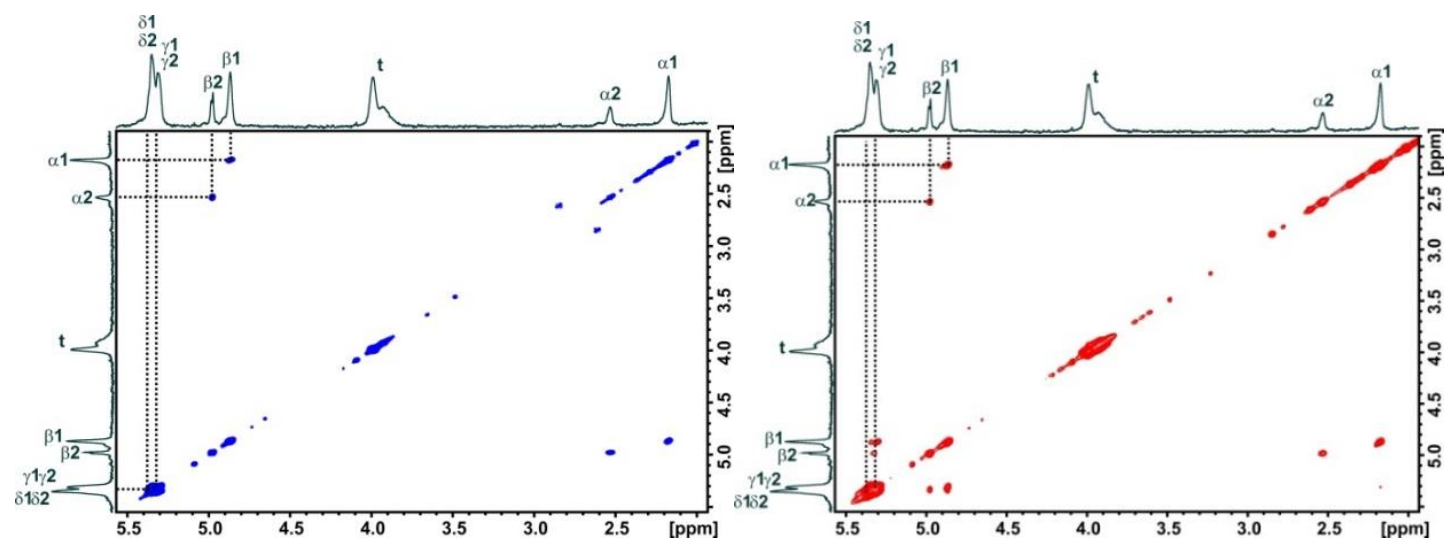

Figure 5.40: (left) COSY correlations between $\alpha 2-\beta 2$ and $\alpha 1-\beta 1$. (right) NOESY correlation between $\beta 2-\gamma 2$ and $\beta 1-\gamma 1$.

Lastly, the assignments for the spectrum of $\boldsymbol{b}$-P14 will be discussed. Without the templates, all porphyrins are free to rotate around the butadiynes which resulted in a simpler spectrum. Most notably, the splitting in alkoxy aryl protons has disappeared as seen in Figure 5.41 between 7.7 and 7.0 ppm. 


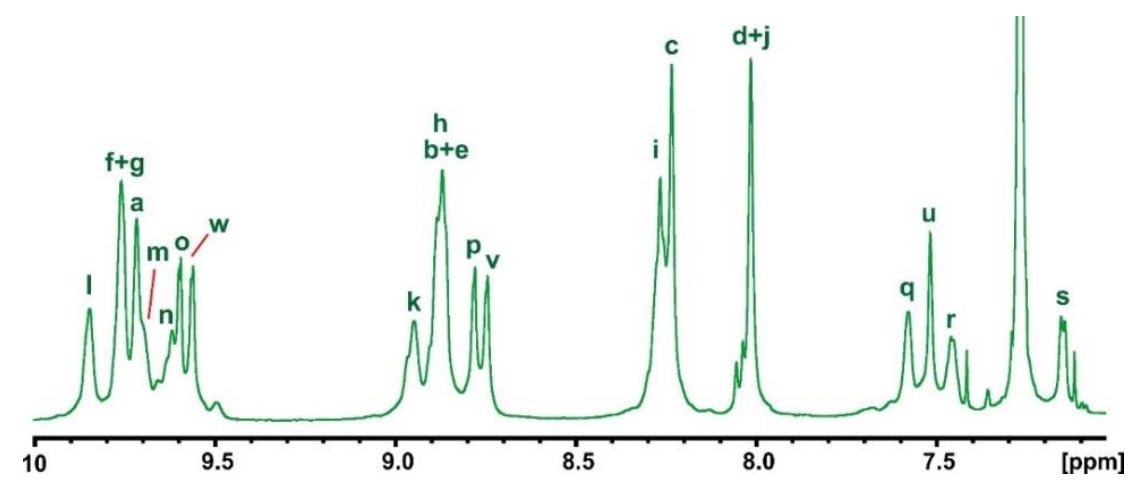

Figure 5.41: The aromatic region of the ${ }^{1} \mathrm{H}$ NMR spectrum of $b$-P14.

By analogy to the previous assignments, the signals for $\mathbf{m}$ and $\mathbf{n}$ were identified as a 16-proton multiplet resonating between 9.71 and $9.61 \mathrm{ppm}$. Only the protons $\mathbf{m}$ and $\mathrm{n}$ are expected to show a COSY correlation in the region between 10 and $9.5 \mathrm{ppm}$. This observation was used to confirm the signals for these protons (Figure 5.42).

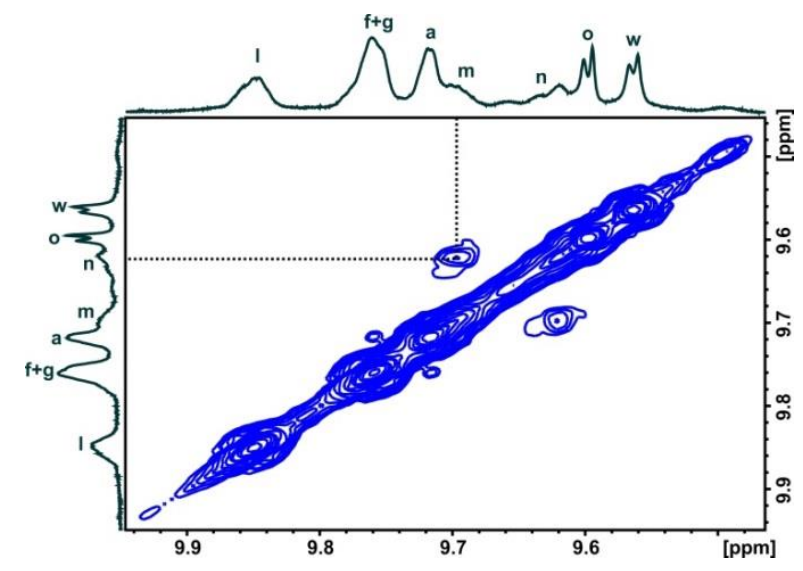

Figure 5.42: COSY correlation between the $\mathrm{m}$ and $\mathrm{n}$ protons.

Due to the removal of the T6 template, the alkoxy-porphyrin D can now freely rotate, as a result there is no longer a splitting for the signals corresponding to the aryl group pointing towards the inside and the periphery of the ball. The signal corresponding to $\mathbf{t}$, found at $3.84 \mathrm{ppm}$, is shifted downfield with respect to the other solubilising protons (EtH) due to the deshielding effect of the oxygen. NOESY correlations from $\mathbf{t}$ were used to identify the signals for $\mathbf{s}$ and $\mathbf{u}$ (Figure 5.43 , left) 

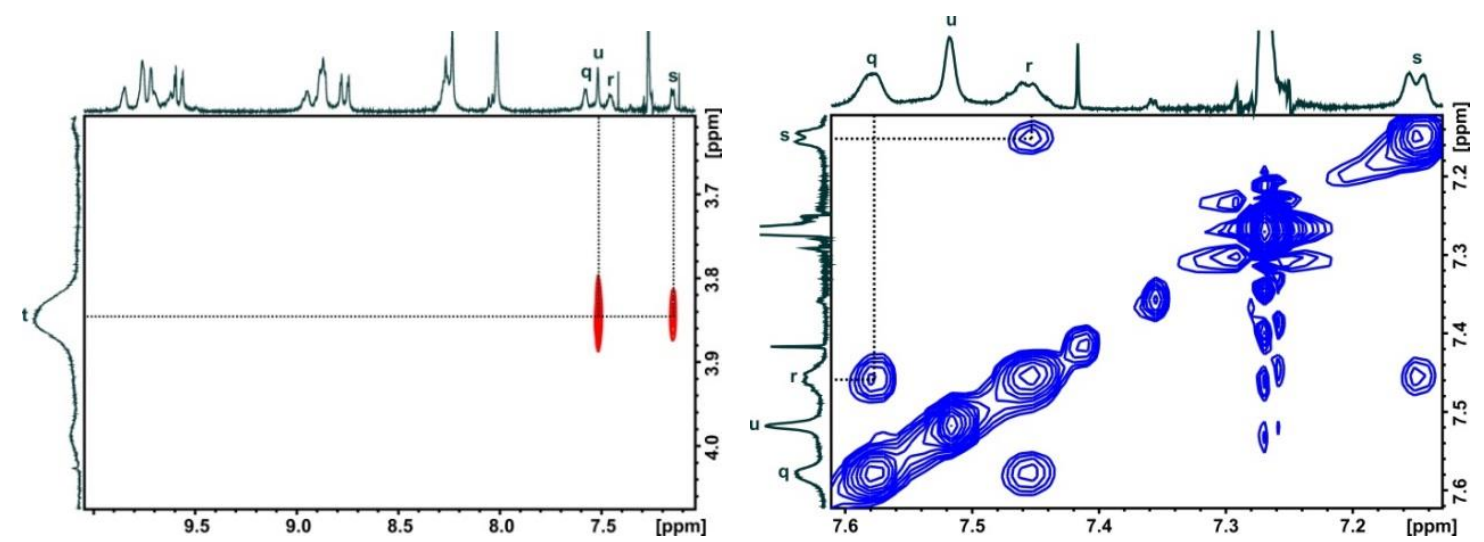

Figure 5.43: (left) NOESY correlation from $t$ to $s$ and $u$. (right) COSY correlation from $r$ to $s$ and $q$, the large signal at $7.27 \mathrm{ppm}$ corresponds to $\mathrm{CDCl}_{3}$.

It was possible to differentiate between $\mathbf{s}$ and $\mathbf{u}$ by interpreting the COSY spectrum (Figure 5.43, right). The protons $\mathbf{u}$ are identified as the 8-proton singlet at $7.52 \mathrm{ppm}$ and show no COSY correlations, while the 8 -proton doublet at $7.15 \mathrm{ppm}$, identified as $\mathbf{s}$, shows a COSY cross-peak with $\mathbf{r}$ (8-proton multiplet at $7.46 \mathrm{ppm}$ ). In addition, $\mathbf{r}$ is coupled to the 8-proton doublet at $7.58 \mathrm{ppm}$ labelled as q. The beta-protons from the alkoxy-porphyrin can be identified through NOESY correlations from $\mathbf{u}$ and $\mathbf{q}$ to the two 8-proton doublets ( $\mathbf{p}$ and $\mathbf{v}$ ) at 8.78 and $8.76 \mathrm{ppm}$, which subsequently show COSY cross-peaks to the two 8-proton doublets at 9.60 and 9.56 ppm corresponding to $\mathbf{o}$ and $\mathbf{w}$ respectively (Figure 5.44 ).
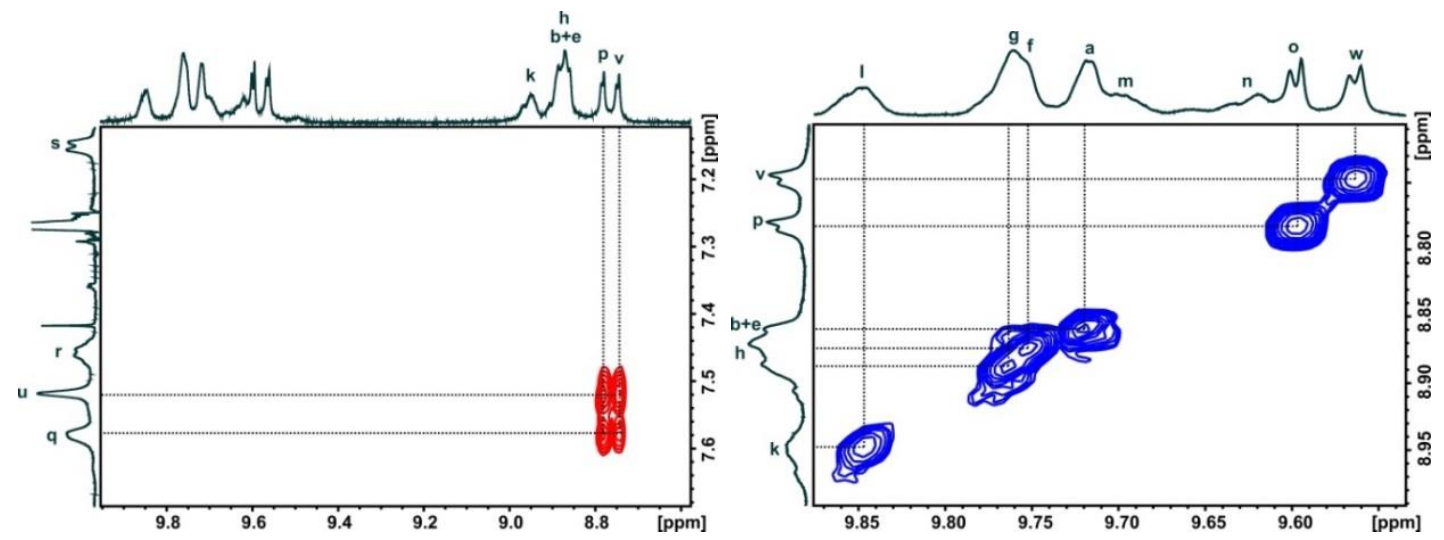

Figure 5.44: (left) NOESY correlations from $q$ and $u$ to $p$ and v. (right) COSY correlations between the porphyrin's beta-protons (for the COSY cross-peak between the $\mathrm{m}$ and $\mathrm{n}$ protons please refer to Figure 5.42).

By analogy to the templated structures, the proton I on the THS-porphyrin A, is identified as an 8-proton multiplet at 9.85 ppm. The COSY cross-peak in Figure 5.44 (right) from I was used to characterise k. The NOESY correlation between the aryl protons and the porphyrins beta-protons are illustrated in Figure 5.45. The NOESY spectrum shows that $\mathbf{g}, \mathbf{h}, \mathbf{k}$ and I are coupled to $\mathbf{i}$. Similar NOESY correlations were 
found for $\mathbf{a}, \mathbf{f}$ and $\mathbf{b}+\mathbf{e}$ to $\mathbf{c}$. Both $\mathbf{c}$ and $\mathbf{i}$ were found to couple to the 16-proton singlet at $8.01 \mathrm{ppm}$ which was assigned to an overlap of $\mathbf{d}$ and $\mathbf{j}$. The correlations between $\mathbf{a}-\mathbf{b}, \mathbf{e}-\mathbf{f}, \mathbf{g}-\mathbf{h}$, and $\mathbf{k}-\mathbf{I}$ were confirmed through their COSY correlations (Figure 5.44 , right).

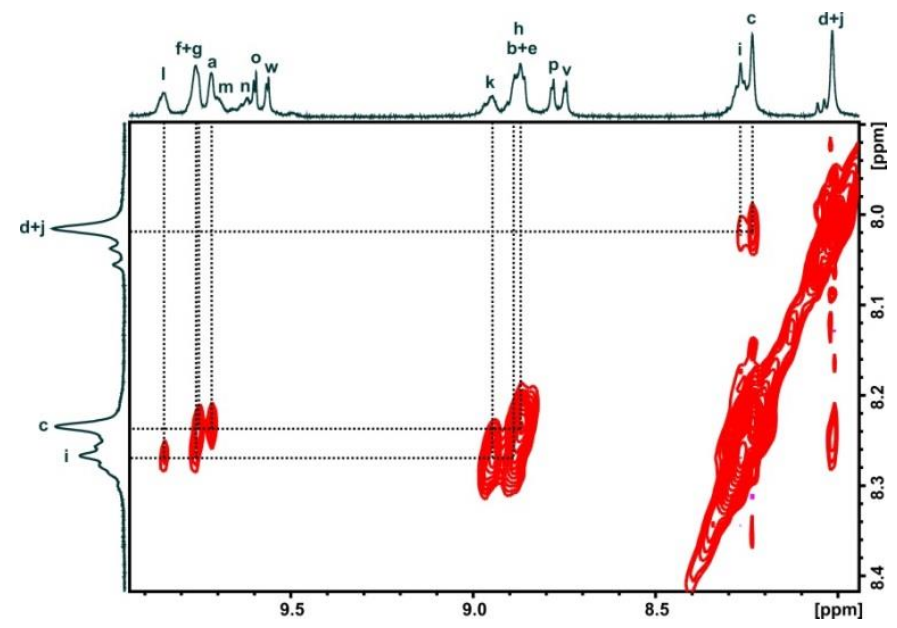

Figure 5.45: NOESY correlations between the aryl protons $\mathrm{c}, \mathrm{d}$, $\mathrm{i}$, and $\mathrm{j}$ to the porphyrin's beta-protons.

\subsubsection{UV-vis-NIR binding studies on $b-\mathrm{P} 14$}

After the characterisation of $\boldsymbol{b}$-P14 and its templated analogues we proceeded to investigate their properties. UV-vis-NIR titrations were performed to quantify the binding behaviour of the templates in the cavity of the ball. In addition the photophysical properties of the ball were investigated to gain insights into the migration of energy between the ring components.

The UV-vis-NIR spectrum of $\boldsymbol{b}$-P14.T6·(T4) $)_{2}$ (Figure 5.46 , black) resembles a combination of the absorption spectra of c-P6.T6 and c-P10.(T5) $\mathbf{2}_{2}$, albeit slightly red-shifted due to its greater conjugation. Similarly to templates bound within porphyrin nanorings, the three templates positioned within the ball can be displaced by the addition of competing ligands. ${ }^{23,60,192}$ Denaturation titrations were performed by titrating a large excess of quinuclidine into a solution of the 1:1:2 (b-P14 : T6 : T4) complex (ca. 0.6 M in toluene at $298 \mathrm{~K}$ ) which displaces the multidentate ligands. We have determined the association constant $K_{\mathrm{f}}$ for both T4 and T6 via their denaturation constants $K_{\mathrm{dn}} \cdot{ }^{55,136}$ Our analysis assumes that the denaturation processes for each template (T4 and T6) are essentially all-or-nothing two state equilibria (i.e. intermediate partially denatured species do not build up to high 
concentrations). This assumption is supported by the isosbestic nature of the UV-vis-NIR titration and the good fits of the curves to the calculated binding isotherm for a two-state equilibrium (Figure 5.47 and Figure 5.48). Denaturation constants were used to calculate the formation constants $K_{\mathrm{f}}$ using the following equation:

$$
K_{\mathrm{f}}=\frac{K_{\mathrm{Q}}^{N}}{K_{\mathrm{dn}}}
$$

where $K_{\mathrm{Q}}$ is a reference binding constant which was approximated to the binding of quinuclidine to a zinc porphyrin monomer (see Section 5.5.4) and $N$ is the number of binding interactions between the template and the host.

Upon the addition of quinuclidine, two distinct processes were observed (Figure 5.46). Due to the weaker binding affinity of T4 in comparison to T6, the T4 templates are selectively removed at a lower concentration of quinuclidine $(0-0.03 \mathrm{M})$ indicated by the disappearance of the sharp characteristic Q-band at $883 \mathrm{~nm}$. Subsequently, T6 is displaced at higher concentration (0.03-0.40 M) evidenced by the disappearance of the distinctive three-finger pattern in the Q-band region. The observed changes in the Q-bands are caused by the greater flexibility of the structure and an increase in the porphyrin-porphyrin dihedral angles upon template removal.
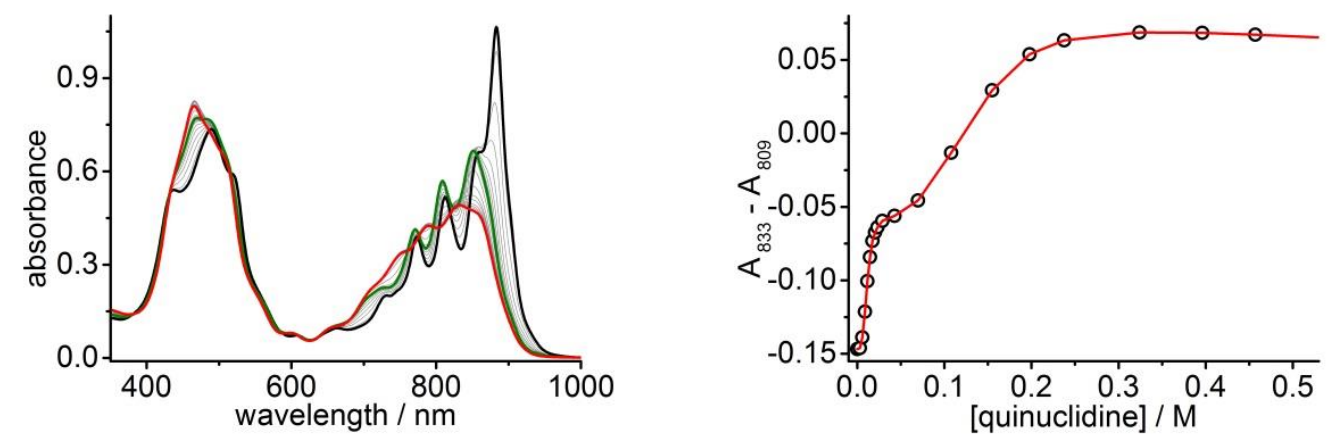

Figure 5.46: UV-vis-NIR denaturation titration of the $b$-P14·T6· $(\mathrm{T} 4)_{2}$ complex with quinuclidine (toluene at 298 K). Initially T4 is removed at lower concentrations (black to green) followed by the displacement of T6 (green to red). On the right the experimental values for the change in absorbance (black circles) are shown connected by a plotted line.

The two independent denaturation processes are split in Figure 5.47 and Figure 5.48 which allowed us to fit the experimental data to a one-to-one binding isotherm. All titrations were performed at least twice (see Section 5.5.4). 

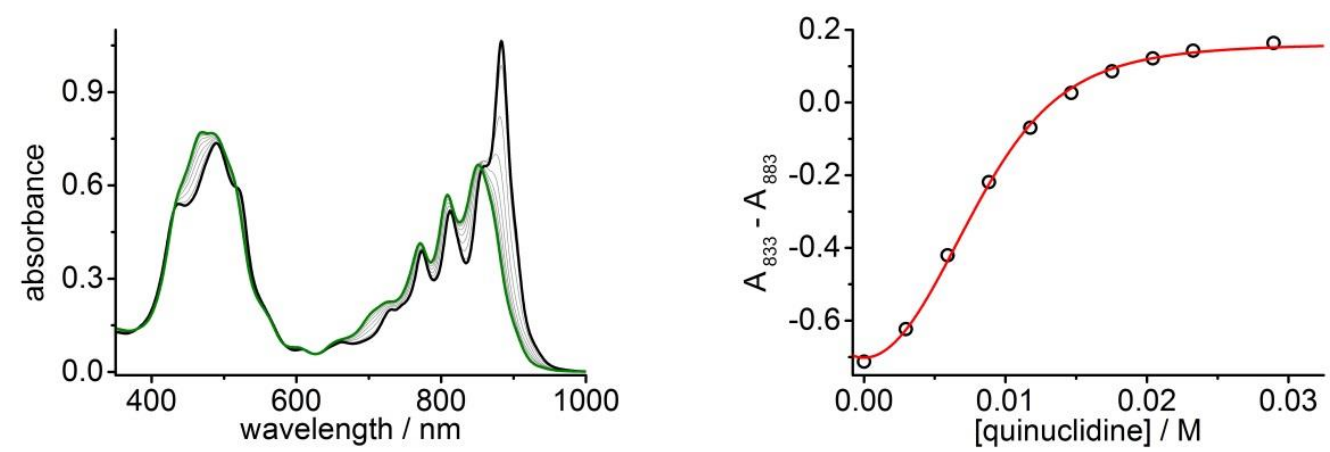

Figure 5.47: First part of the UV-vis-NIR titration of quinuclidine and $b$-P14.T6•(T4) $)_{2}$ illustrating the selective removal of the T4 templates. $R^{2}=0.9986$. (Toluene, 298K, $\left[b-\mathrm{P} 14 \cdot \mathrm{T} 6 \cdot(\mathrm{T} 4)_{2}\right]=0.583 \mu \mathrm{M}, K_{\mathrm{dn}}=1.51 \times 10^{2} \mathrm{M}^{-3}$ ). The effective concentration of the host is doubled in the calculations of the binding isotherm due to the presence of two T4 templates per pall molecule.
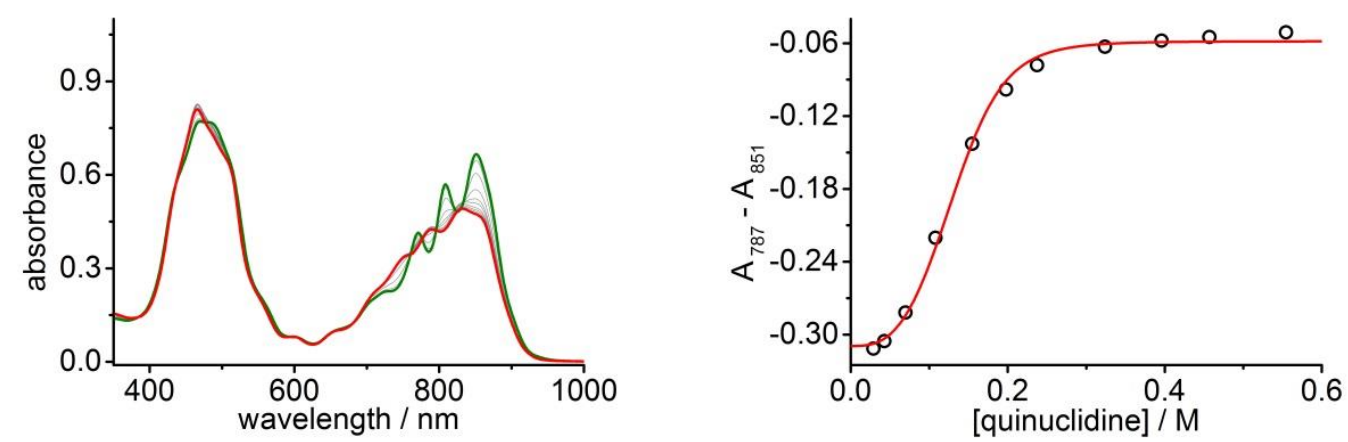

Figure 5.48: Second part of the UV-vis-NIR titration of quinuclidine and $b$-P14·T6-(T4) ${ }_{2}$ illustrating the removal of the T6 template. $R^{2}=0.9972$. (Toluene, 298K, $\left[b-\mathrm{P} 14 \cdot \mathrm{T6} \cdot(\mathrm{T} 4)_{2}\right]=0.583 \mu \mathrm{M}, K_{\mathrm{dn}}=6.64 \times 10^{-2} \mathrm{M}^{-3}$ ).

The stability constants for the templates T4 and T6 were calculated from the denaturation constants and revealed a stability constant of $(1.8 \pm 0.2) \times 10^{22} \mathrm{M}^{-1}$ for T4 and $(5.5 \pm 1.2) \times 10^{37} \mathrm{M}^{-1}$ for T6. The binding strength of T6 is roughly an order of magnitude stronger in the ball than in a comparable 6-ring system. ${ }^{23}$ This increase can be rationalised by the presence of the two shared porphyrins which do not lose any rotational degrees of freedom upon binding the template and which results in an increased binding strength. Similarly to quinuclidine, DABCO can be used to displace the templates from the cavity of the ball. Using concentrated DABCO solutions as the eluent in size exclusion chromatography allows for the preparative removal of the templates to give the empty ball $\boldsymbol{b}$-P14. When a weaker coordinating ligand such as pyridine is used, selective displacement of the T4 templates can be achieved yielding the $\boldsymbol{b}$-P14· $\mathbf{T} 6$ complex.

\subsubsection{Photophysical properties of $b$-P14}

All fluorescence experiments were carried out by Dr Juliane Q. Gong. The UV-vis-NIR absorption and fluorescence spectra of $\boldsymbol{b}$-P14.T6 $(\mathbf{T} 4)_{2}$ and $\boldsymbol{b}$-P14 are compared in 
Figure $5.49 \mathrm{a}, \mathrm{b}$. The more rigid conformation of the template-bound ball is reflected by its sharper and more red-shifted absorption and emission spectra. ${ }^{88,136,195}$ Fluorescence up-conversion spectroscopy experiments revealed that the excited states are fully delocalised across the two perpendicular ring planes in the porphyrin ball on an ultrafast timescale. The fluorescence anisotropy dynamics of $\boldsymbol{b}$-P14, with and without templates, is shown in Figure 5.49c. The template complex, $\boldsymbol{b}$-P14·T6 $(\mathbf{T 4})_{2}$ (black points), exhibits a constant and very low anisotropy $(\gamma=0.02)$ over the time range of this experiment (0-10 ps with a 0.3 ps time resolution), showing that the excited state delocalises in three dimensions within 0.3 ps. In contrast, $\boldsymbol{b}$-P14-T6 (green points) and $\boldsymbol{b}$-P14 (red points) show a fast initial drop in anisotropy within during the first 4 ps from $\gamma=0.1$ to $\gamma=0.03$. This fast depolarisation resembles the anisotropy decay in porphyrin nanorings with $>24$ porphyrin units. ${ }^{189}$ The initial anisotropy of 0.1 suggests that upon excitation, an exciton is delocalised over a full ring and that both absorption and emission transition dipoles are polarised in the ring plane. After ultrafast relaxation, the exciton localises and migrates rapidly around the entire porphyrin ball complex. Contributions from emission components polarised in both planes thus result in a low anisotropy value close to zero. Without the templates, an exciton may be initially more localised in $\boldsymbol{b}$-P14 than in $\boldsymbol{b}$-P14-T6, resulting in the observed anisotropy decays, whereas $\boldsymbol{b}$-P14-T6-(T4) $\mathbf{2}_{2}$ reaches the low anisotropy within the time resolution of the experiment. This behaviour is very different from that of the nanorings c-P6-T6, $\boldsymbol{c - P 6}$ and $\boldsymbol{c - P 1 0 ,}$ which exhibit anisotropies of around 0.1 (which remain constant during $0.3-10$ ps after excitation), in agreement with theoretical predictions for an excited state that is delocalised over a 2D ring. ${ }^{89,125,189,195,196}$ 


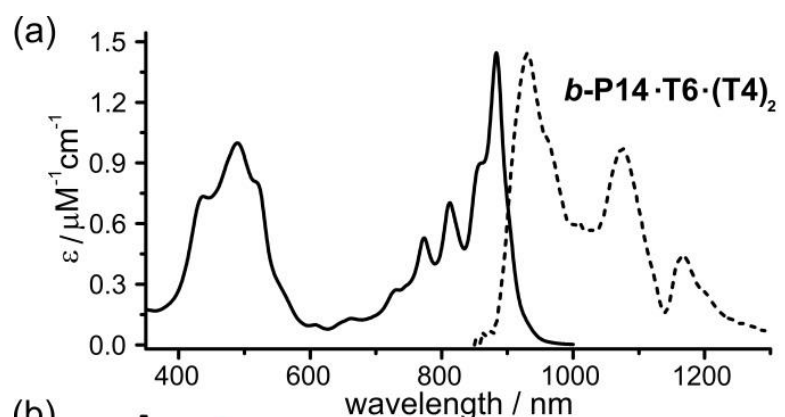

(b)
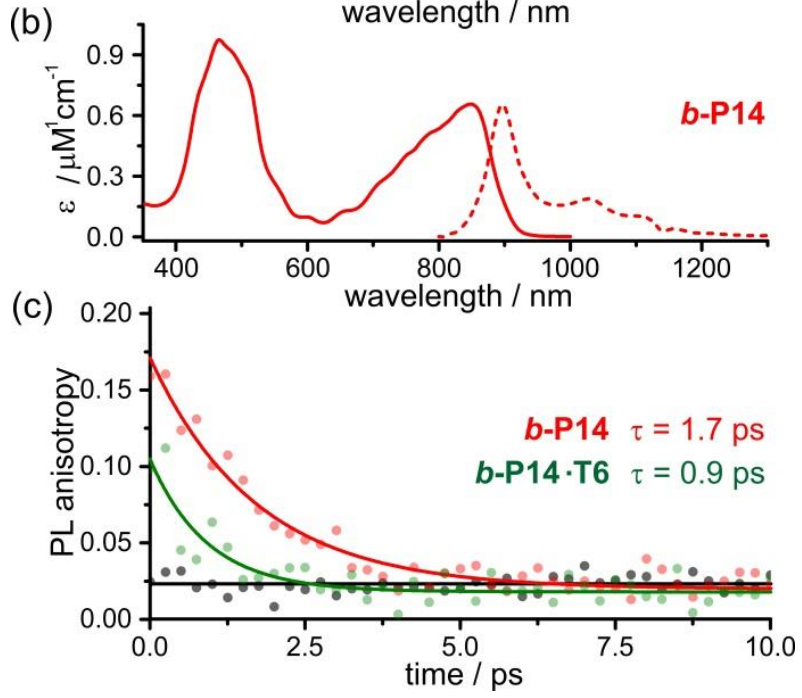

Figure 5.49: UV-vis-NIR absorption (solid line) and fluorescence (dashed line) spectra of $b$-P14·T6·(T4) ${ }_{2}$ (a) and b-P14 (b) recorded in toluene at $298 \mathrm{~K}$. The dip at $1130-1170 \mathrm{~nm}$ corresponds to solvent absorption. (c) Fluorescence anisotropy dynamics. Solutions of $b$-P14-T6-(T4) 2 (black), b-P14-T6 (green) and b-P14 (red) were exited at $820 \mathrm{~nm}$ and emission was detected at $950 \mathrm{~nm}$.

The shape of $\boldsymbol{b}$-P14.T6 $(\mathrm{T4})_{2}$ and $\boldsymbol{b}$-P14 is that of a prolate ball, reminiscent of $\mathrm{C}_{70}$ fullerene. While $\mathrm{C}_{60}$ fullerene has complete polarisation memory loss with zero fluorescence anisotropy, $C_{70}$ displays excitation-wavelength dependent anisotropy values ranging between -0.2 and 0.1 , because emission polarised in the $x-y$ plane is energetically favourable as a result of geometrical deformation. ${ }^{197}$ However, $\boldsymbol{b}$-P14·T6 $\left(\mathrm{TH}_{\mathbf{2}}\right)_{\mathbf{2}}$ and $\boldsymbol{b}$-P14 do not display any significant changes in anisotropy with excitation wavelength in the range of $760-880 \mathrm{~nm}$, probably because the absorption features of their two constituent rings broadly overlap and the emission may be polarised in both constituent ring planes. DFT calculations (B3LYP/6-31G(d)) indicate that the HOMO of $\boldsymbol{b}$-P14 is distributed over the entire $\pi$-system (Figure 5.50) albeit with a higher density on the 6-ring and particularly the spiro-porphyrins. The LUMO and the LUMO+1 are located exclusively on the 6-ring and the 10-ring respectively with nearly identical energies. These calculations are in line with the observation that excitation can migrate between the two rings within the ball. 


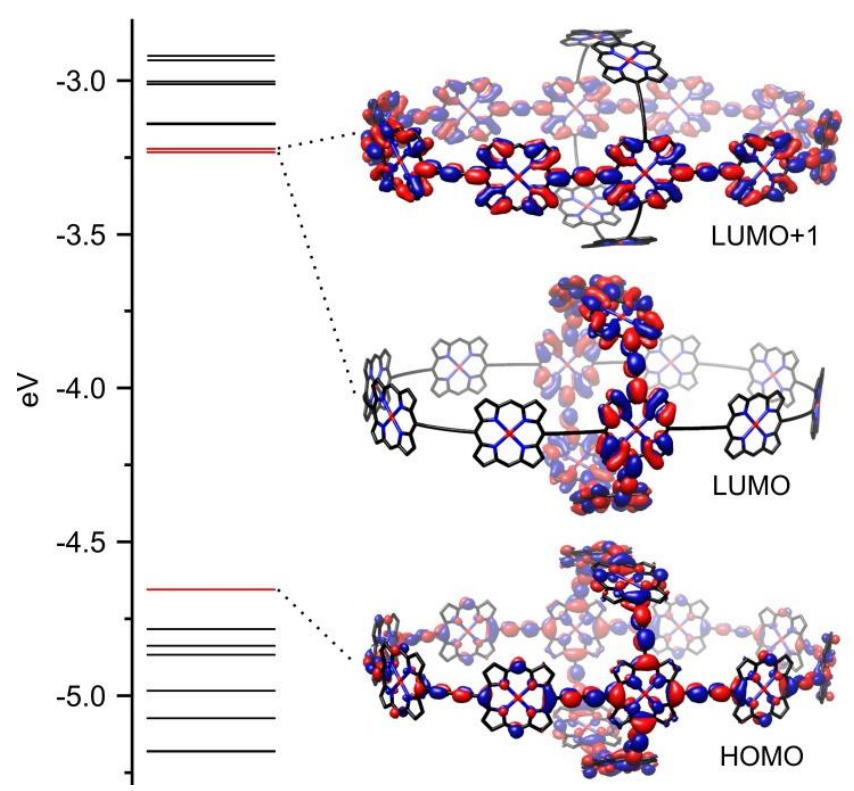

Figure 5.50: LUMO+1, LUMO and HOMO of $b$-P14 calculated at the BLYP/6-31G(d) level of theory shown with a density isovalue of 0.008 together with the corresponding energy levels. Aryl groups bearing solubilising groups were omitted to simplify the calculations.

\subsection{Conclusion}

We have achieved the synthesis and characterisation of a fully $\pi$-conjugated three-dimensional 14-porphyrin nanoball by a template directed approach. UV-vis-NIR titrations showed that the templates bound within the cavity can be removed successively by the addition of a competing ligand. Fluorescence upconversion spectroscopy reveals ultrafast electronic delocalisation between the two perpendicular ring planes in the porphyrin ball. The fluorescence anisotropy approached zero indicate that excitation is delocalised between the two ring components within the nanoball.

\subsection{Experimental procedures and technical details}

\subsubsection{General experimental}

Dry toluene and THF were obtained by passing the solvents through columns of alumina, under nitrogen. Diisopropylamine $\left(i-\mathrm{Pr}_{2} \mathrm{NH}\right)$ was distilled from $\mathrm{CaH}_{2}$ and kept over activated molecular sieves ( $3 \AA$, 8-12 mesh). Unless specified otherwise, all other solvents were used as commercially supplied. Flash chromatography was carried out on silica gel 60 under positive pressure. Analytical thin-layer chromatography was carried out on aluminum-backed silica gel 60 F254 plates. Visualisation was achieved using UV light when necessary. 
All UV-vis-NIR spectra were recorded in solution using a Perkin-Lambda 20 spectrometer ( $1 \mathrm{~cm}$ path length quartz cell). Toluene was used for all titrations without any further purification.

Unless stated otherwise, ${ }^{1} \mathrm{H} /{ }^{13} \mathrm{C}$ NMR spectra were recorded at $298 \mathrm{~K}$ using a Bruker AV400 (400/100 MHz) or Bruker AV700 (700/175 MHz) instrument. ${ }^{1} \mathrm{H}$, and ${ }^{13} \mathrm{C} \mathrm{NMR}$ spectra are reported in ppm; coupling constants are given in Hertz, to the nearest $0.1 \mathrm{~Hz}$. The solvent used was $\mathrm{CDCl}_{3}$.

MALDI-ToF spectra were measured at the EPSRC National Mass Spectrometry service (Swansea) using the Applied Biosystems Voyager DE-STR or at the University of Oxford using Waters MALDI Micro MX spectrometer utilising dithranol as a matrix.

\subsubsection{Synthetic procedures}

\section{Triisopropylsilylpropynal (5.1)}

A solution of triisopropylsilylacetylene $(4.07 \mathrm{~g}, 22.3 \mathrm{mmol})$ in distilled diethyl ether $(19 \mathrm{~mL})$ under a nitrogen atmosphere was cooled to $0{ }^{\circ} \mathrm{C}$.

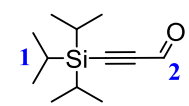
$n$-BuLi (19.5 mL of a 1.6 M solution in $n$-hexane, $31.2 \mathrm{mmol}$ ) was added dropwise and the solution was stirred at $0{ }^{\circ} \mathrm{C}$ for $30 \mathrm{~min}$. The resulting slightly yellow solution was cannulated into a second three-neck flask under a nitrogen atmosphere containing dry $\operatorname{DMF}(5.2 \mathrm{~mL}, 67.0 \mathrm{mmol})$ in $\mathrm{Et}_{2} \mathrm{O}(19 \mathrm{~mL})$ at $-80^{\circ} \mathrm{C}$. Immediately a white precipitate was formed. After stirring the reaction mixture $2 \mathrm{~h}$ between $-80{ }^{\circ} \mathrm{C}$ and $-50{ }^{\circ} \mathrm{C}$ the solution was allowed to reach $0{ }^{\circ} \mathrm{C}$ and was poured onto a $10 \%$ aqueous solution of $\mathrm{H}_{2} \mathrm{SO}_{4}(50 \mathrm{~mL})$. After reaching room temperature, the aqueous layer was extracted 6 times with $\mathrm{Et}_{2} \mathrm{O}$. The organic layers were combined, dried over $\mathrm{MgSO}_{4}$ and the solvent was evaporated to give 5.1 (4.14 g, 88\%) as yellow oil.

${ }^{1} \mathrm{H}$ NMR $\left(400 \mathrm{MHz}, \mathrm{CDCl}_{3}, 298 \mathrm{~K}\right): \delta_{\mathrm{H}}(\mathrm{ppm}) 9.22(1 \mathrm{H}, \mathrm{s}, \mathrm{H} 2), 1.18-1.05(21 \mathrm{H}, \mathrm{m}, \mathrm{H} 1)$. 


\section{Free-base 5,15-bis-((triisopropylsilyl)ethynyl)-porphyrin $(5.2)^{190,199}$}

Dipyrromethane 2.1 (1.47 g, $10.1 \mathrm{mmol}$ ) was dissolved in $\mathrm{CH}_{2} \mathrm{Cl}_{2}$ $(600 \mathrm{~mL})$. The solution was degassed and kept under $\mathrm{N}_{2}$ atmosphere. To this solution triisopropylsilylpropynal $5.1(2.26 \mathrm{~mL}, 10.1 \mathrm{mmol})$ was added followed by $\mathrm{BF}_{3} \cdot \mathrm{Et}_{2} \mathrm{O}(0.40 \mathrm{~mL}, 3.2 \mathrm{mmol})$. The mixture was stirred for $45 \mathrm{~min}$ in the dark under $\mathrm{N}_{2}$ atmosphere, over which time it turned dark blue. DDQ $(3.44 \mathrm{~g}, 15.2 \mathrm{mmol})$ was then added and the

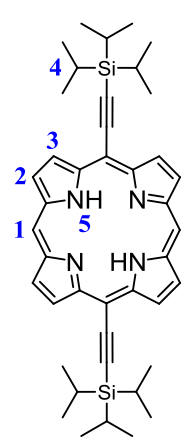
mixture stirred for $10 \mathrm{~min}$. The resulting brown solution was filtered through three consecutive plugs of silica gel with $\mathrm{CH}_{2} \mathrm{Cl}_{2}$ as the eluent, to remove DDQ residues and tar. After removal of the solvent in vacuo, recrystallisation from $\mathrm{CH}_{2} \mathrm{Cl}_{2} /$ methanol yielded the product $(1.1 \mathrm{~g}, 32 \%)$ as a purple solid.

${ }^{1} \mathrm{H}$ NMR $\left(400 \mathrm{MHz}, \mathrm{CDCl}_{3}, 298 \mathrm{~K}\right): \delta_{\mathrm{H}}(\mathrm{ppm}) 10.14(2 \mathrm{H}, \mathrm{s}, \mathrm{H} 1), 9.73(4 \mathrm{H}, \mathrm{d}, J=4.6 \mathrm{~Hz}$, H3), $9.32(4 \mathrm{H}, \mathrm{d}, J=4.6 \mathrm{~Hz}, \mathrm{H} 2), 1.52-1.46(42 \mathrm{H}, \mathrm{m}, \mathrm{H} 4),-2.62(2 \mathrm{H}, \mathrm{s}, \mathrm{H} 5)$.

MALDI-TOF: $m / z=670\left(\mathrm{C}_{42} \mathrm{H}_{54} \mathrm{~N}_{4} \mathrm{Si}_{2}, \mathrm{M}^{+}\right.$requires 670$)$.

\section{Magnesium 5,15-bis-((triisopropylsilyl)ethynyl)-porphyrin (5.3) $)^{190,199}$}

A solution of the free-base porphyrin $5.2\left(248 \mathrm{mg}, 0.37 \mathrm{mmol}\right.$ ) and $\mathrm{MgI}_{2}$ $(515 \mathrm{mg}, 1.8 \mathrm{mmol})$ in pyridine $(30 \mathrm{~mL})$ and $\mathrm{Et}_{3} \mathrm{~N}(1.5 \mathrm{~mL})$ was refluxed overnight under nitrogen atmosphere, during which time the mixture became bright green. The solvent was removed in vacuo and the residue re-dissolved in $\mathrm{CH}_{2} \mathrm{Cl}_{2}$ and filtered over a silica plug (1\% pyridine in $\mathrm{CH}_{2} \mathrm{Cl}_{2}$ ). Solvent evaporation afforded the product $(278 \mathrm{mg}, 97 \%)$ as a

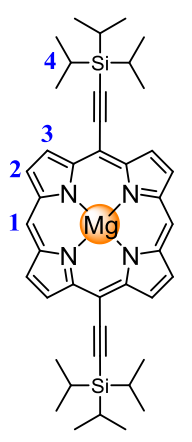
one-to-one complex with pyridine as a dark green/purple solid.

${ }^{1}$ H NMR $\left(400 \mathrm{MHz}, \mathrm{CDCl}_{3}, 298 \mathrm{~K}\right): \delta_{\mathrm{H}}(\mathrm{ppm}) 10.16(2 \mathrm{H}, \mathrm{s}, \mathrm{H} 1), 9.82(4 \mathrm{H}, \mathrm{d}, J=4.3 \mathrm{~Hz}$, H3), $9.34(4 \mathrm{H}, \mathrm{d}, J=4.3 \mathrm{~Hz}, \mathrm{H} 2), 1.54-1.47(42 \mathrm{H}, \mathrm{m}, \mathrm{H} 4)$.

MALDI-TOF: $m / z=692\left(\mathrm{C}_{42} \mathrm{H}_{52} \mathrm{MgN}_{4} \mathrm{Si}_{2}, \mathrm{M}^{+}\right.$requires 692). 
Magnesium 5,15-bis-((triisopropylsilyl)ethynyl)-10,20-dibromo-porphyrin (5.4) $)^{190,199}$ A solution of porphyrin $5.3(278 \mathrm{mg}, 0.36 \mathrm{mmol})$ in dry $\mathrm{CHCl}_{3}(35 \mathrm{~mL})$ and pyridine $(0.4 \mathrm{~mL})$ was bubbled with $\mathrm{N}_{2}$ before $N$-bromosuccinimide (135 $\mathrm{mg}, 0.76 \mathrm{mmol}$ ) was added under magnetic stirring. Upon completion $(1.5 \mathrm{~h})$ the reaction was quenched with acetone $(11 \mathrm{~mL})$. The solvent was removed in vacuo and the residue was purified by column chromatography (4:1 PE

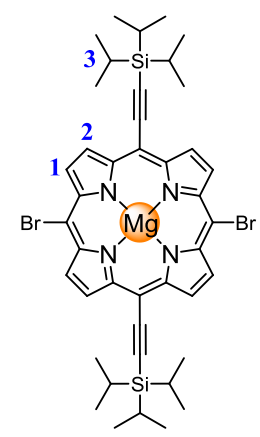
$40-60: \mathrm{CH}_{2} \mathrm{Cl}_{2},+1 \%$ pyridine) affording the product (185 $\mathrm{mg}, 63 \%$ ) as a blue solid.

${ }^{1} \mathbf{H}$ NMR $\left(400 \mathrm{MHz}, \mathrm{CDCl}_{3}, 298 \mathrm{~K}\right): \delta_{\mathrm{H}}(\mathrm{ppm}) 9.65(4 \mathrm{H}, \mathrm{d}, J=4.5 \mathrm{~Hz}, \mathrm{H} 2), 9.61(4 \mathrm{H}, \mathrm{d}$, $J=4.5 \mathrm{~Hz}, \mathrm{H} 1), 1.51-1.45(42 \mathrm{H}, \mathrm{m}, \mathrm{H} 3)$.

MALDI-TOF: $m / z=850\left(\mathrm{C}_{42} \mathrm{H}_{50} \mathrm{Br}_{2} \mathrm{MgN}_{4} \mathrm{Si}_{2}, \mathrm{M}^{+}\right.$requires 850$)$.

\section{Free-base 5,15-bis-((triisopropylsilyl)ethynyl)-10,20-dibromo-porphyrin (5.5)}

To a solution of porphyrin 5.4 (185 mg, $0.23 \mathrm{mmol})$ in $\mathrm{CHCl}_{3}(7 \mathrm{~mL})$ was added trifluoroacetic acid $(90 \mu \mathrm{L}, 1.15 \mathrm{mmol})$ under magnetic stirring. The reaction was monitored by TLC (10:1:1 PE 40-60:EtOAc:pyridine). Upon completion (10 $\mathrm{min}$ ), the solution was washed with water, concentrated and filtered through silica (2:1 PE 40-60: $\mathrm{CH}_{2} \mathrm{Cl}_{2}$ to pure $\mathrm{CH}_{2} \mathrm{Cl}_{2}$ ). The solvent was removed in vacuo to

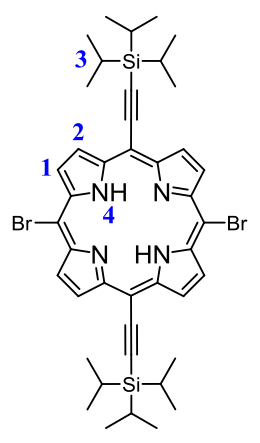
afford the free-base porphyrin (138 $\mathrm{mg}, 72 \%$ ) as a green/purple solid.

${ }^{1} \mathrm{H}$ NMR $\left(400 \mathrm{MHz}, \mathrm{CDCl}_{3}, 298 \mathrm{~K}\right): \delta_{\mathrm{H}}(\mathrm{ppm}) 9.40(4 \mathrm{H}, \mathrm{d}, J=4.6 \mathrm{~Hz}, \mathrm{H} 2), 9.32(4 \mathrm{H}, \mathrm{d}$, $J=4.6 \mathrm{~Hz}, \mathrm{H} 1), 1.58-1.52(42 \mathrm{H}, \mathrm{m}, \mathrm{H} 3),-4.00(2 \mathrm{H}, \mathrm{s}, \mathrm{H} 4)$.

MALDI-TOF: $m / z=828\left(\mathrm{C}_{42} \mathrm{H}_{52} \mathrm{Br}_{2} \mathrm{~N}_{4} \mathrm{Si}_{2}, \mathrm{M}^{+}\right.$requires 828$)$.

Zinc 5,15-bis-((triisopropylsilyl)ethynyl)-10,20-dibromo-porphyrin (5.6) $)^{190,199}$ To a solution porphyrin 5.5 (228 mg, $0.28 \mathrm{mmol})$ in $\mathrm{CHCl}_{3}(28 \mathrm{~mL})$ was added a solution of $\mathrm{Zn}(\mathrm{OAc})_{2} \cdot 2 \mathrm{H}_{2} \mathrm{O}(252 \mathrm{mg}, 1.38 \mathrm{mmol})$ in methanol $(2 \mathrm{~mL})$ and the reaction mixture was refluxed for $2 \mathrm{~h}$. The solution was concentrated and the material filtered over a short silica plug $\left(\mathrm{CHCl}_{3}\right)$. The volatiles were removed in vacuo to afford the zinc porphyrin as a $1: 1$ complex with pyridine $(251 \mathrm{mg}, 94 \%)$ as a

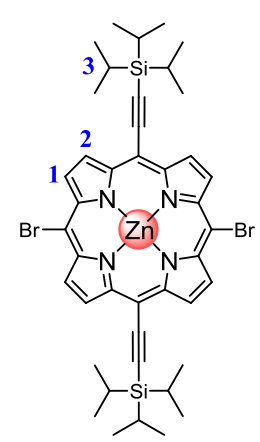
green/purple solid. 
${ }^{1} \mathbf{H}$ NMR $\left(400 \mathrm{MHz} \mathrm{CDCl}_{3}, 298 \mathrm{~K}\right): \delta_{\mathrm{H}}(\mathrm{ppm}) 9.63(4 \mathrm{H}, \mathrm{d}, J=4.6 \mathrm{~Hz}, \mathrm{H} 2), 9.59(4 \mathrm{H}, \mathrm{d}$, $J=4.6 \mathrm{~Hz}, \mathrm{H} 1), 1.52-1.45(42 \mathrm{H}, \mathrm{m}, \mathrm{H} 3)$.

MALDI-TOF: $m / z=892\left(\mathrm{C}_{42} \mathrm{H}_{50} \mathrm{Br}_{2} \mathrm{~N}_{4} \mathrm{Si}_{2} \mathrm{Zn}, \mathrm{M}^{+}\right.$requires 892).

Zinc 5,15-bis-((triisopropylsilyl)ethynyl)-10,20-bis-((trimethylsilyl)ethynyl)porphyrin (5.7) $)^{190,199}$

In a $100-\mathrm{mL}$ two-necked flask were placed porphyrin $\mathbf{5 . 6}$ (251 mg, $0.26 \mathrm{mmol}$ ), $\mathrm{Pd}_{2} \mathrm{dba}_{3}$ (47 mg, $0.052 \mathrm{mmol}$ ), Cul (9.8 $\mathrm{mg}, 0.052 \mathrm{mmol}$ ) and $\mathrm{PPh}_{3}(27 \mathrm{mg}, 0.10 \mathrm{mmol})$. Toluene $(15 \mathrm{~mL})$ and $i-\mathrm{Pr}_{2} \mathrm{NH}(7.5 \mathrm{~mL})$ were added and the mixture was deoxygenated by three freeze-pump-thaw cycles. Trimethylsilylacetylene $(0.11 \mathrm{~mL}, 0.78 \mathrm{mmol})$ was

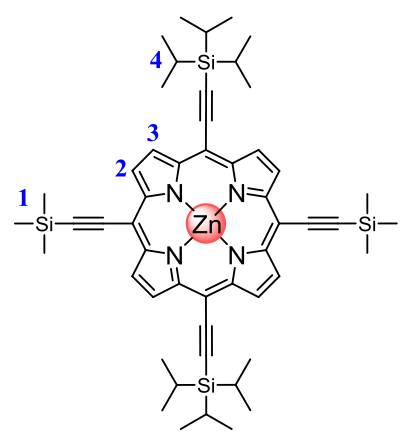
added and the reaction mixture was stirred at $50{ }^{\circ} \mathrm{C}$ for $2 \mathrm{~h}$ after which TLC showed the reaction was completed. The solvent was removed in vacuo and the residue was purified by column chromatography (3:1 to $1: 1 \mathrm{PE}: \mathrm{CH}_{2} \mathrm{Cl}_{2}$ ) affording the desired product (224 mg, 86\%) as a green/purple solid.

${ }^{1} \mathrm{H}$ NMR $\left(400 \mathrm{MHz}, \mathrm{CDCl}_{3}, 298 \mathrm{~K}\right): \delta_{\mathrm{H}}(\mathrm{ppm}) 9.64(4 \mathrm{H}, \mathrm{d}, J=4.5 \mathrm{~Hz}, \mathrm{H} 2$ or $\mathrm{H} 3), 9.61$ $(4 \mathrm{H}, \mathrm{d}, J=4.5 \mathrm{~Hz}, \mathrm{H} 2$ or H3), 1.52-1.45 (42H, m, H4), $0.65(18 \mathrm{H}, \mathrm{s}, \mathrm{H} 1)$.

MALDI-TOF: $m / z=926\left(\mathrm{C}_{52} \mathrm{H}_{68} \mathrm{~N}_{4} \mathrm{Si}_{4} \mathrm{Zn}, \mathrm{M}^{+}\right.$requires 926$)$.

\section{Zinc 5,15-bis-((2-ethylhexyl)oxy-phenyl)-porphyrin (5.9) ${ }^{196}$}

3-((2-Ethylhexyl)oxy)benzaldehyde (5.68 g, $24.2 \mathrm{mmol})$ and Dipyrromethane 2.1 (3.54 g, $24.2 \mathrm{mmol})$ were dissolved in a fresh Winchester bottle of $\mathrm{CH}_{2} \mathrm{Cl}_{2}(2.5 \mathrm{~L})$. The solution was degassed by bubbling the solution with $\mathrm{N}_{2}$ for $20 \mathrm{~min}$ and stirring under nitrogen. Trifluoroacetic acid $(1.06 \mathrm{~mL}$, $33.8 \mathrm{mmol}$ ) was added and the reaction mixture stirred in the

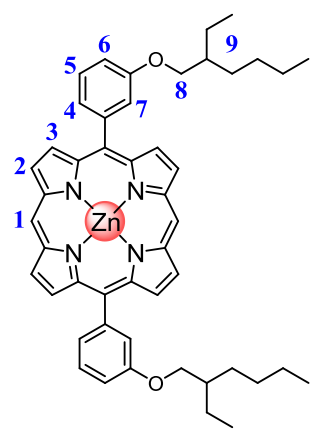
dark for $3 \mathrm{~h}$. DDQ (8.04 g, $35.4 \mathrm{mmol}$ ) was added and stirring continued for $20 \mathrm{~min}$. After quenching of the acid by addition of triethylamine $(63.7 \mathrm{~mL})$ the mixture was concentrated to a volume of $300 \mathrm{~mL}$ on the rotary evaporator, passed over four silica plugs $\left(\mathrm{CH}_{2} \mathrm{Cl}_{2}\right)$ to remove side products and dried under high vacuum. A red oil was obtained which was dissolved in $\mathrm{CHCl}_{3}(175 \mathrm{~mL}) . \mathrm{Zn}(\mathrm{OAc})_{2} \cdot\left(\mathrm{H}_{2} \mathrm{O}\right)_{2}(5.55 \mathrm{~g}, 26.0 \mathrm{mmol})$ was dissolved in methanol $(35 \mathrm{~mL})$ and added to the reaction mixture. The reaction 
was stirred at room temperature for $1 \mathrm{~h}$. The mixture was passed through a short plug of silica gel (1\% pyridine in $\left.\mathrm{CH}_{2} \mathrm{Cl}_{2}\right)$ to give a red oil $(4.17 \mathrm{~g}, 44 \%)$.

${ }^{1} \mathbf{H}$ NMR $\left(400 \mathrm{MHz}, \mathrm{CDCl}_{3}+1 \%\right.$ pyridine- $\left.d_{5}, 298 \mathrm{~K}\right): \delta_{\mathrm{H}}(\mathrm{ppm}) 10.22(2 \mathrm{H}, \mathrm{s}, \mathrm{H} 1), 9.39$ $(4 \mathrm{H}, \mathrm{d}, J=4.5 \mathrm{~Hz}, \mathrm{H} 2), 9.17(4 \mathrm{H}, \mathrm{d}, J=4.5 \mathrm{~Hz}, \mathrm{H} 3), 7.90-7.82(4 \mathrm{H}, \mathrm{m}, \mathrm{H} 6$ and $\mathrm{H} 7), 7.66$ $(2 \mathrm{H}, \mathrm{dd}, J=8.2 \mathrm{~Hz}, J=7.4 \mathrm{~Hz}, \mathrm{H} 5), 7.36(2 \mathrm{H}, \mathrm{dd}, J=8.3 \mathrm{~Hz}, J=2.4 \mathrm{~Hz}, \mathrm{H} 4), 4.10(4 \mathrm{H}, \mathrm{d}$, $J=5.9 \mathrm{~Hz}, \mathrm{H} 8), 2.00-0.80(30 \mathrm{H}, \mathrm{m}, \mathrm{H} 9)$.

${ }^{13} \mathrm{C}$ NMR $\left(100 \mathrm{MHz}, \mathrm{CDCl}_{3}, 298 \mathrm{~K}\right): \delta_{\mathrm{H}}$ (ppm) 157.0, 150.1, 149.6, 143.9, 132.7, 131.8, 127.7, 127.5, 121.4, 120.1, 114.1, 106.3, 71.0, 39.7, 30.8, 29.3, 24.1, 23.2, 14.3, 11.3.

MALDI-TOF: $m / z=780\left(\mathrm{C}_{48} \mathrm{H}_{52} \mathrm{~N}_{4} \mathrm{O}_{2} \mathrm{Zn}, \mathrm{M}^{+}\right.$requires 780$)$.

\section{Zinc 5,15-bis-((2-ethylhexyl)oxy-phenyl)-10,20-dibromo-porphyrin (5.10)}

To a solution of zinc porphyrin $5.9(4.17 \mathrm{~g}, 5.33 \mathrm{mmol})$ in $\mathrm{CHCl}_{3}(200 \mathrm{~mL})$ and pyridine $(4 \mathrm{~mL})$ was added recrystallised $\mathrm{N}$-bromosuccinimide $(1.90 \mathrm{~g}, 10.67 \mathrm{mmol})$ dissolved in $\mathrm{CHCl}_{3}$ $(60 \mathrm{~mL})$. After stirring in the dark for $5 \mathrm{~min}$, acetone $(5 \mathrm{~mL})$ was added to quench the reaction. The mixture was passed through a short plug of silica gel ( $1 \%$ pyridine in $\left.\mathrm{CHCl}_{3}\right)$ to

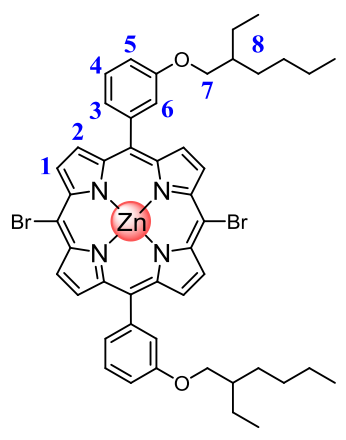
give a purple oil $(4.62 \mathrm{~g}, 92 \%)$.

${ }^{1} \mathrm{H}$ NMR (400 MHz, $\mathrm{CDCl}_{3}+1 \%$ pyridine- $\left.d_{5}, 298 \mathrm{~K}\right): \delta_{\mathrm{H}}(\mathrm{ppm}) 9.64(4 \mathrm{H}, \mathrm{d}, J=4.7 \mathrm{~Hz}$, $\mathrm{H} 1), 8.92(4 \mathrm{H}, \mathrm{d}, J=4.7 \mathrm{~Hz}, \mathrm{H} 2), 7.73-7.68(4 \mathrm{H}, \mathrm{m}, \mathrm{H} 5$ and $\mathrm{H} 6)$, 7.63-7.57 (2H, $\mathrm{m}, \mathrm{H} 4)$, 7.34-7.30 (2H, m, H3), $4.05(4 \mathrm{H}, \mathrm{d}, J=5.8 \mathrm{~Hz}, \mathrm{H} 7), 1.90-0.85(30 \mathrm{H}, \mathrm{m}, \mathrm{H} 8)$.

${ }^{13} \mathrm{C}$ NMR $\left(100 \mathrm{MHz}, \mathrm{CDCl}_{3}, 298 \mathrm{~K}\right): \delta_{\mathrm{H}}$ (ppm) 157.7, 150.8, 150.2, 144.0, 143.7, 136.1, $133.4,132.9,127.3,122.7,121.9,121.5,113.9,104.9,71.0,39.7,30.7,29.3,24.1$, 23.2, 14.3, 11.4 .

MALDI-TOF: $m / z=940\left(\mathrm{C}_{48} \mathrm{H}_{50} \mathrm{Br}_{2} \mathrm{~N}_{4} \mathrm{O}_{2} \mathrm{Zn}, \mathrm{M}^{+}\right.$requires 938). 
Zinc 5,15-bis-((2-ethylhexyl)oxy-phenyl)-10,20-bis-trimethylsilanylethynylporphyrin (5.11)

$\mathrm{Pd}_{2} \mathrm{dba}_{3}$ (292 mg, $0.32 \mathrm{mmol}$ ), Cul (182 mg, $0.96 \mathrm{mmol}$ ), $\mathrm{PPh}_{3}(251 \mathrm{mg}, 0.96 \mathrm{mmol})$ and porphyrin 5.10 (3.00 g, $3.19 \mathrm{mmol}$ ) were placed under argon. Dry toluene $(105 \mathrm{~mL})$ and dry $i-\mathrm{Pr}_{2} \mathrm{NH}(105 \mathrm{~mL})$ were added and the reaction mixture deoxygenated by three vacuum freeze-pump-thaw cycles. Trimethylsilyl acetylene

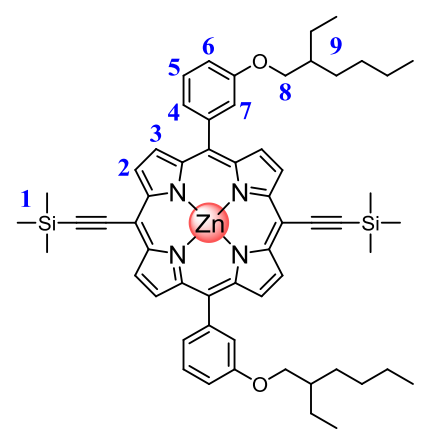
$(1.33 \mathrm{~mL}, 9.57 \mathrm{mmol})$ was added by syringe. The reaction mixture was stirred at $50{ }^{\circ} \mathrm{C}$ for $3 \mathrm{~h}$, and monitored by TLC. The solvents were removed and the residue was passed through a short silica gel column (1\% pyridine in $\mathrm{CHCl}_{3}$ ) to give a green oil (3.01 g, $96 \%)$.

${ }^{1} \mathrm{H}$ NMR $\left(400 \mathrm{MHz}, \mathrm{CDCl}_{3}, 298 \mathrm{~K}\right): \delta_{\mathrm{H}}(\mathrm{ppm}) 9.70(4 \mathrm{H}, \mathrm{d}, J=4.6 \mathrm{~Hz}, \mathrm{H} 2), 8.98(4 \mathrm{H}, \mathrm{d}$, $J=4.6 \mathrm{~Hz}, \mathrm{H} 3), 7.81-7.74(4 \mathrm{H}, \mathrm{m}, \mathrm{H} 6$ and $\mathrm{H} 7), 7.69-7.62(2 \mathrm{H}, \mathrm{m}, \mathrm{H} 5), 7.37-7.31(2 \mathrm{H}, \mathrm{m}$, H4), $4.06(4 \mathrm{H}, \mathrm{d}, J=5.9 \mathrm{~Hz}, \mathrm{H} 8), 1.93-0.90(30 \mathrm{H}, \mathrm{m}, \mathrm{H} 9), 0.64(18 \mathrm{H}, \mathrm{s}, \mathrm{H} 1)$.

MALDI-TOF: $m / z=973\left(\mathrm{C}_{58} \mathrm{H}_{68} \mathrm{~N}_{4} \mathrm{O}_{2} \mathrm{Si}_{2} \mathrm{Zn}, \mathrm{M}^{+}\right.$requires 972).

\section{Zinc 5,15-bis-((triisopropylsilyl)ethynyl)-10,20-bis-ethynyl-porphyrin (5.12) $)^{190}$} Porphyrin 5.7 (0.22 g, $0.24 \mathrm{mmol})$ was dissolved in a mixture of THF (15 mL) and methanol (15 mL). $\mathrm{K}_{2} \mathrm{CO}_{3}(0.67 \mathrm{~g}, 4.84 \mathrm{mmol})$ was added and the mixture was stirred at room temperature for $30 \mathrm{~min}$. The mixture was passed through a short plug of silica gel (1\% pyridine in $\mathrm{CHCl}_{3}$ ) to give a purple solid $(0.18 \mathrm{~g}$, 94\%).

${ }^{1}$ H NMR $\left(400 \mathrm{MHz}, \mathrm{CDCl}_{3}, 298 \mathrm{~K}\right): \delta_{\mathrm{H}}(\mathrm{ppm}) 9.54(4 \mathrm{H}, \mathrm{d}, J=4.6 \mathrm{~Hz}$,

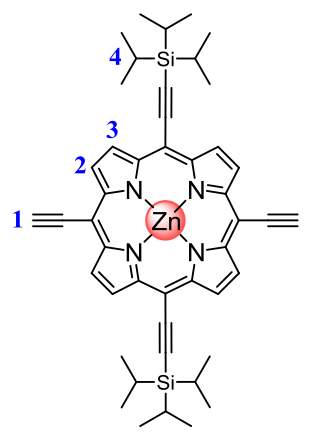
$\mathrm{H} 2$ or H3), $9.49(4 \mathrm{H}, \mathrm{d}, J=4.6 \mathrm{~Hz}, \mathrm{H} 2$ or H3), $4.14(2 \mathrm{H}, \mathrm{s}, \mathrm{H} 1), 1.53-1.49(42 \mathrm{H}, \mathrm{m}, \mathrm{H} 4)$. MALDI-TOF: $m / z=781\left(\mathrm{C}_{46} \mathrm{H}_{52} \mathrm{~N}_{4} \mathrm{Si}_{2} \mathrm{Zn}, \mathrm{M}^{+}\right.$requires 780$)$. 


\section{Zinc 5,15-bis-((2-ethylhexyl)oxy-phenyl)-10-ethynyl-20-trimethylsilanylethynyl-}

porphyrin (5.13)

Porphyrin 5.11 (3.00 g, $3.08 \mathrm{mmol}$ ) was dissolved in $\mathrm{CHCl}_{3}$ $(300 \mathrm{~mL})$ and pyridine $(2 \mathrm{~mL})$. Tetra- $n$-butylammonium fluoride $(1.1 \mathrm{~mL}, 1.0 \mathrm{M}$ solution in THF, $1.1 \mathrm{mmol})$ was added to the stirred solution. The progress of the reaction was monitored by TLC until an optimal product mixture was reached $(5 \mathrm{~min})$. The mixture immediately was passed

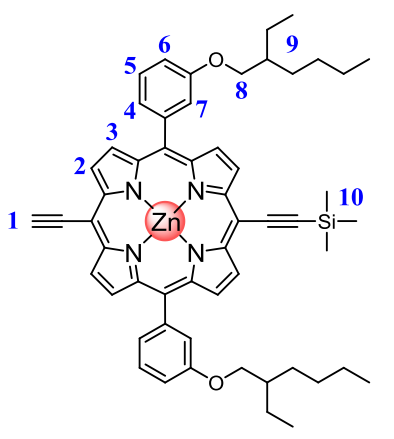
through a short plug of silica gel $\left(\mathrm{CHCl}_{3}\right)$. Column chromatography $(20: 1: 1 \rightarrow$ 10:1:1 $=$ petroleum ether : ethyl acetate : pyridine) gave the title compound as a green oil (0.86 g, 31\%).

${ }^{1} \mathbf{H}$ NMR $\left(400 \mathrm{MHz}, \mathrm{CDCl}_{3}, 298 \mathrm{~K}\right): \delta_{\mathrm{H}}(\mathrm{ppm}) 9.66(4 \mathrm{H}, \mathrm{m}, \mathrm{H} 2), 8.94(4 \mathrm{H}, \mathrm{m}, \mathrm{H} 3)$, 7.79-7.72 (4H, m, H6 and H7), 7.67-7.60 (2H, m, H5), 7.37-7.31 (2H, m, H4), 4.15 (1H, $\mathrm{s}, \mathrm{H} 1), 4.07(4 \mathrm{H}, \mathrm{d}, \mathrm{J}=5.6 \mathrm{~Hz}, \mathrm{H} 8), 1.92-0.88(30 \mathrm{H}, \mathrm{m}, \mathrm{H} 9), 0.61(9 \mathrm{H}, \mathrm{s}, \mathrm{H} 10)$.

MALDI-TOF: $m / z=901\left(\mathrm{C}_{55} \mathrm{H}_{60} \mathrm{~N}_{4} \mathrm{O}_{2} \mathrm{SiZn}, \mathrm{M}^{+}\right.$requires 900).

\section{Porphyrin trimer (5.14)}

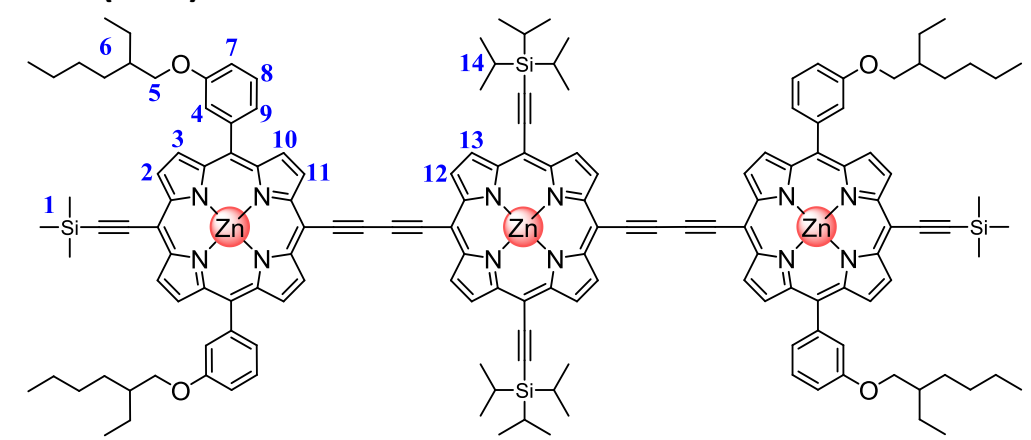

Porphyrin 5.12 (148 mg, $0.19 \mathrm{mmol}$ ) and porphyrin 5.13 (855 mg, $0.95 \mathrm{mmol}$ ) were dissolved in $\mathrm{CHCl}_{3}(120 \mathrm{~mL})$. A catalyst solution was prepared by dissolving $\mathrm{PdCl}_{2}\left(\mathrm{PPh}_{3}\right)_{2}(100 \mathrm{mg}, 0.14 \mathrm{mmol})$, Cul $(100 \mathrm{mg}, 0.52 \mathrm{mmol}$ ) and 1,4-benzoquinone (300 mg, $2.71 \mathrm{mmol})$ in $\mathrm{CHCl}_{3}(65 \mathrm{~mL})$ and $i-\mathrm{Pr}_{2} \mathrm{NH}(3.2 \mathrm{~mL})$. The catalyst solution was added to the porphyrin solution and the reaction mixture was stirred at room temperature for $1 \mathrm{~h}$. The crude reaction mixture was passed through a short plug of silica gel (1\% pyridine in $\mathrm{CHCl}_{3}$ ), passed over a size exclusion column on Biobeads SX-1 (1\% pyridine in toluene), and finally the oligomers were separated by recycling GPC (1\% pyridine in toluene) to give the porphyrin trimer $\mathbf{5 . 1 4}$ as a dark oily solid (227 mg, 47\%). 
${ }^{1} \mathrm{H}$ NMR $\left(400 \mathrm{MHz}, \mathrm{CDCl}_{3}, 298 \mathrm{~K}\right): \delta_{\mathrm{H}}$ (ppm) $9.92(4 \mathrm{H}, \mathrm{d}, J=4.5 \mathrm{~Hz}, \mathrm{H} 2$ or H11 or H12 or H13), $9.90(4 \mathrm{H}, \mathrm{d}, J=4.5 \mathrm{~Hz}, \mathrm{H} 2$ or $\mathrm{H} 11$ or $\mathrm{H} 12$ or $\mathrm{H} 13), 9.76(4 \mathrm{H}, \mathrm{d}, J=4.5 \mathrm{~Hz}, \mathrm{H} 2$ or $\mathrm{H} 11$ or $\mathrm{H} 12$ or $\mathrm{H} 13), 9.67(4 \mathrm{H}, \mathrm{d}, J=4.5 \mathrm{~Hz}, \mathrm{H} 2$ or $\mathrm{H} 11$ or $\mathrm{H} 12$ or $\mathrm{H} 13), 9.06(4 \mathrm{H}, \mathrm{d}$, $J=4.5 \mathrm{~Hz}, \mathrm{H} 3$ or $\mathrm{H} 10), 8.94(4 \mathrm{H}, \mathrm{d}, J=4.5 \mathrm{~Hz}, \mathrm{H} 3$ or $\mathrm{H} 10), 7.83-7.78(8 \mathrm{H}, \mathrm{m}, \mathrm{H} 4$ and $H 7)$, 7.71-7.65 $(4 \mathrm{H}, \mathrm{m}, \mathrm{H} 8), 7.41-7.34(4 \mathrm{H}, \mathrm{m}, \mathrm{H9}), 4.11(8 \mathrm{H}, \mathrm{d}, J=5.9 \mathrm{~Hz}, \mathrm{H} 5)$, 1.95-0.92 (60H, m, H6), 1.53-1.48 (42H, m, H14), $0.63(18 \mathrm{H}, \mathrm{s}, \mathrm{H} 1)$.

MALDI-TOF: $m / z=2,583\left(\mathrm{C}_{156} \mathrm{H}_{168} \mathrm{~N}_{12} \mathrm{O}_{4} \mathrm{Si}_{4} \mathrm{Zn}_{3}, \mathrm{M}^{+}\right.$requires 2,583$)$.

$\lambda_{\text {max }}\left(\mathrm{CHCl}_{3}\right) / \mathrm{nm} \log (\varepsilon): 436$ (5.47), 454 (5.38), 509 (5.35), 584 (4.46), 634 (4.74), 749 (5.11).

Porphyrin trimer $(5.15)^{196}$

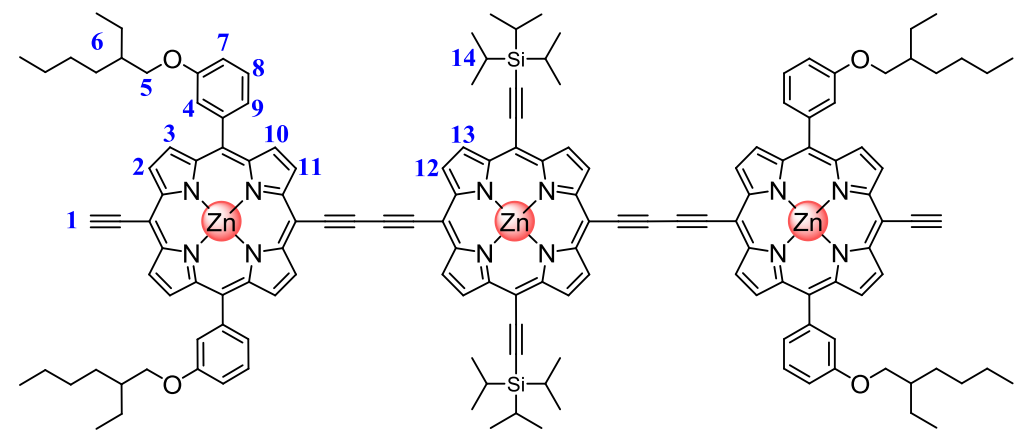

$5.14(227 \mathrm{mg}, 88 \mu \mathrm{mol})$ was dissolved in a mixture of THF $(55 \mathrm{~mL})$ and methanol (25 mL). $\mathrm{K}_{2} \mathrm{CO}_{3}(85 \mathrm{mg}, 0.62 \mathrm{mmol}$ ) was added and the reaction mixture was stirred at room temperature for $1 \mathrm{~h}$. The reaction mixture was passed through a short plug of silica gel $\left(\mathrm{CHCl}_{3}\right)$ to give the title compound as a dark oil (183 $\left.\mathrm{mg}, 85 \%\right)$.

${ }^{1} \mathrm{H}$ NMR $\left(400 \mathrm{MHz}, \mathrm{CDCl}_{3}, 298 \mathrm{~K}\right): \delta_{\mathrm{H}}(\mathrm{ppm}) 9.84(4 \mathrm{H}, \mathrm{d}, J=4.6 \mathrm{~Hz}, \mathrm{H} 2$ or $\mathrm{H} 11$ or $\mathrm{H} 12$ or H13), $9.81(4 \mathrm{H}, \mathrm{d}, J=4.6 \mathrm{~Hz}, \mathrm{H} 2$ or $\mathrm{H} 11$ or $\mathrm{H} 12$ or $\mathrm{H} 13), 9.67(4 \mathrm{H}, \mathrm{d}, J=4.5 \mathrm{~Hz}, \mathrm{H} 2$ or H11 or $\mathrm{H} 12$ or $\mathrm{H} 13), 9.60(4 \mathrm{H}, \mathrm{d}, J=4.6 \mathrm{~Hz}, \mathrm{H} 2$ or $\mathrm{H} 11$ or $\mathrm{H} 12$ or $\mathrm{H} 13), 8.97(4 \mathrm{H}, \mathrm{d}$, $J=4.6 \mathrm{~Hz}, \mathrm{H} 3$ or $\mathrm{H} 10), 8.87(4 \mathrm{H}, \mathrm{d}, J=4.6 \mathrm{~Hz}, \mathrm{H} 3$ or $\mathrm{H} 10), 7.75-7.69(8 \mathrm{H}, \mathrm{m}, \mathrm{H} 4$ and H7), 7.62-7.56 (4H, m, H8), 7.31-7.26 (4H, m, H9), 4.11(2H, s, H1), $4.02(8 \mathrm{H}, \mathrm{d}$, $J=5.8 \mathrm{~Hz}, \mathrm{H} 5), 1.86-0.68(60 \mathrm{H}, \mathrm{m}, \mathrm{H} 6), 1.44-1.40(42 \mathrm{H}, \mathrm{m}, \mathrm{H} 14)$. 


\section{Porphyrin ring $(5.16)^{196}$}

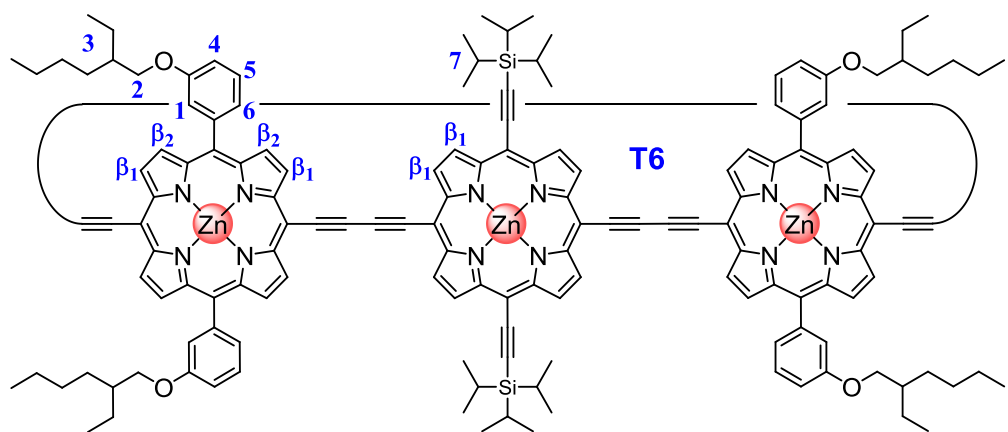

Porphyrin trimer 5.15 (30 mg, $12 \mu \mathrm{mol}$ ) was dissolved in $\mathrm{CHCl}_{3}(10 \mathrm{~mL})$ and $i-\mathrm{Pr}_{2} \mathrm{NH}$ $(0.2 \mathrm{~mL})$. Hexadentate template T6 $(6.1 \mathrm{mg}, 6.2 \mu \mathrm{mol})$ was added and the resulting mixture was sonicated for $0.5 \mathrm{~h}$. A catalyst solution was prepared by dissolving $\mathrm{Pd}\left(\mathrm{PPh}_{3}\right)_{2} \mathrm{Cl}_{2}(2.6 \mathrm{mg}, 3.7 \mu \mathrm{mol})$, $\mathrm{Cul}(7 \mathrm{mg}, 37 \mu \mathrm{mol})$ and 1,4-benzoquinone $(5.3 \mathrm{mg}$, $49 \mu \mathrm{mol})$ in dry $\mathrm{CHCl}_{3}(8 \mathrm{~mL})$ and freshly distilled $i-\mathrm{Pr}_{2} \mathrm{NH}(0.2 \mathrm{~mL})$. This solution was added to the reaction mixture, which was stirred vigorously at room temperature for $3 \mathrm{~h}$. The crude reaction solution was purified by silica column chromatography (1:8 / 40-60 petrol ether: $\mathrm{CH}_{2} \mathrm{Cl}_{2}$ with $1 \%$ of pyridine), size exclusion column on Biobeads SX-1 (1\% pyridine in toluene) and finally precipitated $\left(\mathrm{CH}_{2} \mathrm{Cl}_{2} /\right.$ methanol) to yield the title compound as a red solid ( $28 \mathrm{mg}, 70 \%$ ).

${ }^{1} \mathrm{H}$ NMR $\left(400 \mathrm{MHz}, \mathrm{CDCl}_{3}, 298 \mathrm{~K}\right): \delta \mathrm{H}(\mathrm{ppm}) 9.56\left(18 \mathrm{H}, \mathrm{m}, \beta_{1}\right), 9.48\left(14 \mathrm{H}, \mathrm{m}, \beta_{1}\right)$, 8.85-8.77 $\left(16 \mathrm{H}, \mathrm{m}, \beta_{2}\right), 7.81-7.71(8 \mathrm{H}, \mathrm{m}, \mathrm{H} 1$ or $\mathrm{H} 4), 7.66-7.52(16 \mathrm{H}, \mathrm{m},(\mathrm{H} 1$ or $\mathrm{H} 4)$ and $\mathrm{H} 5), 7.33(8 \mathrm{H}, \mathrm{m}, \mathrm{H} 6), 5.62-5.38(24 \mathrm{H}, \mathrm{m}, \mathrm{T} 6), 5.04-4.93(12 \mathrm{H}, \mathrm{m}, \mathrm{T} 6), 4.18-3.96$ $(16 \mathrm{H}, \mathrm{m}, \mathrm{H} 2), 2.33-2.22(12 \mathrm{H}, \mathrm{m}, \mathrm{T6}), 1.98-0.77(184 \mathrm{H}, \mathrm{m}, \mathrm{H} 3$ and $\mathrm{H} 7)$.

${ }^{13} \mathrm{C}$ NMR $\left(125 \mathrm{MHz}, \mathrm{CDCl}_{3}, 298 \mathrm{~K}\right): \delta_{\mathrm{H}}$ (ppm) 158.0, 157.8, 151.9, 151.8, 151.7, 151.4, $149.9,146.5,146.4,143.6,143.0,142.9,140.1,140.0,138.8,132.7,132.3,132.0$, 131.7, 131.5, 130.7, 130.5, 127.9, 127.7, 127.3, 127.2, 123.9, 123.8, 122.1, 121.0, 119.4, 119.2, 114.4, 113.0, 109.1, 103.7, 101.9, 100.5, 100.0, 99.1, 96.5, 89.4, 71.1, $39.7,30.7,29.3,24.1,23.2,19.3,14.3,12.1,11.4$.

MALDI-TOF: $m / z=5,806\left([5.16]-\mathrm{C}_{3} \mathrm{H}_{7}\right.$ (isopropyl group from TIPS), $\mathrm{C}_{372} \mathrm{H}_{348} \mathrm{~N}_{30} \mathrm{O}_{8} \mathrm{Si}_{4} \mathrm{Zn}_{6},\left[\mathrm{M}^{+}-\mathrm{C}_{3} \mathrm{H}_{7}\right]$ requires 5,827), $\mathrm{m} / \mathrm{z}=4,830\left([5.16-\mathrm{T6}]-\mathrm{C}_{3} \mathrm{H}_{7}\right.$, $\mathrm{C}_{297} \mathrm{H}_{293} \mathrm{~N}_{24} \mathrm{O}_{8} \mathrm{Si}_{4} \mathrm{Zn}_{6},\left[\mathrm{M}^{+}-\mathrm{C}_{3} \mathrm{H}_{7}-\mathrm{T6}\right]$ requires 4,830).

$\lambda_{\text {max }}\left(\mathrm{CHCl}_{3}\right) / \mathrm{nm} \log (\varepsilon): 489$ (5.68), 761 (5.50), 797 (5.68), 837 (5.67). 


\section{Porphyrin ring $(5.17)^{196}$}

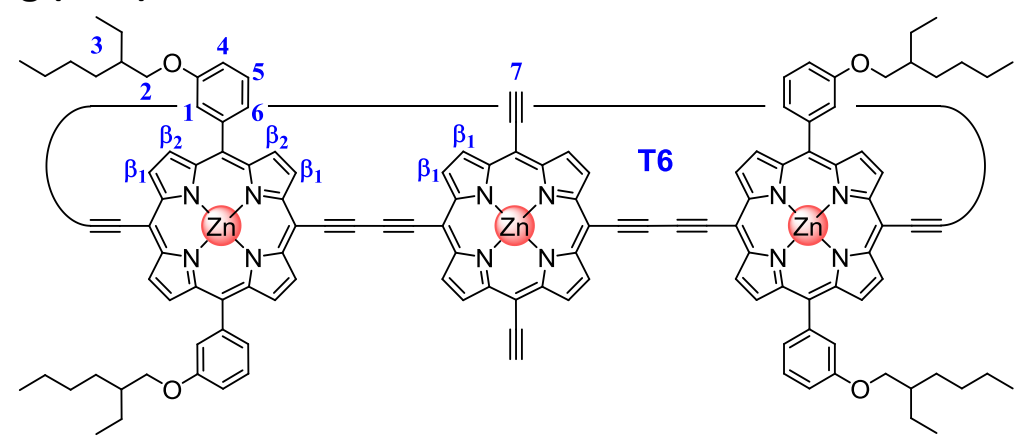

Porphyrin ring $5.16(15.0 \mathrm{mg}, 2.56 \mu \mathrm{mol})$ was dissolved in $\mathrm{CH}_{2} \mathrm{Cl}_{2}(3 \mathrm{~mL})$. Tetra- $n$-butylammonium fluoride $(0.41 \mathrm{~mL}, 1.0 \mathrm{M}$ solution in THF, $0.41 \mathrm{mmol})$ was added and the reaction was stirred for $15 \mathrm{~min}$. The crude reaction mixture was purified by silica column chromatography $\left(\mathrm{CHCl}_{3}\right)$ followed by size exclusion chromatography on Biobeads SX-1 (1\% pyridine in toluene) to yield the title compound as a red solid ( $11.7 \mathrm{mg}, 87 \%)$.

${ }^{1} \mathbf{H}$ NMR $\left(400 \mathrm{MHz}, \mathrm{CDCl}_{3}, 298 \mathrm{~K}\right): \delta \mathrm{H}(\mathrm{ppm})$ 9.60-9.53 (16H, m, $\left.\beta_{1}\right), 9.51(8 \mathrm{H}, \mathrm{d}$, $\left.J=4.5 \mathrm{~Hz}, \beta_{1}\right), 9.47\left(8 \mathrm{H}, \mathrm{d}, J=4.5 \mathrm{~Hz}, \beta_{1}\right), 8.86-8.78\left(16 \mathrm{H}, \mathrm{m}, \beta_{2}\right), 7.82-7.16(32 \mathrm{H}, \mathrm{m}$, $\mathrm{H} 1, \mathrm{H} 4, \mathrm{H} 5$ and $\mathrm{H} 6), 5.57-5.34(24 \mathrm{H}, \mathrm{m}, \mathrm{T6}), 5.04-4.88(12 \mathrm{H}, \mathrm{m}, \mathrm{T} 6), 4.18(4 \mathrm{H}, \mathrm{s}, \mathrm{H} 7)$, 4.17-3.97 (16H, m, H2), 2.32-2.17 (12H, m, T6), 1.93-0.79 (112H, m, H3).

MALDI-TOF: $m / z=5,245\left(\mathrm{C}_{336} \mathrm{H}_{268} \mathrm{~N}_{30} \mathrm{O}_{8} \mathrm{Zn}_{6}, \mathrm{M}^{+}\right.$requires 5,246).

$\lambda_{\max }\left(\mathrm{CHCl}_{3}\right) / \mathrm{nm} \log (\varepsilon): 489$ (5.44), 757 (5.24), 792 (5.38), 833 (5.39).

\section{Porphyrin ring (5.24)}

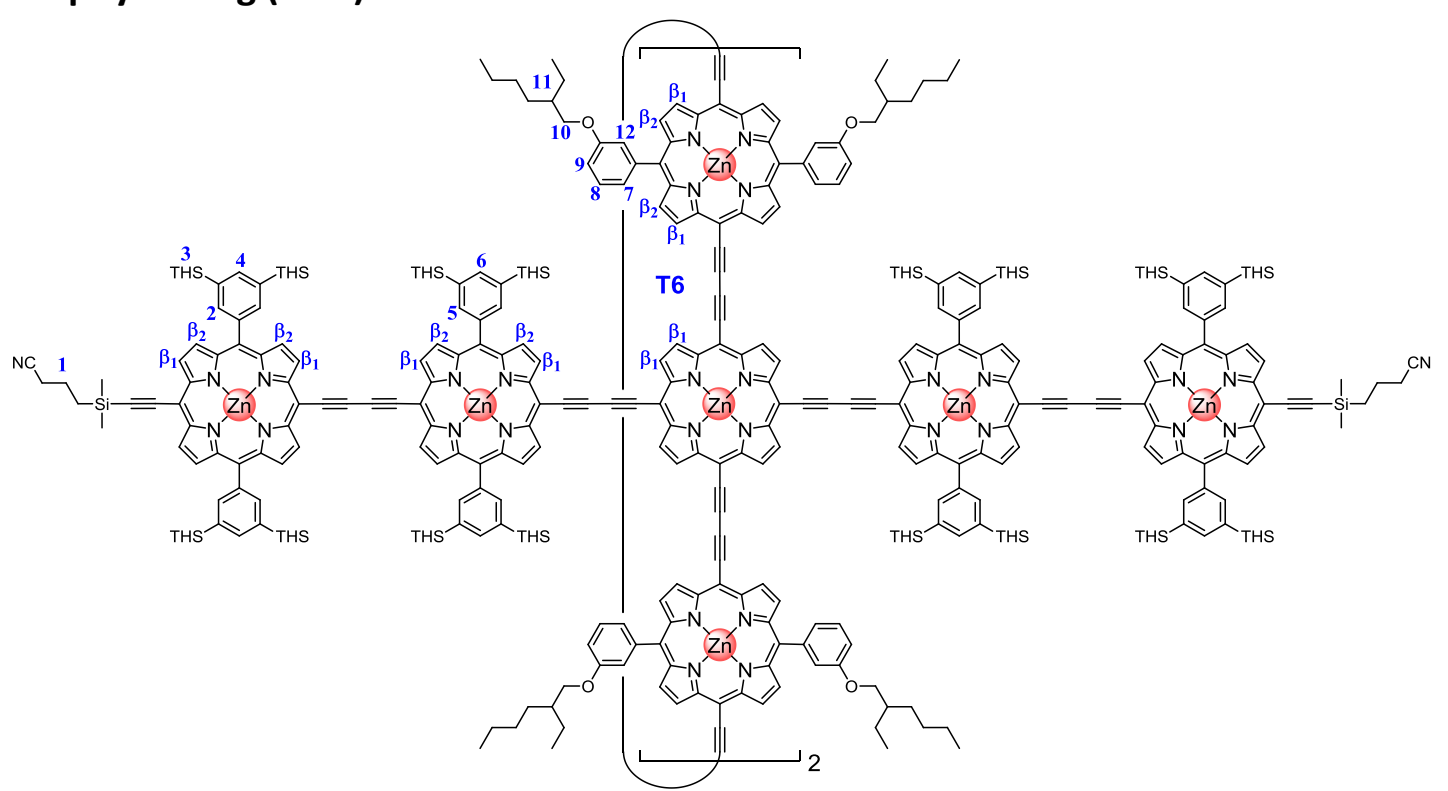


Porphyrin ring 5.17 (6.6 mg, $1.26 \mu \mathrm{mol})$ and a mono-deprotected THS-aryl dimer $\mathbf{5 . 1 9}$ (181.5 mg, $50.59 \mu \mathrm{mol})$ were dissolved in toluene $(10 \mathrm{~mL})$. A catalyst solution was prepared by dissolving $\mathrm{PdCl}_{2}\left(\mathrm{PPh}_{3}\right)_{2}(4.2 \mathrm{mg}, 5.94 \mu \mathrm{mol})$, Cul $(4.2 \mathrm{mg}, 21.88 \mu \mathrm{mol})$ and 1,4-benzoquinone $(12.3 \mathrm{mg}, 0.114 \mathrm{mmol})$ in toluene $(3 \mathrm{~mL})$ and $i-\mathrm{Pr}_{2} \mathrm{NH}$ $(0.25 \mathrm{~mL})$. The catalyst solution was added to the porphyrin solution and the reaction mixture was stirred at room temperature for $35 \mathrm{~min}$. The crude reaction mixture was purified by silica column chromatography (toluene), size exclusion column on Biobeads SX-1 (1\% pyridine in toluene) and finally recycling GPC (1\% pyridine in toluene) to yield the title compound as a red solid (10.0 mg, 41\%).

${ }^{1} \mathrm{H}$ NMR $\left(400 \mathrm{MHz}, \mathrm{CDCl}_{3}, 298 \mathrm{~K}\right): \delta \mathrm{H}(\mathrm{ppm})$ 9.99-9.90 $\left(24 \mathrm{H}, \mathrm{m}, \beta_{1}\right), 9.79-9.54(40 \mathrm{H}$, $\left.m, \beta_{1}\right), 9.08-8.78\left(48 \mathrm{H}, \mathrm{m}, \beta_{2}\right), 8.38(16 \mathrm{H}, \mathrm{m}, \mathrm{H} 2$ or H5), $8.30(16 \mathrm{H}, \mathrm{m}, \mathrm{H} 2$ or H5), 8.09 (8H, m, H4 or H6), $8.04(8 \mathrm{H}, \mathrm{m}, \mathrm{H} 4$ or $\mathrm{H} 6), 7.78(8 \mathrm{H}, \mathrm{m}, \mathrm{H} 9$ or $\mathrm{H} 12), 7.66-7.54(16 \mathrm{H}, \mathrm{m}$, (H9 or H12) and H8), $7.31(8 \mathrm{H}, \mathrm{m}, \mathrm{H7}), 5.67-5.46(24 \mathrm{H}, \mathrm{m}, \mathrm{T6}), 5.17(4 \mathrm{H}, \mathrm{m}, \mathrm{T6}), 5.04$ $(8 \mathrm{H}, \mathrm{m}, \mathrm{T6}), 4.16-3.96(16 \mathrm{H}, \mathrm{m}, \mathrm{H} 10), 2.68(4 \mathrm{H}, \mathrm{m}, \mathrm{T} 6), 2.58(8 \mathrm{H}, \mathrm{t}, J=6.7 \mathrm{~Hz}, \mathrm{H} 1)$, $2.30(8 \mathrm{H}, \mathrm{m}, \mathrm{T} 6), 2.24(8 \mathrm{H}, \mathrm{m}, \mathrm{H} 1), 1.91-0.63(1,424 \mathrm{H}, \mathrm{m}, \mathrm{H} 1$ and $\mathrm{H} 3$ and $\mathrm{H} 11)$.

MALDI-TOF: $m / z=19,600\left(\mathrm{C}_{1240} \mathrm{H}_{1704} \mathrm{~N}_{66} \mathrm{O}_{8} \mathrm{Si}_{36} \mathrm{Zn}_{14}, \mathrm{M}^{+}\right.$requires 19,590).

$\lambda_{\text {max }}\left(\mathrm{CHCl}_{3}\right) / \mathrm{nm} \log (\varepsilon): 456$ (6.25), 493 (6.08), 768 (5.79), 806 (5.93), 847 (5.93).

\section{Porphyrin ring (5.25)}

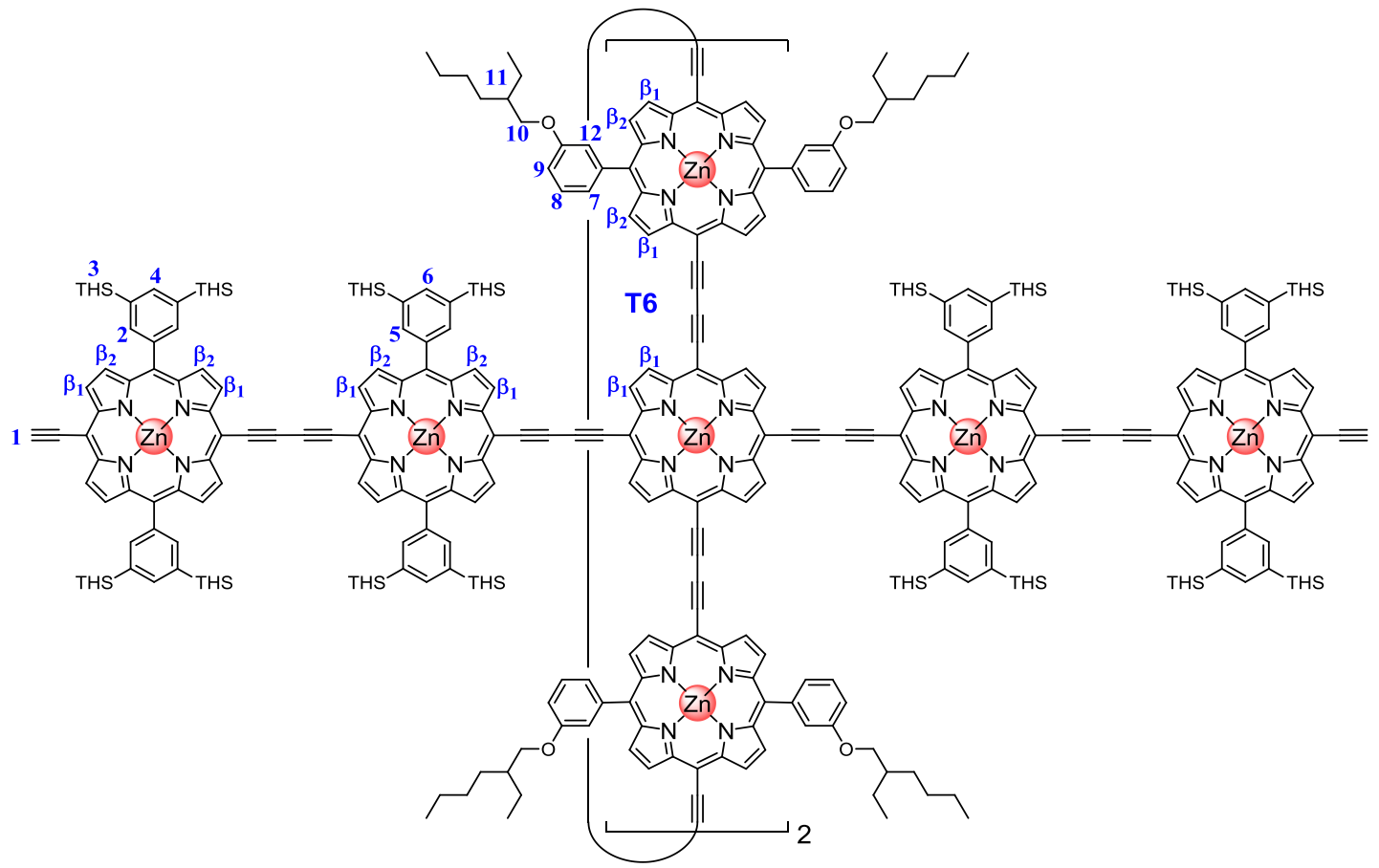


Porphyrin ring $5.24(10.0 \mathrm{mg}, 0.51 \mu \mathrm{mol})$ was dissolved in $\mathrm{CH}_{2} \mathrm{Cl}_{2}(2 \mathrm{~mL})$. Tetra- $n$-butylammonium fluoride ( $82 \mu \mathrm{L}, 1.0 \mathrm{M}$ solution in THF, $82 \mu \mathrm{mol}$ ) was added and the reaction was stirred for $15 \mathrm{~min}$. The crude reaction mixture was purified by silica column chromatography $\left(\mathrm{CHCl}_{3}\right)$ followed by size exclusion chromatography on Biobeads SX-1 (1\% pyridine in toluene) to yield the title compound as a red solid (9.6 mg, 97\%).

${ }^{1}$ H NMR $\left(400 \mathrm{MHz}^{\mathrm{C}} \mathrm{CDCl}_{3}, 298 \mathrm{~K}\right): \delta \mathrm{H}(\mathrm{ppm})$ 9.99-9.88 $\left(24 \mathrm{H}, \mathrm{m}, \beta_{1}\right)$, 9.78-9.53 (40H, $\left.m, \beta_{1}\right), 9.08-8.77\left(48 \mathrm{H}, \mathrm{m}, \beta_{2}\right), 8.38$ (16H, $m, \mathrm{H} 2$ or H5), 8.31 (16H, m, H2 or H5), 8.09 (8H, m, H4 or H6), 8.04 (8H, m, H4 or H6), 7.78 (8H, m, H9 or H12), 7.66-7.54 (16H, m, (H9 or H12) and H8), $7.31(8 \mathrm{H}, \mathrm{m}, \mathrm{H} 7), 5.68-5.45(24 \mathrm{H}, \mathrm{m}, \mathrm{T} 6), 5.16(4 \mathrm{H}, \mathrm{m}, \mathrm{T} 6), 5.04$ $(8 \mathrm{H}, \mathrm{m}, \mathrm{T6}), 4.20(4 \mathrm{H}, \mathrm{s}, \mathrm{H} 1), 4.15-3.95(16 \mathrm{H}, \mathrm{m}, \mathrm{H} 10), 2.68(4 \mathrm{H}, \mathrm{m}, \mathrm{T} 6), 2.31(8 \mathrm{H}, \mathrm{m}$, T6), 1.91-0.63 (1,360H, m, H3 and H11).

MALDI-TOF: $m / z=18,899\left(\mathrm{C}_{1200} \mathrm{H}_{1628} \mathrm{~N}_{62} \mathrm{O}_{8} \mathrm{Si}_{32} \mathrm{Zn}_{14}, \mathrm{M}^{+}\right.$requires 18,864$)$.

$\lambda_{\text {max }}\left(\mathrm{CHCl}_{3}\right) / \mathrm{nm} \log (\varepsilon): 455$ (5.98), 493 (5.85), 768 (5.53), 806 (5.69), 846 (5.70).

\section{Porphyrin ball $b-\mathrm{P} 14 \cdot \mathrm{T} 6 \cdot(\mathrm{T} 4)_{2}$}

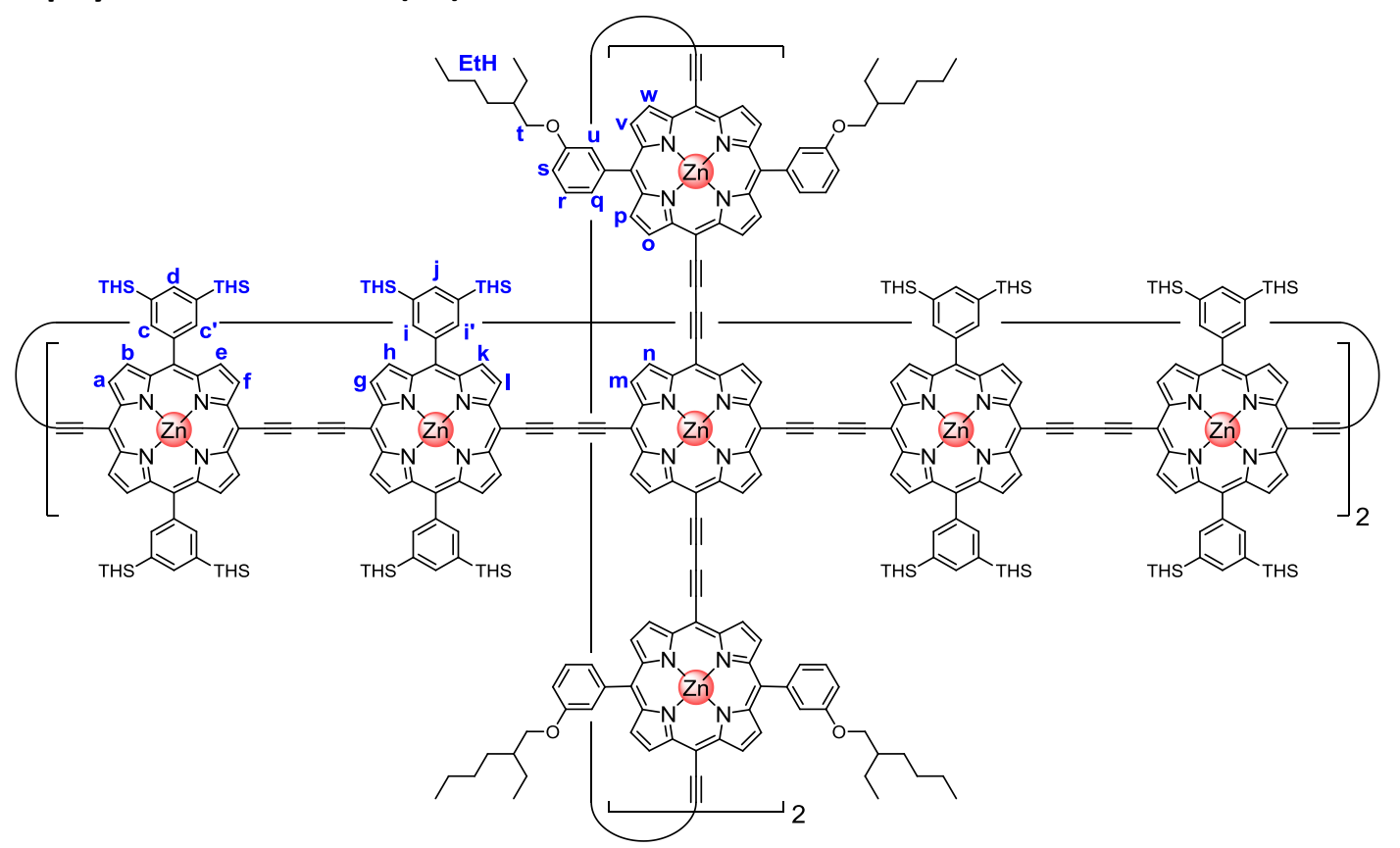



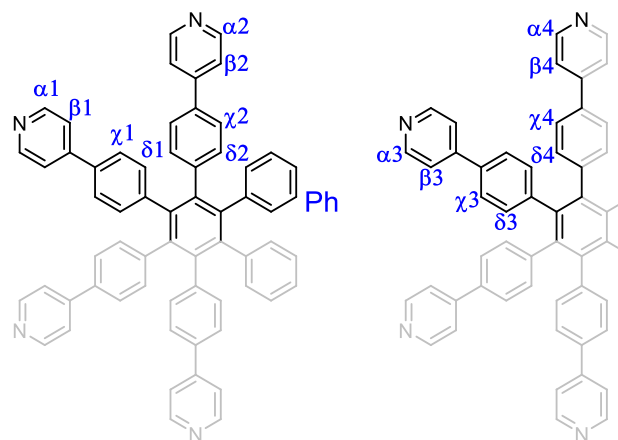

Ball precursor $5.25(9.6 \mathrm{mg}, 0.51 \mu \mathrm{mol})$ and the template $\mathbf{T} 4(0.86 \mathrm{mg}, 1.02 \mu \mathrm{mol})$ were dissolved in $\mathrm{CHCl}_{3}(4.4 \mathrm{~mL})$. UV-vis indicated the formation of the desired two-to-one complex. A catalyst solution was prepared by dissolving $\mathrm{Pd}\left(\mathrm{PPh}_{3}\right)_{2} \mathrm{Cl}_{2}$ (0.88 mg, $1.25 \mu \mathrm{mol})$, Cul (1.20 mg, $6.28 \mu \mathrm{mol})$ and 1,4-benzoquinone (2.78 mg, $25.74 \mu \mathrm{mol})$ in $\mathrm{CHCl}_{3}(0.6 \mathrm{~mL})$ and freshly distilled $i-\mathrm{Pr}_{2} \mathrm{NH}(8.3 \mu \mathrm{L})$. This solution was added to the reaction mixture, which was stirred vigorously at room temperature for $16 \mathrm{~h}$. The crude reaction mixture was purified by silica column chromatography $\left(\mathrm{CHCl}_{3}\right)$, size exclusion column on Biobeads SX-1 (1\% pyridine in toluene) and finally recycling GPC (1\% pyridine in toluene) to yield the title compound as a red solid (5.3 mg, 51\%).

${ }^{1} \mathrm{H}$ NMR (700 MHz, CDCl, $\left.298 \mathrm{~K}\right): \delta_{\mathrm{H}}(\mathrm{ppm}) 9.79(8 \mathrm{H}, \mathrm{m}, \mathrm{l}), 9.66(8 \mathrm{H}, \mathrm{m}, \mathrm{g}), 9.65(16 \mathrm{H}$, m, a/f and m/n), $9.63(8 \mathrm{H}, \mathrm{m}, \mathrm{a} / \mathrm{f}), 9.58(16 \mathrm{H}, \mathrm{m}, \mathrm{o} / \mathrm{w}$ and $\mathrm{m} / \mathrm{n}), 9.51(8 \mathrm{H}, \mathrm{m}, \mathrm{o} / \mathrm{w})$, $8.95(8 \mathrm{H}, \mathrm{m}, \mathrm{k}), 8.86(8 \mathrm{H}, \mathrm{m}, \mathrm{h}), 8.83(16 \mathrm{H}, \mathrm{m}, \mathrm{b} / \mathrm{e}), 8.80(8 \mathrm{H}, \mathrm{m}, \mathrm{p} / \mathrm{v}), 8.76(8 \mathrm{H}, \mathrm{m}$, $p / v), 8.47(8 \mathrm{H}, m, c), 8.43(8 \mathrm{H}, \mathrm{m}, \mathrm{i}), 8.20\left(8 \mathrm{H}, \mathrm{m}, \mathrm{i}^{\prime}\right), 8.11(8 \mathrm{H}, \mathrm{m}, \mathrm{j}), 8.06(16 \mathrm{H}, \mathrm{m}, \mathrm{d}$ and $\left.c^{\prime}\right)$, 7.76-7.58 $(11 \mathrm{H}, \mathrm{m}, \mathrm{q}$ and $\mathrm{u}), 7.57(5 \mathrm{H}, \mathrm{m}, \mathrm{u}), 7.51(6 \mathrm{H}, \mathrm{m}, \mathrm{r}), 7.31(8 \mathrm{H}, \mathrm{m}, \mathrm{s})$, $5.85(44 \mathrm{H}, \mathrm{m}, \delta 1$ and $\delta 2$ and $\gamma 2$ and $\mathrm{Ph}), 5.68(8 \mathrm{H}, \mathrm{m}, \gamma 1), 5.30(32 \mathrm{H}, \mathrm{m}, \beta 2$ and $\gamma 3$ and $\gamma 4$ and $\delta 3$ and $\delta 4), 5.17(8 \mathrm{H}, \mathrm{m}, \beta 1), 4.93(4 \mathrm{H}, \mathrm{m}, \beta 4), 4.83(8 \mathrm{H}, \mathrm{m}, \beta 3), 4.01(16 \mathrm{H}, \mathrm{m}$, t), $2.61(8 \mathrm{H}, \mathrm{m}, \alpha 2), 2.45(12 \mathrm{H}, \mathrm{m}, \alpha 1$ and $\alpha 4), 2.20(8 \mathrm{H}, \mathrm{m}, \alpha 3), 1.98-0.38(1,368 \mathrm{H}$, $m$, THS and EtH).

MALDI-TOF: $m / z=18,861\left(\mathrm{C}_{1200} \mathrm{H}_{1624} \mathrm{~N}_{62} \mathrm{O}_{8} \mathrm{Si}_{32} \mathrm{Zn}_{14}\right.$ (b-P14-T6), $\mathrm{M}^{+}$requires 18,861), $17,865\left(\mathrm{C}_{1128} \mathrm{H}_{1576} \mathrm{~N}_{56} \mathrm{O}_{8} \mathrm{Si}_{32} \mathrm{Zn}_{14}(\boldsymbol{b}-\mathrm{P} 14), \mathrm{M}^{+}\right.$requires 17,863$)$

$\lambda_{\text {max }}$ (toluene) / nm log(ع): 436 (5.87), 490 (6.00), 520 (5.91), 731 (5.44), 773 (5.73), 812 (5.85), 857 (5.96), 883 (6.16). 


\section{Porphyrin ball $b$-P14-T6}

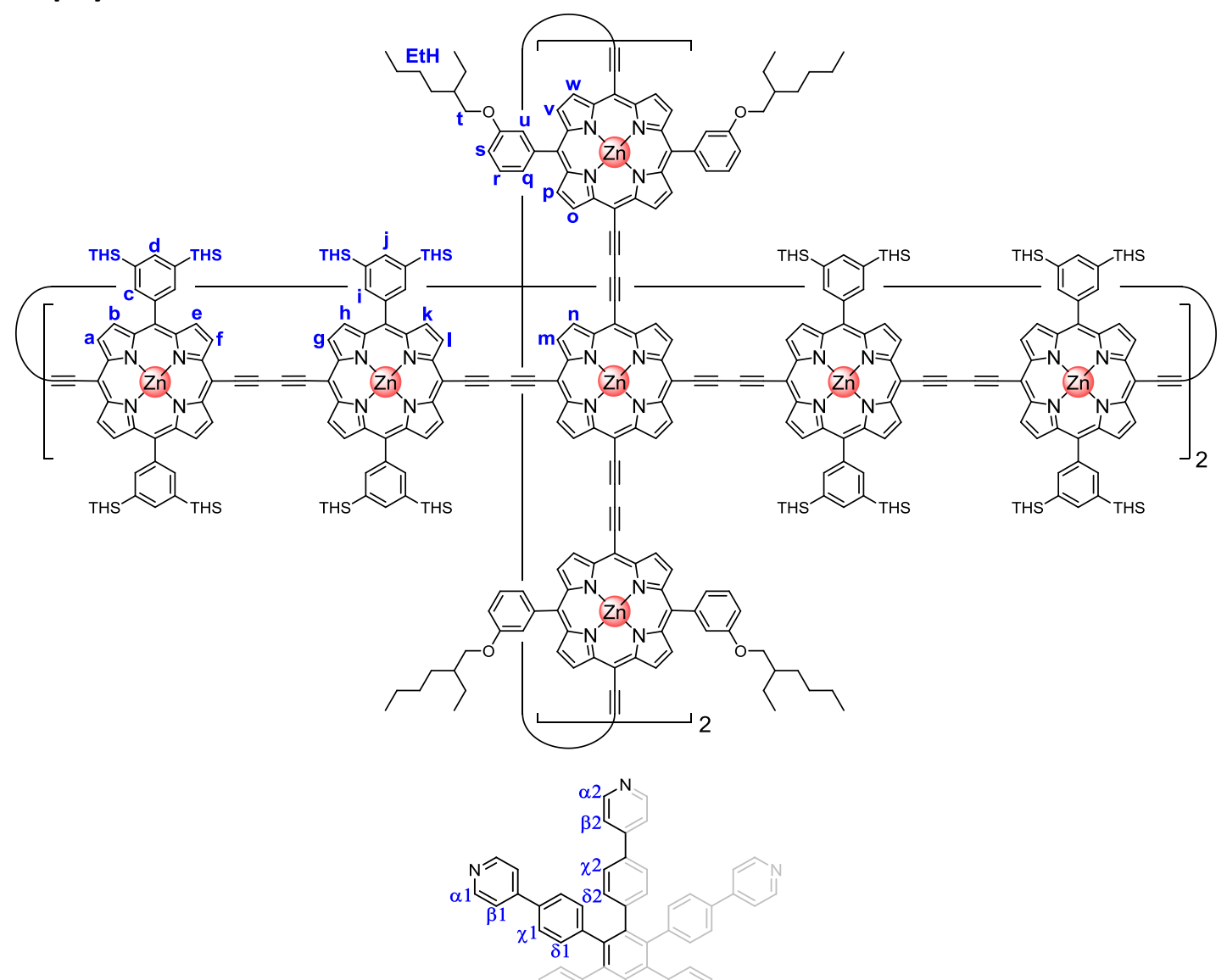

Ball $\boldsymbol{b}$-P14.T6·(T4) $)_{2}(2.0 \mathrm{mg}, 0.10 \mu \mathrm{mol})$ was dissolved in toluene $(0.4 \mathrm{~mL})$ and pyridine $(0.6 \mathrm{~mL})$. The solution was passed over a size exclusion column with Biobeads SX-1 (toluene 40\%, pyridine 60\%). The coloured porphyrin band was collected to yield the title compound as a red solid (1.8 $\mathrm{mg}, 98 \%)$.

${ }^{1} \mathrm{H}$ NMR (700 MHz, $\left.\mathrm{CDCl}_{3}, 298 \mathrm{~K}\right): \delta_{\mathrm{H}}(\mathrm{ppm}) 9.84(8 \mathrm{H}, \mathrm{m}, \mathrm{l}), 9.76(8 \mathrm{H}, \mathrm{m}, \mathrm{g}), 9.75(8 \mathrm{H}$, $m, a / f), 9.70(8 H, m, a / f), 9.66(8 H, m, m / n), 9.55(16 H, m, m / n$ and o/w), $9.50(8 \mathrm{H}$, m, o/w), $8.95(8 \mathrm{H}, \mathrm{m}, \mathrm{k}), 8.89(8 \mathrm{H}, \mathrm{m}, \mathrm{h}), 8.87(8 \mathrm{H}, \mathrm{m}, \mathrm{b} / \mathrm{e}), 8.86(8 \mathrm{H}, \mathrm{m}, \mathrm{b} / \mathrm{e}), 8.76$ $(8 \mathrm{H}, \mathrm{m}, \mathrm{p} / \mathrm{v}), 8.28(16 \mathrm{H}, \mathrm{m}, \mathrm{i}), 8.24(16 \mathrm{H}, \mathrm{m}, \mathrm{c}), 8.05(8 \mathrm{H}, \mathrm{s}, \mathrm{j}), 8.03(8 \mathrm{H}, \mathrm{s}, \mathrm{d}), 7.73-7.61$ $(8 \mathrm{H}, \mathrm{m}, \mathrm{q}), 7.61-7.46(16 \mathrm{H}, \mathrm{m}, \mathrm{r}$ and $\mathrm{u}), 7.32-7.19(8 \mathrm{H}, \mathrm{m}, \mathrm{s}), 5.41-5.26(24 \mathrm{H}, \mathrm{m}, \gamma 1$ and $\gamma 2$ and $\delta 1$ and $\delta 2), 4.98(4 \mathrm{H}, \mathrm{d}, J=7.5 \mathrm{~Hz}, \beta 2), 4.87(8 \mathrm{H}, \mathrm{m}, \beta 1), 4.05-3.80(16 \mathrm{H}$, $m, t), 2.53(4 \mathrm{H}, \mathrm{m}, \alpha 2), 2.22(8 \mathrm{H}, \mathrm{m}, \alpha 1), 1.88-0.32(1,368 \mathrm{H}, \mathrm{m}, \mathrm{THS}$ and $\mathrm{EtH})$. 


\section{Porphyrin ball $b-\mathrm{P} 14$}

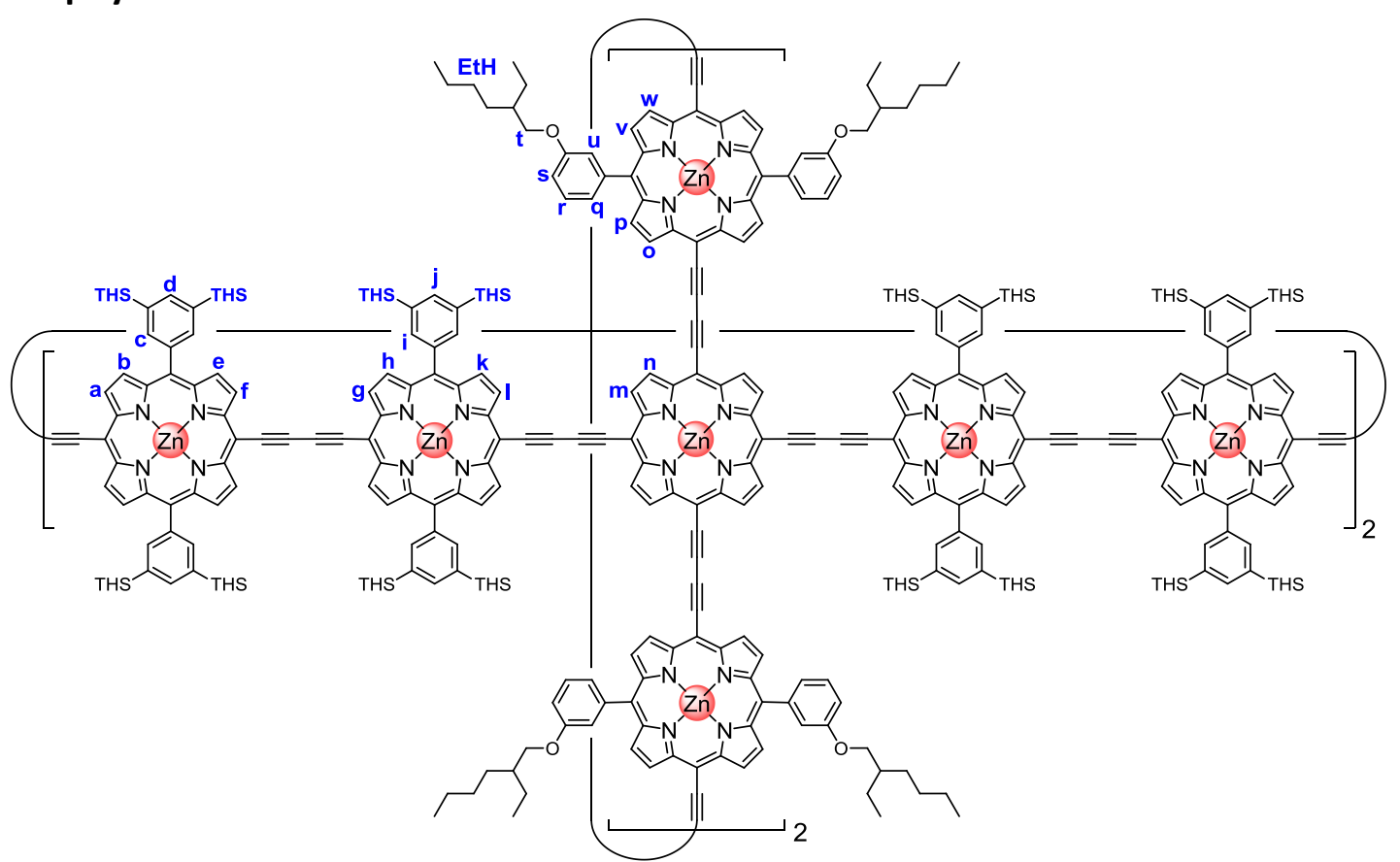

A size exclusion column with Biobeads SX-1 in toluene was prepared. The column was run with a toluene solution saturated with DABCO ( $10 \%$ of the column length) in order to saturate the top of the column with DABCO. Ball $\boldsymbol{b}$-P14-T6-(T4) $\mathbf{2}_{\mathbf{2}}(2.0 \mathrm{mg}$, $0.10 \mu \mathrm{mol})$ was dissolved in the DABCO saturated toluene solution $(1 \mathrm{~mL})$ and loaded on the column. The product was run down the column with the toluene solution saturated with DABCO ( $10 \%$ of the column length) followed by pure toluene. The coloured porphyrin band was collected to yield the template free ball with DABCO coordinating to the zinc centres. The solvent was removed and $\boldsymbol{b}$-P14-(quinuclidine $)_{14}$ was dissolved in toluene $(0.4 \mathrm{~mL})$ and pyridine $(0.6 \mathrm{~mL})$. The solution was passed over a size exclusion column with Biobeads SX-1 (toluene 40\%, pyridine 60\%) in order to remove the quinuclidine. The coloured porphyrin band was collected to yield the title compound as a red solid (1.5 mg, 86\%).

${ }^{1} \mathrm{H}$ NMR (700 MHz, CDCl 3 , $\left.298 \mathrm{~K}\right): \delta_{\mathrm{H}}(\mathrm{ppm}) 9.85(8 \mathrm{H}, \mathrm{m}, \mathrm{l}), 9.79-9.71(24 \mathrm{H}, \mathrm{m}, \mathrm{a} / \mathrm{f}$ and g), 9.71-9.61 (16H, m, m/n), $9.60(8 \mathrm{H}, \mathrm{d}, J=4.4 \mathrm{~Hz}, \mathrm{o} / \mathrm{w}), 9.56(8 \mathrm{H}, \mathrm{d}, J=4.4 \mathrm{~Hz}, \mathrm{o} / \mathrm{w})$, $8.95(8 \mathrm{H}, \mathrm{m}, \mathrm{s}), 8.92-8.84(24 \mathrm{H}, \mathrm{m}, \mathrm{b} / \mathrm{e}$ and $\mathrm{h}), 8.78(8 \mathrm{H}, \mathrm{d}, J=4.4 \mathrm{~Hz}, \mathrm{p} / \mathrm{v}), 8.76(8 \mathrm{H}$, $\mathrm{d}, J=4.4 \mathrm{~Hz}, \mathrm{p} / \mathrm{v}), 8.27(16 \mathrm{H}, \mathrm{m}, \mathrm{i}), 8.23(16 \mathrm{H}, \mathrm{m}, \mathrm{c}), 8.01(16 \mathrm{H}, \mathrm{s}, \mathrm{d}$ and $\mathrm{j}), 7.58(8 \mathrm{H}$, $m, q), 7.52(8 \mathrm{H}, \mathrm{s}, \mathrm{u}), 7.46(8 \mathrm{H}, \mathrm{m}, \mathrm{r}), 7.15(8 \mathrm{H}, \mathrm{d}, J=7.7 \mathrm{~Hz}, \mathrm{~s}), 3.84(16 \mathrm{H}, \mathrm{m}, \mathrm{t})$, 1.64-0.78 $(1,368 \mathrm{H}, \mathrm{m}, \mathrm{THS}$ and $\mathrm{EtH})$. 


\subsubsection{Spectra confirming identity of new compounds}

\section{Porphyrin ring (5.17)}
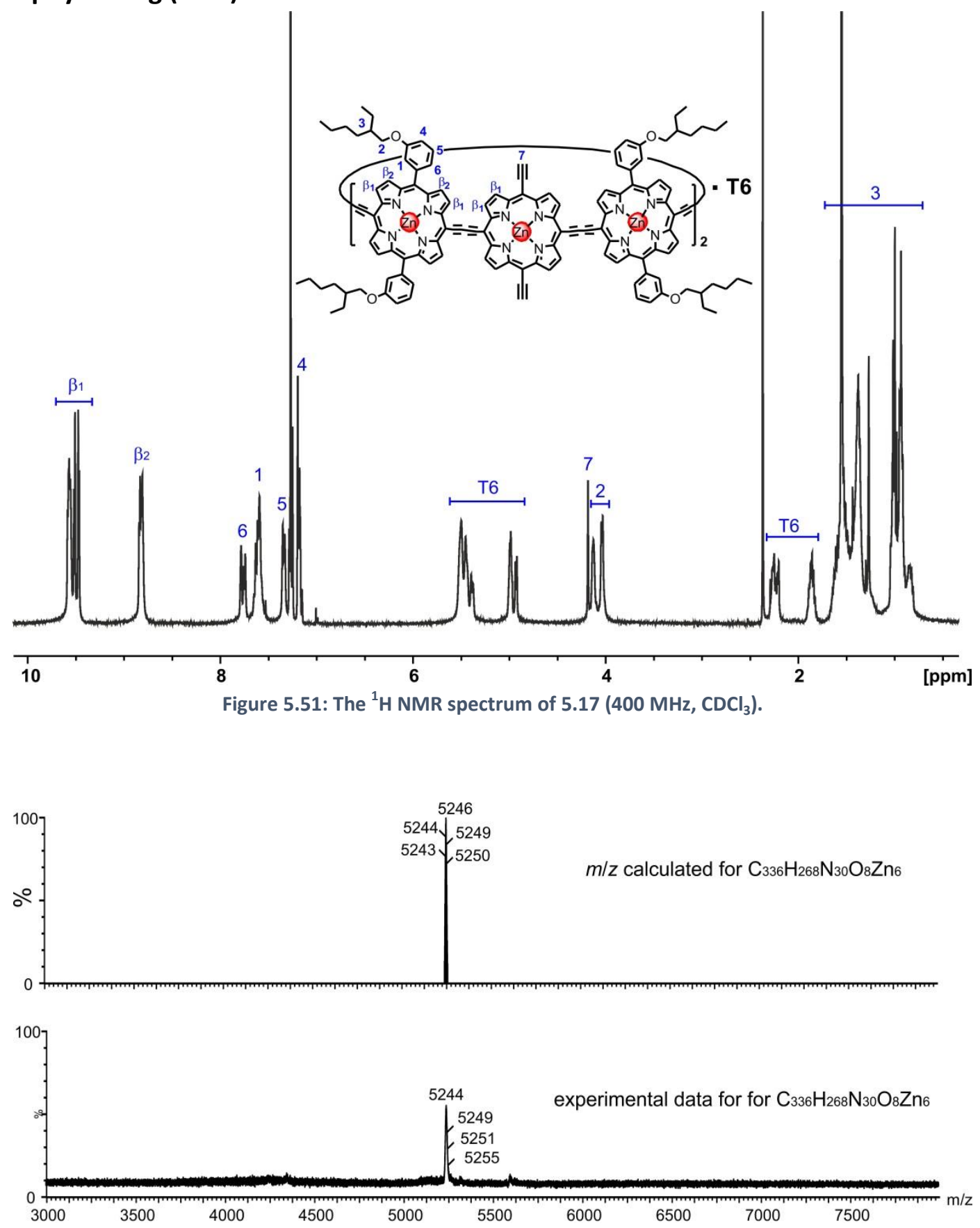

Figure 5.52: The MALDI-MS spectrum of $5.17\left(\mathrm{~m} / \mathrm{z}=5,244\left(\mathrm{C}_{336} \mathrm{H}_{268} \mathrm{~N}_{30} \mathrm{O}_{8} \mathrm{Zn}_{6}, \mathrm{M}^{+}\right.\right.$requires 5,246), matrix: dithranol). 


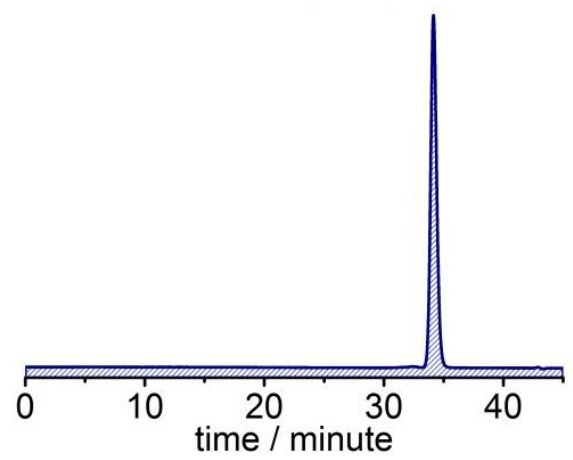

Figure 5.53: Analytical GPC traces (PLGel columns, THF, detection at $800 \mathrm{~nm}$ ) of the pure 5.17.

\section{Ball precursor (5.24)}

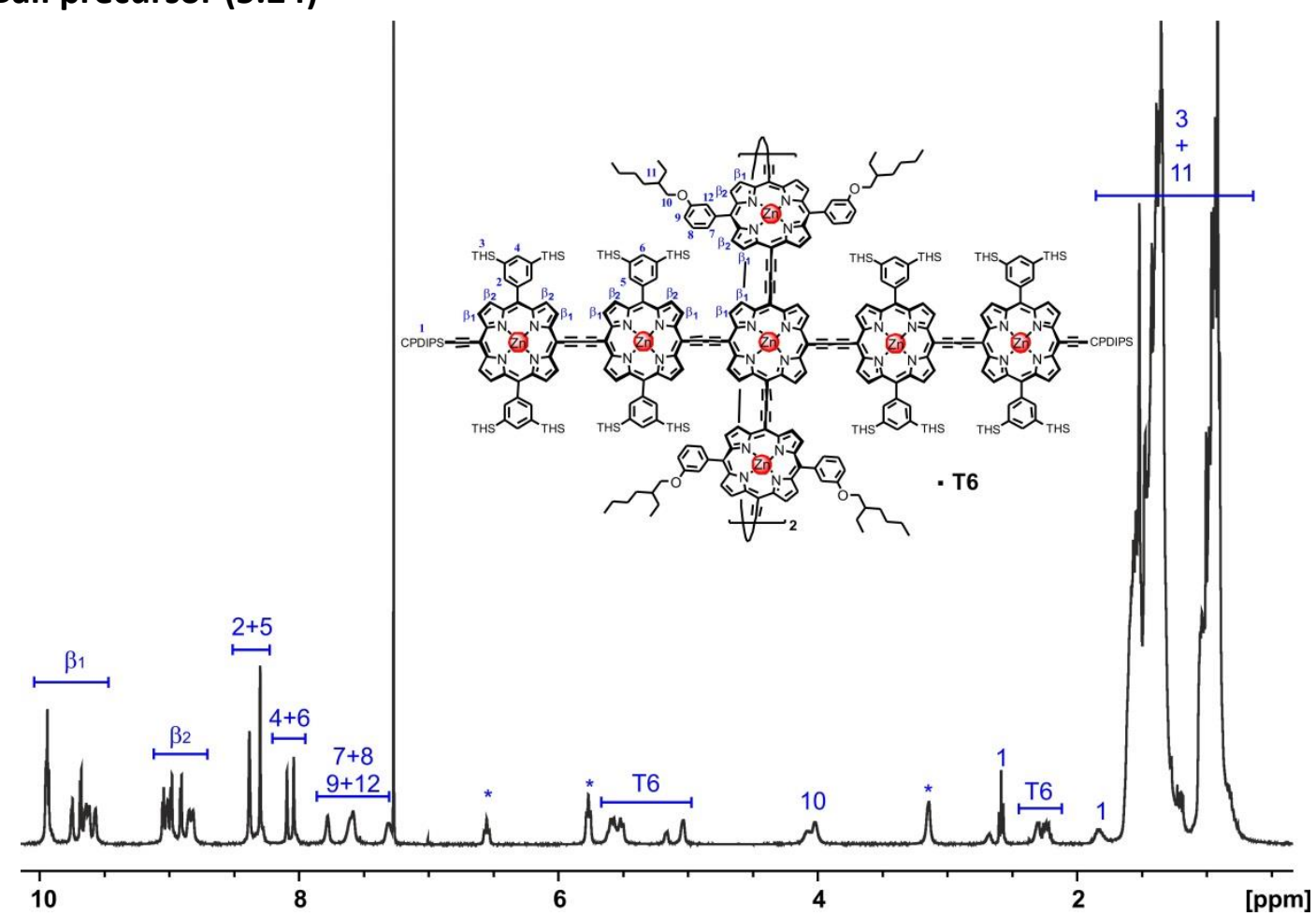

Figure 5.54: The ${ }^{1} \mathrm{H}$ NMR spectrum of $5.24\left(400 \mathrm{MHz}_{2} \mathrm{CDCl}_{3}\right)$. * indicates signals corresponding to pyridine.

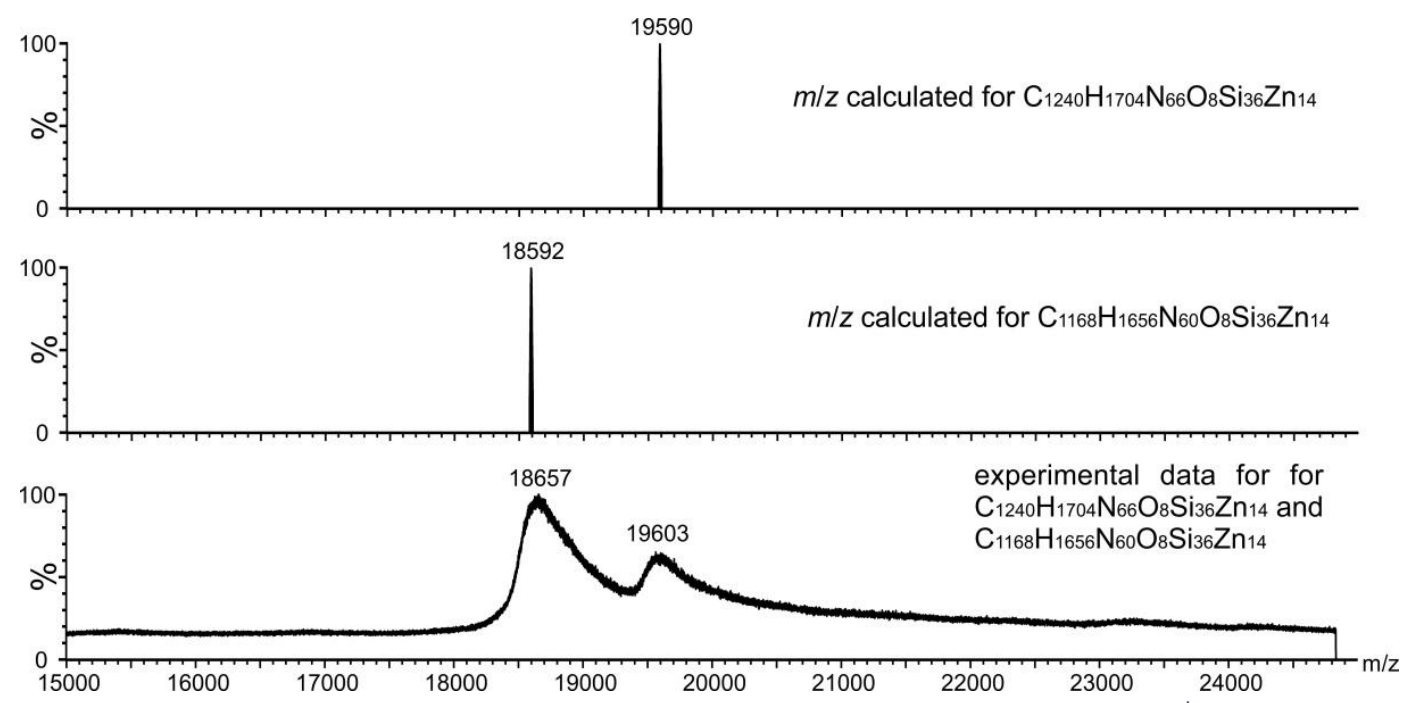

Figure 5.55: The MALDI-MS spectrum of $5.24\left(\mathrm{~m} / z=19,590\left(\mathrm{C}_{1240} \mathrm{H}_{1704} \mathrm{~N}_{66} \mathrm{O}_{8} \mathrm{Si}_{36} \mathrm{Zn}_{14}, \mathrm{M}^{+}\right.\right.$requires 19,590), displacement of T6: $18,592\left(\mathrm{C}_{1168} \mathrm{H}_{1656} \mathrm{~N}_{60} \mathrm{O}_{8} \mathrm{Si}_{36} \mathrm{Zn}_{14}, \mathrm{M}^{+}\right.$requires 18,657), matrix: dithranol). 
Ball Precursor (5.25)

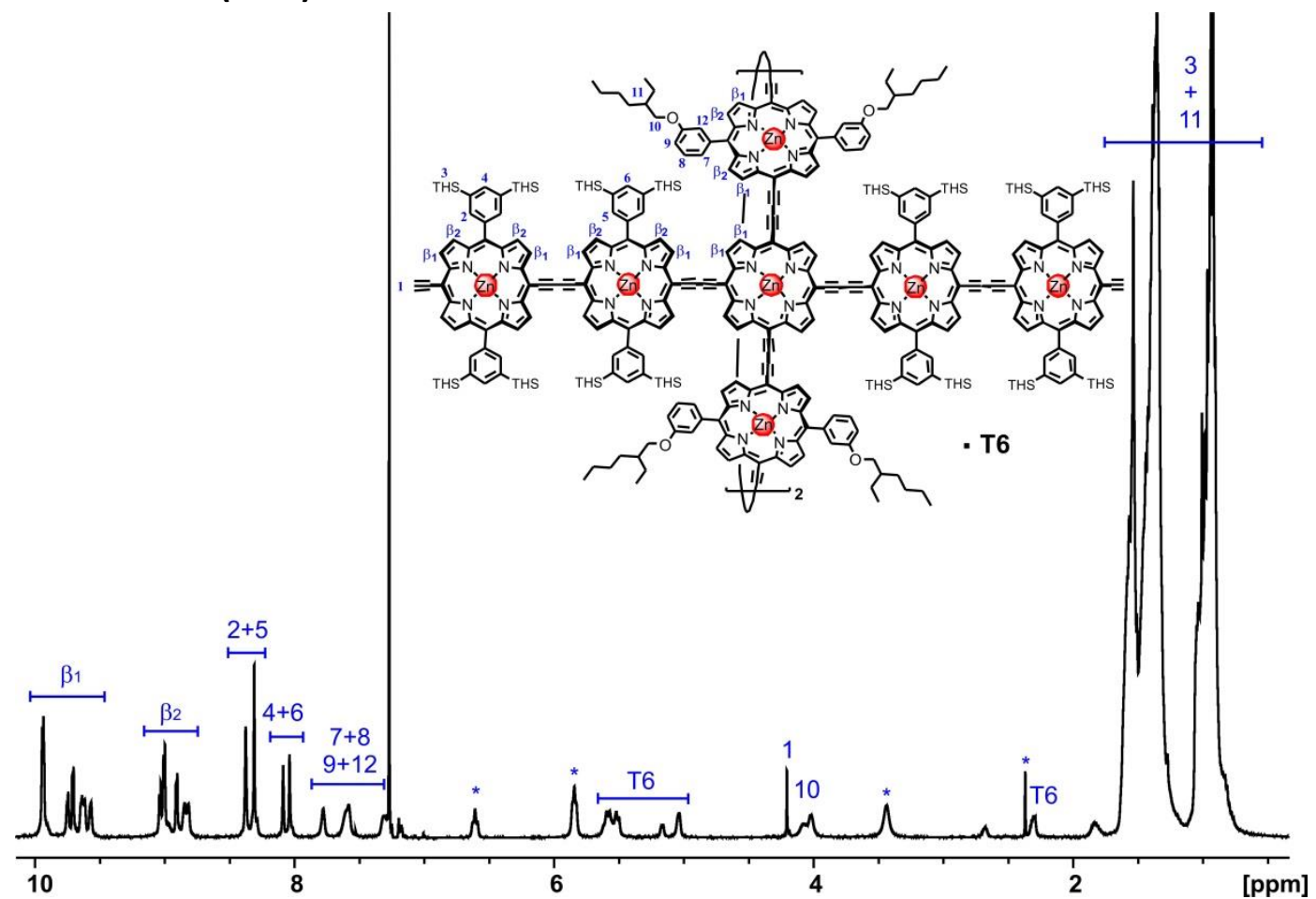

Figure 5.56: The ${ }^{1} \mathrm{H}$ NMR spectrum of $5.25\left(400 \mathrm{MHz}, \mathrm{CDCl}_{3}\right) . *$ indicates signals corresponding to pyridine.
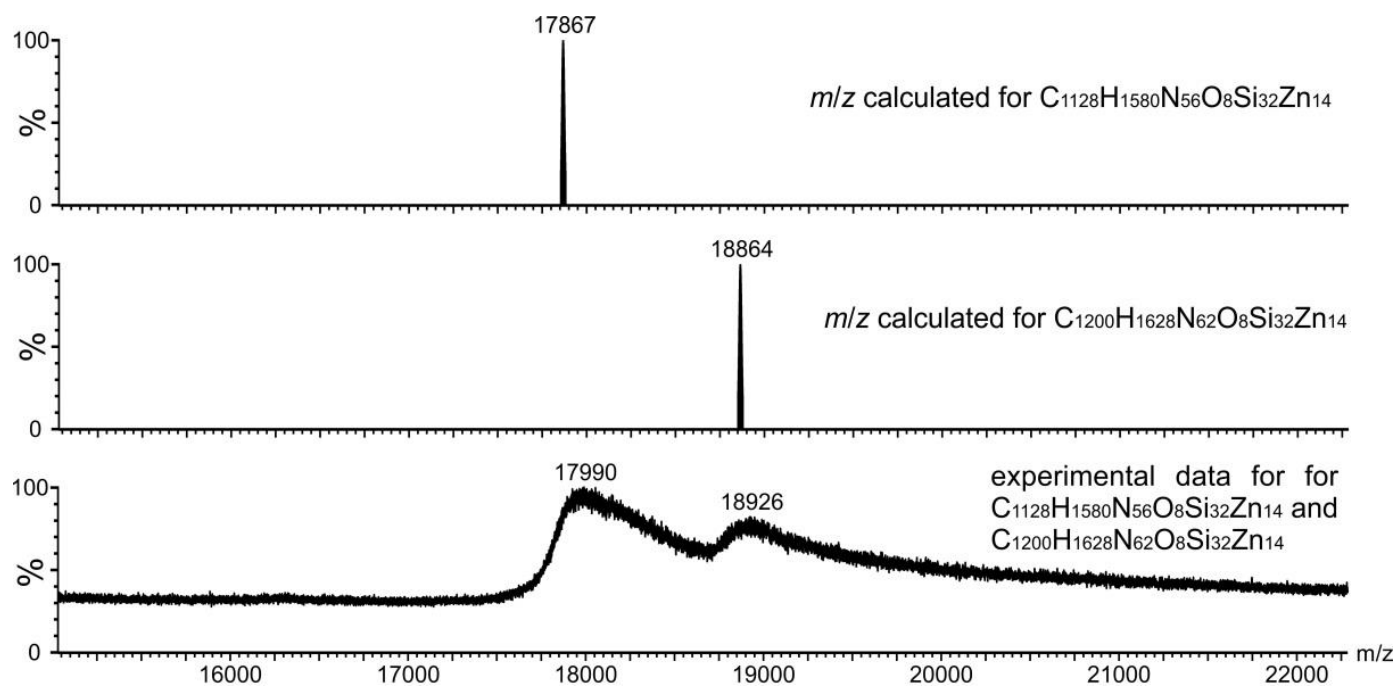

Figure 5.57: The MALDI-MS spectrum of $5.25\left(\mathrm{~m} / \mathrm{z}=18,926\left(\mathrm{C}_{1200} \mathrm{H}_{1628} \mathrm{~N}_{62} \mathrm{O}_{8} \mathrm{Si}_{32} \mathrm{Zn}_{14}, \mathrm{M}^{+}\right.\right.$requires 18,864), displacement of T6: $17,990\left(\mathrm{C}_{1128} \mathrm{H}_{1580} \mathrm{~N}_{56} \mathrm{O}_{8} \mathrm{Si}_{32} \mathrm{Zn}_{14}, \mathrm{M}^{+}\right.$requires 17,867), matrix: dithranol). 


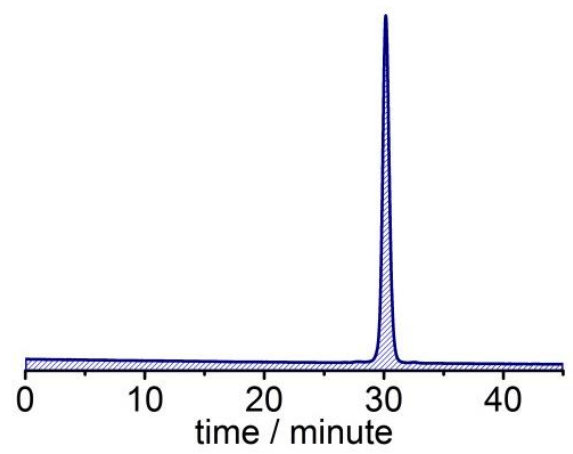

Figure 5.58: Analytical GPC traces (PLGel columns, THF, detection at $800 \mathrm{~nm}$ ) of the pure 5.25.

\section{b-P14·T6·(T4)}

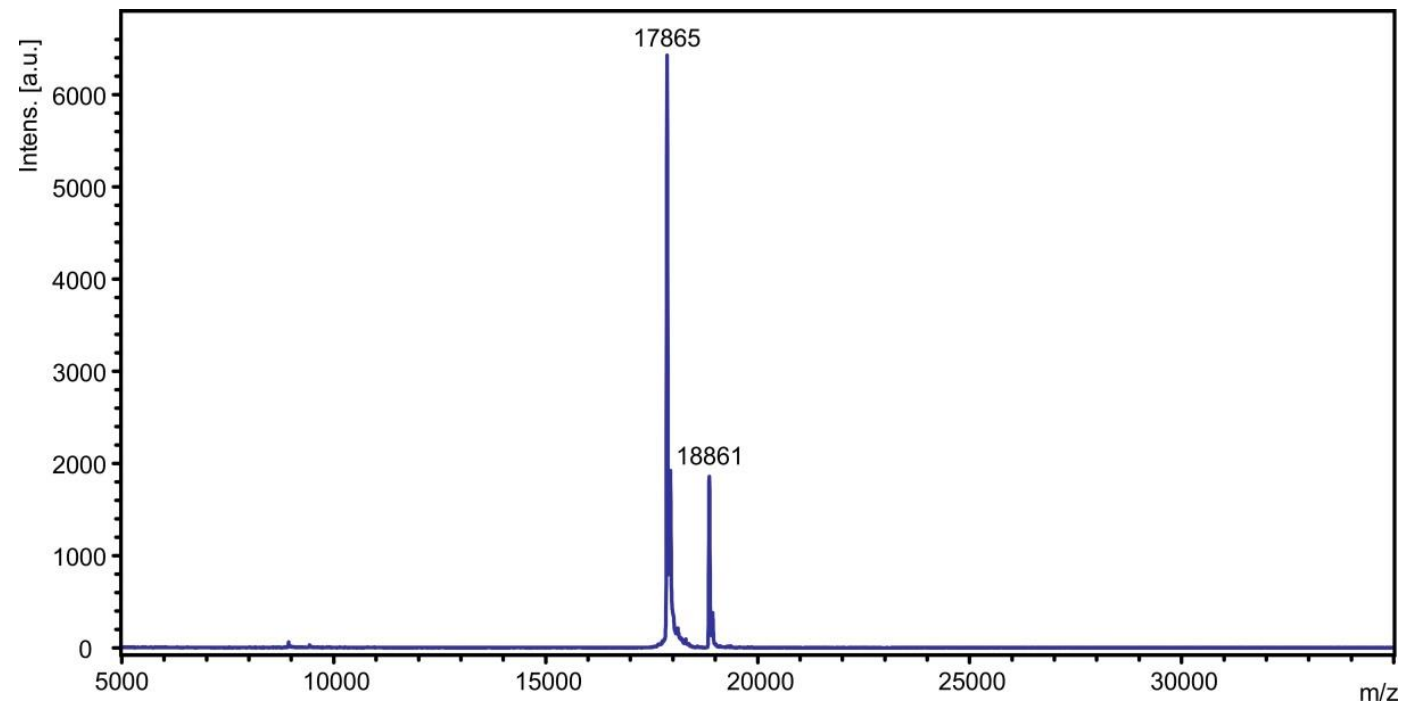

Figure 5.59: MALDI-ToF analysis of $b$-P14·T6•(T4 $)_{2}$ acquired by the EPSRC Mass Spectrometry Centre at Swansea University. DCTB was used as a matrix and samples were dissolved in DCM / 5\% pyridine before mixing with the matrix. Templates are fully/partially displaces upon recording of the spectrum. $b$-P14 $(\mathrm{m} / z=17,865$ $\left(\mathrm{C}_{1128} \mathrm{H}_{1576} \mathrm{~N}_{56} \mathrm{O}_{8} \mathrm{Si}_{32} \mathrm{Zn}_{14}, \mathrm{M}^{+}\right.$requires 17,863), b-P14-T6 (m/z = 18,861 $\left(\mathrm{C}_{1200} \mathrm{H}_{1624} \mathrm{~N}_{62} \mathrm{O}_{8} \mathrm{Si}_{32} \mathrm{Zn}_{14}, \mathrm{M}^{+}\right.$requires $18,861)$.

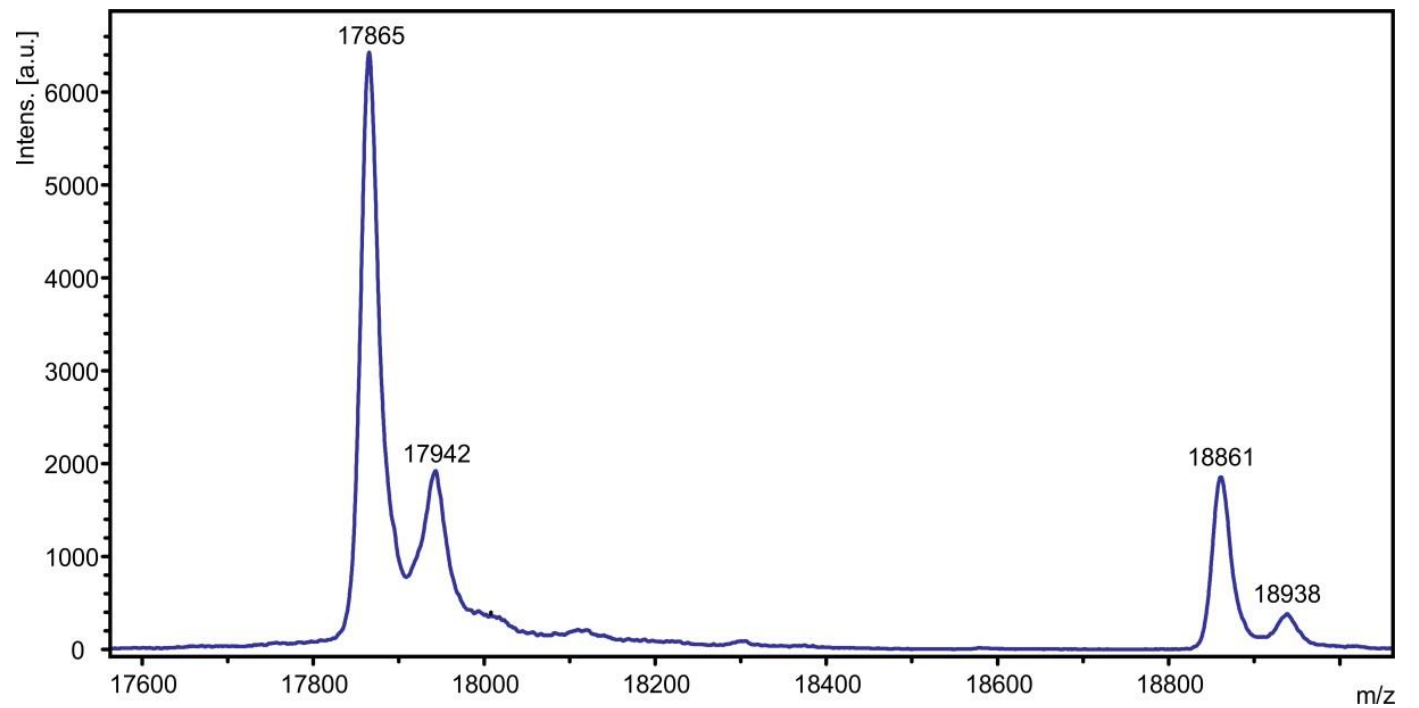

Figure 5.60: A zoom-in of Figure 5.59 reveals shoulders corresponding to complexation with pyridine. b-P14-pyridine $\left(m / z=17,942\left(\mathrm{C}_{113} \mathrm{H}_{1581} \mathrm{~N}_{57} \mathrm{O}_{8} \mathrm{Si}_{32} \mathrm{Zn}_{14}, \mathrm{M}^{+}\right.\right.$requires 17,943), b-P14-T6-pyridine $(\mathrm{m} / \mathrm{z}=18,938$ $\left(\mathrm{C}_{1205} \mathrm{H}_{1629} \mathrm{~N}_{63} \mathrm{O}_{8} \mathrm{Si}_{32} \mathrm{Zn}_{14}, \mathrm{M}^{+}\right.$requires 18,940). 

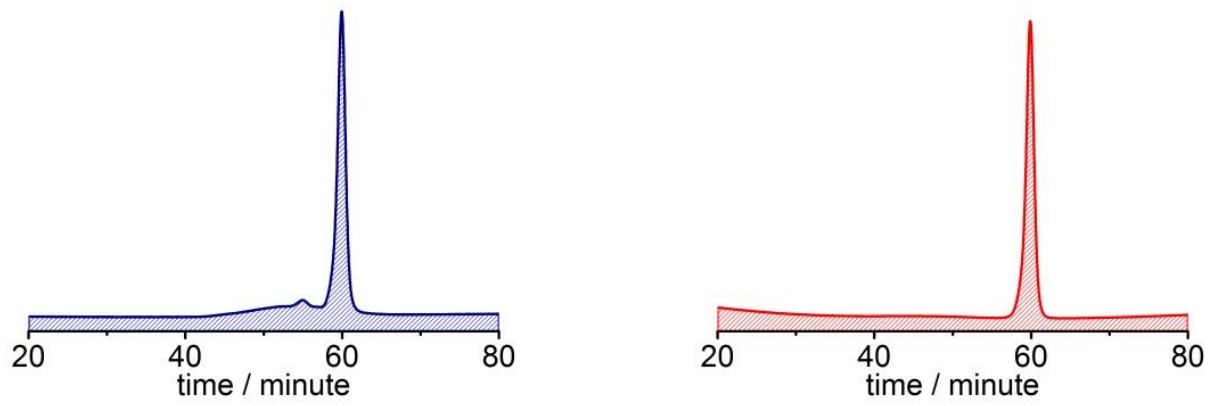

Figure 5.61: Recycling GPC traces (JAIGEL columns, 1st cycle shown, 1\% pyridine in toluene, detection at $800 \mathrm{~nm}$ ) of the crude reaction mixture (left) and the purified $b-\mathrm{P} 14 \cdot \mathrm{T6} \cdot(\mathrm{T4})_{2}$ (right).

\subsubsection{UV-vis-NIR titrations}

\subsubsection{Reference titrations with porphyrin monomers and quinuclidine}

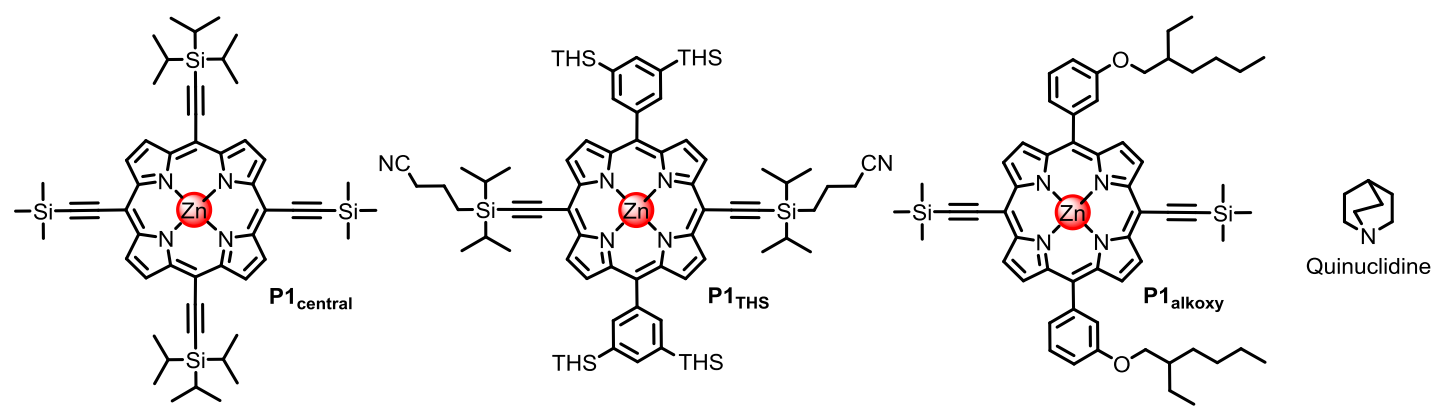

Figure 5.62: Structures of reference porphyrin monomers and the monodentate ligand quinuclidine.

Titrations with porphyrin monomers $\mathbf{P} \mathbf{1}_{\text {central, }} \mathbf{P} \mathbf{1}_{\mathrm{THS}}$ and $\mathbf{P} \mathbf{1}_{\text {alkoxy }}$ with quinuclidine were performed in order to determine reference binding constants ( $K_{\text {central}}, K_{\text {THS }}$, and $\left.K_{\text {alkoxy }}\right)$.

All titrations were performed in toluene at $298 \mathrm{~K}$. Care was taking to keep the porphyrin concentration constant throughout the entire titration by adding porphyrin to the ligand solution before titrations were started. The binding curves were fitted using a 1:1 binding isotherm using the equation:

$$
\frac{A-A_{\text {initial }}}{A_{\infty}-A_{\text {initial }}}=\left(\frac{\left(K_{a}\left([L]+[P]_{0}\right)+1\right)-\sqrt{\left(K_{a}\left([L]+[P]_{0}\right)+1\right)^{2}-4 K_{a}^{2}[P]_{0}[L]}}{2 K_{a}[P]_{0}}\right)
$$

where $A$ is the observed absorption at a specific wavelength or the difference of absorbance between two wavelengths; $A_{\text {initial }}$ is the starting absorption at this wavelength; $A_{\infty}$ is the asymptotic final absorption at this wavelength; $K_{a}$ is the association constant between ligand and porphyrin host; [L] is the concentration of ligand; $[P]_{0}$ is the concentration of porphyrin host. The free variables which were 
adjusted to optimise the fit to the experimental data during the fitting procedure are $A_{\text {initial }}, A_{\infty}$, and $K_{a}$. Fitting analysis was carried out using the Origin software (Figures 5.63-5.68) giving $K_{\text {central }}=(6.45 \pm 0.42) \times 10^{6} \mathrm{M}^{-1}$, $K_{\text {THS }}=(1.30 \pm 0.04) \times 10^{6} \mathrm{M}^{-1}$, and $K_{\text {alkoxy }}=(2.04 \pm 0.09) \times 10^{6} \mathrm{M}^{-1}$.
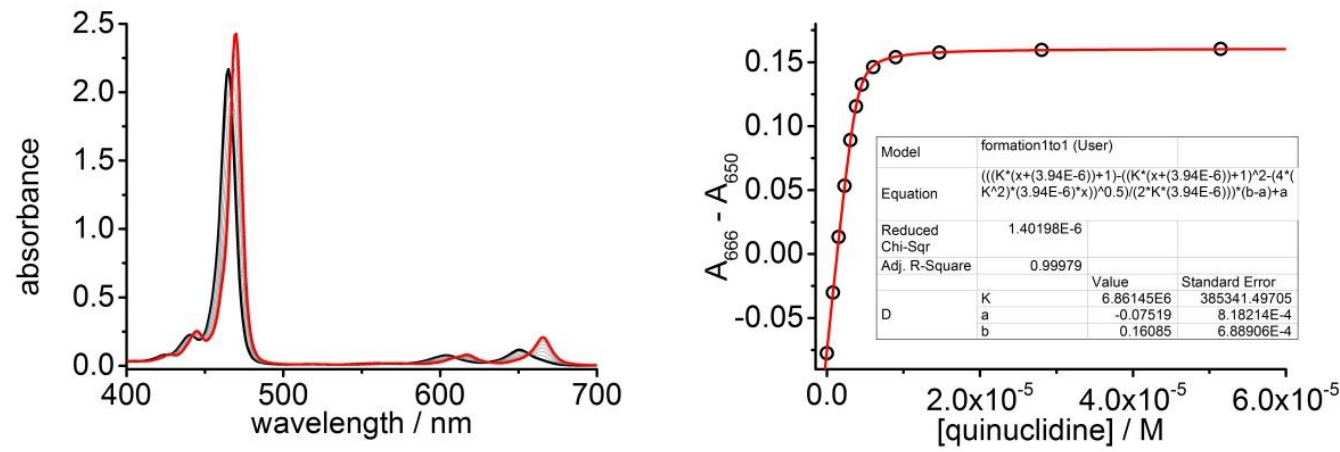

Figure 5.63: UV-vis titration of $\mathrm{P} 1_{\text {central }}$ and quinuclidine, $R^{2}=0.9998$. (Run 1, toluene, $298 \mathrm{~K}$, $\left[P 1_{\text {central }}\right]=3.94 \mu \mathrm{M}, K=6.86 \times 10^{6} \mathrm{M}^{-1}$ ).
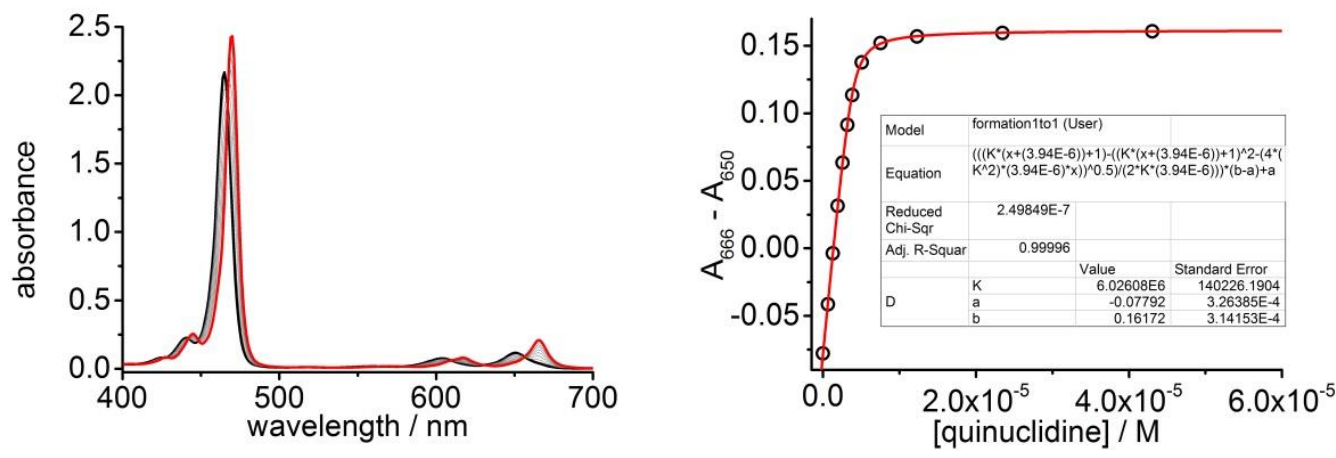

Figure 5.64: UV-vis titration of $P 1_{\text {central }}$ and quinuclidine, $R^{2}=1.0000$. (Run 2, toluene, $298 \mathrm{~K}$, $\left.\left[P 1_{\text {central }}\right]=3.94 \mu \mathrm{M}, K=6.03 \times 10^{6} \mathrm{M}^{-1}\right)$.
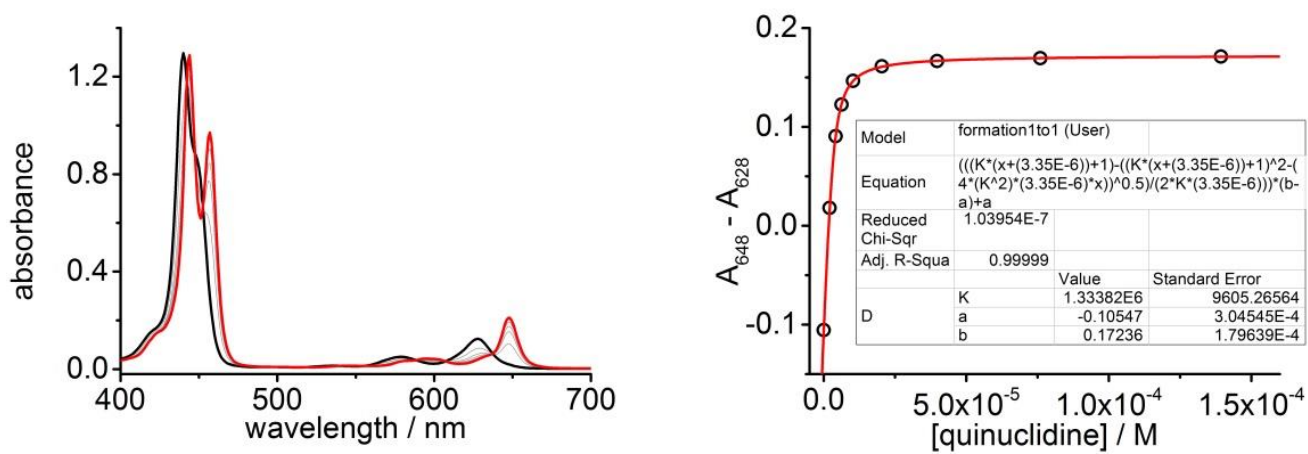

Figure 5.65: UV-vis titration of $\mathrm{P} 1_{\mathrm{THS}}$ and quinuclidine, $R^{2}=1.0000$. (Run 1 , toluene, $298 \mathrm{~K},\left[\mathrm{P} 1_{\mathrm{THS}}\right]=3.35 \mu \mathrm{M}$, $K=1.33 \times 10^{6} \mathrm{M}^{-1}$ ). 

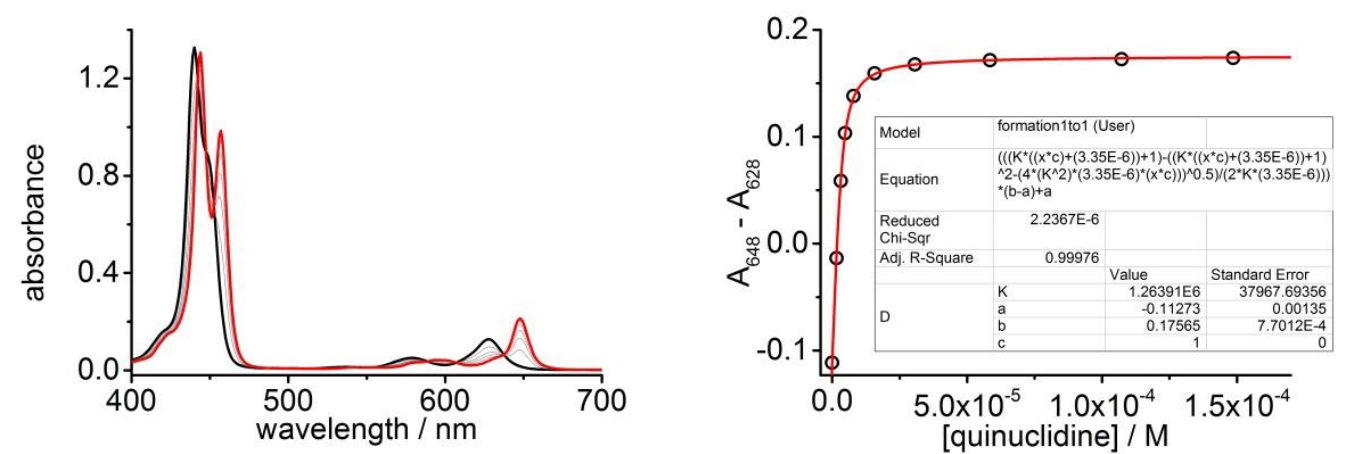

Figure 5.66: UV-vis titration of $\mathrm{P} 1_{\mathrm{THS}}$ and quinuclidine, $R^{2}=0.9998$. (Run 2, toluene, $298 \mathrm{~K},\left[\mathrm{P} 1_{\mathrm{THS}}\right]=3.35 \mu \mathrm{M}$, $K=1.26 \times 10^{6} \mathrm{M}^{-1}$ ).
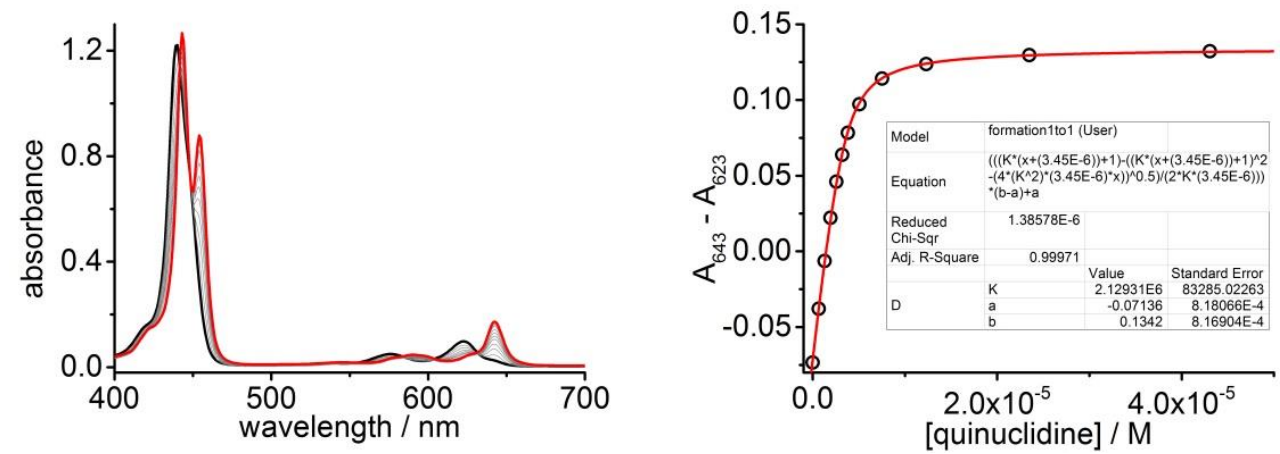

Figure 5.67: UV-vis titration of $\mathrm{P} 1_{\text {alkoxy }}$ and quinuclidine, $R^{2}=0.9997$. (Run 1 , toluene, $298 \mathrm{~K},\left[\mathrm{P} 1_{\text {alkoxy }}\right]=3.45 \mu \mathrm{M}$, $K=2.13 \times 10^{6} \mathrm{M}^{-1}$ ).
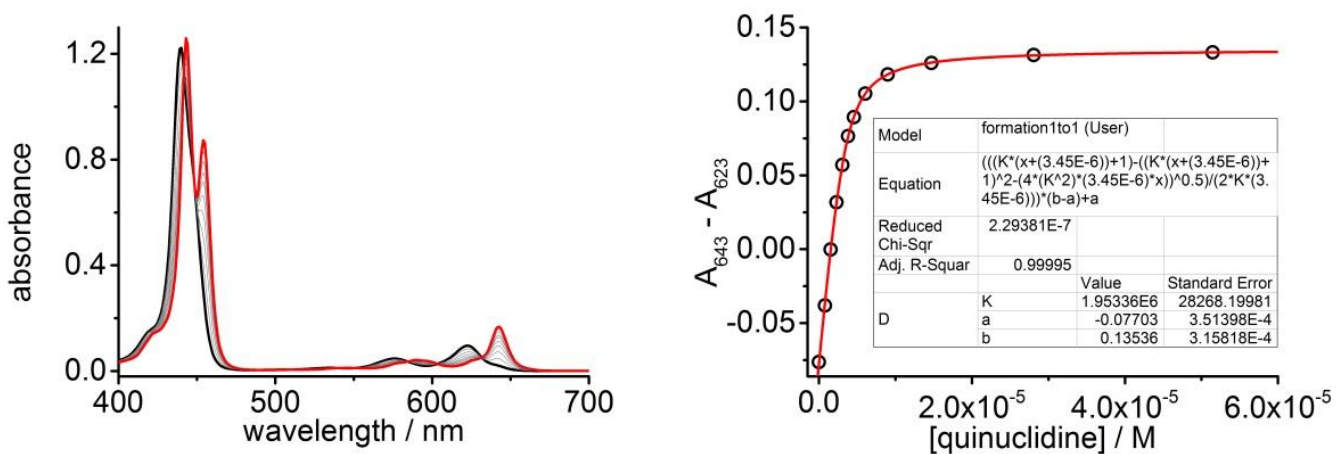

Figure 5.68: UV-vis titration of $\mathrm{P} 1_{\text {alkoxy }}$ and quinuclidine, $R^{2}=1.0000$. (Run 2 , toluene, $298 \mathrm{~K},\left[\mathrm{P} 1_{\text {alkoxy }}\right]=3.45 \mu \mathrm{M}$, $\left.K=1.95 \times 10^{6} \mathrm{M}^{-1}\right)$.

\subsubsection{Denaturation titrations with quinuclidine on $b-\mathrm{P} 14 \cdot \mathrm{T} 6 \cdot(\mathrm{T} 4)_{2}$}

The ball $\boldsymbol{b}$-P14-T6•(T4) $)_{\mathbf{2}}$ was isolated containing the three templates bound strongly within its cavity. In order to derive binding constant for the templates T6 and T4 within the ball, denaturation titrations (break-up titration) were performed with the competing ligand quinuclidine. Using the data from these break-up titrations $\left(K_{\mathrm{dn}}=\right.$ denaturation constant $)$ and the formation constant of the single site binding event of the competing ligand with a zinc porphyrin monomer $\left(K_{\text {central, }}, K_{\mathrm{THS}}, K_{\mathrm{alkoxy}}=\right.$ association constant for quinuclidine to its respective porphyrin monomer $\left(K_{\mathrm{Q}}\right)$ ) 
allows us to derive the formation binding constant $\left(K_{\mathrm{f}}\right)$ between the ball and the templates using the following equation:

$$
K_{\mathrm{f}}=\frac{K_{\mathrm{Q}}^{N}}{K_{\mathrm{dn}}}
$$

in which $N=$ number of zinc porphyrin binding sites, via the thermodynamic cycle shown in Figure 5.69. The displacement of the templates T4 and T6 occurs at distinctly different quinuclidine concentrations, which makes it is valid to split the thermodynamic cycle in two individual cycles.

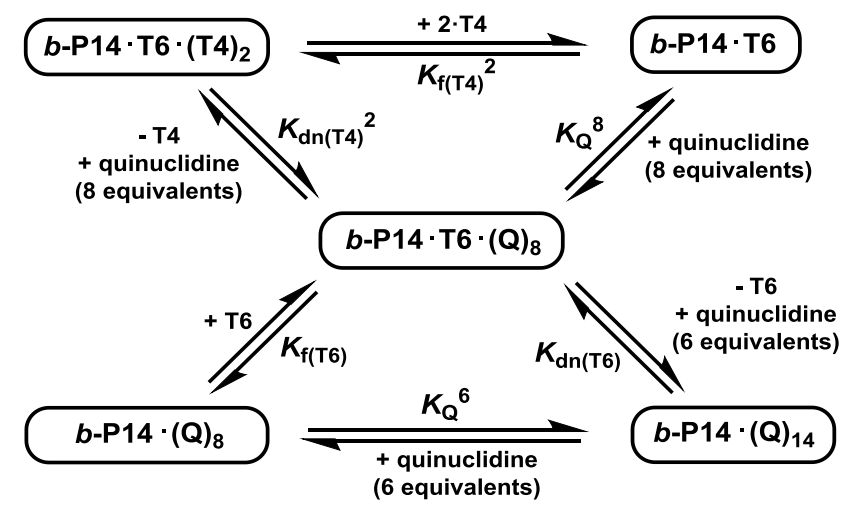

Figure 5.69: Individual thermodynamic cycles relating the formation constant of the template complex $\left(K_{\mathrm{f}}\right)$ to the denaturation constant $\left(K_{\mathrm{dn}}\right)$ and binding constant of each porphyrin unit for quinuclidine $\left(K_{\mathrm{Q}}\right)$.

As a result, the association constants for T4 and T6 within the $\boldsymbol{b}$-P14 can be calculated using the following equations:

$$
K_{\mathrm{f}(\mathrm{T} 4)}=\sqrt{\frac{K_{\mathrm{Q}}^{8}}{K_{\mathrm{dn}(\mathrm{T} 4)}^{2}}} \quad \text { and } \quad K_{\mathrm{f}(\mathrm{T} 6)}=\frac{K_{\mathrm{Q}}^{6}}{K_{\mathrm{dn}(\mathrm{T} 6)}}
$$

Denaturation titrations were performed in toluene at $298 \mathrm{~K}$. All denaturation

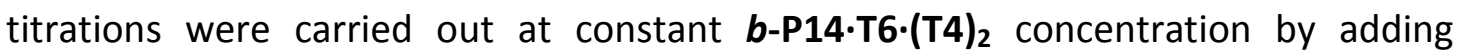
complex to the ligand (quinuclidine) stock solution before titrations started.

Data were fitted to the $\mathrm{N}$-dentate denaturation binding isotherm described in the following equation:

$$
\frac{A-A_{\text {initial }}}{A_{\infty}-A_{\text {initial }}}=\left(\frac{-K_{d n}[L]^{N}+\sqrt{K_{d n}^{2}[L]^{2 N}+4 K_{d n}[L]^{N}[P]_{0}}}{2[P]_{0}}\right)
$$


where $A$ is the observed absorption at a specific wavelength or difference of absorption between two wavelengths; $A_{\text {initial }}$ is the starting absorption at a specific wavelength or difference between absorption in two wavelengths; $A_{\infty}$ is the asymptotic absorption at a specific wavelength or difference of absorption in two wavelengths; $K_{\mathrm{dn}}$ is the denaturation constant between ligand and porphyrin oligomer complex on titrating with quinuclidine, $[L]$ is the concentration of quinuclidine; $[\mathrm{P}]_{0}$ is the concentration of porphyrin oligomer complex, $N$ is the number of binding sites in the complex. The titration curves and fittings are shown below.

Table 5.1: Results from UV-vis-NIR titrations in Figure 5.70-5.75.

\begin{tabular}{|c|c|c|c|c|c|}
\hline complex & $K_{\mathrm{dn}}$ & $K_{\mathrm{f}}\left(\mathrm{M}^{-1}\right)$ & $K_{\sigma}$ & $K_{\text {chem }}\left(M^{-1}\right)$ & $\log K_{\text {chem }}\left(M^{-1}\right)$ \\
\hline $\begin{array}{c}b \text {-P14·T6·(T4) } \\
\leftrightarrow b-\mathrm{P} 14 \cdot \mathrm{T} 6\end{array}$ & $\begin{array}{c}(1.57 \pm 0.07) \\
\times 10^{2} \mathrm{M}^{-3}\end{array}$ & $\begin{array}{c}(1.82 \pm 0.24) \\
\times 10^{22}\end{array}$ & 32 & $\begin{array}{c}(5.68 \pm 0.74) \\
\times 10^{20}\end{array}$ & $20.8 \pm 0.1$ \\
\hline $\begin{array}{l}b-\mathrm{P} 14 \cdot \mathrm{T} 6 \\
\leftrightarrow b-\mathrm{P} 14\end{array}$ & $\begin{array}{c}(6.84 \pm 0.20) \\
\times 10^{-2} \mathrm{M}^{-5}\end{array}$ & $\begin{array}{c}(1.05 \pm 0.23) \\
\times 10^{40}\end{array}$ & 192 & $\begin{array}{c}(5.47 \pm 1.21) \\
\times 10^{37}\end{array}$ & $37.7 \pm 0.1$ \\
\hline
\end{tabular}
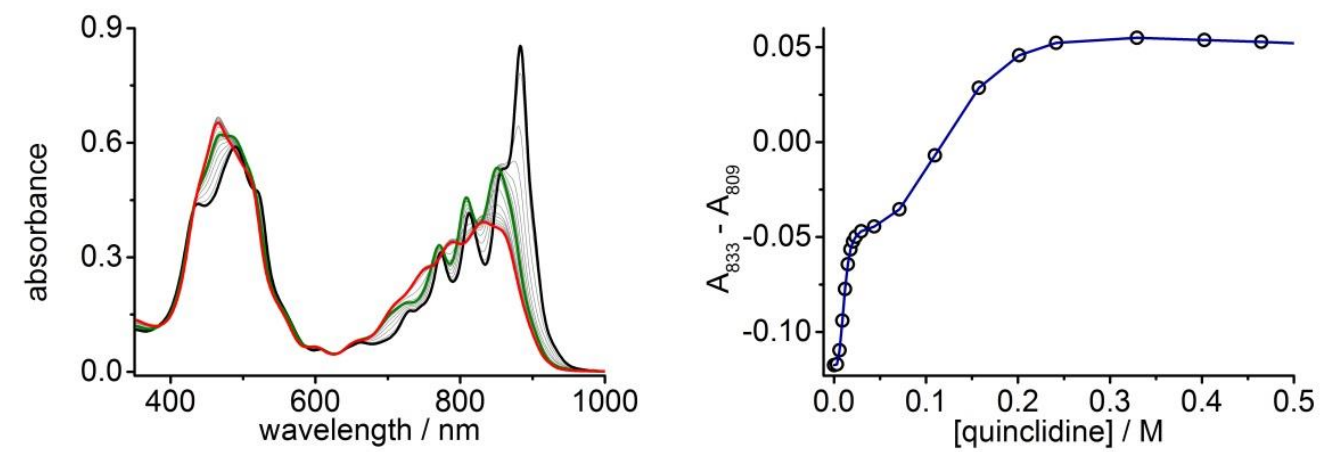

Figure 5.70: UV-vis-NIR titration of quinuclidine and b-P14.T6.(T4) $)_{2}$ (Run 1 , toluene, 298K, $\left.\left[b-\mathrm{P} 14 \cdot \mathrm{T6} \cdot(\mathrm{T} 4)_{2}\right]=0.583 \mu \mathrm{M}\right)$. Two stages of the titration can be observed: black to green shows the removal of the T4 templates; green to red shows the removal of T6. On the right the experimental data (black circles) and a plotted line (blue line) are shown.
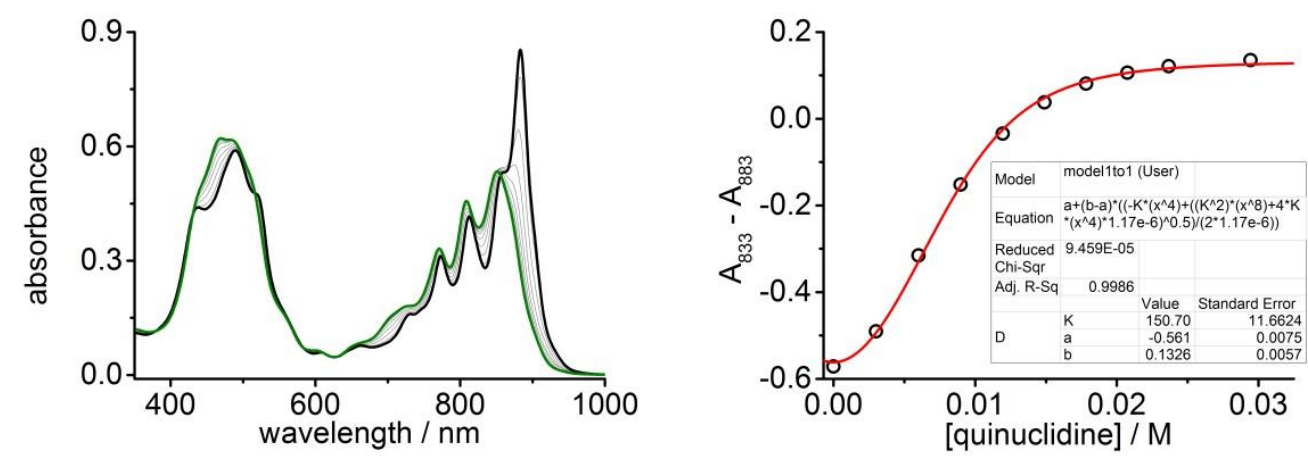

Figure 5.71: First part of the UV-vis-NIR titration of quinuclidine and $b$-P14.T6·(T4 $)_{2}$ illustrating the selective removal of the T4 templates. $R^{2}=0.9986$. (Run 1, toluene, 298K, $\left[b-\mathrm{P} 14 \cdot \mathrm{T6} \cdot(\mathrm{T} 4)_{2}\right]=0.583 \mu \mathrm{M}$, $\left.K_{\mathrm{dn}}=1.51 \times 10^{2} \mathrm{M}^{-3}\right)$. The effective concentration of the host is doubled in the calculation of the binding isotherm due to the presence of two T4 templates per ball molecule. 

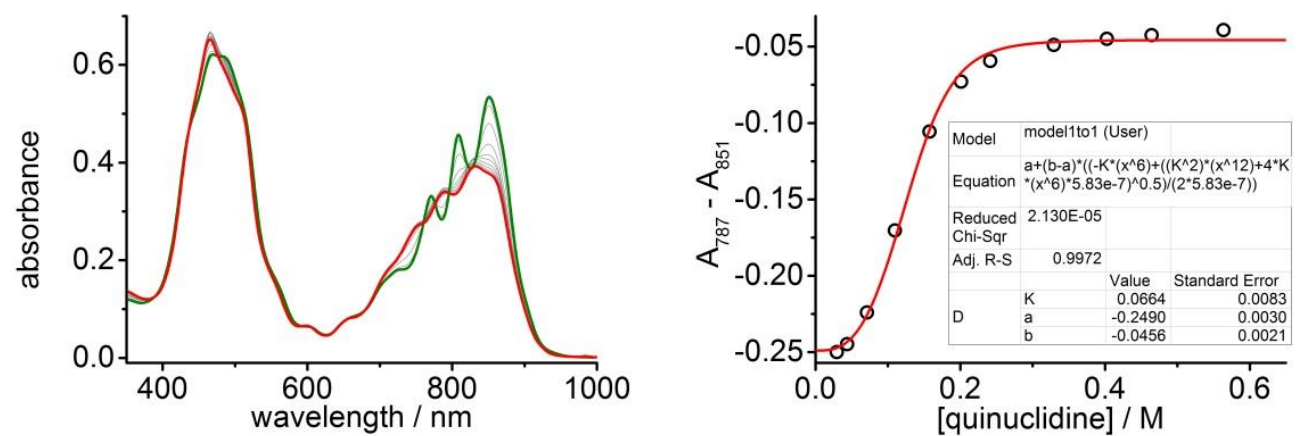

Figure 5.72: Second part of the UV-vis-NIR titration of quinuclidine and $b$-P14.T6-(T4), illustrating the removal of the T6 template. $R^{2}=0.9972$. (Run 1, toluene, 298K, $\left[b-\mathrm{P} 14 \cdot \mathrm{T} 6 \cdot(\mathrm{T} 4)_{2}\right]=0.583 \mu \mathrm{M}, K_{\mathrm{dn}}=6.64 \times 10^{-2} \mathrm{M}^{-3}$ ).
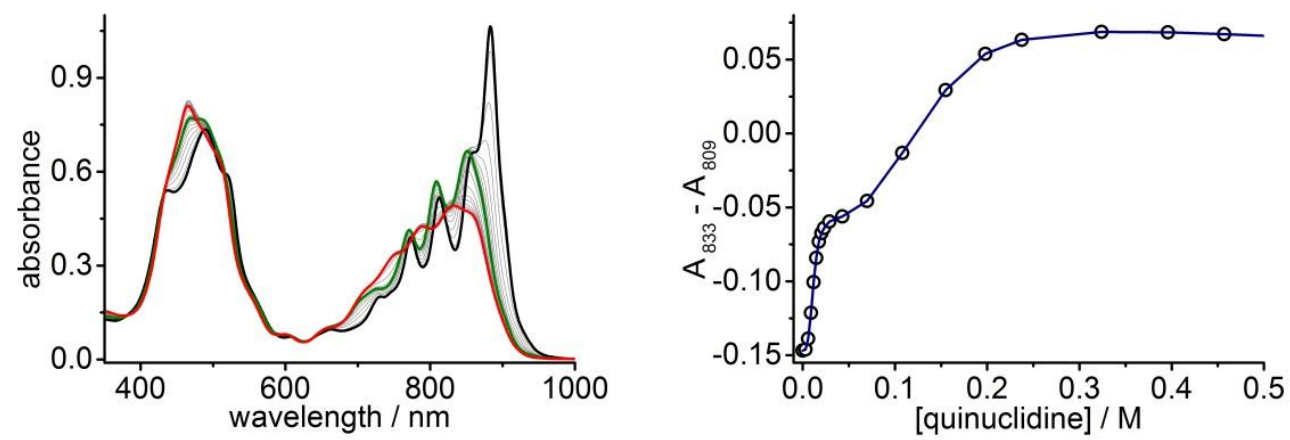

Figure 5.73: UV-vis-NIR titration of quinuclidine and b-P14·T6.(T4) $)_{2}$ (Run 2, toluene, 298K, [b-P14-T6·(T4 $\left.\left.)_{2}\right]=0.728 \mu \mathrm{M}\right)$. Two stages of the titration can be observed: black to green shows the removal of the T4 templates; green to red shows the removal of T6. On the right the experimental data (black circles) and a plotted line (blue line) are shown.
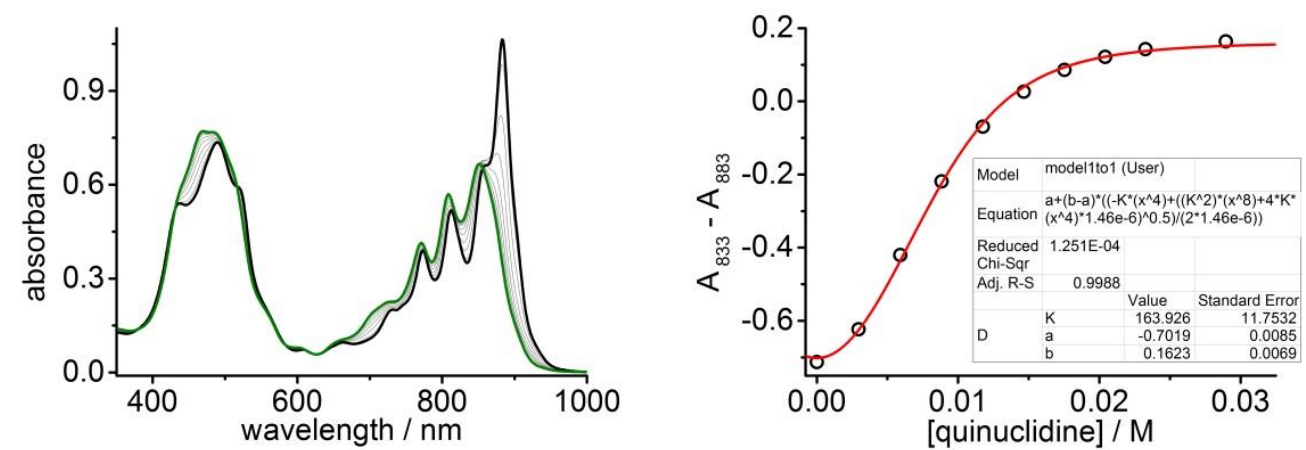

Figure 5.74: First part of the UV-vis-NIR titration of quinuclidine and $b$-P14·T6· $(\mathrm{T} 4)_{2}$ illustrating the selective removal of the T4 templates. $R^{2}=0.9988$. (Run 2, toluene, 298K, $\left[b-\mathrm{P} 14 \cdot \mathrm{T} 6 \cdot(\mathrm{T} 4)_{2}\right]=0.728 \mu \mathrm{M}$, $\left.K_{\mathrm{dn}}=1.64 \times 10^{2} \mathrm{M}^{-3}\right)$. The effective concentration of the host is doubled in the calculation of the binding isotherm due to the presence of two T4 templates per ball molecule. 

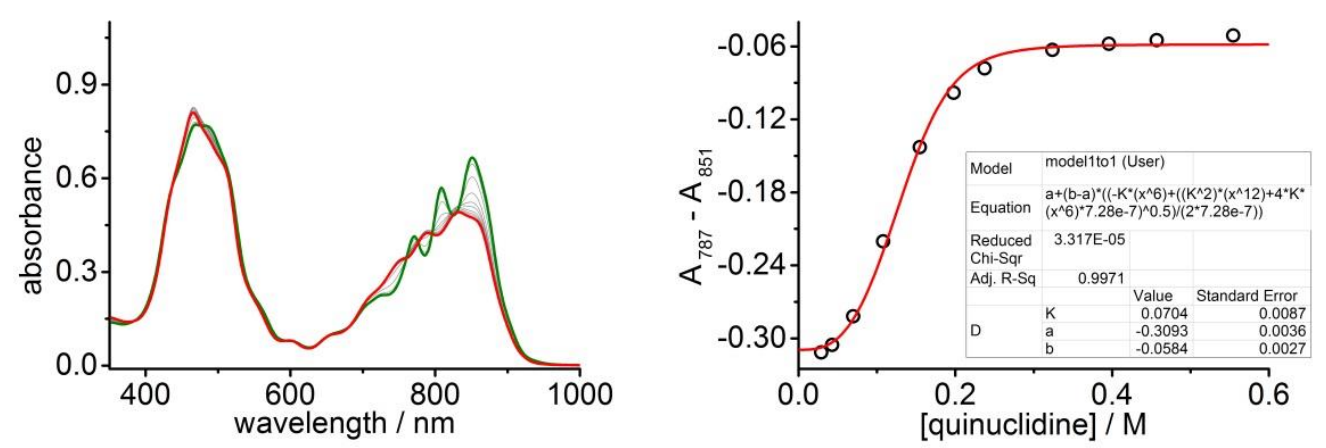

Figure 5.75: Second part of the UV-vis-NIR titration of quinuclidine and $b$-P14·T6•(T4) ${ }_{2}$ illustrating the removal of the T6 template. $R^{2}=0.9971$. (Run 2, toluene, 298K, $\left[b-\mathrm{P} 14 \cdot \mathrm{T} 6 \cdot(\mathrm{T} 4)_{2}\right]=0.728 \mu \mathrm{M}, K_{\mathrm{dn}}=7.04 \times 10^{-2} \mathrm{M}^{-3}$ ).

\subsubsection{Calculation of statistical factors}

To understand the stability constants of different complexes, it is useful to factor out statistical contributions. A measured equilibrium constant $K_{\text {eq }}$ can be factorised into its statistical component $K_{\sigma}$ and its statistically corrected value $K_{\text {chem }}$ according to the following equation:

$$
\begin{gathered}
w \mathrm{~A}+x \mathrm{~B} \rightleftharpoons y \mathrm{C}+z \mathrm{D} \\
K_{\mathrm{eq}}=\frac{Q_{\mathrm{C}}^{y} Q_{\mathrm{D}}^{z}}{Q_{\mathrm{A}}^{w} Q_{\mathrm{C}}^{x}}=\frac{Q_{\mathrm{A}}^{w} Q_{\mathrm{B}}^{x}}{Q_{\mathrm{C}}^{y} Q_{\mathrm{D}}^{z}} \frac{Q_{\mathrm{C}}^{\prime y} Q_{\mathrm{D}}^{\prime z}}{Q_{\mathrm{A}}^{\prime} Q_{\mathrm{C}}^{\prime x}}=K_{\sigma} K_{\text {chem }}
\end{gathered}
$$

where for each species $i, Q_{i}$ is the partition coefficient, $Q^{\prime}{ }_{i}$ is the statistically corrected partition coefficient, and $\sigma_{i}$ is the symmetry number. ${ }^{23,27,136}$

Values of $K_{\sigma}$ were calculated using Benson's symmetry number method. ${ }^{137,138}$ The symmetry number $(\sigma)$ of each species is the product of its external symmetry number, $\sigma_{\text {ext }}$ (calculated from the point group of the molecule) and its internal symmetry number, $\sigma_{\text {int }}$ (calculated from the number of degenerate internal rotors). The values of $\sigma$ for all the complexes involved are shown in Table 5.2. The external symmetry number is defined as the number of different but indistinguishable atomic arrangements that can be obtained by rotating a given molecule as a whole as a rigid object. The internal symmetry number is defined as the number of different but indistinguishable atomic arrangements that can be obtained by internal rotations around single bonds. 
Table 5.2: Internal, external and total symmetry numbers for each component.*

\begin{tabular}{|c|c|c|c|c|}
\hline component & point group & $\sigma_{\text {int }}$ & $\sigma_{\text {ext }}$ & $\sigma$ \\
\hline quinuclidine & $\mathrm{C}_{3 \mathrm{v}}$ & 1 & 3 & 3 \\
\hline T4 & $\mathrm{C}_{2 \mathrm{v}}$ & 1 & 2 & 2 \\
\hline T6 & $\mathrm{D}_{6 \mathrm{~h}}$ & 1 & 12 & 12 \\
\hline $\mathbf{P 1}_{\text {central }}$ & $\mathrm{D}_{2 \mathrm{~h}}$ & 1 & 4 & 4 \\
\hline $\mathbf{P 1}_{\text {THS }}$ & $\mathrm{D}_{2 \mathrm{~h}}$ & 1 & 4 & 4 \\
\hline $\mathbf{P 1}_{\text {alkoxy }}$ & $\mathrm{D}_{2 \mathrm{~h}}$ & 1 & 4 & 4 \\
\hline $\boldsymbol{b}$-P14-T6·(T4) & $\mathrm{D}_{2 \mathrm{~h}}$ & 1 & 4 & 4 \\
\hline $\boldsymbol{b}-\mathbf{P 1 4}$ 146 & $\mathrm{D}_{2 \mathrm{~h}}$ & 256 & 4 & 1,024 \\
\hline $\boldsymbol{b}$-P14 & $\mathrm{D}_{2 \mathrm{~h}}$ & 16 & 4 & 64 \\
\hline
\end{tabular}

[* Note that when counting internal rotations and calculating $\sigma_{i n t}$, we do not include rotors which are unaffected by the binding process, such as the para-phenylene links in T5 because if a rotor is unaffected by the binding process it has no influence on $K_{\sigma}$.]

The binding isotherm used to calculate the denaturation constants for T4 and T6 described in Section 5.5.4.2, assume the break-up of a 1:1 porphyrin oligomer template complexes. Since the displacement of the ligands T4 and T6 occurs at vastly different quinuclidine concentrations, we can fit the data for both processes independently to obtain both $K_{\mathrm{dn}(\mathbf{T} 4)}$ and $K_{\mathrm{dn}(\mathbf{T 6})}$. In order to factor out statistical contributions we have calculated the statistical factors for all the species involved in the process (Figure 5.76). 


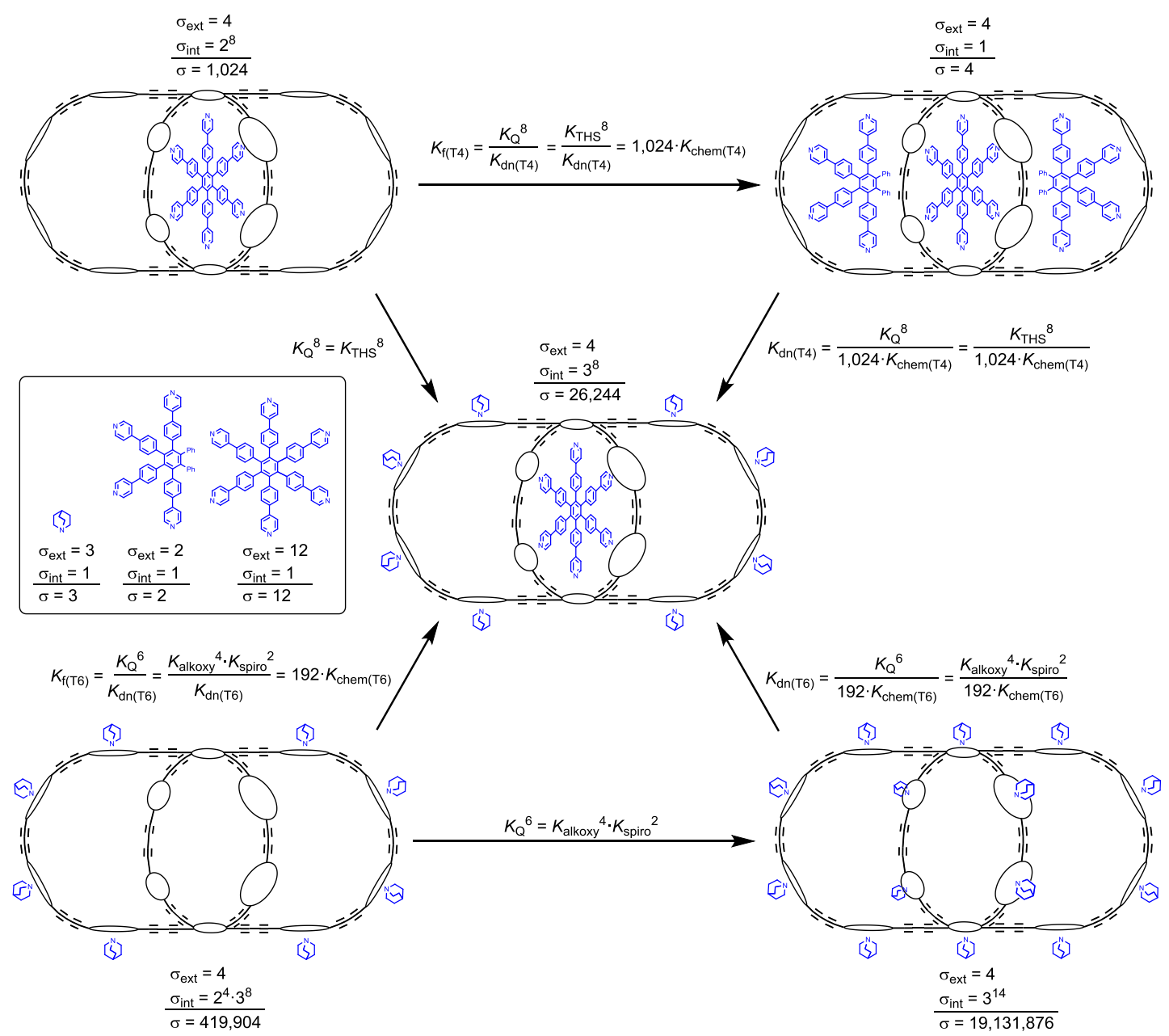

Figure 5.76: Statistical factors involved in the formation and denaturation (quinuclidine) of the $b$-P14.T6-(T4) complex.

Since we assume the denaturation of a 1:1 porphyrin oligomer template complex, we need to assume that both halves of the 10-porphyrin nanoring containing the T4 templates act independently. This observation drastically changes the statistical factors involved in the process and subsequently changes the equations used to determine the formation constant for the T4 template (Figure 5.77). 


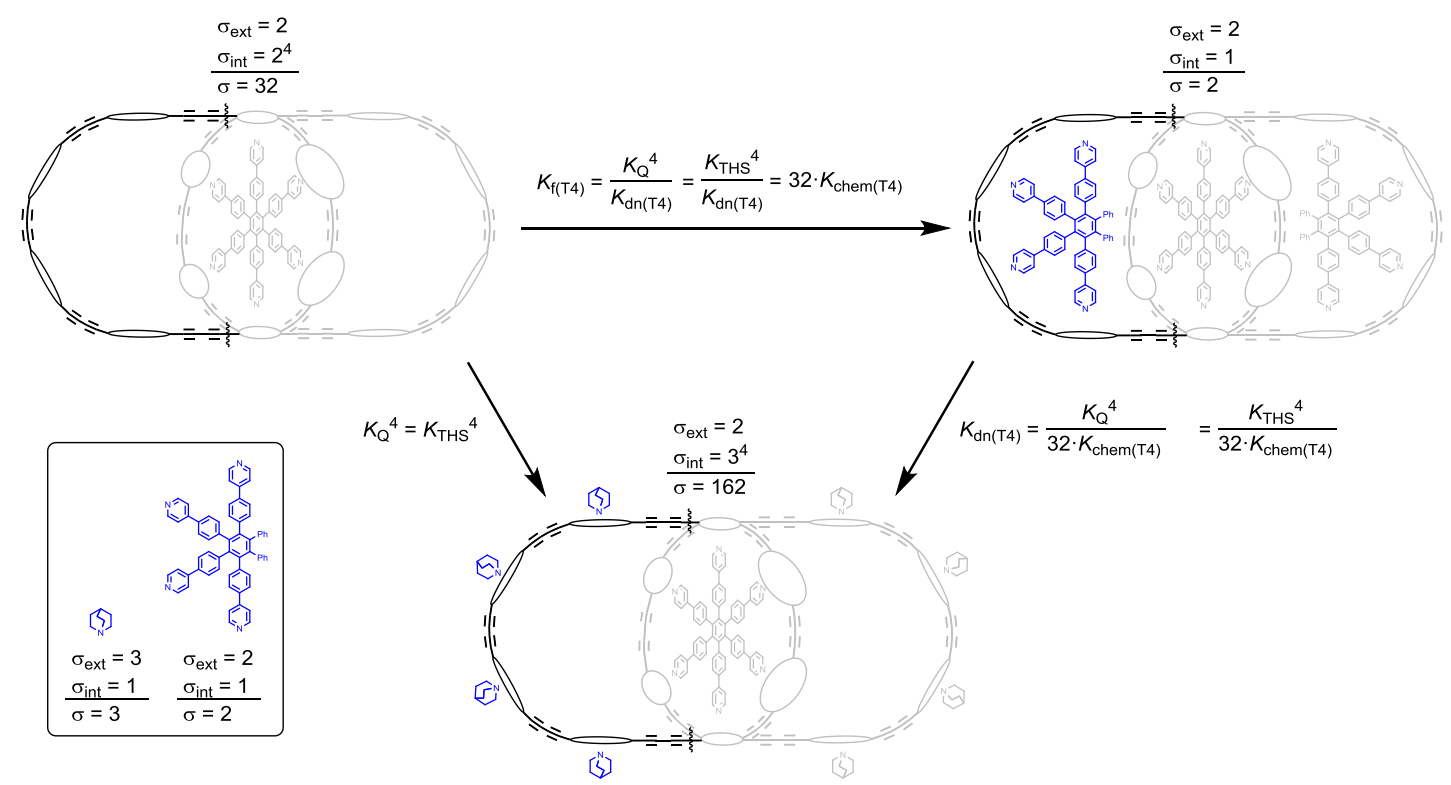

Figure 5.77: Statistical factors involved in the removal of the T4 templates from the $b$-P14.T6·(T4 $)_{2}$ complex with quinuclidine.

When calculating the statistical factors involved with the removal of the T6 template, $\sigma_{\text {int }}$ is drastically reduced in size by not including the rotors which are unaffected by the binding process, such as the rotations of the eight quinuclidine units bound to the 10-porphyrin nanoring (Figure 5.78). Since these rotors are identical in all three complexes, they have no influence on $K_{\sigma}$.
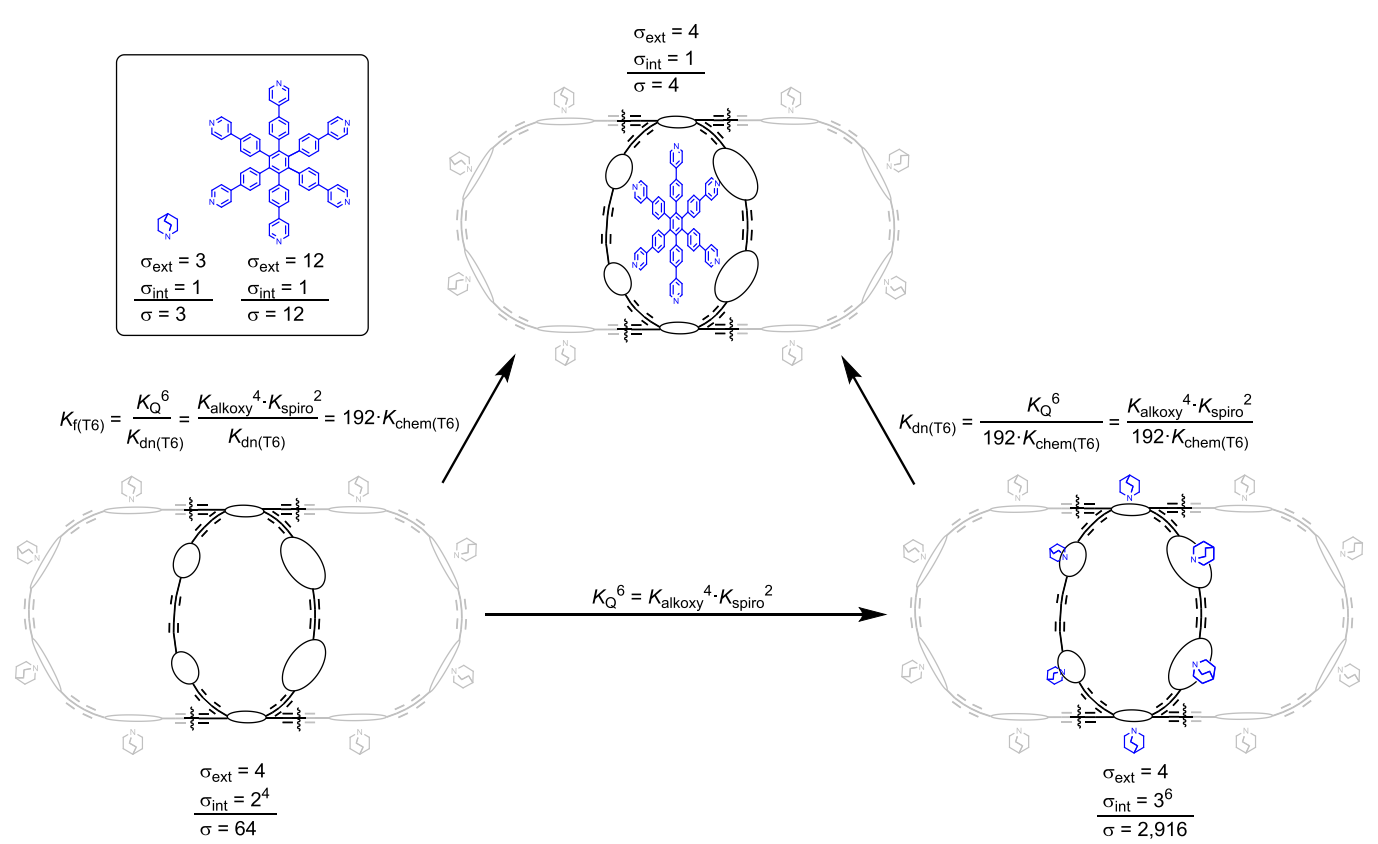

Figure 5.78: Statistical factors involved in the removal of the T6 template from the $b$-P14-T6 complex with quinuclidine. 


\subsubsection{Computational chemistry}

DFT calculations were carried out by Renee Haver. Starting coordinates for $\boldsymbol{b}$-P14 were derived by combining fragments from the previously optimised $\boldsymbol{c}$-P6 porphyrin nanoring. ${ }^{165}$ All DFT calculations were carried out using Gaussian 09/D.01. ${ }^{200}$ The computational model of $\boldsymbol{b}$-P14 does not include template molecules, and the aryl side-groups are truncated to $-\mathrm{H}$.

The optimised geometry of $\boldsymbol{b}$-P14 was calculated at the B3LYP ${ }^{201}$ level of theory employing the $6-31 \mathrm{G}(\mathrm{d})$ basis set $^{202-205}$, with the addition of Grimme's empirical dispersion correction (GD3). ${ }^{206}$ The initial symmetry was $C_{2 v}$ but during optimisation, symmetry was lost $\left(C_{1}\right)$. Tight convergence criteria were used for the geometry optimisation. The minimum nature of the optimised geometry was confirmed by the absence of imaginary vibrational frequencies. The computed energy of $\boldsymbol{b}$-P14 is -41164.8070698 a.u. (B3LYP/6-31G* GD3).

\subsubsection{Photophysical measurements}

\subsubsection{Absorption and emission spectra}

Measurements were carried out on $\boldsymbol{b}$-P14.T6 $(\mathbf{T 4})_{2}$ and $\boldsymbol{b}$-P14, and were referenced to three control samples to compare photophysical properties of the ball complexes to their constituent 6- and 10-porphyrin rings (Figure 5.79).
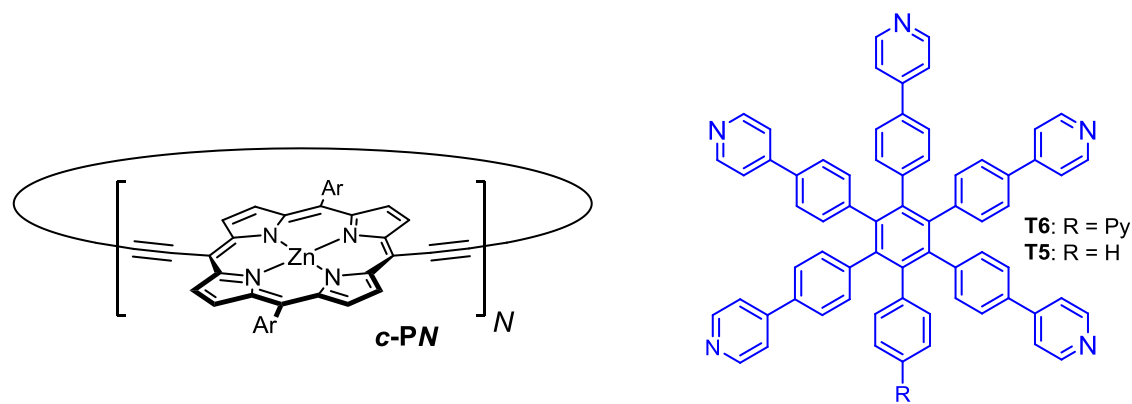

Figure 5.79: Structure of reference samples and their templates. ${ }^{88,136}$ ( $\mathrm{Ar}=3,5$-bis-trihexylsilyl-phenyl).

Figure 5.80 shows normalised absorption and emission spectra at room temperature

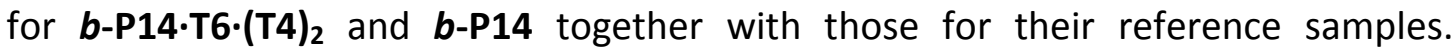
Analysis of the peak ratios of the three prominent features in the Q-band in Figure 5.80a shows that the mixture $\boldsymbol{c}-\mathbf{P 6} \cdot \mathbf{T 6}+\boldsymbol{c}-\mathbf{P} \mathbf{1 0} \cdot(\mathbf{T 5})_{\mathbf{2}}$ has a narrow and intense peak at the long-wavelength end due to contribution of $c-\mathbf{P 1 0} \cdot(\mathbf{T 5})_{2}$, whereas features attributed to c-P6-T6 dominate at the short-wavelength end. In comparison, 
porphyrin ball $\boldsymbol{b}$-P14-T6·(T4) $)_{2}$ demonstrates a sharpening and red shift in the Q-band features, but still adopts a similar peak ratio as the mixture $c-\mathbf{P 6} \cdot \mathbf{T 6}+c-\mathbf{P 1 0}(\mathrm{T5})_{2}$. This observation suggests that with longer excitation wavelength in the Q-band region, absorption into a $\boldsymbol{c}$-P10·(T5) ${ }_{2}$-like state increasingly dominates over absorption into the $\boldsymbol{c}$-P6-T6-like state. For $\boldsymbol{b}$-P14 the Q-band of its reference samples are broadly overlapping and lack distinct features. By examining the PL spectra in Figure 5.80, it becomes apparent that emission features from both reference mixtures are largely dominated by $c-\mathrm{P} 10 / \mathrm{c}-\mathrm{P} \mathbf{1 0} \cdot(\mathrm{T} 5)_{2}$ components, most likely because PL quantum yield of those components is almost an order of magnitude larger than those of $\mathbf{c - P 6 / c - P 6 \cdot T 6 .}{ }^{89}$

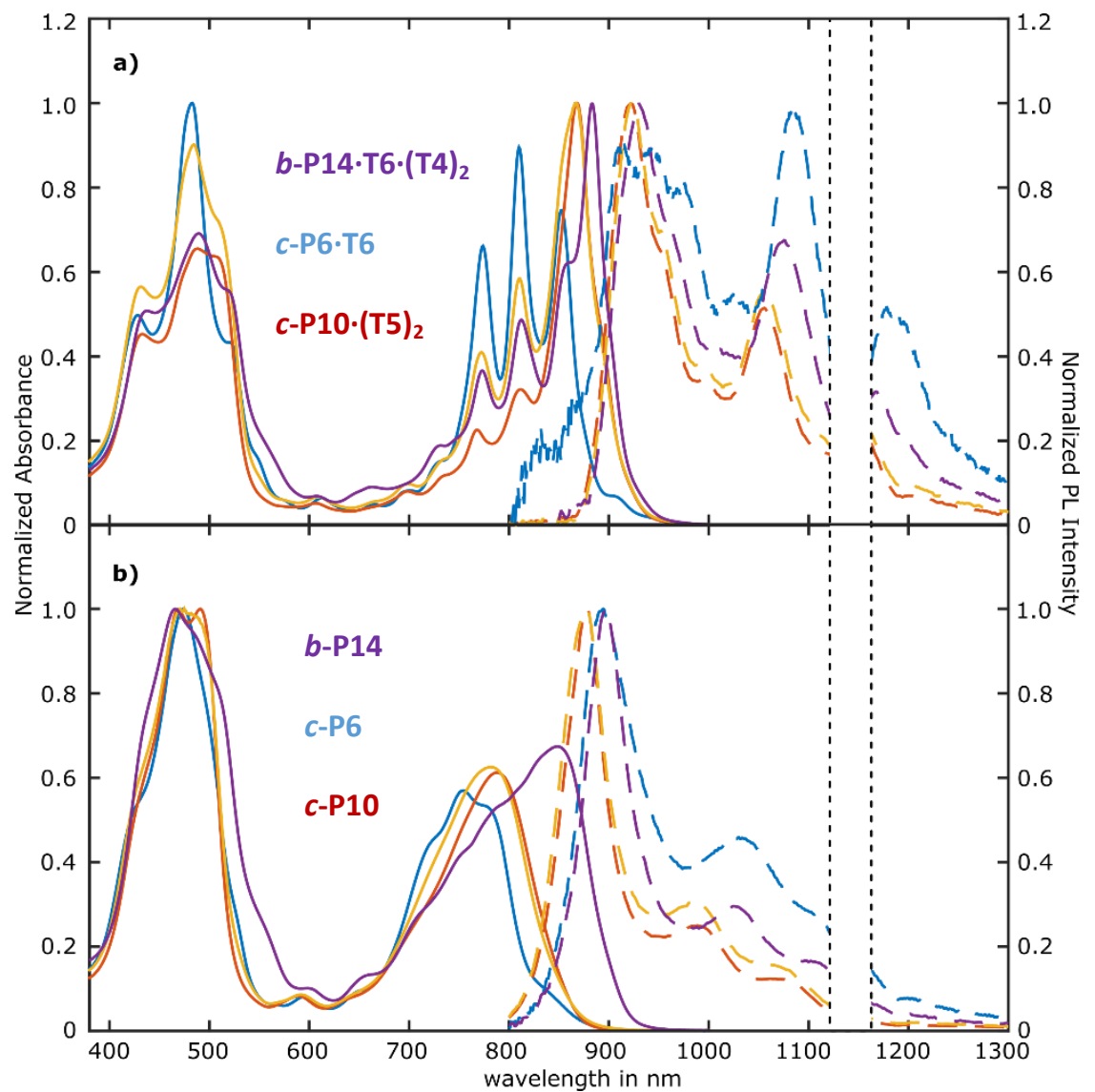

Figure 5.80 (a) Normalised absorbance (solid line) and PL intensity (dashed line) of $b$-P14.T6-(T4), (purple), c-P6-T6 (blue), c-P10·(T5) 2 (red) and control mixture c-P6.T6 + c-P10·(T5) $)_{2}$ (yellow); (b) normalised absorbance (solid line) and PL intensity (dashed line) of $b$-P14 (purple), c-P6 (blue), c-P10 (red) and control mixture $c$-P6 + c-P10 (yellow). Data points between dotted vertical lines at 1120-1160 nm in the PL intensity spectrum are excluded because of solvent absorption effects.

\subsubsection{PL anisotropy}

The photoluminescence $(\mathrm{PL})$ upconversion technique was engaged to investigate the $\mathrm{PL}$ and anisotropy dynamics of sample solutions held in quartz cuvettes. An 
excitation pulse was generated by a mode-locked Ti:Sapphire laser with pulse duration of $100 \mathrm{fs}$ and a repetition rate of $80 \mathrm{MHz}$. PL is collected and optically gated in a beta-barium-borate ( $\mathrm{BBO}$ ) crystal by a vertically polarised time-delayed gate beam. The upconverted signal, which consists of sum-frequency photons from the gate pulse and the vertical component of the $\mathrm{PL}$, was collected, dispersed in a monochromator and detected using a nitrogen-cooled CCD. Using a combination of a half-wave plate and a Glan-Thompson polariser, the polarisation of the excitation pulse was varied and the PL intensity dynamics were recorded separately for components polarised parallel $\left(I_{\|}\right)$and perpendicular $\left(I_{\perp}\right)$ to the excitation pulse polarisation as shown in Figure 5.81a-c. The PL anisotropy is defined using:

$$
\gamma=\left(I_{\|}-I_{\perp}\right) /\left(I_{\|}+2 I_{\perp}\right)
$$

and calculated from the measured components. The full-width-half-maximum of the instrumental response function was measured to be $270 \mathrm{fs}$, which gives the time-resolution limit of the system. The detection wavelength was chosen to be 900 $\mathrm{nm}$ for all the samples and kept constant for all excitation wavelengths.

For $\boldsymbol{b}$-P14-T6-(T4) 2 , the PL anisotropy is found to be constant at $\gamma=0.02 \pm 0.01$ within at least the first 20 ps after excitation as shown in Figure $5.81 \mathrm{~d}$. $\boldsymbol{b}$-P14-T6 and $\boldsymbol{b}$-P14, however, exhibit a fast initial drop in anisotropy within the first 4 ps from $\gamma=0.1$ to $\gamma=0.03 \pm 0.01$ which remains constant thereafter within the observation window of the first 20 ps after excitation (Figure 5.81e-f). In a solution of randomly oriented molecules, an anisotropy value of $\gamma=0.1$ is expected for complete depolarisation of an excited state transition dipole moment within a 2D plane, e.g. for a cyclic porphyrin ring structure with an exciton delocalised over the entire ring. ${ }^{189,207}$ When the emission transition dipole moment is oriented perpendicular to polarisation direction of the absorbing state, $\gamma=-0.2$ is expected. ${ }^{54,207}$ Because the porphyrin ball possesses two ring planes with perpendicular orientations, contributions from these two effects may yield an anisotropy value close to zero. The almost complete polarisation memory loss therefore suggests that an excitation can access any segment on the ball complex. 
The fast initial drop resembles the anisotropy decay dynamics of large porphyrin nanorings with $n>20$ porphyrin units that has previously been observed. ${ }^{189}$ The initial anisotropy of 0.1 suggests that upon excitation, an exciton is delocalised over a full ring and both absorption and emission transition dipoles are polarised in this ring plane. After ultrafast relaxation, the exciton localises and migrates rapidly around the entire porphyrin ball complex. Contributions from emission components polarised in both planes thus result in the low anisotropy value close to zero. Due to the lack of templates, an exciton may be initially more localised in $\boldsymbol{b}$-P14 and $\boldsymbol{b}$-P14-T6, resulting in the initial observed anisotropy drop, whereas $b$-P14·T6-(T4) 2 reaches the low anisotropy within the time resolution of the system.

To obtain a representative value for the PL anisotropy, ten PL intensity values were independently recorded at 5 ps delay after excitation for each polarisation direction, averaged and the equations above was applied. This process was repeated several times for a range of excitation wavelengths between $760 \mathrm{~nm}$ and $880 \mathrm{~nm}$. The anisotropy values were averaged and presented in Table 5.3. The reference sample c-P6-T6 and the mixture c-P6 + c-P10 exhibit anisotropy values of about 0.1 , as expected for a ring system sustaining delocalised excitations. ${ }^{189}$ c-P10.(T5) ${ }_{2}$ demonstrates a somewhat lower anisotropy, because it adapts a twisted geometry with out-of-plane bending which is not present in the rigid $\boldsymbol{b}$-P14.T6.(T4) $)_{2}$; this anisotropy-lowering effect has been extensively investigated and reported in the literature. $^{195}$

Table 5.3: PL anisotropy of porphyrin balls and reference samples 5 ps after excitation.

\begin{tabular}{|c|c|}
\hline Sample & PL Anisotropy \\
\hline $\boldsymbol{b}$-P14·T6·(T4) ${ }_{2}$ & $0.02 \pm 0.01$ \\
\hline $\boldsymbol{c}-\mathrm{P6}$-T6 & $0.10 \pm 0.01$ \\
\hline $\boldsymbol{c}$-P10·(T5) 2 & $0.05 \pm 0.02$ \\
\hline $\boldsymbol{b}-\mathrm{P} 14$ & $0.03 \pm 0.01$ \\
\hline $\boldsymbol{c}-\mathrm{P6}+\boldsymbol{c}-\mathrm{P} 10$ & $0.11 \pm 0.01$ \\
\hline $\boldsymbol{b}-\mathrm{P} 14 \cdot \mathrm{T6}$ & $0.03 \pm 0.01$ \\
\hline
\end{tabular}




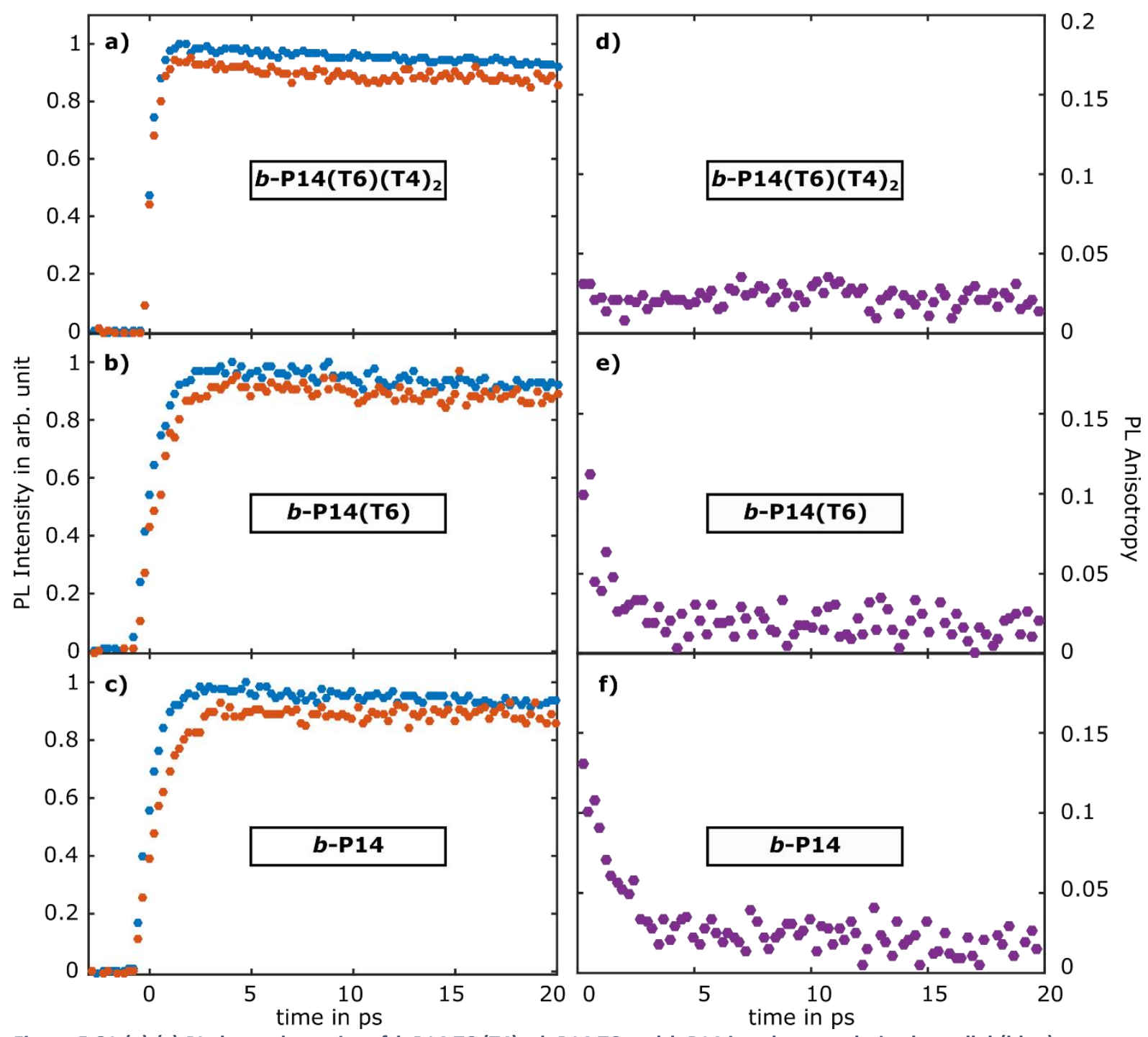

Figure 5.81 (a)-(c) PL decay dynamics of $b$-P14-T6·(T4) $)_{2}, b$-P14-T6 and $b$-P14 in toluene polarised parallel (blue) and perpendicular (red) to the excitation laser pulse. (d)-(e) PL anisotropy dynamics derived from PL intensity. Samples were excited at $820 \mathrm{~nm}$ and detected at $950 \mathrm{~nm}$. 


\section{The Synthesis of a $D_{4 \mathrm{~h}}$ Symmetric Porphyrin Nanoball using Selective Demetallation}

\section{Contents}

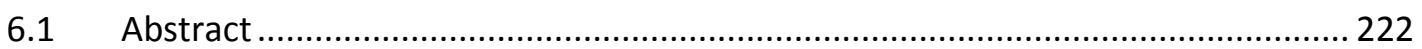

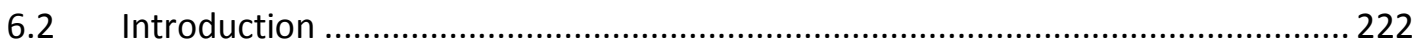

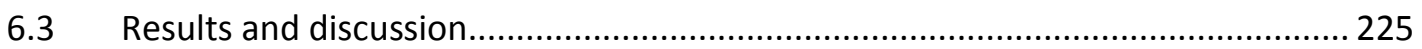

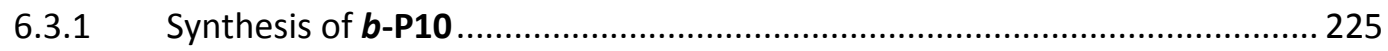

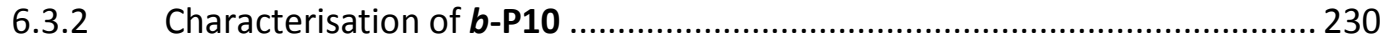

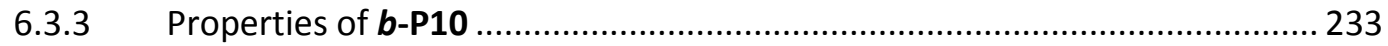

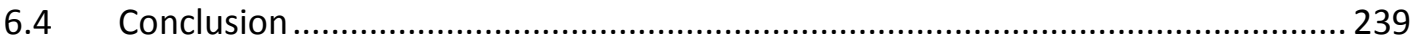

6.5 Experimental procedures and technical details.................................................. 239

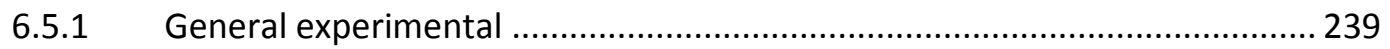

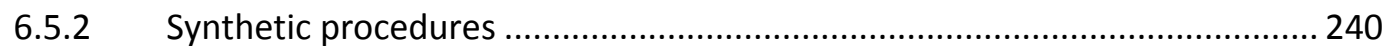




\subsection{Abstract}

The synthesis of heterometallated zinc and magnesium porphyrin oligomers allowed selective demetallation to be used as a synthetic tool to prepare a 10-porphyrin nanoball. The T6 template and a rationally designed 10-legged T10 template were used to investigate the binding properties of the $D_{4 h}$ symmetric structure by UV-vis-NIR.

\subsection{Introduction}

Chapter 5 described the linear synthesis of the 14-porphyrin nanoball $\boldsymbol{b}$-P14. The rationally designed synthetic route allowed for the successive use of two simple templates to prepare the 3D-conjugated structure. This chapter describes the synthesis of a $D_{4 \mathrm{~h}}$ symmetric analogue of the porphyrin nanoball. The structure $\boldsymbol{b}$-P10, consists of ten porphyrin units arranged as two perpendicular 6-porphyrin nanorings intersecting at two porphyrins (Figure 6.1). Due to its smaller number of porphyrin units and its higher symmetry, the structure might appear to be a simpler synthetic target than $\boldsymbol{b}$-P14, however, a more complex synthetic route was required.
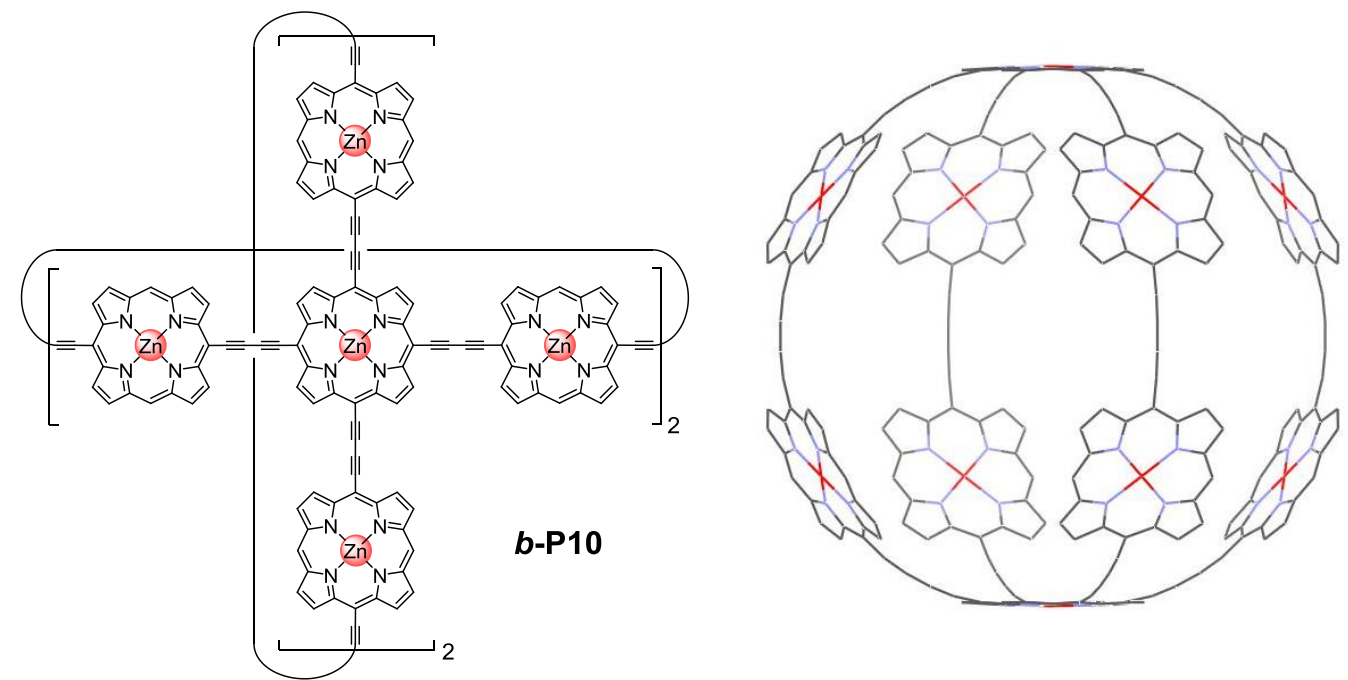

Figure 6.1: The chemical structure of the $\mathrm{D}_{4 h}$ symmetric 10-porphyrin nanoball $b$-P10 and the calculated structure at the BLYP/6-31G(d) level of theory shown with a density isovalue of 0.008 . Hydrogen atoms and aryl groups bearing solubilising side chains were omitted to simplify the calculations.

In the synthesis of $\boldsymbol{b}$-P14, a subsequent templating strategy was used. A similar strategy could not be used to prepare $\boldsymbol{b}$-P10 since the presence of the T6 template occupying the cavity of the initial 6-ring intermediate would prevent the use of additional templates to further pre-organise the system and form the second ring. Without this fundamental pre-organisation of the system, polymerisation would 
dominate in the ring closing reaction. In order to overcome this issue, previous members in the Anderson group have prepared an ingenious but laborious three-dimensional ten-legged template (Figure 6.2). The T10 template contains 10 pre-arranged binding sites with a geometry potentially capable of preparing $\boldsymbol{b}$-P10 directly from simple subcomponents. Regrettably, all attempts towards $\boldsymbol{b}$-P10 using the T10 template were unsuccessful both from simple and more elaborate starting materials.
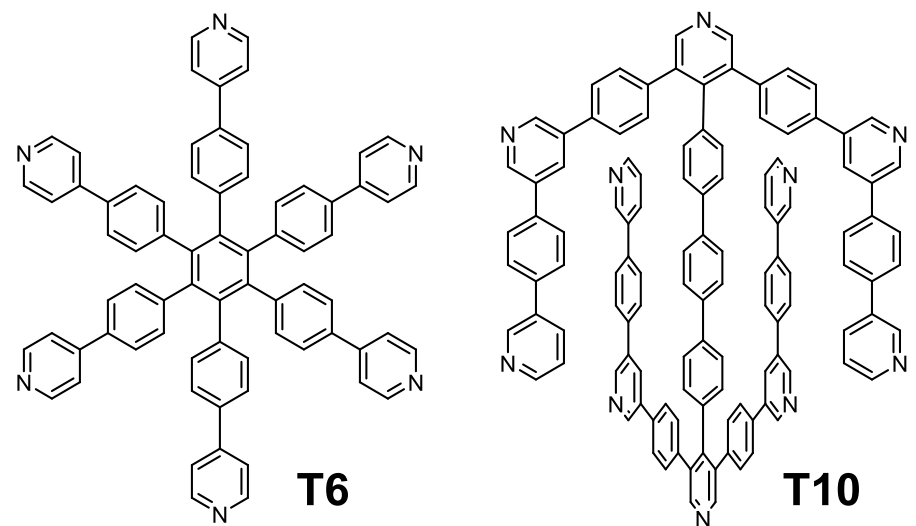

Figure 6.2: Chemical structure of T6 and the T10 template designed and prepared previously in an attempt to prepare the elusive $b$-P10 target.

Since the presence of the T6 template in the first ring hinders the binding of any additional templates, it seems reasonable to simply remove the template. Unfortunately, any template that would theoretically be capable of pre-organising the second ring before subjecting the complex to oxidative coupling conditions would have a stronger binding affinity to the first existing ring. As a result, the template would simply insert into the first ring and not pre-organise the system.

In Chapter 3 we have shown that a linear $\mathrm{Zn}-\mathrm{Cu}-\mathrm{Zn}$ trimer can be used to prepare a 6-porphyrin nanoring with copper centres in opposing positions of the ring (Figure 6.3a). Despite the virtually non-existent binding affinity of the central copper centre to the template, the binding of the two adjacent zinc porphyrins provided sufficient binding strength to the T6 template to allow formation of the ring from the two-to-one complex. This result inspired a route in which a ball precursor was prepared consisting of a free-base 6-porphyrin nanoring coupled to four zinc porphyrin monomers (Figure 6.3b). We hypothesised that the presence of four zinc porphyrins would provide sufficient binding affinity to the T6 template, which will 
now no longer insert into the first ring due to the absence of zinc centres, to prepare a $\boldsymbol{b}$-P10 nanoball target.

(a)

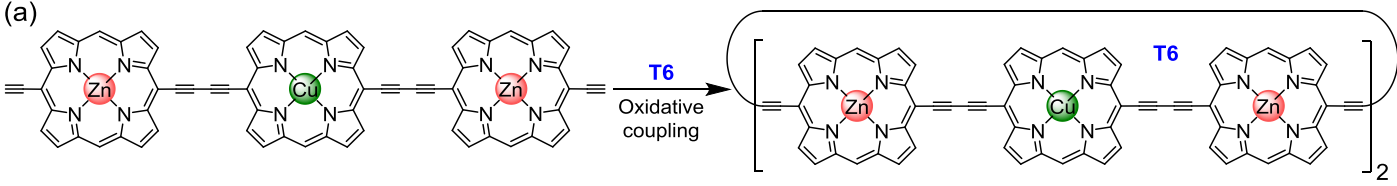

(b)

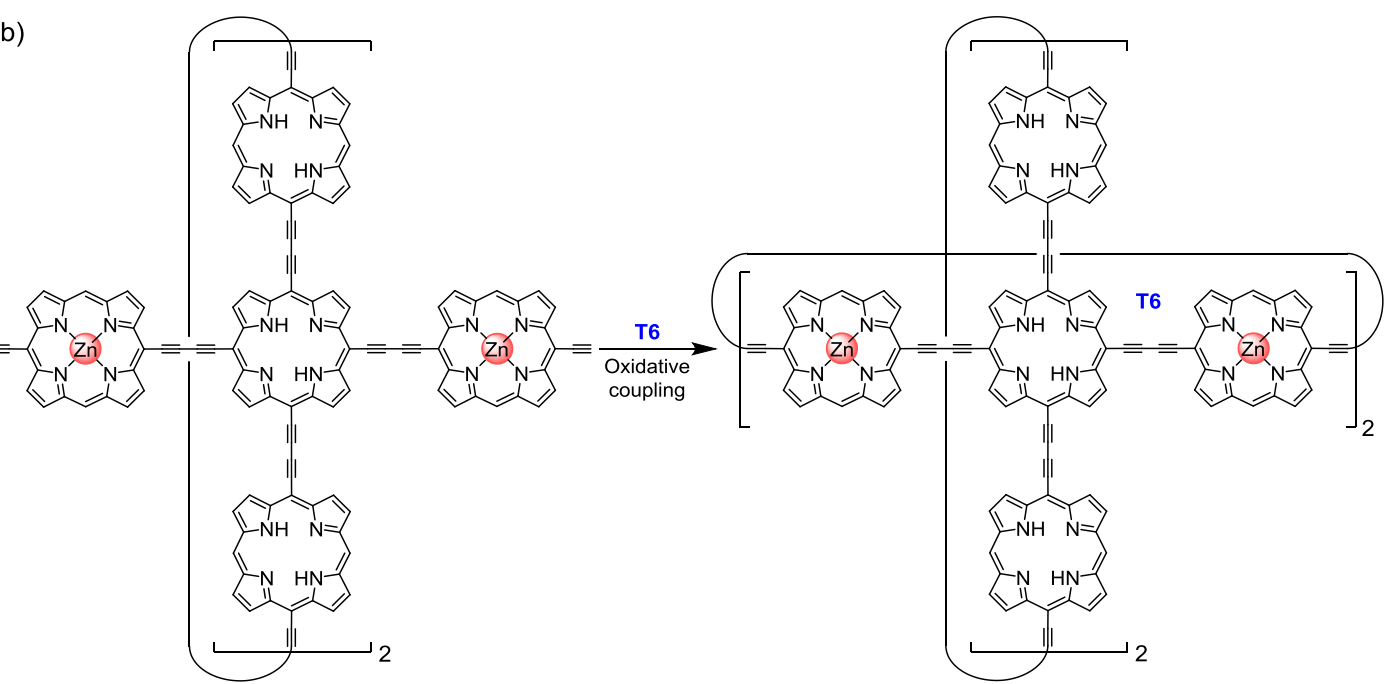

Figure 6.3: (a) Reaction scheme of a linear zinc-copper-zinc trimer forming a 6-porphyrin nanoring. (b) Proposed reaction scheme to make a porphyrin nanoball from a free-base 6-ring intermediate. The absence of binding sites for T6 in the initial ring could allow the template to rotate and pre-organise the second ring before subjecting the mixture to reaction conditions. Aryl solubilising groups are omitted for clarity.

Despite substantial synthetic effort, the route going through a free-base 6-ring intermediate was unsuccessful. In the absence of the central zinc porphyrin binding site, the binding to the T6 template appeared too weak to pre-organise the system and prepare the target compound. In order to increase the binding strength between the precursor and the template, we investigated synthetic methods to prepare a precursor which still contains the zinc centre in the tetraalkynylporphyin in addition to the adjacent zinc porphyrins.

Similar to zinc porphyrins, magnesium porphyrins are known to have a strong axial binding interaction with pyridyl ligands. As a result magnesium porphyrins can be utilised in the synthesis of porphyrin nanorings via standard template directed methods. Interestingly, the stability of magnesium in the plane of a porphyrin is lower than the stability of zinc. While a strong acid, such as trifluoroacetic acid (TFA) is required to demetallate zinc porphyrins, a weaker acid (e.g. acetic acid) is sufficient to remove magnesium from a porphyrin. The different stability of the metal centres 
in the porphyrin plane allows for the selective removal of magnesium from a heterometallated dimer, while leaving the zinc porphyrins in place (Figure 6.4).

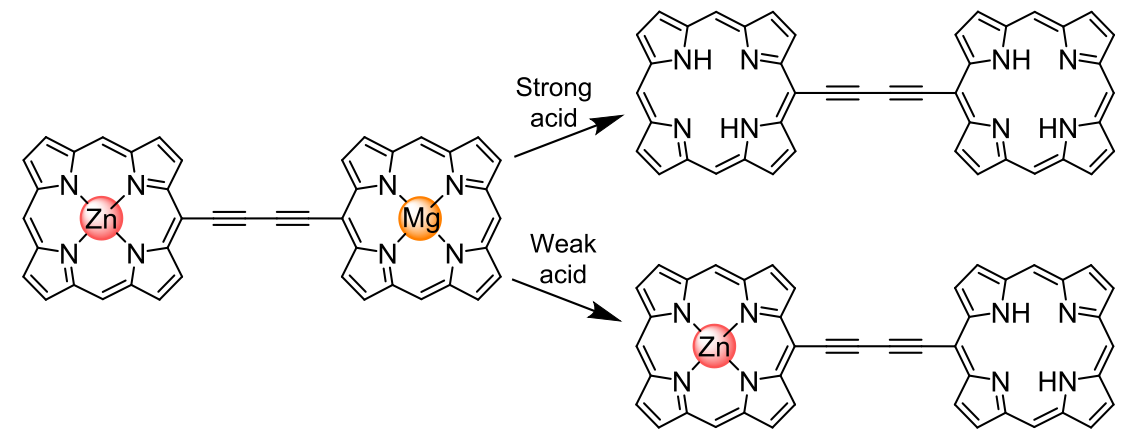

Figure 6.4: When a Mg-Zn mixed porphyrin dimer is subjected to strongly acidic reaction conditions, both metal centres are removed. When milder reaction conditions are used, the magnesium centre is selectively removed from the dimer.

The following section describes the synthesis of the 10-porphyrin nanoball $\boldsymbol{b}$-P10 by utilising a heterometallated zinc-magnesium 6-ring intermediate, which in turn allows for the use of a selective demetallation strategy.

\subsection{Results and discussion}

\subsubsection{Synthesis of $b-\mathrm{P} 10$}

The aryl groups of the porphyrins adjacent to the central unit are expected to come in close proximity to each other, potentially resulting in high steric congestion (Figure 6.5). In the synthesis of $\boldsymbol{b}$-P14, a combination of solubilising aryl groups was used to strike a balance between the steric congestion of the molecule and its solubility in organic solvents. To preserve the overall symmetry of $\boldsymbol{b}$-P10, it was decided to use homogeneous solubilising groups on all porphyrins.

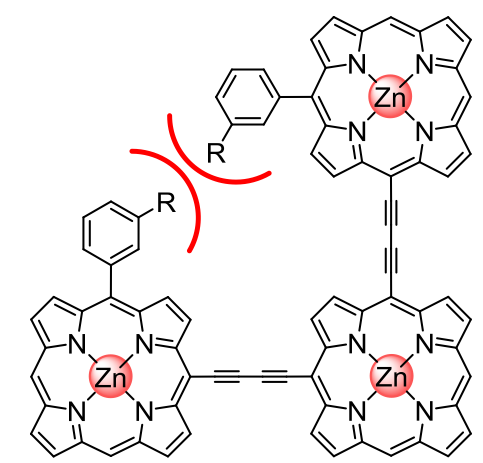

Figure 6.5: Illustration of the steric congestion of the aryl groups on the porphyrins adjacent to the central porphyrin. The solubilising groups are expected to point towards the periphery of the ball, minimising the steric clash of the R-groups with the templates present in the centre of the ball.

By utilising a single solubilising chain in the meta position of the aryl group, which we assume will point to the periphery of the ball, steric congestion towards the centre of 
the ball between the chains and the templates is expected to be minimised. An alternative strategy to minimise steric congestion is to use solubilising chains in the ortho-positions of the aryl group instead of the meta-position. During the course of this project, three different aryl groups were utilised towards the preparation of $\boldsymbol{b}$-P10 (Figure 6.6). The entire synthesis described below was initially attempted with the branched meta-alkoxy aryl $\left(\mathbf{A r}_{1}\right)$ and the ortho-bis-octyloxy aryl $\left(\mathbf{A r}_{2}\right)$ but both resulted in solubility problems at the final stage of the synthesis and did not yield the desired product. To increase solubility a mono-substituted analogue of the meta-bis-trihexylsilyl aryl was prepared $\left(\mathrm{Ar}_{3}\right)$ which proved to sufficiently increase the solubility in order to prepare the target nanoball as detailed below.

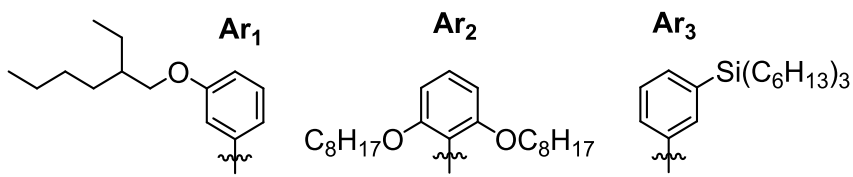

Figure 6.6: Chemical structure of the three different aryl solubilising groups attempted in the synthesis of $b$-P10.

The synthesis was started with the preparation of zinc and magnesium analogues of the mono-THS porphyrin (6.4 and 6.7). The mono-trihexylsilyl (THS) aldehyde 6.1 was prepared in a 55\% yield over two steps from 1,3-dibromobenzene. Dipyrromethane 2.1 and the aldehyde 6.1 were allowed to react in a porphyrin condensation reaction followed by zinc insertion to give porphyrin 6.2 in a $62 \%$ overall yield. The meso-positions of the porphyrin were brominated with NBS in an $82 \%$ yield after which cyanopropyldimethylsilyl (CPDMS) protected acetylenes were coupled onto the porphyrin via a Sonogashira to give 6.4 in a 93\% yield (Figure 6.7). The magnesium analogue was prepared in three steps from 6.3. The porphyrin was demetallated by the addition of trifluoroacetic acid in an $83 \%$ yield after which magnesium was inserted to give $\mathbf{6 . 6}$ in an $\mathbf{8 8 \%}$ yield. Finally a Sonogashira coupling gave 6.7 in nearly quantitative yields. Due to the acid sensitivity of the CPDMS protecting groups, it is not possible to directly demetallate and remetallate 6.4 to give 6.7. 


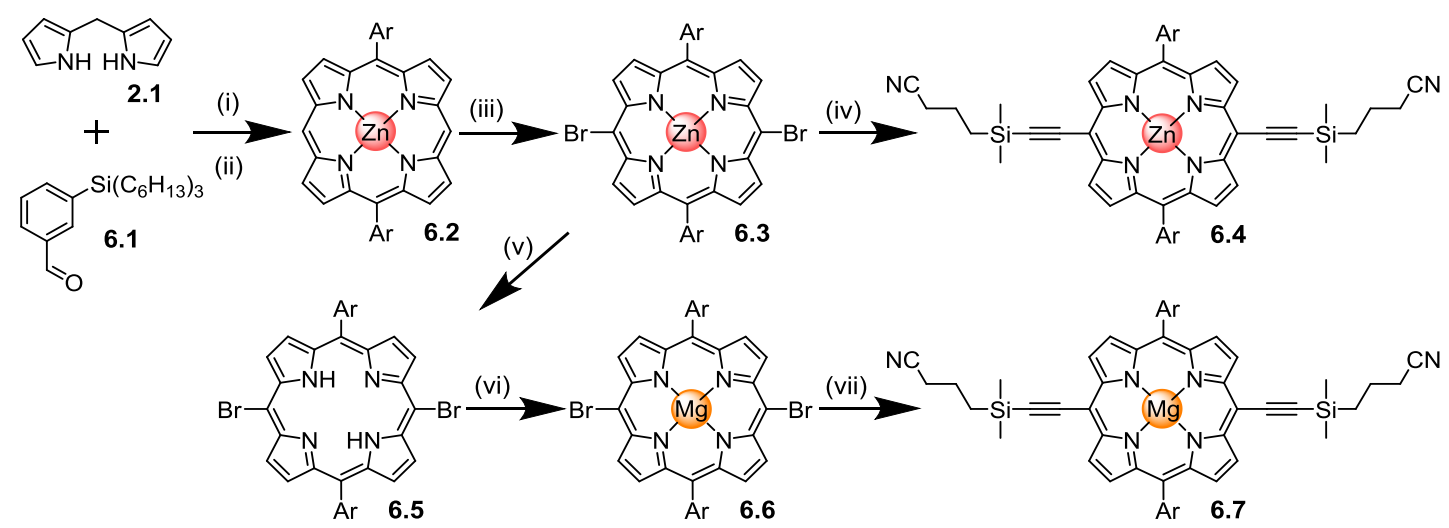

Figure 6.7: Synthetic route to porphyrins 6.4 and 6.7: (i) TFA, DDQ, $\mathrm{CH}_{2} \mathrm{Cl}_{2}$ then (ii) $\mathrm{Zn}(\mathrm{OAc})_{2} \cdot\left(\mathrm{H}_{2} \mathrm{O}\right)_{2}, \mathrm{CHCl}_{3}, 62 \%$; (iii) NBS, $\mathrm{CHCl}_{3} /$ pyridine, 81\%; (iv) $\mathrm{Pd}_{2}(\mathrm{dba})_{3}, \mathrm{Cul}, \mathrm{PPh}_{3}$, ethynylcyanopropyldimethylsilane, toluene/i- $\mathrm{Pr}_{2} \mathrm{NH}$, 93\%; (v) TFA, $\mathrm{CHCl}_{3}, 83 \%$; (vi) $\mathrm{Mgl}_{2}, \mathrm{CHCl}_{3} / \mathrm{Et}_{3} \mathrm{~N}, 88 \%$; $\mathrm{Pd}_{2}(\mathrm{dba})_{3}, \mathrm{Cul}, \mathrm{PPh}_{3}$, ethynylcyanopropyldimethylsilane, toluene/i- $\mathrm{Pr}_{2} \mathrm{NH}, 97 \%$. Ar = 3-(trihexylsilyl)phenyl.

One of the protecting groups on the acetylenes of 6.7 was statistically removed by the careful addition of TBAF to give the mono-deprotected porphyrin 6.8 in a $21 \%$ yield (Figure 6.8). In order to prepare the heterometallated trimer, 6.8 was mixed in a five-to-one ratio with the bis-deprotected central porphyrin 5.12, after which oxidative coupling conditions were used to form a statistical mixture of oligomers. The reaction mixture was purified by silica gel chromatography, size exclusion chromatography and finally recycling GPC to give trimer 6.9 in a $28 \%$ yield (Figure 6.8).

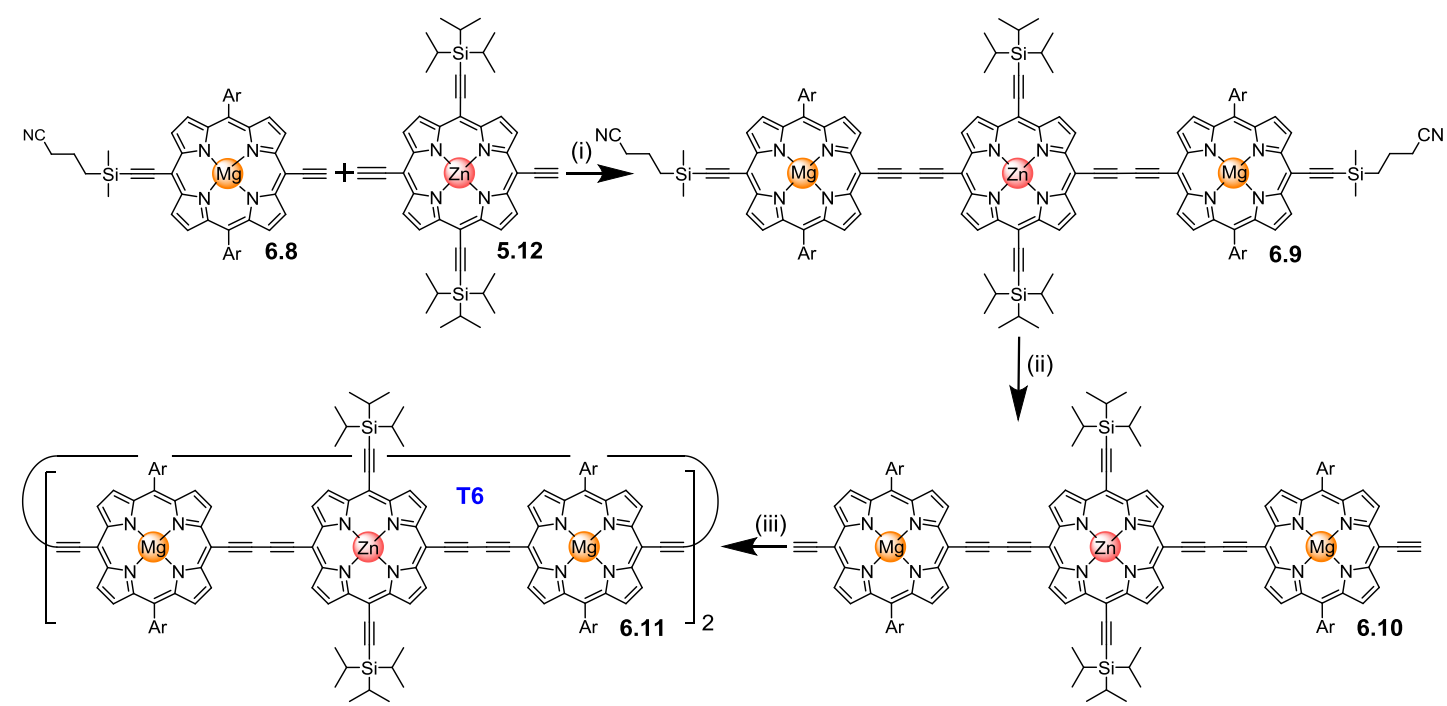

Figure 6.8: Synthetic route to porphyrin ring 6.11. (i) $\mathrm{PdCl}_{2}\left(\mathrm{PPh}_{3}\right)_{2}, \mathrm{Cul}, 1,4$-benzoquinone, $\mathrm{CHCl}_{3} / \mathrm{i}-\mathrm{Pr}_{2} \mathrm{NH}, 28 \%$; (ii) $\mathrm{K}_{2} \mathrm{CO}_{3}, \mathrm{THF} / \mathrm{methanol}, 97 \%$; (iii) $\mathrm{T6}, \mathrm{Pd}\left(\mathrm{PPh}_{3}\right)_{2} \mathrm{Cl}_{2}, \mathrm{Cul}, 1,4-$ benzoquinone, $\mathrm{CHCl}_{3} / \mathrm{i}-\mathrm{Pr}_{2} \mathrm{NH}, 26 \%$. $\mathrm{Ar}=3$-(trihexylsilyl)phenyl.

The CPDMS protecting groups on the acetylenes of the magnesium porphyrins were selectively removed with $\mathrm{K}_{2} \mathrm{CO}_{3}$, giving 6.10 in a $97 \%$ yield. Both the zinc and magnesium centres in the trimer have a strong axial binding affinity for pyridyl 
ligands. As a result, the T6 template could be used to form a two-to-one complex before subjecting the mixture to oxidative coupling conditions to give the heterometallated porphyrin nanoring $\mathbf{6 . 1 1}$ in a $26 \%$ yield after purification by recycling GPC.

The essence of the synthetic strategy is illustrated in the next synthetic step. By using a heterometallated 6-ring intermediate, it was possible to selectively remove the magnesium centres and the template from the ring. As a result, the cavity of the ring is available for template binding in later stages of the synthesis.

While a simple magnesium porphyrin monomer could be demetallated by the addition of acetic acid, no reaction was observed when 6.11 was subjected to identical reaction conditions. In a porphyrin monomer, the magnesium centre is stabilised by four $\mathrm{N} \cdots \mathrm{Mg}$ interactions in the plane of the porphyrin. The additional axial $\mathrm{N} \cdots \mathrm{Mg}$ interaction to the template in the porphyrin nanoring, appears to render the magnesium centres more stable. To circumvent this issue, it was decided to initially remove the T6 template from $\mathbf{6 . 1 1}$ by the addition of a large excess of the competing ligand $\mathrm{DABCO}$, after which the magnesium centres were be selectively removed by the addition of acetic acid to give $\mathbf{6 . 1 2}$ in a 74\% yield over two steps.

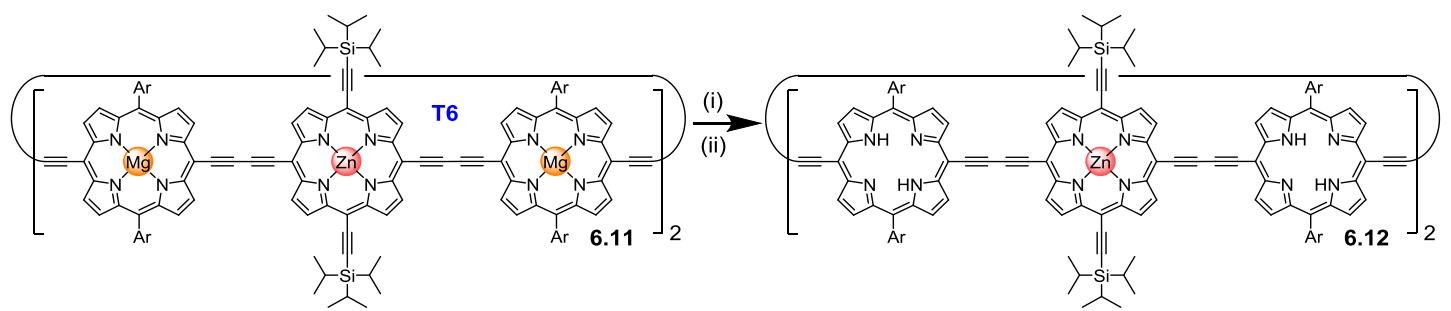

Figure 6.9: Synthetic route to porphyrin ring 6.12 (i) DABCO, pyridine, size exclusion chromatography, then (ii) acetic acid, DCM, 74\%. Ar = 3-(trihexylsilyl)phenyl.

The synthesis was continued by removing the TIPS protecting groups on the central porphyrin by the addition of TBAF, yielding 6.13 in an $86 \%$ yield. Zinc porphyrin 6.4 was statistically deprotected by the careful addition of TBAF to give the mono-deprotected 6.14 in a $21 \%$ yield. The bis-zinc nanoring 6.13 was subjected to oxidative coupling conditions in the presence of a large excess of 6.14 to form the extended nanoring 6.15 in a $56 \%$ yield from which the CPDMS protecting groups were subsequently removed by the addition of TBAF in a quantitative yield to give 6.16 (Figure 6.10). 


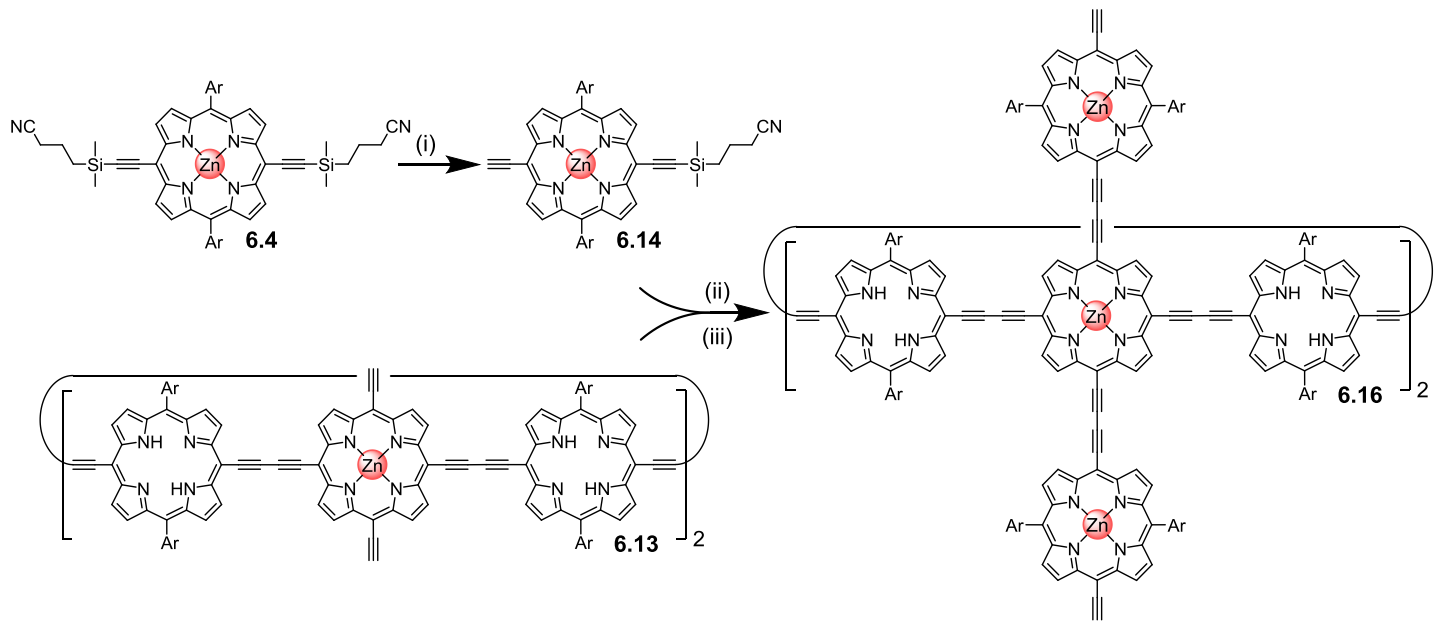

Figure 6.10: Synthetic route to porphyrin ring 6.16 (i) TBAF, $\mathrm{CHCl}_{3} / \mathrm{EtOH}, 21 \%$; (ii) $\mathrm{PdCl}_{2}\left(\mathrm{PPh}_{3}\right)_{2}, \mathrm{Cul}$, 1,4-benzoquinone, $\mathrm{CHCl}_{3} / i-\mathrm{Pr}_{2} \mathrm{NH}, 56 \%$; (iii) $\mathrm{TBAF}, \mathrm{CH}_{2} \mathrm{Cl}_{2}, 100 \%$. $\mathrm{Ar}=3$-(trihexylsilyl)phenyl.

The binding of T6 to 6.16 was investigated by UV-vis-NIR titrations. Upon the addition of small aliquots of T6, a strong red-shift in the Q-bands was observed as a result of the formation of the desired one-to-one complex (Figure 6.11). A linear, isosbestic change in the absorption spectrum was observed upon the addition of one equivalent T6, indicating the strong formation of the complex (black to green). Upon the addition of more ligand, the spectra continue to change, likely due to the formation of a two-to-one complex between T6 and 6.16 (green to red).
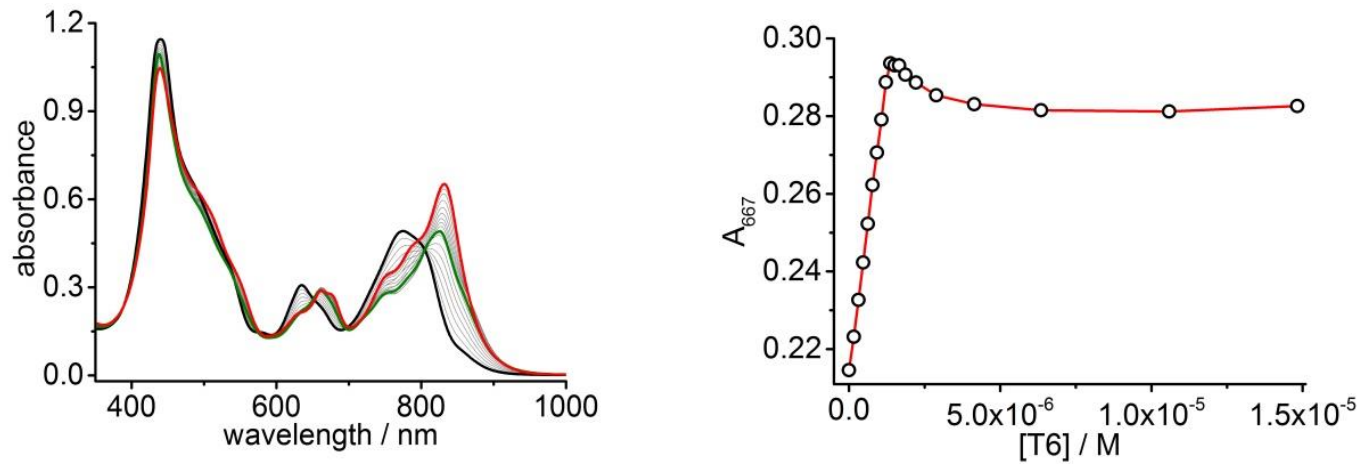

Figure 6.11: UV-vis-NIR formation titration of 6.16 and the template T6 indicating the formation of a one-to-one complex (black to green). The complex denaturates to the two-to-one complex upon the addition of more T6 (green to red).

With the confirmation of the formation of the one-to-one complex, we continued the synthesis to form the ball 6.17. The ball precursor 6.16 and T6 were mixed in a one-to-one ratio after which the reaction mixture was subjected to oxidative coupling conditions. If the copper(I) in the reaction mixture is oxidised to copper(II), the four free-base porphyrins in $\mathbf{6 . 1 6}$ are at risk of copper insertion and therefore the reaction time was kept to a minimum. The reaction progress was closely monitored by UV-vis-NIR spectroscopy which revealed a characteristic sharpening of the 
Q-bands indicating the formation of a templated 6-porphyrin nanoring (Figure 6.12a). The crude reaction mixture was purified by silica gel chromatography, SEC and recycling GPC to give the nanoball 6.17 in a $7 \%$ yield (Figure $6.12 b$ and $c$ ). A large amount of insoluble material was formed in this reaction which is possibly due to polymerisation of 6.17 , explaining the low yield in the reaction.

(a)

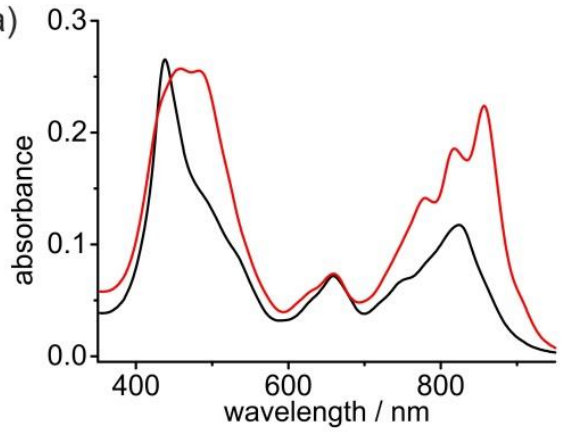

(b)

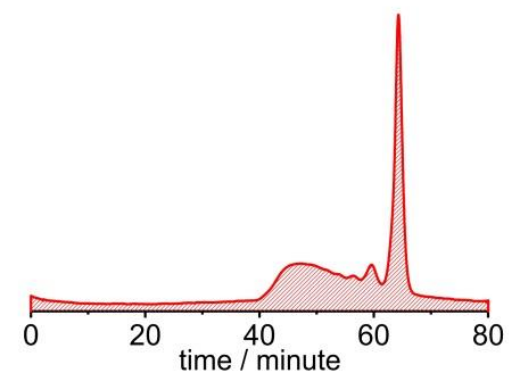

(c)

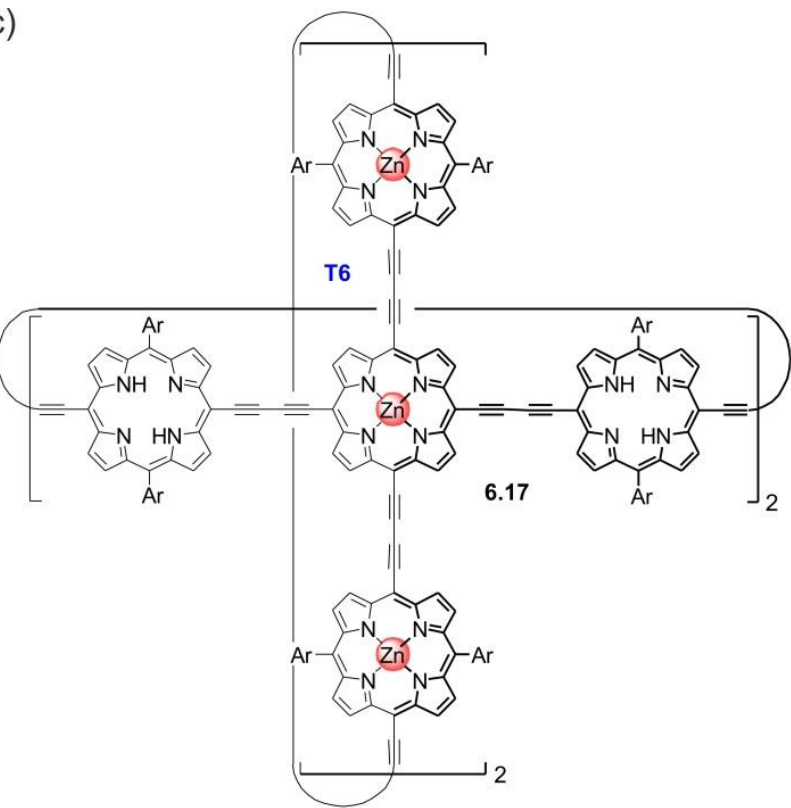

Figure 6.12: (a) UV-vis-NIR spectra of the starting material (black) and the crude reaction mixture (red). (b) Crude reaction GPC trace (JAIGEL columns, $1^{\text {st }}$ cycle shown, $1 \%$ pyridine in toluene, detection at $800 \mathrm{~nm}$ ) showing the product at 63 minutes. (c) Chemical structure of 6.17. Ar = 3-(trihexylsilyl)phenyl.

In the final steps of the synthesis, the template was removed by the addition of a large excess of the competing ligand $\mathrm{DABCO}$, followed by zinc insertion to give the highly symmetric $\boldsymbol{b}$-P10 target molecule in nearly quantitative yield.

\subsubsection{Characterisation of $b$-P10}

The structure of $\boldsymbol{b}$-P10 was confirmed by ${ }^{1} \mathrm{H}$ NMR, MALDI, UV-Vis-NIR and analytical GPC. All ${ }^{1} \mathrm{H}$ NMR spectra were recorded at $323 \mathrm{~K}$ using a Bruker AV700 (700 MHz) instrument with $\mathrm{CDCl}_{3}$ as the solvent. The 2D-NMR technique NOESY was used to achieve full assignment of the signals. In the molecular structure and in the crosspeaks, NOESY correlations are indicated in red (Figure 6.13). 


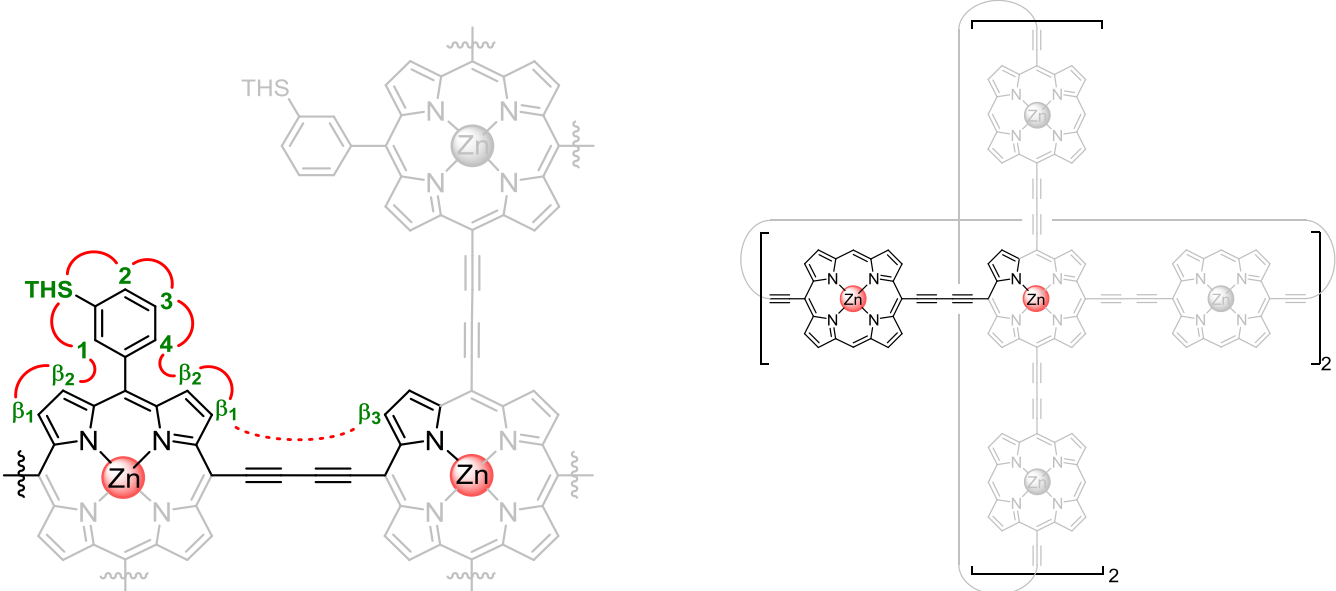

Figure 6.13: The symmetric unit of the porphyrins in $b-\mathrm{P} 10$ with proton labelling and a representation of its position within the ball. NOESY correlations have been indicated in red. The dotted red line indicates a potential NOE which was not observed. (THS = trihexylsilyl).

As a result of the $D_{4 \mathrm{~h}}$ symmetry of the molecule the ${ }^{1} \mathrm{H}$ NMR spectrum is remarkably simple. The symmetric unit of $\boldsymbol{b}$-P10 represents $1 / 16^{\text {th }}$ of the molecule, with merely nine resonances in the aromatic region (Figure 6.14). The only other signal was a broad unresolved envelope of 624 protons, corresponding to the trihexylsilyl (THS) solubilising groups on the aryls, resonating between 1.6 and $0.6 \mathrm{ppm}$.

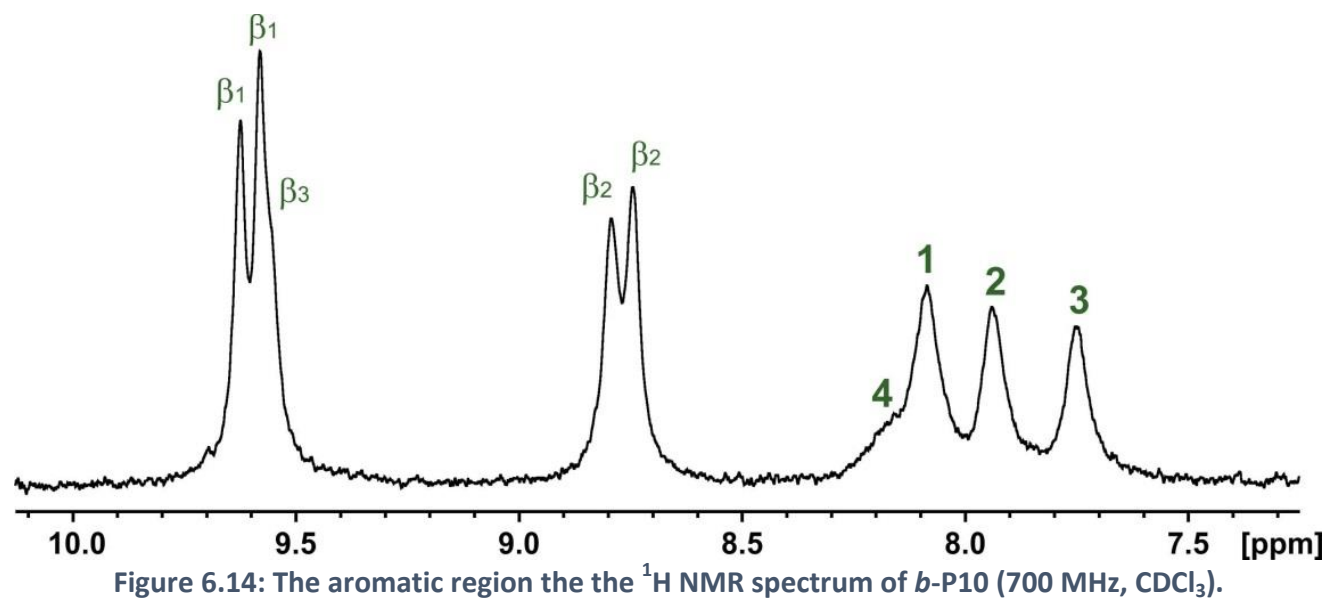

The four aromatic protons on the aryl group were distinguished from each other through their NOESY interactions. Proton 3 resonates at $7.74 \mathrm{ppm}$ and shows correlations to both protons 2 and 4 as can be seen in Figure 6.15 (left). The signal at 7.93 was identified as proton 2 by its correlation to the THS protons (Figure 6.15, right) and lack of correlation to the $\boldsymbol{\beta}_{\mathbf{2}}$ protons. Proton $\mathbf{1}$ and $\mathbf{4}$ are found to as two partially overlapping signals at 8.08 and $8.16 \mathrm{ppm}$ respectively. Both protons show a correlation to the $\boldsymbol{\beta}_{\mathbf{2}}$ protons but only $\mathbf{1}$ shows a correlation to the THS protons. 

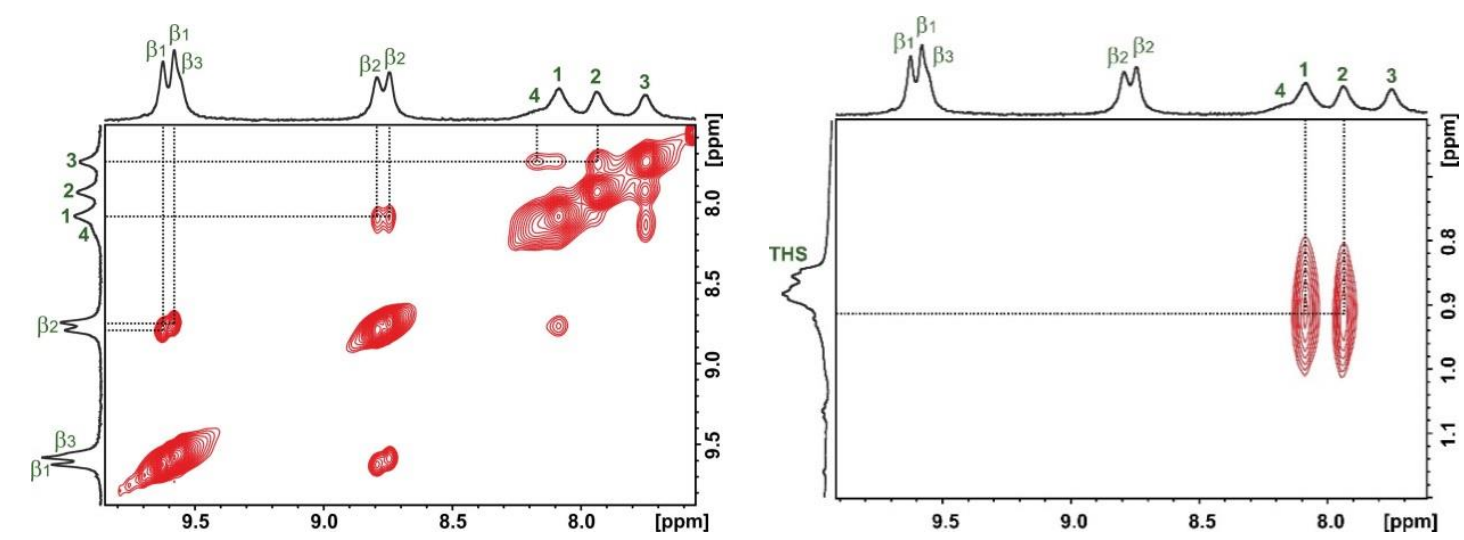

Figure 6.15: (left) NOESY correlations in the aromatic region of $b$-P10. (right) NOESY cross-peaks between protons 1 and 2 to the THS solubilising chains.

The beta-protons gave rise to five broad signals between 10 and $8.5 \mathrm{ppm}$. The beta-protons in close proximity to the acetylenes were found at higher chemical shift than the protons close to the aryls. The $\beta_{1}$ and $\beta_{\mathbf{2}}$ protons show NOESY correlations to each other, allowing $\beta_{3}$ to be identified as the shoulder at $9.55 \mathrm{ppm}$ due to its absence of NOESY correlations. Selective 1D-NOESY experiments were performed in order to show the correlation between the proton $\beta_{3}$ and one of the $\beta_{1}$ protons across the butadiyne. This was an attempt to distinguish between the different $\boldsymbol{\beta}_{1}$ 's and $\beta_{2}$ 's, but due to the broadness of the signals no such correlation were observed.

When zooming in on the NOESY spectrum, relayed NOEs between proton $\mathbf{3}$ can be observed to $\boldsymbol{\beta}_{\mathbf{2}}$ through proton $\mathbf{4}$. Similarly a relayed correlation can be observed between proton 1 and the $\boldsymbol{\beta}_{\mathbf{1}}$ protons through the protons $\boldsymbol{\beta}_{\mathbf{2}}$ (Figure 6.16 , left). In addition, relayed NOEs were observed from the THS protons, through the aryl protons to the beta-protons of the porphyrin (Figure 6.16, right). The lack of correlation to $\beta_{3}$ is in agreement with the assignment described above.
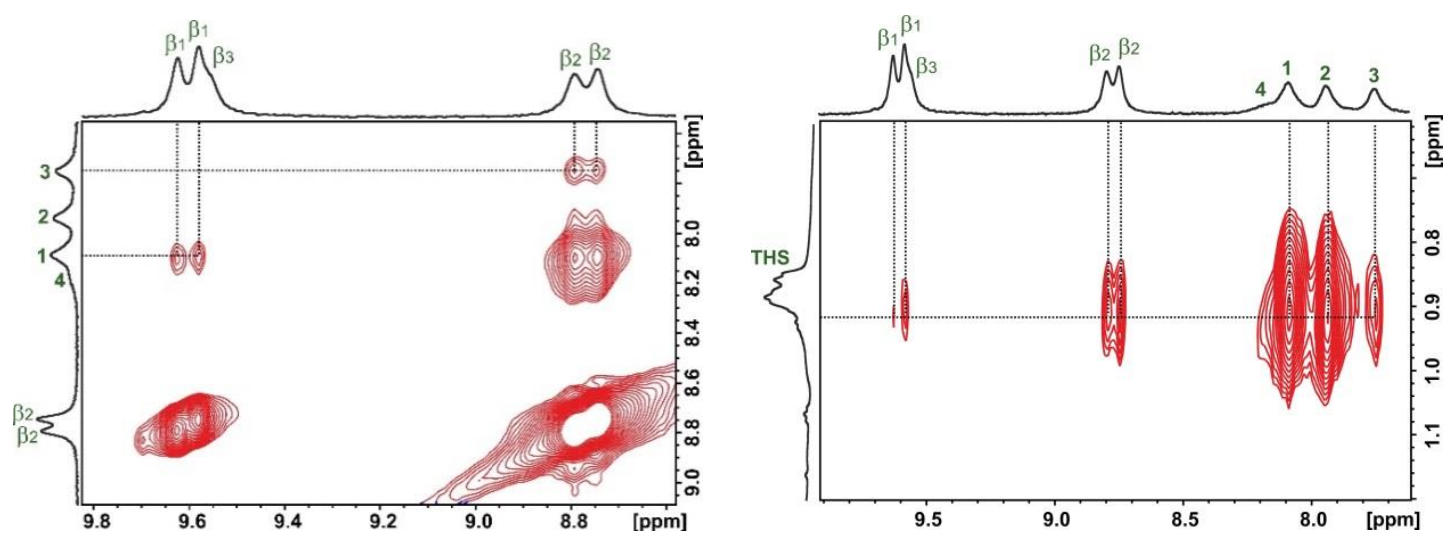

Figure 6.16: NOESY correlations illustrated in Figure 6.15 zoomed in to a greater depth to show the relayed NOEs in $b$-P10. 


\subsubsection{Properties of $b-\mathrm{P} 10$}

After the characterisation of $\boldsymbol{b}$-P10 we proceeded to investigate its properties. UV-vis-NIR titrations were performed in order to investigate the binding of templates within the cavity of the ball. As can be seen in the DFT optimised structure the windows of the ball are relatively narrow and might not allow for the encapsulation of large guest molecules (Figure 6.17). However, the edges of the structure can wiggle slightly in order to potentially allow guests to slip in.
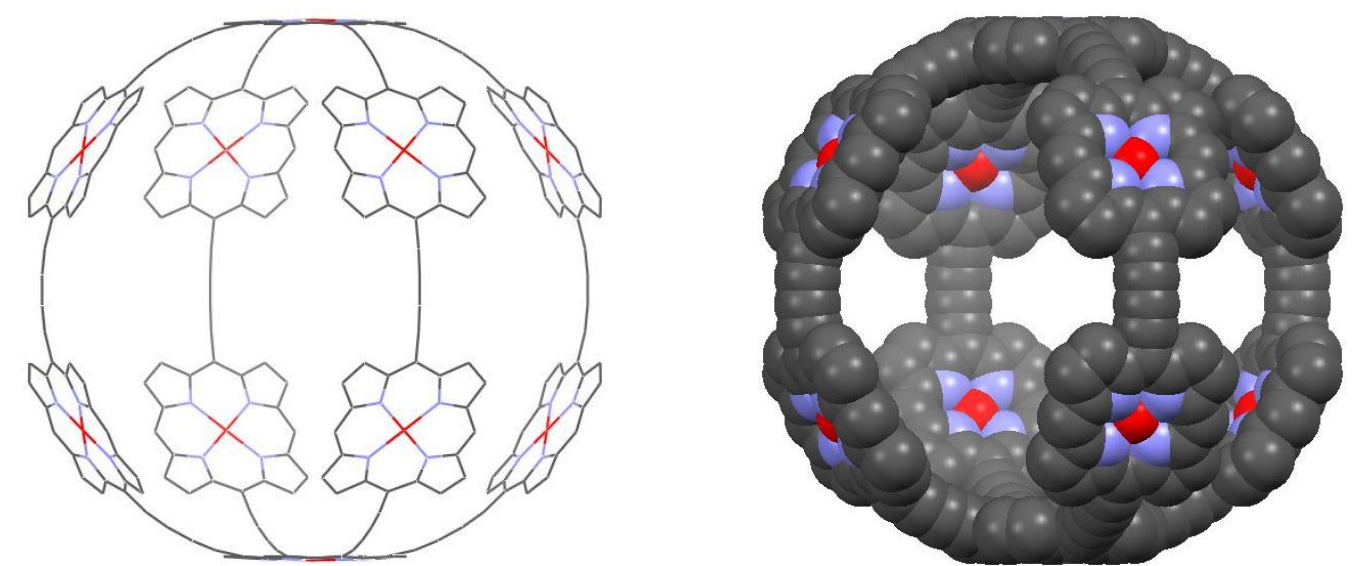

Figure 6.17: Structure of $b-\mathrm{P} 10$ with calculated at the BLYP/6-31G(d) level of theory shown with a density isovalue of 0.008 . Hydrogen atoms and aryl groups bearing solubilising side chains were omitted to simplify the calculations.

We initially investigated the binding of the T6 template. The UV-vis-NIR spectrum of $\boldsymbol{b}$-P10 (Figure 6.18, black) resembles the absorption spectrum of template-free $\boldsymbol{c}$-P6, albeit slightly red-shifted due to its greater conjugation. In addition, $\boldsymbol{b}$-P10 has a slightly more pronounced three-finger Q-band pattern than is generally observed for c-P6, potentially due to the more restricted rotation of the individual porphyrins.

Upon the addition of T6, a linear change in the absorption spectrum with a sharp end point was observed indicating the formation of a one-to-one complex. The absorption spectrum of $\boldsymbol{b}$-P10.T6 is slightly more red-shifted as the template free analogue. In addition, a sharpening of the Q-band features is observed as a result of the more rigid structure and reduced porphyrin-porphyrin dihedral angles upon templation. Only a marginal change in shape of the UV-vis-NIR spectrum is observed during the formation titration which indicates that even without a template, $\boldsymbol{b}$-P10 is quite rigid. 

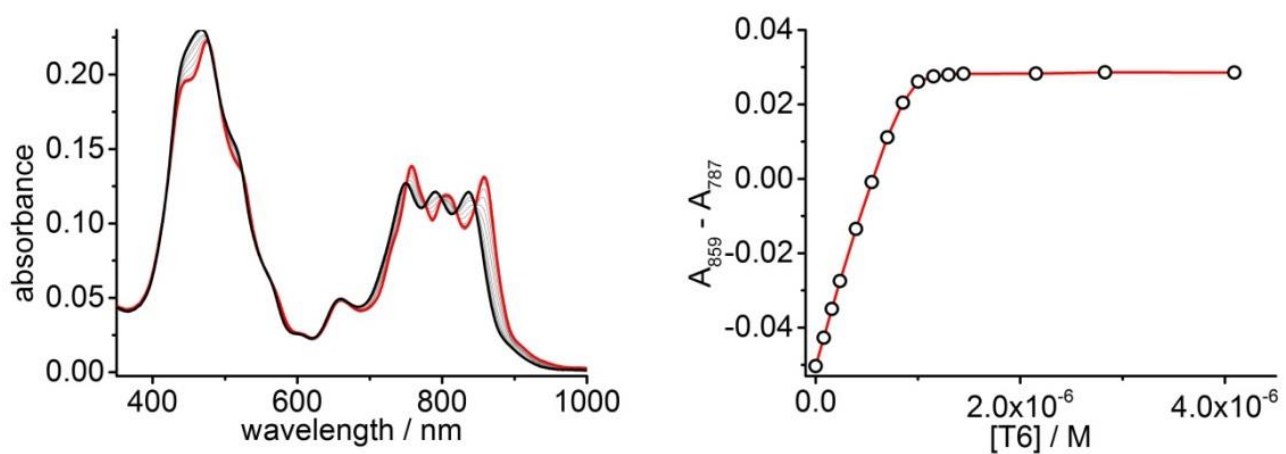

Figure 6.18: The UV-vis-NIR formation titration of $b$-P10 (black line) with T6 (toluene at $298 \mathrm{~K}$ ) forming the one-to-one complex $b$-P10.T6 (red line). On the right, the change in absorption as a function of the T6 concentration is shown, indication the formation of a strong one-to-one complex with a red line plotted through the experimental data point (black cicles).

The one-to-one complex between $\boldsymbol{b}$-P10 and T6 is too stable to determine the association constant directly from the formation curve. Therefore, the complex was denaturated with quinuclidine to gain insights into the binding strength of the template. A large excess of quinuclidine was titrated into a solution of the one-to-one complex ( $c a .0 .6 \mathrm{M}$ in toluene at $298 \mathrm{~K}$ ) to displace the multidentate ligand (Figure 6.19).
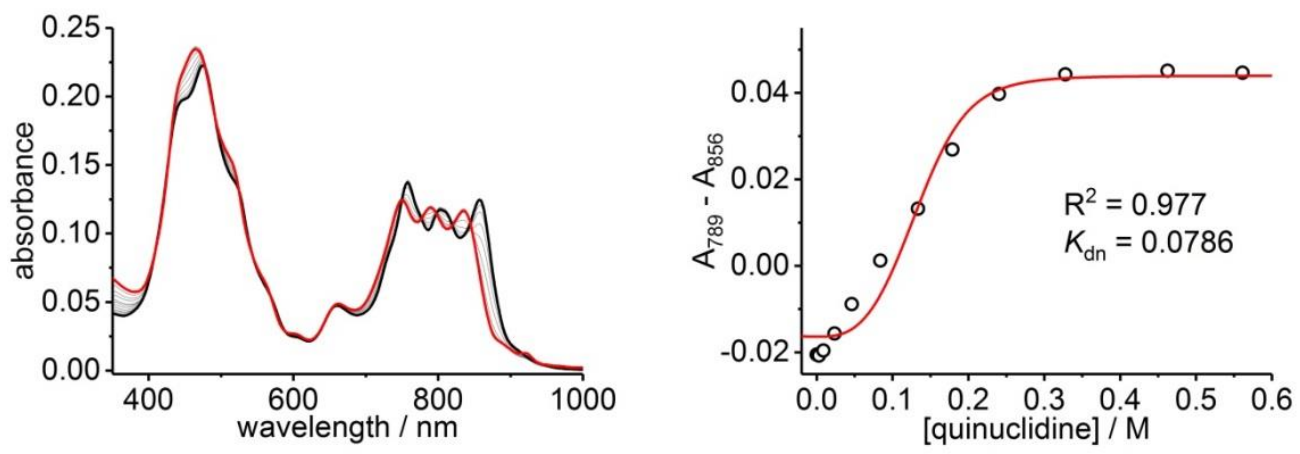

Figure 6.19: The UV-vis-NIR denaturation titration of $b$-P10.T6 with quinuclidine. $R^{2}=0.977$. (Run 1 , toluene, $298 \mathrm{~K},[b-\mathrm{P} 10 \cdot \mathrm{T} 6]=0.901 \mu \mathrm{M}, K=0.0786 \mathrm{M}^{-5}$ ).

Generally, the formation constant $K_{\mathrm{f}}$ is determined via the denaturation constant $K_{\mathrm{dn}}$. This analysis assumes an essentially all-or-nothing two state equilibrium (i.e. intermediate partially denatured species do not build up to high concentrations) and is generally supported by the isosbestic nature of the UV-vis-NIR titration and the good fits of the curves to the calculated binding isotherm for a two-state equilibrium. While the titration is isosbestic, the fit to the binding isotherm is not ideal, potentially due to the template rotating in the cavity of $\boldsymbol{b}$-P10. An estimate of the denaturation constant $K_{\mathrm{dn}}$ can be obtained at the half-way point of the denaturation titration via the formula: 


$$
K_{\mathrm{dn}}=\frac{\left[\mathrm{HQ}_{6}\right] \cdot[\mathrm{L}]}{[\mathrm{HL}] \cdot[\mathrm{Q}]^{6}}
$$

in which $\left[\mathrm{HQ}_{6}\right]$ is the concentration of quinuclidine saturated host, $[\mathrm{L}]$ and $[\mathrm{Q}]$ are the concentration free ligand T6 and quinuclidine respectively, and [HL] is the concentration host-guest complex. At the half-way denaturation point, we know $\left[\mathrm{HQ}_{6}\right]=[\mathrm{HL}]$, and we assume that $[\mathrm{Q}]$ equals the total amount of added quinuclidine which allows us to simplify the equation to:

$$
K_{\mathrm{dn}}=\frac{[\mathrm{L}]}{[\mathrm{Q}]^{6}}
$$

in which [L] equals half the concentration of available host, allowing $K_{\mathrm{dn}}$ to be estimated:

$$
K_{\mathrm{dn}}=\frac{\left[4.5 \times 10^{-7}\right]}{[0.13]^{6}}=9.3 \cdot 10^{-2} \mathrm{M}^{-5}
$$

which is in reasonable agreement with the denaturation constant found when fitting the data to the binding isotherm shown in Figure 6.19. The denaturation constant was used to calculate the formation constant $K_{\mathrm{f}}$ using the equation:

$$
K_{\mathrm{f}}=\frac{K_{\mathrm{Q}}^{N}}{K_{\mathrm{dn}}}=\frac{K_{\mathrm{THS}}^{4} \cdot K_{\mathrm{cen}}^{2}}{K_{\mathrm{dn}}}=\frac{\left(1.30 \cdot 10^{6}\right)^{4} \cdot\left(6.45 \cdot 10^{6}\right)^{2}}{9.3 \cdot 10^{-2}}=1.28 \cdot 10^{39} \mathrm{M}^{-1}
$$

After correcting the formation constant for statistical factors, $K_{\mathrm{f}}=5.32 \cdot 10^{37} \mathrm{M}^{-1}$ which is in good agreement with T6 binding to $c$-P6 indicating that the binding of T6 in $\boldsymbol{b}$-P10 is hardly influenced by the presence of the two central-porphyrins which do not lose rotational degrees of freedom upon complex formation. In addition, the presence of the second ring does not influence the binding which indicates that T6 is relatively free to move in and out of the cavity.

While so far, the T10 template depicted in Figure 6.2 has not been used successfully in the template directed synthesis of $\boldsymbol{b}$-P10, its shape, size and number of binding sites make it a potential guest for the ball. The probe the feasibility of T10 insertion, a MALDI-MS spectrum of $\boldsymbol{b}$-P10 was recorded before and after exposing the compound to an excess of the T10 template. Encouragingly, the spectra revealed the quantitative insertion of the template (Figure 6.20). 


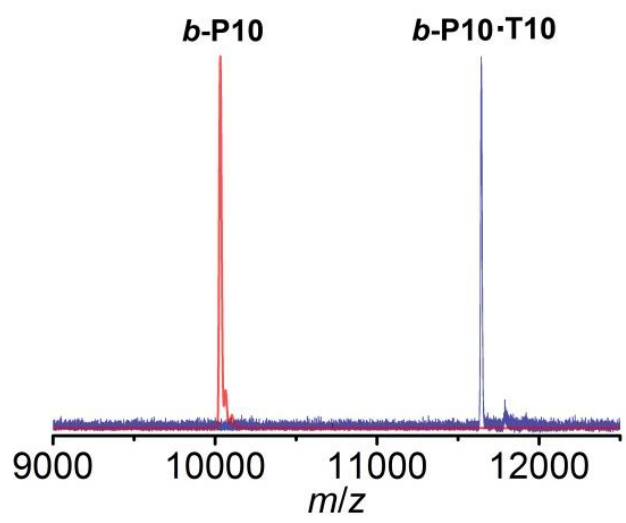

Figure 6.20: Overlay of the MALDI-MS spectra of $b$-P10 (red) and $b$-P10.T10 (blue) demonstrating the quantitative insertion of the template.

The binding of T10 was then investigated by UV-vis-NIR titrations. Upon addition of T10 to a solution of $\boldsymbol{b}-\mathbf{P} 10$, a similar change in the Q-band absorbance was observed as was the case for the addition of T6 (Figure 6.21). While the formation of the complex between $\boldsymbol{b}$-P10 and T6 was nearly instantaneously, the insertion of T10 needed to equilibrate for several minutes between additions, indicating that the insertion process is slower, potentially due to the larger size of the template making it more difficult for the template to slip through the windows of the ball.
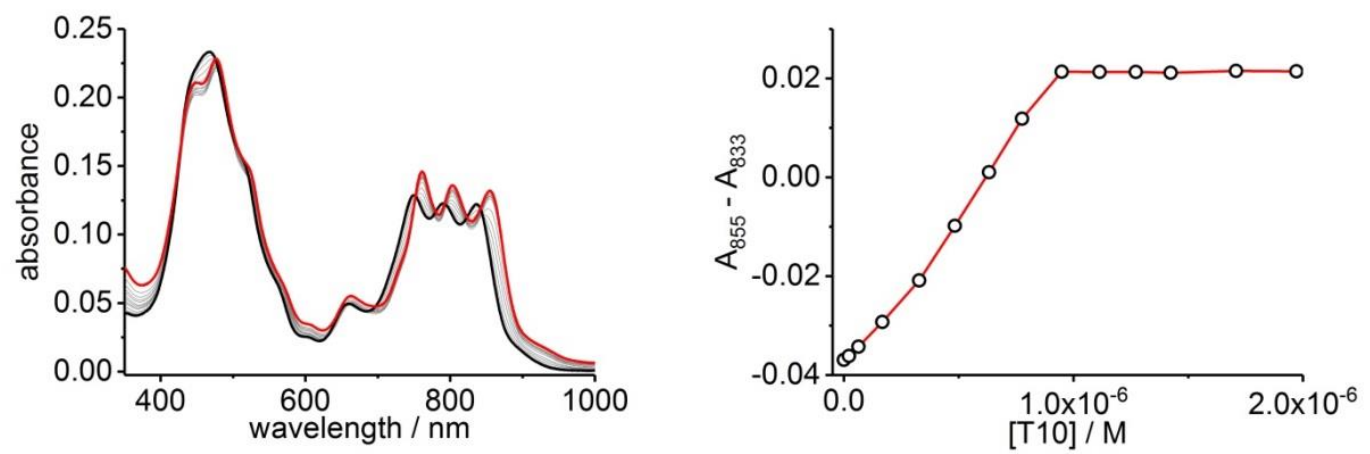

Figure 6.21: The UV-vis-NIR formation titration of $b$-P10 (black line) with T10 (toluene at $298 \mathrm{~K}$ ) forming the one-to-one complex, b-P10-T10 (red line). On the right, the change in absorption as a function of the T10 concentration is shown, indication the formation of a strong one-to-one complex with a red line plotted through the experimental data point (black cicles).

The sharp end point of the titration shows that the one-to-one complex between $\boldsymbol{b}$-P10 and T10 is too stable to determine the association constant directly from the formation curve. Therefore, the complex was denaturated with quinuclidine to determine the binding strength of the template. A large excess of quinuclidine was titrated into a solution of the one-to-one complex ( $c a .0 .3 \mathrm{M}$ in toluene at $298 \mathrm{~K}$ ) to displace the multidentate ligand (Figure 6.22). 

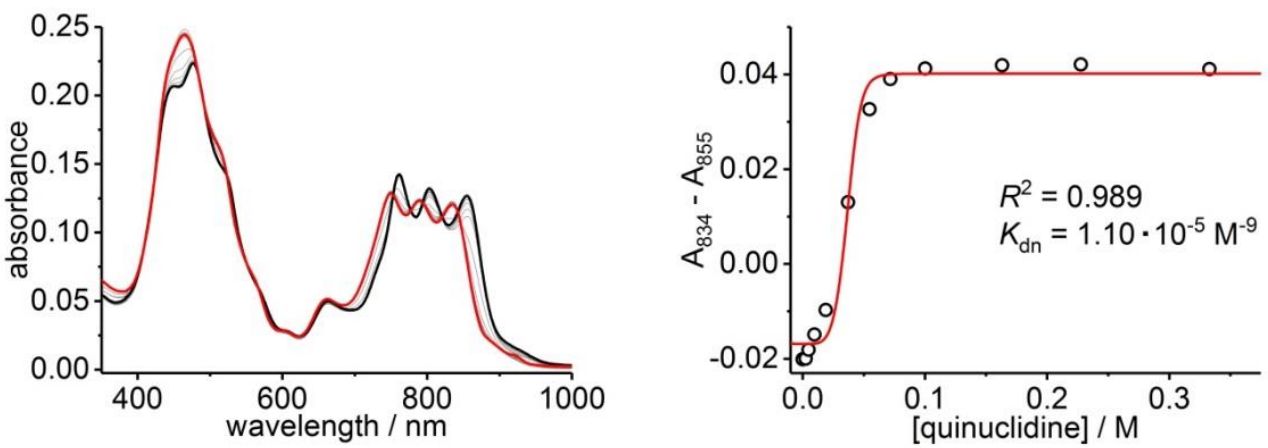

Figure 6.22: The UV-vis-NIR denaturation titration of $b$-P10.T10 with quinuclidine. $R^{2}=0.989$. (Toluene, $298 \mathrm{~K}$, $[b-\mathrm{P} 10 \cdot \mathrm{T} 10]=0.925 \mu \mathrm{M}, N=10, K=1.10 \cdot 10^{-5} \mathrm{M}^{-9}$ ).

When plotting the change in absorbance as a function of the quinuclidine concentration, the fit of the experimental data points to the one-to-one binding isotherm, assuming ten binding sites $(N)$, is far from satisfactory. The bad fit to the experimental data indicates that the model used or the assumptions made, result in an inaccuracy.

The assumption of an all-or-nothing two state equilibrium could be inaccurate since a decent amount of flexibility is conceivable in the T10 template which might allow for the build-up of partially denaturated species to non-negligible quantities. In addition, a closer inspection of the experimental data, especially the shape of the curve, appears to be more in line with a lower number of binding sites in the denaturation process. When fitting the data assuming five binding sites, a more satisfactory fit to the experimental data is observed but this observation cannot be logically explained and should not provide an accurate measure of $K_{\mathrm{dn}}$ (Figure 6.23 , left).
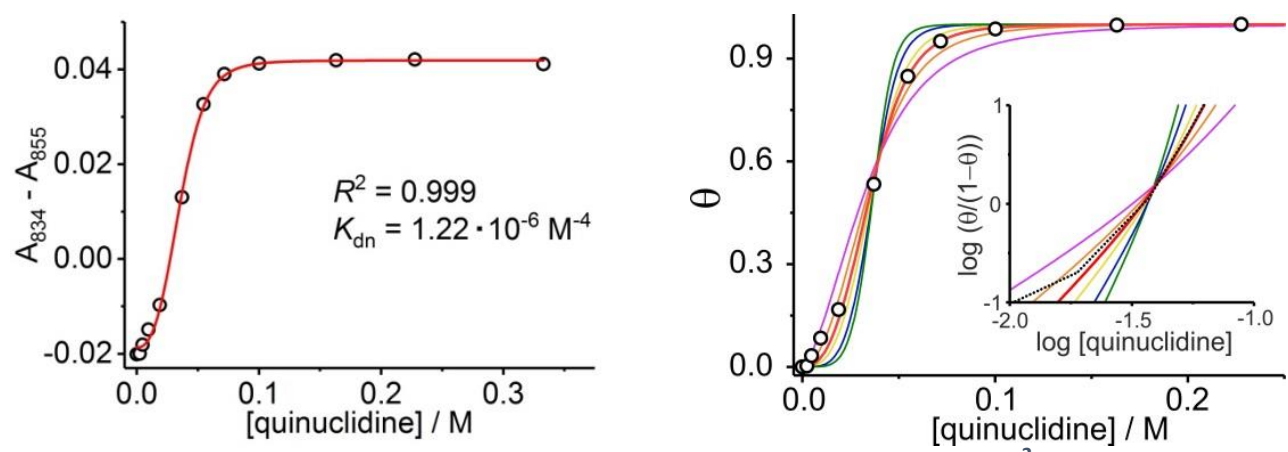

Figure 6.23: The UV-vis-NIR denaturation titration of $b$-P10.T10 with quinuclidine. $R^{2}=0.999$. (Toluene, $298 \mathrm{~K}$, $\left.[b-\mathrm{P} 10 \cdot \mathrm{T} 10]=0.925 \mu \mathrm{M}, \mathrm{n}=5, K=1.22 \cdot 10^{-6} \mathrm{M}^{-4}\right)$ and the extent of denaturation of $b$-P10.T10 $(\theta)$ derived from the absorption difference at $834-855 \mathrm{~nm}$ (black dots) fitted to the calculated curves for $N=10$ (green line), 8 (blue line), 6 (yellow line), 5 (red line), 4 (orange line), and 3 (purple line) binding sites. The inset shows the Hill plot with fits for the same values of $N$. 
To investigate the cooperativity in the system, the Hill coefficient was determined (Figure 6.23, right). ${ }^{208,209}$ The Hill coefficient is commonly used to estimate the number of ligand binding sites on a host. However, the conditions under which the Hill coefficient provides an accurate estimate are strict. Therefore it is more appropriate to use the Hill coefficient as an interaction coefficient, reflecting cooperativity rather than as a reliable estimate of the number of binding sites. In the case of positive cooperativity ( $n>1$ ), the Hill coefficient can be used to determine a minimum estimate of the number of binding sites present. A positive Hill coefficient of 3.44 was calculated for the denaturation of $\boldsymbol{b}$-P10.T10 with $N=5$ binding sites. While this is not a quantitative number, when compared to the denaturation of c-P6-T6, for which a value of 3.94 was found, it shows that the level of cooperativity for T10 binding to $\boldsymbol{b}$-P10 is lower, indicating that the ten binding sites do not bind to the host efficiently.

While the data do not provide an accurate measure of the binding strength between $\boldsymbol{b}$-P10 and T10, the concentration quinuclidine required to denaturate the $\boldsymbol{b}$-P10.T10 is approximately four-fold smaller than the concentration needed to denaturate b-P10.T6 indicating a weaker binding interaction. The fact that the cooperativity of T10 to $b$-P10 is lower than for T6, in addition to the weaker binding affinity reveals that despite the higher number of binding sites, T10 is less ideal guest for the ball potentially due to its lower degree of pre-organisation which could account for the discovery that T10 failed to efficiently prepare $\boldsymbol{b}$-P10 from simple precursors. However, it is likely that the formation of $\boldsymbol{b}-\mathbf{P} 10$ via the template directed synthesis with T10 failed due to the choice in solubilising groups and this hypothesis is currently under investigation.

DFT calculations (B3LYP/6-31G(d)) indicate that the HOMO of $\boldsymbol{b}$-P10 is located most prominently on the tetraalkynylporphyrin with some electron density spreading to the neighbouring porphyrins. The LUMOs are degenerate and located on the two individual rings exclusively. Since the two orbitals are degenerate in energy, the electron density is distributed equally over the entire $\pi$-system. Photophysical studies are currently underway. 


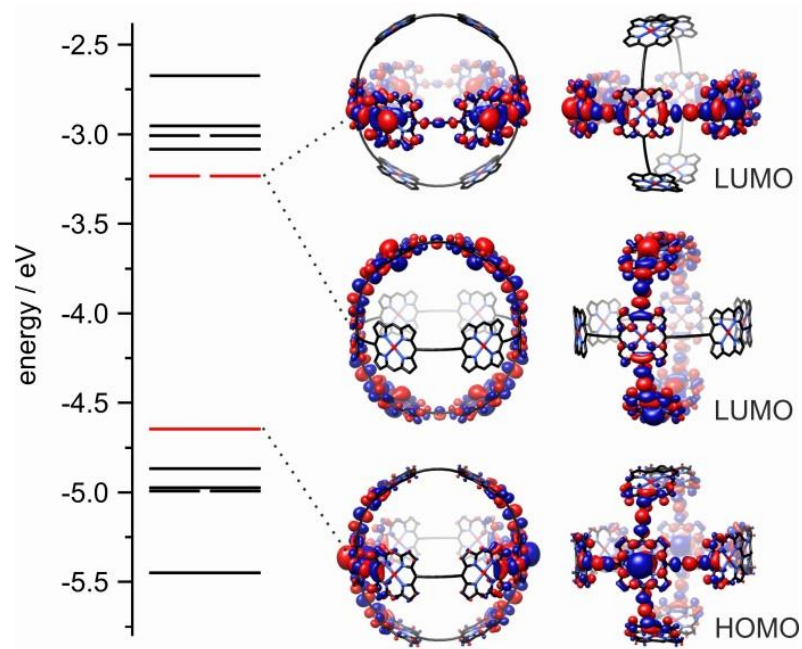

Figure 6.24: Degenerate LUMOs and HOMO of $b$-P10 calculated at the B3LYP/6-31G(d) level of theory shown with a density isovalue of 0.008 together with their corresponding energy levels (degenerate energy levels are indicated by broken lines). Aryl groups were omitted to simplify the calculations.

\subsection{Conclusion}

The $\mathrm{D}_{4 h}$ symmetric 10 -porphyrin nanoball $\boldsymbol{b}$-P10 was prepared through a rationally designed, template directed synthetic strategy. We utilised a combination of zinc and magnesium metal centres, which allowed for the selective demetallation of the magnesium porphyrins, to prepare the target nanoball. The high symmetry in the molecule resulted in a remarkably simple ${ }^{1} \mathrm{H}$ NMR spectrum which was fully assigned with the aid of 2D-NOESY experiments. The accessibility of the cavity was probed by UV-vis-NIR titrations which revealed the insertion of both the T6 template and the three-dimensional T10 template. Interestingly, the flexibility of the T10 template resulted in a weaker binding interaction despite the high number of binding sites.

Photophysical measurements are currently underway and might provide insights into the migration of energy between the two rings.

\subsection{Experimental procedures and technical details}

\subsubsection{General experimental}

Dry toluene and THF were obtained by passing the solvents through columns of alumina, under nitrogen. Diisopropylamine $\left(i-\operatorname{Pr}_{2} \mathrm{NH}\right)$ was distilled from $\mathrm{CaH}_{2}$ and kept over activated molecular sieves (3 $\AA, 8-12$ mesh). Unless specified otherwise, all other solvents were used as commercially supplied. Flash chromatography was 
carried out on silica gel 60 under positive pressure. Analytical thin-layer chromatography was carried out on aluminum-backed silica gel 60 F254 plates. Visualisation was achieved using UV light when necessary.

All UV-vis-NIR spectra were recorded in solution using a Perkin-Lambda 20 spectrometer $(1 \mathrm{~cm}$ path length quartz cell). Toluene was used for all titrations without any further purification.

Unless stated otherwise, ${ }^{1} \mathrm{H} /{ }^{13} \mathrm{C}$ NMR spectra were recorded at $298 \mathrm{~K}$ using a Bruker AV400 (400/100 MHz) instrument. ${ }^{1} \mathrm{H}$, and ${ }^{13} \mathrm{C}$ NMR spectra are reported in ppm; coupling constants are given in Hertz, to the nearest $0.1 \mathrm{~Hz}$. The solvent used was $\mathrm{CDCl}_{3}$.

MALDI-ToF spectra were measured at the EPSRC National Mass Spectrometry service (Swansea) using the Applied Biosystems Voyager DE-STR or at the University of Oxford using Waters MALDI Micro MX spectrometer utilising dithranol as a matrix.

\subsubsection{Synthetic procedures}

\section{Zinc 5,15-(meta-trihexylsilyl-phenyl)-porphyrin (6.2)}

3-(Trihexylsilyl)benzaldehyde 6.1 (5.30 g, $13.7 \mathrm{mmol})$ and dipyrromethane $2.1(2.00 \mathrm{~g}, 13.7 \mathrm{mmol})$ were dissolved in a fresh Winchester bottle of $\mathrm{CH}_{2} \mathrm{Cl}_{2}(2.5 \mathrm{~L})$. The solution was degassed by bubbling the solution with $\mathrm{N}_{2}$ for $20 \mathrm{~min}$ and stirring under nitrogen. Trifluoroacetic acid $(1.8 \mathrm{~mL}, 23.4 \mathrm{mmol})$ was added and the reaction mixture stirred in the dark for $3 \mathrm{~h}$. DDQ (5.36 g, $23.6 \mathrm{mmol})$ was added and stirring continued for $\left(\mathrm{C}_{6} \mathrm{H}_{13}\right)_{3} \mathrm{Si}$

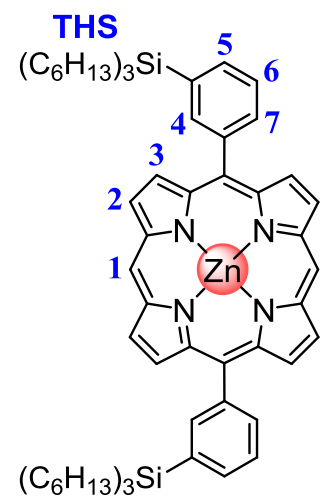
$20 \mathrm{~min}$. After quenching of the acid by addition of triethylamine $(14 \mathrm{~mL})$ the mixture was concentrated to a volume of about $300 \mathrm{~mL}$ on the rotary evaporator, passed over a silica plug $\left(\mathrm{CH}_{2} \mathrm{Cl}_{2}\right)$. The product was redissolved in $\mathrm{CHCl}_{3}(300 \mathrm{~mL})$. $\mathrm{Zn}(\mathrm{OAc})_{2} \cdot 2 \mathrm{H}_{2} \mathrm{O}(4.04 \mathrm{~g}, 19.0 \mathrm{mmol})$ was dissolved in methanol $(44 \mathrm{~mL})$ and added to the reaction mixture. The reaction was stirred at $40{ }^{\circ} \mathrm{C}$ for $2 \mathrm{~h}$. The mixture was passed through a short plug of silica gel (1\% pyridine in $\mathrm{CH}_{2} \mathrm{Cl}_{2}$ ) to give 6.2 as a pink oil $(4.58 \mathrm{~g}, 62 \%)$. 
${ }^{1}$ H NMR $\left(400 \mathrm{MHz}, \mathrm{CDCl}_{3}, 298 \mathrm{~K}\right): \delta_{\mathrm{H}}(\mathrm{ppm}) 10.24(2 \mathrm{H}, \mathrm{s}, \mathrm{H} 1), 9.39(4 \mathrm{H}, \mathrm{d}, J=4.4 \mathrm{~Hz}$, H2), $9.09(4 \mathrm{H}, \mathrm{d}, J=4.4 \mathrm{~Hz}, \mathrm{H} 3), 8.39(2 \mathrm{H}, \mathrm{m}, \mathrm{H} 7), 8.22(2 \mathrm{H}, \mathrm{m}, \mathrm{H} 4), 7.90(2 \mathrm{H}, \mathrm{m}, \mathrm{H} 5)$, $7.75(2 \mathrm{H}, \mathrm{m}, \mathrm{H} 6), 1.63-0.80(78 \mathrm{H}, \mathrm{m}, \mathrm{THS})$.

MALDI-TOF: $m / z=1,091\left(\mathrm{C}_{68} \mathrm{H}_{96} \mathrm{~N}_{4} \mathrm{Si}_{2} \mathrm{Zn}, \mathrm{M}^{+}\right.$requires 1,091$)$.

\section{Zinc 5,15- (meta-trihexylsilyl-phenyl)-10,20-dibromo-porphyrin (6.3)}

To a solution of 6.2 (4.58 g, $4.20 \mathrm{mmol})$ in $\mathrm{CHCl}_{3}(650 \mathrm{~mL})$ and pyridine $(65 \mathrm{~mL})$ was added freshly recrystallised $\mathrm{N}$-bromosuccinimide $(1.49 \mathrm{~g}, 8.40 \mathrm{mmol})$. After stirring for $5 \mathrm{~min}$, acetone $(100 \mathrm{~mL})$ was added to quench the reaction. The mixture was passed through a short plug of silica gel $\left(\mathrm{CHCl}_{3}\right)$ to give 6.3 as a green oil $(4.26 \mathrm{~g}, 81 \%)$.

${ }^{1}$ H NMR $\left(400 \mathrm{MHz}, \mathrm{CDCl}_{3}, 298 \mathrm{~K}\right): \delta_{\mathrm{H}}(\mathrm{ppm}) 9.66(4 \mathrm{H}, \mathrm{d}$,

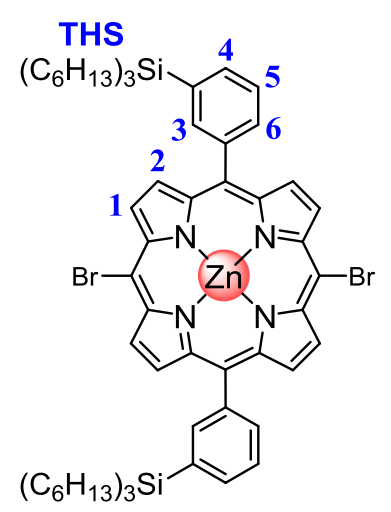
$J=4.7 \mathrm{~Hz}, \mathrm{H} 1), 8.87(4 \mathrm{H}, \mathrm{d}, J=4.7 \mathrm{~Hz}, \mathrm{H} 2), 8.25(2 \mathrm{H}, \mathrm{m}, \mathrm{H} 6), 8.12(2 \mathrm{H}, \mathrm{m}, \mathrm{H} 3), 7.88$ $(2 \mathrm{H}, \mathrm{m}, \mathrm{H} 4), 7.72(2 \mathrm{H}, \mathrm{m}, \mathrm{H} 5), 1.55-0.81(78 \mathrm{H}, \mathrm{m}, \mathrm{THS})$.

MALDI-TOF: $m / z=1,249\left(\mathrm{C}_{68} \mathrm{H}_{94} \mathrm{Br}_{2} \mathrm{~N}_{4} \mathrm{Si}_{2} \mathrm{Zn}, \mathrm{M}^{+}\right.$requires 1,248).

\section{Zinc 5,15-bis-(meta-trihexylsilyl-phenyl)-10,20-bis-} cyanopropyldimethylsilanylethynyl-porphyrin (6.4)

$\mathrm{Pd}_{2} \mathrm{dba}_{3}$ (312 $\mathrm{mg}, 0.34 \mathrm{mmol}$ ), Cul (195 mg, $1.02 \mathrm{mmol}), \mathrm{PPh}_{3}(268 \mathrm{mg}$, $1.02 \mathrm{mmol})$ and $6.3(4.26 \mathrm{~g}, 3.41 \mathrm{mmol})$ were placed under argon. Dry toluene $(112 \mathrm{~mL})$ and dry $i-\operatorname{Pr}_{2} \mathrm{NH}(112 \mathrm{~mL})$ were added and the reaction mixture deoxygenated [vacuum freeze/thaw

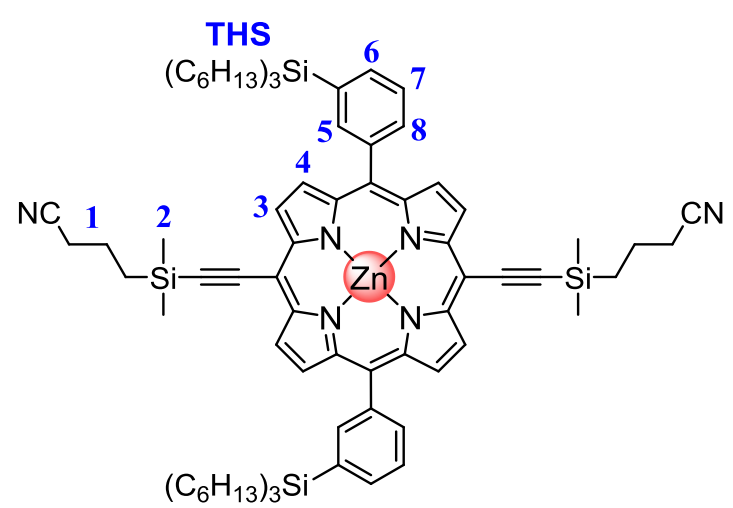
3 times]. cyanopropyldimethylsilyl acetylene $(1.55 \mathrm{~g}, 10.23 \mathrm{mmol})$ was added by syringe. The reaction mixture was stirred at $50{ }^{\circ} \mathrm{C}$ for $3 \mathrm{~h}$. The solvents were removed and the residue was passed through a short silica gel column ( $1 \%$ pyridine in $\mathrm{CHCl}_{3}$ ) to give 6.4 as a green oil ( $4.43 \mathrm{~g}, 93 \%)$.

${ }^{1} \mathrm{H}$ NMR $\left(400 \mathrm{MHz}, \mathrm{CDCl}_{3}, 298 \mathrm{~K}\right): \delta_{\mathrm{H}}(\mathrm{ppm}) 9.59(4 \mathrm{H}, \mathrm{d}, J=4.5 \mathrm{~Hz}, \mathrm{H} 3), 8.86(4 \mathrm{H}, \mathrm{d}$, $J=4.5 \mathrm{~Hz}, \mathrm{H} 4), 8.27(2 \mathrm{H}, \mathrm{m}, \mathrm{H} 8), 8.12(2 \mathrm{H}, \mathrm{m}, \mathrm{H} 5), 7.89(2 \mathrm{H}, \mathrm{m}, \mathrm{H} 6), 7.73(2 \mathrm{H}, \mathrm{m}, \mathrm{H} 7)$, 
$2.58(4 \mathrm{H}, \mathrm{t}, J=7.0 \mathrm{~Hz}, \mathrm{H} 1), 2.15(4 \mathrm{H}, \mathrm{m}, \mathrm{H} 1), 1.54-0.83(82 \mathrm{H}, \mathrm{m}, \mathrm{H} 1$ and THS $), 0.60$ $(12 \mathrm{H}, \mathrm{s}, \mathrm{H} 2)$.

Free base 5,15- (meta-trihexylsilyl-phenyl)-10,20-dibromo-porphyrin (6.5)

6.3 (2.24 g, $1.79 \mathrm{mmol})$ was dissolved in $\mathrm{CHCl}_{3}(300 \mathrm{~mL})$.

Trifluoroacetic acid $(1.4 \mathrm{~mL})$ was mixed with $\mathrm{CHCl}_{3}(14 \mathrm{~mL})$ to give a $10 \%$ solution). The TFA solution was added dropwise to the porphyrin solution and the reaction mixture was stirred at room temperature under nitrogen for $15 \mathrm{~min}$. UV and TLC indicated the completion of the reaction and the mixture was passed immediately through a short plug of

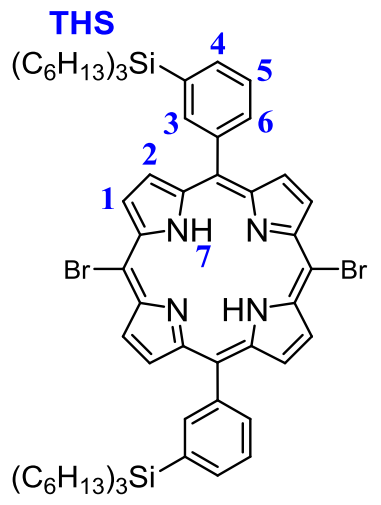
silica gel (1\% pyridine in $\mathrm{CHCl}_{3}$ ) to give 6.5 as a brown oil $(1.77 \mathrm{~g}, 83 \%)$.

${ }^{1} \mathrm{H}$ NMR $\left(400 \mathrm{MHz}, \mathrm{CDCl}_{3}, 298 \mathrm{~K}\right): \delta_{\mathrm{H}}(\mathrm{ppm}) 9.65(4 \mathrm{H}, \mathrm{d}, J=4.4 \mathrm{~Hz}, \mathrm{H1}), 8.89(4 \mathrm{H}, \mathrm{d}$, $J=4.4 \mathrm{~Hz}, \mathrm{H} 2), 8.34(2 \mathrm{H}, \mathrm{m}, \mathrm{H} 6), 8.18(2 \mathrm{H}, \mathrm{m}, \mathrm{H} 3), 7.98(2 \mathrm{H}, \mathrm{m}, \mathrm{H} 4), 7.80(2 \mathrm{H}, \mathrm{m}, \mathrm{H} 5)$, 1.62-0.89 (78H, m, THS).

MALDI-TOF: $m / z=1,187\left(\mathrm{C}_{68} \mathrm{H}_{96} \mathrm{Br}_{2} \mathrm{~N}_{4} \mathrm{Si}_{2}, \mathrm{M}^{+}\right.$requires 1,185$)$.

\section{Magnesium 5,15- (meta-trihexylsilyl-phenyl)-10,20-dibromo-porphyrin (6.6)}

6.5 (1.58 g, $1.33 \mathrm{mmol}$ ) was dissolved in $\mathrm{CHCl}_{3}(180 \mathrm{~mL})$ and triethylamine $(1.8 \mathrm{~mL})$. Magnesium iodide $(1.87 \mathrm{~g}$, $6.66 \mathrm{mmol}$ ) was added and the solution was stirred for $2 \mathrm{~h}$. The organic phase was washed with water $(3 \times 300 \mathrm{~mL})$, dried $\left(\mathrm{MgSO}_{4}\right)$ and filtered. The product was passed through a short plug of silica gel (1\% pyridine in $\mathrm{CHCl}_{3}$ ) to give 6.6 as a green oil (1.41 g, 88\%).

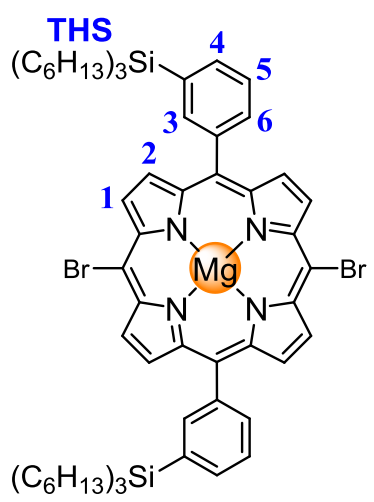

${ }^{1} \mathrm{H}$ NMR $\left(400 \mathrm{MHz}, \mathrm{CDCl}_{3}, 298 \mathrm{~K}\right): \delta_{\mathrm{H}}(\mathrm{ppm}) 9.68(4 \mathrm{H}, \mathrm{d}, J=4.6 \mathrm{~Hz}, \mathrm{H} 1), 8.89(4 \mathrm{H}, \mathrm{d}$, $J=4.6 \mathrm{~Hz}, \mathrm{H} 2), 8.29(2 \mathrm{H}, \mathrm{m}, \mathrm{H} 6), 8.15(2 \mathrm{H}, \mathrm{m}, \mathrm{H} 3), 7.91(2 \mathrm{H}, \mathrm{m}, \mathrm{H} 4), 7.74(2 \mathrm{H}, \mathrm{m}, \mathrm{H} 5)$, $1.58-0.80(78 \mathrm{H}, \mathrm{m}, \mathrm{THS})$.

MALDI-TOF: $m / z=1,207\left(\mathrm{C}_{68} \mathrm{H}_{94} \mathrm{Br}_{2} \mathrm{MgN}_{4} \mathrm{Si}_{4}, \mathrm{M}^{+}\right.$requires 1,207). 
Magnesium 5,15-bis-(meta-trihexylsilyl-phenyl)-10,20-bis-

cyanopropyldimethylsilanylethynyl-porphyrin (6.7)

$\mathrm{Pd}_{2} \mathrm{dba}_{3}$ (107 mg, $0.12 \mathrm{mmol}$ ), Cul

(67 mg, $0.35 \mathrm{mmol}), \mathrm{PPh}_{3}(92 \mathrm{mg}$,

$0.35 \mathrm{mmol})$ and $6.6(1.41 \mathrm{~g}, 1.17 \mathrm{mmol})$ were placed under argon. Dry toluene $(37.8 \mathrm{~mL})$ and dry $i-\mathrm{Pr}_{2} \mathrm{NH}(37.8 \mathrm{~mL})$ were added and the reaction mixture deoxygenated [vacuum freeze/thaw

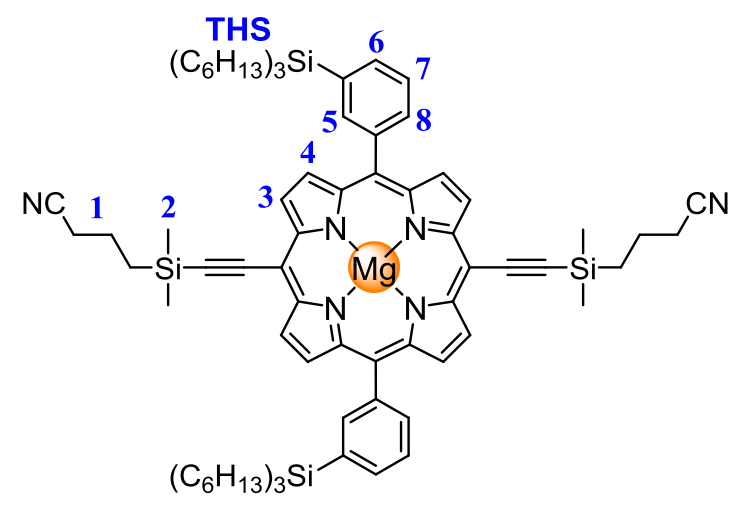
3 times]. cyanopropyldimethylsilyl acetylene $(566 \mathrm{mg}, 3.50 \mathrm{mmol}$ ) was added by syringe. The reaction mixture was stirred at $50{ }^{\circ} \mathrm{C}$ for $3 \mathrm{~h}$. The solvents were removed and the residue was passed through a short silica gel column ( $1 \%$ pyridine in $\mathrm{CHCl}_{3}$ ) to give 6.7 as a green oil (1.53 g, 97\%).

${ }^{1} \mathrm{H}$ NMR $\left(400 \mathrm{MHz}, \mathrm{CDCl}_{3}, 298 \mathrm{~K}\right): \delta_{\mathrm{H}}(\mathrm{ppm}) 9.59(4 \mathrm{H}, \mathrm{d}, J=4.5 \mathrm{~Hz}, \mathrm{H3}), 8.86(4 \mathrm{H}, \mathrm{d}$, $J=4.5 \mathrm{~Hz}, \mathrm{H} 4), 8.28(2 \mathrm{H}, \mathrm{m}, \mathrm{H} 8), 8.12(2 \mathrm{H}, \mathrm{m}, \mathrm{H} 5), 7.88(2 \mathrm{H}, \mathrm{m}, \mathrm{H} 6), 7.72(2 \mathrm{H}, \mathrm{m}, \mathrm{H} 7)$, $2.57(4 \mathrm{H}, \mathrm{t}, J=7.0 \mathrm{~Hz}, \mathrm{H} 1), 2.15(4 \mathrm{H}, \mathrm{m}, \mathrm{H} 1), 1.54-0.83(82 \mathrm{H}, \mathrm{m}, \mathrm{H} 1$ and THS), 0.60 $(12 \mathrm{H}, \mathrm{s}, \mathrm{H} 2)$.

MALDI-TOF: $m / z=1,349\left(\mathrm{C}_{84} \mathrm{H}_{118} \mathrm{MgN}_{6} \mathrm{Si}_{4}, \mathrm{M}^{+}\right.$requires 1,348$)$.

\section{Magnesium 5,15-bis-(meta-trihexylsilyl-phenyl)-10-ethynyl-20-} cyanopropyldimethylsilanylethynyl-porphyrin (6.8)

6.7 (0.86 g, $0.63 \mathrm{mmol}$ ) was dissolved in $\mathrm{CHCl}_{3}$ $(55 \mathrm{~mL})$ and ethanol (0.55 mL).

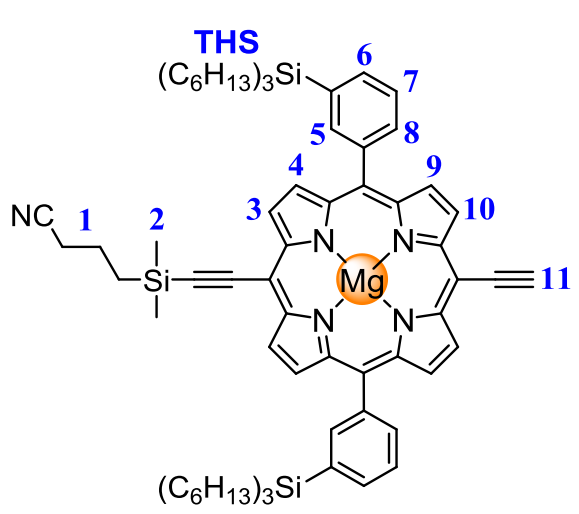
optimal product mixture was reached. The mixture immediately was passed through a short plug of silica gel (1\% pyridine in
$\mathrm{CHCl}_{3}$ ). Column chromatography $(10: 1: 1 \rightarrow 6: 1: 1=$ petroleum ether : ethyl acetate : pyridine) to give 6.8 as a green oil $(0.17 \mathrm{~g}, 21 \%)$. 
${ }^{1} \mathbf{H}$ NMR $\left(400 \mathrm{MHz} \mathrm{CDCl}_{3}, 298 \mathrm{~K}\right): \delta_{\mathrm{H}}(\mathrm{ppm}) 9.67(2 \mathrm{H}, \mathrm{d}, J=4.6 \mathrm{~Hz}, \mathrm{H} 3$ or $\mathrm{H} 10), 9.60$ $(2 \mathrm{H}, \mathrm{d}, J=4.6 \mathrm{~Hz}, \mathrm{H} 3$ or H10), $8.87(4 \mathrm{H}, \mathrm{d}, J=4.6 \mathrm{~Hz}, \mathrm{H} 4$ and $\mathrm{H} 9), 8.28(2 \mathrm{H}, \mathrm{m}, \mathrm{H} 8)$, $8.13(2 \mathrm{H}, \mathrm{m}, \mathrm{H} 5), 7.88(2 \mathrm{H}, \mathrm{m}, \mathrm{H} 6), 7.72(2 \mathrm{H}, \mathrm{m}, \mathrm{H} 7), 4.07(1 \mathrm{H}, \mathrm{s}, \mathrm{H} 11), 2.57(2 \mathrm{H}, \mathrm{t}$, $J=6.9 \mathrm{~Hz}, \mathrm{H} 1), 2.16(2 \mathrm{H}, \mathrm{m}, \mathrm{H} 1), 1.56-0.82(80 \mathrm{H}, \mathrm{m}, \mathrm{H} 1$ and THS), $0.61(6 \mathrm{H}, \mathrm{s}, \mathrm{H} 2)$.

\section{Porphyrin trimer (6.9)}

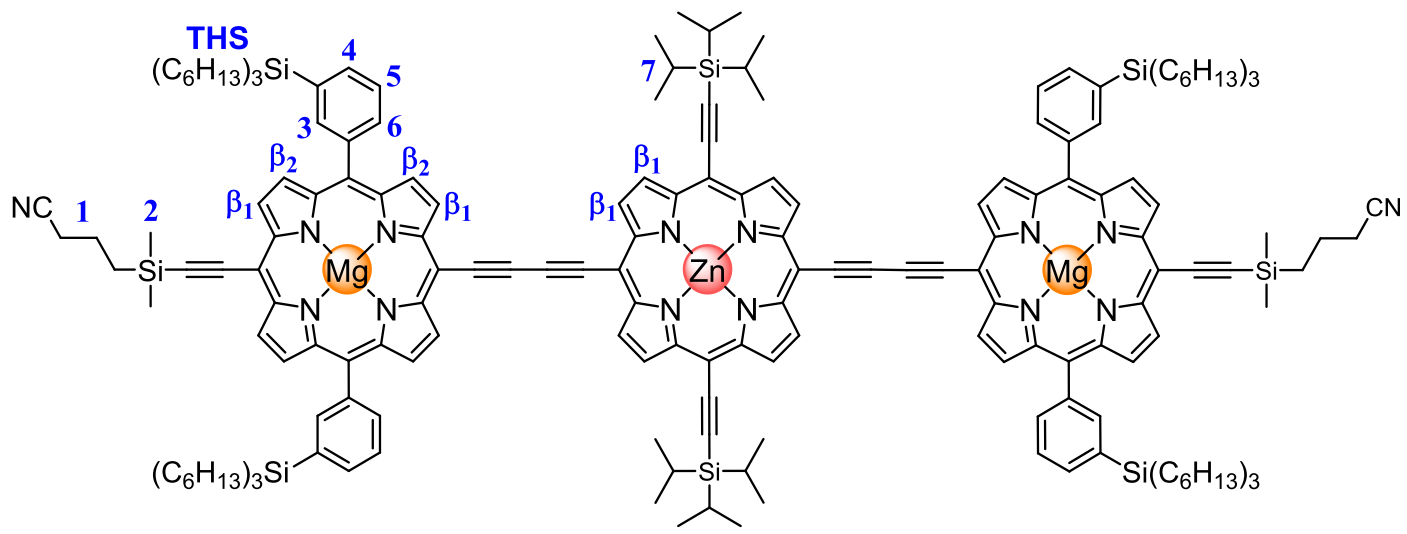

Porphyrin 6.8 (1.29 g, $1.05 \mathrm{mmol})$ and porphyrin 5.12 (165 mg, $0.21 \mathrm{mmol})$ were dissolved in $\mathrm{CHCl}_{3}(140 \mathrm{~mL})$. A catalyst solution was prepared by dissolving $\mathrm{PdCl}_{2}\left(\mathrm{PPh}_{3}\right)_{2}$ (111 mg, $\left.0.16 \mathrm{mmol}\right)$, Cul (110 mg, $0.58 \mathrm{mmol}$ ) and 1,4-benzoquinone (326 mg, $3.02 \mathrm{mmol})$ in $\mathrm{CHCl}_{3}(75 \mathrm{~mL})$ and $i-\mathrm{Pr}_{2} \mathrm{NH}(3.7 \mathrm{~mL})$. The catalyst solution was added to the porphyrin solution and the reaction mixture was stirred at room temperature for $1 \mathrm{~h}$. The crude reaction mixture was passed through a short plug of silica gel ( $5 \%$ pyridine in $\mathrm{CH}_{2} \mathrm{Cl}_{2}$ ), passed over a size exclusion column on Biobeads SX-1 (1\% pyridine in toluene), and finally the oligomers were separated by recycling GPC (1\% pyridine in toluene) to give the porphyrin trimer 6.9 as a dark oily solid (190 mg, 28\%).

${ }^{1} \mathrm{H}$ NMR $\left(400 \mathrm{MHz}, \mathrm{CDCl}_{3}, 298 \mathrm{~K}\right): \delta_{\mathrm{H}}(\mathrm{ppm}) 9.92\left(4 \mathrm{H}, \mathrm{d}, J=4.5 \mathrm{~Hz}, \mathrm{H} \beta_{1}\right), 9.89(4 \mathrm{H}, \mathrm{d}$, $\left.J=4.5 \mathrm{~Hz}, H \beta_{1}\right), 9.75\left(4 \mathrm{H}, \mathrm{d}, J=4.5 \mathrm{~Hz}, H \beta_{1}\right), 9.62\left(4 \mathrm{H}, \mathrm{d}, J=4.5 \mathrm{~Hz}, H \beta_{1}\right), 8.90(4 \mathrm{H}, \mathrm{d}$, $\left.J=4.5 \mathrm{~Hz}, H \beta_{2}\right), 8.89\left(4 \mathrm{H}, \mathrm{d}, J=4.5 \mathrm{~Hz}, \mathrm{H} \beta_{2}\right), 8.36(4 \mathrm{H}, \mathrm{d}, J=7.3 \mathrm{~Hz}, \mathrm{H} 3), 8.21(4 \mathrm{H}, \mathrm{m}$, H6), $7.93(4 \mathrm{H}, \mathrm{d}, J=7.3 \mathrm{~Hz}, \mathrm{H} 4), 7.79(4 \mathrm{H}, \mathrm{t}, J=7.3 \mathrm{~Hz}, \mathrm{H} 5), 2.61(4 \mathrm{H}, \mathrm{t}, J=6.9 \mathrm{~Hz}, \mathrm{H} 1)$, $2.19(4 \mathrm{H}, \mathrm{m}, \mathrm{H} 1), 1.59-0.85(202 \mathrm{H}, \mathrm{m}, \mathrm{H} 1$ and $\mathrm{H} 7$ and $\mathrm{THS}), 0.64(12 \mathrm{H}, \mathrm{s}, \mathrm{H} 2)$.

MALDI-TOF: $m / z=3,225\left(\mathrm{C}_{202} \mathrm{H}_{262} \mathrm{Mg}_{2} \mathrm{~N}_{14} \mathrm{Si}_{8} \mathrm{Zn}, \mathrm{M}^{+}\right.$requires 3,225). 


\section{Deprotected trimer (6.10)}

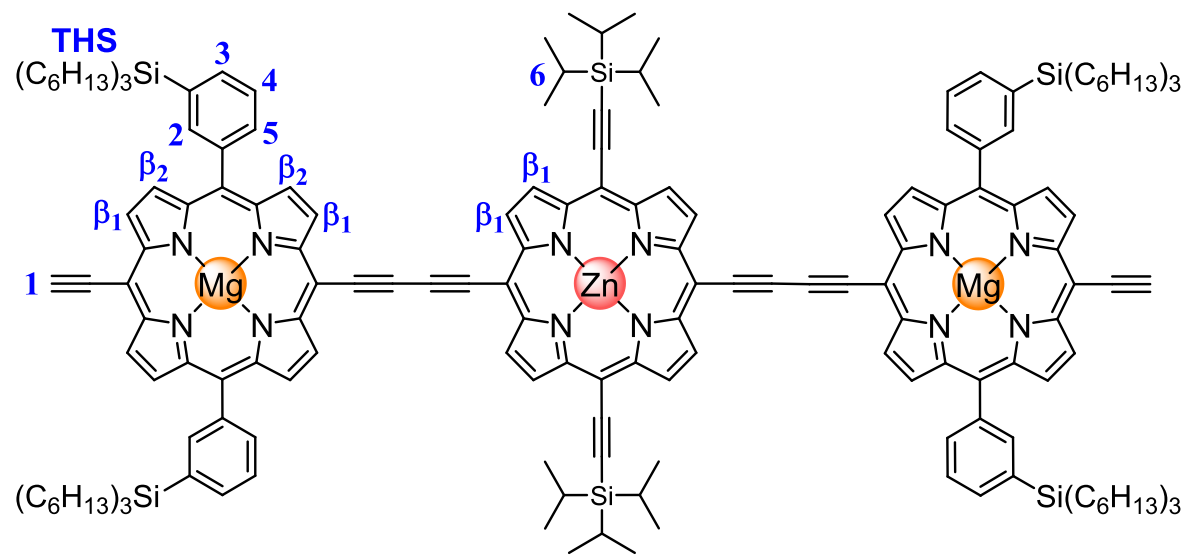

6.9 (190 mg, $59 \mu \mathrm{mol})$ was dissolved in THF $(40 \mathrm{~mL}) . \mathrm{K}_{2} \mathrm{CO}_{3}(163 \mathrm{mg}, 1.18 \mathrm{mmol})$ was dissolved in methanol $(17 \mathrm{~mL})$. The solutions were combined and the reaction mixture was stirred at room temperature for $1 \mathrm{~h}$. The reaction mixture was passed through a short plug of silica gel (5\% pyridine in $\mathrm{CH}_{2} \mathrm{Cl}_{2}$ ) gave 6.10 as a dark oil (160 mg, 97\%).

${ }^{1} \mathrm{H}$ NMR $\left(400 \mathrm{MHz}, \mathrm{CDCl}_{3}, 298 \mathrm{~K}\right): \delta_{\mathrm{H}}(\mathrm{ppm}) 9.93\left(4 \mathrm{H}, \mathrm{d}, J=4.5 \mathrm{~Hz}, \mathrm{H} \beta_{1}\right), 9.89(4 \mathrm{H}, \mathrm{d}$, $\left.J=4.5 \mathrm{~Hz}, H \beta_{1}\right), 9.75\left(4 \mathrm{H}, \mathrm{d}, J=4.5 \mathrm{~Hz}, \mathrm{H} \beta_{1}\right), 9.69\left(4 \mathrm{H}, \mathrm{d}, J=4.5 \mathrm{~Hz}, \mathrm{H} \beta_{1}\right), 9.01(4 \mathrm{H}, \mathrm{d}$, $\left.J=4.5 \mathrm{~Hz}, \mathrm{H} \beta_{2}\right), 8.90\left(4 \mathrm{H}, \mathrm{d}, J=4.5 \mathrm{~Hz}, \mathrm{H} \beta_{2}\right), 8.36(4 \mathrm{H}, \mathrm{d}, J=6.7 \mathrm{~Hz}, \mathrm{H} 2), 8.22(4 \mathrm{H}, \mathrm{m}$, H5), $7.93(4 \mathrm{H}, \mathrm{d}, J=7.5 \mathrm{~Hz}, \mathrm{H} 3), 7.79(4 \mathrm{H}, \mathrm{t}, J=7.4 \mathrm{~Hz}, \mathrm{H} 4), 4.21(2 \mathrm{H}, \mathrm{s}, \mathrm{H} 1), 1.60-0.86$ (198H, m, H6 and THS).

MALDI-TOF: $m / z=2,975\left(\mathrm{C}_{190} \mathrm{H}_{240} \mathrm{Mg}_{2} \mathrm{~N}_{12} \mathrm{Si}_{6} \mathrm{Zn}, \mathrm{M}^{+}\right.$requires 2,975$)$.

\section{Ring (6.11)}

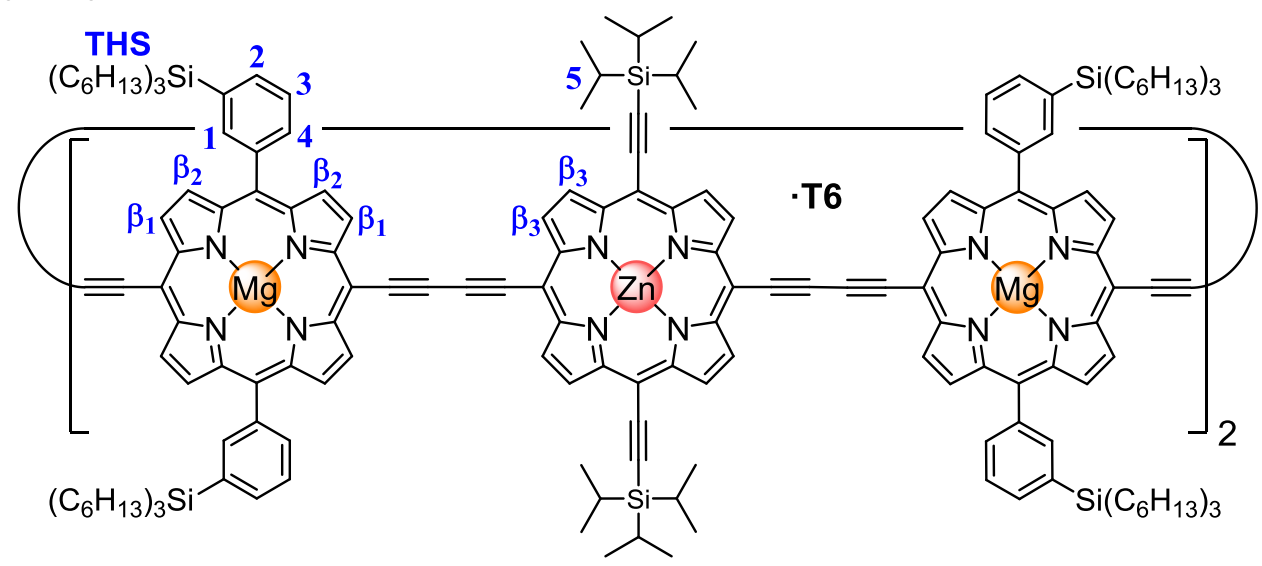

Fully deprotected trimer 6.10 (160 mg, $57 \mu \mathrm{mol}$ ) was dissolved in $\mathrm{CHCl}_{3}(44 \mathrm{~mL})$. Hexadentate template T6 (29 mg, $29 \mu \mathrm{mol}$ ) was added and the resulting mixture was sonicated for $0.5 \mathrm{~h}$. The solvent was removed, dried under vacuum and redissolved in 
$\mathrm{CHCl}_{3}$ (44 mL). A catalyst solution was prepared by dissolving $\mathrm{Pd}\left(\mathrm{PPh}_{3}\right)_{2} \mathrm{Cl}_{2}(12 \mathrm{mg}$, $17 \mu \mathrm{mol}$ ), Cul (33 mg, $172 \mu \mathrm{mol}$ ) and 1,4-benzoquinone (25 mg, $229 \mu \mathrm{mol}$ ) in $\mathrm{CHCl}_{3}$ $(37 \mathrm{~mL})$ and freshly distilled $i-\mathrm{Pr}_{2} \mathrm{NH}(2.0 \mathrm{~mL})$. This solution was added to the reaction mixture, which was stirred vigorously at room temperature for $16 \mathrm{~h}$. The crude reaction solution was purified by silica column chromatography $(5 \%$ pyridine in $\mathrm{CH}_{2} \mathrm{Cl}_{2}$ ), size exclusion column on Biobeads SX-1 (1\% pyridine in toluene), and finally by recycling GPC (1\% pyridine in toluene) to give 6.11 as a red solid (52 $\mathrm{mg}, 26 \%$ ).

${ }^{1} \mathrm{H}$ NMR $\left(400 \mathrm{MHz}, \mathrm{CDCl}_{3}, 298 \mathrm{~K}\right): \delta_{\mathrm{H}}(\mathrm{ppm})$ 9.61-9.55 (16H, m, $\left.\mathrm{H} \beta_{1}\right)$, 9.51-9.45 (16H, $\left.m, H \beta_{3}\right), 8.82-8.72\left(16 \mathrm{H}, \mathrm{m}, \mathrm{H} \beta_{2}\right), 8.32-8.25\left(3 \mathrm{H}, \mathrm{m}, \mathrm{H} 1^{*}\right), 8.21-8.12(9 \mathrm{H}, \mathrm{m}, \mathrm{H} 4$ and $\left.H 4^{*}\right)$, 8.05-7.97 (3H, m, H1), 7.93-7.86 (8H, m, H2), 7.79-7.69 (8H, m, H3), 5.56-5.48 $\left(12 \mathrm{H}, \mathrm{m}, \mathrm{T} 6_{\delta}\right), 5.48-5.38\left(12 \mathrm{H}, \mathrm{m}, \mathrm{T} 6_{\chi}\right), 5.02-4.93\left(12 \mathrm{H}, \mathrm{m}, \mathrm{T} 6_{\beta}\right), 2.41-2.27(12 \mathrm{H}, \mathrm{m}$, T6 $\alpha$ ), 1.58-0.82 $(396 \mathrm{H}, \mathrm{m}, \mathrm{THS}$ and H5). (Signals denoted with * correspond to aryl protons with the THS solubilising chain pointing towards the centre of the ring).

MALDI-TOF: $m / z=6,945\left(\mathrm{C}_{452} \mathrm{H}_{524} \mathrm{Mg}_{4} \mathrm{~N}_{30} \mathrm{Si}_{12} \mathrm{Zn}_{2}, \mathrm{M}^{+}\right.$requires 6,942).

$\lambda_{\text {max }}\left(\mathrm{CHCl}_{3}\right) / \mathrm{nm} \log (\varepsilon): 432$ (5.72), 490 (5.95), 770 (5.78), 806 (5.95), 849 (5.90).

\section{Ring (6.12)}

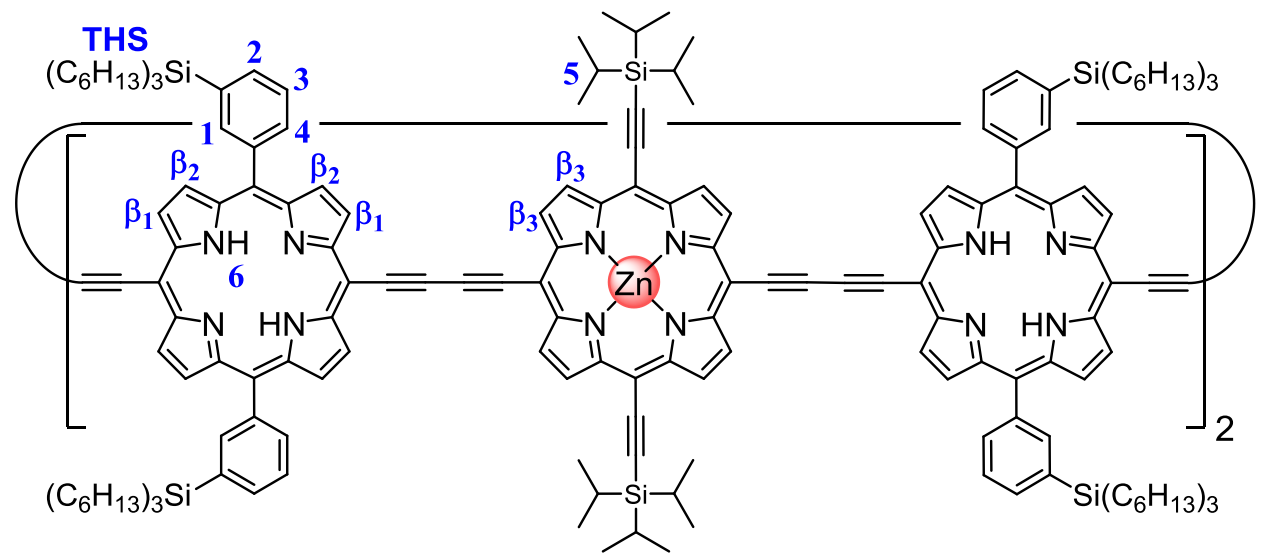

Step 1: A size exclusion column (300 mm length, $20 \mathrm{~mm}$ diameter) with Biobeads SX-1 was prepared in pyridine. A saturated DABCO solution in pyridine was prepared which was used to saturate the top of the column with DABCO (ca. $30 \%$ of the column length). 6.11 (52 mg, $7.5 \mu \mathrm{mol}$ ) was dissolved in the minimal amount of pyridine and loaded on the column. The product was eluted with the saturated pyridine/DABCO solution ( $c a .10 \%$ of the column length) followed by eluting with 
pure pyridine. The colored band corresponding to a mixture of starting ring 6.11 and template free $6.11^{*}$ was collected and the solvent was removed.

Step 2: The mixture was dissolved in DCM $(50 \mathrm{~mL})$ and glacial acetic acid $(50 \mathrm{~mL})$ was added. The reaction mixture was stirred for $30 \mathrm{~min}$. after which the organic phase was diluted with chloroform $(50 \mathrm{~mL})$ and washed with water $(2 \times 200 \mathrm{~mL})$, saturated sodium carbonate solution (aq. $200 \mathrm{~mL}$ ), and brine $(100 \mathrm{~mL}$ ). The solution was dried with sodium sulfate and the solvent was removed.

Approximately $30 \%$ of the template is removed in Step 1 but we cannot simply repeat the SEC column since the T6 template that is removed is picked up by the ring not containing the template which only results in a marginal improvement of the yield. Step 2 solely removes the magnesium centres from porphyrins not complexed to T6. As a result, only the template free ring is demetallated. When Step 1 is now repeated, the T6 that is removed in the course of the SEC column cannot be taken up by template free ring because there are not enough metal centres to bind the template. Therefore, repeating Step 1 and 2 several times results in full removal of the templates and magnesium centres. Step 1 and 2 were repeated 6 times to remove all traces of the template and magnesium centres to yield the target $\mathbf{4 . 1 2}$ (32 mg, 74\%).
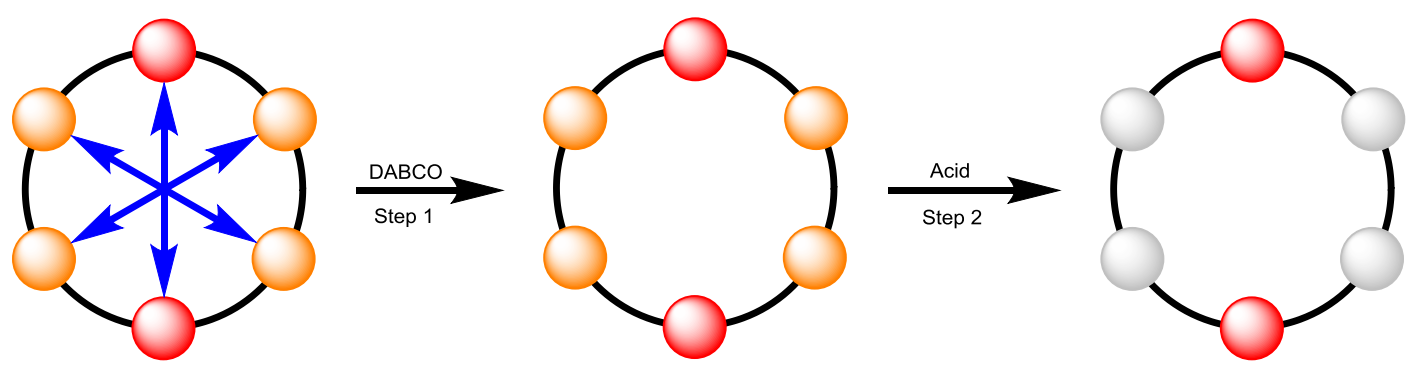

${ }^{1} \mathrm{H}$ NMR $\left(400 \mathrm{MHz}, \mathrm{CDCl}_{3}, 298 \mathrm{~K}\right): \delta_{\mathrm{H}}(\mathrm{ppm}) 9.62\left(8 \mathrm{H}, \mathrm{d}, J=4.7 \mathrm{~Hz}, \mathrm{H} \beta_{1}\right), 9.59(8 \mathrm{H}, \mathrm{d}$, $\left.J=4.7 \mathrm{~Hz}, H \beta_{1}\right), 9.51\left(8 \mathrm{H}, \mathrm{d}, J=4.3 \mathrm{~Hz}, \mathrm{H} \beta_{2}\right), 9.48\left(8 \mathrm{H}, \mathrm{d}, J=4.3 \mathrm{~Hz}, \mathrm{H} \beta_{2}\right), 8.77(8 \mathrm{H}, \mathrm{d}$, $\left.J=4.7 \mathrm{~Hz}, \mathrm{H} \beta_{3}\right), 8.75\left(8 \mathrm{H}, \mathrm{d}, J=4.7 \mathrm{~Hz}, \mathrm{H} \beta_{3}\right), 8.21(8 \mathrm{H}, \mathrm{m}, \mathrm{H} 1$ or $\mathrm{H} 4), 8.08(8 \mathrm{H}, \mathrm{m}, \mathrm{H} 1$ or H4), $7.89(8 \mathrm{H}, \mathrm{d}, J=7.3 \mathrm{~Hz}, \mathrm{H} 2), 7.73(8 \mathrm{H}, \mathrm{t}, J=7.3 \mathrm{~Hz}, \mathrm{H} 3), 1.55-0.75(396 \mathrm{H}, \mathrm{m}$, THS and H5), $-1.32(8 \mathrm{H}, \mathrm{s}, \mathrm{H} 6)$.

MALDI-TOF: $m / z=5,856\left(\mathrm{C}_{380} \mathrm{H}_{484} \mathrm{~N}_{24} \mathrm{Si}_{12} \mathrm{Zn}_{2}, \mathrm{M}^{+}\right.$requires 5,856).

$\lambda_{\max }$ (toluene) / nm log(ع): 482 (5.91), 608 (5.15), 661 (5.30), 770 (5.71), 798 (5.71). 


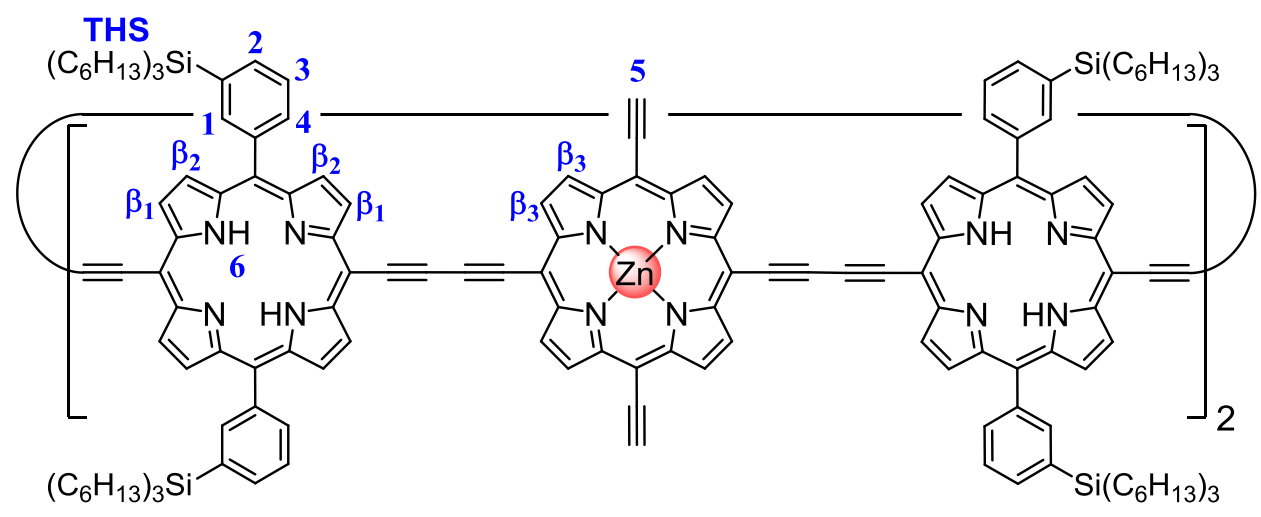

Ring 6.12 (32 mg, $5.5 \mu \mathrm{mol}$ ) was dissolved in $\mathrm{CH}_{2} \mathrm{Cl}_{2}(6.6 \mathrm{~mL})$ and pyridine $(0.33 \mathrm{~mL})$. Tetra- $n$-butylammonium fluoride $(0.22 \mathrm{~mL}, 1.0 \mathrm{M}$ solution in THF, $0.22 \mathrm{mmol})$ was added and the reaction was stirred for $15 \mathrm{~min}$. The crude reaction mixture was purified by silica column chromatography (10\% pyridine in $\mathrm{CHCl}_{3}$ ) followed by size exclusion chromatography on Biobead SX-1 (5\% pyridine in toluene) to give $\mathbf{6 . 1 3}$ as a red solid (24.5 mg, 86\%).

${ }^{1} \mathbf{H}$ NMR $\left(400 \mathrm{MHz}, \mathrm{CDCl}_{3}, 298 \mathrm{~K}\right): \delta_{\mathrm{H}}(\mathrm{ppm})$ 9.69-9.50 (32H, m, $\left.\mathrm{H} \beta_{1}\right)$, 8.82-8.71 (16H, $\left.m, H \beta_{2}\right), 8.21(8 \mathrm{H}, \mathrm{m}, \mathrm{H} 1$ or $\mathrm{H} 4), 8.09(8 \mathrm{H}, \mathrm{m}, \mathrm{H} 1$ or $\mathrm{H} 4), 7.89(8 \mathrm{H}, \mathrm{d}, J=7.7 \mathrm{~Hz}, \mathrm{H} 2)$, $7.73(8 \mathrm{H}, \mathrm{t}, J=7.7 \mathrm{~Hz}, \mathrm{H} 3), 4.13(4 \mathrm{H}, \mathrm{s}, \mathrm{H} 5), 1.53-0.71(312 \mathrm{H}, \mathrm{m}, \mathrm{THS}),-1.31(8 \mathrm{H}, \mathrm{s}$, H6).

$\lambda_{\max }$ (toluene) / nm log(ع): 479 (5.92), 604 (5.17), 657 (5.31), 765 (5.72), 793 (5.73).

Zinc 5,15-bis-(meta-trihexylsilyl-phenyl)-10-ethynyl-20cyanopropyldimethylsilanylethynyl-porphyrin (6.14)

Porphyrin 6.4 (0.86 g, $0.63 \mathrm{mmol}$ ) was dissolved in $\mathrm{CHCl}_{3}(55 \mathrm{~mL})$ and ethanol $(0.55 \mathrm{~mL})$. Tetra- $n$-butylammonium fluoride $(0.63 \mathrm{~mL}$, 1.0 $\mathrm{M}$ solution in THF, $0.63 \mathrm{mmol}$ ) was added dropwise to the stirred solution. The progress of the reaction was monitored by TLC until an optimal product mixture was reached. The

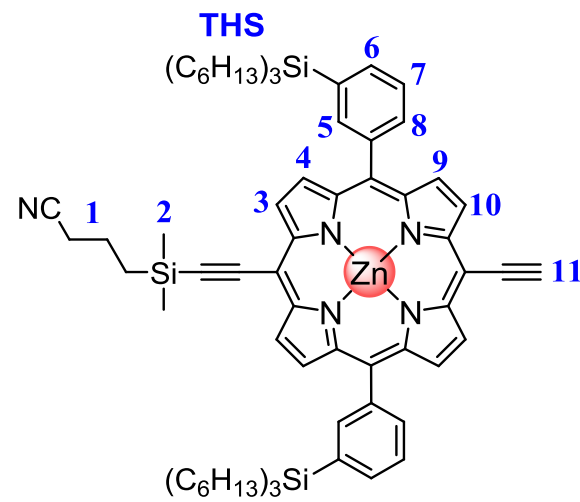
mixture immediately was passed through a short plug of silica gel (1\% pyridine in $\left.\mathrm{CHCl}_{3}\right)$. Column chromatography $(10: 1: 1 \rightarrow 6: 1: 1=$ petroleum ether $:$ ethyl acetate : pyridine) gave 6.14 as a green oil $(0.17 \mathrm{~g}, 21 \%)$. 
${ }^{1} \mathrm{H}$ NMR $\left(400 \mathrm{MHz}, \mathrm{CDCl}_{3}, 298 \mathrm{~K}\right): \delta_{\mathrm{H}}(\mathrm{ppm}) 9.66(2 \mathrm{H}, \mathrm{d}, J=4.6 \mathrm{~Hz}, \mathrm{H} 3$ or H10), 9.60 $(2 \mathrm{H}, \mathrm{d}, J=4.6 \mathrm{~Hz}, \mathrm{H} 3$ or H10), $8.87(4 \mathrm{H}, \mathrm{d}, J=4.6 \mathrm{~Hz}, \mathrm{H} 4$ and $\mathrm{H9}), 8.27(2 \mathrm{H}, \mathrm{m}, \mathrm{H} 8)$, $8.13(2 \mathrm{H}, \mathrm{m}, \mathrm{H} 5), 7.88(2 \mathrm{H}, \mathrm{m}, \mathrm{H} 6), 7.73(2 \mathrm{H}, \mathrm{m}, \mathrm{H} 7), 4.15(1 \mathrm{H}, \mathrm{s}, \mathrm{H} 11), 2.58(2 \mathrm{H}, \mathrm{t}$, $J=6.9 \mathrm{~Hz}, \mathrm{H} 1), 2.16(2 \mathrm{H}, \mathrm{m}, \mathrm{H} 1), 1.54-0.82(80 \mathrm{H}, \mathrm{m}, \mathrm{H} 1$ and THS), $0.60(6 \mathrm{H}, \mathrm{s}, \mathrm{H} 2)$.

\section{Ring 6.15}

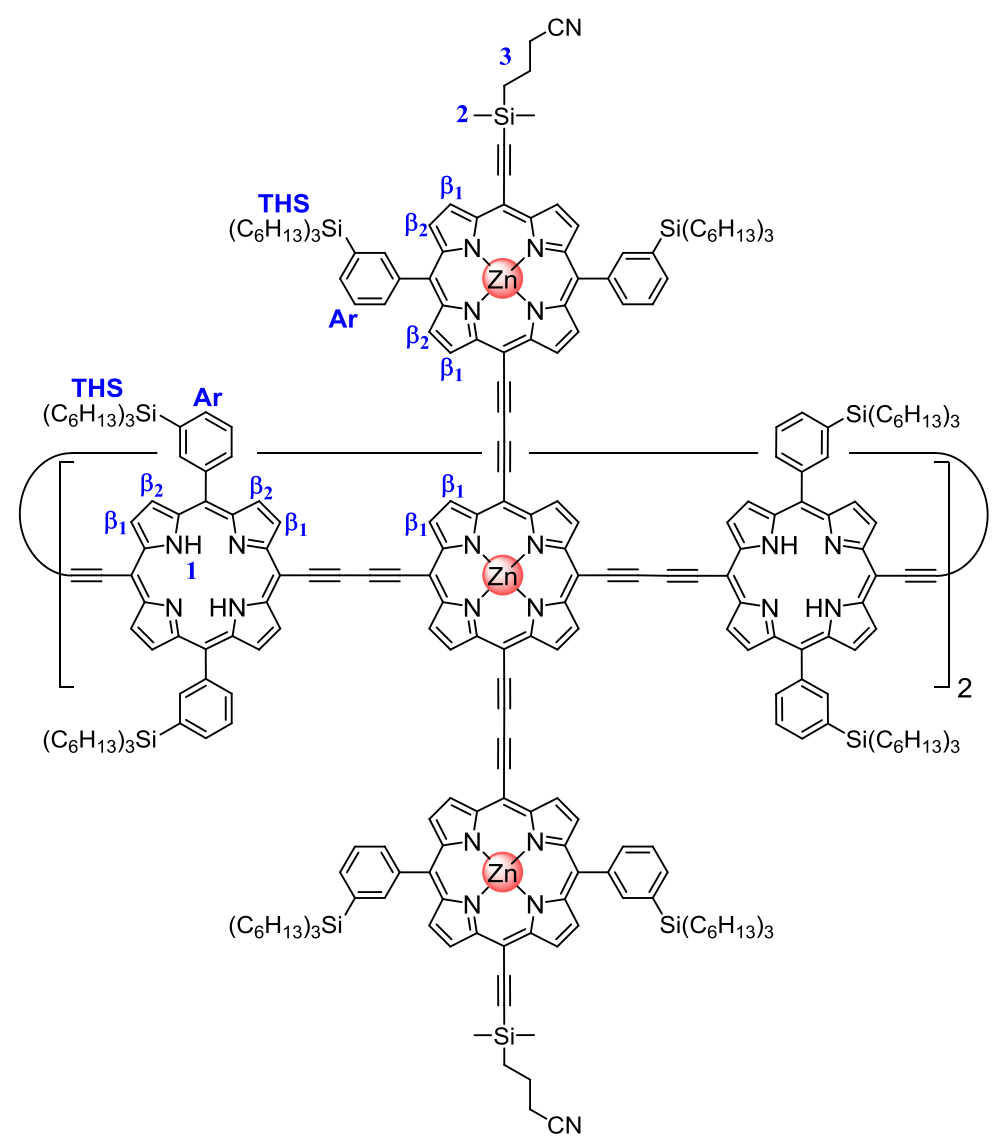

Ring 6.13 (24.5 mg, $4.7 \mu \mathrm{mol})$ and $6.14\left(237 \mathrm{mg}, 0.187 \mathrm{mmol}\right.$ ) were dissolved in $\mathrm{CHCl}_{3}$ (38.6 mL). A catalyst solution was prepared by dissolving $\mathrm{PdCl}_{2}\left(\mathrm{PPh}_{3}\right)_{2}(15.5 \mathrm{mg}$, $22.0 \mu \mathrm{mol})$, Cul (15.4 mg, $81.0 \mu \mathrm{mol})$ and 1,4-benzoquinone $(45.5 \mathrm{mg}, 0.421 \mathrm{mmol})$ in $\mathrm{CHCl}_{3}(11.4 \mathrm{~mL})$ and $i-\mathrm{Pr}_{2} \mathrm{NH}(0.96 \mathrm{~mL})$. The catalyst solution was added to the porphyrin solution and the reaction mixture was stirred at room temperature for $10 \mathrm{~min}$. The crude reaction mixture was purified by silica column chromatography (10\% pyridine in $\mathrm{CHCl}_{3}$ ), size exclusion column on Biobeads $\mathrm{SX}-1$ (5\% pyridine in toluene) and finally recycling GPC (1\% pyridine in toluene) to give 6.15 as a red solid (27.0 mg, 56\%).

${ }^{1} \mathrm{H}$ NMR $\left(400 \mathrm{MHz}, \mathrm{CDCl}_{3}, 298 \mathrm{~K}\right): \delta_{\mathrm{H}}(\mathrm{ppm}) 9.89\left(8 \mathrm{H}, \mathrm{d}, J=4.5 \mathrm{~Hz}, \mathrm{H} \beta_{1}\right), 9.77(8 \mathrm{H}, \mathrm{d}$, $\left.J=4.1 \mathrm{~Hz}, H \beta_{1}\right), 9.68\left(8 \mathrm{H}, \mathrm{d}, J=4.2 \mathrm{~Hz}, \mathrm{H} \beta_{1}\right), 9.66\left(8 \mathrm{H}, \mathrm{d}, J=4.5 \mathrm{~Hz}, H \beta_{1}\right), 9.63(8 \mathrm{H}, \mathrm{d}$, 
$\left.J=4.2 \mathrm{~Hz}, H \beta_{1}\right), 9.59\left(8 \mathrm{H}, \mathrm{d}, J=4.5 \mathrm{~Hz}, \mathrm{H} \beta_{1}\right), 8.98\left(8 \mathrm{H}, \mathrm{d}, J=4.5 \mathrm{~Hz}, \mathrm{H} \beta_{2}\right), 8.90(8 \mathrm{H}, \mathrm{d}$, $\left.J=4.5 \mathrm{~Hz}, \mathrm{H} \beta_{2}\right), 8.78\left(8 \mathrm{H}, \mathrm{d}, J=4.5 \mathrm{~Hz}, \mathrm{H} \beta_{2}\right), 8.74\left(8 \mathrm{H}, \mathrm{d}, J=4.5 \mathrm{~Hz}, \mathrm{H} \beta_{2}\right), 8.33(8 \mathrm{H}, \mathrm{m}$, $\left.\mathrm{H}_{\mathrm{Ar}}\right), 8.24-8.16\left(16 \mathrm{H}, \mathrm{m}, \mathrm{H}_{\mathrm{Ar}}\right), 8.10\left(8 \mathrm{H}, \mathrm{m}, \mathrm{H}_{\mathrm{Ar}}\right), 7.93\left(8 \mathrm{H}, \mathrm{m}, \mathrm{H}_{\mathrm{Ar}}\right), 7.85\left(8 \mathrm{H}, \mathrm{m}, \mathrm{H}_{\mathrm{Ar}}\right)$, $7.78\left(8 \mathrm{H}, \mathrm{m}, \mathrm{H}_{\mathrm{Ar}}\right), 7.71\left(8 \mathrm{H}, \mathrm{m}, \mathrm{H}_{\mathrm{Ar}}\right), 2.62(8 \mathrm{H}, \mathrm{m}, \mathrm{H} 3), 2.19(8 \mathrm{H}, \mathrm{m}, \mathrm{H} 3), 1.62-0.72$ (632H, m, THS), $0.64(24 \mathrm{H}, \mathrm{s}, \mathrm{H} 2),-1.31(8 \mathrm{H}, \mathrm{s}, \mathrm{H} 1)$.

MALDI-TOF: $m / z=10,277\left(\mathrm{C}_{656} \mathrm{H}_{824} \mathrm{~N}_{44} \mathrm{Si}_{20} \mathrm{Zn}_{6}, \mathrm{M}^{+}\right.$requires 10,280$)$.

$\lambda_{\text {max }}$ (toluene) / nm log(ع): 445 (5.91), 652 (5.38), 775 (5.56), 800 (5.54).

\section{Ring (6.16)}

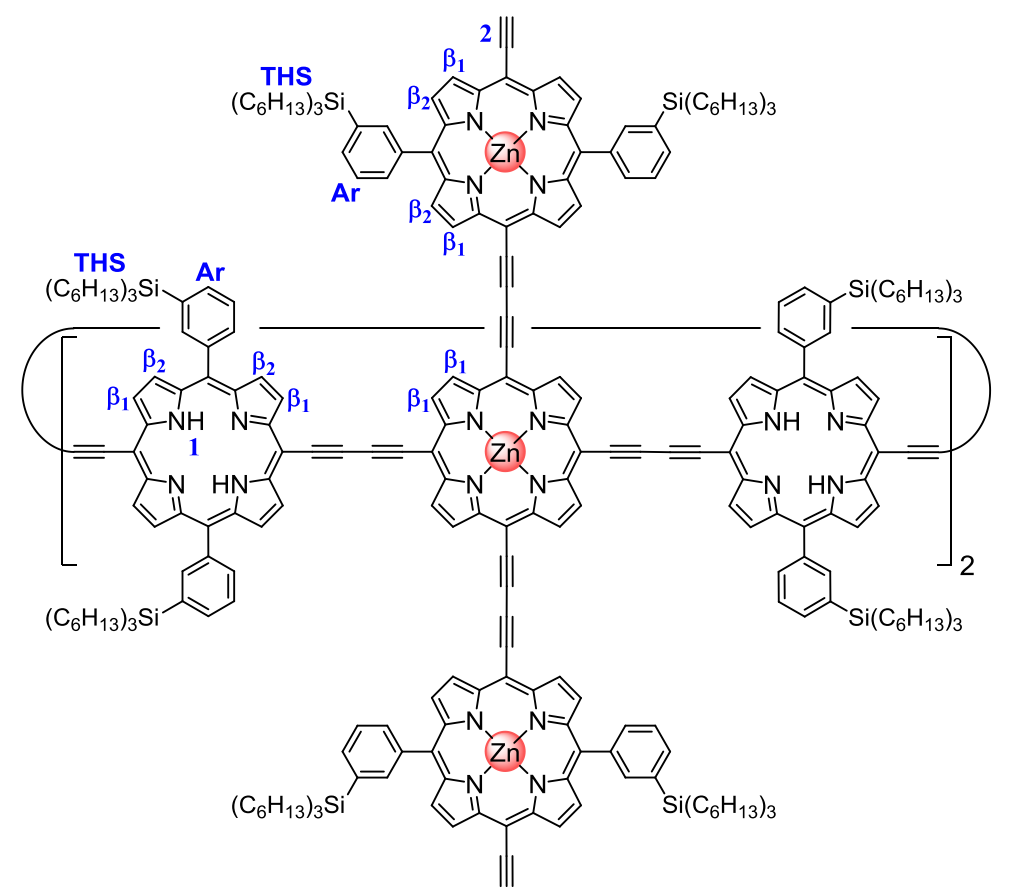

Ring 6.15 (16.2 mg, $1.58 \mu \mathrm{mol})$ was dissolved in $\mathrm{CH}_{2} \mathrm{Cl}_{2}(12 \mathrm{~mL})$. Tetra-n-butylammonium fluoride ( $63 \mu \mathrm{L}, 1.0 \mathrm{M}$ solution in THF, $82 \mu \mathrm{mol}$ ) was added and the reaction was stirred for $15 \mathrm{~min}$. The crude reaction mixture was purified by silica column chromatography ( $5 \%$ pyridine in $\mathrm{CHCl}_{3}$ ) followed by size exclusion chromatography on Biobeads SX-1 (5\% pyridine in toluene) to give ring 6.16 as a red solid (16.2 mg, 100\%).

${ }^{1} \mathbf{H}$ NMR $\left(400 \mathrm{MHz}, \mathrm{CDCl}_{3}, 298 \mathrm{~K}\right): \delta_{\mathrm{H}}(\mathrm{ppm}) 9.90\left(8 \mathrm{H}, \mathrm{m}, \mathrm{H} \beta_{1}\right), 9.76\left(8 \mathrm{H}, \mathrm{m}, \mathrm{H} \beta_{1}\right)$, 9.73-9.63 (24H, m, H $\left.\beta_{1}\right), 9.58\left(8 \mathrm{H}, \mathrm{m}, \mathrm{H} \beta_{1}\right), 8.99\left(8 \mathrm{H}, \mathrm{m}, \mathrm{H} \beta_{2}\right), 8.90\left(8 \mathrm{H}, \mathrm{m}, \mathrm{H} \beta_{2}\right), 8.78$ $\left(8 \mathrm{H}, \mathrm{d}, J=4.5 \mathrm{~Hz}, \mathrm{H} \beta_{2}\right), 8.74\left(8 \mathrm{H}, \mathrm{d}, J=4.5 \mathrm{~Hz}, \mathrm{H} \beta_{2}\right), 8.32\left(8 \mathrm{H}, \mathrm{m}, \mathrm{H}_{\mathrm{Ar}}\right), 8.25-8.14(16 \mathrm{H}$, $\left.m, H_{A r}\right), 8.10\left(8 \mathrm{H}, m, H_{A r}\right), 7.93\left(8 H, m, H_{A r}\right), 7.85\left(8 H, m, H_{A r}\right), 7.78\left(8 H, m, H_{A r}\right), 7.70$ $\left(8 \mathrm{H}, \mathrm{m}, \mathrm{H}_{\mathrm{Ar}}\right), 4.42(4 \mathrm{H}, \mathrm{s}, \mathrm{H} 2), 1.65-0.69(624 \mathrm{H}, \mathrm{m}, \mathrm{THS}),-1.31(8 \mathrm{H}, \mathrm{s}, \mathrm{H} 1)$. 
$\lambda_{\text {max }}$ (toluene) / nm log(ع): 440 (5.97), 635 (5.39), 663 (5.27), 774 (5.60), 803 (5.55).

\section{Ball (6.17)}

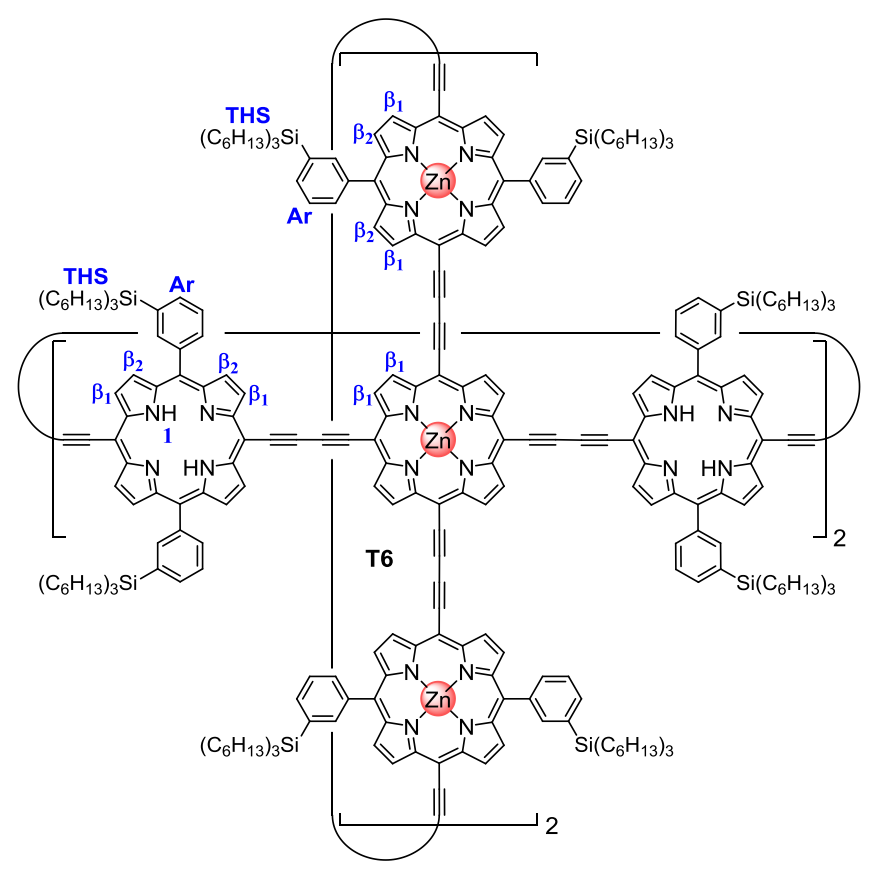

Ring 6.16 (16.2 mg, $1.66 \mu \mathrm{mol})$ and template T6 (1.82 mg, $1.82 \mu \mathrm{mol})$ were dissolved in $\mathrm{CHCl}_{3}(16 \mathrm{~mL})$. UV-vis indicated the formation of the desired one-to-one complex. A catalyst solution was prepared by dissolving $\mathrm{Pd}\left(\mathrm{PPh}_{3}\right)_{2} \mathrm{Cl}_{2}(2.86 \mathrm{mg}, 4.08 \mu \mathrm{mol}), \mathrm{Cul}$ (3.90 mg, $20.46 \mu \mathrm{mol}$ ) and 1,4- benzoquinone (9.06 mg, $83.81 \mu \mathrm{mol}$ ) in $\mathrm{CHCl}_{3}$ $(1.97 \mathrm{~mL})$ and freshly distilled $i-\operatorname{Pr}_{2} \mathrm{NH}(27 \mu \mathrm{L})$. This solution was added to the reaction mixture, which was stirred vigorously at room temperature for $2 \mathrm{~h}$. The crude reaction mixture was purified by silica column chromatography (10\% pyridine in $\mathrm{CHCl}_{3}$ ), size exclusion column on Biobeads SX-1 (5\% pyridine in toluene) and finally recycling GPC (1\% pyridine in toluene) to give the ball 6.17 as a red solid (1.3 $\mathrm{mg}$, $7 \%)$.

${ }^{1} \mathbf{H}$ NMR $\left(400 \mathrm{MHz}, \mathrm{CDCl}_{3}, 298 \mathrm{~K}\right): \delta_{\mathrm{H}}(\mathrm{ppm})$ 9.69-9.24 $\left(48 \mathrm{H}, \mathrm{m}, \mathrm{H} \beta_{1}\right), 8.87-8.53(32 \mathrm{H}$, $\left.m, H \beta_{2}\right)$, 8.39-7.59 $\left(64 \mathrm{H}, \mathrm{m}, \mathrm{H}_{\mathrm{Ar}}\right), 5.09-4.29\left(36 \mathrm{H}, \mathrm{m}, \mathbf{T 6}_{\beta-\gamma-\delta}\right), 2.06\left(12 \mathrm{H}, \mathrm{m}, \mathbf{T 6 _ { \alpha }}\right)$, $1.62-0.71(624 \mathrm{H}, \mathrm{m}, \mathrm{THS}),-1.34(8 \mathrm{H}, \mathrm{s}, \mathrm{H} 1)$.

MALDI-TOF: $m / z=10,786\left(C_{704} \mathrm{H}_{824} \mathrm{~N}_{46} \mathrm{Si}_{16} \mathrm{Zn}_{6}, \mathrm{M}^{+}\right.$requires 10,772$)$.

$\lambda_{\text {max }}$ (toluene) / nm log(ع): 467 (5.27), 662 (4.75), 759 (5.07), 808 (4.99), 856 (5.02). 
Ball (6.18)

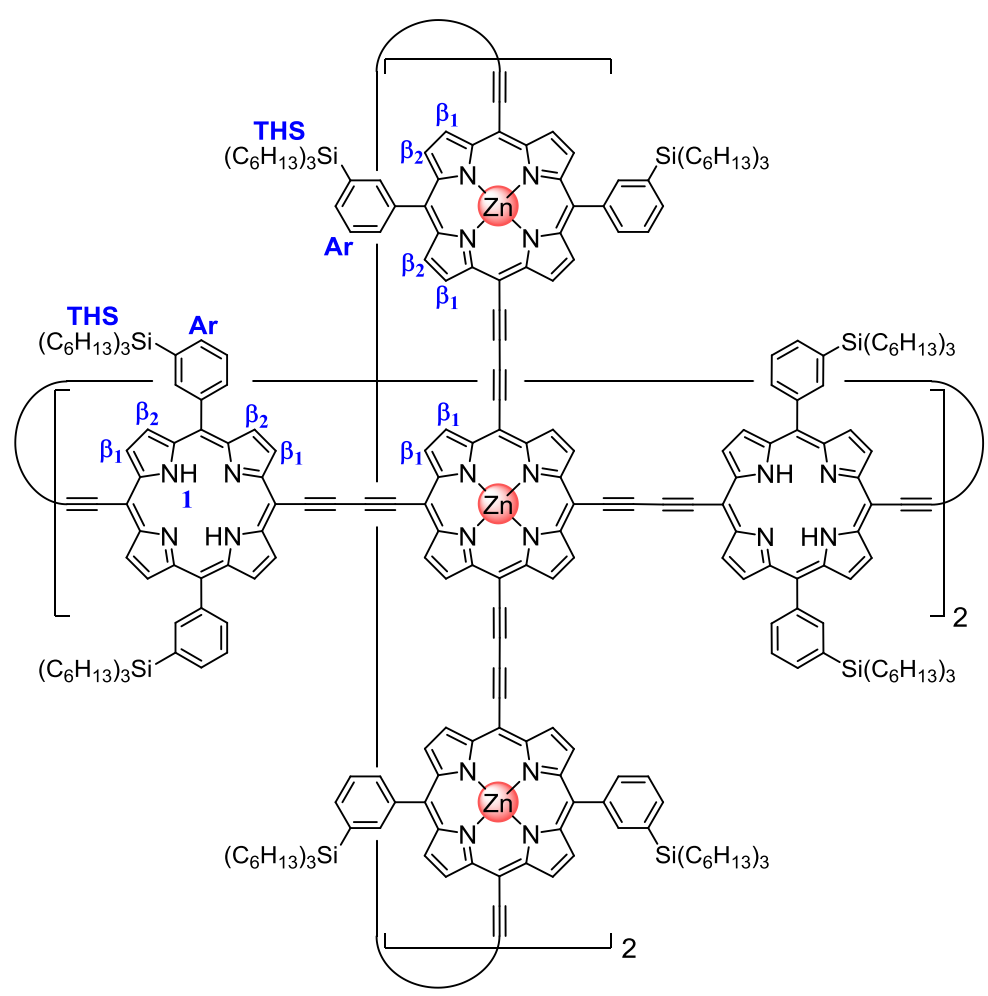

A size exclusion column with Biobeads SX-1 was prepared $(50 \mathrm{~cm}$ by $1 \mathrm{~cm}, 1 \%$ pyridine in toluene). The column was run with a toluene solution saturated with DABCO ( $10 \%$ of the column length) in order to saturate the top of the column with DABCO. 6.17 (2.1 mg, $0.20 \mu \mathrm{mol})$ was dissolved in the $1 \%$ pyridine in toluene $(0.5 \mathrm{~mL})$ and loaded on the column. The product was eluted with the toluene solution saturated with DABCO ( $10 \%$ of the column length) followed by eluting with $1 \%$ pyridine in toluene. The coloured porphyrin band was collected to yield the template free ball with DABCO coordinating to the zinc centres. The solvent was removed and the solid was dissolved in $1 \%$ pyridine in toluene $(0.4 \mathrm{~mL})$. The solution was passed over a size exclusion column with Biobeads SX-1 (10\% pyridine in toluene) in order to remove the DABCO. The coloured porphyrin band was collected to yield the template free ball 6.18 as a red solid (1.94 $\mathrm{mg}, 100 \%)$.

${ }^{1} \mathrm{H}$ NMR $\left(400 \mathrm{MHz}, \mathrm{CDCl}_{3}, 298 \mathrm{~K}\right): \delta_{\mathrm{H}}(\mathrm{ppm}) 9.59\left(48 \mathrm{H}, \mathrm{m}, \mathrm{H} \beta_{1}\right), 8.77\left(32 \mathrm{H}, \mathrm{m}, \mathrm{H} \beta_{2}\right)$, $8.10\left(32 \mathrm{H}, \mathrm{m}, \mathrm{H}_{\mathrm{Ar}}\right), 7.93\left(16 \mathrm{H}, \mathrm{m}, \mathrm{H}_{\mathrm{Ar}}\right), 7.75\left(16 \mathrm{H}, \mathrm{m}, \mathrm{H}_{\mathrm{Ar}}\right), 1.62-0.71(624 \mathrm{H}, \mathrm{m}$, THS), $-1.34(8 \mathrm{H}, \mathrm{s}, \mathrm{H} 1)$.

$\lambda_{\max }$ (toluene) / nm log(ع): 456 (5.38), 658 (4.80), 748 (5.05), 784 (5.03), 832 (5.02). 


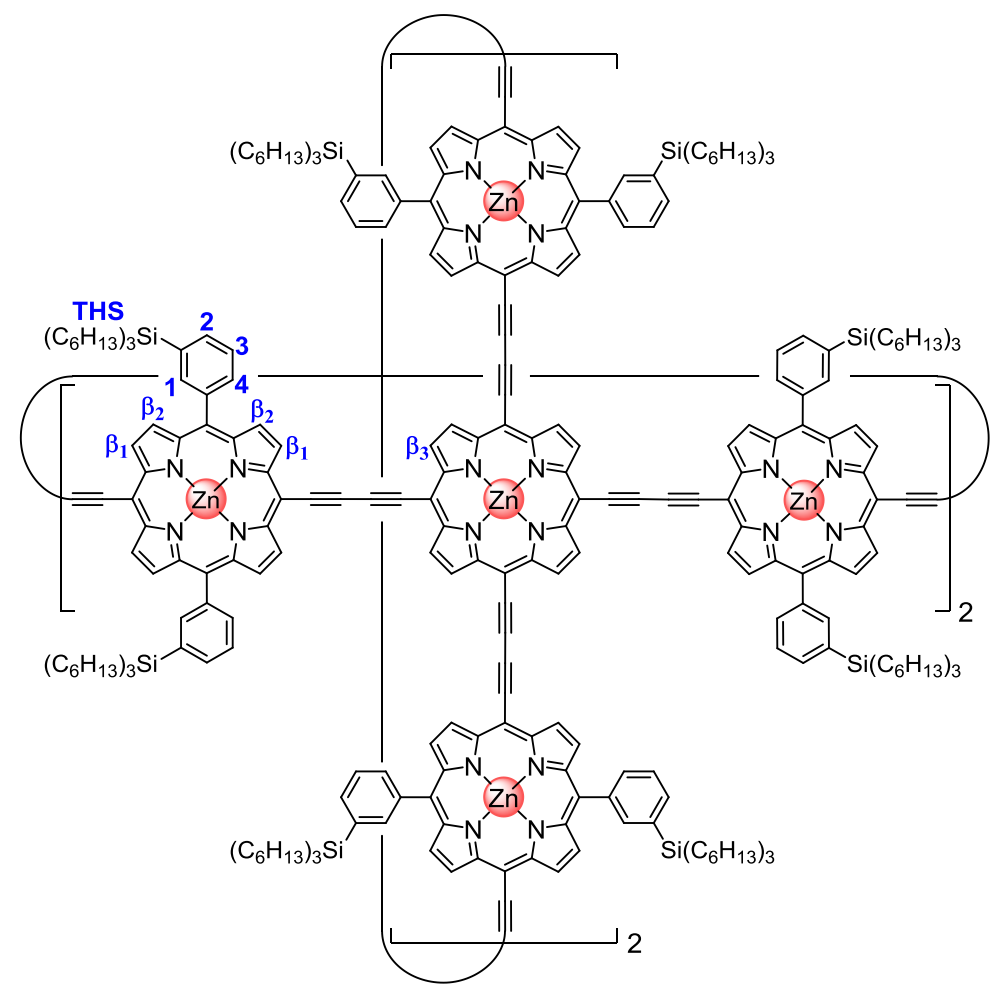

Ball 6.18 (1.94 mg, $0.20 \mu \mathrm{mol})$ was dissolved in $\mathrm{CH}_{2} \mathrm{Cl}_{2}(2 \mathrm{~mL})$ and pyridine $(0.2 \mathrm{~mL})$. $\mathrm{Zn}(\mathrm{OAC})_{2} \cdot\left(\mathrm{H}_{2} \mathrm{O}\right)_{2}(8.7 \mathrm{mg}, 39.69 \mu \mathrm{mol})$ was dissolved in methanol $(0.2 \mathrm{~mL})$. The solutions were combined and the reaction mixture was stirred for $4 \mathrm{~h}$. The crude reaction mixture was purified by silica column chromatography (10\% pyridine in $\mathrm{CHCl}_{3}$ to give the ball $\boldsymbol{b}$-P10 as a red solid (1.83 $\left.\mathrm{mg}, 92 \%\right)$.

${ }^{1} \mathbf{H}$ NMR (700 MHz, $\left.\mathrm{CDCl}_{3}, 313 \mathrm{~K}\right): \delta_{\mathrm{H}}(\mathrm{ppm}) 9.61\left(16 \mathrm{H}, \mathrm{m}, \mathrm{H} \beta_{1}\right), 9.57\left(16 \mathrm{H}, \mathrm{m}, \mathrm{H} \beta_{1}\right)$, $9.55\left(16 \mathrm{H}, \mathrm{m}, \mathrm{H} \beta_{3}\right), 8.78\left(16 \mathrm{H}, \mathrm{m}, \mathrm{H} \beta_{2}\right), 8.73\left(16 \mathrm{H}, \mathrm{m}, \mathrm{H} \beta_{2}\right), 8.16(16 \mathrm{H}, \mathrm{m}, \mathrm{H} 4), 8.08$ $(16 \mathrm{H}, \mathrm{m}, \mathrm{H} 1), 7.93(16 \mathrm{H}, \mathrm{m}, \mathrm{H} 2), 7.74(16 \mathrm{H}, \mathrm{m}, \mathrm{H3}), 1.71-0.50$ (624H, m, THS).

MALDI-TOF: $m / z=10,032\left(\mathrm{C}_{632} \mathrm{H}_{768} \mathrm{~N}_{40} \mathrm{Si}_{16} \mathrm{Zn}_{10}, \mathrm{M}^{+}\right.$requires 10,029$)$.

$\lambda_{\text {max }}$ (toluene) / nm log(ع): 467 (5.14), 659 (4.74), 750 (5.15), 791 (5.13), 837 (5.13). 


\section{References}

(1) Willstätter, R. J. Am. Chem. Soc. 1915, 37, 323.

(2) McDermott, G.; Prince, S. M.; Freer, A. A.; Hawthornthwaite-Lawless, A. M.; Papiz, M. Z.; Cogdell, R. J.; Isaacs, N. W. Nature 1995, 374, 517.

(3) Cogdell, R. J.; Fyfe, P. K.; Barrett, S. J.; Prince, S. M.; Freer, A. A.; Isaacs, N. W.; McGlynn, P.; Hunter, C. N. Photosynth. Res. 1996, 48, 55.

(4) Jimenez, R.; Dikshit, S. N.; Bradforth, S. E.; Fleming, G. R. J. Phys. Chem. 1996, 100, 6825.

(5) Omura, T.; Sato, R. J. Biol. Chem. 1964, 239, 2370.

(6) Senge, M. O.; Davis, M. JPP 2010, 14, 557.

(7) Venkataramani, S.; Jana, U.; Dommaschk, M.; Sönnichsen, F. D.; Tuczek, F.; Herges, R. Science 2011, 331, 445.

(8) Bowen, A. M.; Jones, M. W.; Lovett, J. E.; Gaule, T. G.; McPherson, M. J.; Dilworth, J. R.; Timmel, C. R.; Harmer, J. R. PCCP 2016, 18, 5981.

(9) Wang, S.-P.; Shen, Y.-F.; Zhu, B.-Y.; Wu, J.; Li, S. Chem. Commun. 2016, $52,10205$.

(10) Anderson, H. L. Chem. Commun. 1999, 2323.

(11) Terazima, M.; Shimizu, H.; Osuka, A. J. Appl. Phys. 1997, 81, 2946.

(12) Nakano, A.; Osuka, A.; Yamazaki, I.; Yamazaki, T.; Nishimura, Y. Angew. Chem. Int. Ed. 1998, 37, 3023.

(13) Osuka, A.; Tanabe, N.; Nakajima, S.; Maruyama, K. J. Chem. Soc., Perkin Trans. 2 1996, 199.

(14) Wagner, R. W.; Johnson, T. E.; Lindsey, J. S. J. Am. Chem. Soc. 1996, 118, 11166.

(15) Seth, J.; Palaniappan, V.; Johnson, T. E.; Prathapan, S.; Lindsey, J. S.; Bocian, D. F. J. Am. Chem. Soc. 1994, 116, 10578.

(16) Arnold, D. P.; Johnson, A. W.; Mahendran, M. J. Chem. Soc., Perkin Trans. 1 1978, 366.

(17) Arnold, D. P.; Nitschinsk, L. J. Tetrahedron 1992, 48, 8781.

(18) Arnold, D. P. Synlett 2000, 2000, 296.

(19) Anderson, H. L. Inorg. Chem. 1994, 33, 972.

(20) Winters, M. U.; Kärnbratt, J.; Eng, M.; Wilson, C. J.; Anderson, H. L.; Albinsson, B. J. Phys. Chem. C 2007, 111, 7192.

(21) Winters, M. U.; Dahlstedt, E.; Blades, H. E.; Wilson, C. J.; Frampton, M. J.; Anderson, H. L.; Albinsson, B. J. Am. Chem. Soc. 2007, 129, 4291.

(22) Taylor, P. N.; Anderson, H. L. J. Am. Chem. Soc. 1999, 121, 11538.

(23) Hogben, H. J.; Sprafke, J. K.; Hoffmann, M.; Pawlicki, M.; Anderson, H. L. J. Am. Chem. Soc. 2011, 133, 20962.

(24) Bhyrappa, P.; Krishnan, V.; Nethaji, M. J. Chem. Soc. Dalton. Trans. 1993, 1901.

(25) Whitty, A. Nat. Chem. Biol. 2008, 4, 435.

(26) Perutz, M. F. Q. Rev. Biophys. 2009, 22, 139.

(27) Hunter, C. A.; Anderson, H. L. Angew. Chem. Int. Ed. 2009, 48, 7488.

(28) Busch, D. H. J. Inclusion Phenom. Mol. Recognit. Chem. 1992, 12, 389.

(29) Fatin-Rouge, N.; Blanc, S.; Pfeil, A.; Rigault, A.; Albrecht-Gary, A.-M.; Lehn, J.-M. Helv. Chim. Acta 2001, 84, 1694.

(30) Lehn, J.-M. Angew. Chem. Int. Ed. 1988, 27, 89.

(31) Lehn, J.-M.; Rigault, A. Angew. Chem. Int. Ed. 1988, 27, 1095.

(32) Lehn, J.-M. Science 2002, 295, 2400.

(33) Núñez-Villanueva, D.; ladevaia, G.; Stross, A. E.; Jinks, M. A.; Swain, J. A.; Hunter, C. A. J. Am. Chem. Soc. 2017, 139, 6654.

(34) Hunter, C. A. Angew. Chem. Int. Ed. 2004, 43, 5310. 
(35) Adams, H.; Chekmeneva, E.; Hunter, C. A.; Misuraca, M. C.; Navarro, C.; Turega, S. M. J. Am. Chem. Soc. 2013, 135, 1853.

(36) von Krbek, L. K. S.; Achazi, A. J.; Solleder, M.; Weber, M.; Paulus, B.; Schalley, C. A. Chem. Eur. J. 2016, 22, 15475.

(37) Anderson, S.; Anderson, H. L.; Sanders, J. K. M. Acc. Chem. Res. 1993, 26, 469.

(38) Hoffmann, M.; Kärnbratt, J.; Chang, M.-H.; Herz, L. M.; Albinsson, B.; Anderson, H. L. Angew. Chem. Int. Ed. 2008, 47, 4993.

(39) O'Sullivan, M. C.; Sprafke, J. K.; Kondratuk, D. V.; Rinfray, C.; Claridge, T. D.; Saywell, A.; Blunt, M. O.; O'Shea, J. N.; Beton, P. H.; Malfois, M.; Anderson, H. L. Nature 2011, $469,72$.

(40) Durot, S.; Taesch, J.; Heitz, V. Chem. Rev. 2014, 114, 8542.

(41) Anderson, H. L.; Sanders, J. K. M. Angew. Chem. Int. Ed. 1990, 29, 1400.

(42) Li, J.; Ambroise, A.; Yang, S. I.; Diers, J. R.; Seth, J.; Wack, C. R.; Bocian, D. F.; Holten, D.; Lindsey, J. S. J. Am. Chem. Soc. 1999, 121, 8927.

(43) Yu, L.; Lindsey, J. S. J. Org. Chem. 2001, 66, 7402.

(44) Tomizaki, K.-y.; Yu, L.; Wei, L.; Bocian, D. F.; Lindsey, J. S. J. Org. Chem. 2003, 68, 8199.

(45) Mongin, O.; Schuwey, A.; Vallot, M.-A.; Gossauer, A. Tetrahedron Lett. 1999, 40, 8347.

(46) Rucareanu, S.; Schuwey, A.; Gossauer, A. J. Am. Chem. Soc. 2006, 128, 3396.

(47) Tashiro, K.; Aida, T.; Zheng, J.-Y.; Kinbara, K.; Saigo, K.; Sakamoto, S.; Yamaguchi, K. J. Am. Chem. Soc. 1999, 121, 9477.

(48) Yanagisawa, M.; Tashiro, K.; Yamasaki, M.; Aida, T. J. Am. Chem. Soc. 2007, 129, 11912.

(49) Tashiro, K.; Hirabayashi, Y.; Aida, T.; Saigo, K.; Fujiwara, K.; Komatsu, K.; Sakamoto, S.; Yamaguchi, K. J. Am. Chem. Soc. 2002, 124, 12086.

(50) Hoffmann, M.; Wilson, C. J.; Odell, B.; Anderson, H. L. Angew. Chem. Int. Ed. 2007, $46,3122$.

(51) Kondratuk, D. V.; Perdigao, L. M. A.; O'Sullivan, M. C.; Svatek, S.; Smith, G.; O'Shea, J. N.; Beton, P. H.; Anderson, H. L. Angew. Chem. Int. Ed. 2012, 51, 6696.

(52) Kamonsutthipaijit, N.; Anderson, H. L. Chem. Sci. 2017, 8, 2729.

(53) Kondratuk, D. V.; Perdigão, L. M. A.; Esmail, A. M. S.; O'Shea, J. N.; Beton, P. H.; Anderson, H. L. Nat. Chem. 2015, 7, 317.

(54) Neuhaus, P.; Cnossen, A.; Gong, J. Q.; Herz, L. M.; Anderson, H. L. Angew. Chem. Int. Ed. 2015, 54, 7344.

(55) Favereau, L.; Cnossen, A.; Kelber, J. B.; Gong, J. Q.; Oetterli, R. M.; Cremers, J.; Herz, L. M.; Anderson, H. L. J. Am. Chem. Soc. 2015, 137, 14256.

(56) Stulz, E.; Scott, S. M.; Ng, Y.-F.; Bond, A. D.; Teat, S. J.; Darling, S. L.; Feeder, N.; Sanders, J. K. M. Inorg. Chem. 2003, 42, 6564.

(57) Haycock, R. A.; Hunter, C. A.; James, D. A.; Michelsen, U.; Sutton, L. R. Org. Lett. 2000, 2, 2435.

(58) Stulz, E.; Ng, Y.-F.; Scott, S. M.; Sanders, J. K. M. Chem. Commun. 2002, 524.

(59) Davidson, G. J. E.; Tong, L. H.; Raithby, P. R.; Sanders, J. K. M. Chem. Commun. 2006, 3087.

(60) Rousseaux, S. A. L.; Gong, J. Q.; Haver, R.; Odell, B.; Claridge, T. D. W.; Herz, L. M.; Anderson, H. L. J. Am. Chem. Soc. 2015, 137, 12713.

(61) Borovkov, V.; M. Lintuluoto, J.; Inoue, Y. Synlett, 1998; 768.

(62) Borovkov, V.; M. Lintuluoto, J.; Inoue, Y. Helv. Chim. Acta 1999, 82, 919.

(63) Brun, A. M.; Harriman, A.; Heitz, V.; Sauvage, J. P. J. Am. Chem. Soc. 1991, 113, 8657.

(64) Brun, A. M.; Atherton, S. J.; Harriman, A.; Heitz, V.; Sauvage, J. P. J. Am. Chem. Soc. 1992, 114, 4632. 
(65) Harriman, A.; Heitz, V.; Chambron, J.-C.; Sauvage, J.-P. Coord. Chem. Rev. 1994, 132, 229.

(66) Andersson, M.; Linke, M.; Chambron, J.-C.; Davidsson, J.; Heitz, V.; Hammarström, L.; Sauvage, J.-P. J. Am. Chem. Soc. 2002, 124, 4347.

(67) Linke, M.; Fujita, N.; Chambron, J.-C.; Heitz, V.; Sauvage, J.-P. New J. Chem. 2001, 25, 790.

(68) Andréasson, J.; Kodis, G.; Ljungdahl, T.; Moore, A. L.; Moore, T. A.; Gust, D.; Mårtensson, J.; Albinsson, B. J. Phys. Chem. A 2003, 107, 8825.

(69) Pettersson, K.; Wiberg, J.; Ljungdahl, T.; Mårtensson, J.; Albinsson, B. J. Phys. Chem. A 2006, 110, 319.

(70) Fortage, J.; Boixel, J.; Blart, E.; Hammarström, L.; Becker, H. C.; Odobel, F. Chem. Eur. J. 2008, 14, 3467.

(71) Fortage, J.; Boixel, J.; Blart, E.; Becker, H. C.; Odobel, F. Inorg. Chem. 2009, 48, 518.

(72) Fortage, J.; Scarpaci, A.; Viau, L.; Pellegrin, Y.; Blart, E.; Falkenström, M.; Hammarström, L.; Asselberghs, I.; Kellens, R.; Libaers, W.; Clays, K.; Eng, M. P.; Odobel, F. Chem. Eur. J. 2009, 15, 9058.

(73) Boixel, J.; Fortage, J.; Blart, E.; Pellegrin, Y.; Hammarstrom, L.; Becker, H.-C.; Odobel, F. Dalton Trans. 2010, 39, 1450.

(74) Larsen, R. G.; Singel, D. J. J. Chem. Phys. 1993, 98, 5134.

(75) Schiemann, O.; Prisner, T. F. Q Rev Biophys 2007, 40, 1.

(76) Lovett, J. E.; Hoffmann, M.; Cnossen, A.; Shutter, A. T. J.; Hogben, H. J.; Warren, J. E.; Pascu, S. I.; Kay, C. W. M.; Timmel, C. R.; Anderson, H. L. J. Am. Chem. Soc. 2009, 131, 13852.

(77) Richert, S.; Kuprov, I.; Peeks, M. D.; Suturina, E. A.; Cremers, J.; Anderson, H. L.; Timmel, C. R. PCCP 2017, 19, 16057.

(78) Lambert, C. J. Chem. Soc. Rev. 2015, 44, 875.

(79) Magoga, M.; Joachim, C. Phys. Rev. B 1999, 59, 16011.

(80) Patoux, C.; Coudret, C.; Launay, J.-P.; Joachim, C.; Gourdon, A. Inorg. Chem. 1997, 36, 5037.

(81) Li, Z.; Smeu, M.; Rives, A.; Maraval, V.; Chauvin, R.; Ratner, M. A.; Borguet, E. Nat. Commun. 2015, 6, 6321.

(82) Chichak, K. S.; Cantrill, S. J.; Pease, A. R.; Chiu, S.-H.; Cave, G. W. V.; Atwood, J. L.; Stoddart, J. F. Science 2004, 304, 1308.

(83) Suzuki, K.; Sato, S.; Fujita, M. Nat. Chem. 2010, 2, 25.

(84) Guo, J.; Mayers, P. C.; Breault, G. A.; Hunter, C. A. Nat. Chem. 2010, 2, 218.

(85) Beves, J. E.; Blight, B. A.; Campbell, C. J.; Leigh, D. A.; McBurney, R. T. Angew. Chem. Int. Ed. 2011, 50, 9260.

(86) van Gerven, P. C. M.; Elemans, J. A. A. W.; Gerritsen, J. W.; Speller, S.; Nolte, R. J. M.; Rowan, A. E. Chem. Commun. 2005, 3535.

(87) Zhu, B.; Chen, H.; Lin, W.; Ye, Y.; Wu, J.; Li, S. J. Am. Chem. Soc. 2014, 136, 15126.

(88) Liu, S.; Kondratuk, D. V.; Rousseaux, S. A. L.; Gil-Ramírez, G.; O'Sullivan, M. C.; Cremers, J.; Claridge, T. D. W.; Anderson, H. L. Angew. Chem. Int. Ed. 2015, $54,5355$.

(89) Yong, C.-K.; Parkinson, P.; Kondratuk, D. V.; Chen, W.-H.; Stannard, A.; Summerfield, A.; Sprafke, J. K.; O'Sullivan, M. C.; Beton, P. H.; Anderson, H. L.; Herz, L. M. Chem. Sci. 2015, 6, 181.

(90) Tait, C. E.; Neuhaus, P.; Peeks, M. D.; Anderson, H. L.; Timmel, C. R. J. Am. Chem. Soc. 2015, 137, 8284.

(91) Harriman, A.; Heitz, V.; Sauvage, J. P. J. Phys. Chem. 1993, 97, 5940.

(92) Brookfield, R. L.; Ellul, H.; Harriman, A. J. Chem. Soc., Faraday Trans. 2 1985, 81, 1837.

(93) Borovkov, V. V.; Lintuluoto, J. M.; Inoue, Y. Helv. Chim. Acta 1999, 82, 919. 
(94) Asano-Someda, M.; van der Est, A.; Krüger, U.; Stehlik, D.; Kaizu, Y.; Levanon, H. J. Phys. Chem. A 1999, 103, 6704.

(95) Vidal-Ferran, A.; Bampos, N.; Sanders, J. K. M. Inorg. Chem. 1997, 36, 6117.

(96) Webb, S. J.; Sanders, J. K. M. Inorg. Chem. 2000, 39, 5912.

(97) Rucareanu, S.; Mongin, O.; Schuwey, A.; Hoyler, N.; Gossauer, A.; Amrein, W.; Hediger, H.-U. J. Org. Chem. 2001, 66, 4973.

(98) Kim, D.; Holten, D.; Gouterman, M. J. Am. Chem. Soc. 1984, 106, 2793.

(99) Asano, M.; Kaizu, Y.; Kobayashi, H. J. Chem. Phys. 1988, 89, 6567.

(100) Liu, F.; Cunningham, K. L.; Uphues, W.; Fink, G. W.; Schmolt, J.; McMillin, D. R. Inorg. Chem. 1995, 34, 2015.

(101) Szintay, G.; Horváth, A. Inorg. Chim. Acta. 2001, 324, 278.

(102) Asano-Someda, M.; van der Est, A.; Krüger, U.; Stehlik, D.; Kaizu, Y.; Levanon, H. J. Phys. Chem. A 1999, 103, 6704.

(103) Toyama, N.; Asano-Someda, M.; Ichino, T.; Kaizu, Y. J. Phys. Chem. A 2000, 104, 4857.

(104) Nguyen, T.; Hakansson, P.; Edge, R.; Collison, D.; Goodman, B. A.; Burns, J. R.; Stulz, E. New J. Chem. 2014, 38, 5254.

(105) Jeschke, G. ChemPhysChem 2002, 3, 927.

(106) Jeschke, G.; Polyhach, Y. PCCP 2007, 9, 1895.

(107) Becker, J. S.; Saxena, S. Chem. Phys. Lett. 2005, 414, 248.

(108) Yang, Z.; Kise, D.; Saxena, S. J. Phys. Chem. B 2010, 114, 6165.

(109) Richert, S.; Cremers, J.; Anderson, H. L.; Timmel, C. R. Chem. Sci. 2016, 7, 6952.

(110) Miller, J. R.; Dorough, G. D. J. Am. Chem. Soc. 1952, 74, 3977.

(111) Abraham, R. J.; Leighton, P.; Sanders, J. K. M. J. Am. Chem. Soc. 1985, 107, 3472.

(112) Tabushi, I.; Kugimiya, S.; Kinnaird, M. G.; Sasaki, T. J. Am. Chem. Soc. 1985, 107, 4192.

(113) Beletskaya, I.; Tyurin, V. S.; Tsivadze, A. Y.; Guilard, R.; Stern, C. Chem. Rev. 2009, 109, 1659.

(114) Hambright, P. Chem. Commun. 1967, 470.

(115) Cockroft, S. L.; Hunter, C. A. Chem. Soc. Rev. 2007, 36, 172.

(116) Camara-Campos, A.; Musumeci, D.; Hunter, C. A.; Turega, S. J. Am. Chem. Soc. 2009, $131,18518$.

(117) Ahlrichs, R.; Bär, M.; Häser, M.; Horn, H.; Kölmel, C. Chem. Phys. Lett. 1989, 162, 165.

(118) Schäfer, A.; Huber, C.; Ahlrichs, R. J. Chem. Phys. 1994, 100, 5829.

(119) Eichkorn, K.; Weigend, F.; Treutler, O.; Ahlrichs, R. Theor. Chem. Acc. 1997, 97, 119.

(120) Grimme, S. J. Comput. Chem. 2004, 25, 1463.

(121) Grimme, S. J. Comput. Chem. 2006, 27, 1787.

(122) Lipstman, S.; Goldberg, I. Cryst. Growth Des. 2010, 10, 4596.

(123) Renner, M. W.; Barkigia, K. M.; Fajer, J. Inorg. Chim. Acta. 1997, 263, 181.

(124) Zimmer, B.; Hutin, M.; Bulach, V.; Hosseini, M. W.; De Cian, A.; Kyritsakas, N. New J. Chem. 2002, 26, 1532.

(125) Sprafke, J. K.; Kondratuk, D. V.; Wykes, M.; Thompson, A. L.; Hoffmann, M.; Drevinskas, R.; Chen, W.-H.; Yong, C. K.; Kärnbratt, J.; Bullock, J. E.; Malfois, M.; Wasielewski, M. R.; Albinsson, B.; Herz, L. M.; Zigmantas, D.; Beljonne, D.; Anderson, H. L. J. Am. Chem. Soc. 2011, 133, 17262.

(126) Wagner, R. W.; Johnson, T. E.; Li, F.; Lindsey, J. S. J. Org. Chem. 1995, 60, 5266.

(127) Sun, H.; Hunter, C. A.; Llamas, E. M. Chem. Sci. 2015, 6, 1444.

(128) Sun, H.; Hunter, C. A.; Navarro, C.; Turega, S. J. Am. Chem. Soc. 2013, 135, 13129.

(129) Williams, D. H.; Stephens, E.; O'Brien, D. P.; Zhou, M. Angew. Chem. Int. Ed. 2004, 43, 6596.

(130) Cole, S. J.; Curthoys, G. C.; Magnusson, E. A.; Phillips, J. N. Inorg. Chem. 1972, 11, 1024.

(131) Vogel, G. C.; Stahlbush, J. R. Inorg. Chem. 1977, 16, 950. 
(132) Walker, F. A.; Benson, M. J. Am. Chem. Soc. 1980, 102, 5530.

(133) Ercolani, G.; Schiaffino, L. Angew. Chem. Int. Ed. 2011, 50, 1762.

(134) N. Taylor, P.; Huuskonen, J.; T. Aplin, R.; L. Anderson, H.; Rumbles, G.; Williams, E. Chem. Commun. 1998, 909.

(135) Littler, B. J.; Miller, M. A.; Hung, C.-H.; Wagner, R. W.; O'Shea, D. F.; Boyle, P. D.; Lindsey, J. S. J. Org. Chem. 1999, 64, 1391.

(136) Cremers, J.; Richert, S.; Kondratuk, D. V.; Claridge, T. D. W.; Timmel, C. R.; Anderson, H. L. Chem. Sci. 2016, 7, 6961.

(137) Benson, S. W. J. Am. Chem. Soc. 1958, 80, 5151.

(138) Ercolani, G.; Piguet, C.; Borkovec, M.; Hamacek, J. J. Phys. Chem. B 2007, 111, 12195.

(139) Balabin, I. A.; Onuchic, J. N. Science 2000, 290, 114.

(140) Beratan, D. N.; Skourtis, S. S.; Balabin, I. A.; Balaeff, A.; Keinan, S.; Venkatramani, R.; Xiao, D. Acc. Chem. Res. 2009, 42, 1669.

(141) Lande, A. d. I.; Babcock, N. S.; Rezac, J.; Levy, B.; Sanders, B. C.; Salahub, D. R. PCCP 2012, 14, 5902.

(142) Hansen, T.; Solomon, G. C. J. Phys. Chem. C 2016, 120, 6295.

(143) Goldsmith, R. H.; Wasielewski, M. R.; Ratner, M. A. J. Phys. Chem. B 2006, 110, 20258.

(144) Kocherzhenko, A. A.; Grozema, F. C.; Siebbeles, L. D. A. PCCP 2011, 13, 2096.

(145) Vazquez, H.; Skouta, R.; Schneebeli, S.; Kamenetska, M.; Breslow, R.; Venkataraman, L.; Hybertsen, M. S. Nat. Tech. 2012, 7, 663.

(146) Xiang, D.; Wang, X.; Jia, C.; Lee, T.; Guo, X. Chem. Rev. 2016, 116, 4318.

(147) Marqués-González, S.; Low, P. J. Aust. J. Chem. 2016, 69, 244.

(148) Aradhya, S. V.; Venkataraman, L. Nat. Nanotech. 2013, 8, 399.

(149) Godziela, G. M.; Goff, H. M. J. Am. Chem. Soc. 1986, 108, 2237.

(150) Renner, M. W.; Barkigia, K. M.; Zhang, Y.; Medforth, C. J.; Smith, K. M.; Fajer, J. J. Am. Chem. Soc. 1994, 116, 8582.

(151) Schwarzhans, K. E. Angew. Chem. Int. Ed. 1970, 9, 946.

(152) Claridge, T. D. W. Elsevier Science: Oxford 2009, High-Resolution NMR Techniques in Organic Chemistry, 2nd ed.

(153) Koehler, J.; Meiler, J. Prog. Nucl. Magn. Reson. Spectrosc. 2011, 59, 360.

(154) Bertini, I.; Luchinat, C.; Parigi, G. Prog. Nucl. Magn. Reson. Spectrosc. 2002, 40, 249.

(155) Bertini, I.; Luchinat, C.; Parigi, G.; Pierattelli, R. ChemBioChem 2005, 6, 1536.

(156) Pardo, E.; Faus, J.; Julve, M.; Lloret, F.; Muñoz, M. C.; Cano, J.; Ottenwaelder, X.; Journaux, Y.; Carrasco, R.; Blay, G.; Fernández, I.; Ruiz-García, R. J. Am. Chem. Soc. 2003, 125, 10770.

(157) Castellano, M.; Ruiz-García, R.; Cano, J.; Ferrando-Soria, J.; Pardo, E.; Fortea-Pérez, F. R.; Stiriba, S.-E.; Julve, M.; Lloret, F. Acc. Chem. Res. 2015, 48, 510.

(158) Jeschke, G. Macromol. Rapid. Commun. 2002, 23, 227.

(159) Jeschke, G. Annu. Rev. Phys. Chem. 2012, 63, 419.

(160) Richert, S.; Cremers, J.; Kuprov, I.; Peeks, M. D.; Anderson, H. L.; Timmel, C. R. Nat. Commun. 2017, 8, 14842.

(161) Wautelet, P.; Bieber, A.; Turek, P.; Moigne, J. L.; André, J.-J. Mol. Cryst. Liq. Cryst. Sci. Technol., Sect. A 1997, 305, 55.

(162) Nishizawa, S.; Hasegawa, J.-y.; Matsuda, K. J. Phys. Chem. C 2013, 117, 26280.

(163) Herrmann, C.; Elmisz, J. Chem. Commun. 2013, 49, 10456.

(164) Grozema, F. C.; Houarner-Rassin, C.; Prins, P.; Siebbeles, L. D. A.; Anderson, H. L. J. Am. Chem. Soc. 2007, 129, 13370.

(165) Peeks, M. D.; Claridge, T. D. W.; Anderson, H. L. Nature 2017, 541, 200.

(166) Parkinson, P.; Knappke, C. E. I.; Kamonsutthipaijit, N.; Sirithip, K.; Matichak, J. D.; Anderson, H. L.; Herz, L. M. J. Am. Chem. Soc. 2014, 136, 8217. 
(167) Kroto, H. W.; Heath, J. R.; O'Brien, S. C.; Curl, R. F.; Smalley, R. E. Nature 1985, 318, 162.

(168) Scott, L. T.; Boorum, M. M.; McMahon, B. J.; Hagen, S.; Mack, J.; Blank, J.; Wegner, H.; de Meijere, A. Science 2002, 295, 1500.

(169) Kabdulov, M.; Jansen, M.; Amsharov, K. Y. Chem. Eur. J. 2013, 19, 17262.

(170) Greisch, J.-F.; Amsharov, K. Y.; Weippert, J.; Weis, P.; Böttcher, A.; Kappes, M. M. J. Am. Chem. Soc. 2016, 138, 11254.

(171) Matsui, K.; Segawa, Y.; Namikawa, T.; Kamada, K.; Itami, K. Chem. Sci. 2013, 4, 84.

(172) Kayahara, E.; Iwamoto, T.; Takaya, H.; Suzuki, T.; Fujitsuka, M.; Majima, T.; Yasuda, N.; Matsuyama, N.; Seki, S.; Yamago, S. Nat. Commun. 2013, 4, 2694.

(173) Matsui, K.; Segawa, Y.; Itami, K. J. Am. Chem. Soc. 2014, 136, 16452.

(174) Ikemoto, K.; Kobayashi, R.; Sato, S.; Isobe, H. Angew. Chem. Int. Ed. 2017, 56, 6511.

(175) Gutzler, R.; Perepichka, D. F. J. Am. Chem. Soc. 2013, 135, 16585.

(176) Peeks, M. D.; Tait, C. E.; Neuhaus, P.; Fischer, G. M.; Hoffmann, M.; Haver, R.; Cnossen, A.; Harmer, J. R.; Timmel, C. R.; Anderson, H. L. J. Am. Chem. Soc. 2017, 139, 10461.

(177) Ball, M.; Zhong, Y.; Fowler, B.; Zhang, B.; Li, P.; Etkin, G.; Paley, D. W.; Decatur, J.; Dalsania, A. K.; Li, H.; Xiao, S.; Ng, F.; Steigerwald, M. L.; Nuckolls, C. J. Am. Chem. Soc. 2016, 138, 12861.

(178) Zhang, G.; Mastalerz, M. Chem. Soc. Rev. 2014, 43, 1934.

(179) Hasell, T.; Cooper, A. I. Nat. Rev. Mater. 2016, 1, 16053.

(180) Olenyuk, B.; Levin, M. D.; Whiteford, J. A.; Shield, J. E.; Stang, P. J. J. Am. Chem. Soc. 1999, 121, 10434.

(181) Sun, Q.-F.; Iwasa, J.; Ogawa, D.; Ishido, Y.; Sato, S.; Ozeki, T.; Sei, Y.; Yamaguchi, K.; Fujita, M. Science 2010, 328, 1144.

(182) Zarra, S.; Wood, D. M.; Roberts, D. A.; Nitschke, J. R. Chem. Soc. Rev. 2015, 44, 419.

(183) Zhang, G.; Presly, O.; White, F.; Oppel, I. M.; Mastalerz, M. Angew. Chem. Int. Ed. 2014, 53, 5126.

(184) Rue, N. M.; Sun, J.; Warmuth, R. Isr. J. Chem. 2011, 51, 743.

(185) Zhang, C.; Wang, Q.; Long, H.; Zhang, W. J. Am. Chem. Soc. 2011, 133, 20995.

(186) Lee, S.; Yang, A.; Moneypenny, T. P.; Moore, J. S. J. Am. Chem. Soc. 2016, 138, 2182.

(187) Wasielewski, M. R. Acc. Chem. Res. 2009, 42, 1910.

(188) Aratani, N.; Kim, D.; Osuka, A. Acc. Chem. Res. 2009, 42, 1922.

(189) Parkinson, P.; Kondratuk, D. V.; Menelaou, C.; Gong, J. Q.; Anderson, H. L.; Herz, L. M. J. Phys. Chem. Lett. 2014, 5, 4356.

(190) Screen, T. E. O.; Lawton, K. B.; Wilson, G. S.; Dolney, N.; Ispasoiu, R.; Goodson III, T.; Martin, S. J.; Bradley, D. D. C.; Anderson, H. L. J. Mater. Chem. 2001, 11, 312.

(191) Scott Wilson, G.; L. Anderson, H. Chem. Commun. 1999, 1539.

(192) Liu, P.; Neuhaus, P.; Kondratuk, D. V.; Balaban, T. S.; Anderson, H. L. Angew. Chem. Int. Ed. 2014, 53, 7770.

(193) Robinson, P. T.; Pham, T. N.; Uhrín, D. J. Magn. Reson. 2004, 170, 97.

(194) Duncan, S. J.; Lewis, R.; Bernstein, M. A.; Sandor, P. Magn. Reson. Chem. 2007, 45, 283.

(195) Gong, J. Q.; Parkinson, P.; Kondratuk, D. V.; Gil-Ramírez, G.; Anderson, H. L.; Herz, L. M. J. Phys. Chem. C 2015, 119, 6414.

(196) Gong, J. Q.; Favereau, L.; Anderson, H. L.; Herz, L. M. J. Phys. Chem. Lett. 2016, 7, 332.

(197) Fedorov, A.; Berberan-Santos, M. N.; Lefèvre, J.-P.; Valeur, B. Chem. Phys. Lett. 1997, 267, 467.

(198) Kerisit, N.; Toupet, L.; Larini, P.; Perrin, L.; Guillemin, J.-C.; Trolez, Y. Chem. Eur. J. 2015, 21, 6042. 
(199) Scott Wilson, G.; Anderson, H. Chem. Commun. 1996, 1996, 1039.

(200) Frisch, M. J.; Trucks, G. W.; Schlegel, H. B.; Scuseria, G. E.; Robb, M. A.; Cheeseman, J. R.; Scalmani, G.; Barone, V.; Petersson, G. A.; Nakatsuji, H.; Li, X.; Caricato, M.; Marenich, A. V.; Bloino, J.; Janesko, B. G.; Gomperts, R.; Mennucci, B.; Hratchian, H. P.; Ortiz, J. V.; Izmaylov, A. F.; Sonnenberg, J. L.; Williams; Ding, F.; Lipparini, F.; Egidi, F.; Goings, J.; Peng, B.; Petrone, A.; Henderson, T.; Ranasinghe, D.; Zakrzewski, V. G.; Gao, J.; Rega, N.; Zheng, G.; Liang, W.; Hada, M.; Ehara, M.; Toyota, K.; Fukuda, R.; Hasegawa, J.; Ishida, M.; Nakajima, T.; Honda, Y.; Kitao, O.; Nakai, H.; Vreven, T.; Throssell, K.; Montgomery Jr., J. A.; Peralta, J. E.; Ogliaro, F.; Bearpark, M. J.; Heyd, J. J.; Brothers, E. N.; Kudin, K. N.; Staroverov, V. N.; Keith, T. A.; Kobayashi, R.; Normand, J.; Raghavachari, K.; Rendell, A. P.; Burant, J. C.; Iyengar, S. S.; Tomasi, J.; Cossi, M.; Millam, J. M.; Klene, M.; Adamo, C.; Cammi, R.; Ochterski, J. W.; Martin, R. L.; Morokuma, K.; Farkas, O.; Foresman, J. B.; Fox, D. J. Gaussian 09, Revision D.01, software, Wallingford CT 2013.

(201) Becke, A. D. J. Chem. Phys. 1993, 98, 5648.

(202) Ditchfield, R.; Hehre, W. J.; Pople, J. A. J. Chem. Phys. 1971, 54, 724.

(203) Hehre, W. J.; Ditchfield, R.; Pople, J. A. J. Chem. Phys. 1972, 56, 2257.

(204) Hariharan, P. C.; Pople, J. A. Theor. Chem. Acc. 1973, 28, 213.

(205) Rassolov, V. A.; Pople, J. A.; Ratner, M. A.; Windus, T. L. J. Chem. Phys. 1998, 109, 1223.

(206) Grimme, S.; Antony, J.; Ehrlich, S.; Krieg, H. J. Chem. Phys. 2010, 132, 154104.

(207) Valeur, P. B.-S., M. N. Molecular fluorescence: principles and applications $2012 \mathrm{John}$ Wiley \& Sons.

(208) Hill, A. V. Biochem. J. 1913, 7, 471.

(209) Weiss, J. N. FASEB J. 1997, 11, 835. 